Date Submitted: $\quad \underline{6 / 26 / 08}$

Originator: J.M. Capron

Phone: $\quad 372-9227$

\section{WASTE SITE RECLASSIFICATION FORM}

Operable Unit(s): 100-FR-1

Waste Site Code: $\quad 128-\mathrm{F}-2$

Type of Reclassification Action:

Closed Out $\square \quad$ Interim Closed Out $\otimes$ No Action $\square$

RCRA Postclosure $\square \quad$ Rejected $\square \quad$ Consolidated $\square$

Control Number: 2008-031
P72-9227

\section{.}

$=-1=1$

This form documents agreement among parties listed authorizing classification of the subject unit as Closed Out, Interim Closed Out, No Action, RCRA Postclosure, Rejected, or Consolidated. This form also authorizes backfill of the waste management unit, if appropriate, for Closed Out and Interim Closed Out units. Final removal from the NPL of No Action and Closed Out waste management units will occur at a future date.

Description of current waste site condition:

The 128-F-2 waste site consisted of multiple burn and debris filled pits located directly east of the 107-F Retention Basin and approximately $30.5 \mathrm{~m}(100 \mathrm{ft})$ east of the northeast corner of the 100-F Area perimeter road that runs along the riverbank. The burn pits were used for incinerating nonradioactive, combustible materials from 1945 to 1965. During remediation, brick, wood, fiberglass, wire, sheet metal, rebar, burned paper, plastic, concrete, cement blocks, pipe, burned tires, steel, electrical conduit, lead weights, nails, bolts, and other unspecified building materials were also found within the waste site. The waste site is located adjacent to the Columbia River and has been remediated to the elevation of the ordinary high water mark (OHWM). The waste site was divided into areas A, B, and C for verification sampling. Area A includes the river sideslope above the OHWM, area B includes the upland portion west of area $\mathrm{A}$, and area $\mathrm{C}$ includes a riparian area below the OHWM to the east of area $\mathrm{A}$. Only area $\mathrm{A}$ and area $\mathrm{B}$ are included in the reclassification. One portion of the waste site (area $\mathrm{B}$ ) has been backfilled with regulatory agency concurrence.

The area below the OHWM (area C) is known to contain metal and organic contaminants above the soil RAGs. This riparian area will be designated as a new waste site and undergo further remediation to remove material to the depth of the mean low water level. Remediation below the OHWM will be performed at a time of anticipated low river flow rates and will extend to the east of area $\mathrm{C}$ to the point where visible surface debris is no longer present. Remediation will extend to the southeast of area $\mathrm{C}$ to an elevation of $115.5 \mathrm{~m}(378.9 \mathrm{ft})$. Excavation in this new waste site will proceed to a depth at which water is encountered.

Following remediation, sampling to document the as left condition of the sediment surrounding the excavated area will be performed. Since the vadose zone will not be present, obtaining soil samples from the base of the excavation will not be possible. Samples will be obtained from locations at the perimeter of the excavation. After the remediation and sampling of the perimeter are completed, the new waste site will be interim closed. If the sampling results indicate areas of contamination remain, these areas will be addressed as a discovery waste site.

Remediation and verification sampling of the 128-F-2 waste site have been performed in accordance with remedial action objectives and goals established by the Interim Action Record of Decision for the 100-BC-1, 100-BC-2, 100 DR-1, 100-DR-2, 100-FR-1, 100-FR-2, 100-HR-1, 100-HR-2, 100-KR-1, 100-KR-2, 100-IU-2, 100-IU-6, and 200-CW-3 Operable Units, Hanford Site, Benton County, Washington (Remaining Sites ROD), U.S. Environmental Protection Agency, Region 10, Seattle, Washington. The selected action involved: (1) evaluating the site using available process information, (2) remediating the site, (3) demonstrating through verification sampling that cleanup goals have been achieved, and (4) proposing the site for reclassification to Interim Closed Out.

Basis for reclassification:

In accordance with this evaluation, the verification sampling results support a reclassification of the 128 -F-2 site to Interim Closed Out. The current site conditions achieve the remedial action objectives and the corresponding remedial action goals established in the Remaining Sites ROD. The results of verification sampling show that residual contaminant concentrations do not preclude any future uses (as bounded by the rural-residential scenario) and allow for unrestricted use of shallow-zone soils

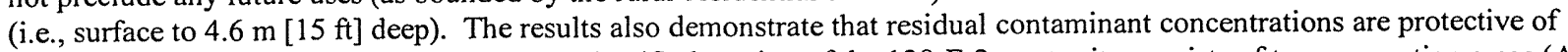
groundwater and the Columbia River. The reclassified portion of the 128-F-2 waste site consists of two excavation areas (Areas $\mathrm{A}$ and $\mathrm{B}$ ). Area A consists of the river sideslope extending to the OHWM while area B consists of the upland portion of the site. Area $\mathrm{C}$ includes the area that was excavated to an elevation below the OHWM. However contamination above soil RAGs remains in this area. Therefore, area $\mathrm{C}$ is not included in this reclassification of the 128-F-2 waste site.

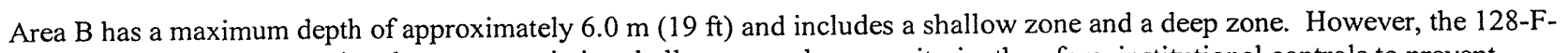
2 waste site is closed out using the more restrictive shallow zone cleanup criteria; therefore, institutional controls to prevent uncontrolled drilling or excavation into the deep zone are not required. The basis for reclassification is described in detail in the Remaining Sites Verification Package for the 128-F-2, 100-F-Burning Pit (attached). 
Waste Site Controls:

Engineered Controls: Yes $\square$ No $\square \quad$ Institutional Controls: Yes $\square$ No $\square \quad$ O\&M requirements: Yes $\square$ No $\square$

If any of the Waste Site Controls are checked Yes specify control requirements including reference to the Record of Decision,

TSD Closure Letter, or other relevant documents.

M. S. French

DOE Federal Project Director (printed)

N/A

Ecology Project Manager (printed)

R. A. Lobos

EPA Project Manager (printed)
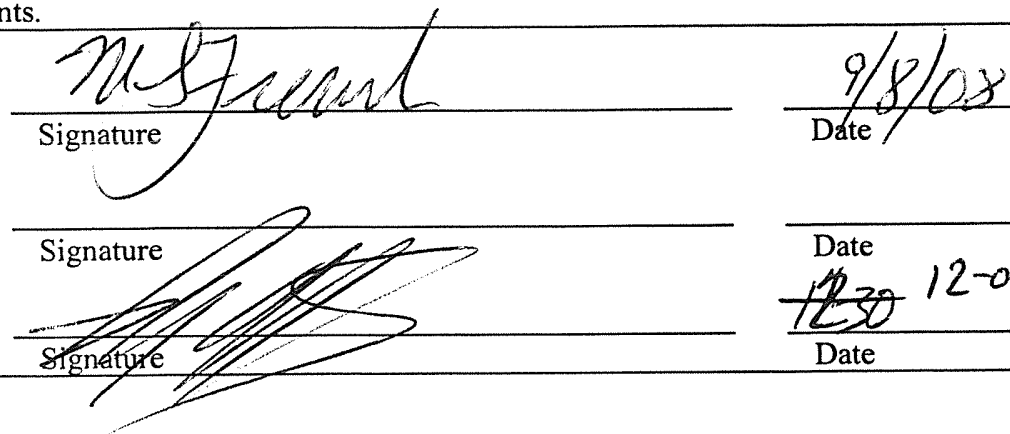

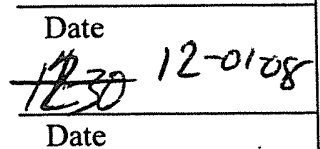




\title{
REMAINING SITES VERIFICATION PACKAGE FOR THE 128-F-2, 100-F BURNING PIT WASTE SITE
}

Attachment to Waste Site Reclassification Form 2008-031

\author{
June 2008
}


Rev. 0 


\section{REMAINING SITES VERIFICATION PACKAGE FOR THE 128-F-2, 100-F BURNING PIT WASTE SITE}

\section{EXECUTIVE SUMMARY}

The 128-F-2 waste site is located adjacent to the Columbia River within the 100-FR-1 Operable Unit on the Hanford Site and includes the former 100-F Burning Pit. The waste site was an irregularly shaped depression that was used for incinerating nonradioactive, combustible materials (e.g., vegetation, office waste, paint waste, and chemical solvents) from 1945 to 1965. During Hanford Site cleanup operations in the late 1970s, unknown quantities of debris were removed from the burn pit and the site was backfilled (UNI 1980). The site is approximately $30.5 \mathrm{~m}$ (100 ft) east of the northeast corner of the 100-F Area perimeter road that runs along the riverbank and is directly east of the 107-F Retention Basin.

Confirmatory sampling of the 128-F-2 waste site began on December 3, 2004. A focused sampling approach was used and one test trench was excavated in each of four stratified sample areas. Based on the results of the confirmatory sampling, the entire waste site was determined to require remediation. The results of confirmatory sampling were used to determine the contaminants of concern (COCs) and contaminants of potential concern (COPCs) for verification sampling. Metals (including hexavalent chromium and mercury), semivolatile organic compounds (inclusive of the polycyclic aromatic hydrocarbons detected in confirmatory samples), chlorinated pesticides, total petroleum hydrocarbons, polychlorinated biphenyls, and asbestos were considered COCs/COPCs for verification sampling. Additionally, because radiological activity was detected above background levels during confirmatory sampling by field instrumentation, laboratory radiological analysis was performed on verification samples to verify the absence of radiological contamination.

Remediation of the waste site was initially performed from August through October 2005 with a total of 21,900 metric tons (24,100 US tons) of material removed and disposed. Other debris unearthed in the waste site included brick, wood, fiberglass, wire, sheet metal, rebar, burned paper, plastic, concrete, cement blocks, pipe, burned tires, steel, electrical conduit, lead weights, nails, bolts, and other unspecified building materials. Additional debris and ash related to the burn pit waste site were still visible on the Columbia River shoreline in areas outside the waste site boundary. In October 2006, further remediation was conducted that removed material on the riverbank slope but left a soil berm to protect against encroachment of the river. However, in-process sample results from October and November 2006 showed that the residual metal concentrations in the excavated area and soil berm exceeded soil remedial action goals (RAGs). After meeting with the regulatory agency, it was agreed that additional excavation would be conducted on the shoreline below the ordinary high water mark (OHWM). This excavation was completed in February 2007 and was followed by immediate collection verification samples and gravel stabilization at the site prior to the onset of higher spring river levels.

The 128-F-2 waste site was divided into five decision units (areas A, B, C, D, and E) for verification sampling. The verification sampling for the specific area of the 128-F-2 waste site 
below the OHWM (designated area C) was performed in February 2007, to determine if the soil RAGs had been met. Analytical results showed the concentrations of multiple metals in area $\mathrm{C}$ were above the soil RAGs. Therefore, area $\mathrm{C}$ is not considered for interim closeout via this document. An agreement with the lead regulatory agency allowed area $\mathrm{C}$ to be designated as a new waste site. Area $\mathrm{C}$ was combined with adjoining riparian areas to the east and southeast that are known to contain concentrations of metals exceeding soil RAGs. This site is accepted in the Waste Information Data System (WIDS) as 100-F-59, Riparian Area Contamination Originating from 128-F-2.

The remaining portions of the 128-F-2 waste site, areas A, B, D, and E, are discussed in this document for interim closure. Area $\mathrm{A}$ is located adjacent to, and upslope of, area $\mathrm{C}$ and includes riverbank areas above the OHWM. Area B is located west of area A and is separate from the steep grade of the riverbank area. Area D is the waste staging pile area. Area E is a small area within area $\mathrm{D}$ in which asbestos-containing material was stored.

Verification sampling in the 128-F-2 waste site occurred on various dates in 2007 and 2008. In two cases, a second round of verification sampling was required because elevated levels of contaminants necessitated additional remediation. In the first case, a second sample design was created for Area B after initial verification sampling showed the soils contained elevated hexavalent chromium concentrations. The area underwent additional excavation and was then sampled for this constituent only using the second design. In the second case, results from a single sample location in area A showed detectable levels of some organics. Although none of the results exceed direct exposure RAGs, this area underwent additional remediation due to its proximity to the Columbia River. This single location was then re-sampled for all COCs/COPCs to verify site cleanup.

A summary of the cleanup evaluation for the soil results against the applicable criteria is presented in Table ES-1. The results of the verification sampling are used to make reclassification decisions for the 128-F-2 waste site in accordance with the TPA-MP-14 (DOE-RL 2007) procedure.

Table ES-1. Summary of Remedial Action Goals for the 128-F-2 Waste Site.

\begin{tabular}{|c|c|c|c|}
\hline $\begin{array}{l}\text { Regulatory } \\
\text { Requirement }\end{array}$ & Remedial Action Goals & Results & $\begin{array}{l}\text { Remedial Action } \\
\text { Objectives } \\
\text { Attained? }\end{array}$ \\
\hline $\begin{array}{l}\text { Direct Exposure - } \\
\text { Radionuclides }\end{array}$ & $\begin{array}{l}\text { Attain } 15-\mathrm{mrem} / \mathrm{yr} \text { dose rate above } \\
\text { background over } 1,000 \text { years. }\end{array}$ & $\begin{array}{l}\text { A single detection of } 0.218 \mathrm{pCi} / \mathrm{g} \\
\text { cesium-137 was reported for area A. } \\
\text { This amount is below the levels for } \\
\text { direct exposure. }\end{array}$ & Yes \\
\hline $\begin{array}{l}\text { Direct Exposure - } \\
\text { Nonradionuclides }\end{array}$ & Attain individual COPC RAGs. & $\begin{array}{l}\text { All individual COPC concentrations } \\
\text { are below the direct exposure criteria. }\end{array}$ & Yes \\
\hline \multirow[t]{2}{*}{$\begin{array}{l}\text { Risk Requirements - } \\
\text { Nonradionuclides }\end{array}$} & $\begin{array}{l}\text { Attain a hazard quotient of }<1 \text { for } \\
\text { all individual noncarcinogens. }\end{array}$ & $\begin{array}{l}\text { All hazard quotients in each of areas } \\
\text { A, B, and D are less than } 1 .\end{array}$ & \multirow[t]{2}{*}{ Yes } \\
\hline & $\begin{array}{l}\text { Attain a cumulative hazard } \\
\text { quotient of }<1 \text { for noncarcinogens. }\end{array}$ & $\begin{array}{l}\text { The cumulative hazard quotients of } \\
\text { areas } \mathrm{A}\left(1.2 \times 10^{-2}\right) \text {, B }\left(8.1 \times 10^{-2}\right) \text {, and } \\
\mathrm{D}\left(5.3 \times 10^{-2}\right) \text { are each less than } 1 .\end{array}$ & \\
\hline
\end{tabular}


Table ES-1. Summary of Remedial Action Goals for the 128-F-2 Waste Site.

\begin{tabular}{|c|c|c|c|}
\hline $\begin{array}{l}\text { Regulatory } \\
\text { Requirement }\end{array}$ & Remedial Action Goals & Results & $\begin{array}{c}\text { Remedial Action } \\
\text { Objectives } \\
\text { Attained? }\end{array}$ \\
\hline & $\begin{array}{l}\text { Attain an excess cancer risk of } \\
<1 \times 10^{-6} \text { for individual } \\
\text { carcinogens. }\end{array}$ & $\begin{array}{l}\text { The excess cancer risk for carcinogens } \\
\text { in each of areas A, B, and D is less } \\
\text { than } 1 \times 10^{-6} \text {. }\end{array}$ & \\
\hline & $\begin{array}{l}\text { Attain a cumulative excess cancer } \\
\text { risk of }<1 \times 10^{-5} \text { for carcinogens. }\end{array}$ & $\begin{array}{l}\text { The total excess cancer risks of areas } \\
\text { A }\left(6.7 \times 10^{-7}\right) \text {, B }\left(4.3 \times 10^{-6}\right) \text {, and D } \\
\left(3.6 \times 10^{-6}\right) \text { are each less than } 1 \times 10^{-5} \text {. }\end{array}$ & \\
\hline \multirow{4}{*}{$\begin{array}{l}\text { Groundwater/River } \\
\text { Protection - } \\
\text { Radionuclides }\end{array}$} & $\begin{array}{l}\text { Attain single-COPC groundwater } \\
\text { and river protection RAGs. }\end{array}$ & \multirow{4}{*}{$\begin{array}{l}\text { A single detection of } 0.218 \mathrm{pCi} / \mathrm{g} \\
\text { cesium-137 was reported for area A. } \\
\text { This amount is below the levels for } \\
\text { groundwater and river protection. }{ }^{\text {c }}\end{array}$} & \multirow{4}{*}{ Yes } \\
\hline & $\begin{array}{l}\text { Attain national primary drinking } \\
\text { water standards: } 4 \text { mrem/yr } \\
\text { (beta/gamma) dose rate to target } \\
\text { receptor/organs. }\end{array}$ & & \\
\hline & $\begin{array}{l}\text { Meet drinking water standards for } \\
\text { alpha emitters: the most stringent } \\
\text { of } 15 \text { pCi/L MCL or } 1 / 25 \text { th of the } \\
\text { derived concentration guides from } \\
\text { DOE Order } 5400.5 \text {. }\end{array}$ & & \\
\hline & $\begin{array}{l}\text { Meet total uranium standard of } \\
30 \mu \mathrm{g} / \mathrm{L}(21.2 \mathrm{pCi} / \mathrm{L}) \text {. }\end{array}$ & & \\
\hline $\begin{array}{l}\text { Groundwater/River } \\
\text { Protection - } \\
\text { Nonradionuclides }\end{array}$ & $\begin{array}{l}\text { Attain individual nonradionuclide } \\
\text { groundwater and river cleanup } \\
\text { requirements. }\end{array}$ & $\begin{array}{l}\text { Maximum detected results for } \\
\text { nonradionuclides are below } \\
\text { groundwater and river protection } \\
\text { RAGs. }\end{array}$ & Yes \\
\hline
\end{tabular}

a “National Primary Drinking Water Regulations” (40 Code of Federal Regulations 141).

b Radiation Protection of the Public and the Environment (DOE Order 5400.5).

c Revised lookup value per 100 Area Radionuclide and Nonradionuclide Lookup Values for the 1995 Interim Remedial Action Record of Decision (BHI 2004a).

COPC = contaminant of potential concern

MCL = maximum contaminant level (drinking water standard)

RAG = remedial action goal

In accordance with this evaluation, the verification sampling results support a reclassification of this site to interim closed out. The current site conditions achieve the remedial action objectives and the corresponding RAGs established in the Remedial Design Report/Remedial Action Work Plan for the 100 Area (DOE-RL 2005) and the Interim Action Record of Decision for the 100-BC-1, 100-BC-2, 100-DR-1, 100-DR-2, 100-FR-1, 100-FR-2, 100-HR-1, 100-HR-2, 100-KR-1, 100-KR-2, 100-IU-2, 100-IU-6, and 200-CW-3 Operable Units, Hanford Site, Benton County, Washington (Remaining Sites ROD) (EPA 1999). These results show that residual soil concentrations support future land uses that can be represented (or bounded) by a rural-residential scenario. The results also demonstrate that residual contaminant concentrations support unrestricted future use of shallow zone soil (i.e., surface to $4.6 \mathrm{~m}[15 \mathrm{ft}]$ ) and contaminant levels remaining in the soil are protective of groundwater and the Columbia River. This site does not have a deep zone; therefore, no deep zone institutional controls are required. 
A comparison against ecological risk screening levels has been made for the site contaminants of concern and other constituents. Using a conservative approach, the highest values from each of the three applicable areas within the waste site, areas A, B, and D, were summed together. Using the combined values, screening levels were exceeded for antimony, arsenic, barium, boron, chromium, cobalt, copper, lead, manganese, mercury, nickel, selenium, vanadium, zinc, high molecular weight polycyclic aromatic hydrocarbons (HMW-PAHs), and DDT combined with its degradation products. Exceedance of screening values does not necessarily indicate the existence of risk to ecological receptors. It is believed that the presence of arsenic, antimony, manganese, mercury, and vanadium does not pose a risk to ecological receptors because their concentrations below site background levels. Boron concentrations are consistent with those seen elsewhere at the Hanford Site (no established background value is available for boron). If the highest value from each of the three areas was evaluated independently, then only lead, selenium, zinc, HMW-PAHs, and DDT plus its degradation products would exceed the screening levels. These constituents will be evaluated in the context of additional lines of evidence for ecological effects as part of the final closeout decision for this site. 


\section{REMAINING SITES VERIFICATION PACKAGE FOR THE 128-F-2, 100-F BURNING PIT WASTE SITE}

\section{STATEMENT OF PROTECTIVENESS}

This report demonstrates that the 128-F-2 waste site, the 100-F Burning Pit, meets the objectives for interim closure as established in the Remedial Design Report/Remedial Action Work Plan for the 100 Area (RDR/RAWP) (DOE-RL 2005) and the Interim Action Record of Decision for the 100-BC-1, 100-BC-2, 100-DR-1, 100-DR-2, 100-FR-1, 100-FR-2, 100-HR-1, 100-HR-2, 100-KR-1, 100-KR-2, 100-IU-2, 100-IU-6, and 200-CW-3 Operable Units, Hanford Site, Benton County, Washington (Remaining Sites ROD) (EPA 1999). These results show that residual soil concentrations support future land uses that can be represented (or bounded) by a rural-residential scenario. The results also demonstrate that residual contaminant concentrations support unrestricted future use of shallow zone soil (i.e., surface to $4.6 \mathrm{~m}$ [15 ft]) and contaminant levels remaining in the soil are protective of groundwater and the Columbia River. This site does not have a deep zone; therefore, no deep zone institutional controls are required.

A comparison against ecological risk screening levels has been made for the site contaminants of concern and other constituents. Using a conservative approach, the highest values from each of the three applicable areas within the waste site, areas A, B, and D, were summed together. Using the combined values, screening levels were exceeded for antimony, arsenic, barium, boron, chromium, cobalt, copper, lead, manganese, mercury, nickel, selenium, vanadium, zinc, high molecular weight polycyclic aromatic hydrocarbons (HMW-PAHs), and DDT combined with its degradation products. Exceedance of screening values does not necessarily indicate the existence of risk to ecological receptors. It is believed that the presence of arsenic, antimony, manganese, mercury, and vanadium does not pose a risk to ecological receptors because their concentrations below site background levels. Boron concentrations are consistent with those seen elsewhere at the Hanford Site (no established background value is available for boron). If the highest value from each of the three areas was evaluated independently, then only lead, selenium, zinc, HMW-PAHs, and DDT plus its degradation products would exceed the screening levels. These constituents will be evaluated in the context of additional lines of evidence for ecological effects as part of the final closeout decision for this site.

\section{GENERAL SITE INFORMATION AND BACKGROUND}

The site is located in the 100-FR-1 Operable Unit of the Hanford Site and is approximately $30.5 \mathrm{~m}(100 \mathrm{ft})$ east of the northeast corner of the 100-F Area perimeter road that runs along the riverbank. The site is located directly east of the 107-F Retention Basin (Figure 1).

According to the Waste Information Data System (WIDS), the 128-F-2 Burning Pit was an irregularly shaped depression that was used for incinerating nonradioactive, combustible materials (e.g., vegetation, office waste, paint waste, and chemical solvents). The burning pit was operational from 1945 to 1965. 
Figure 1. 128-F-2 Waste Site Location Map.

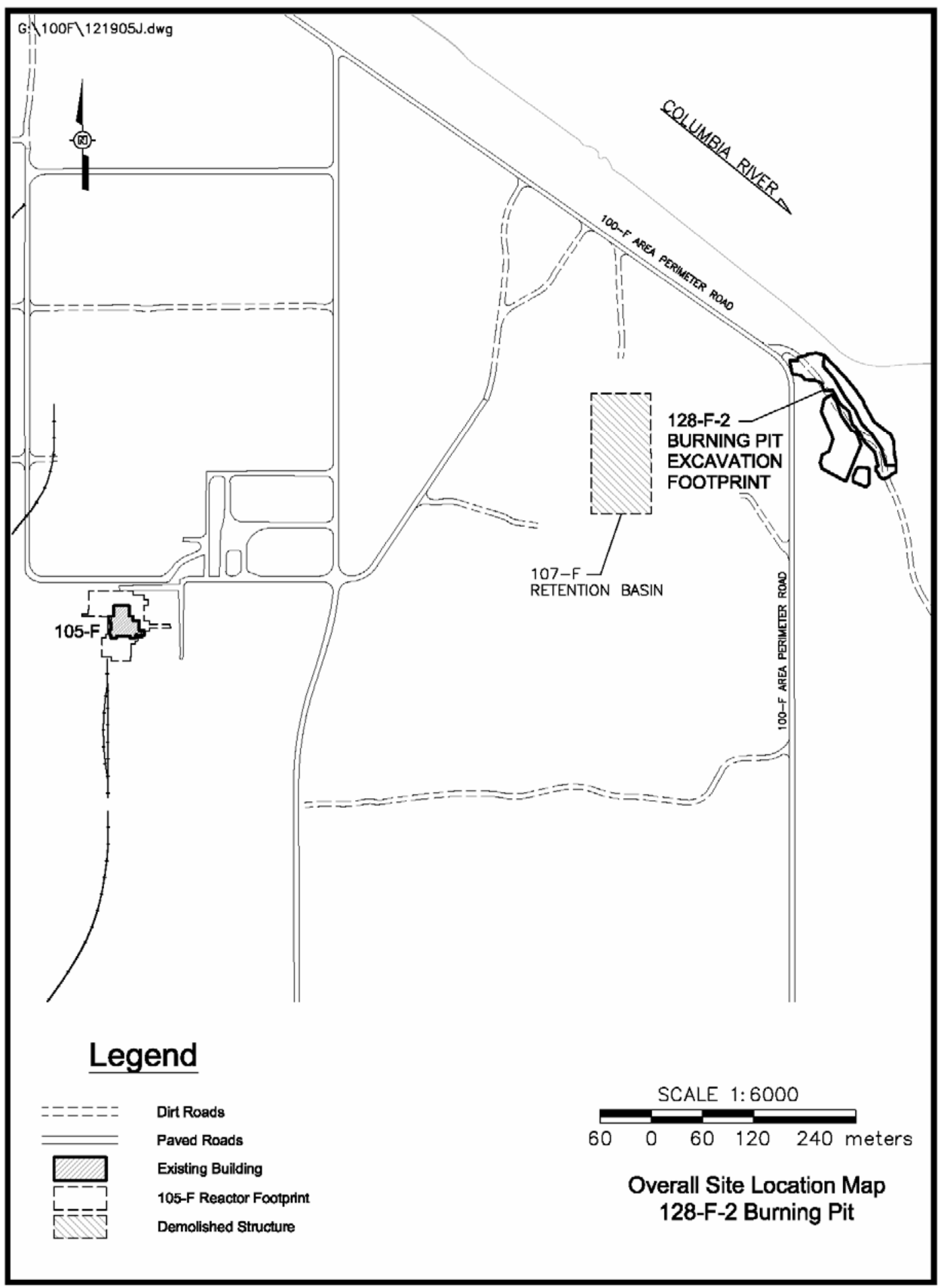


During Hanford Site cleanup operations in the late 1970s, unknown quantities of debris were removed from the burn pit and the site was backfilled (UNI 1980). Historical documentation (WHC 1994) suggested that multiple burn pits were present at the site, with each pit having the dimensions of 31 by 31 by $3 \mathrm{~m}$ ( 100 by 100 by $10 \mathrm{ft}$ ) deep. According to WIDS, the waste site was $46 \mathrm{~m}(151 \mathrm{ft})$ by $18 \mathrm{~m}(60 \mathrm{ft})$ and was situated on a steep grade near the shoreline of the Columbia River (Figure 2).

\section{PRE-REMEDIATION ACTIVITIES}

\section{Nonintrusive Investigation Results}

Geophysical investigations identified several anomalous zones adjacent to the WIDS site boundary (BHI 2004d). At the time, it was unknown if these buried anomalies were part of the 128-F-2 waste site or if the anomalies represented a new, previously undocumented waste site. Based on historical photographs (Figure 3 [DOE 1962]) and the geophysical investigation, it was believed that the waste site boundaries extend beyond those areas sampled during the confirmatory sampling effort. Because the waste site was located in close proximity to the Columbia River and the shoreline of the river is known to contain many culturally sensitive areas, confirmatory sampling activities were restricted to the excavation boundaries identified in the archaeological subsurface testing report for the 128-F-2 waste site (BHI 2004b).

\section{Confirmatory Sample Design}

A stratified sampling design with a focused sampling approach, biased towards probable worstcase contaminant locations, was selected for the confirmatory sampling effort. Historical photographs, geophysical surveys, and site visit information were used to focus sampling locations to those areas that were likely to contain the highest quantities of buried debris. The locations selected for confirmatory sampling extended beyond the boundary originally designated in WIDS.

Table 1 provides a summary of confirmatory sampling activities at the 128-F-2 waste site and Figure 4 provides the confirmatory sampling locations.

\section{Confirmatory Sample Results}

Confirmatory samples were analyzed using analytical methods approved by the U.S. Environmental Protection Agency (EPA). The results are stored in the Environmental Restoration (ENRE) project-specific database prior to archiving in the Hanford Environmental Information System (HEIS) and are included in Appendix A (Table A-1). 
Figure 2. Original WIDS Boundary and Topography of the 128-F-2 Waste Site.

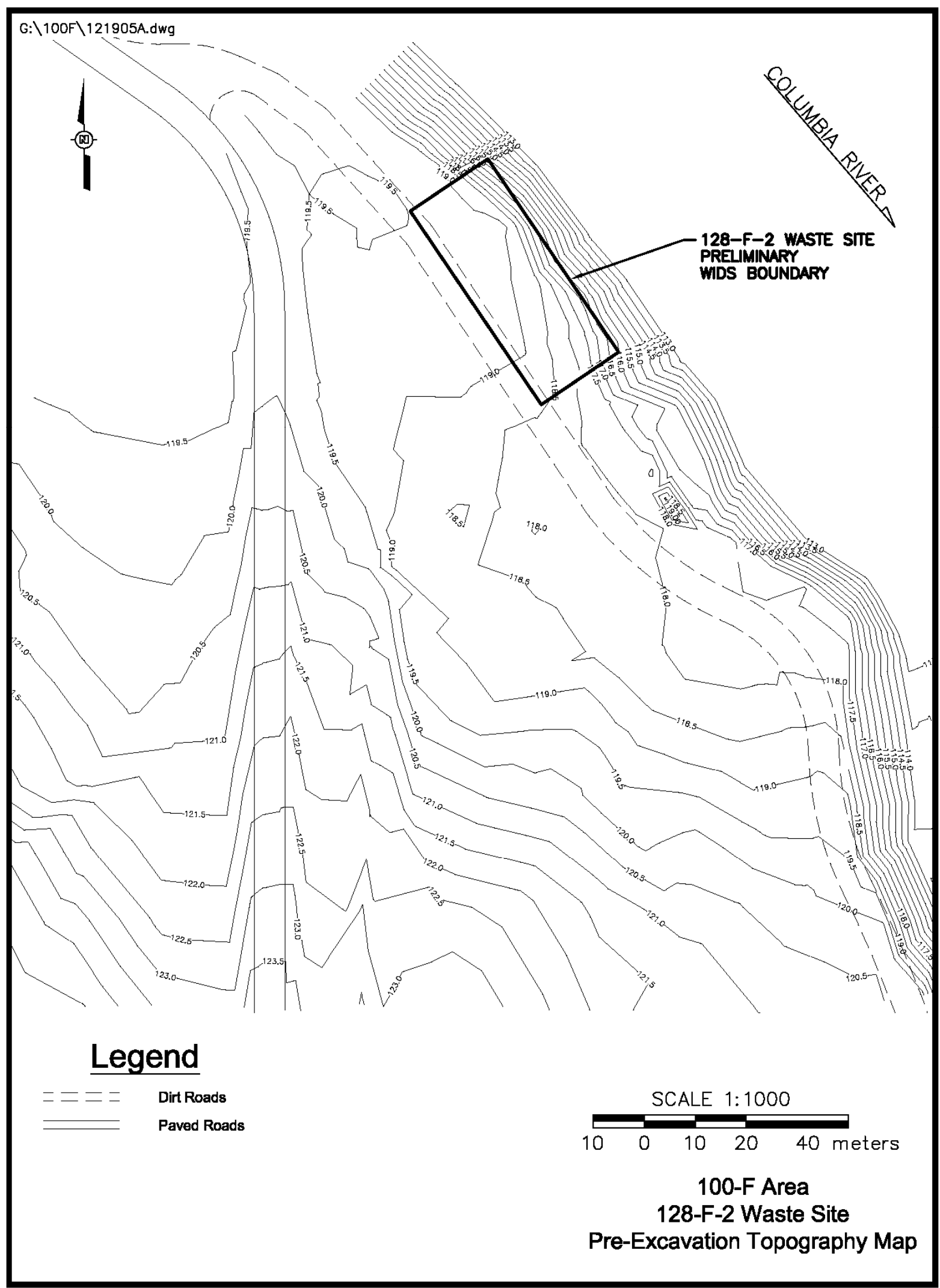




\section{Figure 3. Historical Photograph of the 128-F-2 Burn Pit.}

\section{8-F2-H1 Burn Pit 6451-5}

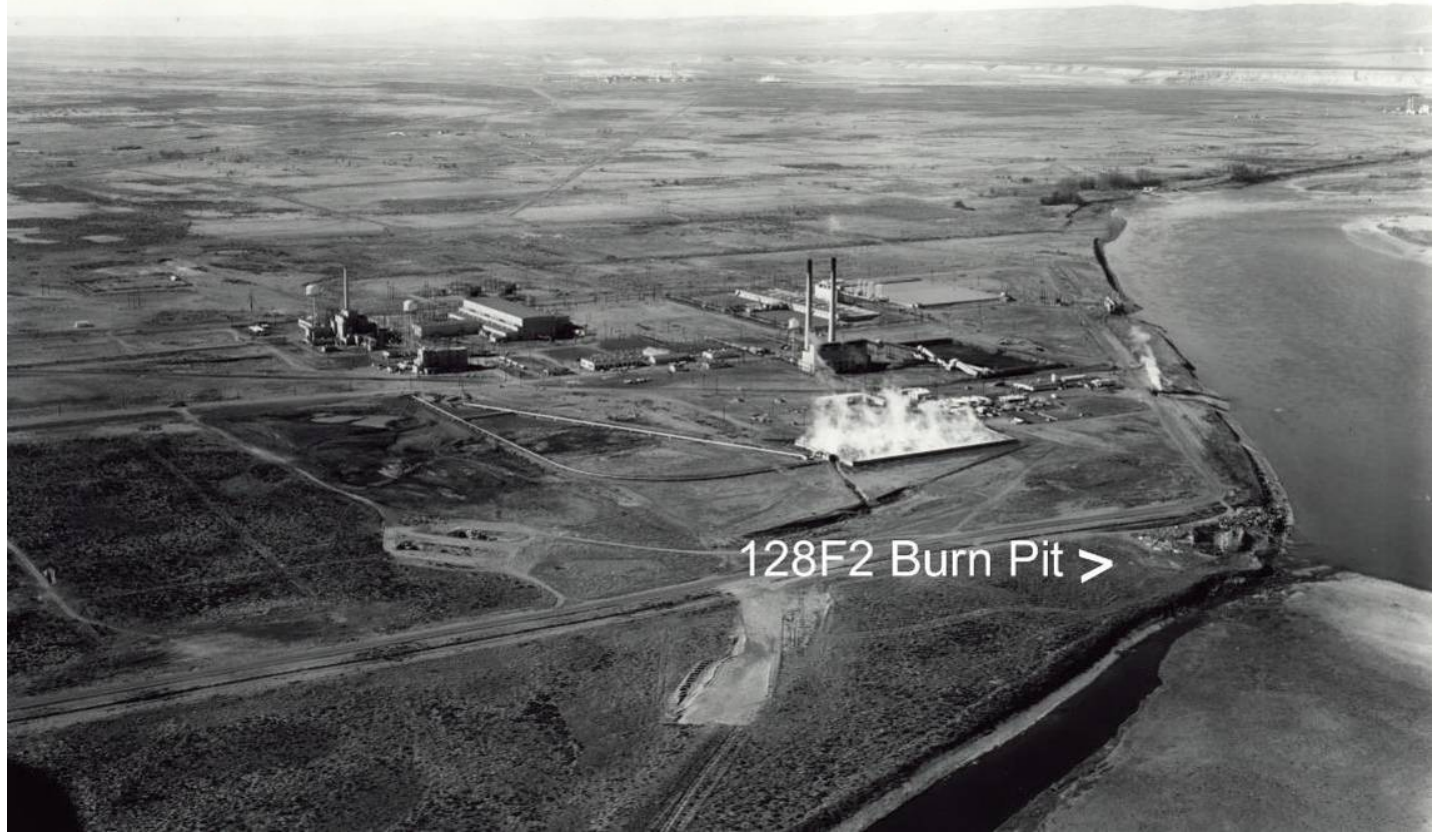

Table 1. Confirmatory Sample Summary for the 128-F-2 Site. (2 Pages)

\begin{tabular}{|c|c|c|c|c|c|}
\hline $\begin{array}{l}\text { Sample } \\
\text { Location }\end{array}$ & $\begin{array}{l}\text { Sample } \\
\text { Media }\end{array}$ & $\begin{array}{c}\text { HEIS Sample } \\
\text { Number }\end{array}$ & $\begin{array}{l}\text { Coordinate } \\
\text { Locations }\end{array}$ & $\begin{array}{l}\text { Depth } \\
\text { (m bgs) }\end{array}$ & Sample Analyses \\
\hline \multirow{4}{*}{ Area 1} & $\begin{array}{l}\text { Tan fibrous } \\
\text { PACM }\end{array}$ & J022J4 & $\begin{array}{l}\text { N } 147896 \\
\text { E } 581260 \\
\end{array}$ & 1.5 & Asbestos \\
\hline & \begin{tabular}{|l} 
Golden and \\
purple soil
\end{tabular} & J024R1 & $\begin{array}{l}\text { N } 147896 \\
\text { E } 581260\end{array}$ & 1.5 & $\begin{array}{l}\text { Pesticides, PCB, SVOA, PAH, TPH, } \\
\text { ICP metals, mercury, hexavalent chromium }\end{array}$ \\
\hline & \begin{tabular}{|l|} 
Light whitish- \\
tan soil
\end{tabular} & J024R2 & $\begin{array}{l}\text { N } 147896 \\
\text { E } 581257\end{array}$ & 1 & $\begin{array}{l}\text { Pesticides, PCB, SVOA, PAH, TPH, } \\
\text { ICP metals, mercury, hexavalent chromium }\end{array}$ \\
\hline & $\begin{array}{l}\text { Bottom of } \\
\text { trench soil }\end{array}$ & J024R3 & $\begin{array}{l}\text { N } 147896 \\
\text { E } 581255\end{array}$ & 2 to 2.5 & $\begin{array}{l}\text { Pesticides, PCB, SVOA, PAH, TPH, } \\
\text { ICP metals, mercury, hexavalent chromium }\end{array}$ \\
\hline \multirow{4}{*}{ Area 2} & $\begin{array}{l}\text { White chalky } \\
\text { PACM }\end{array}$ & J022J6 & $\begin{array}{l}\text { N } 147834 \\
\text { E } 581272\end{array}$ & 1.5 & Asbestos \\
\hline & $\begin{array}{l}\text { Reddish-tan } \\
\text { PACM }\end{array}$ & J022J5 & $\begin{array}{l}\text { N } 147834 \\
\text { E } 581272\end{array}$ & 1.5 & Asbestos \\
\hline & Ash & J024R7 & $\begin{array}{l}\text { N } 147836 \\
\text { E } 581271\end{array}$ & 1.2 to 1.8 & $\begin{array}{l}\text { Pesticides, PCB, SVOA, PAH, TPH, } \\
\text { ICP metals, mercury }\end{array}$ \\
\hline & Ash & J024R8 & $\begin{array}{l}\text { N } 147836 \\
\text { E } 581271\end{array}$ & 2.7 & $\begin{array}{l}\text { Pesticides, PCB, SVOA, PAH, TPH, } \\
\text { ICP metals, mercury }\end{array}$ \\
\hline
\end{tabular}


Table 1. Confirmatory Sample Summary for the 128-F-2 Site. (2 Pages)

\begin{tabular}{|c|c|c|c|c|c|}
\hline $\begin{array}{c}\text { Sample } \\
\text { Location }\end{array}$ & $\begin{array}{l}\text { Sample } \\
\text { Media }\end{array}$ & $\begin{array}{c}\text { HEIS Sample } \\
\text { Number }\end{array}$ & $\begin{array}{l}\text { Coordinate } \\
\text { Locations }\end{array}$ & $\begin{array}{c}\text { Depth } \\
\text { (m bgs) }\end{array}$ & Sample Analyses \\
\hline & \begin{tabular}{|l|} 
Cream- \\
colored paint
\end{tabular} & J022M8 & \multirow{2}{*}{$\begin{array}{l}\text { N } 147842 \\
\text { E } 581279\end{array}$} & \multirow{2}{*}{\begin{tabular}{|l|} 
Depth and location \\
unknown, paint \\
bucket found in \\
spoil pile 0.3 to 2.1 \\
\end{tabular}} & Hexavalent chromium \\
\hline & $\begin{array}{l}\text { Cream- } \\
\text { colored paint }\end{array}$ & J02581 & & & $\begin{array}{l}\text { Pesticides, PCB, SVOA, PAH, TPH, } \\
\text { ICP metals, mercury }\end{array}$ \\
\hline & $\begin{array}{l}\text { Bottom of } \\
\text { trench soil }\end{array}$ & J024X3 & $\begin{array}{l}\text { N } 147838 \\
\text { E } 581271\end{array}$ & 3 & $\begin{array}{l}\text { Pesticides, PCB, SVOA, PAH, TPH, } \\
\text { ICP metals, mercury, hexavalent chromium }\end{array}$ \\
\hline \multirow{4}{*}{ Area 3} & PACM & J022K0 & $\begin{array}{l}\text { N } 147808 \\
\text { E } 581271\end{array}$ & 0.9 to 1.5 & Asbestos \\
\hline & \begin{tabular}{|l|} 
Black roofing \\
felt
\end{tabular} & J02578 & $\begin{array}{l}\text { N } 147808 \\
\text { E } 581271\end{array}$ & 0.9 to 1.5 & Asbestos \\
\hline & Oily sediment & J024T0 & $\begin{array}{l}\text { N } 147808 \\
\text { E } 581271\end{array}$ & 0.9 to 1.5 & PCB, PAH, ICP metals, mercury \\
\hline & \begin{tabular}{|l|} 
Bottom of \\
trench soil
\end{tabular} & J024X2 & $\begin{array}{l}\text { N } 147815 \\
\text { E } 581280\end{array}$ & 3 to 4.6 & $\begin{array}{l}\text { Pesticides, PCB, SVOA, PAH, TPH, } \\
\text { ICP metals, mercury, hexavalent chromium }\end{array}$ \\
\hline \multirow{6}{*}{ Area 4} & \begin{tabular}{|l|}
$\begin{array}{l}\text { Rusty stained } \\
\text { soil }\end{array}$ \\
\end{tabular} & J024R4 & $\begin{array}{l}\text { N } 147805 \\
\text { E } 581321\end{array}$ & 0.8 & $\begin{array}{l}\text { Pesticides, PCB, SVOA, PAH, TPH, } \\
\text { ICP metals, mercury, hexavalent chromium }\end{array}$ \\
\hline & $\begin{array}{l}\text { Roofing } \\
\text { material }\end{array}$ & J022J7 & $\begin{array}{l}\text { N } 147805 \\
\text { E } 581321\end{array}$ & 0.9 to 1.5 & Asbestos \\
\hline & Transite & J022J8 & $\begin{array}{l}\text { N } 147805 \\
\text { E } 581321\end{array}$ & 2.4 to 3 & Asbestos \\
\hline & Welding rod & J022J9 & $\begin{array}{l}\text { N } 147805 \\
\text { E } 581321\end{array}$ & 4.3 & Asbestos \\
\hline & Oily soil & J024R5 & $\begin{array}{l}\text { N } 147805 \\
\text { E } 581321\end{array}$ & 4.3 & $\begin{array}{l}\text { Pesticides, PCB, SVOA, PAH, TPH, } \\
\text { ICP metals, mercury, hexavalent chromium }\end{array}$ \\
\hline & \begin{tabular}{|l|} 
Bottom of \\
trench soil
\end{tabular} & J024R6 & $\begin{array}{l}\text { N } 147810 \\
\text { E } 581318\end{array}$ & 4.6 & $\begin{array}{l}\text { Pesticides, PCB, SVOA, PAH, TPH, } \\
\text { ICP metals, mercury, hexavalent chromium }\end{array}$ \\
\hline Duplicate & Soil & J024X0 & $\begin{array}{l}\text { N } 147810 \\
\text { E } 581318 \\
\end{array}$ & 4.6 & $\begin{array}{l}\text { Pesticides, PCB, SVOA, PAH, TPH, } \\
\text { ICP metals, mercury, hexavalent chromium }\end{array}$ \\
\hline $\begin{array}{l}\text { Equipment } \\
\text { blank }\end{array}$ & Silica sand & J024X1 & NA & NA & ICP metals, mercury \\
\hline
\end{tabular}

Source: Remaining Sites Field Sampling, Logbook El-1578-5 (BHI 2004c).

$\begin{array}{lll}\text { bgs } & =\text { below ground surface } & \text { PAH }=\text { polycyclic aromatic hydrocarbon } \\ \text { HEIS } & =\text { Hanford Environmental Information System } & \text { PCB }=\text { polychlorinated biphenyl } \\ \text { ICP } & \text { inductively coupled plasma } & \text { SVOA = semivolatile organic analysis } \\ \text { NA } & =\text { not applicable } & \text { TPH }=\text { total petroleum hydrocarbon } \\ \text { PACM } & =\text { potential asbestos-containing material } & \end{array}$


Figure 4. Confirmatory Sampling Locations and Sample Areas at the 128-F-2 Waste Site.

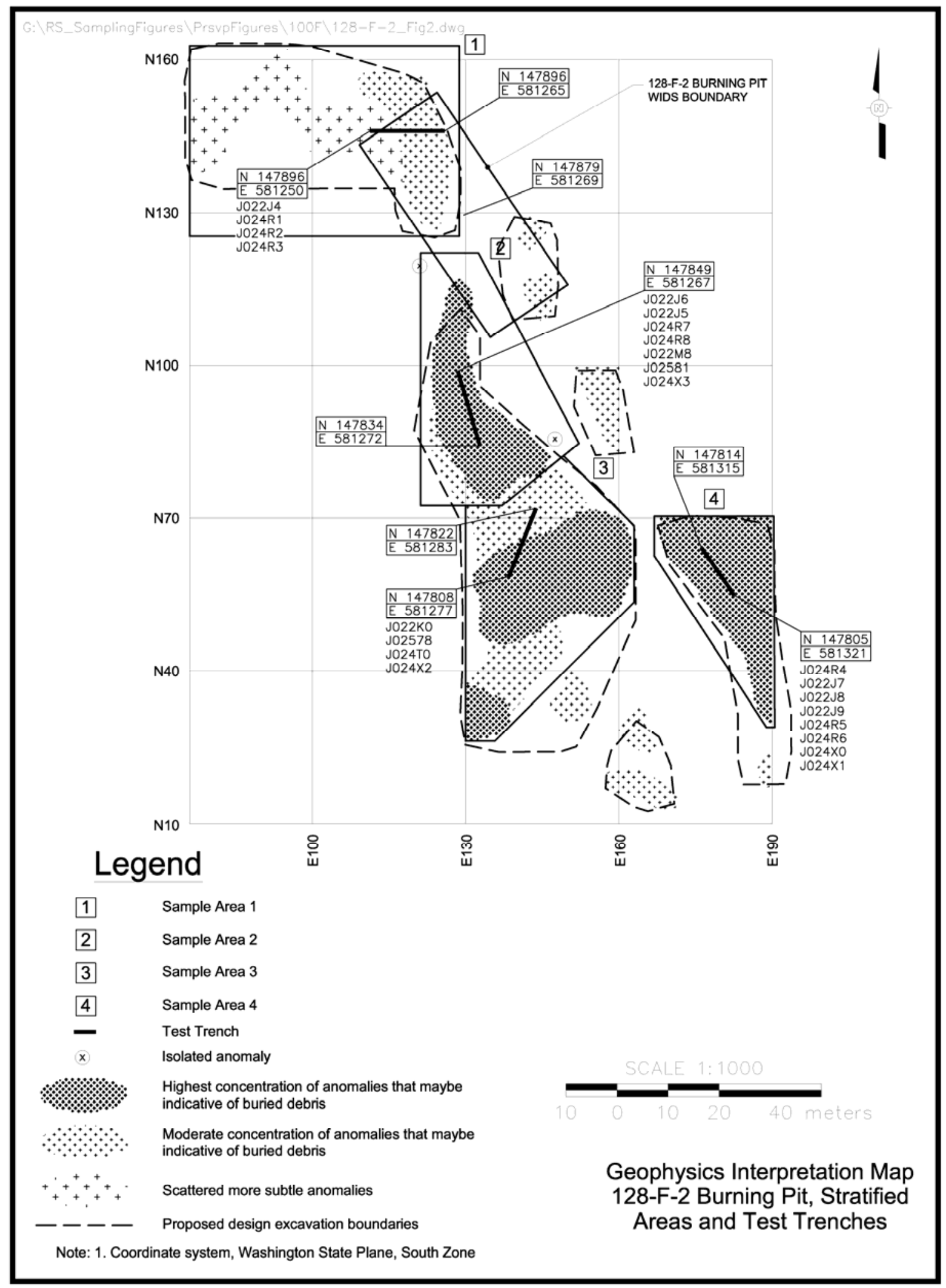


Confirmatory sampling of the 128-F-2 site occurred in December 2004. A focused sampling approach was used and one test trench was excavated in each of four stratified sample areas (Figure 2). Test trench 1 was excavated in sample area 1 to a depth of $2 \mathrm{~m}(8 \mathrm{ft})$ below ground surface (bgs). One sample of fibrous potential asbestos-containing material (PACM), two stained soil samples, and one native soil sample were collected. Test trench 2 was excavated in sample area 2 to a depth of $3 \mathrm{~m}$ (10 ft) bgs. Two PACM samples, two ash material samples, two paint samples, and one native soil sample were collected. Test trench 3 was excavated in sample area 3 to a depth of $5 \mathrm{~m}(15 \mathrm{ft})$ bgs. One PACM sample, one PACM roofing material sample, one stained soil sample, and one native soil sample were collected. Test trench 4 was excavated in sample area

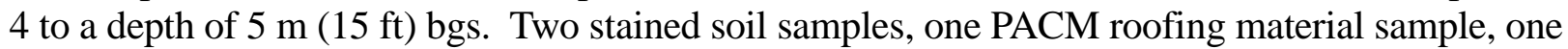
PACM welding rod sample, one transite PACM sample, one native soil sample, and one field duplicate of the native soil sample were collected. Selected photographs of the sampling activities are presented in Appendix B.

Additional debris discovered during excavation of the four test trenches included brick, wood, fiberglass, wire, sheet metal, rebar, burned paper, plastic, concrete, cement blocks, pipe, burned tires, steel, electrical conduit, lead weights, nails, bolts, and other unspecified building materials (BHI 2004c). Field volatile organic compound (VOC) surveys were conducted during excavation; no elevated detections were noted in the logbook (BHI 2004c). As a result, VOCs were excluded as contaminants of potential concern (COPCs) and no volatile organic analysis was performed on samples. No radiological contamination was detected above background with the exception of material in trench 4 with activity approximately 1,000 cpm above background.

Tables 2a, 2b, 2c, and 2d compare the maximum detected results for COPCs that were identified for each of the four confirmatory sampling areas for the 128-F-2 site with the remedial action objectives and the corresponding remedial action goals (RAGs) established in the Remaining Sites ROD (EPA 1999). Calculated cleanup levels are not presented in the Model Toxics Control Act Cleanup Levels and Risk Calculations table under Washington Administrative Code (WAC) 173-340-740(3) for aluminum, calcium, iron, magnesium, potassium, silicon, and sodium, and therefore these constituents are not considered COPCs. Contaminants that were not detected above the practical quantitation limits or minimum detectable activities are excluded from these tables.

Table 2a. Confirmatory Sample Area 1 - Comparison of Maximum Values to Action Levels at the 128-F-2 Waste Site. (3 Pages)

\begin{tabular}{|c|c|c|c|c|c|c|}
\hline \multirow[b]{2}{*}{ СОРС } & \multirow[b]{2}{*}{$\begin{array}{c}\text { Maximum } \\
\text { Result (mg/kg) }\end{array}$} & \multicolumn{3}{|c|}{ Soil Cleanup Levels, (mg/kg) ${ }^{a}$} & \multirow{2}{*}{$\begin{array}{c}\text { Does the } \\
\text { Maximum } \\
\text { Result Exceed } \\
\text { RAGs? }\end{array}$} & \multirow{2}{*}{$\begin{array}{c}\text { Does the } \\
\text { Maximum } \\
\text { Result Pass } \\
\text { RESRAD } \\
\text { Modeling? }\end{array}$} \\
\hline & & $\begin{array}{c}\text { Direct } \\
\text { Exposure }\end{array}$ & $\begin{array}{c}\text { Groundwater } \\
\text { Protection }\end{array}$ & $\begin{array}{c}\text { River } \\
\text { Protection }\end{array}$ & & \\
\hline Antimony & $0.59(<\mathrm{BG})$ & 32 & $5^{b}$ & $5^{b}$ & No & -- \\
\hline Arsenic & 59.7 & 20 & 20 & 20 & Yes & $--^{c}$ \\
\hline Barium & 83.9 & 5,600 & $132^{b}$ & 224 & No & -- \\
\hline Beryllium & $0.24(<\mathrm{BG})$ & $10.4^{\mathrm{d}}$ & $1.51^{\mathrm{b}}$ & $1.51^{b}$ & No & -- \\
\hline Boron $^{e}$ & 3.2 & 16,000 & 320 & NA & No & -- \\
\hline
\end{tabular}


Table 2a. Confirmatory Sample Area 1 - Comparison of Maximum Values to Action Levels at the 128-F-2 Waste Site. (3 Pages)

\begin{tabular}{|c|c|c|c|c|c|c|}
\hline \multirow[b]{2}{*}{ COPC } & \multirow[b]{2}{*}{$\begin{array}{c}\text { Maximum } \\
\text { Result (mg/kg) }\end{array}$} & \multicolumn{3}{|c|}{ Soil Cleanup Levels, (mg/kg) ${ }^{a}$} & \multirow{2}{*}{$\begin{array}{c}\text { Does the } \\
\text { Maximum } \\
\text { Result Exceed } \\
\text { RAGs? }\end{array}$} & \multirow{2}{*}{$\begin{array}{c}\text { Does the } \\
\text { Maximum } \\
\text { Result Pass } \\
\text { RESRAD } \\
\text { Modeling? }\end{array}$} \\
\hline & & $\begin{array}{c}\text { Direct } \\
\text { Exposure }\end{array}$ & $\begin{array}{c}\text { Groundwater } \\
\text { Protection }\end{array}$ & $\begin{array}{c}\text { River } \\
\text { Protection }\end{array}$ & & \\
\hline Cadmium & $0.41(<\mathrm{BG})$ & $13.9^{\mathrm{d}}$ & $0.81^{b}$ & $0.81^{b}$ & No & -- \\
\hline Chromium, total & 137 & 80,000 & $18.5^{b}$ & $18.5^{b}$ & Yes & $--^{f}$ \\
\hline Chromium, hexavalent $^{\mathrm{e}}$ & 6.8 & $2.1^{\mathrm{d}}$ & 4.8 & 2 & Yes & $--^{c}$ \\
\hline Cobalt & 26 & 1,600 & 32 & NA & No & -- \\
\hline Copper & 52.9 & 2,960 & 59.2 & $22.0^{b}$ & Yes & $--^{f}$ \\
\hline Lead & 417 & 353 & $10.2^{b}$ & $10.2^{b}$ & Yes & $--^{c}$ \\
\hline Manganese & $361(<\mathrm{BG})$ & 11,200 & $512^{b}$ & $512^{b}$ & No & -- \\
\hline Mercury & 0.45 & 24 & $0.33^{b}$ & $0.33^{b}$ & Yes & $--^{f}$ \\
\hline Molybdenum $^{\mathrm{e}}$ & 0.69 & 400 & 8 & NA & No & -- \\
\hline Nickel & 336 & 1,600 & $19.1^{b}$ & 27.4 & Yes & $--^{f}$ \\
\hline Selenium & $0.62(<\mathrm{BG})$ & 400 & 5 & 1 & No & -- \\
\hline Silver & 2.1 & 400 & 8 & $0.73^{b}$ & Yes & $--^{f}$ \\
\hline Vanadium & $33.3(<\mathrm{BG})$ & 560 & $85.1^{b}$ & NA & No & -- \\
\hline Zinc & $40.3(<\mathrm{BG})$ & 24,000 & 480 & $67.8^{b}$ & No & -- \\
\hline Anthracene & 0.018 & 24,000 & 240 & 1,920 & No & -- \\
\hline Benzo(a)anthracene & 0.092 & $1.37^{\mathrm{g}}$ & $0.015^{\mathrm{h}}$ & $0.015^{\mathrm{h}}$ & Yes & -- \\
\hline Benzo(a)pyrene & 0.077 & 0.137 & $0.015^{\mathrm{h}}$ & $0.015^{\mathrm{h}}$ & Yes & -- \\
\hline Benzo(b)fluoranthene & 0.065 & $1.37^{\mathrm{g}}$ & $0.015^{\mathrm{h}}$ & $0.015^{\mathrm{h}}$ & Yes & - \\
\hline Benzo(g,h,i)perylene ${ }^{\mathrm{i}}$ & 0.040 & 2,400 & 48 & 192 & No & - \\
\hline Benzo(k)fluoranthene & 0.032 & $13.7^{\mathrm{g}}$ & $0.015^{\mathrm{h}}$ & $0.015^{\mathrm{h}}$ & Yes & -- \\
\hline Bis(2-ethylhexyl)phthalate & 0.16 & 71.4 & 0.625 & 0.36 & No & -- \\
\hline Chrysene & 0.074 & $137^{\mathrm{g}}$ & $0.1^{\mathrm{h}}$ & $0.1^{\mathrm{h}}$ & No & -- \\
\hline Dibenzo(a,h)anthracene & 0.010 & 0.137 & $0.03^{h}$ & $0.03^{h}$ & No & -- \\
\hline Indeno(1,2,3-cd)pyrene & 0.048 & 1.37 & $0.33^{h}$ & $0.33^{h}$ & No & -- \\
\hline Fluoranthene & 0.12 & 3,200 & 64 & 18.0 & No & -- \\
\hline Fluorene & 0.020 & 3,200 & 64 & 260 & No & -- \\
\hline Phenanthrene $^{\mathrm{i}}$ & 0.10 & 24,000 & 240 & 1,920 & No & -- \\
\hline Pyrene & 0.20 & 2,400 & 48 & 192 & No & -- \\
\hline
\end{tabular}


Table 2a. Confirmatory Sample Area 1 - Comparison of Maximum Values to Action Levels at the 128-F-2 Waste Site. (3 Pages)

\begin{tabular}{|l|c|c|c|c|c|c|}
\hline \multirow{2}{*}{ COPC } & \multirow{2}{*}{$\begin{array}{c}\text { Maximum } \\
\text { Result (mg/kg) }\end{array}$} & $\begin{array}{c}\text { Soil Cleanup Levels, (mg/kg) } \\
\text { Direct } \\
\text { Exposure }\end{array}$ & $\begin{array}{c}\text { Groundwater } \\
\text { Protection }\end{array}$ & $\begin{array}{c}\text { Does the } \\
\text { River } \\
\text { Protection }\end{array}$ & $\begin{array}{c}\text { Does the } \\
\text { Maximum } \\
\text { Result Exceed } \\
\text { RAGs? }\end{array}$ & $\begin{array}{c}\text { Maximum } \\
\text { Result Pass } \\
\text { RESRAD } \\
\text { Modeling? }\end{array}$ \\
\hline DDE, 4,4'- & 0.42 & 2.94 & 0.0257 & $0.0033^{\mathrm{h}}$ & Yes & $-\mathrm{f}^{\mathrm{f}}$ \\
\hline DDT, 4,4'- & 2.2 & 2.94 & 0.0257 & $0.0033^{\mathrm{h}}$ & Yes & $-\mathrm{-}^{\mathrm{f}}$ \\
\hline Total petroleum hydrocarbon & 257 & NA & 200 & 200 & Yes & $--^{\mathrm{f}}$ \\
\hline
\end{tabular}

${ }^{a}$ Lookup values and RAGs obtained from the Remedial Design Report/Remedial Action Work Plan for the 100 Area (DOE-RL 2005) or calculated per WAC 173-340-720, WAC 173-340-730, and WAC 173-340-740, Method B, unless otherwise noted.

${ }^{\mathrm{b}}$ Where cleanup levels are less than background, cleanup levels default to background per WAC 173-340-700[4][d].

${ }^{\mathrm{c}}$ RESRAD evaluation not performed; failed direct exposure RAG.

${ }^{\mathrm{d}}$ Carcinogenic cleanup level calculated based on the inhalation exposure pathway (WAC 173-340-750[3]) using an airborne particulate mass-loading rate of $0.0001 \mathrm{~g} / \mathrm{m}^{3}$ (WDOH 1997).

e No Hanford Site-specific or Washington State background value available.

${ }^{\mathrm{f}}$ The groundwater and river protection evaluations were not completed because one or more COPCs failed direct exposure cleanup criteria and the site was remediated

${ }^{g}$ Cleanup level calculated with updated toxicity values using the appropriate formulas from WAC 173-340-740. Updated toxicity values are available from the EPA Integrated Risk Information System (IRIS) at < http://www.epa.gov/iris $>$ or from the Risk Assessment Information System database of the Oak Ridge National Laboratory on the Internet at $<$ http://risk.lsd.ornl.gov $>$.

${ }^{\mathrm{h}}$ Where cleanup levels are less than RDLs, cleanup levels default to RDLs per WAC 173-340-707(2) and DOE-RL (2005). Values for benzo(a)anthracene, benzo(a)pyrene, benzo(b)fluoranthene, benzo(k)fluoranthene, chrysene, and dibenzo(a,h)anthracene are based on EPA method 8310 RDLs.

${ }^{\mathrm{i}}$ Toxicity data for this chemical are not available. Cleanup levels are based on surrogate chemicals:

Contaminant: benzo(g,h,i)perylene; surrogate: pyrene

Contaminant: phenathrene; surrogate: anthracene.

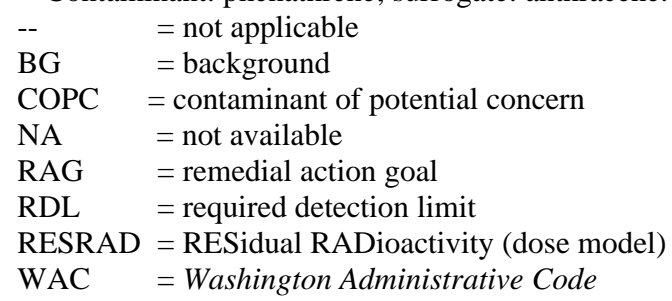

Table 2b. Confirmatory Sample Area 2 - Comparison of Maximum Values to Action Levels at the 128-F-2 Site. (3 Pages)

\begin{tabular}{|c|c|c|c|c|c|c|}
\hline \multirow[b]{2}{*}{ COPC } & \multirow[b]{2}{*}{$\begin{array}{c}\text { Maximum } \\
\text { Result (mg/kg) }\end{array}$} & \multicolumn{3}{|c|}{ Soil Cleanup Levels, $(\mathrm{mg} / \mathrm{kg})^{\mathrm{a}}$} & \multirow{2}{*}{$\begin{array}{c}\text { Does the } \\
\text { Maximum } \\
\text { Result Exceed } \\
\text { RAGs? }\end{array}$} & \multirow{2}{*}{$\begin{array}{c}\text { Does the } \\
\text { Maximum } \\
\text { Result Pass } \\
\text { RESRAD } \\
\text { Modeling? }\end{array}$} \\
\hline & & $\begin{array}{c}\text { Direct } \\
\text { Exposure }\end{array}$ & $\begin{array}{l}\text { Groundwater } \\
\text { Protection }\end{array}$ & $\begin{array}{c}\text { River } \\
\text { Protection }\end{array}$ & & \\
\hline Antimony & $3.1(<\mathrm{BG})$ & 32 & $5^{b}$ & $5^{b}$ & No & -- \\
\hline Arsenic & $5.7(<\mathrm{BG})$ & 20 & 20 & 20 & No & -- \\
\hline Barium & 233 & 5,600 & $132^{\mathrm{b}}$ & 224 & Yes & $--^{c}$ \\
\hline Beryllium & $0.32(<\mathrm{BG})$ & $10.4^{\mathrm{d}}$ & $1.5^{b}$ & $1.51^{\mathrm{b}}$ & No & -- \\
\hline Boron $^{e}$ & 22.1 & 16,000 & 320 & NA & No & -- \\
\hline Cadmium & 2.9 & $13.9^{\mathrm{d}}$ & $0.81^{\mathrm{b}}$ & $0.81^{\mathrm{b}}$ & Yes & $--^{c}$ \\
\hline Chromium, total & 242 & 80,000 & $18.5^{b}$ & $18.5^{b}$ & Yes & $--^{c}$ \\
\hline
\end{tabular}


Table 2b. Confirmatory Sample Area 2 - Comparison of Maximum Values to Action Levels at the 128-F-2 Site. (3 Pages)

\begin{tabular}{|c|c|c|c|c|c|c|}
\hline \multirow[b]{2}{*}{ COPC } & \multirow[b]{2}{*}{$\begin{array}{c}\text { Maximum } \\
\text { Result (mg/kg) }\end{array}$} & \multicolumn{3}{|c|}{ Soil Cleanup Levels, (mg/kg) ${ }^{a}$} & \multirow{2}{*}{$\begin{array}{c}\text { Does the } \\
\text { Maximum } \\
\text { Result Exceed } \\
\text { RAGs? }\end{array}$} & \multirow{2}{*}{$\begin{array}{c}\text { Does the } \\
\text { Maximum } \\
\text { Result Pass } \\
\text { RESRAD } \\
\text { Modeling? }\end{array}$} \\
\hline & & $\begin{array}{c}\text { Direct } \\
\text { Exposure }\end{array}$ & $\begin{array}{c}\text { Groundwater } \\
\text { Protection }\end{array}$ & $\begin{array}{c}\text { River } \\
\text { Protection }\end{array}$ & & \\
\hline Chromium, hexavalent $^{\text {e }}$ & 1.9 & $2.1^{\mathrm{d}}$ & 4.8 & 2 & No & -- \\
\hline Cobalt & $8.4(<\mathrm{BG})$ & 1,600 & 32 & NA & No & -- \\
\hline Copper & 2,240 & 2,960 & 59.2 & $22.0^{b}$ & Yes & $--^{c}$ \\
\hline Lead & 386 & 353 & $10.2^{b}$ & $10.2^{b}$ & Yes & $-{ }^{f}$ \\
\hline Manganese & 775 & 11,200 & $512^{b}$ & $512^{b}$ & Yes & $-{ }^{c}$ \\
\hline Mercury & 1.5 & 24 & $0.33^{b}$ & $0.33^{b}$ & Yes & $-{ }^{c}$ \\
\hline Molybdenum $^{\mathrm{e}}$ & 2.7 & 400 & 8 & NA & No & -- \\
\hline Nickel & 79.3 & 1,600 & $19.1^{b}$ & 27.4 & Yes & $--^{c}$ \\
\hline Silver & 1.8 & 400 & 8 & $0.73^{b}$ & Yes & $-{ }^{c}$ \\
\hline Vanadium & $35.2(<\mathrm{BG})$ & 560 & $85.1^{b}$ & NA & No & -- \\
\hline Zinc & 1,030 & 24,000 & 480 & $67.8^{b}$ & Yes & $--^{c}$ \\
\hline Acenaphthene & 190 & 4,800 & 96 & 129 & Yes & $--^{c}$ \\
\hline Acenapthylene ${ }^{\mathrm{g}}$ & 70 & 4,800 & 96 & 129 & No & -- \\
\hline Anthracene & 330 & 24,000 & 240 & 1,920 & Yes & $--^{c}$ \\
\hline Benzo(a)anthracene & 620 & $1.37^{\mathrm{h}}$ & $0.015^{\mathrm{i}}$ & $0.015^{\mathrm{i}}$ & Yes & $-{ }^{f}$ \\
\hline Benzo(a)pyrene & 430 & $0.33^{\mathrm{i}}$ & $0.33^{\mathrm{i}}$ & $0.33^{\mathrm{i}}$ & Yes & $-{ }^{f}$ \\
\hline Benzo(b)fluoranthene & 330 & $1.37^{\mathrm{h}}$ & $0.33^{\mathrm{i}}$ & $0.33^{\mathrm{i}}$ & Yes & $-{ }^{f}$ \\
\hline Benzo(g,h,i)perylene ${ }^{g}$ & 250 & 2,400 & 48 & 192 & Yes & $--^{c}$ \\
\hline Benzo(k)fluoranthene & 360 & $13.7^{\mathrm{h}}$ & $0.33^{\mathrm{i}}$ & $0.33^{\mathrm{i}}$ & Yes & $-{ }^{f}$ \\
\hline Bis(2-ethylhexyl)phthalate & 0.20 & 71.4 & 0.625 & 0.36 & No & -- \\
\hline Carbazole & 180 & 50 & 0.437 & NA & Yes & $-{ }^{f}$ \\
\hline Chrysene & 970 & $137^{\mathrm{h}}$ & $0.1^{\mathrm{i}}$ & $0.1^{\mathrm{i}}$ & Yes & $-{ }^{f}$ \\
\hline Dibenzo(a,h)anthracene & 120 & $0.33^{\mathrm{i}}$ & $0.33^{\mathrm{i}}$ & $0.33^{\mathrm{i}}$ & Yes & $-{ }^{f}$ \\
\hline Dibenzofuran & 86 & 160 & 3.20 & NA & Yes & $--^{c}$ \\
\hline Di-n-butylphthalate & 0.11 & 8,000 & 160 & 540 & No & -- \\
\hline Fluoranthene & 1,010 & 3,200 & 64 & 18.0 & Yes & $--^{c}$ \\
\hline Fluorene & 161 & 3,200 & 64 & 260 & Yes & $--^{c}$ \\
\hline Indeno(1,2,3-cd)pyrene & 260 & 1.37 & $0.03^{\mathrm{i}}$ & $0.03^{\mathrm{i}}$ & Yes & $-{ }^{f}$ \\
\hline Methylnaphthalene;2- & 26 & 320 & 3.2 & NA & Yes & $--^{c}$ \\
\hline Naphthalene & 325 & 1,600 & 16.0 & 988 & Yes & $--^{c}$ \\
\hline Phenanthrene $^{\mathrm{g}}$ & 1,090 & 24,000 & 240 & 1,920 & Yes & $--^{c}$ \\
\hline Pyrene & 1,220 & 2,400 & 48 & 192 & Yes & $--^{\mathrm{C}}$ \\
\hline Endosulfan II & 0.11 & 480 & 9.6 & 0.0112 & Yes & $--^{c}$ \\
\hline Endosulfan sulfate & 0.24 & 480 & 9.6 & 0.0112 & Yes & $--^{c}$ \\
\hline
\end{tabular}


Table 2b. Confirmatory Sample Area 2 - Comparison of Maximum Values to Action Levels at the 128-F-2 Site. (3 Pages)

\begin{tabular}{|l|c|c|c|c|c|c|}
\hline \multirow{2}{*}{ COPC } & \multirow{2}{*}{$\begin{array}{c}\text { Maximum } \\
\text { Result (mg/kg) }\end{array}$} & $\begin{array}{c}\text { Soil Cleanup Levels, (mg/kg) } \\
\text { Direct } \\
\text { Exposure }\end{array}$ & $\begin{array}{c}\text { Groundwater } \\
\text { Protection }\end{array}$ & $\begin{array}{c}\text { Does the } \\
\text { River } \\
\text { Protection }\end{array}$ & $\begin{array}{c}\text { Does the } \\
\text { Maximum } \\
\text { Result Exceed } \\
\text { RAGs? }\end{array}$ & $\begin{array}{c}\text { Mesult Pass } \\
\text { RESRAD } \\
\text { Modeling? }\end{array}$ \\
\hline DDD, 4,4'- & 0.047 & 4.17 & 0.0365 & $0.0033^{\mathrm{i}}$ & Yes & ${--^{\mathrm{c}}}^{\mathrm{i}}$ \\
\hline DDT, 4,4'- & 0.031 & 2.94 & 0.0257 & $0.0033^{\mathrm{i}}$ & Yes & ${--^{\mathrm{c}}}^{\mathrm{i}}$ \\
\hline Total petroleum hydrocarbon & 762 & NA & 200 & 200 & Yes & ${--^{\mathrm{c}}}^{\mathrm{c}}$ \\
\hline
\end{tabular}

${ }^{\mathrm{a}}$ Lookup values and RAGs obtained from the Remedial Design Report/Remedial Action Work Plan for the 100 Area (DOE-RL 2005) or calculated per WAC 173-340-720, WAC 173-340-730, and WAC 173-340-740, Method B, unless otherwise noted.

${ }^{\mathrm{b}}$ Where cleanup levels are less than background, cleanup levels default to background per WAC 173-340-700[4][d].

${ }^{\mathrm{c}}$ The groundwater and river protection evaluations were not completed because one or more COPCs failed direct exposure cleanup criteria and the site was remediated.

${ }^{\mathrm{d}}$ Carcinogenic cleanup level calculated based on the inhalation exposure pathway (WAC 173-340-750[3]) using an airborne particulate mass-loading rate of $0.0001 \mathrm{~g} / \mathrm{m}^{3}$ (WDOH 1997).

e No Hanford Site-specific or Washington State background value available.

${ }^{\mathrm{f}}$ RESRAD evaluation not performed; failed direct exposure RAG.

${ }^{g}$ Toxicity data for this chemical are not available. Cleanup levels are based on surrogate chemicals: Contaminant: acenapthylene; surrogate: acenapthene

Contaminant: benzo(g,h,i)perylene; surrogate: pyrene

Contaminant: phenathrene; surrogate: anthracene.

${ }^{\mathrm{h}}$ Cleanup level calculated with updated toxicity values using the appropriate formulas from WAC 173-340-740. Updated toxicity values are available from the EPA Integrated Risk Information System (IRIS) at < http://www.epa.gov/iris > or from the Risk Assessment Information System database of the Oak Ridge National Laboratory on the Internet at $<$ http://risk.lsd.ornl.gov $>$.

${ }^{\mathrm{i}}$ Where cleanup levels are less than RDLs, cleanup levels default to RDLs per WAC 173-340-707(2) and DOE-RL (2005). Values for benzo(a)anthracene and chrysene are based on EPA method 8310 RDLs. Values for benzo(a)pyrene, benzo(b)fluoranthene, benzo(k)fluoranthene, and dibenzo(a,h)anthracene RDL based on EPA method 8270.

- $\quad=$ not applicable

BG = background

COPC = contaminant of potential concern

NA $=$ not available

RAG = remedial action goal

RDL = required detection limit

RESRAD $=$ RESidual RADioactivity (dose model)

WAC = Washington Administrative Code

Table 2c. Confirmatory Sample Area 3 - Comparison of Maximum Values to Action Levels at the 128-F-2 Site. (3 Pages)

\begin{tabular}{|c|c|c|c|c|c|c|}
\hline \multirow[b]{2}{*}{ COPC } & \multirow[b]{2}{*}{$\begin{array}{c}\text { Maximum } \\
\text { Result }(\mathrm{mg} / \mathrm{kg})\end{array}$} & \multicolumn{3}{|c|}{ Soil Cleanup Levels, $(\mathrm{mg} / \mathrm{kg})^{\text {a }}$} & \multirow{2}{*}{$\begin{array}{c}\text { Does the } \\
\text { Maximum } \\
\text { Result Exceed } \\
\text { RAGs? }\end{array}$} & \multirow{2}{*}{$\begin{array}{c}\text { Does the } \\
\text { Maximum } \\
\text { Result Pass } \\
\text { RESRAD } \\
\text { Modeling? }\end{array}$} \\
\hline & & $\begin{array}{c}\text { Direct } \\
\text { Exposure }\end{array}$ & $\begin{array}{l}\text { Groundwater } \\
\text { Protection }\end{array}$ & $\begin{array}{c}\text { River } \\
\text { Protection }\end{array}$ & & \\
\hline Antimony & 15 & 32 & $5^{b}$ & $5^{b}$ & Yes & $--^{c}$ \\
\hline Arsenic & $6.7(<\mathrm{BG})$ & 20 & 20 & 20 & No & -- \\
\hline Barium & 498 & 5,600 & $132^{b}$ & 224 & Yes & $--^{c}$ \\
\hline Beryllium & $0.36(<\mathrm{BG})$ & $10.4^{\mathrm{d}}$ & $1.51^{\mathrm{b}}$ & $1.51^{\mathrm{b}}$ & No & -- \\
\hline Boron $^{e}$ & 1,260 & 16,000 & 320 & NA & Yes & $--^{c}$ \\
\hline Cadmium & 16.8 & $13.9^{\mathrm{d}}$ & $0.81^{\mathrm{b}}$ & $0.81^{\mathrm{b}}$ & Yes & $--^{f}$ \\
\hline
\end{tabular}


Table 2c. Confirmatory Sample Area 3 - Comparison of Maximum Values to Action Levels at the 128-F-2 Site. (3 Pages)

\begin{tabular}{|c|c|c|c|c|c|c|}
\hline \multirow[b]{2}{*}{ COPC } & \multirow[b]{2}{*}{$\begin{array}{c}\text { Maximum } \\
\text { Result (mg/kg) }\end{array}$} & \multicolumn{3}{|c|}{ Soil Cleanup Levels, (mg/kg) } & \multirow{2}{*}{$\begin{array}{c}\text { Does the } \\
\text { Maximum } \\
\text { Result Exceed } \\
\text { RAGs? }\end{array}$} & \multirow{2}{*}{$\begin{array}{l}\text { Does the } \\
\text { Maximum } \\
\text { Result Pass } \\
\text { RESRAD } \\
\text { Modeling? }\end{array}$} \\
\hline & & $\begin{array}{c}\text { Direct } \\
\text { Exposure }\end{array}$ & $\begin{array}{c}\text { Groundwater } \\
\text { Protection }\end{array}$ & $\begin{array}{c}\text { River } \\
\text { Protection }\end{array}$ & & \\
\hline Chromium, total & 106 & 80,000 & $18.5^{b}$ & $18.5^{b}$ & Yes & $-{ }^{c}$ \\
\hline Chromium, hexavalent $^{\mathrm{e}}$ & 1.5 & $2.1^{\mathrm{d}}$ & 4.8 & 2 & No & - \\
\hline Cobalt & 46.7 & 1,600 & 32 & NA & Yes & $--^{c}$ \\
\hline Copper & 138 & 2,960 & 59.2 & $22.0^{b}$ & Yes & $-{ }^{c}$ \\
\hline Lead & 226 & 353 & $10.2^{b}$ & $10.2^{b}$ & Yes & $-{ }^{c}$ \\
\hline Manganese & $401(<\mathrm{BG})$ & 11,200 & $512^{b}$ & $512^{b}$ & No & $-{ }^{c}$ \\
\hline Mercury & 9.8 & 24 & $0.33^{b}$ & $0.33^{b}$ & Yes & $--^{c}$ \\
\hline Molybdenum $^{\mathrm{e}}$ & 0.42 & 400 & 8 & NA & No & -- \\
\hline Nickel & 106 & 1,600 & $19.1^{b}$ & $27.4^{c}$ & Yes & $-{ }^{c}$ \\
\hline Silver & 1.6 & 400 & 8 & $0.73^{b}$ & Yes & $-{ }^{c}$ \\
\hline Vanadium & $36.9(<\mathrm{BG})$ & 560 & $85.1^{b}$ & NA & No & -- \\
\hline Zinc & 2,310 & 24,000 & 480 & $67.8^{b}$ & Yes & $--^{c}$ \\
\hline Acenaphthene & 2.2 & 4,800 & 96 & 129 & No & -- \\
\hline Anthracene & 9.0 & 24,000 & 240 & 1,920 & No & -- \\
\hline Benzo(a)anthracene & 17 & $1.37^{\mathrm{g}}$ & $0.015^{\mathrm{h}}$ & $0.015^{\mathrm{h}}$ & Yes & $-{ }^{f}$ \\
\hline Benzo(a)pyrene & 16 & 0.137 & $0.015^{\mathrm{h}}$ & $0.015^{\mathrm{h}}$ & Yes & $-\mathrm{f}^{\mathrm{f}}$ \\
\hline Benzo(b)fluoranthene & 13 & $1.37^{\mathrm{g}}$ & $0.015^{h}$ & $0.015^{\mathrm{h}}$ & Yes & $-{ }^{f}$ \\
\hline Benzo(k)fluoranthene & 8.3 & $13.7^{\mathrm{g}}$ & $0.015^{\mathrm{h}}$ & $0.015^{\mathrm{h}}$ & Yes & $--^{c}$ \\
\hline Bis(2-ethylhexyl)phthalate & 0.33 & 71.4 & 0.625 & 0.36 & No & -- \\
\hline Chrysene & 22 & $137^{\mathrm{g}}$ & $0.1^{\mathrm{h}}$ & $0.1^{\mathrm{h}}$ & Yes & $--^{c}$ \\
\hline Dibenzo(a,h)anthracene & 2.5 & 0.137 & $0.03^{\mathrm{h}}$ & $0.03^{\mathrm{h}}$ & Yes & $-{ }^{f}$ \\
\hline Dibenzofuran & 0.94 & 160 & 3.20 & NA & No & -- \\
\hline Indeno(1,2,3-cd)pyrene & 12 & 1.37 & $0.33^{\mathrm{h}}$ & $0.33^{h}$ & Yes & $-{ }_{-}^{f}$ \\
\hline Benzo(g,h,i)perylene ${ }^{\mathrm{i}}$ & 8.5 & 2,400 & 48 & 192 & No & -- \\
\hline Fluoranthene & 53 & 3,200 & 64 & 18.0 & Yes & $--^{c}$ \\
\hline Fluorene & 8.4 & 3,200 & 64 & 260 & No & -- \\
\hline Methylnaphthalene;2- & 0.28 & 320 & 3.2 & NA & No & -- \\
\hline Naphthalene & 0.73 & 1,600 & 16.0 & 1,976 & No & -- \\
\hline Phenanthrene $^{\mathrm{i}}$ & 49 & 24,000 & 240 & 1,920 & No & -- \\
\hline Pyrene & 63 & 2,400 & 48 & 1,920 & Yes & $--^{c}$ \\
\hline Aroclor-1254 & 8.7 & 0.5 & $0.017^{\mathrm{h}}$ & $0.017^{\mathrm{h}}$ & Yes & $-{ }_{--} \mathrm{g}$ \\
\hline
\end{tabular}


Table 2c. Confirmatory Sample Area 3 - Comparison of Maximum Values to Action Levels at the 128-F-2 Site. (3 Pages)

\begin{tabular}{|l|c|c|c|c|c|c|}
\hline \multirow{2}{*}{ COPC } & $\begin{array}{c}\text { Maximum } \\
\text { Result (mg/kg) }\end{array}$ & $\begin{array}{c}\text { Soil Cleanup Levels, (mg/kg) } \\
\text { Direct } \\
\text { Exposure }\end{array}$ & $\begin{array}{c}\text { Groundwater } \\
\text { Protection }\end{array}$ & $\begin{array}{c}\text { Roes the } \\
\text { River } \\
\text { Protection }\end{array}$ & $\begin{array}{c}\text { Does the } \\
\text { Maximum } \\
\text { Result Exceed } \\
\text { RAGs? }\end{array}$ & $\begin{array}{c}\text { Maximum } \\
\text { Result Pass } \\
\text { RESRAD } \\
\text { Modeling? }\end{array}$ \\
\hline DDT, 4,4'- & 0.070 & 2.94 & 0.0365 & $0.0033^{\mathrm{h}}$ & Yes & $-^{\mathrm{c}}$ \\
\hline
\end{tabular}

${ }^{a}$ Lookup values and RAGs obtained from the 100 Area Remedial Design Report/Remedial Action Work Plan for the 100 Area (DOE-RL 2005) or calculated per WAC 173-340-720, WAC 173-340-730, and WAC 173-340-740, Method B, unless otherwise noted.

${ }^{\mathrm{b}}$ Where cleanup levels are less than background, cleanup levels default to background per WAC 173-340-700[4][d].

${ }^{c}$ The groundwater and river protection evaluations were not completed because one or more COPCs failed direct exposure cleanup criteria and the site was remediated.

${ }^{\mathrm{d}}$ Carcinogenic cleanup level calculated based on the inhalation exposure pathway (WAC 173-340-750[3]) using an airborne particulate mass-loading rate of $0.0001 \mathrm{~g} / \mathrm{m}^{3}$ (WDOH 1997).

e No Hanford Site-specific or Washington State background value available.

${ }^{\mathrm{f}}$ RESRAD evaluation not performed; failed direct exposure RAG.

${ }^{g}$ Cleanup level calculated with updated toxicity values using the appropriate formulas from WAC 173-340-740. Updated toxicity values are available from the EPA Integrated Risk Information System (IRIS) at < http://www.epa.gov/iris $>$ or from the Risk Assessment Information System database of the Oak Ridge National Laboratory on the Internet at $<$ http://risk.lsd.ornl.gov $>$.

${ }^{\mathrm{h}}$ Where cleanup levels are less than RDLs, cleanup levels default to RDLs per WAC 173-340-707(2) and DOE-RL (2005). Values for benzo(a)anthracene, benzo(a)pyrene, benzo(b)fluoranthene, benzo(k)fluoranthene, chrysene, and dibenzo(a,h)anthracene are based on EPA method 8310 RDLs.

${ }^{\mathrm{i}}$ Toxicity data for this chemical are not available. Cleanup levels are based on surrogate chemicals:

Contaminant: benzo(g,h,i)perylene; surrogate: pyrene

Contaminant: phenathrene; surrogate: anthracene.

-- $\quad=$ not applicable

BG = background

$\mathrm{COPC}=$ contaminant of potential concern

NA $=$ not available

RAG = remedial action goal

RDL = required detection limit

RESRAD = RESidual RADioactivity (dose model)

WAC = Washington Administrative Code

Table 2d. Confirmatory Sample Area 4-Comparison of Maximum Values to Action Levels at the 128-F-2 Site. (2 Pages)

\begin{tabular}{|c|c|c|c|c|c|c|}
\hline \multirow[b]{2}{*}{ COPC } & \multirow[b]{2}{*}{$\begin{array}{c}\text { Maximum Result } \\
(\mathrm{mg} / \mathrm{kg})\end{array}$} & \multicolumn{3}{|c|}{ Soil Cleanup Levels, (mg/kg) ${ }^{a}$} & \multirow{2}{*}{$\begin{array}{c}\text { Does the } \\
\text { Maximum } \\
\text { Result Exceed } \\
\text { RAGs? }\end{array}$} & \multirow{2}{*}{$\begin{array}{l}\text { Does the } \\
\text { Maximum } \\
\text { Result Pass } \\
\text { RESRAD } \\
\text { Modeling? }\end{array}$} \\
\hline & & $\begin{array}{c}\text { Direct } \\
\text { Exposure }\end{array}$ & $\begin{array}{c}\text { Groundwater } \\
\text { Protection }\end{array}$ & $\begin{array}{c}\text { River } \\
\text { Protection }\end{array}$ & & \\
\hline Antimony & $0.49(<\mathrm{BG})$ & 32 & $5^{b}$ & $5^{b}$ & No & -- \\
\hline Arsenic & 20.8 & 20 & 20 & 20 & Yes & $--^{c}$ \\
\hline Barium & 659 & 5,600 & $132^{b}$ & 224 & Yes & $-{ }^{d}$ \\
\hline Beryllium & $0.40(<\mathrm{BG})$ & $10.4^{\mathrm{e}}$ & $1.51^{\mathrm{b}}$ & $1.51^{\mathrm{b}}$ & No & -- \\
\hline Boron $^{f}$ & 2.1 & 16,000 & 320 & NA & No & -- \\
\hline Cadmium & $0.75(<\mathrm{BG})$ & $13.9^{\mathrm{e}}$ & $0.81^{\mathrm{b}}$ & $0.81^{\mathrm{b}}$ & No & -- \\
\hline Chromium, total & 65.9 & 80,000 & $18.5^{b}$ & $18.5^{b}$ & Yes & $--^{d}$ \\
\hline Chromium, hexavalent $^{\mathrm{f}}$ & 8.5 & $2.1^{\mathrm{e}}$ & 4.8 & 2 & Yes & $--^{c}$ \\
\hline Cobalt & $14.9(<\mathrm{BG})$ & 1,600 & 32 & NA & No & -- \\
\hline Copper & 42.9 & 2,960 & 59.2 & $22.0^{b}$ & Yes & $-{ }^{d}$ \\
\hline Lead & $9.8(<\mathrm{BG})$ & 353 & $10.2^{b}$ & $10.2^{b}$ & No & -- \\
\hline
\end{tabular}


Table 2d. Confirmatory Sample Area 4-Comparison of Maximum Values to Action Levels at the 128-F-2 Site. (2 Pages)

\begin{tabular}{|c|c|c|c|c|c|c|}
\hline \multirow[b]{2}{*}{ COPC } & \multirow[b]{2}{*}{$\begin{array}{c}\text { Maximum Result } \\
(\mathrm{mg} / \mathrm{kg})\end{array}$} & \multicolumn{3}{|c|}{ Soil Cleanup Levels, (mg/kg) ${ }^{\text {a }}$} & \multirow{2}{*}{\begin{tabular}{|c|} 
Does the \\
Maximum \\
Result Exceed \\
RAGs?
\end{tabular}} & \multirow{2}{*}{$\begin{array}{c}\text { Does the } \\
\text { Maximum } \\
\text { Result Pass } \\
\text { RESRAD } \\
\text { Modeling? }\end{array}$} \\
\hline & & $\begin{array}{c}\text { Direct } \\
\text { Exposure }\end{array}$ & $\begin{array}{c}\text { Groundwater } \\
\text { Protection }\end{array}$ & $\begin{array}{c}\text { River } \\
\text { Protection }\end{array}$ & & \\
\hline Manganese & 4,540 & 11,200 & $512^{b}$ & $512^{b}$ & Yes & $-{ }^{d}$ \\
\hline Molybdenum $^{\mathrm{f}}$ & 2.1 & 400 & 8 & NA & No & -- \\
\hline Nickel & 41.1 & 1,600 & $19.1^{b}$ & 27.4 & Yes & $-{ }^{\mathrm{d}}$ \\
\hline Selenium & $0.65(<\mathrm{BG})$ & 400 & 5 & 1 & No & -- \\
\hline Silver & 14.9 & 400 & 8 & $0.73^{b}$ & Yes & $-{ }^{d}$ \\
\hline Vanadium & $62.3(<\mathrm{BG})$ & 560 & $85.1^{b}$ & NA & No & -- \\
\hline Zinc & $52.1(<\mathrm{BG})$ & 24,000 & 480 & $67.8^{\mathrm{b}}$ & No & -- \\
\hline Acenaphthylene $^{\mathrm{g}}$ & 0.078 & 4,800 & 96 & 129 & No & -- \\
\hline Acenaphthene & 0.085 & 4,800 & 96 & 129 & No & -- \\
\hline Bis(2-ethylhexyl)phthalate & 0.028 & 71.4 & 0.625 & 0.36 & No & -- \\
\hline Fluorene & 0.035 & 3,200 & 64 & 260 & No & -- \\
\hline Naphthalene & 0.60 & 1,600 & 16.0 & 988 & No & -- \\
\hline Phenanthrene $^{\mathrm{g}}$ & 0.078 & 24,000 & 240 & 1,920 & No & -- \\
\hline
\end{tabular}

${ }^{a}$ Lookup values and RAGs obtained from the 100 Area RDR/RAWP (DOE-RL 2005) or calculated per WAC-173-340-720, WAC-173340-730, and WAC-173-340-740, Method B, unless otherwise noted.

${ }^{\mathrm{b}}$ Where cleanup levels are less than background, cleanup levels default to background per WAC 173-340-700[4][d].

${ }^{\mathrm{c}}$ RESRAD evaluation not performed; failed direct exposure RAG.

${ }^{\mathrm{d}}$ The groundwater/ river protection evaluation was not completed because one or more COPCs failed direct exposure cleanup criteria and the site was remediated.

${ }^{\text {e }}$ Carcinogenic cleanup level calculated based on the inhalation exposure pathway (WAC 173-340-750[3]) using an airborne particulate mass-loading rate of $0.0001 \mathrm{~g} / \mathrm{m}^{3}$ (WDOH 1997).

${ }^{\mathrm{f}}$ No Hanford Site-specific or Washington State background value available.

${ }^{\mathrm{g}}$ Toxicity data for this chemical are not available. Cleanup levels are based on surrogate chemicals:

Contaminant: acenapthylene; surrogate: acenapthene

Contaminant: phenathrene; surrogate: anthracene.

-- $\quad=$ not applicable

BG = background

COPC = contaminant of potential concern

NA $\quad=$ not available

RAG = remedial action goal

RDL = required detection limit

RESRAD = RESidual RADioactivity (dose model)

WAC $=$ Washington Administrative Code

Analytical results from the inductively coupled plasma metals, mercury, semivolatile organic compound (SVOC), PAH, pesticides, and total petroleum hydrocarbon (TPH) analyses indicated that multiple constituents in each of the four sample areas exceeded the lookup values for direct exposure, groundwater, and/or river protection. Because direct exposure cleanup levels were exceeded for one or more constituents in all four sample areas it was determined that all of the sample areas would undergo remediation. Therefore, the evaluation of groundwater and river protection to determine which constituents may not reach groundwater within 1,000 years was not completed. 
Although no RAGs are established in the Remaining Sites ROD (EPA 1999) for asbestos, an agreement between the U.S. Department of Energy (DOE) and the EPA implies that total friable asbestos concentrations in building or facility materials on the Hanford Site must be less than $1 \%$. At least one sample of PACM collected in each of the four sample areas contained asbestiform minerals at concentrations exceeding $1 \%$. As a result, asbestos was considered a COC for the entire site. The asbestos concentrations in samples collected from each sample area are included in Appendix A (Table A-1).

\section{Confirmatory Sampling Summary}

The results of confirmatory sampling were used to determine the COCs and COPCs for verification sampling. Metals (including hexavalent chromium and mercury), SVOCs (inclusive of the PAH detected in confirmatory samples), chlorinated pesticides, TPH, PCBs, and asbestos were considered COCs/COPCs for verification sampling. Additionally, because radiological activity was detected above background levels during confirmatory sampling by field instrumentation, laboratory radiological analyses were performed on verification samples to verify the absence of radiological contamination.

\section{REMEDIAL ACTION SUMMARY}

\section{Phase I Cleanup Activities}

Remediation of the 128-F-2 waste site was initially performed from August 17 through October 3, 2005, with 21,900 metric tons (24,100 US tons) of material excavated and disposed at the Environmental Restoration Disposal Facility (ERDF). Scrap metal, concrete debris, bottles, laboratory glassware, ash, potentially contaminated soil, asbestos debris, and other miscellaneous materials were removed from the waste site. Excavated materials were sorted and segregated based on visual inspection and location of origin and then staged onsite before disposal at ERDF. Samples of soil and debris were collected as needed to support waste characterization. A silt fence supported by hay bales was installed at the river edge of the excavation to prevent materials from the excavation from moving downslope toward the river prior to backfill or other stabilization of the excavation.

On August 19, 2005, soon after the onset of remediation, an area of cultural significance was discovered in the northwestern end of the riverside excavation (WCH 2006a). The first discovery was a thin concentration of mussel shell and bone fragments in the wall of the excavation. Additional artifacts (subsistence bone, chipped stone tools, knives, several rough stone tools, and trade beads) were discovered on the surface in nearby recently scraped areas on August 22, 2005. During a follow-up inspection on August 29, 2005, a hearth was discovered. The area of cultural significance as shown in Figure 5 was excluded from disturbance during remediation activities and was backfilled between February 20 and February 28, 2008. 
Figure 5. Area of Cultural Significance Discovered within the 128-F-2 Waste Site.

G: \100F\121905K.dwg

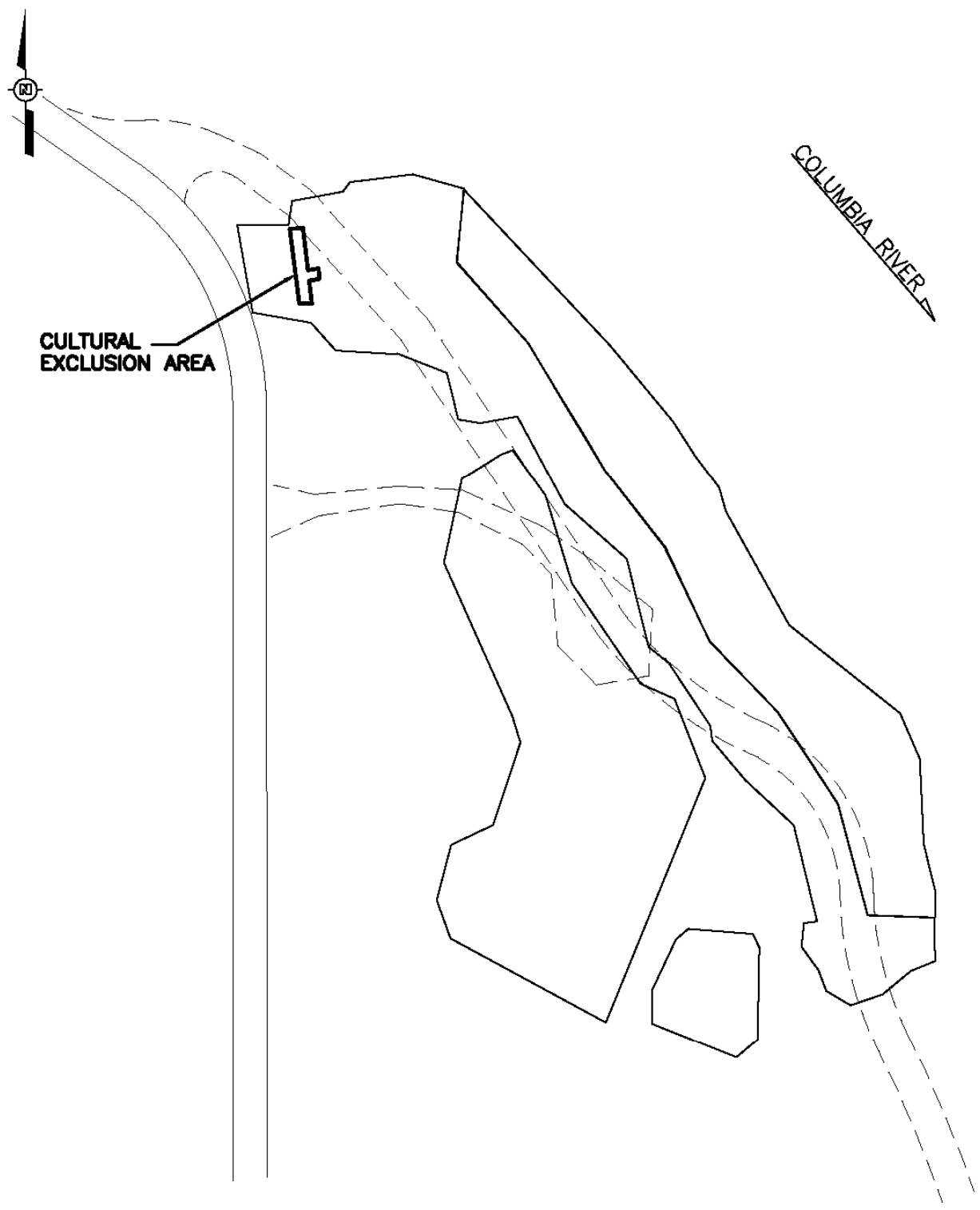

\section{Legend}

\section{ニ二ニニ Dirt Roads}

Paved Roads 


\section{Phase II Cleanup Activities}

During the initial 2005 remediation activities, it was observed that debris and ash material extended beyond the excavation design boundaries of the site toward the river shoreline and appeared to be present below the OHWM. Further excavation was delayed until October 2006 to take advantage of lower flows of the Columbia River and avoid impacts to endangered Upper Columbia River (UCR) spring Chinook salmon and steelhead. On September 14, 2006, Washington Closure Hanford (WCH) notified the National Marine Fisheries Service (NMFS) of the proposed additional excavation (WCH 2006b) that was to stop at the OHWM.

Removal of the silt fence and excavation of the soil containing debris and ash within the slope extending to the OHWM was resumed October 2006. The remediation proceeded to the shoreline, leaving a low berm of unexcavated soil between the excavation and the river to protect against site flooding in the event of higher river stages. Figure 6 provides a cross-section schematic of the additional soil and debris removal and photographs are presented in Appendix B. Soil samples were collected from the berm to evaluate if it could be left in place or required removal (Figure 7). The data showed individual samples contained levels of hexavalent chromium and lead that exceeded direct exposure RAGs and levels of barium, cadmium, chromium, copper, manganese, mercury, nickel, silver, zinc, PAHs, and PCBs that exceeded the criteria for protection of groundwater and the Columbia River. The results of the berm soil samples are provided in Appendix A (Table A-2).

Due to unexpectedly high river flows during Phase II remediation, water infiltration was observed in the low-lying areas within the excavation adjacent to the berm. To expedite potential backfilling in the event the area required stabilization, 10 soil samples were collected within the low-lying area in late October and early November 2006 and submitted for metals analysis only, as other in-process data results for the area indicated that residual metal contamination is the primary risk driver for the site (Figure 8). Grab samples were also collected from random locations within the remaining portion of the riverside excavation (area A) and at the adjacent exposed river bed substrate during low water. The data showed individual samples taken within area C (J13PC0, J13PC1, and J13PB1 through J13PB9) contained levels of hexavalent chromium that exceeded direct exposure RAGs and levels of barium, cadmium, chromium, copper, lead, manganese, nickel, and zinc that exceeded the soil criteria for protection of groundwater and the Columbia River. In contrast, data from individual grab samples taken from the exposed river bed and from within the boundary of area A (J13PC2 through J13PC7) showed levels of lead and/or chromium slightly higher than the soil criteria for protection of groundwater and the Columbia River. The laboratory analytical results for these samples are provided in Appendix A (Table A-3), and photographs of the excavation are presented in Appendix B. 
Figure 6. Cross-Section Schematic of Phase II Remediation at the 128-F-2 Waste Site.

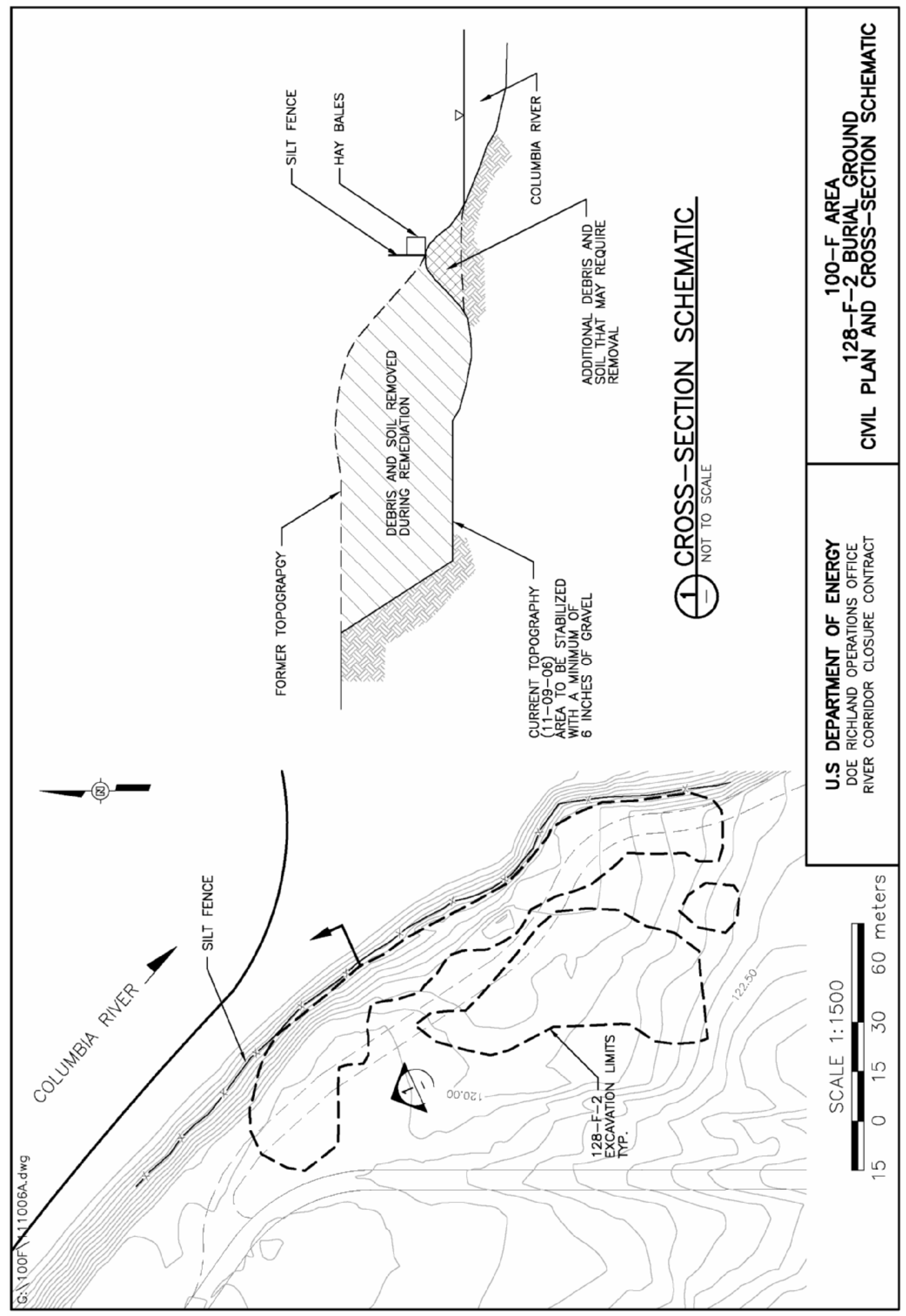


Figure 7. Locations of In-Process Berm Samples (Overlay Shows Excavation of Areas A, B, and C).

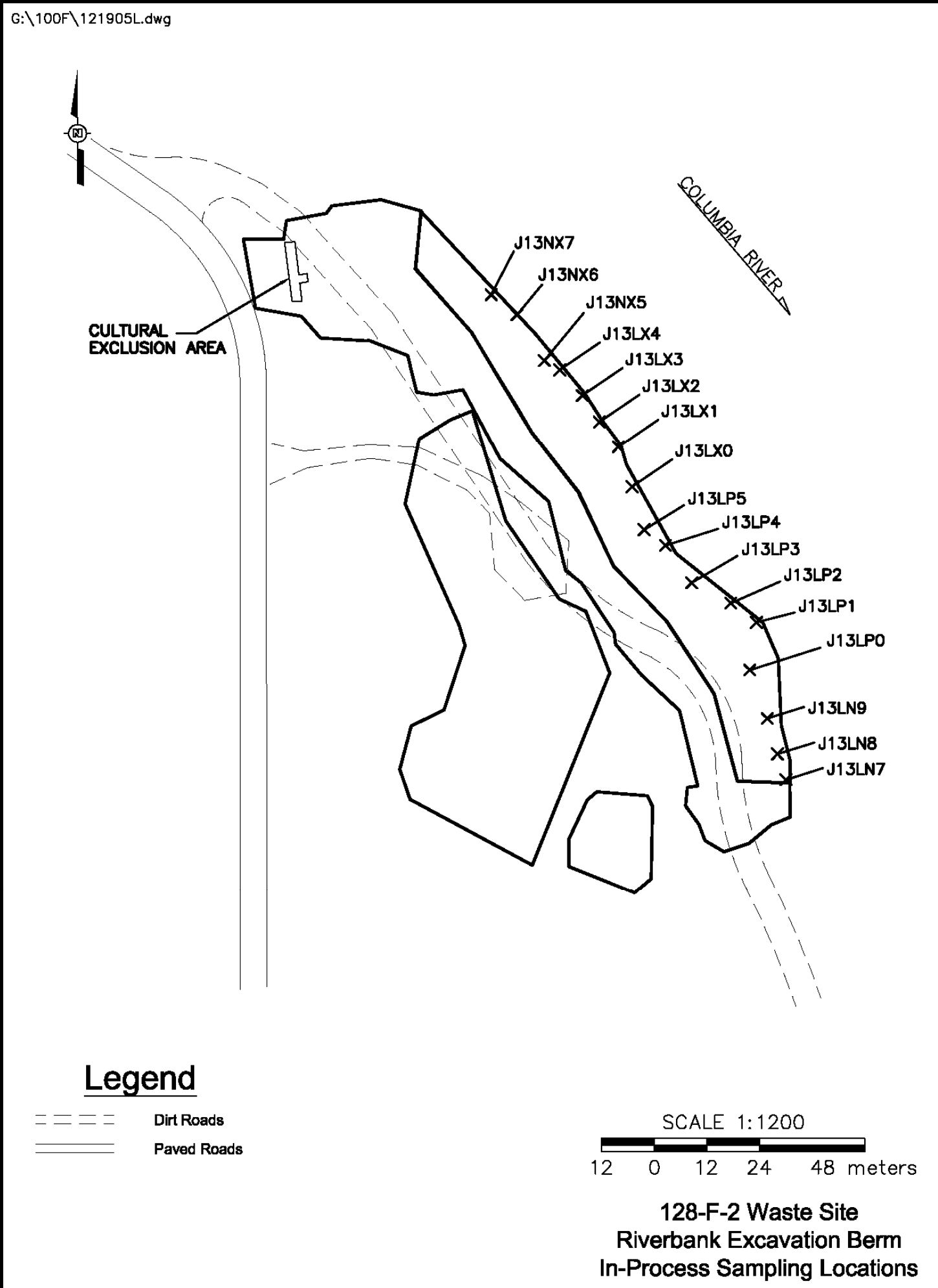


Figure 8. Locations of In-Process Low-Lying Area Samples (Overlay Shows Excavation of Areas A, B, and C).

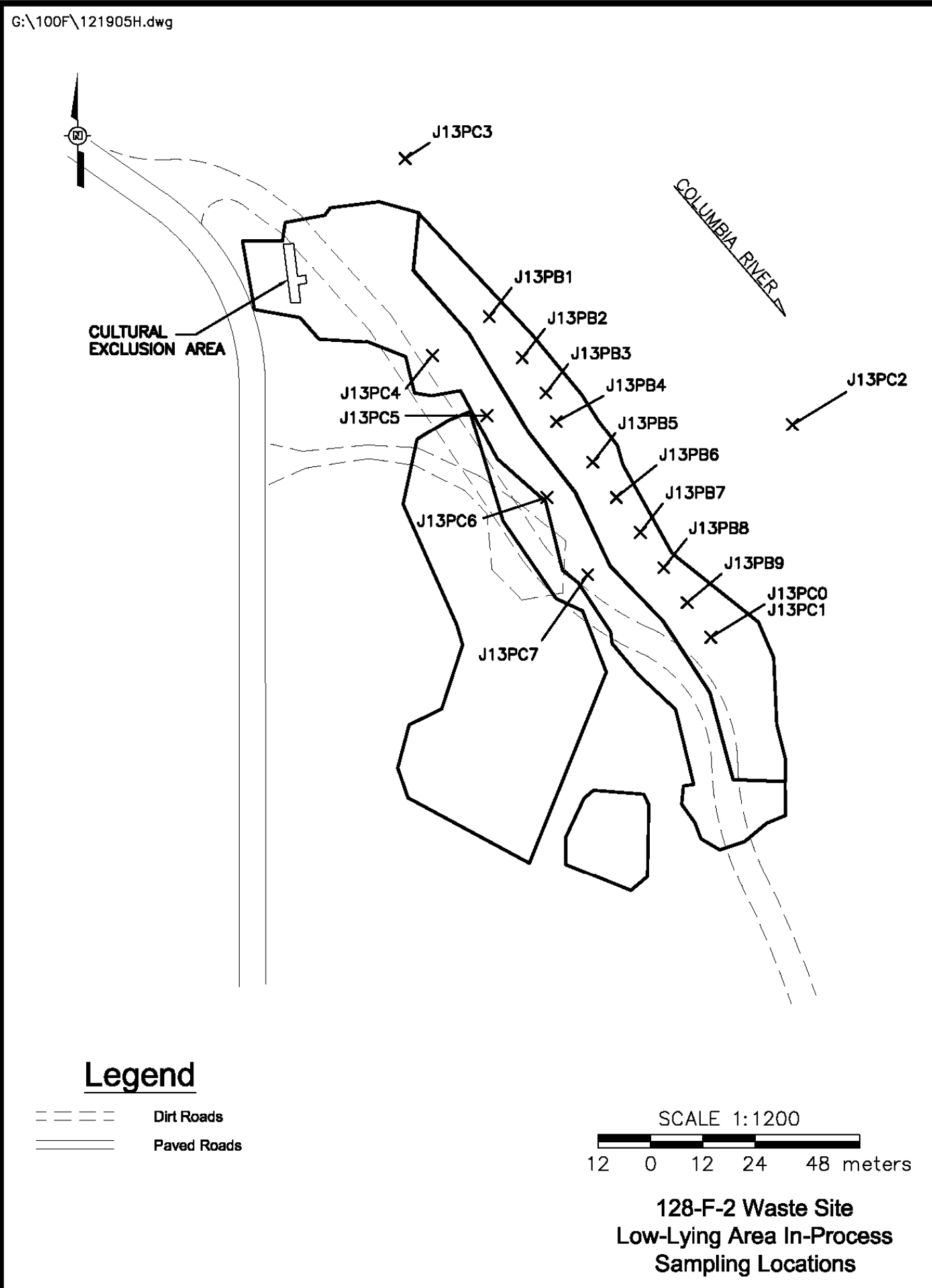


Given these results from the in-process samples, a letter requesting consultation under Section 7 of the Endangered Species Act of 1973, and concurrence on the approach for excavating the 128-F-2 site beyond the OHWM, was submitted to NMFS on November 7, 2006 (DOE-RL 2006). The NMFS provided concurrence (NMFS 2006) that the proposed action was not likely to adversely affect the UCR spring Chinook salmon and steelhead or their critical habitat contingent upon full implementation of the minimization measures proposed by $\mathrm{WCH}$ and DOE (DOE-RL 2006). The NMFS also agreed that the conservation methods proposed by DOE to address the ESA concerns are also adequate to avoid, minimize, or otherwise offset potential adverse effects to designated Essential Fish Habitat as defined by the Magnuson-Stevens Fishery Conservation and Management Act.

\section{In-Process Sample Results}

Laboratory analytical results for in-process soil samples collected from the low-lying areas within the Phase II excavation and from the soil berm indicated the presence of residual metals contamination exceeding the cleanup criteria for protection of groundwater and the Columbia River. Barium, cadmium, chromium, copper, hexavalent chromium, lead, manganese, and zinc were measured in soil samples at levels exceeding the criteria for protection of groundwater and the Columbia River. Mercury and silver were also detected below statistical background levels in several of the soil samples collected from the berm.

Data evaluation indicated that residual metals contamination in area $\mathrm{C}$ was likely to be present in soil directly in contact with groundwater. A meeting was held with the EPA on December 12, 2006 to discuss the sampling results and determine a path forward for site closeout. It was decided to excavate and remove both the soil berm and additional underlying soil below the OHWM and then perform verification sampling within this low-lying area along the river shore. Additionally, three test pits were to be dug to collect soil samples to further evaluate the vertical distribution of metals contamination near the river shore.

This excavation, which included the soil berm, underlying soil, and additional soil from the low-lying area, was performed in February 2007 (photographs are presented in Appendix B). Excavation activities did not extend into groundwater or into the river bed. Because the excavation extended below the OHWM, verification sampling and backfilling of this area were performed nearly simultaneously in order to stabilize the river bank prior to increased spring river flows. As such, the backfilling of the low-lying area was performed prior to receipt of the verification data. Due to the presence of groundwater just below the excavated surface, only one of the three planned test pits could be sampled.

The results of the February 2007 area C verification samples showed exceedances of direct exposure soil RAGs for arsenic and hexavalent chromium based on individual sample results. By the same measure, groundwater and river protection soil RAGs were exceeded for barium, cadmium, chromium, cobalt, copper, lead, manganese, nickel, aroclor-1260, DDT, and zinc at several sample locations. The sample results are presented in Appendix A (Table A-4).

An agreement was reached in April 2008 between the EPA, DOE, Richland Operations Office, and $\mathrm{WCH}$ that further characterization would be conducted in area $\mathrm{C}$ to determine the nature and 
extent of the contamination. It was also agreed that area $C$ would become a new waste site due to the inherent differences and complexities of remediation in a riparian zone as opposed to nonriparian areas. In July, 2008, Area C was combined with adjoining riparian areas to the east and southeast known to have levels of metals in excess of soil RAGs and submitted to WIDS as a discovery site, 100-F-59, Riparian Area Contamination Originating from 128-F-2. Therefore, sample results from area $\mathrm{C}$ are not further discussed in this Remaining Sites Verification Package (RSVP).

Additional remediation was also conducted in a small section of area A in March 2008. The area was the location of a verification sample (A-7) that had slightly elevated levels of several metals and PAHs (Appendix A, Table A-7). Although the levels of metals and PAHs did not exceed direct exposure RAGs, additional material was removed as a conservative measure due to the proximity of the site to the Columbia River. A replacement verification sample was taken at the same coordinates of the original sample and the results were used in closeout calculations and comparisons. Figure 9 presents the post-excavation civil survey for the waste site.

\section{VERIFICATION SAMPLING ACTIVITIES}

Remedial action goals are the specific numeric goals against which the cleanup verification data are evaluated to demonstrate attainment of the remedial action objectives for the site. The final verification sampling for the 128-F-2 waste site was performed on February 28, 2007, March 1, 2007, and March 5, 2008 for area A; March 6 and August 16, 2007 for area B; and December 11, 2007 for areas D and E (WCH 2007a, 2007b, 2007c, 2008) to collect data to determine if the RAGs had been met.

\section{Contaminants of Concern/Contaminants of Potential Concern}

The results of confirmatory sampling were used to determine the COCs and contaminants of potential concern COPCs for verification sampling. Metals (including hexavalent chromium and mercury), SVOCs (inclusive of the PAHs detected in confirmatory samples), chlorinated pesticides, TPH, PCBs, and asbestos were considered COCs/COPCs for verification sampling. Additionally, because radiological activity was detected above background levels during confirmatory sampling by field instrumentation, laboratory radiological analysis was performed on verification samples to verify the absence of radiological contamination.

\section{Verification Sample Design}

This section describes the basis for selection of an appropriate sample design and determination of the number of verification samples to collect. The excavation boundary survey, incorporating Phase I and Phase II excavation, was used to divide the 128-F-2 site into five sampling areas for the purpose of verification sampling, as listed in Table 3. A statistical sampling approach was implemented for sampling areas A, B, and C, as described in the work instruction (WCH 2007d). The sampling designs for sampling areas $\mathrm{D}$ and $\mathrm{E}$ were based on professional judgment in consideration of conditions particular to these decision units. Three test pits were planned for excavation within area $C$ to collect soil samples at $0.3 \mathrm{~m}(1-\mathrm{ft})$ intervals bgs until groundwater was reached. However, only one of the three pits was in fact dug and sampled due to the high groundwater level (less than $0.3 \mathrm{~m}[1-\mathrm{ft}] \mathrm{bgs}$ ). 
Figure 9. Post-Remediation Civil Survey of the 128-F-2 Waste Site.

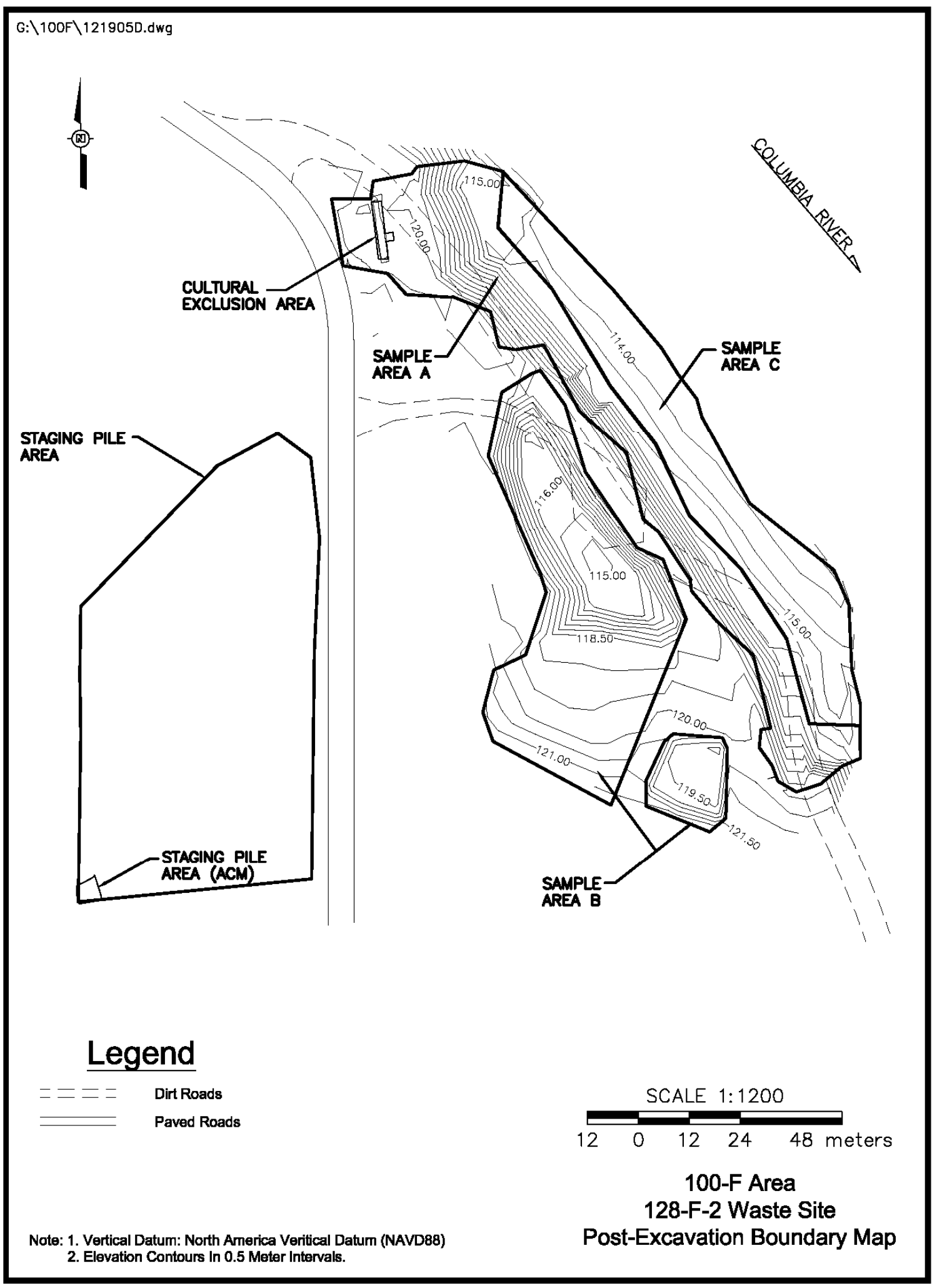


Table 3. Verification Sampling Areas for the 128-F-2 Waste Site.

\begin{tabular}{|l|}
\hline A. Phase I excavation between the river and the upland excavated areas \\
\hline B. Upland excavation \\
\hline C. Phase II excavation \\
\hline D. General waste staging area footprint \\
\hline E. Asbestos-containing material waste staging pile footprint \\
\hline
\end{tabular}

\section{Verification Sampling - Excavated Areas}

The decision rule for demonstrating compliance with the cleanup criteria requires comparison of the true population mean, as estimated by the 95\% upper confidence limit (UCL) on the sample mean, with the cleanup level. Therefore, a statistical sampling design is the preferred verification sampling approach for this site because the distribution of potential residual soil contamination over the site is uncertain. The Washington State Department of Ecology publication Guidance on Sampling and Data Analysis Methods (Ecology 1995) recommends that systematic sampling with sample locations distributed over the entire study area be used. This sampling approach is referred to by the Washington State Department of Ecology as "area-wide sampling."

Statistical parameters (i.e., standard deviation within the populations) for residual contaminant levels at the 128-F-2 site were unknown. For the upland excavated area, the standard deviations of the residual contaminant populations were assumed to be less than $25 \%$ of the corresponding decision thresholds for the populations. Greater heterogeneity may exist within the excavation along the river shoreline due to limitations imposed on excavation in consideration of the proximity of the river; the standard deviations for residual contaminant populations within this excavation (sampling areas A and B) were, therefore, assumed to be up to $30 \%$ of their respective decision thresholds.

Sampling areas A, B, and C were delineated in Visual Sample Plan ${ }^{1}$ (VSP) and used as the basis for location of a random-start systematic grid for verification soil sample collection. Ten samples were collected on the grid associated with area B, and 12 samples were collected on the grid associated with each of sampling areas A and C, as shown in Figure 10. Triangular grids were selected based on studies indicating triangular grids are superior to square grids (Gilbert 1987). Additional details concerning the use of VSP to develop the statistical sampling design and derive the number of verification samples to collect are discussed in the verification work instruction (WCH 2007d).

\footnotetext{
${ }^{1}$ Visual Sample Plan is a site map-based user-interface program that may be downloaded at http://dqo.pnl.gov.
} 
Figure 10. 128-F-2 Verification Sample Locations.

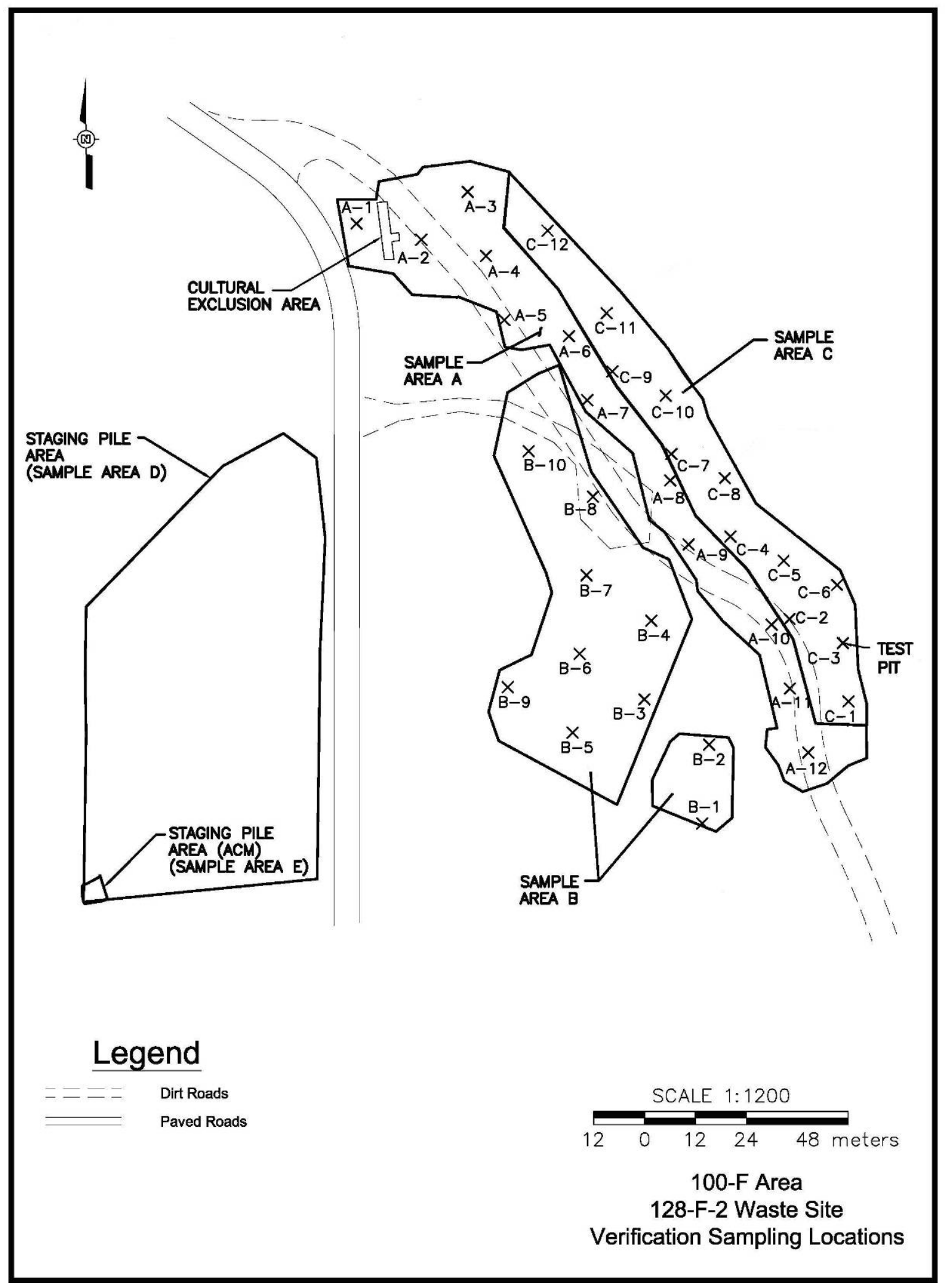


A second sampling design was prepared in VSP for area B after the verification sample results showed elevated levels of hexavalent chromium (Appendix C). After excavation and removal of additional material, verification samples for hexavalent chromium analysis were taken at the locations determined in the second sampling design.

\section{Verification Sampling - Waste Staging Areas}

Waste staged onsite during remedial activities consisted of soil and debris. The waste was completely removed and disposed at the ERDF, along with a layer of underlying soil. There was very little potential for contaminant migration into soils underlying the waste staging piles; therefore, statistical sampling designs were not warranted for the staging pile footprints and professional judgment was used to develop the sampling design. Sampling at the primary waste staging area consisted of dividing the area into 3 regions (northern, central, and southern) of approximately equal size, collecting 30 aliquots of soil distributed across the surface of each region, and combining into 1 sample per region for laboratory analysis. Sampling at the asbestos-containing material (ACM) staging area consisted of collecting 30 aliquots of soil distributed within the footprint and combining into 1 sample for laboratory asbestos analysis only.

\section{Verification Sampling Results}

Verification samples were analyzed using EPA-approved analytical methods. The laboratoryreported data results for all constituents are stored in the ENRE project-specific database prior to archival in the HEIS database and are presented in Attachment 1 of the 95\% UCL calculation for the respective sampling area (Appendix D).

As previously described, the 128-F-2 waste site was divided into five sampling areas for verification sampling: (1) area A (Phase I excavated area between Columbia River and the upland excavation), (2) area B (upland excavation), (3) area C (phase II excavation), (4) area D (waste staging area), and (5) area E (ACM staging area).

Verification data from the excavation areas A and B were evaluated independently by calculating the 95\% UCL on the true population mean for residual concentrations of COPCs as specified by the RDR/RAWP (DOE-RL 2005). These calculations are provided in Appendix D.

The area C excavation is not being closed under this RSVP due to uncertainty regarding the nature and extent of remaining contaminants and the need to determine an appropriate path forward for this riparian area. The verification data from area $\mathrm{C}$ is presented in Appendix A, but is not discussed in this RSVP.

Evaluation of the verification data from area $\mathrm{D}$ was performed by direct comparison of the sample results against cleanup criteria. Evaluation of the sample results from area $\mathrm{E}$ was performed by direct evaluation of the amount of asbestos fibers present in the sample. Sample results from area $\mathrm{D}$ are presented in Appendix A (Table A-5). Verification sample results for asbestos from all five areas are presented in Appendix A (Table A-6). 
A complete listing of samples, coordinates, and COCs/COPCs is presented in Table 4.

Table 4. Verification Sample Summary for Areas A, B, D, and E of the 128-F-2 Waste Site. ${ }^{a}$ (5 Pages)

\begin{tabular}{|c|c|c|c|c|}
\hline \multirow{2}{*}{$\begin{array}{c}\text { Sample } \\
\text { Location }\end{array}$} & \multirow{2}{*}{$\begin{array}{l}\text { Sample } \\
\text { Media }\end{array}$} & $\begin{array}{c}\text { Actual } \\
\text { Coordinates }\end{array}$ & \multirow{2}{*}{$\begin{array}{c}\text { HEIS } \\
\text { Number }\end{array}$} & \multirow{2}{*}{ Sample Analysis } \\
\hline & & $\begin{array}{c}\text { Northing } \\
\text { Easting }\end{array}$ & & \\
\hline A1 & Soil & $\begin{array}{l}\text { N } 147901.6 \\
\text { E } 581222.9\end{array}$ & J14KB8 & Bulk asbestos \\
\hline A2 & Soil & $\begin{array}{l}\text { N } 147897.8 \\
\text { E } 581238.1\end{array}$ & J14KB9 & Bulk asbestos \\
\hline A3 & Soil & $\begin{array}{l}\text { N } 147909.1 \\
\text { E } 581249.1\end{array}$ & J14KC0 & Bulk asbestos \\
\hline A4 & Soil & $\begin{array}{l}\text { N } 147894.0 \\
\text { E } 581253.4\end{array}$ & J14KC1 & Bulk asbestos \\
\hline A5 & Soil & $\begin{array}{l}\text { N } 147878.9 \\
\text { E } 581257.7\end{array}$ & J14KC2 & Bulk asbestos \\
\hline A6 & Soil & $\begin{array}{l}\text { N } 147875.1 \\
\text { E } 581272.9\end{array}$ & J14KC3 & Bulk asbestos \\
\hline A7 & Soil & $\begin{array}{l}\text { N } 147860.0 \\
\text { E } 581277.0\end{array}$ & J16DF4 & Bulk asbestos \\
\hline A8 & Soil & $\begin{array}{l}\text { N } 147841.1 \\
\text { E } 581296.8\end{array}$ & J14KC5 & Bulk asbestos \\
\hline A9 & Soil & $\begin{array}{l}\text { N } 147825.9 \\
\text { E } 581301.1\end{array}$ & J14KC6 & Bulk asbestos \\
\hline A10 & Soil & $\begin{array}{l}\text { N } 147807.0 \\
\text { E } 581320.7\end{array}$ & J14KC7 & Bulk asbestos \\
\hline A11 & Soil & $\begin{array}{l}\text { N } 147791.9 \\
\text { E } 581325.0\end{array}$ & J14KC8 & Bulk asbestos \\
\hline A12 & Soil & $\begin{array}{l}\text { N } 147776.8 \\
\text { E } 581329.3\end{array}$ & $\mathrm{~J} 14 \mathrm{KD} 0$ & Bulk asbestos \\
\hline A1 & Soil & $\begin{array}{l}\text { N } 147901.6 \\
\text { E } 581222.9\end{array}$ & J14K95 & $\begin{array}{l}\text { GEA, gross alpha, gross beta, ICP metals, } \\
\text { hexavalent chromium, mercury, PCB, SVOA, } \\
\text { pesticides, and TPH }\end{array}$ \\
\hline A2 & Soil & $\begin{array}{l}\text { N } 147897.8 \\
\text { E 581238.1 }\end{array}$ & J14K96 & $\begin{array}{l}\text { GEA, gross alpha, gross beta, ICP metals, } \\
\text { hexavalent chromium, mercury, PCB, SVOA, } \\
\text { pesticides, and TPH }\end{array}$ \\
\hline A3 & Soil & $\begin{array}{l}\text { N } 147909.1 \\
\text { E } 581249.1\end{array}$ & J14K97 & $\begin{array}{l}\text { GEA, gross alpha, gross beta, ICP metals, } \\
\text { hexavalent chromium, mercury, PCB, SVOA, } \\
\text { pesticides, and TPH }\end{array}$ \\
\hline
\end{tabular}


Table 4. Verification Sample Summary for Areas A, B, D, and E of the 128-F-2 Waste Site. ${ }^{\mathrm{a}}$ (5 Pages)

\begin{tabular}{|c|c|c|c|c|}
\hline \multirow{2}{*}{$\begin{array}{c}\text { Sample } \\
\text { Location }\end{array}$} & \multirow{2}{*}{$\begin{array}{l}\text { Sample } \\
\text { Media }\end{array}$} & $\begin{array}{c}\text { Actual } \\
\text { Coordinates }^{\mathbf{b}} \\
\end{array}$ & \multirow{2}{*}{$\begin{array}{c}\text { HEIS } \\
\text { Number }\end{array}$} & \multirow{2}{*}{ Sample Analysis } \\
\hline & & $\begin{array}{l}\text { Northing } \\
\text { Easting }\end{array}$ & & \\
\hline A4 & Soil & $\begin{array}{l}\text { N } 147894.0 \\
\text { E } 581253.4\end{array}$ & J14K98 & $\begin{array}{l}\text { GEA, gross alpha, gross beta, ICP metals, } \\
\text { hexavalent chromium, mercury, PCB, SVOA, } \\
\text { pesticides, and TPH }\end{array}$ \\
\hline A5 & Soil & $\begin{array}{l}\text { N } 147878.9 \\
\text { E } 581257.7\end{array}$ & J14K99 & $\begin{array}{l}\text { GEA, gross alpha, gross beta, ICP metals, } \\
\text { hexavalent chromium, mercury, PCB, SVOA, } \\
\text { pesticides, and TPH }\end{array}$ \\
\hline A6 & Soil & $\begin{array}{l}\text { N } 147875.1 \\
\text { E } 581272.9\end{array}$ & J14KB0 & $\begin{array}{l}\text { GEA, gross alpha, gross beta, ICP metals, } \\
\text { hexavalent chromium, mercury, PCB, SVOA, } \\
\text { pesticides, and TPH }\end{array}$ \\
\hline A7 & Soil & $\begin{array}{l}\text { N } 147860.0 \\
\text { E } 581277.0\end{array}$ & J16DF5 & $\begin{array}{l}\text { GEA, gross alpha, gross beta, ICP metals, } \\
\text { hexavalent chromium, mercury, PCB, SVOA, } \\
\text { pesticides, and TPH }\end{array}$ \\
\hline A8 & Soil & $\begin{array}{l}\text { N } 147841.1 \\
\text { E } 581296.8\end{array}$ & J14KB2 & $\begin{array}{l}\text { GEA, gross alpha, gross beta, ICP metals, } \\
\text { hexavalent chromium, mercury, PCB, SVOA, } \\
\text { pesticides, and TPH }\end{array}$ \\
\hline A9 & Soil & $\begin{array}{l}\text { N } 147825.9 \\
\text { E } 581301.1\end{array}$ & J14KB3 & $\begin{array}{l}\text { GEA, gross alpha, gross beta, ICP metals, } \\
\text { hexavalent chromium, mercury, PCB, SVOA, } \\
\text { pesticides, and TPH }\end{array}$ \\
\hline A10 & Soil & $\begin{array}{l}\text { N } 147807.0 \\
\text { E } 581320.7\end{array}$ & J14KB4 & $\begin{array}{l}\text { GEA, gross alpha, gross beta, ICP metals, } \\
\text { hexavalent chromium, mercury, PCB, SVOA, } \\
\text { pesticides, and TPH }\end{array}$ \\
\hline A11 & Soil & $\begin{array}{l}\text { N } 147791.9 \\
\text { E } 581325.0\end{array}$ & J14KB5 & $\begin{array}{l}\text { GEA, gross alpha, gross beta, ICP metals, } \\
\text { hexavalent chromium, mercury, PCB, SVOA, } \\
\text { pesticides, and TPH }\end{array}$ \\
\hline A12 & Soil & $\begin{array}{l}\text { N } 147776.8 \\
\text { E } 581329.3\end{array}$ & J14KB7 & $\begin{array}{l}\text { GEA, gross alpha, gross beta, ICP metals, } \\
\text { hexavalent chromium, mercury, PCB, SVOA, } \\
\text { pesticides, and TPH }\end{array}$ \\
\hline B1 & Soil & $\begin{array}{l}\text { N } 147760.1 \\
\text { E } 581309.3\end{array}$ & J14L15 & Bulk asbestos \\
\hline B2 & Soil & $\begin{array}{l}\text { N } 147778.7 \\
\text { E } 581305.9\end{array}$ & J14L16 & Bulk asbestos \\
\hline B3 & Soil & $\begin{array}{l}\text { N } 147789.4 \\
\text { E } 581290.7\end{array}$ & J14L17 & Bulk asbestos \\
\hline B4 & Soil & $\begin{array}{l}\text { N } 147808.0 \\
\text { E } 581292.3\end{array}$ & J14L18 & Bulk asbestos \\
\hline B5 & Soil & $\begin{array}{l}\text { N } 147781.5 \\
\text { E } 581273.8\end{array}$ & J14L19 & Bulk asbestos \\
\hline B6 & Soil & $\begin{array}{l}\text { N } 147800.1 \\
\text { E } 581275.4\end{array}$ & J14L21 & Bulk asbestos \\
\hline
\end{tabular}


Table 4. Verification Sample Summary for Areas A, B, D, and E of the 128-F-2 Waste Site. ${ }^{\mathrm{a}}$ (5 Pages)

\begin{tabular}{|c|c|c|c|c|}
\hline \multirow{2}{*}{$\begin{array}{l}\text { Sample } \\
\text { Location }\end{array}$} & \multirow{2}{*}{$\begin{array}{l}\text { Sample } \\
\text { Media }\end{array}$} & $\begin{array}{c}\text { Actual } \\
\text { Coordinates }^{b}\end{array}$ & \multirow{2}{*}{$\begin{array}{c}\text { HEIS } \\
\text { Number }\end{array}$} & \multirow{2}{*}{ Sample Analysis } \\
\hline & & $\begin{array}{l}\text { Northing } \\
\text { Easting }\end{array}$ & & \\
\hline B7 & Soil & $\begin{array}{l}\text { N } 147818.7 \\
\text { E } 581277.1\end{array}$ & J14L22 & Bulk asbestos \\
\hline B8 & Soil & $\begin{array}{l}\text { N } 147837.2 \\
\text { E } 581278.7\end{array}$ & J14L23 & Bulk asbestos \\
\hline B9 & Soil & $\begin{array}{l}\text { N } 147792.2 \\
\text { E } 581258.5\end{array}$ & J14L24 & Bulk asbestos \\
\hline B10 & Soil & $\begin{array}{l}\text { N } 147847.9 \\
\text { E } 581263.4\end{array}$ & J14L25 & Bulk asbestos \\
\hline B1 & Soil & $\begin{array}{l}\text { N } 147760.1 \\
\text { E } 581309.3\end{array}$ & J14L26 & $\begin{array}{l}\text { GEA, gross alpha, gross beta, ICP metals, } \\
\text { mercury, PCB, SVOA, pesticides, and TPH }\end{array}$ \\
\hline B2 & Soil & $\begin{array}{l}\text { N } 147778.7 \\
\text { E } 581305.9\end{array}$ & J14L27 & $\begin{array}{l}\text { GEA, gross alpha, gross beta, ICP metals, } \\
\text { mercury, PCB, SVOA, pesticides, and TPH }\end{array}$ \\
\hline B3 & Soil & $\begin{array}{l}\text { N } 147789.4 \\
\text { E } 581290.7\end{array}$ & J14L28 & $\begin{array}{l}\text { GEA, gross alpha, gross beta, ICP metals, } \\
\text { mercury, PCB, SVOA, pesticides, and TPH }\end{array}$ \\
\hline B4 & Soil & $\begin{array}{l}\text { N } 147808.0 \\
\text { E } 581292.3\end{array}$ & J14L29 & $\begin{array}{l}\text { GEA, gross alpha, gross beta, ICP metals, } \\
\text { mercury, PCB, SVOA, pesticides, and TPH }\end{array}$ \\
\hline B5 & Soil & $\begin{array}{l}\text { N } 147781.5 \\
\text { E } 581273.8\end{array}$ & J14L30 & $\begin{array}{l}\text { GEA, gross alpha, gross beta, ICP metals, } \\
\text { mercury, PCB, SVOA, pesticides, and TPH }\end{array}$ \\
\hline B6 & Soil & $\begin{array}{l}\text { N } 147800.1 \\
\text { E } 581275.4\end{array}$ & J14L32 & $\begin{array}{l}\text { GEA, gross alpha, gross beta, ICP metals, } \\
\text { mercury, PCB, SVOA, pesticides, and TPH }\end{array}$ \\
\hline B7 & Soil & $\begin{array}{l}\text { N } 147818.7 \\
\text { E } 581277.1\end{array}$ & J14L33 & $\begin{array}{l}\text { GEA, gross alpha, gross beta, ICP metals, } \\
\text { mercury, PCB, SVOA, pesticides, and TPH }\end{array}$ \\
\hline B8 & Soil & $\begin{array}{l}\text { N } 147837.2 \\
\text { E } 581278.7\end{array}$ & J14L34 & $\begin{array}{l}\text { GEA, gross alpha, gross beta, ICP metals, } \\
\text { mercury, PCB, SVOA, pesticides, and TPH }\end{array}$ \\
\hline B9 & Soil & $\begin{array}{l}\text { N } 147792.2 \\
\text { E } 581258.5\end{array}$ & J14L35 & $\begin{array}{l}\text { GEA, gross alpha, gross beta, ICP metals, } \\
\text { mercury, PCB, SVOA, pesticides, and TPH }\end{array}$ \\
\hline B10 & Soil & $\begin{array}{l}\text { N } 147847.9 \\
\text { E } 581263.4\end{array}$ & J14L36 & $\begin{array}{l}\text { GEA, gross alpha, gross beta, ICP metals, } \\
\text { mercury, PCB, SVOA, pesticides, and TPH }\end{array}$ \\
\hline B1 & Soil & $\begin{array}{l}\text { N } 147864.2 \\
\text { E } 581268.7 \\
\end{array}$ & J15FD0 & Hexavalent chromium \\
\hline B2 & Soil & $\begin{array}{l}\text { N } 147845.7 \\
\text { E } 581266.2\end{array}$ & J15FD1 & Hexavalent chromium \\
\hline B3 & Soil & $\begin{array}{l}\text { N } 147790.3 \\
\text { E } 581258.7\end{array}$ & J15FD2 & Hexavalent chromium \\
\hline B4 & Soil & $\begin{array}{l}\text { N } 147834.3 \\
\text { E } 581281.0\end{array}$ & J15FD3 & Hexavalent chromium \\
\hline B5 & Soil & $\begin{array}{l}\text { N } 147815.9 \\
\text { E } 581278.5 \\
\end{array}$ & J15FD4 & Hexavalent chromium \\
\hline B6 & Soil & $\begin{array}{l}\text { N } 147797.4 \\
\text { E } 581276.0 \\
\end{array}$ & J15FD5 & Hexavalent chromium \\
\hline
\end{tabular}


Table 4. Verification Sample Summary for Areas A, B, D, and E of the 128-F-2 Waste Site. ${ }^{\mathrm{a}}$ (5 Pages)

\begin{tabular}{|c|c|c|c|c|}
\hline \multirow{2}{*}{$\begin{array}{c}\text { Sample } \\
\text { Location }\end{array}$} & \multirow{2}{*}{$\begin{array}{l}\text { Sample } \\
\text { Media }\end{array}$} & $\begin{array}{c}\text { Actual } \\
\text { Coordinates }^{b}\end{array}$ & \multirow{2}{*}{$\begin{array}{c}\text { HEIS } \\
\text { Number }\end{array}$} & \multirow{2}{*}{ Sample Analysis } \\
\hline & & $\begin{array}{l}\text { Northing } \\
\text { Easting }\end{array}$ & & \\
\hline B7 & Soil & $\begin{array}{l}\text { N } 147779.0 \\
\text { E } 581273.5\end{array}$ & J15FD6 & Hexavalent chromium \\
\hline B8 & Soil & $\begin{array}{l}\text { N } 147804.5 \\
\text { E } 581293.2\end{array}$ & J15FD7 & Hexavalent chromium \\
\hline B9 & Soil & $\begin{array}{l}\text { N } 147786.0 \\
\text { E } 581290.7 \\
\end{array}$ & J15FD8 & Hexavalent chromium \\
\hline B10 & Soil & $\begin{array}{l}\text { N } 147774.6 \\
\text { E } 581305.5\end{array}$ & J15FD9 & Hexavalent chromium \\
\hline $\begin{array}{l}\text { Waste staging } \\
\text { area-north }\end{array}$ & Soil & Composite & J163R5 & ICP metals, mercury, and hexavalent chromium \\
\hline $\begin{array}{l}\text { Waste staging } \\
\text { area-central }\end{array}$ & Soil & Composite & J163R6 & ICP metals, mercury, and hexavalent chromium \\
\hline $\begin{array}{l}\text { Waste staging } \\
\text { area-south }\end{array}$ & Soil & Composite & J163R7 & ICP metals, mercury, and hexavalent chromium \\
\hline $\begin{array}{l}\text { Waste staging } \\
\text { area-north } \\
\text { Area D }\end{array}$ & Soil & Composite & J163R2 & Bulk asbestos \\
\hline $\begin{array}{l}\text { Waste staging } \\
\text { area-central } \\
\text { Area D }\end{array}$ & Soil & Composite & J163R3 & Bulk asbestos \\
\hline $\begin{array}{l}\text { Waste staging } \\
\text { area-south } \\
\text { Area D }\end{array}$ & Soil & Composite & J163R4 & Bulk asbestos \\
\hline $\begin{array}{l}\text { ACM staging } \\
\text { area (Area E) }\end{array}$ & Soil & Composite & J163T1 & Bulk asbestos \\
\hline $\begin{array}{l}\text { Duplicate of } \\
\text { J14KC8 }\end{array}$ & Soil & $\begin{array}{l}\text { N } 147791.9 \\
\text { E } 581325.0\end{array}$ & J14KC9 & Bulk asbestos \\
\hline $\begin{array}{l}\text { Duplicate of } \\
\text { J14L19 }\end{array}$ & Soil & $\begin{array}{l}\text { N } 147800.1 \\
\text { E } 581275.4 \\
\end{array}$ & J14L20 & Bulk asbestos \\
\hline $\begin{array}{l}\text { Duplicate of } \\
\text { J14KB5 }\end{array}$ & Soil & $\begin{array}{l}\text { N } 147791.9 \\
\text { E } 581325.0\end{array}$ & J14KB6 & $\begin{array}{l}\text { GEA, gross alpha, gross beta, ICP metals, } \\
\text { hexavalent chromium, mercury, PCB, SVOA, } \\
\text { pesticides, and TPH }\end{array}$ \\
\hline $\begin{array}{l}\text { Duplicate of } \\
\text { J14L30 }\end{array}$ & Soil & $\begin{array}{c}\text { N } 147781.5 \\
\text { E } 581273.8\end{array}$ & J14L31 & $\begin{array}{l}\text { GEA, gross alpha, gross beta, ICP metals, } \\
\text { hexavalent chromium, mercury, PCB, SVOA, } \\
\text { pesticides, and TPH }\end{array}$ \\
\hline
\end{tabular}


Table 4. Verification Sample Summary for Areas A, B, D, and E of the 128-F-2 Waste Site. ${ }^{\text {a }}$ (5 Pages)

\begin{tabular}{|c|c|c|c|c|}
\hline \multirow{2}{*}{$\begin{array}{l}\text { Sample } \\
\text { Location }\end{array}$} & \multirow{2}{*}{$\begin{array}{l}\text { Sample } \\
\text { Media }\end{array}$} & $\begin{array}{c}\text { Actual } \\
\text { Coordinates }^{b}\end{array}$ & \multirow{2}{*}{$\begin{array}{c}\text { HEIS } \\
\text { Number }\end{array}$} & \multirow{2}{*}{ Sample Analysis } \\
\hline & & $\begin{array}{l}\text { Northing } \\
\text { Easting }\end{array}$ & & \\
\hline $\begin{array}{l}\text { Duplicate of } \\
\text { J163R7 }\end{array}$ & Soil & Composite & J163R8 & $\begin{array}{l}\text { GEA, gross alpha, gross beta, ICP metals, } \\
\text { hexavalent chromium, mercury, PCB, SVOA, } \\
\text { pesticides, and TPH }\end{array}$ \\
\hline $\begin{array}{l}\text { Duplicate of } \\
\text { J163R4 }\end{array}$ & Soil & Composite & J163T0 & Bulk asbestos \\
\hline $\begin{array}{l}\text { Equipment } \\
\text { blank }\end{array}$ & Silica sand & NA & J14JT6 & ICP metals, hexavalent chromium, mercury \\
\hline $\begin{array}{l}\text { Equipment } \\
\text { blank }\end{array}$ & Silica sand & NA & J14K94 & ICP metals, hexavalent chromium, mercury \\
\hline $\begin{array}{l}\text { Equipment } \\
\text { blank }\end{array}$ & Silica sand & NA & J163R9 & ICP metal and SVOA \\
\hline
\end{tabular}

a Source: Field logbooks EFL-1174-2, EFL-1174-3, and EFL-1174-4 (WCH 2007a, 2007b, 2007c, and 2008).

b Washington State Plane (meters).

GEA = gamma spectroscopy

ICP = inductively coupled plasma

PCB = polychlorinated biphenyl

SVOA $=$ semivolatile organic analysis

$\mathrm{TPH}=$ total petroleum hydrocarbons

Comparisons of the statistical and maximum results for COPCs with the shallow zone RAGs for the excavated areas A and B along with the waste staging area are summarized in Tables 5a, 5b, and $5 c$, respectively. All three decision units are evaluated using the more restrictive shallow zone cleanup criteria. Contaminants that were not detected by laboratory analysis are excluded from these tables. Calculated cleanup levels are not presented in the Cleanup Levels and Risk Calculations Database (Ecology 2005) under Washington Administrative Code (WAC) 173-340-740(3) for aluminum, calcium, iron, magnesium, potassium, silicon, and sodium; therefore, these constituents are not considered site COPCs. Potassium-40, radium-226, radium-228, thorium-228, and thorium-232 were detected in samples collected at the site, but are not considered within statistical calculations or the following tables, as these isotopes are not related to the operational history of the site and were detected below background levels (based on an assumption of secular equilibrium, the background activities for radium-228 and thorium-228 are equal to the statistical background activity of $1.32 \mathrm{pCi} / \mathrm{g}$ for thorium-232 provided in DOE-RL [1996]).

All asbestos results are reported in Appendix A, Table A-6. All samples results reported either trace amount or no detection. All asbestos results are considered acceptable for interim closeout. 
Table 5a. Comparison of Maximum or Statistical Contaminant Concentrations to Action Levels for the 128-F-2 Area A Verification Sampling (2 Pages)

\begin{tabular}{|c|c|c|c|c|c|c|}
\hline \multirow[b]{2}{*}{ COPC } & \multirow[b]{2}{*}{$\begin{array}{c}\text { Maximum } \\
\text { Result (pCi/g) }\end{array}$} & \multicolumn{3}{|c|}{ Generic Site Lookup Values ${ }^{\mathrm{a}}$ (pCi/g) } & \multirow[b]{2}{*}{$\begin{array}{c}\text { Does the } \\
\text { Maximum } \\
\text { Result Exceed } \\
\text { RAGs? }\end{array}$} & \multirow[b]{2}{*}{$\begin{array}{c}\text { Does the } \\
\text { Result Pass } \\
\text { RESRAD } \\
\text { Modeling? }\end{array}$} \\
\hline & & $\begin{array}{l}\text { Shallow } \\
\text { Zone } \\
\text { Lookup } \\
\text { Value } \\
\end{array}$ & $\begin{array}{l}\text { Groundwater } \\
\text { Protection } \\
\text { Lookup Value }\end{array}$ & $\begin{array}{c}\text { River } \\
\text { Protection } \\
\text { Lookup } \\
\text { Value } \\
\end{array}$ & & \\
\hline Cesium-137 & 0.218 & 6.2 & $1,465^{b}$ & $1,465^{b}$ & No & -- \\
\hline \multirow[b]{2}{*}{ COPC } & \multirow{2}{*}{$\begin{array}{l}\text { Maximum or } \\
\text { Statistical } \\
\text { Result (mg/kg) }\end{array}$} & \multicolumn{3}{|c|}{ Remedial Action Goals $(\mathrm{mg} / \mathrm{kg})$} & \multirow{2}{*}{$\begin{array}{l}\text { Does the } \\
\text { Maximum } \\
\text { Exceed } \\
\text { RAGs? } \\
\end{array}$} & \multirow{2}{*}{$\begin{array}{c}\text { Does the } \\
\text { Result Pass } \\
\text { RESRAD } \\
\text { Modeling? }\end{array}$} \\
\hline & & $\begin{array}{c}\text { Direct } \\
\text { Exposure }\end{array}$ & $\begin{array}{l}\text { Protective of } \\
\text { Groundwater }\end{array}$ & $\begin{array}{c}\text { Protective of } \\
\text { the River }\end{array}$ & & \\
\hline Antimony & $0.92(<\mathrm{BG})$ & 32 & $5^{d}$ & $5^{d}$ & No & -- \\
\hline Arsenic & $4.4(<\mathrm{BG})$ & 20 & 20 & 20 & No & -- \\
\hline Barium & $84.9(<\mathrm{BG})$ & 5,600 & $132^{\mathrm{d}}$ & 224 & No & -- \\
\hline Beryllium & $0.26(<\mathrm{BG})$ & $10.4^{\mathrm{e}}$ & $1.51^{\mathrm{d}}$ & $1.51^{\mathrm{d}}$ & No & -- \\
\hline Boron $^{f}$ & 1.8 & 16,000 & 320 & NA & No & -- \\
\hline Cadmium & $0.22(<\mathrm{BG})$ & $13.9^{\mathrm{e}}$ & $0.81^{\mathrm{d}}$ & $0.81^{\mathrm{d}}$ & No & -- \\
\hline Chromium, Total & 23.5 & 80,000 & $18.5^{\mathrm{d}}$ & $18.5^{\mathrm{d}}$ & Yes & Yes $^{g}$ \\
\hline Cobalt & $6.0(<\mathrm{BG})$ & 1,600 & 32 & NA & No & -- \\
\hline Copper & 23.4 & 2,960 & 59.2 & $22.0^{\mathrm{d}}$ & Yes & Yes $^{\mathrm{h}}$ \\
\hline Hexavalent chromium ${ }^{\mathrm{f}}$ & 0.53 & $2.1^{\mathrm{e}}$ & 4.8 & 2 & No & -- \\
\hline Lead & $9.0(<\mathrm{BG})$ & 353 & $10.2^{\mathrm{d}}$ & $10.2^{\mathrm{d}}$ & No & -- \\
\hline Manganese & $304(<\mathrm{BG})$ & 11,200 & $512^{d}$ & $512^{d}$ & No & -- \\
\hline Mercury & $0.04(<\mathrm{BG})$ & 24 & $0.33^{d}$ & $0.33^{d}$ & No & -- \\
\hline Vanadium & $34.2(<\mathrm{BG})$ & 560 & $85.1^{\mathrm{d}}$ & NA & No & -- \\
\hline Zinc & $47.2(<\mathrm{BG})$ & 24,000 & 480 & $67.8^{\mathrm{d}}$ & No & -- \\
\hline $\begin{array}{l}\text { Bis(2-ethylhexyl) } \\
\text { phthalate }\end{array}$ & 0.085 & 71.4 & 0.625 & 0.36 & No & -- \\
\hline Dibenz(a,h)anthracene & 0.048 & $0.33^{\mathrm{i}}$ & $0.33^{\mathrm{i}}$ & $0.33^{\mathrm{i}}$ & Yes & Yes $^{g}$ \\
\hline Aldrin & 0.0022 & 0.0588 & $0.002^{\mathrm{i}}$ & $0.002^{\mathrm{i}}$ & Yes & Yes $^{g}$ \\
\hline Beta-BHC & 0.0047 & 0.556 & 0.00486 & 0.00554 & No & -- \\
\hline 4,4'-DDE & 0.0050 & 2.94 & 0.0257 & $0.0033^{\mathrm{i}}$ & Yes & Yes $^{g}$ \\
\hline 4,4'-DDT & 0.0055 & 2.94 & 0.0257 & $0.0033^{i}$ & Yes & Yes $^{g}$ \\
\hline Endrin aldehyde & 0.0051 & 24 & 0.2 & 0.039 & No & -- \\
\hline Heptachlor & 0.0033 & 0.222 & $0.002^{i}$ & $0.002^{\mathrm{i}}$ & Yes & Yes $^{j}$ \\
\hline
\end{tabular}




\section{Table 5a. Comparison of Maximum or Statistical Contaminant Concentrations to Action} Levels for the 128-F-2 Area A Verification Sampling (2 Pages)

\footnotetext{
${ }^{a}$ Soil activity obtained from the Remedial Design Report/Remedial Action Work Plan for the 100 Area (100 Area RDR/RAWP) (DOE-RL 2005) or calculated using RESRAD version 6.3 with generic site input parameters from Table B-1 of the 100 Area RDR/RAWP (DOE-RL 2005).

b Revised lookup value per 100 Area Radionuclide and Nonradionuclide Lookup Values for the 1995 Interim Remedial Action Record of Decision (BHI 2004a).

c Lookup values and RAGs obtained from the 100 Area RDR/RAWP (DOE-RL 2005) or calculated per WAC 173-340-720, WAC 173-340-730, and WAC 173-340-740, Method B, 1996, unless otherwise noted.

d Where cleanup levels are less than background, cleanup levels default to background (WAC 173-340-700[4][d], 1996).

e Carcinogenic cleanup level calculated based on the inhalation exposure pathway (WAC 173-340-750[3]) using an airborne particulate mass-loading rate of $0.0001 \mathrm{~g} / \mathrm{m}^{3}$ (WDOH 1997).

f No Hanford Site-specific or Washington State background value available.

g Based on the 100 Area Analogous Sites RESRAD Calculations (BHI 2005), residual concentrations of chromium, dibenz(a,h)anthracene, aldrin, 4,4'-DDE, and 4,4'-DDT are not expected to migrate more than $1 \mathrm{~m}$ (3.3 ft) vertically in 1,000 years (based on the lowest soil-partitioning distribution coefficient [aldrin] of $48.7 \mathrm{~mL} / \mathrm{g}$ ). The vadose zone underlying the remediation

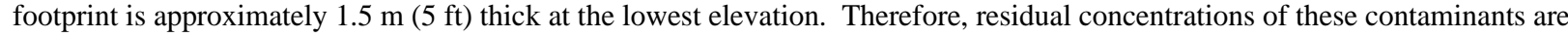
predicted to be protective of groundwater and the Columbia River.

h The river protection RAG for copper is set at the Hanford site background of $22.0 \mathrm{mg} / \mathrm{kg}$. The statistical value for copper in area A is slightly above the RAG at $23.4 \mathrm{mg} / \mathrm{kg}$. However, the actual values for copper collected in the verification sampling range from 9.3 to $33.9 \mathrm{mg} / \mathrm{kg}$. The Hanford Site background data for copper ranges from 8.1 to $36.1 \mathrm{mg} / \mathrm{kg}$ (DOE-RL 1995). All the copper concentrations measured in the verification samples fall within the range of copper values from background samples. Hence, the concentration of copper in these samples is indistinguishable from the concentrations measured in the background samples, and is therefore, predicted to be protective of groundwater and the Columbia River.

i Where cleanup levels are less than RDLs, cleanup levels default to RDLs per WAC 173-340-707(2) and DOE-RL (2005). The value for the dibenzo(a,h)anthracene RDL is based on EPA method 8270.

$\mathrm{j}$ The maximum, and only, detection of heptachlor in the verification samples is $0.0033 \mathrm{mg} / \mathrm{kg}$. The groundwater and river protection RAG for heptachlor is based on the RDL $(0.002 \mathrm{mg} / \mathrm{kg})$. Due to the fact that the contaminant was only detected in 1 out of 12 samples, and at a level less than 2 times the RDL, there will not be a sufficient mass of the chemical to exceed the RAG for heptachlor at the point of compliance. Therefore, these results are predicted to be protective of groundwater and the Columbia River.

-- $\quad=$ not applicable

$\mathrm{BG}=$ background

COPC = contaminant of potential concern

$\mathrm{EPA}=$ Environmental Protection Agency

$$
\begin{array}{ll}
\text { RESRAD } & =\text { RESidual RADioactivity (dose assessment model) } \\
\text { RDL } & =\text { required detection limit } \\
\text { WAC } & =\text { Washington Administrative Code }
\end{array}
$$$$
\text { RAG = remedial action goal }
$$

\begin{tabular}{|c|c|c|c|c|c|c|}
\hline \multirow[b]{2}{*}{ COC/COPC } & \multirow[b]{2}{*}{$\begin{array}{c}\text { Statistical or } \\
\text { Maximum } \\
\text { Result } \\
\text { (mg/kg) }\end{array}$} & \multicolumn{3}{|c|}{ Remedial Action Goals ${ }^{\mathrm{a}}$ (mg/kg) } & \multirow[b]{2}{*}{$\begin{array}{l}\text { Does the } \\
\text { Result } \\
\text { Exceed } \\
\text { RAGs? }\end{array}$} & \multirow[b]{2}{*}{$\begin{array}{c}\text { Does the } \\
\text { Result Pass } \\
\text { RESRAD } \\
\text { Modeling? }\end{array}$} \\
\hline & & $\begin{array}{c}\text { Direct } \\
\text { Exposure }\end{array}$ & $\begin{array}{l}\text { Soil Cleanup } \\
\text { Level for } \\
\text { Groundwater } \\
\text { Protection }\end{array}$ & $\begin{array}{l}\text { Soil Cleanup } \\
\text { Level for } \\
\text { River } \\
\text { Protection }\end{array}$ & & \\
\hline Arsenic & $3.5(<\mathrm{BG})$ & 20 & 20 & 20 & No & -- \\
\hline Barium & $72.9(<\mathrm{BG})$ & 5,600 & $132^{b}$ & 224 & No & -- \\
\hline Beryllium & $0.39(<\mathrm{BG})$ & $10.4^{c}$ & $1.51^{b}$ & $1.51^{b}$ & No & -- \\
\hline Boron $^{\mathrm{d}}$ & 2.1 & 16,000 & 320 & NA & No & -- \\
\hline Chromium (total) & 26.7 & 80,000 & $18.5^{\mathrm{b}}$ & $18.5^{\mathrm{b}}$ & Yes & Yes $^{\mathrm{e}}$ \\
\hline
\end{tabular}

Table 5b. Comparison of Contaminant Concentrations to Action Levels for the 128-F-2 Upland (Area B) Verification Sampling Event. (4 Pages) 
Table 5b. Comparison of Contaminant Concentrations to Action Levels for the 128-F-2 Upland (Area B) Verification Sampling Event. (4 Pages)

\begin{tabular}{|c|c|c|c|c|c|c|}
\hline \multirow[b]{2}{*}{ COC/COPC } & \multirow[b]{2}{*}{$\begin{array}{l}\text { Statistical or } \\
\text { Maximum } \\
\text { Result } \\
\text { (mg/kg) }\end{array}$} & \multicolumn{3}{|c|}{ Remedial Action Goals ${ }^{\mathrm{a}}$ (mg/kg) } & \multirow[b]{2}{*}{$\begin{array}{l}\text { Does the } \\
\text { Result } \\
\text { Exceed } \\
\text { RAGs? }\end{array}$} & \multirow[b]{2}{*}{$\begin{array}{c}\text { Does the } \\
\text { Result Pass } \\
\text { RESRAD } \\
\text { Modeling? }\end{array}$} \\
\hline & & $\begin{array}{c}\text { Direct } \\
\text { Exposure }\end{array}$ & $\begin{array}{l}\text { Soil Cleanup } \\
\text { Level for } \\
\text { Groundwater } \\
\text { Protection }\end{array}$ & $\begin{array}{l}\text { Soil Cleanup } \\
\text { Level for } \\
\text { River } \\
\text { Protection }\end{array}$ & & \\
\hline Cobalt & $6.4(<\mathrm{BG})$ & 1,600 & 32 & NA & No & -- \\
\hline Copper & 39.6 & 2,960 & 59.2 & $22.0^{b}$ & Yes & $--^{f}$ \\
\hline Hexavalent chromium & 0.80 & $2.1^{\mathrm{c}}$ & 4.8 & 2 & No & -- \\
\hline Lead & 10.8 & 353 & $10.2^{b}$ & $10.2^{b}$ & Yes & Yes ${ }^{e}$ \\
\hline Manganese & $275(<\mathrm{BG})$ & 11,200 & $512^{b}$ & $512^{b}$ & No & -- \\
\hline Mercury & $0.07(<\mathrm{BG})$ & 24 & $0.33^{b}$ & $0.33^{b}$ & No & -- \\
\hline Molybdenum $^{\mathrm{d}}$ & 0.67 & 400 & 8 & NA & No & -- \\
\hline Nickel & $13.0(<\mathrm{BG})$ & 1,600 & $19.1^{b}$ & 27.4 & No & -- \\
\hline Zinc & $38.7(<\mathrm{BG})$ & 24,000 & 480 & $67.8^{b}$ & No & -- \\
\hline Aroclor-1254 & 0.044 & 0.5 & $0.017^{\mathrm{g}}$ & $0.017^{\mathrm{g}}$ & Yes & Yes $^{\text {e }}$ \\
\hline $\mathrm{TPH}$ & 48.7 & NA & 200 & 200 & No & -- \\
\hline Acenaphthene & 0.063 & 4,800 & 96 & 129 & No & -- \\
\hline Anthracene & 0.12 & 24,000 & 240 & 1,920 & No & -- \\
\hline Benzo(a)anthracene & 0.35 & $1.37^{\mathrm{h}}$ & $0.33^{\mathrm{g}}$ & $0.33^{g}$ & Yes $^{\mathrm{i}}$ & -- \\
\hline Benzo(a)pyrene & 0.29 & $0.33^{g}$ & $0.33^{g}$ & $0.33^{g}$ & No & -- \\
\hline Benzo(b)fluoranthene & 0.23 & $1.37^{\mathrm{h}}$ & $0.33^{g}$ & $0.33^{g}$ & No & -- \\
\hline Benzo(k)fluoranthene & 0.30 & $13.7^{\mathrm{h}}$ & $0.33^{g}$ & $0.33^{\mathrm{g}}$ & No & -- \\
\hline Benzo(ghi)perylene ${ }^{\mathrm{j}}$ & 0.19 & 2,400 & 48 & 192 & No & -- \\
\hline Bis(2-ethylhexyl) phthalate & 0.080 & 71.4 & 0.625 & 0.36 & No & -- \\
\hline Carbazole & 0.062 & $50^{c}$ & 0.437 & NA & No & -- \\
\hline Chrysene & 0.39 & $137^{\mathrm{h}}$ & $1.2^{\mathrm{h}}$ & $0.33^{g}$ & Yes $^{i}$ & -- \\
\hline Dibenzo(a,h)anthracene & 0.143 & $0.33^{\mathrm{g}}$ & $0.33^{g}$ & $0.33^{g}$ & No & -- \\
\hline Diethylphthalate & 0.099 & 64,000 & 1,280 & 4,600 & No & -- \\
\hline Dimethylphthalate & 0.021 & 80,000 & 1,600 & 14,400 & No & -- \\
\hline Fluoranthene & 0.356 & 3,200 & 64 & 18.0 & No & -- \\
\hline Fluorene & 0.046 & 3,200 & 64 & 260 & No & -- \\
\hline Indeno(1,2,3-cd) pyrene & 0.18 & $1.37^{\mathrm{c}}$ & $0.33^{g}$ & $0.33^{\mathrm{g}}$ & No & - \\
\hline Phenanthrene $^{\mathrm{j}}$ & 0.264 & 24,000 & 240 & 1,920 & No & -- \\
\hline Pyrene & 0.381 & 2,400 & 48 & 192 & No & -- \\
\hline Aldrin & 0.0017 & $0.0588^{c}$ & 0.00165 & $0.00165^{\mathrm{g}}$ & Yes & Yes $^{\text {e }}$ \\
\hline
\end{tabular}


Table 5b. Comparison of Contaminant Concentrations to Action Levels for the 128-F-2 Upland (Area B) Verification Sampling Event. (4 Pages)

\begin{tabular}{|c|c|c|c|c|c|c|}
\hline \multirow[b]{2}{*}{ СОС/СОРС } & \multirow[b]{2}{*}{$\begin{array}{c}\text { Statistical or } \\
\text { Maximum } \\
\text { Result } \\
\text { (mg/kg) }\end{array}$} & \multicolumn{3}{|c|}{ Remedial Action Goals (mg/kg) } & \multirow[b]{2}{*}{$\begin{array}{l}\text { Does the } \\
\text { Result } \\
\text { Exceed } \\
\text { RAGs? }\end{array}$} & \multirow[b]{2}{*}{$\begin{array}{c}\text { Does the } \\
\text { Result Pass } \\
\text { RESRAD } \\
\text { Modeling? }\end{array}$} \\
\hline & & $\begin{array}{c}\text { Direct } \\
\text { Exposure }\end{array}$ & $\begin{array}{l}\text { Soil Cleanup } \\
\text { Level for } \\
\text { Groundwater } \\
\text { Protection }\end{array}$ & $\begin{array}{l}\text { Soil Cleanup } \\
\text { Level for } \\
\text { River } \\
\text { Protection }\end{array}$ & & \\
\hline $\begin{array}{l}\text { BHC, beta } \\
\text { (Hexachlorocyclohexane) }\end{array}$ & 0.027 & 0.556 & 0.00486 & 0.00554 & Yes & $--^{k}$ \\
\hline Chlordane (alpha, gamma) & 0.0101 & 2.86 & 0.0165 & $0.0165^{\mathrm{g}}$ & No & -- \\
\hline 4,4'-DDD & 0.003 & $4.17^{\mathrm{c}}$ & 0.0365 & $0.005^{\mathrm{g}}$ & No & -- \\
\hline 4,4'-DDE & 0.0064 & $2.94^{\mathrm{c}}$ & 0.0257 & $0.005^{\mathrm{g}}$ & Yes & Yes $^{\text {e }}$ \\
\hline 4,4'-DDT & 0.045 & $2.94^{\mathrm{c}}$ & 0.0257 & $0.005^{g}$ & Yes & Yes $^{\mathrm{e}}$ \\
\hline Endosulfan (I, II, sulfate) & 0.0061 & 480 & 9.6 & 0.0112 & No & -- \\
\hline $\begin{array}{l}\text { Endrin, Endrin ketone, and } \\
\text { Endrin aldehyde) }\end{array}$ & 0.0261 & 24 & 0.2 & 0.039 & No & -- \\
\hline
\end{tabular}

${ }^{a}$ Lookup values and RAGs obtained from the Remedial Design Report/Remedial Action Work Plan for the 100 Area (100 Area RDR/RAWP (DOE-RL 2005) or calculated per WAC 173-340-720, WAC 173-340-730, and WAC 173-340-740, Method B, 1996, unless otherwise noted.

b Where cleanup levels are less than background, cleanup levels default to background (WAC 173-340-700[4][d], 1996).

c Carcinogenic cleanup level calculated based on the inhalation exposure pathway (WAC 173-340-750[3], 1996) and an airborne particulate mass-loading rate of $0.0001 \mathrm{~g} / \mathrm{m}^{3}$ (WDOH 1997).

d No Hanford Site-specific or Washington State background value available.

e Based on the 100 Area Analogous Sites RESRAD Calculations (BHI 2005), residual concentrations are not expected to migrate more than $2 \mathrm{~m}$ (6.6 ft) vertically in 1,000 years (based on the lowest soil-partitioning distribution coefficient [lead] of

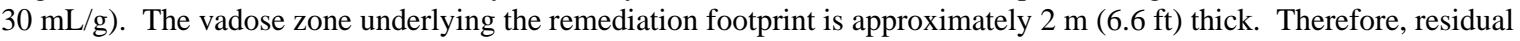
concentrations of lead, aldrin, DDE, and DDT are predicted to be protective of groundwater and the Columbia River.

f Copper was quantified above the soil RAG (22 mg/kg) for groundwater protection in one sample (110 mg/kg) in area B; the remaining nine sample results were all less than background $(22 \mathrm{mg} / \mathrm{kg}$ ). The soil-partitioning coefficient for copper (22 $\mathrm{mL} / \mathrm{g}$ ) prevents use of conservative migration modeling to demonstrate protectiveness. However, modeling of the hydraulic conductivity and hydraulic gradient for the $100-\mathrm{F}$ Area shows that there is a soil-to-groundwater dilution factor of approximately 6 in the soils underlying the 128-F-2 site, which indicates that copper will not leach to groundwater at concentrations exceeding the most restrictive WAC 173-340-720 cleanup level for groundwater (592 $\mu \mathrm{g} / \mathrm{L}$ ). Further, copper has not been detected in groundwater samples collected from nearby monitoring well 199-F-42. The fact that the only detection of copper above background was in a single sample suggests that there is insufficient residual mass of copper present to migrate through the $2 \mathrm{~m}(6.6 \mathrm{ft})$ of underlying vadose zone soil at this sample location to reach groundwater and result in concentrations exceeding groundwater RAG.

g Where cleanup levels are less than RDLs, cleanup levels default to RDLs per WAC 173-340-707(2) and DOE-RL (2005). Values for benzo(a)anthracene and chrysene are based on EPA method 8310 RDLs. Values for benzo(a)anthracene, benzo(a)pyrene, benzo(b)fluoranthene, benzo(k)fluoranthene, chrysene, and dibenzo(a,h)anthracene RDL based on EPA method 8270.

${ }^{\mathrm{h}}$ Cleanup level calculated with updated toxicity values using the appropriate formulas from WAC 173-340-740. Updated toxicity values are available from the EPA Integrated Risk Information System (IRIS) at < http://www.epa.gov/iris > or from the Risk Assessment Information System database of the Oak Ridge National Laboratory on the Internet at $<$ http://risk.lsd.ornl.gov >.

${ }^{\mathrm{i}}$ PAH associated with asphalt excluded as a dangerous waste (see Table 6).

j Toxicity data for this chemical are not available. Cleanup levels are based on surrogate chemicals: Contaminant: benzo(g,h,i)perylene; surrogate: pyrene Contaminant: phenathrene; surrogate: anthracene 
Table 5b. Comparison of Contaminant Concentrations to Action Levels for the 128-F-2 Upland (Area B) Verification Sampling Event. (4 Pages)

\begin{tabular}{|c|c|c|c|c|c|c|}
\hline \multirow[b]{2}{*}{ COC/COPC } & \multirow[b]{2}{*}{$\begin{array}{c}\text { Statistical or } \\
\text { Maximum } \\
\text { Result } \\
\text { (mg/kg) }\end{array}$} & \multicolumn{3}{|c|}{ Remedial Action Goals ${ }^{\mathrm{a}}$ (mg/kg) } & \multirow[b]{2}{*}{$\begin{array}{l}\text { Does the } \\
\text { Result } \\
\text { Exceed } \\
\text { RAGs? }\end{array}$} & \multirow[b]{2}{*}{$\begin{array}{l}\text { Does the } \\
\text { Result Pass } \\
\text { RESRAD } \\
\text { Modeling? }\end{array}$} \\
\hline & & $\begin{array}{c}\text { Direct } \\
\text { Exposure }\end{array}$ & $\begin{array}{l}\text { Soil Cleanup } \\
\text { Level for } \\
\text { Groundwater } \\
\text { Protection }\end{array}$ & $\begin{array}{l}\text { Soil Cleanup } \\
\text { Level for } \\
\text { River } \\
\text { Protection }\end{array}$ & & \\
\hline
\end{tabular}

The pesticide beta-BHC was quantified slightly above the soil RAGs for groundwater and river protection in three of the ten soil samples. The soil-partitioning coefficient for this contaminant $(2.14 \mathrm{~mL} / \mathrm{g})$ prevents use of conservative migration modeling to demonstrate protectiveness. However, modeling of the hydraulic conductivity and gradient for the 100-F Area shows that there is a soil-to-groundwater dilution factor of approximately 6 in the soils underlying the 128-F-2 site, which indicates that beta-BHC will not leach to groundwater at concentrations exceeding the most restrictive WAC 173-340-720 cleanup level for groundwater $(0.049 \mu \mathrm{g} / \mathrm{L})$. Further, beta-BHC has not been detected in groundwater samples collected from nearby monitoring well 199-F-42.

$\begin{array}{llll}-- & \text { not applicable } & \text { PAH } & =\text { polycyclic aromatic hydrocarbon } \\ \text { BG } & =\text { background } & \text { RAG } & =\text { remedial action goal } \\ \text { COC } & =\text { contaminant of concern } & \text { RESRAD } & =\text { RESidual RADioactivity (dose assessment model) } \\ \text { COPC } & =\text { contaminant of potential concern } & \text { RDL } & =\text { required detection limit } \\ \text { NA } & =\text { not available } & \text { WAC } & =\text { Washington Administrative Code } \\ \text { ND } & =\text { not detected } & & \end{array}$

Table 5c. Comparison of Maximum or Statistical Contaminant Concentrations to Action Levels for the 128-F-2 Waste Site Staging Pile Footprint Sampling Results. (3 Pages)

\begin{tabular}{|c|c|c|c|c|c|c|}
\hline \multirow{2}{*}{ COPC } & \multirow{2}{*}{$\begin{array}{c}\text { Maximum } \\
\text { Result }(\mathrm{mg} / \mathrm{kg})\end{array}$} & \multicolumn{3}{|c|}{$\begin{array}{c}\text { Remedial Action Goals } \\
(\mathrm{mg} / \mathrm{kg})^{\mathrm{a}}\end{array}$} & \multirow{2}{*}{\begin{tabular}{|c|} 
Does the \\
Maximum \\
Result Exceed \\
RAGs?
\end{tabular}} & \multirow{2}{*}{$\begin{array}{l}\text { Does the } \\
\text { Maximum } \\
\text { Result Pass } \\
\text { RESRAD } \\
\text { Modeling? }\end{array}$} \\
\hline & & $\begin{array}{c}\text { Direct } \\
\text { Exposure }\end{array}$ & $\begin{array}{c}\text { Groundwater } \\
\text { Protection }\end{array}$ & $\begin{array}{c}\text { River } \\
\text { Protection }\end{array}$ & & \\
\hline Antimony & $4.2(<\mathrm{BG})$ & 32 & $5^{b}$ & $5^{b}$ & No & -- \\
\hline Arsenic & $5.2(<\mathrm{BG})$ & 20 & 20 & 20 & No & -- \\
\hline Barium & $68.2(<\mathrm{BG})$ & 5,600 & $132^{b}$ & 224 & No & -- \\
\hline Chromium, total & 32.2 & 80,000 & $18.5^{b}$ & $18.5^{\mathrm{b}}$ & Yes & Yes $^{c}$ \\
\hline Chromium, hexavalent $^{\mathrm{d}}$ & 0.73 & $2.1^{\mathrm{e}}$ & 4.8 & 2 & No & -- \\
\hline Cobalt & $6.3(<\mathrm{BG})$ & 1,600 & 32 & NA & No & -- \\
\hline Copper & 23.9 & 2,960 & 59.2 & $22^{\mathrm{b}}$ & Yes & Yes $^{c}$ \\
\hline Lead & 24.8 & 353 & $10.2^{\mathrm{b}}$ & $10.2^{\mathrm{b}}$ & Yes & Yes $^{c}$ \\
\hline Manganese & $277(<\mathrm{BG})$ & 11,200 & $512^{b}$ & $512^{b}$ & No & -- \\
\hline Mercury & $0.05(<\mathrm{BG})$ & 24 & $0.33^{b}$ & $0.33^{\mathrm{b}}$ & No & -- \\
\hline Molybdenum $^{\mathrm{d}}$ & 1.3 & 400 & 8 & NA & No & -- \\
\hline Nickel & $11.7(<\mathrm{BG})$ & 1,600 & $19.1^{\mathrm{b}}$ & 27.4 & No & -- \\
\hline Selenium & 0.95 & 400 & 5 & 1 & No & -- \\
\hline Vanadium & $42.7(<\mathrm{BG})$ & 560 & $85.1^{\mathrm{b}}$ & NA & No & -- \\
\hline Zinc & 124 & 24,000 & 480 & $67.8^{\mathrm{b}}$ & Yes & Yes $^{c}$ \\
\hline
\end{tabular}


Table 5c. Comparison of Maximum or Statistical Contaminant Concentrations to Action Levels for the 128-F-2 Waste Site Staging Pile Footprint Sampling Results. (3 Pages)

\begin{tabular}{|c|c|c|c|c|c|c|}
\hline \multirow[b]{2}{*}{ COPC } & \multirow{2}{*}{$\begin{array}{c}\text { Maximum } \\
\text { Result (mg/kg) }\end{array}$} & \multicolumn{3}{|c|}{$\begin{array}{c}\text { Remedial Action Goals } \\
(\mathrm{mg} / \mathrm{kg})^{\mathrm{a}}\end{array}$} & \multirow{2}{*}{$\begin{array}{c}\text { Does the } \\
\text { Maximum } \\
\text { Result Exceed } \\
\text { RAGs? }\end{array}$} & \multirow{2}{*}{$\begin{array}{l}\text { Does the } \\
\text { Maximum } \\
\text { Result Pass } \\
\text { RESRAD } \\
\text { Modeling? }\end{array}$} \\
\hline & & $\begin{array}{c}\text { Direct } \\
\text { Exposure }\end{array}$ & $\begin{array}{l}\text { Groundwater } \\
\text { Protection }\end{array}$ & $\begin{array}{c}\text { River } \\
\text { Protection }\end{array}$ & & \\
\hline Aroclor-1254 & 0.048 & 0.5 & $0.017^{\mathrm{f}}$ & $0.017^{\mathrm{f}}$ & Yes & Yes $^{c}$ \\
\hline Acenaphthene & 0.027 & 4,800 & 96 & 129 & No & -- \\
\hline Anthracene & 0.06 & 24,000 & 240 & 1,920 & No & -- \\
\hline Benzo(a)anthracene & 0.32 & $1.37^{\mathrm{g}}$ & $0.33^{f}$ & $0.33^{f}$ & No & -- \\
\hline Benzo(a)pyrene & 0.28 & $0.33^{f}$ & $0.33^{f}$ & $0.33^{f}$ & No & -- \\
\hline Benzo(b)fluoranthene & 0.21 & $1.37^{\mathrm{g}}$ & $0.33^{f}$ & $0.33^{f}$ & No & -- \\
\hline Benzo(k)fluoranthene & 0.23 & $13.7^{\mathrm{g}}$ & $0.33^{\mathrm{f}}$ & $0.33^{f}$ & No & -- \\
\hline Benzo(g,h,i)perylene ${ }^{\text {h }}$ & 0.16 & 2,400 & 48 & 192 & No & -- \\
\hline Bis(2-ethylhexyl)phthalate & 0.11 & 71.4 & 0.625 & 0.36 & No & -- \\
\hline Carbazole & 0.030 & 50 & 0.437 & NA & No & -- \\
\hline Chrysene & 0.37 & 137 & $1.2^{\mathrm{g}}$ & $0.33^{\mathrm{g}}$ & Yes & Yes $^{c}$ \\
\hline Dibenzo(a,h)anthracene & 0.077 & $0.33^{f}$ & $0.33^{f}$ & $0.33^{f}$ & No & -- \\
\hline Indeno(1,2,3-cd)pyrene & 0.15 & 1.37 & $0.33^{f}$ & $0.33^{f}$ & No & -- \\
\hline Fluoranthene & 0.67 & 3,200 & 64 & 18.0 & No & -- \\
\hline Fluorene & 0.019 & 3,200 & 64 & 260 & No & -- \\
\hline Phenanthrene ${ }^{\mathrm{h}}$ & 0.31 & 24,000 & 240 & 1,920 & No & -- \\
\hline Pyrene & 0.79 & 2,400 & 48 & 192 & No & -- \\
\hline
\end{tabular}


Table 5c. Comparison of Maximum or Statistical Contaminant Concentrations to Action Levels for the 128-F-2 Waste Site Staging Pile Footprint Sampling Results. (3 Pages)

\begin{tabular}{|c|c|c|c|c|c|c|}
\hline \multirow[b]{2}{*}{ СОРC } & \multirow{2}{*}{$\begin{array}{c}\text { Maximum } \\
\text { Result (mg/kg) }\end{array}$} & \multicolumn{3}{|c|}{$\begin{array}{c}\text { Remedial Action Goals } \\
(\mathrm{mg} / \mathrm{kg})^{\mathrm{a}}\end{array}$} & \multirow{2}{*}{$\begin{array}{c}\text { Does the } \\
\text { Maximum } \\
\text { Result Exceed } \\
\text { RAGs? }\end{array}$} & \multirow{2}{*}{$\begin{array}{c}\text { Does the } \\
\text { Maximum } \\
\text { Result Pass } \\
\text { RESRAD } \\
\text { Modeling? }\end{array}$} \\
\hline & & $\begin{array}{c}\text { Direct } \\
\text { Exposure }\end{array}$ & $\begin{array}{l}\text { Groundwater } \\
\text { Protection }\end{array}$ & $\begin{array}{c}\text { River } \\
\text { Protection }\end{array}$ & & \\
\hline 4,4'-DDE & 0.0022 & 2.94 & $0.017^{\mathrm{f}}$ & $0.017^{\mathrm{f}}$ & No & -- \\
\hline 4,4'-DDT & 0.0066 & 2.94 & $0.017^{f}$ & $0.017^{\mathrm{f}}$ & No & -- \\
\hline
\end{tabular}

${ }^{\mathrm{a}}$ Lookup values and RAGs obtained from the Remedial Design Report/Remedial Action Work Plan for the 100 Area (100 Area RDR/RAWP) (DOE-RL 2005) or calculated per WAC 173-340-720, WAC 173-340-730, and WAC 173-340-740, Method B, unless otherwise noted.

${ }^{\mathrm{b}}$ Where cleanup levels are less than background, cleanup levels default to background (WAC 173-340-700[4][d]).

${ }^{\mathrm{c}}$ Based on the 100 Area Analogous Sites RESRAD Calculations (BHI 2005), residual concentrations are not expected to migrate more than $3 \mathrm{~m}$ (10 ft) vertically in 1,000 years (based on the lowest soil-partitioning distribution coefficient [copper] of $22 \mathrm{~mL} / \mathrm{g}$ ). The

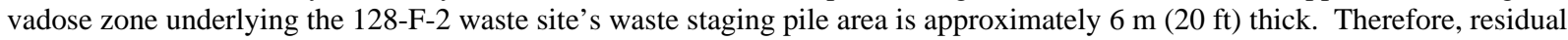
concentrations of chromium, copper, lead, zinc, aroclor-1254, chrysene, DDE, and DDT are predicted to be protective of groundwater and the Columbia River.

${ }^{\mathrm{d}}$ No Hanford Site-specific or Washington State background value available.

${ }^{\text {e }}$ Carcinogenic cleanup level calculated based on the inhalation exposure pathway (WAC 173-340-750[3]) using an airborne particulate mass-loading rate of $0.0001 \mathrm{~g} / \mathrm{m}^{3}$ (WDOH 1997).

${ }^{\mathrm{f}}$ Where cleanup levels are less than RDLs, cleanup levels default to RDLs per WAC 173-340-707(2) and DOE-RL (2005).

${ }^{g}$ Calculated using the appropriate formulas from WAC 173-340-740, with toxicity values updated through April 11, 2007, from the EPA Integrated Risk Information System (IRIS) at http://www.epa.gov/iris or from the Risk Assessment Information System (RAIS) database of the Oak Ridge National Laboratory on the Internet at http://risk.lsd.ornl.gov.

${ }^{\mathrm{h}}$ Toxicity data for this chemical are not available. Cleanup levels are based on surrogate chemicals: Contaminant: benzo(g,h,i)perylene; surrogate: pyrene

Contaminant: phenathrene; surrogate: anthracene

$--\quad=$ not applicable

$\mathrm{BG} \quad=$ background

COPC = contaminant of potential concern

NA $\quad=$ not applicable

RAG = remedial action goal

RDL = required detection limit

RESRAD = RESidual RADioactivity (dose model)

WAC = Washington Administrative Code

A comparison of the detected PAHs in the verification data set to a known asphalt sample (Table 6) shows a good correlation, as indicated by the Ratio column. Asphalt is listed as an inert waste in WAC 173-350-990(2)(b), and, therefore, does not present a significant risk to human health or the environment. The verification data set for 128-F-2 site is therefore considered to achieve soil RAGs for PAHs.

Table 6. Comparison of 128-F-2 Polyaromatic Hydrocarbon Verification Data to Asphalt Data. (2 Pages)

\begin{tabular}{|l|c|c|c|}
\hline \multicolumn{1}{|c|}{ Analyte } & $\begin{array}{c}\text { Asphalt Sample Result } \\
(\mathbf{m g} / \mathbf{k g})\end{array}$ & $\begin{array}{c}\text { Maximum 128-F-2 Staging } \\
\text { Area Result (mg/kg) }\end{array}$ & Ratio $^{\mathbf{a}}\left(\mathbf{X ~ 1 0}^{-4}\right)$ \\
\hline 2-Methylnaphthalene & 394 & ND & -- \\
\hline Acenaphthene & 1,783 & 0.063 & 0.35 \\
\hline
\end{tabular}


Table 6. Comparison of 128-F-2 Polyaromatic Hydrocarbon Verification Data to Asphalt Data. (2 Pages)

\begin{tabular}{|c|c|c|c|}
\hline Analyte & $\begin{array}{c}\text { Asphalt Sample Result } \\
\text { (mg/kg) }\end{array}$ & $\begin{array}{c}\text { Maximum 128-F-2 Staging } \\
\text { Area Result (mg/kg) }\end{array}$ & Ratio $^{\mathrm{a}}\left(\mathrm{X} \mathrm{10}^{-4}\right)$ \\
\hline Anthracene & 3,699 & 0.12 & 0.32 \\
\hline Benzo(a)anthracene & 5,792 & 0.35 & 0.60 \\
\hline Benzo(a)pyrene & 5,533 & 0.29 & 0.52 \\
\hline Benzo(b)fluoranthene & 4,619 & 0.23 & 0.50 \\
\hline Benzo(g,h,i)perylene & 2,839 & 0.19 & 0.67 \\
\hline Benzo(k)fluoranthene & 4,527 & 0.30 & 0.66 \\
\hline Carbazole & 2,049 & 0.062 & 0.30 \\
\hline Chrysene & 5,580 & 0.39 & 0.70 \\
\hline Dibenzo(a,h)anthracene & 1,531 & 0.143 & 0.93 \\
\hline Dibenzofuran & 1,135 & ND & -- \\
\hline Fluoranthene & 10,665 & 0.356 & 0.33 \\
\hline Fluorene & 1,756 & 0.046 & 0.26 \\
\hline Indeno(1,2,3-cd) pyrene & 2,751 & 0.18 & 0.65 \\
\hline Naphthalene & 1,917 & ND & - \\
\hline Phenanthrene & 10,975 & 0.264 & 0.24 \\
\hline Pyrene & 10,205 & 0.381 & 0.37 \\
\hline
\end{tabular}

${ }^{a}$ Determined by dividing the 128-F-2 Area B statistical or maximum result by the asphalt sample result.

$\mathrm{ND}=$ not detected (in any verification sample)

-- $\quad$ = not applicable

\section{DATA EVALUATION}

\section{Radionuclides}

Evaluation of RAG attainment for radionuclides was performed using the single-radionuclide dose-equivalence lookup values. The model used to develop these dose-equivalence lookup values is presented in the RDR/RAWP (DOE-RL 2005). Cesium-137, the only detected radionuclide, was quantified at $0.218 \mathrm{pCi} / \mathrm{g}$. This value is significantly less than the single radionuclide $15 \mathrm{mrem} / \mathrm{yr}$ dose equivalence lookup value for cesium-137 of $6.2 \mathrm{pCi} / \mathrm{g}$,

\section{Nonradionuclides}

All verification sample nonradionuclide COPCs achieved compliance with direct exposure, groundwater, and river protection RAGs. When using a statistical sampling approach, a RAG requirement for nonradionuclides is the WAC 173-340-740(7)(e) three-part test. The application of the three-part test for the 128-F-2 waste site is included in the statistical calculations 
(Appendix D). All residual COPC concentrations for the 128-F-2 waste site pass the three-part test.

Assessment of the risk requirements for the 128-F-2 waste site, the 100-F Burning Pit, is determined by calculation of the hazard quotient and carcinogenic (excess cancer) risk values for nonradionuclides. These calculations are located in Appendix D. The requirements include an individual hazard quotient of less than 1.0, a cumulative hazard quotient of less than 1.0, an individual contaminant carcinogenic risk of less than $1 \times 10^{-6}$, and a cumulative excess carcinogenic risk of less than $1 \times 10^{-5}$. These risk values were conservatively calculated for each of the three decision units of the128-F-2 waste site in which nonradionuclides were sampled (area E was not included as it was sampled for asbestos only). Risk values were not calculated for constituents that were not detected or were detected at concentrations below Hanford Site or Washington State background values. The calculations indicated that all individual hazard quotients for noncarcinogenic constituents are less than 1.0. All individual cumulative carcinogenic risk values are less than $1 \times 10^{-6}$. The cumulative hazard quotients and carcinogenic risk values for each of areas A, B, and D are presented in Table 7. All cumulative hazard quotients are less than 1.0 and all cumulative carcinogenic risk values are less than $1 \times 10^{-5}$. Therefore, nonradionuclide risk requirements are met.

Table 7. Cumulative Hazard Quotient and Carcinogenic Risk Calculation Results for Areas A, B, and D of the 128-F-2 Waste Site.

\begin{tabular}{|c|c|c|}
\hline Area & Cumulative Hazard Quotient & Cumulative Carcinogenic Risk \\
\hline A & $1.2 \times 10^{-2}$ & $6.7 \times 10^{-7}$ \\
\hline B & $8.1 \times 10^{-2}$ & $4.3 \times 10^{-6}$ \\
\hline D & $5.3 \times 10^{-2}$ & $3.6 \times 10^{-6}$ \\
\hline
\end{tabular}

\section{VERIFICATION SAMPLING DATA QUALITY ASSESSMENT}

A data quality assessment (DQA) is performed to compare the verification sampling approach, the field logbook (WCH 2007a, 2007b, 2007c and 2008), and resulting analytical data with the sampling and data quality requirements specified by the project objectives and performance specifications.

The DQA for the 128-F-2 waste site established that the data are of the right type, quality, and quantity to support site verification decisions within specified error tolerances. All analytical data were found to be acceptable for decision-making purposes. The evaluation verified that the sample design was sufficient for the purpose of clean site verification. The cleanup verification sample analytical data are stored in the ENRE project-specific database for data evaluation prior to its archival in the HEIS and are summarized in Attachment 1 of the appropriate 95\% UCL calculations in Appendix D. The detailed DQA is presented in Appendix E. 


\section{SUMMARY FOR INTERIM CLOSURE}

1. The area within the final boundary of the 128-F-2 waste site, the 100-F Burning Pit, which does not include area $\mathrm{C}$ below the OHWM, has been remediated in accordance with the Remaining Sites ROD (EPA 1999) and the RDR/RAWP (DOE-RL 2005). The site was remediated by removing approximately 16,222 bank cubic meters of material for disposal at the ERDF. Statistical sampling to verify the completeness of remediation was performed and analytical results for the decision units (areas A, B, D, and E) were shown to meet the cleanup objectives for direct exposure, groundwater protection, and river protection. Accordingly, an interim closure reclassification is supported for the 128-F-2 waste site. The site does not have a deep zone or residual contaminant concentrations that would require any institutional controls.

2. The portion of the 128-F-2 site that was excavated to a point below the OHWM but did not meet soil RAGs for waste site closeout (area C) was combined with adjoining riparian areas to the east and southeast known to contain levels of metals in excess of soil RAGs. This site, 100-F-59, Riparian Area Contamination Originating from 128-F-2, is accepted in WIDS as a discovery site.

\section{REFERENCES}

40 CFR 141, "National Primary Drinking Water Regulations," Code of Federal Regulations, as amended.

BHI, 2004a, 100 Area Radionuclide and Nonradionuclide Lookup Values for the 1995 Interim Remedial Action Record of Decision, 0100X-CA-V0046, Rev. 0, Bechtel Hanford, Inc., Richland, Washington.

BHI, 2004b, Archaeological Subsurface Testing Report: Remediation of Waste Sites 128-F-2 and 1607-F-5 on the Hanford Site, Benton County, Washington, BHI-01743, Rev. 0, Bechtel Hanford, Inc., Richland, Washington.

BHI, 2004c, Remaining Sites Field Sampling, Logbook EL-1578-5, Bechtel Hanford, Inc., Richland, Washington.

BHI, 2004d, Results of Geophysical Investigation at the 100-F Area Remaining Sites, CCN 112477 from K. A. Bergstrom to R. A. Carlson, dated May 27, Bechtel Hanford, Inc., Richland, Washington.

BHI, 2005, 100 Area Analogous Sites RESRAD Calculations, Calculation No. 0100X-CAV0050, Rev. 0, Bechtel Hanford, Inc., Richland, Washington.

DOE, 1962, Photograph No. 6451, U.S. Department of Energy, Richland Operations Office, Richland, Washington. 
DOE Order 5400.5, Radiation Protection of the Public and the Environment, as amended, U.S. Department of Energy, Washington, D.C.

DOE-RL, 1995, Hanford Site Background: Part 1, Soil Background for Nonradioactive Analytes, DOE/RL-92-24, Rev. 3, U.S. Department of Energy, Richland Operations Office, Richland, Washington.

DOE-RL, 1996, Hanford Site Background: Part 2, Soil Background for Radionuclides, DOE/RL-96-12, Rev. 0, U.S. Department of Energy, Richland Operations Office, Richland, Washington.

DOE-RL, 2005, Remedial Design Report/Remedial Action Work Plan for the 100 Area, DOE/RL-96-17, Rev. 5, U.S. Department of Energy, Richland Operations Office, Richland, Washington.

DOE-RL, 2006, Consultation for 128-F-2 Waste Site on the Hanford Site, 07-AMRC-0029, CCN 131076, Letter to F. D. Bambrick, dated November 7, 2006, U.S. Department of Energy, Richland Operations Office, Richland, Washington.

DOE-RL, 2007, Tri-Party Agreement Handbook Management Procedures, RL-TPA-90-0001, Guideline Number TPA-MP-14, "Maintenance of the Waste Information Data System (WIDS),” Rev. 1, U.S. Department of Energy, Richland Operations Office, Richland, Washington.

Ecology, 1995, Washington State Department of Ecology Toxics Cleanup Program - Guidance on Sampling and Data Analysis Methods, Publication No. 94-49, Washington State Department of Ecology, Olympia, Washington.

Ecology, 2005, Cleanup Levels and Risk Calculations (CLARC) Database, Washington State Department of Ecology, Olympia, Washington, $<$ https://fortress.wa.gov/ecy/clarc/CLARCHome.aspx>

Endangered Species Act of 1973, 16 U.S.C. 1531, et seq.

ENV-1, Environmental Monitoring \& Management, Washington Closure Hanford, Richland, Washington.

EPA, 1999, Interim Action Record of Decision for the 100-BC-1, 100-BC-2, 100-DR-1, 100-DR-2, 100-FR-1, 100-FR-2, 100-HR-1, 100-HR-2, 100-KR-1, 100-KR-2, 100-IU-2, 100-IU-6, and 200-CW-3 Operable Units, Hanford Site, Benton County, Washington, U.S. Environmental Protection Agency, Region 10, Seattle, Washington.

EPA, 2006, Integrated Risk Information System (IRIS), U.S. Environmental Protection Agency, Washington, D.C., available at <http://www.epa.gov/iris>. 
Gilbert, R. O., 1987, Statistical Methods for Environmental Pollution Monitoring, Wiley \& Sons, Inc., New York, New York.

Magnuson-Stevens Fishery Conservation and Management Act, 16 U.S.C. 1801-1882, et seq.

NMFS, 2006, Endangered Species Act Section 7 Informal Consultation and Magnuson-Stevens Fishery Conservation and Management Act Essential Fish Habitat Consultation for the Removal of Non-radioactive Waste at 128-F-2 Waste Site Near Hanford, Washington (Sixth Field HUC - Columbia River-Coyote Rapids, 170200160405), NMFS Tracking No. 2006/06126, CCN 131222, Letter to J. R. Franco, dated December 6, 2006, National Marine Fisheries Service, Seattle, Washington.

UNI, 1980, Hanford Site Cleanup Program FY 1979 Pictorial Review, UNI-1488, United Nuclear Industries, Richland, Washington.

WAC 173-340, “Model Toxics Control Act -- Cleanup,” Washington Administrative Code, amended 1996.

WCH, 2006a, Archaeological Activity Report: Post-Review Discoveries Within $45 B N 431$ at Solid Waste Site 128-F-2, WCH-142, Rev. 0, Washington Closure Hanford, Richland, Washington.

WCH, 2006b, Completion of Excavation of the 128-F-2 Burn Pit, CCN 130506, Email to F. D. Bambrick from K. A. Gano, dated September 14, 2006, Washington Closure Hanford, Richland, Washington.

WCH, 2007a, 100 F Area Remedial Sampling, Logbook EFL-1174-2, pp. 62-71, Washington Closure Hanford, Richland, Washington.

WCH, 2007b, 100 F Area Remedial Sampling, Logbook EFL-1174-3, pp. 57-59, Washington Closure Hanford, Richland, Washington.

WCH, 2007c, 100 F Area Remedial Sampling, Logbook EFL-1174-4, pp. 34-35, Washington Closure Hanford, Richland, Washington.

WCH, 2007d, Work Instruction for Verification Sampling of the 128-F-2 Waste Site, 100-F Burning Pit, 0100F-WI-G0037, Rev. 0, Washington Closure Hanford, Richland, Washington.

WCH, 2008, 100-F Remedial Sampling, Logbook ELF-1174-4, pp. 83-85, Washington Closure Hanford, Richland, Washington.

WHC, 1994, Hanford Surplus Facilities Programs Facilities Listings and Descriptions, WHC-SP-0331, Westinghouse Hanford Company, Richland, Washington.

WDOH, 1997, Hanford Guidance for Radiological Cleanup, WDOH/320-015, Rev. 1, Washington State Department of Health, Olympia, Washington. 


\section{APPENDIX A \\ CONFIRMATORY, IN-PROCESS, AREA C VERIFICATION, STOCKPILE VERIFICATION, AND ASBESTOS VERIFICATION SAMPLE RESULTS FOR THE 128-F-2 WASTE SITE}


Table A-1. 128-F-2 Confirmatory Sample Results. (12 Pages)

\begin{tabular}{|c|c|c|c|c|c|c|c|c|c|c|c|c|c|c|c|c|c|c|c|c|}
\hline \multirow{2}{*}{ Sample Location } & \multirow{2}{*}{$\begin{array}{c}\text { HEIS } \\
\text { Number }\end{array}$} & \multirow{2}{*}{\begin{tabular}{|c|} 
Sample \\
Date
\end{tabular}} & \multicolumn{3}{|c|}{ Aluminum } & \multicolumn{3}{|c|}{ Antimony } & \multicolumn{3}{|c|}{ Arsenic } & \multicolumn{3}{|c|}{ Barium } & \multicolumn{3}{|c|}{ Beryllium } & \multicolumn{3}{|c|}{ Boron } \\
\hline & & & $\mathrm{mg} / \mathrm{kg}$ & $\mathbf{Q}$ & PQL & $\mathrm{mg} / \mathrm{kg}$ & $\mathbf{Q}$ & PQL & $\mathrm{mg} / \mathrm{kg}$ & $\mathbf{Q}$ & PQL & $\mathrm{mg} / \mathrm{kg}$ & $\mathbf{Q}$ & PQL & $\mathrm{mg} / \mathrm{kg}$ & $\mathbf{Q}$ & PQL & $\mathrm{mg} / \mathrm{kg}$ & Q & PQL \\
\hline Area 1 Golden Soil & J024R1 & $12 / 03 / 04$ & 2240 & & 2.4 & 0.59 & & 0.21 & 8 & & 0.2 & 83.9 & $\mathrm{C}$ & 0.03 & 0.06 & & 0.008 & 3.2 & & 0.23 \\
\hline Area 1 Tan Soil & J024R2 & $12 / 03 / 04$ & 4430 & & 3.1 & 0.27 & $\mathrm{U}$ & 0.27 & 59.7 & & 0.25 & 42 & & 0.04 & 0.17 & $\mathrm{C}$ & 0.01 & 2.7 & & 0.29 \\
\hline Area 1 Trench Soil & J024R3 & $12 / 03 / 04$ & 6830 & & 3 & 0.27 & $\mathrm{U}$ & 0.27 & 4.1 & & 0.25 & 50.8 & $\mathrm{C}$ & 0.04 & 0.24 & & 0.01 & 1.6 & & 0.29 \\
\hline Area 4 Rusty Soil & J024R4 & $12 / 06 / 04$ & 8110 & & 3.9 & 0.49 & & 0.35 & 4.4 & & 0.33 & 71.1 & \begin{tabular}{|l|l}
$\mathrm{C}$ \\
\end{tabular} & 0.05 & 0.28 & & 0.01 & 2.1 & & 0.38 \\
\hline Area 4 Oily Soil & J024R5 & $12 / 06 / 04$ & 8580 & & 2.8 & 0.38 & & 0.25 & 20.8 & & 0.24 & 659 & $\mathrm{C}$ & 0.04 & 0.4 & & 0.009 & 0.5 & & 0.27 \\
\hline Area 4 Trench Soil & J024R6 & $12 / 06 / 04$ & 9780 & & 3.4 & 0.3 & $\mathrm{U}$ & 0.3 & 7.6 & & 0.28 & 166 & $\mathrm{C}$ & 0.04 & 0.33 & & 0.01 & 0.64 & & 0.32 \\
\hline \begin{tabular}{|l|} 
Duplicate of J024R6 \\
\end{tabular} & J024X0 & $12 / 06 / 04$ & 9320 & & 3.4 & 0.31 & $\mathrm{U}$ & 0.31 & 7.2 & & 0.28 & 158 & $\mathrm{C}$ & 0.04 & 0.32 & & 0.01 & 0.49 & & 0.33 \\
\hline Equipment Blank & $\mathrm{J} 024 \mathrm{X} 1$ & $12 / 06 / 04$ & 44 & & 2.3 & 0.21 & $\mathrm{U}$ & 0.21 & 0.4 & & 0.19 & 0.94 & $\mathrm{U}$ & 0.03 & 0.01 & C & 0.007 & 0.22 & & 0.22 \\
\hline Area 3 Trench Soil & $\mathrm{J} 024 \mathrm{X} 2$ & $12 / 07 / 04$ & 8210 & & 2.3 & 1.3 & & 0.2 & 2.9 & & 0.19 & 76.6 & $\mathrm{C}$ & 0.03 & 0.36 & & 0.007 & 1.1 & & 0.22 \\
\hline Area 2 Trench Soil & J024X3 & $12 / 07 / 04$ & 6360 & & 3.3 & 0.86 & & 0.29 & 3.4 & & 0.27 & 100 & $\mathrm{C}$ & 0.04 & 0.25 & & 0.01 & 1.9 & & 0.31 \\
\hline Area 2 Ash & J024R7 & $12 / 03 / 04$ & 5340 & & 20.9 & 1.87 & $\mathrm{U}$ & 1.9 & 2.1 & & 1.7 & 142 & & 0.27 & 0.3 & & 0.07 & 22.1 & & 2 \\
\hline Area 2 Ash & J024R8 & \begin{tabular}{|l|}
$12 / 03 / 04$ \\
\end{tabular} & 7690 & & 18.6 & 3.1 & & 1.7 & 5.7 & & 1.5 & 233 & & 0.24 & 0.315 & & 0.06 & 6.9 & & 1.8 \\
\hline Area 3 Oily Sediment & J024T0 & $12 / 07 / 04$ & 8310 & & 21.9 & 15 & & 2 & 6.7 & & 1.8 & 498 & & 0.28 & 0.336 & & 0.07 & 1260 & & 2.1 \\
\hline Area 2 Paint & J02581 & \begin{tabular}{|l|}
$12 / 07 / 04$ \\
\end{tabular} & 1060 & & 18.6 & 1.66 & $\mathrm{U}$ & 1.7 & 1.54 & $\mathrm{U}$ & 1.5 & 1.9 & & 0.24 & 0.059 & $\mathrm{U}$ & 0.06 & 1.78 & & 1.8 \\
\hline
\end{tabular}

\begin{tabular}{|c|c|c|c|c|c|c|c|c|c|c|c|c|c|c|c|c|c|c|c|c|}
\hline \multirow[t]{2}{*}{ Sample Location } & \multirow{2}{*}{$\begin{array}{c}\text { HEIS } \\
\text { Number }\end{array}$} & \multirow{2}{*}{$\begin{array}{c}\text { Sample } \\
\text { Date }\end{array}$} & \multicolumn{3}{|c|}{ Cadmium } & \multicolumn{3}{|c|}{ Calcium } & \multicolumn{3}{|c|}{ Chromium } & \multicolumn{3}{|c|}{ Cobalt } & \multicolumn{3}{|c|}{ Copper } & \multicolumn{3}{|c|}{$\begin{array}{l}\text { Hexavalent } \\
\text { Chromium }\end{array}$} \\
\hline & & & $\mathrm{mg} / \mathrm{kg}$ & $\mathbf{Q}$ & PQL & $\mathrm{mg} / \mathrm{kg}$ & $\mathbf{Q}$ & PQL & $\mathrm{mg} / \mathrm{kg}$ & $\mathbf{Q}$ & PQL & $\mathrm{mg} / \mathrm{kg}$ & Q & PQL & $\mathrm{mg} / \mathrm{kg}$ & $\mathbf{Q}$ & PQL & $\mathrm{mg} / \mathrm{kg}$ & $\mathbf{Q}$ & PQL \\
\hline Area 1 Golden Soil & J024R1 & $12 / 03 / 04$ & 0.41 & & 0.02 & 2410 & & 2.1 & 11.5 & & 0.06 & 3.4 & & 0.05 & 52.9 & & 0.11 & 6.6 & & 2.1 \\
\hline Area 1 Tan Soil & J024R2 & $12 / 03 / 04$ & 0.33 & & 0.03 & 3500 & & 2.7 & 137 & & 0.08 & 26 & & 0.07 & 14.4 & & 0.14 & 6.8 & & 2.1 \\
\hline Area 1 Trench Soil & J024R3 & $12 / 03 / 04$ & 0.18 & & 0.03 & 10900 & & 2.7 & 14.9 & & 0.08 & 5.4 & & 0.07 & 12.5 & & 0.13 & 0.2 & $\mathrm{U}$ & 0.2 \\
\hline Area 4 Rusty Soil & J024R4 & $12 / 06 / 04$ & 0.28 & & 0.04 & 8740 & & 3.5 & 16 & & 0.1 & 6.8 & & 0.09 & 15.4 & & 0.18 & 8.5 & & 2.7 \\
\hline Area 4 Oily Soil & J024R5 & $12 / 06 / 04$ & 0.75 & & 0.03 & 3050 & & 2.5 & 65.9 & & 0.07 & 14.9 & & 0.06 & 42.9 & & 0.13 & 5 & & 0.21 \\
\hline Area 4 Trench Soil & J024R6 & $12 / 06 / 04$ & 0.21 & & 0.03 & 3300 & & 3 & 61.5 & & 0.09 & 6.2 & & 0.08 & 22.3 & & 0.15 & 5.5 & & 0.22 \\
\hline Duplicate of J024R6 & J024X0 & $12 / 06 / 04$ & 0.2 & & 0.03 & 3130 & & 3.1 & 55.6 & & 0.09 & 6.2 & & 0.08 & 22.6 & & 0.15 & 2.4 & & 0.22 \\
\hline Equipment Blank & J024X1 & $12 / 06 / 04$ & 0.02 & $\mathrm{U}$ & 0.02 & 18.7 & & 2.1 & 0.13 & & 0.06 & 0.05 & $\mathrm{U}$ & 0.05 & 0.17 & & 0.1 & & & \\
\hline Area 3 Trench Soil & J024X2 & $12 / 07 / 04$ & 0.71 & & 0.02 & 3650 & & 2 & 106 & & 0.06 & 6.1 & & 0.05 & 44.6 & & 0.1 & 1.5 & & 0.23 \\
\hline Area 2 Trench Soil & J024X3 & $12 / 07 / 04$ & 0.97 & & 0.03 & 3650 & & 2.9 & 223 & & 0.08 & 6.6 & & 0.07 & 2240 & & 0.15 & 1.9 & & 0.22 \\
\hline Area 2 Ash & J024R7 & $12 / 03 / 04$ & 0.38 & & 0.2 & 63900 & & 18.6 & 8.7 & & 0.53 & 3.5 & & 0.47 & 15 & $\mathrm{C}$ & 0.93 & & & \\
\hline Area 2 Ash & J024R8 & $12 / 03 / 04$ & 2.9 & & 0.18 & 7320 & & 16.6 & 242 & & 0.48 & 8.4 & & 0.42 & 1240 & $\mathrm{C}$ & 0.83 & & & \\
\hline Area 3 Oily Sediment & J024T0 & $12 / 07 / 04$ & 16.8 & & 0.21 & 14300 & & 19.6 & 74.6 & & 0.56 & 46.7 & & 0.49 & 138 & $\mathrm{C}$ & 0.98 & & & \\
\hline Area 2 Paint & J02581 & $12 / 07 / 04$ & 0.178 & \begin{tabular}{|l|}
$\mathrm{U}$ \\
\end{tabular} & 0.18 & 74 & & 16.6 & 1.3 & & 0.47 & 0.415 & \begin{tabular}{|l|l}
$\mathrm{U}$ \\
\end{tabular} & 0.42 & 1.9 & $\mathrm{C}$ & 0.83 & & & \\
\hline
\end{tabular}

Area 2 Paint

Acronyms and note apply to all tables in this appendix.

Note: Data qualified with B, C, and/or J, are considered real values.

$\mathrm{J}=$ estimate

$\mathrm{B}=$ blank contamination (organic constituents)

minimum detectable activity

$\mathrm{C}=$ blank contamination (inorganic constituents)

$\mathrm{PQL}=$ practical quantitation limit

$\mathrm{D}=$ diluted

GEA = gamma energy analysis

HEIS = Hanford Environmental Information System

$\mathrm{TPH}=$ total petroleum hydrocarbons

$\mathrm{Q}=$ qualifier 
Table A-1. 128-F-2 Confirmatory Sample Results. (12 Pages)

\begin{tabular}{|c|c|c|c|c|c|c|c|c|c|c|c|c|c|c|c|c|c|c|c|c|}
\hline \multirow{2}{*}{ Sample Location } & \multirow{2}{*}{$\begin{array}{c}\text { HEIS } \\
\text { Number }\end{array}$} & \multirow{2}{*}{$\begin{array}{c}\text { Sample } \\
\text { Date }\end{array}$} & \multicolumn{3}{|c|}{ Iron } & \multicolumn{3}{|c|}{ Lead } & \multicolumn{3}{|c|}{ Magnesium } & \multicolumn{3}{|c|}{ Manganese } & \multicolumn{3}{|c|}{ Mercury } & \multicolumn{3}{|c|}{ Molybdenum } \\
\hline & & & $\mathrm{mg} / \mathrm{kg}$ & $\mathbf{Q}$ & PQL & $\mathrm{mg} / \mathrm{kg}$ & $\mathbf{Q}$ & PQL & $\mathrm{mg} / \mathrm{kg}$ & $\mathbf{Q}$ & PQL & $\mathrm{mg} / \mathrm{kg}$ & $\mathbf{Q}$ & PQL & $\mathrm{mg} / \mathrm{kg}$ & $\mathbf{Q}$ & PQL & $\mathrm{mg} / \mathrm{kg}$ & $\mathbf{Q}$ & PQL \\
\hline Area 1 Golden Soil & J024R1 & $12 / 03 / 04$ & 35100 & & 2.1 & 417 & & 0.17 & 1460 & & 0.52 & 71 & & 0.02 & 0.45 & & 0.02 & 0.69 & & 0.17 \\
\hline Area 1 Tan Soil & J024R2 & $12 / 03 / 04$ & 22500 & & 2.7 & 5.1 & & 0.21 & 30000 & & 0.67 & 361 & & 0.03 & 0.16 & & 0.02 & 0.22 & $\mathrm{U}$ & 0.22 \\
\hline Area 1 Trench Soil & J024R3 & $12 / 03 / 04$ & 17300 & & 2.7 & 4.4 & & 0.21 & 5240 & & 0.66 & 230 & & 0.03 & 0.02 & $\mathrm{U}$ & 0.02 & 0.22 & & 0.22 \\
\hline Area 4 Rusty Soil & J024R4 & $12 / 06 / 04$ & 21300 & & 3.54 & 9.8 & & 0.28 & 5560 & & 0.87 & 301 & & 0.04 & 0.02 & $\mathrm{U}$ & 0.02 & 0.29 & $\mathrm{U}$ & 0.29 \\
\hline Area 4 Oily Soil & J024R5 & $12 / 06 / 04$ & 28500 & & 3.5 & 6.1 & & 0.2 & 4150 & & 0.63 & 4540 & & 0.16 & 0.02 & $\mathrm{U}$ & 0.02 & 2.1 & & 0.21 \\
\hline Area 4 Trench Soil & J024R6 & $12 / 06 / 04$ & 20000 & & 2.5 & 7.3 & & 0.24 & 4130 & & 0.74 & 492 & & 0.03 & 0.02 & $\mathrm{U}$ & 0.02 & 0.44 & & 0.25 \\
\hline Duplicate of J024R6 & J024X0 & $12 / 06 / 04$ & 19500 & & 3 & 7.2 & & 0.24 & 4010 & & 0.76 & 473 & & 0.03 & 0.02 & $\mathrm{U}$ & 0.02 & 0.37 & & 0.25 \\
\hline Equipment Blank & J024X1 & $12 / 06 / 04$ & 116 & & 3.1 & 0.2 & & 0.21 & 6.4 & & 0.51 & 2.8 & & 0.02 & 0.02 & $\mathrm{U}$ & 0.02 & 0.17 & $\mathrm{U}$ & 0.17 \\
\hline Area 3 Trench Soil & $\mathrm{J} 024 \mathrm{X} 2$ & $12 / 07 / 04$ & 17200 & & 2.1 & 39.4 & & 0.16 & 4690 & & 0.5 & 351 & & 0.02 & 0.11 & & 0.02 & 0.42 & & 0.17 \\
\hline Area 2 Trench Soil & J024X3 & $12 / 07 / 04$ & 23100 & & 2.9 & 100 & & 0.23 & 4210 & & 0.72 & 318 & & 0.03 & 1.5 & & 0.02 & 2.7 & & 0.24 \\
\hline Area 2 Ash & J024R7 & $12 / 03 / 04$ & 9940 & & 18.6 & 19.8 & & 1.5 & 6410 & & 4.6 & 146 & & 0.2 & 0.097 & & 0.02 & 1.53 & $\mathrm{U}$ & 1.5 \\
\hline Area 2 Ash & J024R8 & $12 / 03 / 04$ & 30100 & & 16.6 & 386 & & 1.3 & 5450 & & 4.1 & 775 & & 0.18 & 0.879 & & 0.02 & 1.9 & & 1.4 \\
\hline \begin{tabular}{|l|} 
Area 3 Oily Sediment \\
\end{tabular} & J024T0 & $12 / 07 / 04$ & 52800 & & 19.6 & 226 & & 2 & 5920 & & 4.8 & 401 & & 0.21 & 9.75 & & 0.02 & 1.61 & $\mathrm{U}$ & 1.6 \\
\hline Area 2 Paint & J02581 & $12 / 07 / 04$ & 293 & & 16.6 & 4 & & 1.3 & 18.3 & & 4.1 & 2.8 & & 0.18 & 0.016 & $\mathrm{U}$ & 0.02 & 1.36 & $\mathrm{U}$ & 1.4 \\
\hline
\end{tabular}

\begin{tabular}{|c|c|c|c|c|c|c|c|c|c|c|c|c|c|c|c|c|c|c|c|c|}
\hline \multirow{2}{*}{ Sample Location } & \multirow{2}{*}{$\begin{array}{c}\text { HEIS } \\
\text { Number }\end{array}$} & \multirow{2}{*}{\begin{tabular}{c|c|} 
Sample \\
Date
\end{tabular}} & \multicolumn{3}{|c|}{ Nickel } & \multicolumn{3}{|c|}{ Potassium } & \multicolumn{3}{|c|}{ Selenium } & \multicolumn{3}{|c|}{ Silicon } & \multicolumn{3}{|c|}{ Silver } & \multicolumn{3}{|c|}{ Sodium } \\
\hline & & & $\mathrm{mg} / \mathrm{kg}$ & $\mathbf{Q}$ & PQL & $\mathrm{mg} / \mathrm{kg}$ & $\mathbf{Q}$ & PQL & $\mathrm{mg} / \mathrm{kg}$ & $\mathbf{Q}$ & $\mathbf{P Q L}$ & $\mathrm{mg} / \mathrm{kg}$ & \begin{tabular}{l|l}
$\mathbf{Q}$ \\
\end{tabular} & PQL & $\mathrm{mg} / \mathrm{kg}$ & $\mathbf{Q}$ & PQL & $\mathrm{mg} / \mathrm{kg}$ & $\mathbf{Q}$ & PQL \\
\hline Area 1 Golden Soil & J024R1 & $12 / 03 / 04$ & 4.7 & & 0.09 & 2390 & $\mathrm{C}$ & 1.4 & 0.62 & & 0.28 & 117 & & 1.1 & 2.1 & & 0.08 & 680 & $\mathrm{C}$ & 0.39 \\
\hline \begin{tabular}{|l|} 
Area 1 Tan Soil \\
\end{tabular} & J024R2 & $12 / 03 / 04$ & 336 & & 0.12 & 908 & $\mathrm{C}$ & 1.8 & 0.52 & & 0.36 & 157 & & 1.4 & 0.1 & $\mathrm{U}$ & 0.1 & 169 & & 0.5 \\
\hline Area 1 Trench Soil & J024R3 & $12 / 03 / 04$ & 13.1 & & 0.11 & 2460 & $\mathrm{C}$ & 1.8 & 0.37 & & 0.35 & 185 & & 1.3 & 0.1 & $\mathrm{U}$ & 0.1 & 313 & $\mathrm{C}$ & 0.49 \\
\hline Area 4 Rusty Soil & J024R4 & $12 / 06 / 04$ & 14.1 & & 0.15 & 1820 & $\mathrm{C}$ & 2.4 & 0.65 & & 0.47 & 187 & & 1.8 & 14.9 & & 0.13 & 337 & $\mathrm{C}$ & 0.64 \\
\hline Area 4 Oily Soil & J024R5 & $12 / 06 / 04$ & 41.1 & & 0.11 & 1020 & $\mathrm{C}$ & 1.7 & 0.62 & & 0.34 & 171 & & 1.3 & 0.23 & & 0.09 & 92.2 & $\mathrm{C}$ & 0.46 \\
\hline \begin{tabular}{|l} 
Area 4 Trench Soil \\
\end{tabular} & J024R6 & $12 / 06 / 04$ & 22.9 & & 0.13 & 1230 & $\mathrm{C}$ & 2 & 0.4 & $\mathrm{U}$ & 0.4 & 172 & & 1.5 & 0.11 & $\mathrm{U}$ & 0.11 & 131 & $\mathrm{C}$ & 0.55 \\
\hline Duplicate of J024R6 & J024X0 & $12 / 06 / 04$ & 21.8 & & 0.13 & 922 & $\mathrm{C}$ & 2.1 & 0.41 & $\mathrm{U}$ & 0.41 & 125 & & 1.5 & 0.11 & $\mathrm{U}$ & 0.11 & 119 & $\mathrm{C}$ & 0.56 \\
\hline Equipment Blank & J024X1 & 12/06/04 & 0.12 & & 0.09 & 20.1 & $\mathrm{C}$ & 1.4 & 0.27 & $\mathrm{U}$ & 0.27 & 33.9 & & 1 & 0.07 & $\mathrm{U}$ & 0.07 & 5.7 & $\mathrm{C}$ & 0.38 \\
\hline Area 3 Trench Soil & J024X2 & $12 / 07 / 04$ & 15.5 & & 0.09 & 1050 & $\mathrm{C}$ & 1.4 & 0.27 & $\mathrm{U}$ & 0.27 & 153 & & 1 & 0.08 & & 0.07 & 147 & $\mathrm{C}$ & 0.37 \\
\hline Area 2 Trench Soil & J024X3 & $12 / 07 / 04$ & 79.3 & & 0.13 & 833 & $\mathrm{C}$ & 2 & 0.39 & $\mathrm{U}$ & 0.39 & 276 & & 1.5 & 0.42 & & 0.1 & 188 & $\mathrm{C}$ & 0.53 \\
\hline Area 2 Ash & J024R7 & $12 / 03 / 04$ & 8.4 & & 0.8 & 1260 & $\mathrm{C}$ & 12.6 & 2.47 & $\mathrm{U}$ & 2.5 & 209 & & 9.3 & 0.667 & $\mathrm{U}$ & 0.67 & 2010 & $\mathrm{C}$ & 3.4 \\
\hline Area 2 Ash & J024R8 & $12 / 03 / 04$ & 32 & & 0.71 & 1150 & $\mathrm{C}$ & 11.2 & 2.2 & $\mathrm{U}$ & 2.2 & 470 & & 8.3 & 1.8 & & 0.589 & 538 & $\mathrm{C}$ & 3 \\
\hline Area 3 Oily Sediment & J024T0 & $12 / 07 / 04$ & 106 & & 0.84 & 1790 & $\mathrm{C}$ & 13.2 & 2.59 & $\mathrm{U}$ & 2.6 & 468 & & 9.8 & 1.6 & & 0.7 & 3710 & $\mathrm{C}$ & 3.6 \\
\hline Area 2 Paint & J02581 & $12 / 07 / 04$ & 4.2 & & 0.71 & 60.8 & $\mathrm{C}$ & 11.2 & 2.2 & $\mathrm{U}$ & 2.2 & 257 & & 8.3 & 0.593 & $\mathrm{U}$ & 0.59 & 20.5 & $\mathrm{C}$ & 3 \\
\hline
\end{tabular}


Table A-1. 128-F-2 Confirmatory Sample Results. (12 Pages)

\begin{tabular}{|c|c|c|c|c|c|c|c|c|c|c|c|}
\hline \multirow[t]{2}{*}{ Sample Location } & \multirow{2}{*}{$\begin{array}{c}\text { HEIS } \\
\text { Number }\end{array}$} & \multirow{2}{*}{$\begin{array}{c}\text { Sample } \\
\text { Date }\end{array}$} & \multicolumn{3}{|c|}{ Vanadium } & \multicolumn{3}{|c|}{ Zinc } & \multicolumn{3}{|c|}{$\begin{array}{c}\text { Total Petroleum } \\
\text { Hydrocarbons }\end{array}$} \\
\hline & & & $\mathrm{mg} / \mathrm{kg}$ & $\mathbf{Q}$ & PQL & $\mathrm{mg} / \mathrm{kg}$ & $\mathbf{Q}$ & PQL & $\mathrm{mg} / \mathrm{kg}$ & $\mathbf{Q}$ & PQL \\
\hline Area 1 Golden Soil & J024R1 & $12 / 03 / 04$ & 19.8 & & 0.05 & 40.3 & & 0.1 & 257 & & 34.3 \\
\hline Area 1 Tan Soil & J024R2 & $12 / 03 / 04$ & 31.7 & & 0.07 & 31.5 & & 0.13 & 55.9 & & 34 \\
\hline Area 1 Trench Soil & J024R3 & $12 / 03 / 04$ & 33.3 & & 0.07 & 35.5 & & 0.12 & 34.1 & $\mathrm{U}$ & 34.1 \\
\hline Area 4 Rusty Soil & J024R4 & $12 / 06 / 04$ & 46.6 & & 0.09 & 52.1 & & 0.16 & 45.3 & $\mathrm{U}$ & 45.3 \\
\hline Area 4 Oily Soil & J024R5 & $12 / 06 / 04$ & 62.3 & & 0.06 & 37 & & 0.12 & 34.8 & $\mathrm{U}$ & 34.8 \\
\hline Area 4 Trench Soil & J024R6 & $12 / 06 / 04$ & 40.3 & & 0.08 & 47 & & 0.14 & 36.5 & $\mathrm{U}$ & 36.5 \\
\hline Duplicate of J024R6 & J024X0 & $12 / 06 / 04$ & 39.3 & & 0.08 & 44 & & 0.14 & 36.4 & $\mathrm{U}$ & 36.4 \\
\hline Equipment Blank & J024X1 & $12 / 06 / 04$ & 0.11 & & 0.05 & 0.85 & & 0.1 & & & \\
\hline Area 3 Trench Soil & J024X2 & $12 / 07 / 04$ & 30.4 & & 0.05 & 207 & & 0.09 & 33.1 & $\mathrm{U}$ & 33.1 \\
\hline Area 2 Trench Soil & J024X3 & $12 / 07 / 04$ & 32.9 & & 0.07 & 289 & & 0.14 & 37.6 & $\mathrm{U}$ & 37.6 \\
\hline Area 2 Ash & J024R7 & $12 / 03 / 04$ & 21.3 & & 0.47 & 65.1 & & 0.87 & 201 & & 38.5 \\
\hline Area 2 Ash & J024R8 & $12 / 03 / 04$ & 35.2 & & 0.42 & 1030 & & 0.77 & 71.3 & & 36.3 \\
\hline Area 3 Oily Sediment & J024T0 & $12 / 07 / 04$ & 36.9 & & 0.49 & 2310 & & 0.91 & & & \\
\hline Area 2 Paint & J02581 & $12 / 07 / 04$ & 3 & & 0.42 & 342 & & 0.77 & 762 & & 187 \\
\hline
\end{tabular}

Table A-1. 128-F-2 Asbestos Confirmatory Sample Results. (12 Pages)

\begin{tabular}{|l|c|c|l|}
\hline \multicolumn{1}{|c|}{ Sample Area } & $\begin{array}{c}\text { HEIS } \\
\text { Number }\end{array}$ & $\begin{array}{c}\text { Sample } \\
\text { Date }\end{array}$ & \multicolumn{1}{c|}{ Asbestos Result } \\
\hline Area 1 Tan Material & J022J4 & $12 / 03 / 04$ & Between 5\% and 10\% total asbestos. \\
\hline Area 2 Chalky Material & J022J5 & $12 / 03 / 04$ & None detected. \\
\hline Area 2 Red Material & J022J6 & $12 / 03 / 04$ & Between 13\% and 25\% total asbestos. \\
\hline Area 3 Felt & J02578 & $12 / 07 / 04$ & Between 10\% and 20\% total asbestos. \\
\hline Area 3 Black Material & J02579 & $12 / 07 / 04$ & Between 3\% and 5\% total asbestos. \\
\hline Area 3 Chalky Material & J022K0 & $12 / 07 / 04$ & None detected. \\
\hline Area 4 Roofing Material & J022J7 & $12 / 06 / 04$ & None detected. \\
\hline Area 4 Transite & J022J8 & $12 / 06 / 04$ & Between 10\% and 20\% total asbestos. \\
\hline Area 4 Welding Rod & J022J9 & $12 / 06 / 04$ & Between 1\% and 3\% total asbestos. \\
\hline
\end{tabular}


Table A-1. 128-F-2 Confirmatory Sample Results. (12 Pages)

\begin{tabular}{|c|c|c|c|c|c|c|c|c|c|c|c|c|}
\hline \multirow[t]{2}{*}{ Constituent } & \multicolumn{3}{|c|}{$\begin{array}{c}\text { J024R1 } \\
\text { Area 1 Golden Soil } \\
\text { Sample Date 12/3/04 }\end{array}$} & \multicolumn{3}{|c|}{$\begin{array}{c}\text { J024R2 } \\
\text { Area } 1 \text { Tan Soil } \\
\text { Sample Date 12/3/04 }\end{array}$} & \multicolumn{3}{|c|}{$\begin{array}{c}\text { J024R3 } \\
\text { Area } 1 \text { Trench Soil } \\
\text { Sample Date 12/3/04 }\end{array}$} & \multicolumn{3}{|c|}{$\begin{array}{c}\text { J024R4 } \\
\text { Area 4 Rusty Soil } \\
\text { Sample Date 12/6/04 }\end{array}$} \\
\hline & $\mu g / \mathrm{kg}$ & $\mathbf{Q}$ & PQL & $\mu \mathrm{g} / \mathrm{kg}$ & $\mathbf{Q}$ & $\overline{\text { PQL }}$ & $\mu g / k g$ & $\mathbf{Q}$ & PQL & $\mu g / k g$ & $\mathbf{Q}$ & PQL \\
\hline \multicolumn{13}{|c|}{ Polycyclic Aromatic Hydrocarbons } \\
\hline Acenaphthene & 51.7 & $\mathrm{U}$ & 51.7 & 51.3 & $\mathrm{U}$ & 51.3 & 51.3 & $\mathrm{U}$ & 51.3 & 77.7 & & 68.5 \\
\hline Acenaphthylene & 51.7 & $\mathrm{U}$ & 51.7 & 51.3 & $\mathrm{U}$ & 51.3 & 51.3 & $\mathrm{U}$ & 51.3 & 84.9 & & 68.5 \\
\hline Anthracene & 5.17 & $\mathrm{U}$ & 5.17 & 18.2 & & 5.13 & 5.13 & $\mathrm{U}$ & 5.13 & 6.85 & $\mathrm{U}$ & 6.85 \\
\hline Benzo(a)anthracene & 5.17 & $\mathrm{U}$ & 5.17 & 91.5 & & 5.13 & 5.13 & $\mathrm{U}$ & 5.13 & 6.85 & $\mathrm{U}$ & 6.85 \\
\hline Benzo(a)pyrene & 5.17 & $\mathrm{U}$ & 5.17 & 76.9 & & 5.13 & 5.13 & $\mathrm{U}$ & 5.13 & 6.85 & $\mathrm{U}$ & 6.85 \\
\hline Benzo(b)fluoranthene & 11.4 & & 5.17 & 64.6 & & 5.13 & 5.13 & $\mathrm{U}$ & 5.13 & 6.85 & $\mathrm{U}$ & 6.85 \\
\hline Benzo(ghi)perylene & 9.6 & & 5.17 & 40.0 & & 5.13 & 5.13 & $\mathrm{U}$ & 5.13 & 6.85 & $\mathrm{U}$ & 6.85 \\
\hline Benzo(k)fluoranthene & 4.9 & $\mathrm{~J}$ & 5.17 & 32.3 & & 5.13 & 5.13 & $\mathrm{U}$ & 5.13 & 6.85 & $\mathrm{U}$ & 6.85 \\
\hline Chrysene & 5.17 & $\mathrm{U}$ & 5.17 & 73.6 & & 5.13 & 5.13 & $\mathrm{U}$ & 5.13 & 6.85 & $\mathrm{U}$ & 6.85 \\
\hline Dibenz[a,h]anthracene & 5.17 & $\mathrm{U}$ & 5.17 & 10.3 & & 5.13 & 5.13 & $\mathrm{U}$ & 5.13 & 6.85 & $\mathrm{U}$ & 6.85 \\
\hline Fluoranthene & 10.3 & $\mathrm{U}$ & 10.3 & 124.9 & & 10.2 & 10.2 & $\mathrm{U}$ & 10.2 & 13.7 & $\mathrm{U}$ & 13.7 \\
\hline Fluorene & 5.17 & $\mathrm{U}$ & 5.17 & 20.3 & & 5.13 & 5.13 & $\mathrm{U}$ & 5.13 & 34.9 & & 6.85 \\
\hline Indeno(1,2,3-cd)pyrene & 5.17 & $\mathrm{U}$ & 5.17 & 47.9 & & 5.13 & 5.13 & $\mathrm{U}$ & 5.13 & 6.85 & $\mathrm{U}$ & 6.85 \\
\hline Naphthalene & 51.7 & $\mathrm{U}$ & 51.7 & 51.3 & $\mathrm{U}$ & 51.3 & 51.3 & $\mathrm{U}$ & 51.3 & 597.3 & & 68.5 \\
\hline Phenanthrene & 5.17 & $\mathrm{U}$ & 5.17 & 99.2 & & 5.13 & 5.13 & $\mathrm{U}$ & 5.13 & 78.0 & & 6.85 \\
\hline Pyrene & 10.3 & $\mathrm{U}$ & 10.3 & 197.4 & & 10.2 & 10.2 & $\mathrm{U}$ & 10.2 & 13.7 & $\mathrm{U}$ & 13.7 \\
\hline \multicolumn{13}{|c|}{ PCBs (Polychlorinated biphenyls) } \\
\hline Aroclor-1016 & 69 & $\mathrm{U}$ & 69 & 68 & $\mathrm{U}$ & 68 & 68 & $\mathrm{U}$ & 68 & 91 & $\mathrm{U}$ & 91 \\
\hline \begin{tabular}{|l} 
Aroclor-1221 \\
\end{tabular} & 69 & $\mathrm{U}$ & 69 & 68 & $\mathrm{U}$ & 68 & 68 & $\mathrm{U}$ & 68 & 91 & $\mathrm{U}$ & 91 \\
\hline Aroclor-1232 & 69 & $\mathrm{U}$ & 69 & 68 & $\mathrm{U}$ & 68 & 68 & $\mathrm{U}$ & 68 & 91 & $\mathrm{U}$ & 91 \\
\hline Aroclor-1242 & 69 & $\mathrm{U}$ & 69 & 68 & $\mathrm{U}$ & 68 & 68 & $\mathrm{U}$ & 68 & 91 & $\mathrm{U}$ & 91 \\
\hline \begin{tabular}{|l} 
Aroclor-1248 \\
\end{tabular} & 69 & $\mathrm{U}$ & 69 & 68 & $\mathrm{U}$ & 68 & 68 & $\mathrm{U}$ & 68 & 91 & $\mathrm{U}$ & 91 \\
\hline Aroclor-1254 & 69 & $\mathrm{U}$ & 69 & 68 & $\mathrm{U}$ & 68 & 68 & $\mathrm{U}$ & 68 & 91 & $\mathrm{U}$ & 91 \\
\hline Aroclor-1260 & 69 & $\mathrm{U}$ & 69 & 68 & U & 68 & 68 & $\mathrm{U}$ & 68 & 91 & $\mathrm{U}$ & 91 \\
\hline \multicolumn{13}{|c|}{ Pesticides } \\
\hline Aldrin & 34 & UD & 34 & 34 & UD & 34 & 1.7 & $\mathrm{U}$ & 1.7 & 46 & \begin{tabular}{|l|} 
UD \\
\end{tabular} & 46 \\
\hline Alpha-BHC & 34 & UD & 34 & 34 & UD & 34 & 1.7 & $\mathrm{U}$ & 1.7 & 46 & UD & 46 \\
\hline alpha-Chlordane & 34 & UD & 34 & 34 & UD & 34 & 1.7 & $\mathrm{U}$ & 1.7 & 34 & UD & 34 \\
\hline $\begin{array}{l}\text { beta-1,2,3,4,5,6- } \\
\text { Hexachlorocyclohexane }\end{array}$ & 34 & UD & 34 & 34 & UD & 34 & 1.7 & $\mathrm{U}$ & 1.7 & 46 & UD & 46 \\
\hline Delta-BHC & 34 & UD & 34 & 34 & UD & 34 & 1.7 & $\mathrm{U}$ & 1.7 & 46 & UD & 46 \\
\hline Dichlorodiphenyldichloroethane & 69 & UD & 69 & 68 & UD & 68 & 3.4 & $\mathrm{U}$ & 3.4 & 91 & UD & 91 \\
\hline Dichlorodiphenyldichloroethylene & 69 & UD & 69 & 420 & $\mathrm{D}$ & 68 & 3.4 & $\mathrm{U}$ & 3.4 & 91 & UD & 91 \\
\hline Dichlorodiphenyltrichloroethane & 69 & UD & 69 & 2200 & $\mathrm{D}$ & 68 & 3.4 & $\mathrm{U}$ & 3.4 & 91 & UD & 91 \\
\hline Dieldrin & 69 & UD & 69 & 68 & UD & 68 & 3.4 & $\mathrm{U}$ & 3.4 & 91 & UD & 91 \\
\hline Endosulfan I & 34 & UD & 34 & 34 & UD & 34 & 1.7 & $\mathrm{U}$ & 1.7 & 46 & UD & 46 \\
\hline Endosulfan II & 69 & UD & 69 & 68 & UD & 68 & 3.4 & $\mathrm{U}$ & 3.4 & 91 & UD & 91 \\
\hline Endosulfan sulfate & 69 & UD & 69 & 68 & UD & 68 & 3.4 & $\mathrm{U}$ & 3.4 & 91 & UD & 91 \\
\hline Endrin & 69 & UD & 69 & 68 & UD & 68 & 3.4 & $\mathrm{U}$ & 3.4 & 91 & UD & 91 \\
\hline Endrin aldehyde & 69 & UD & 69 & 68 & UD & 68 & 3.4 & $\mathrm{U}$ & 3.4 & 69 & UD & 69 \\
\hline Endrin ketone & 69 & UD & 69 & 68 & UD & 68 & 3.4 & $\mathrm{U}$ & 3.4 & 69 & UD & 69 \\
\hline Gamma-BHC (Lindane) & 34 & UD & 34 & 34 & UD & 34 & 1.7 & $\mathrm{U}$ & 1.7 & 46 & UD & 46 \\
\hline gamma-Chlordane & 34 & UD & 34 & 34 & UD & 34 & 1.7 & $\mathrm{U}$ & 1.7 & 34 & UD & 34 \\
\hline Heptachlor & 34 & UD & 34 & 34 & UD & 34 & 1.7 & $\mathrm{U}$ & 1.7 & 46 & UD & 46 \\
\hline Heptachlor epoxide & 34 & UD & 34 & 34 & UD & 34 & 1.7 & $\mathrm{U}$ & 1.7 & 46 & UD & 46 \\
\hline Methoxychlor & 340 & UD & 340 & 340 & UD & 340 & 17 & $\mathrm{U}$ & 17 & 460 & UD & 460 \\
\hline Toxaphene & 3400 & UD & 3400 & 3400 & UD & 3400 & 170 & $\mathrm{U}$ & 170 & 4600 & UD & 4600 \\
\hline
\end{tabular}


Table A-1. 128-F-2 Confirmatory Sample Results. (12 Pages)

\begin{tabular}{|c|c|c|c|c|c|c|c|c|c|c|c|c|}
\hline \multirow[t]{2}{*}{ Constituent } & \multicolumn{3}{|c|}{$\begin{array}{c}\text { J024R1 } \\
\text { Area 1 Golden Soil } \\
\text { Sample Date 12/3/04 }\end{array}$} & \multicolumn{3}{|c|}{$\begin{array}{c}\text { J024R2 } \\
\text { Area } 1 \text { Tan Soil } \\
\text { Sample Date 12/3/04 }\end{array}$} & \multicolumn{3}{|c|}{$\begin{array}{c}\text { J024R3 } \\
\text { Area } 1 \text { Trench Soil } \\
\text { Sample Date 12/3/04 }\end{array}$} & \multicolumn{3}{|c|}{$\begin{array}{c}\text { J024R4 } \\
\text { Area } 4 \text { Rusty Soil } \\
\text { Sample Date 12/6/04 }\end{array}$} \\
\hline & $\mu \mathrm{g} / \mathrm{kg}$ & $\mathbf{Q}$ & PQL & $\mu \mathrm{g} / \mathrm{kg}$ & $\mathbf{Q}$ & PQL & $\mu \mathrm{g} / \mathrm{kg}$ & $\mathbf{Q}$ & PQL & $\mu \mathrm{g} / \mathrm{kg}$ & $\mathbf{Q}$ & PQL \\
\hline \multicolumn{13}{|c|}{ SVOAs (Semivolatile organics) } \\
\hline 1,2,4-Trichlorobenzene & 340 & $\mathrm{U}$ & 340 & 340 & $\mathrm{U}$ & 340 & 340 & $\mathrm{U}$ & 340 & 460 & $\mathrm{U}$ & 460 \\
\hline 1,2-Dichlorobenzene & 340 & $\mathrm{U}$ & 340 & 340 & $\mathrm{U}$ & 340 & 340 & $\mathrm{U}$ & 340 & 460 & $\mathrm{U}$ & 460 \\
\hline 1,3-Dichlorobenzene & 340 & $\mathrm{U}$ & 340 & 340 & $\mathrm{U}$ & 340 & 340 & $\mathrm{U}$ & 340 & 460 & $\mathrm{U}$ & 460 \\
\hline 1,4-Dichlorobenzene & 340 & $\mathrm{U}$ & 340 & 340 & $\mathrm{U}$ & 340 & 340 & $\mathrm{U}$ & 340 & 460 & $\mathrm{U}$ & 460 \\
\hline 2,4,5-Trichlorophenol & 860 & $\mathrm{U}$ & 860 & 860 & $\mathrm{U}$ & 860 & 860 & $\mathrm{U}$ & 860 & 1100 & $\mathrm{U}$ & 1100 \\
\hline 2,4,6-Trichlorophenol & 340 & $\mathrm{U}$ & 340 & 340 & $\mathrm{U}$ & 340 & 340 & $\mathrm{U}$ & 340 & 460 & $\mathrm{U}$ & 460 \\
\hline 2,4-Dichlorophenol & 340 & $\mathrm{U}$ & 340 & 340 & $\mathrm{U}$ & 340 & 340 & $\bar{U}$ & 340 & 460 & $\mathrm{U}$ & 460 \\
\hline 2,4-Dimethylphenol & 340 & $\mathrm{U}$ & 340 & 340 & $\mathrm{U}$ & 340 & 340 & $\mathrm{U}$ & 340 & 460 & $\mathrm{U}$ & 460 \\
\hline 2,4-Dinitrophenol & 860 & $\mathrm{U}$ & 860 & 860 & $\mathrm{U}$ & 860 & 860 & $\mathrm{U}$ & 860 & 1100 & $\mathrm{U}$ & 1100 \\
\hline 2,4-Dinitrotoluene & 340 & $\mathrm{U}$ & 340 & 340 & $\mathrm{U}$ & 340 & 340 & $\bar{U}$ & 340 & 460 & $\mathrm{U}$ & 460 \\
\hline 2,6-Dinitrotoluene & 340 & $\mathrm{U}$ & 340 & 340 & $\mathrm{U}$ & 340 & 340 & $\mathrm{U}$ & 340 & 460 & $\mathrm{U}$ & 460 \\
\hline 2-Chloronaphthalene & 340 & $\mathrm{U}$ & 340 & 340 & $\mathrm{U}$ & 340 & 340 & $\mathrm{U}$ & 340 & 460 & $\mathrm{U}$ & 460 \\
\hline 2-Chlorophenol & 340 & $\mathrm{U}$ & 340 & 340 & $\mathrm{U}$ & 340 & 340 & $\mathrm{U}$ & 340 & 460 & $\mathrm{U}$ & 460 \\
\hline 2-Methylnaphthalene & 340 & $\mathrm{U}$ & 340 & 340 & $\mathrm{U}$ & 340 & 340 & $\mathrm{U}$ & 340 & 460 & $\mathrm{U}$ & 460 \\
\hline 2-Methylphenol (cresol, o-) & 340 & $\mathrm{U}$ & 340 & 340 & $\mathrm{U}$ & 340 & 340 & $\mathrm{U}$ & 340 & 460 & $\mathrm{U}$ & 460 \\
\hline 2-Nitroaniline & 860 & $\mathrm{U}$ & 860 & 860 & $\mathrm{U}$ & 860 & 860 & $\mathrm{U}$ & 860 & 1100 & $\mathrm{U}$ & 1100 \\
\hline 2-Nitrophenol & 340 & $\mathrm{U}$ & 340 & 340 & $\mathrm{U}$ & 340 & 340 & $\mathrm{U}$ & 340 & 460 & $\mathrm{U}$ & 460 \\
\hline 3+4 Methylphenol (cresol, m+p) & 340 & $\mathrm{U}$ & 340 & 340 & $\mathrm{U}$ & 340 & 340 & $\mathrm{U}$ & 340 & 460 & $\mathrm{U}$ & 460 \\
\hline 3,3'-Dichlorobenzidine & 340 & $\mathrm{U}$ & 340 & 340 & $\mathrm{U}$ & 340 & 340 & $\mathrm{U}$ & 340 & 460 & $\mathrm{U}$ & 460 \\
\hline 3-Nitroaniline & 860 & $\mathrm{U}$ & 860 & 860 & $\mathrm{U}$ & 860 & 860 & $\mathrm{U}$ & 860 & 1100 & $\mathrm{U}$ & 1100 \\
\hline 4,6-Dinitro-2-methylphenol & 860 & $\mathrm{U}$ & 860 & 860 & $\mathrm{U}$ & 860 & 860 & $\mathrm{U}$ & 860 & 1100 & $\mathrm{U}$ & 1100 \\
\hline 4-Bromophenylphenyl ether & 340 & $\mathrm{U}$ & 340 & 340 & $\mathrm{U}$ & 340 & 340 & $\mathrm{U}$ & 340 & 460 & $\mathrm{U}$ & 460 \\
\hline 4-Chloro-3-methylphenol & 340 & $\mathrm{U}$ & 340 & 340 & $\mathrm{U}$ & 340 & 340 & $\mathrm{U}$ & 340 & 460 & $\mathrm{U}$ & 460 \\
\hline 4-Chloroaniline & 340 & $\mathrm{U}$ & 340 & 340 & $\mathrm{U}$ & 340 & 340 & $\mathrm{U}$ & 340 & 460 & $\mathrm{U}$ & 460 \\
\hline 4-Chlorophenylphenyl ether & 340 & $\mathrm{U}$ & 340 & 340 & $\mathrm{U}$ & 340 & 340 & $\mathrm{U}$ & 340 & 460 & $\mathrm{U}$ & 460 \\
\hline 4-Nitroaniline & 860 & $\mathrm{U}$ & 860 & 860 & $\mathrm{U}$ & 860 & 860 & $\mathrm{U}$ & 860 & 1100 & $\mathrm{U}$ & 1100 \\
\hline 4-Nitrophenol & 860 & $\mathrm{U}$ & 860 & 860 & $\mathrm{U}$ & 860 & 860 & $\mathrm{U}$ & 860 & 1100 & $\mathrm{U}$ & 1100 \\
\hline Acenaphthene & 340 & $\mathrm{U}$ & 340 & 340 & $\mathrm{U}$ & 340 & 340 & $\mathrm{U}$ & 340 & 460 & $\mathrm{U}$ & 460 \\
\hline Acenaphthylene & 340 & $\mathrm{U}$ & 340 & 340 & $\mathrm{U}$ & 340 & 340 & $\mathrm{U}$ & 340 & 460 & $\mathrm{U}$ & 460 \\
\hline Anthracene & 340 & $\mathrm{U}$ & 340 & 340 & $\mathrm{U}$ & 340 & 340 & $\mathrm{U}$ & 340 & 460 & $\mathrm{U}$ & 460 \\
\hline Benzo(a)anthracene & 340 & $\mathrm{U}$ & 340 & 33 & $\mathrm{~J}$ & 340 & 340 & $\mathrm{U}$ & 340 & 460 & $\mathrm{U}$ & 460 \\
\hline Benzo(a)pyrene & 340 & $\mathrm{U}$ & 340 & 32 & $\mathrm{~J}$ & 340 & 340 & $\mathrm{U}$ & 340 & 460 & $\mathrm{U}$ & 460 \\
\hline Benzo(b)fluoranthene & 340 & $\mathrm{U}$ & 340 & 23 & $\mathrm{~J}$ & 340 & 340 & $\mathrm{U}$ & 340 & 460 & $\mathrm{U}$ & 460 \\
\hline Benzo(ghi)perylene & 340 & $\mathrm{U}$ & 340 & 20 & $\mathrm{~J}$ & 340 & 340 & $\mathrm{U}$ & 340 & 460 & $\mathrm{U}$ & 460 \\
\hline Benzo(k)fluoranthene & 340 & $\mathrm{U}$ & 340 & 29 & $\mathrm{~J}$ & 340 & 340 & $\mathrm{U}$ & 340 & 460 & $\mathrm{U}$ & 460 \\
\hline Bis(2-chloro-1-methylethyl)ether & 340 & $\mathrm{U}$ & 340 & 340 & $\mathrm{U}$ & 340 & 340 & $\mathrm{U}$ & 340 & 460 & $\mathrm{U}$ & 460 \\
\hline Bis(2-Chloroethoxy)methane & 340 & $\mathrm{U}$ & 340 & 340 & $\mathrm{U}$ & 340 & 340 & $\mathrm{U}$ & 340 & 460 & $\mathrm{U}$ & 460 \\
\hline Bis(2-chloroethyl) ether & 340 & $\mathrm{U}$ & 340 & 340 & $\mathrm{U}$ & 340 & 340 & $\mathrm{U}$ & 340 & 460 & $\mathrm{U}$ & 460 \\
\hline Bis(2-ethylhexyl) phthalate & 22 & JB & 340 & 160 & JB & 340 & 340 & $\mathrm{U}$ & 340 & 26 & JB & 460 \\
\hline Butylbenzylphthalate & 340 & $\mathrm{U}$ & 340 & 340 & $\mathrm{U}$ & 340 & 340 & $\mathrm{U}$ & 340 & 460 & $\mathrm{U}$ & 460 \\
\hline Carbazole & 340 & $\mathrm{U}$ & 340 & 340 & $\mathrm{U}$ & 340 & 340 & $\mathrm{U}$ & 340 & 460 & $\mathrm{U}$ & 460 \\
\hline Chrysene & 340 & $\mathrm{U}$ & 340 & 40 & $\mathrm{~J}$ & 340 & 340 & $\mathrm{U}$ & 340 & 460 & $\mathrm{U}$ & 460 \\
\hline Di-n-butylphthalate & 340 & $\mathrm{U}$ & 340 & 340 & $\mathrm{U}$ & 340 & 340 & $\mathrm{U}$ & 340 & 460 & $\mathrm{U}$ & 460 \\
\hline Di-n-octylphthalate & 340 & $\mathrm{U}$ & 340 & 340 & $\mathrm{U}$ & 340 & 340 & $\mathrm{U}$ & 340 & 460 & $\mathrm{U}$ & 460 \\
\hline Dibenz[a,h]anthracene & 340 & $\mathrm{U}$ & 340 & 340 & $\mathrm{U}$ & 340 & 340 & $\mathrm{U}$ & 340 & 460 & $\mathrm{U}$ & 460 \\
\hline Dibenzofuran & 340 & $\mathrm{U}$ & 340 & 340 & $\mathrm{U}$ & 340 & 340 & $\mathrm{U}$ & 340 & 460 & $\mathrm{U}$ & 460 \\
\hline Diethylphthalate & 340 & $\mathrm{U}$ & 340 & 340 & $\mathrm{U}$ & 340 & 340 & $\mathrm{U}$ & 340 & 460 & $\mathrm{U}$ & 460 \\
\hline Dimethyl phthalate & 340 & $\mathrm{U}$ & 340 & 340 & $\mathrm{U}$ & 340 & 340 & $\mathrm{U}$ & 340 & 460 & $\mathrm{U}$ & 460 \\
\hline Fluoranthene & 340 & $\mathrm{U}$ & 340 & 53 & $\mathrm{~J}$ & 340 & 340 & $\mathrm{U}$ & 340 & 460 & $\mathrm{U}$ & 460 \\
\hline Fluorene & 340 & $\mathrm{U}$ & 340 & 340 & $\mathrm{U}$ & 340 & 340 & $\mathrm{U}$ & 340 & 460 & $\mathrm{U}$ & 460 \\
\hline
\end{tabular}


Table A-1. 128-F-2 Confirmatory Sample Results. (12 Pages)

\begin{tabular}{|c|c|c|c|c|c|c|c|c|c|c|c|c|}
\hline \multirow[t]{2}{*}{ Constituent } & \multicolumn{3}{|c|}{$\begin{array}{c}\text { J024R1 } \\
\text { Area } 1 \text { Golden Soil } \\
\text { Sample Date 12/3/04 }\end{array}$} & \multicolumn{3}{|c|}{$\begin{array}{c}\text { J024R2 } \\
\text { Area } 1 \text { Tan Soil } \\
\text { Sample Date 12/3/04 }\end{array}$} & \multicolumn{3}{|c|}{$\begin{array}{c}\text { J024R3 } \\
\text { Area } 1 \text { Trench Soil } \\
\text { Sample Date 12/3/04 }\end{array}$} & \multicolumn{3}{|c|}{$\begin{array}{c}\text { J024R4 } \\
\text { Area } 4 \text { Rusty Soil } \\
\text { Sample Date 12/6/04 }\end{array}$} \\
\hline & $\mu \mathrm{g} / \mathrm{kg}$ & $\mathbf{Q}$ & PQL & $\mu \mathrm{g} / \mathrm{kg}$ & $\mathbf{Q}$ & PQL & $\mu \mathrm{g} / \mathrm{kg}$ & $\mathbf{Q}$ & PQL & $\mu \mathrm{g} / \mathrm{kg}$ & $\mathbf{Q}$ & PQL \\
\hline \multicolumn{13}{|c|}{ SVOAs (Semivolatile organics) (continued) } \\
\hline Hexachlorobenzene & 340 & $\mathrm{U}$ & 340 & 340 & $\mathrm{U}$ & 340 & 340 & $\mathrm{U}$ & 340 & 460 & $\mathrm{U}$ & 460 \\
\hline Hexachlorobutadiene & 340 & $\mathrm{U}$ & 340 & 340 & $\mathrm{U}$ & 340 & 340 & $\mathrm{U}$ & 340 & 460 & $\mathrm{U}$ & 460 \\
\hline Hexachlorocyclopentadiene & 340 & $\mathrm{U}$ & 340 & 340 & $\mathrm{U}$ & 340 & 340 & U & 340 & 460 & $\mathrm{U}$ & 460 \\
\hline Hexachloroethane & 340 & $\mathrm{U}$ & 340 & 340 & $\mathrm{U}$ & 340 & 340 & $\mathrm{U}$ & 340 & 460 & $\mathrm{U}$ & 460 \\
\hline Indeno(1,2,3-cd)pyrene & 340 & $\mathrm{U}$ & 340 & 18 & $\mathrm{~J}$ & 340 & 340 & $\mathrm{U}$ & 340 & 460 & $\mathrm{U}$ & 460 \\
\hline Isophorone & 340 & $\mathrm{U}$ & 340 & 340 & $\mathrm{U}$ & 340 & 340 & $\mathrm{U}$ & 340 & 460 & $\mathrm{U}$ & 460 \\
\hline N-Nitroso-di-n-dipropylamine & 340 & $\mathrm{U}$ & 340 & 340 & $\mathrm{U}$ & 340 & 340 & $\mathrm{U}$ & 340 & 460 & $\mathrm{U}$ & 460 \\
\hline N-Nitrosodiphenylamine & 340 & $\mathrm{U}$ & 340 & 340 & $\mathrm{U}$ & 340 & 340 & $\mathrm{U}$ & 340 & 460 & $\mathrm{U}$ & 460 \\
\hline Naphthalene & 340 & $\mathrm{U}$ & 340 & 340 & $\mathrm{U}$ & 340 & 340 & $\mathrm{U}$ & 340 & 460 & $\mathrm{U}$ & 460 \\
\hline Nitrobenzene & 340 & $\mathrm{U}$ & 340 & 340 & $\mathrm{U}$ & 340 & 340 & $\mathrm{U}$ & 340 & 460 & $\mathrm{U}$ & 460 \\
\hline Pentachlorophenol & 860 & $\mathrm{U}$ & 860 & 860 & $\mathrm{U}$ & 860 & 860 & $\mathrm{U}$ & 860 & 1100 & $\mathrm{U}$ & 1100 \\
\hline Phenanthrene & 340 & $\mathrm{U}$ & 340 & 31 & $\mathrm{~J}$ & 340 & 340 & U & 340 & 460 & $\mathrm{U}$ & 460 \\
\hline Phenol & 340 & $\mathrm{U}$ & 340 & 340 & $\mathrm{U}$ & 340 & 340 & $\mathrm{U}$ & 340 & 460 & $\mathrm{U}$ & 460 \\
\hline Pyrene & 340 & $\mathrm{U}$ & 340 & 58.26 & $\mathrm{~J}$ & 340 & 340 & $\mathrm{U}$ & 340 & 460 & $\mathrm{U}$ & 460 \\
\hline
\end{tabular}

\begin{tabular}{|c|c|c|c|c|c|c|c|c|c|c|c|c|}
\hline \multirow[t]{2}{*}{ Constituent } & \multicolumn{3}{|c|}{$\begin{array}{c}\text { J024R5 } \\
\text { Area } 4 \text { Oily Soil } \\
\text { Sample Date 12/6/04 }\end{array}$} & \multicolumn{3}{|c|}{$\begin{array}{c}\text { J024R6 } \\
\text { Area } 4 \text { Trench Soil } \\
\text { Sample Date 12/6/04 }\end{array}$} & \multicolumn{3}{|c|}{$\begin{array}{c}\text { J024X0 } \\
\text { Duplicate of J024R6 } \\
\text { Sample Date 12/6/04 }\end{array}$} & \multicolumn{3}{|c|}{$\begin{array}{c}\text { J024X2 } \\
\text { Area } 3 \text { Trench Soil } \\
\text { Sample Date 12/7/04 }\end{array}$} \\
\hline & $\mu g / \mathrm{kg}$ & $\mathbf{Q}$ & PQL & $\mu \mathrm{g} / \mathrm{kg}$ & $\mathbf{Q}$ & PQL & $\mu g / \mathrm{kg}$ & $\mathbf{Q}$ & PQL & $\mu \mathrm{g} / \mathrm{kg}$ & $\mathbf{Q}$ & $\overline{\text { PQL }}$ \\
\hline \multicolumn{13}{|c|}{ Polycyclic Aromatic Hydrocarbons } \\
\hline Acenaphthene & 52.3 & $\mathrm{U}$ & 52.3 & 54.8 & $\mathrm{U}$ & 54.8 & 54.8 & $\mathrm{U}$ & 54.8 & 56.8 & $\bar{U}$ & 56.8 \\
\hline Acenaphthylene & 52.3 & $\mathrm{U}$ & 52.3 & 54.8 & $\mathrm{U}$ & 54.8 & 54.8 & $\mathrm{U}$ & 54.8 & 56.8 & $\mathrm{U}$ & 56.8 \\
\hline Anthracene & 5.23 & $\mathrm{U}$ & 5.23 & 5.47 & $\mathrm{U}$ & 5.47 & 5.48 & $\mathrm{U}$ & 5.48 & 788 & & 5.68 \\
\hline Benzo(a)anthracene & 5.23 & $\mathrm{U}$ & 5.23 & 5.47 & $\mathrm{U}$ & 5.47 & 5.48 & $\mathrm{U}$ & 5.48 & 2200 & & 5.68 \\
\hline Benzo(a)pyrene & 5.23 & $\mathrm{U}$ & 5.23 & 5.47 & $\mathrm{U}$ & 5.47 & 5.48 & $\mathrm{U}$ & 5.48 & 1400 & & 5.68 \\
\hline Benzo(b)fluoranthene & 5.23 & $\mathrm{U}$ & 5.23 & 5.47 & $\mathrm{U}$ & 5.47 & 5.48 & $\mathrm{U}$ & 5.48 & 1360 & & 5.68 \\
\hline Benzo(ghi)perylene & 5.23 & $\mathrm{U}$ & 5.23 & 5.47 & $\mathrm{U}$ & 5.47 & 5.48 & $\mathrm{U}$ & 5.48 & 942 & & 5.68 \\
\hline Benzo(k)fluoranthene & 5.23 & $\mathrm{U}$ & 5.23 & 5.47 & $\mathrm{U}$ & 5.47 & 5.48 & $\mathrm{U}$ & 5.48 & 711 & & 5.68 \\
\hline Chrysene & 5.23 & $\mathrm{U}$ & 5.23 & 5.47 & $\mathrm{U}$ & 5.47 & 5.48 & $\mathrm{U}$ & 5.48 & 2160 & & 5.68 \\
\hline Dibenz[a,h]anthracene & 5.23 & $\mathrm{U}$ & 5.23 & 5.47 & $\mathrm{U}$ & 5.47 & 5.48 & $\mathrm{U}$ & 5.48 & 257 & & 5.68 \\
\hline Fluoranthene & 10.5 & $\mathrm{U}$ & 10.5 & 10.9 & $\mathrm{U}$ & 10.9 & 10.5 & $\mathrm{U}$ & 10.5 & 4760 & $\mathrm{D}$ & 114 \\
\hline Fluorene & 5.23 & $\mathrm{U}$ & 5.23 & 5.47 & $\mathrm{U}$ & 5.47 & 5.48 & $\mathrm{U}$ & 5.48 & 715 & & 5.68 \\
\hline Indeno(1,2,3-cd)pyrene & 5.23 & $\mathrm{U}$ & 5.23 & 5.47 & $\mathrm{U}$ & 5.47 & 5.48 & $\mathrm{U}$ & 5.48 & 1230 & & 5.68 \\
\hline Naphthalene & 52.3 & $\mathrm{U}$ & 52.3 & 54.8 & $\mathrm{U}$ & 54.8 & 54.8 & $\mathrm{U}$ & 54.8 & 56.8 & $\mathrm{U}$ & 56.8 \\
\hline Phenanthrene & 5.23 & $\mathrm{U}$ & 5.23 & 5.47 & $\mathrm{U}$ & 5.47 & 5.48 & $\mathrm{U}$ & 5.48 & 4630 & $\mathrm{D}$ & 56.8 \\
\hline Pyrene & 10.5 & $\mathrm{U}$ & 10.5 & 10.9 & $\mathrm{U}$ & 10.9 & 10.5 & $\mathrm{U}$ & 10.5 & 5720 & $\mathrm{D}$ & 114 \\
\hline \multicolumn{13}{|c|}{ PCBs (Polychlorinated biphenyls) } \\
\hline Aroclor-1016 & 70 & $\mathrm{U}$ & 70 & 73 & $\mathrm{U}$ & 73 & 73 & $\mathrm{U}$ & 73 & 76 & $\bar{U}$ & 76 \\
\hline Aroclor-1221 & 70 & $\mathrm{U}$ & 70 & 73 & $\mathrm{U}$ & 73 & 73 & $\mathrm{U}$ & 73 & 76 & $\mathrm{U}$ & 76 \\
\hline Aroclor-1232 & 70 & $\mathrm{U}$ & 70 & 73 & $\mathrm{U}$ & 73 & 73 & $\mathrm{U}$ & 73 & 76 & $\mathrm{U}$ & 76 \\
\hline Aroclor-1242 & 70 & $\mathrm{U}$ & 70 & 73 & $\mathrm{U}$ & 73 & 73 & $\mathrm{U}$ & 73 & 76 & $\mathrm{U}$ & 76 \\
\hline Aroclor-1248 & 70 & $\mathrm{U}$ & 70 & 73 & $\mathrm{U}$ & 73 & 73 & $\mathrm{U}$ & 73 & 76 & $\mathrm{U}$ & 76 \\
\hline Aroclor-1254 & 70 & $\mathrm{U}$ & 70 & 73 & $\mathrm{U}$ & 73 & 73 & $\mathrm{U}$ & 73 & 940 & & 76 \\
\hline Aroclor-1260 & 70 & $\mathrm{U}$ & 70 & 73 & $\mathrm{U}$ & 73 & 73 & $\mathrm{U}$ & 73 & 76 & $\mathrm{U}$ & 76 \\
\hline \multicolumn{13}{|c|}{ Pesticides } \\
\hline Aldrin & 1.7 & $\mathrm{U}$ & 1.7 & 36 & UD & 36 & 1.8 & $\mathrm{U}$ & 1.8 & 38 & UD & 38 \\
\hline Alpha-BHC & 1.7 & $\mathrm{U}$ & 1.7 & 36 & UD & 36 & 1.8 & $\mathrm{U}$ & 1.8 & 38 & UD & 38 \\
\hline alpha-Chlordane & 1.7 & $\mathrm{U}$ & 1.7 & 36 & UD & 36 & 1.8 & $\mathrm{U}$ & 1.8 & 38 & UD & 38 \\
\hline
\end{tabular}


Table A-1. 128-F-2 Confirmatory Sample Results. (12 Pages)

\begin{tabular}{|c|c|c|c|c|c|c|c|c|c|c|c|c|}
\hline \multirow[t]{2}{*}{ Constituent } & \multicolumn{3}{|c|}{$\begin{array}{c}\text { J024R5 } \\
\text { Area } 4 \text { Oily Soil } \\
\text { Sample Date 12/6/04 }\end{array}$} & \multicolumn{3}{|c|}{$\begin{array}{c}\text { J024R6 } \\
\text { Area } 4 \text { Trench Soil } \\
\text { Sample Date 12/6/04 }\end{array}$} & \multicolumn{3}{|c|}{$\begin{array}{c}\text { J024X0 } \\
\text { Duplicate of J024R6 } \\
\text { Sample Date 12/6/04 }\end{array}$} & \multicolumn{3}{|c|}{$\begin{array}{c}\text { J024X2 } \\
\text { Area } 3 \text { Trench Soil } \\
\text { Sample Date 12/7/04 }\end{array}$} \\
\hline & $\mu \mathrm{g} / \mathrm{kg}$ & $\mathbf{Q}$ & PQL & $\mu g / k g$ & $\mathbf{Q}$ & PQL & $\mu g / \mathrm{kg}$ & $\mathbf{Q}$ & PQL & $\mu g / \mathrm{kg}$ & $\mathbf{Q}$ & PQL \\
\hline \multicolumn{13}{|c|}{ Pesticides (continued) } \\
\hline $\begin{array}{l}\text { beta-1,2,3,4,5,6- } \\
\text { Hexachlorocyclohexane }\end{array}$ & 1.7 & $\mathrm{U}$ & 1.7 & 36 & UD & 36 & 1.8 & $\mathrm{U}$ & 1.8 & 38 & UD & 38 \\
\hline Delta-BHC & 1.7 & $\mathrm{U}$ & 1.7 & 36 & UD & 36 & 1.8 & $\mathrm{U}$ & 1.8 & 38 & UD & 38 \\
\hline Dichlorodiphenyldichloroethane & 3.5 & $\mathrm{U}$ & 3.5 & 73 & UD & 73 & 3.7 & $\mathrm{U}$ & 3.7 & 76 & UD & 76 \\
\hline Dichlorodiphenyldichloroethylene & 3.5 & $\mathrm{U}$ & 3.5 & 73 & UD & 73 & 3.7 & $\mathrm{U}$ & 3.7 & 76 & UD & 76 \\
\hline Dichlorodiphenyltrichloroethane & 3.5 & $\mathrm{U}$ & 3.5 & 73 & UD & 73 & 3.7 & $\mathrm{U}$ & 3.7 & 70 & JD & 70 \\
\hline Dieldrin & 3.5 & $\mathrm{U}$ & 3.5 & 73 & $\mathrm{UD}$ & 73 & 3.7 & $\mathrm{U}$ & 3.7 & 76 & UD & 76 \\
\hline Endosulfan I & 1.7 & $\mathrm{U}$ & 1.7 & 36 & UD & 36 & 1.8 & $\mathrm{U}$ & 1.8 & 38 & UD & 38 \\
\hline Endosulfan II & 3.5 & $\mathrm{U}$ & 3.5 & 73 & UD & 73 & 3.7 & $\mathrm{U}$ & 3.7 & 76 & UD & 76 \\
\hline Endosulfan sulfate & 3.5 & $\mathrm{U}$ & 3.5 & 73 & UD & 73 & 3.7 & $\mathrm{U}$ & 3.7 & 76 & UD & 76 \\
\hline Endrin & 3.5 & $\mathrm{U}$ & 3.5 & 73 & UD & 73 & 3.7 & $\mathrm{U}$ & 3.7 & 76 & UD & 76 \\
\hline Endrin aldehyde & 3.5 & $\mathrm{U}$ & 3.5 & 73 & UD & 73 & 3.7 & $\mathrm{U}$ & 3.7 & 76 & UD & 76 \\
\hline Endrin ketone & 3.5 & $\mathrm{U}$ & 3.5 & 73 & UD & 73 & 3.7 & $\mathrm{U}$ & 3.7 & 76 & UD & 76 \\
\hline Gamma-BHC (Lindane) & 1.7 & $\mathrm{U}$ & 1.7 & 36 & UD & 36 & 1.8 & $\mathrm{U}$ & 1.8 & 38 & UD & 38 \\
\hline gamma-Chlordane & 1.7 & $\mathrm{U}$ & 1.7 & 36 & UD & 36 & 1.8 & $\mathrm{U}$ & 1.8 & 38 & UD & 38 \\
\hline Heptachlor & 1.7 & $\mathrm{U}$ & 1.7 & 36 & UD & 36 & 1.8 & $\mathrm{U}$ & 1.8 & 38 & UD & 38 \\
\hline Heptachlor epoxide & 1.7 & $\mathrm{U}$ & 1.7 & 36 & UD & 36 & 1.8 & $\mathrm{U}$ & 1.8 & 38 & UD & 38 \\
\hline Methoxychlor & 17 & $\mathrm{U}$ & 17 & 360 & UD & 360 & 18 & $\mathrm{U}$ & 18 & 380 & UD & 380 \\
\hline Toxaphene & 170 & $\mathrm{U}$ & 170 & 3600 & UD & 3600 & 180 & $\mathrm{U}$ & 180 & 3800 & UD & 3800 \\
\hline \multicolumn{13}{|c|}{ SVOAs (Semivolatile organics) } \\
\hline 1,2,4-Trichlorobenzene & 350 & $\mathrm{U}$ & 350 & 360 & $\mathrm{U}$ & 360 & 370 & $\mathrm{U}$ & 370 & 3800 & UD & 3800 \\
\hline 1,2-Dichlorobenzene & 350 & $\mathrm{U}$ & 350 & 360 & $\mathrm{U}$ & 360 & 370 & $\mathrm{U}$ & 370 & 3800 & UD & 3800 \\
\hline 1,3-Dichlorobenzene & 350 & $\mathrm{U}$ & 350 & 360 & $\mathrm{U}$ & 360 & 370 & $\mathrm{U}$ & 370 & 3800 & UD & 3800 \\
\hline 1,4-Dichlorobenzene & 350 & $\mathrm{U}$ & 350 & 360 & $\mathrm{U}$ & 360 & 370 & $\mathrm{U}$ & 370 & 3800 & UD & 3800 \\
\hline 2,4,5-Trichlorophenol & 870 & $\mathrm{U}$ & 870 & 910 & $\mathrm{U}$ & 910 & 910 & $\mathrm{U}$ & 910 & 9500 & UD & 9500 \\
\hline 2,4,6-Trichlorophenol & 350 & $\mathrm{U}$ & 350 & 360 & $\bar{U}$ & 360 & 370 & $\mathrm{U}$ & 370 & 3800 & UD & 3800 \\
\hline 2,4-Dichlorophenol & 350 & $\mathrm{U}$ & 350 & 360 & $\mathrm{U}$ & 360 & 370 & $\mathrm{U}$ & 370 & 3800 & UD & 3800 \\
\hline 2,4-Dimethylphenol & 350 & $\mathrm{U}$ & 350 & 360 & $\mathrm{U}$ & 360 & 370 & $\mathrm{U}$ & 370 & 3800 & UD & 3800 \\
\hline 2,4-Dinitrophenol & 870 & $\bar{U}$ & 870 & 910 & $\mathrm{U}$ & 910 & 910 & $\bar{U}$ & 910 & 9500 & UD & 9500 \\
\hline 2,4-Dinitrotoluene & 350 & $\mathrm{U}$ & 350 & 360 & $\mathrm{U}$ & 360 & 370 & $\mathrm{U}$ & 370 & 3800 & UD & 3800 \\
\hline 2,6-Dinitrotoluene & 350 & $\mathrm{U}$ & 350 & 360 & $\mathrm{U}$ & 360 & 370 & $\mathrm{U}$ & 370 & 3800 & UD & 3800 \\
\hline 2-Chloronaphthalene & 350 & $\mathrm{U}$ & 350 & 360 & $\mathrm{U}$ & 360 & 370 & $\mathrm{U}$ & 370 & 3800 & UD & 3800 \\
\hline 2-Chlorophenol & 350 & $\mathrm{U}$ & 350 & 360 & $\mathrm{U}$ & 360 & 370 & $\mathrm{U}$ & 370 & 3800 & UD & 3800 \\
\hline 2-Methylnaphthalene & 350 & $\mathrm{U}$ & 350 & 360 & $\mathrm{U}$ & 360 & 370 & $\mathrm{U}$ & 370 & 280 & JD & 3800 \\
\hline 2-Methylphenol (cresol, o-) & 350 & $\mathrm{U}$ & 350 & 360 & $\mathrm{U}$ & 360 & 370 & $\mathrm{U}$ & 370 & 3800 & \begin{tabular}{|l|} 
UD \\
\end{tabular} & 3800 \\
\hline 2-Nitroaniline & 870 & $\mathrm{U}$ & 870 & 910 & $\mathrm{U}$ & 910 & 910 & $\mathrm{U}$ & 910 & 9500 & UD & 9500 \\
\hline 2-Nitrophenol & 350 & $\mathrm{U}$ & 350 & 360 & $\mathrm{U}$ & 360 & 370 & $\mathrm{U}$ & 370 & 3800 & UD & 3800 \\
\hline 3+4 Methylphenol (cresol, $\mathrm{m}+\mathrm{p}$ ) & 350 & $\mathrm{U}$ & 350 & 360 & $\mathrm{U}$ & 360 & 370 & $\mathrm{U}$ & 370 & 3800 & UD & 3800 \\
\hline 3,3'-Dichlorobenzidine & 350 & $\mathrm{U}$ & 350 & 360 & $\mathrm{U}$ & 360 & 370 & $\mathrm{U}$ & 370 & 3800 & UD & 3800 \\
\hline 3-Nitroaniline & 870 & $\mathrm{U}$ & 870 & 910 & $\mathrm{U}$ & 910 & 910 & $\mathrm{U}$ & 910 & 9500 & UD & 9500 \\
\hline 4,6-Dinitro-2-methylphenol & 870 & $\mathrm{U}$ & 870 & 910 & $\mathrm{U}$ & 910 & 910 & $\mathrm{U}$ & 910 & 9500 & UD & 9500 \\
\hline 4-Bromophenylphenyl ether & 350 & $\mathrm{U}$ & 350 & 360 & $\mathrm{U}$ & 360 & 370 & $\mathrm{U}$ & 370 & 3800 & \begin{tabular}{|l|}
$U D$ \\
\end{tabular} & 3800 \\
\hline 4-Chloro-3-methylphenol & 350 & $\mathrm{U}$ & 350 & 360 & $\mathrm{U}$ & 360 & 370 & $\mathrm{U}$ & 370 & 3800 & UD & 3800 \\
\hline 4-Chloroaniline & 350 & $\mathrm{U}$ & 350 & 360 & $\mathrm{U}$ & 360 & 370 & $\mathrm{U}$ & 370 & 3800 & UD & 3800 \\
\hline 4-Chlorophenylphenyl ether & 350 & $\mathrm{U}$ & 350 & 360 & $\mathrm{U}$ & 360 & 370 & $\mathrm{U}$ & 370 & 3800 & UD & 3800 \\
\hline 4-Nitroaniline & 870 & $\mathrm{U}$ & 870 & 910 & $\mathrm{U}$ & 910 & 910 & $\mathrm{U}$ & 910 & 9500 & UD & 9500 \\
\hline 4-Nitrophenol & 870 & $\mathrm{U}$ & 870 & 910 & $\mathrm{U}$ & 910 & 910 & $\mathrm{U}$ & 910 & 9500 & \begin{tabular}{|l|} 
UD \\
\end{tabular} & 9500 \\
\hline Acenaphthene & 350 & $\mathrm{U}$ & 350 & 360 & $\mathrm{U}$ & 360 & 370 & $\mathrm{U}$ & 370 & 2200 & JD & 3800 \\
\hline Acenaphthylene & 350 & $\mathrm{U}$ & 350 & 360 & $\mathrm{U}$ & 360 & 370 & $\bar{U}$ & 370 & 3800 & UD & 3800 \\
\hline Anthracene & 350 & $\mathrm{U}$ & 350 & 360 & $\mathrm{U}$ & 360 & 370 & $\mathrm{U}$ & 370 & 3400 & JD & 3800 \\
\hline Benzo(a)anthracene & 350 & $\mathrm{U}$ & 350 & 360 & $\mathrm{U}$ & 360 & 370 & $\mathrm{U}$ & 370 & 6600 & $\mathrm{D}$ & 3800 \\
\hline Benzo(a)pyrene & 350 & $\mathrm{U}$ & 350 & 360 & $\mathrm{U}$ & 360 & 370 & $\mathrm{U}$ & 370 & 5200 & $\mathrm{D}$ & 3800 \\
\hline
\end{tabular}


Table A-1. 128-F-2 Confirmatory Sample Results. (12 Pages)

\begin{tabular}{|c|c|c|c|c|c|c|c|c|c|c|c|c|}
\hline \multirow[t]{2}{*}{ Constituent } & \multicolumn{3}{|c|}{$\begin{array}{c}\text { J024R5 } \\
\text { Area } 4 \text { Oily Soil } \\
\text { Sample Date 12/6/04 }\end{array}$} & \multicolumn{3}{|c|}{$\begin{array}{c}\text { J024R6 } \\
\text { Area } 4 \text { Trench Soil } \\
\text { Sample Date 12/6/04 }\end{array}$} & \multicolumn{3}{|c|}{$\begin{array}{c}\text { J024X0 } \\
\text { Duplicate of J024R6 } \\
\text { Sample Date 12/6/04 } \\
\end{array}$} & \multicolumn{3}{|c|}{$\begin{array}{c}\mathrm{J024X2} \\
\text { Area } 3 \text { Trench Soil } \\
\text { Sample Date 12/7/04 }\end{array}$} \\
\hline & $\mu g / k g$ & $\mathbf{Q}$ & $\overline{P Q L}$ & $\mu g / \mathrm{kg}$ & $\mathbf{Q}$ & $\overline{P Q L}$ & $\mu \mathrm{g} / \mathrm{kg}$ & $\mathbf{Q}$ & PQL & $\mu \mathrm{g} / \mathrm{kg}$ & $\bar{Q}$ & PQL \\
\hline \multicolumn{13}{|c|}{ SVOAs (Semivolatile organics) (continued) } \\
\hline Benzo(b)fluoranthene & 350 & $\mathrm{U}$ & 350 & 360 & $\mathrm{U}$ & 360 & 370 & $\mathrm{U}$ & 370 & 4000 & $\mathrm{D}$ & 3800 \\
\hline Benzo(ghi)perylene & 350 & $\mathrm{U}$ & 350 & 360 & $\mathrm{U}$ & 360 & 370 & $\mathrm{U}$ & 370 & 2800 & $\mathrm{JD}$ & 3800 \\
\hline Benzo(k)fluoranthene & 350 & $\mathrm{U}$ & 350 & 360 & $\mathrm{U}$ & 360 & 370 & $\mathrm{U}$ & 370 & 4500 & $\mathrm{D}$ & 3800 \\
\hline Bis(2-chloro-1-methylethyl)ether & 350 & $\mathrm{U}$ & 350 & 360 & $\mathrm{U}$ & 360 & 370 & $\mathrm{U}$ & 370 & 3800 & UD & 3800 \\
\hline Bis(2-Chloroethoxy)methane & 350 & $\mathrm{U}$ & 350 & 360 & $\mathrm{U}$ & 360 & 370 & $\mathrm{U}$ & 370 & 3800 & UD & 3800 \\
\hline Bis(2-chloroethyl) ether & 350 & $\mathrm{U}$ & 350 & 360 & $\mathrm{U}$ & 360 & 370 & $\mathrm{U}$ & 370 & 3800 & UD & 3800 \\
\hline Bis(2-ethylhexyl) phthalate & 350 & $\mathrm{U}$ & 350 & 28 & $\mathrm{JB}$ & 360 & 22 & $\mathrm{JB}$ & 370 & 330 & JBD & 3800 \\
\hline Butylbenzylphthalate & 350 & $\mathrm{U}$ & 350 & 360 & $\mathrm{U}$ & 360 & 370 & $\mathrm{U}$ & 370 & 3800 & UD & 3800 \\
\hline Carbazole & 350 & $\mathrm{U}$ & 350 & 360 & $\mathrm{U}$ & 360 & 370 & $\mathrm{U}$ & 370 & 1900 & JD & 3800 \\
\hline Chrysene & 350 & $\mathrm{U}$ & 350 & 360 & $\mathrm{U}$ & 360 & 370 & $\mathrm{U}$ & 370 & 7700 & $\mathrm{D}$ & 3800 \\
\hline Di-n-butylphthalate & 350 & $\mathrm{U}$ & 350 & 360 & $\mathrm{U}$ & 360 & 370 & $\mathrm{U}$ & 370 & 3800 & UD & 3800 \\
\hline Di-n-octylphthalate & 350 & $\mathrm{U}$ & 350 & 360 & $\mathrm{U}$ & 360 & 370 & $\mathrm{U}$ & 370 & 3800 & UD & 3800 \\
\hline Dibenz[a,h]anthracene & 350 & $\mathrm{U}$ & 350 & 360 & $\mathrm{U}$ & 360 & 370 & $\mathrm{U}$ & 370 & 1500 & JD & 3800 \\
\hline Dibenzofuran & 350 & $\mathrm{U}$ & 350 & 360 & $\mathrm{U}$ & 360 & 370 & $\mathrm{U}$ & 370 & 940 & JD & 3800 \\
\hline Diethylphthalate & 350 & $\mathrm{U}$ & 350 & 360 & $\mathrm{U}$ & 360 & 370 & $\mathrm{U}$ & 370 & 3800 & UD & 3800 \\
\hline Dimethyl phthalate & 350 & $\mathrm{U}$ & 350 & 360 & $\mathrm{U}$ & 360 & 370 & $\mathrm{U}$ & 370 & 3800 & UD & 3800 \\
\hline Fluoranthene & 350 & $\mathrm{U}$ & 350 & 360 & $\mathrm{U}$ & 360 & 370 & $\mathrm{U}$ & 370 & 15000 & $\mathrm{D}$ & 3800 \\
\hline Fluorene & 350 & $\mathrm{U}$ & 350 & 360 & $\mathrm{U}$ & 360 & 370 & $\mathrm{U}$ & 370 & 1700 & JD & 3800 \\
\hline Hexachlorobenzene & 350 & $\mathrm{U}$ & 350 & 360 & $\mathrm{U}$ & 360 & 370 & $\mathrm{U}$ & 370 & 3800 & UD & 3800 \\
\hline Hexachlorobutadiene & 350 & $\mathrm{U}$ & 350 & 360 & $\mathrm{U}$ & 360 & 370 & $\mathrm{U}$ & 370 & 3800 & UD & 3800 \\
\hline Hexachlorocyclopentadiene & 350 & $\mathrm{U}$ & 350 & 360 & $\mathrm{U}$ & 360 & 370 & $\mathrm{U}$ & 370 & 3800 & UD & 3800 \\
\hline Hexachloroethane & 350 & $\mathrm{U}$ & 350 & 360 & $\mathrm{U}$ & 360 & 370 & $\mathrm{U}$ & 370 & 3800 & UD & 3800 \\
\hline Indeno(1,2,3-cd)pyrene & 350 & $\mathrm{U}$ & 350 & 360 & $\mathrm{U}$ & 360 & 370 & $\mathrm{U}$ & 370 & 2800 & JD & 3800 \\
\hline Isophorone & 350 & $\mathrm{U}$ & 350 & 360 & $\mathrm{U}$ & 360 & 370 & $\mathrm{U}$ & 370 & 3800 & UD & 3800 \\
\hline N-Nitroso-di-n-dipropylamine & 350 & $\mathrm{U}$ & 350 & 360 & $\mathrm{U}$ & 360 & 370 & $\mathrm{U}$ & 370 & 3800 & UD & 3800 \\
\hline N-Nitrosodiphenylamine & 350 & $\bar{U}$ & 350 & 360 & $\mathrm{U}$ & 360 & 370 & $\mathrm{U}$ & 370 & 3800 & UD & 3800 \\
\hline Naphthalene & 350 & $\mathrm{U}$ & 350 & 360 & $\mathrm{U}$ & 360 & 370 & $\mathrm{U}$ & 370 & 760 & JD & 3800 \\
\hline Nitrobenzene & 350 & $\mathrm{U}$ & 350 & 360 & $\mathrm{U}$ & 360 & 370 & $\mathrm{U}$ & 370 & 3800 & UD & 3800 \\
\hline Pentachlorophenol & 870 & $\mathrm{U}$ & 870 & 910 & $\mathrm{U}$ & 910 & 910 & $\mathrm{U}$ & 910 & 9500 & UD & 9500 \\
\hline Phenanthrene & 350 & $\mathrm{U}$ & 350 & 360 & $\mathrm{U}$ & 360 & 370 & $\mathrm{U}$ & 370 & 15000 & $\mathrm{D}$ & 3800 \\
\hline Phenol & 350 & $\mathrm{U}$ & 350 & 360 & $\mathrm{U}$ & 360 & 370 & $\mathrm{U}$ & 370 & 3800 & UD & 3800 \\
\hline Pyrene & 350 & $\mathrm{U}$ & 350 & 360 & $\mathrm{U}$ & 360 & 370 & $\mathrm{U}$ & 370 & 15000 & $\mathrm{D}$ & 3800 \\
\hline
\end{tabular}

\begin{tabular}{|c|c|c|c|c|c|c|c|c|c|c|c|c|}
\hline \multirow[t]{2}{*}{ Constituent } & \multicolumn{3}{|c|}{$\begin{array}{c}\text { J024X3 } \\
\text { Area } 2 \text { Trench Soil } \\
\text { Sample Date 12/7/04 }\end{array}$} & \multicolumn{3}{|c|}{$\begin{array}{c}\text { J024R7 } \\
\text { Area } 2 \text { Ash } \\
\text { Sample Date 12/3/04 }\end{array}$} & \multicolumn{3}{|c|}{$\begin{array}{c}\text { J024R8 } \\
\text { Area } 2 \text { Ash } \\
\text { Sample Date 12/3/04 }\end{array}$} & \multicolumn{3}{|c|}{$\begin{array}{c}\text { J024T0 } \\
\text { Area } 3 \text { Oily Sediment } \\
\text { Sample Date } 12 / 7 / 04\end{array}$} \\
\hline & $\mu \mathrm{g} / \mathrm{kg}$ & $\mathbf{Q}$ & PQL & $\mu \mathrm{g} / \mathrm{kg}$ & $\mathbf{Q}$ & PQL & $\mu \mathrm{g} / \mathrm{kg}$ & $\mathbf{Q}$ & PQL & $\mu \mathrm{g} / \mathrm{kg}$ & $\mathbf{Q}$ & PQL \\
\hline \multicolumn{13}{|c|}{ Polycyclic Aromatic Hydrocarbons } \\
\hline Acenaphthene & 54.5 & $\mathrm{U}$ & 54.5 & 61700 & $\mathrm{D}$ & 54000 & 54.5 & $\mathrm{U}$ & 54.5 & 730 & UD & 730 \\
\hline Acenaphthylene & 54.5 & $\mathrm{U}$ & 54.5 & 70200 & $\mathrm{D}$ & 10800 & 54.5 & $\mathrm{U}$ & 54.5 & 730 & UD & 730 \\
\hline Anthracene & 170 & & 5.45 & 234000 & $\mathrm{D}$ & 5400 & 29.7 & & 5.45 & 9010 & $\mathrm{D}$ & 73 \\
\hline Benzo(a)anthracene & 469 & & 5.45 & 619000 & $\mathrm{D}$ & 5400 & 84.5 & & 5.45 & 17200 & $\mathrm{D}$ & 73 \\
\hline Benzo(a)pyrene & 307 & & 5.45 & 328000 & $\mathrm{D}$ & 5400 & 55.4 & & 5.45 & 15700 & $\mathrm{D}$ & 73 \\
\hline Benzo(b)fluoranthene & 243 & & 5.45 & 250000 & $\mathrm{D}$ & 5400 & 56.2 & & 5.45 & 13300 & $\mathrm{D}$ & 73 \\
\hline Benzo(ghi)perylene & 171 & & 5.45 & 76400 & $\mathrm{D}$ & 5400 & 36.5 & & 5.45 & 8450 & $\mathrm{D}$ & 73 \\
\hline Benzo(k)fluoranthene & 149 & & 5.45 & 79400 & $\mathrm{D}$ & 5400 & 27.8 & & 5.45 & 8330 & $\mathrm{D}$ & 73 \\
\hline \begin{tabular}{|l|} 
Chrysene \\
\end{tabular} & 417 & & 5.45 & 965000 & $\mathrm{D}$ & 10800 & 55.4 & & 5.45 & 22000 & $\mathrm{D}$ & 73 \\
\hline Dibenz[a,h]anthracene & 72 & & 5.45 & 19800 & $\mathrm{D}$ & 5400 & 12.5 & & 5.45 & 2490 & $\mathrm{D}$ & 73 \\
\hline Fluoranthene & 764 & & 10.9 & 1010000 & $\mathrm{D}$ & 10800 & 162 & & 10.9 & 53000 & $\mathrm{D}$ & 1490 \\
\hline Fluorene & 132 & & 5.45 & 161000 & $\mathrm{D}$ & 5400 & 14.5 & & 5.45 & 8430 & $\mathrm{D}$ & 73 \\
\hline
\end{tabular}


Table A-1. 128-F-2 Confirmatory Sample Results. (12 Pages)

\begin{tabular}{|c|c|c|c|c|c|c|c|c|c|c|c|c|}
\hline \multirow[t]{2}{*}{ Constituent } & \multicolumn{3}{|c|}{$\begin{array}{c}\text { J024X3 } \\
\text { Area } 2 \text { Trench Soil } \\
\text { Sample Date 12/7/04 }\end{array}$} & \multicolumn{3}{|c|}{$\begin{array}{c}\text { J024R7 } \\
\text { Area } 2 \text { Ash } \\
\text { Sample Date 12/3/04 }\end{array}$} & \multicolumn{3}{|c|}{$\begin{array}{c}\text { J024R8 } \\
\text { Area } 2 \text { Ash } \\
\text { Sample Date 12/3/04 }\end{array}$} & \multicolumn{3}{|c|}{$\begin{array}{c}\text { J024T0 } \\
\text { Area } 3 \text { Oily Sediment } \\
\text { Sample Date } 12 / 7 / 04 \\
\end{array}$} \\
\hline & $\mu \mathrm{g} / \mathrm{kg}$ & $\mathbf{Q}$ & PQL & $\mu \mathrm{g} / \mathrm{kg}$ & $\mathbf{Q}$ & PQL & $\mu \mathrm{g} / \mathrm{kg}$ & $\mathbf{Q}$ & PQL & $\mu \mathrm{g} / \mathrm{kg}$ & $\mathbf{Q}$ & PQL \\
\hline \multicolumn{13}{|c|}{ Polycyclic Aromatic Hydrocarbons (continued) } \\
\hline Indeno(1,2,3-cd)pyrene & 259 & & 5.45 & 133000 & $\mathrm{D}$ & 5400 & 63.3 & & 5.45 & 11700 & $\mathrm{D}$ & 73 \\
\hline Naphthalene & 54.5 & $\mathrm{U}$ & 54.5 & 66500 & $\mathrm{D}$ & 54000 & 54.5 & $\mathrm{U}$ & 54.5 & 730 & UD & 730 \\
\hline Phenanthrene & 776 & & 5.45 & 1090000 & $\mathrm{D}$ & 5400 & 171 & & 5.45 & 49100 & $\mathrm{D}$ & 730 \\
\hline Pyrene & 10.9 & $\mathrm{U}$ & 10.9 & 1220000 & $\mathrm{D}$ & 108000 & 173 & $\mathrm{U}$ & 10.9 & 62800 & $\mathrm{D}$ & 1490 \\
\hline \multicolumn{13}{|c|}{ PCBs (Polychlorinated biphenyls) } \\
\hline Aroclor-1016 & 73 & $\mathrm{U}$ & 73 & 77 & $\mathrm{U}$ & 77 & 77 & $\mathrm{U}$ & 77 & 970 & UD & 970 \\
\hline Aroclor-1221 & 73 & $\mathrm{U}$ & 73 & 77 & $\mathrm{U}$ & 77 & 77 & $\mathrm{U}$ & 77 & 970 & UD & 970 \\
\hline Aroclor-1232 & 73 & $\mathrm{U}$ & 73 & 77 & $\mathrm{U}$ & 77 & 77 & $\mathrm{U}$ & 77 & 970 & UD & 970 \\
\hline Aroclor-1242 & 73 & $\mathrm{U}$ & 73 & 77 & $\mathrm{U}$ & 77 & 77 & $\mathrm{U}$ & 77 & 970 & UD & 970 \\
\hline Aroclor-1248 & 73 & $\mathrm{U}$ & 73 & 77 & $\mathrm{U}$ & 77 & 77 & $\mathrm{U}$ & 77 & 970 & UD & 970 \\
\hline Aroclor-1254 & 73 & $\mathrm{U}$ & 73 & 77 & $\mathrm{U}$ & 77 & 77 & $\mathrm{U}$ & 77 & 8700 & $\mathrm{D}$ & 970 \\
\hline Aroclor-1260 & 73 & $\mathrm{U}$ & 73 & 77 & $\mathrm{U}$ & 77 & 77 & $\mathrm{U}$ & 77 & 970 & UD & 970 \\
\hline \multicolumn{10}{|c|}{ Pesticides } & & & \\
\hline Aldrin & 36 & UD & 36 & 39 & UD & 39 & 0.05 & UD & 0.05 & & & \\
\hline Alpha-BHC & 36 & UD & 36 & 39 & UD & 39 & 0.05 & UD & 0.05 & & & \\
\hline alpha-Chlordane & 36 & UD & 36 & 39 & UD & 39 & 0.05 & UD & 0.05 & & & \\
\hline $\begin{array}{l}\text { beta-1,2,3,4,5,6- } \\
\text { Hexachlorocyclohexane }\end{array}$ & 36 & UD & 36 & 39 & UD & 39 & 0.05 & UD & 0.05 & & & \\
\hline Delta-BHC & 36 & UD & 36 & 39 & UD & 39 & 0.05 & UD & 0.05 & & & \\
\hline Dichlorodiphenyldichloroethane & 73 & UD & 73 & 47 & JD & 47 & 18 & UD & 18 & & & \\
\hline Dichlorodiphenyldichloroethylene & 73 & UD & 73 & 77 & UD & 77 & 18 & UD & 18 & & & \\
\hline Dichlorodiphenyltrichloroethane & 73 & UD & 73 & 77 & UD & 77 & 31 & JD & 18 & & & \\
\hline Dieldrin & 73 & UD & 73 & 77 & UD & 77 & 18 & UD & 18 & & & \\
\hline Endosulfan I & 36 & UD & 36 & 39 & UD & 39 & 0.05 & UD & 0.05 & & & \\
\hline Endosulfan II & 73 & UD & 73 & 110 & $\mathrm{D}$ & 77 & 18 & UD & 18 & & & \\
\hline Endosulfan sulfate & 73 & UD & 73 & 240 & $\mathrm{D}$ & 77 & 18 & UD & 18 & & & \\
\hline Endrin & 73 & UD & 73 & 77 & UD & 77 & 18 & UD & 18 & & & \\
\hline Endrin aldehyde & 73 & UD & 73 & 77 & UD & 77 & 18 & UD & 18 & & & \\
\hline Endrin ketone & 73 & UD & 73 & 77 & UD & 77 & 18 & UD & 18 & & & \\
\hline Gamma-BHC (Lindane) & 36 & UD & 36 & 39 & UD & 39 & 0.05 & UD & 0.05 & & & \\
\hline gamma-Chlordane & 36 & UD & 36 & 39 & UD & 39 & 0.05 & UD & 0.05 & & & \\
\hline Heptachlor & 36 & UD & 36 & 39 & UD & 39 & 0.05 & UD & 0.05 & & & \\
\hline Heptachlor epoxide & 36 & UD & 36 & 39 & UD & 39 & 0.05 & UD & 0.05 & & & \\
\hline Methoxychlor & 360 & UD & 360 & 390 & UD & 390 & 0.5 & UD & 0.5 & & & \\
\hline Toxaphene & 3600 & UD & 3600 & 3900 & UD & 3900 & 5 & UD & 5 & & & \\
\hline \multicolumn{10}{|c|}{ SVOAs (Semivolatile organics) } & & & \\
\hline 1,2,4-Trichlorobenzene & 1800 & UD & 1800 & 230000 & UD & 230000 & 360 & $\mathrm{U}$ & 360 & & & \\
\hline 1,2-Dichlorobenzene & 1800 & UD & 1800 & 230000 & UD & 230000 & 360 & $\mathrm{U}$ & 360 & & & \\
\hline 1,3-Dichlorobenzene & 1800 & UD & 1800 & 230000 & UD & 230000 & 360 & $\mathrm{U}$ & 360 & & & \\
\hline 1,4-Dichlorobenzene & 1800 & UD & 1800 & 230000 & UD & 230000 & 360 & $\mathrm{U}$ & 360 & & & \\
\hline 2,4,5-Trichlorophenol & 4500 & UD & 4500 & 580000 & UD & 580000 & 910 & $\mathrm{U}$ & 910 & & & \\
\hline 2,4,6-Trichlorophenol & 1800 & UD & 1800 & 230000 & UD & 230000 & 360 & $\mathrm{U}$ & 360 & & & \\
\hline 2,4-Dichlorophenol & 1800 & UD & 1800 & 230000 & UD & 230000 & 360 & $\mathrm{U}$ & 360 & & & \\
\hline 2,4-Dimethylphenol & 1800 & UD & 1800 & 230000 & UD & 230000 & 360 & $\mathrm{U}$ & 360 & & & \\
\hline 2,4-Dinitrophenol & 4500 & UD & 4500 & 580000 & $\mathrm{UD}$ & 580000 & 910 & $\mathrm{U}$ & 910 & & & \\
\hline 2,4-Dinitrotoluene & 1800 & UD & 1800 & 230000 & UD & 230000 & 360 & $\mathrm{U}$ & 360 & & & \\
\hline 2,6-Dinitrotoluene & 1800 & UD & 1800 & 230000 & UD & 230000 & 360 & $\mathrm{U}$ & 360 & & & \\
\hline 2-Chloronaphthalene & 1800 & UD & 1800 & 230000 & UD & 230000 & 360 & $\mathrm{U}$ & 360 & & & \\
\hline 2-Chlorophenol & 1800 & UD & 1800 & 230000 & UD & 230000 & 360 & $\mathrm{U}$ & 360 & & & \\
\hline 2-Methylnaphthalene & 1800 & UD & 1800 & 26000 & JD & 230000 & 360 & $\mathrm{U}$ & 360 & & & \\
\hline 2-Methylphenol (cresol, o-) & 1800 & UD & 1800 & 230000 & UD & 230000 & 360 & $\mathrm{U}$ & 360 & & & \\
\hline 2-Nitroaniline & 4500 & UD & 4500 & 580000 & UD & 580000 & 910 & $\mathrm{U}$ & 910 & & & \\
\hline 2-Nitrophenol & 1800 & UD & 1800 & 230000 & UD & 230000 & 360 & $\mathrm{U}$ & 360 & & & \\
\hline
\end{tabular}


Table A-1. 128-F-2 Confirmatory Sample Results. (12 Pages)

\begin{tabular}{|c|c|c|c|c|c|c|c|c|c|}
\hline \multirow[t]{2}{*}{ Constituent } & \multicolumn{3}{|c|}{$\begin{array}{c}\text { J024X3 } \\
\text { Area } 2 \text { Trench Soil } \\
\text { Sample Date 12/7/04 }\end{array}$} & \multicolumn{3}{|c|}{$\begin{array}{c}\text { J024R7 } \\
\text { Area } 2 \text { Ash } \\
\text { Sample Date 12/3/04 }\end{array}$} & \multicolumn{3}{|c|}{$\begin{array}{c}\text { J024R8 } \\
\text { Area } 2 \text { Ash } \\
\text { Sample Date 12/3/04 }\end{array}$} \\
\hline & $\mu g / k g$ & $\mathbf{Q}$ & PQL & $\mu \mathrm{g} / \mathrm{kg}$ & $\mathbf{Q}$ & PQL & $\mu \mathrm{g} / \mathrm{kg}$ & $\mathbf{Q}$ & PQL \\
\hline \multicolumn{10}{|c|}{ SVOAs (continued) } \\
\hline 3+4 Methylphenol (cresol, m+p) & 1800 & UD & 1800 & 230000 & UD & 230000 & 360 & $\mathrm{U}$ & 360 \\
\hline 3,3'-Dichlorobenzidine & 1800 & $\mathrm{UD}$ & 1800 & 230000 & UD & 230000 & 360 & $\mathrm{U}$ & 360 \\
\hline 3-Nitroaniline & 4500 & UD & 4500 & 580000 & UD & 580000 & 910 & $\mathrm{U}$ & 910 \\
\hline 4,6-Dinitro-2-methylphenol & 4500 & $\mathrm{UD}$ & 4500 & 580000 & UD & 580000 & 910 & $\mathrm{U}$ & 910 \\
\hline 4-Bromophenylphenyl ether & 1800 & $\mathrm{UD}$ & 1800 & 230000 & $\mathrm{UD}$ & 230000 & 360 & $\mathrm{U}$ & 360 \\
\hline 4-Chloro-3-methylphenol & 1800 & $\mathrm{UD}$ & 1800 & 230000 & $\mathrm{UD}$ & 230000 & 360 & $\mathrm{U}$ & 360 \\
\hline 4-Chloroaniline & 1800 & UD & 1800 & 230000 & UD & 230000 & 360 & $\mathrm{U}$ & 360 \\
\hline 4-Chlorophenylphenyl ether & 1800 & $\mathrm{UD}$ & 1800 & 230000 & $\mathrm{UD}$ & 230000 & 360 & $\mathrm{U}$ & 360 \\
\hline 4-Nitroaniline & 4500 & $\mathrm{UD}$ & 4500 & 580000 & $\mathrm{UD}$ & 580000 & 910 & $\mathrm{U}$ & 910 \\
\hline 4-Nitrophenol & 4500 & $\mathrm{UD}$ & 4500 & 580000 & $\mathrm{UD}$ & 580000 & 910 & $\mathrm{U}$ & 910 \\
\hline Acenaphthene & 500 & $\mathrm{JD}$ & 1800 & 190000 & $\mathrm{JD}$ & 230000 & 23 & $\mathrm{~J}$ & 360 \\
\hline Acenaphthylene & 1800 & UD & 1800 & 230000 & UD & 230000 & 360 & $\mathrm{U}$ & 360 \\
\hline Anthracene & 2000 & $\mathrm{D}$ & 1800 & 330000 & $\mathrm{D}$ & 230000 & 38 & $\mathrm{~J}$ & 360 \\
\hline Benzo(a)anthracene & 7400 & $\mathrm{D}$ & 1800 & 550000 & $\mathrm{D}$ & 230000 & 100 & $\mathrm{~J}$ & 360 \\
\hline Benzo(a)pyrene & 5900 & $\mathrm{D}$ & 1800 & 430000 & $\mathrm{D}$ & 230000 & 83 & $\mathrm{~J}$ & 360 \\
\hline Benzo(b)fluoranthene & 4300 & $\mathrm{D}$ & 1800 & 330000 & $\mathrm{D}$ & 230000 & 77 & $\mathrm{~J}$ & 360 \\
\hline Benzo(ghi)perylene & 3100 & $\mathrm{D}$ & 1800 & 250000 & $\mathrm{D}$ & 230000 & 66 & $\mathrm{~J}$ & 360 \\
\hline Benzo(k)fluoranthene & 5200 & $\mathrm{D}$ & 1800 & 360000 & $\mathrm{D}$ & 230000 & 73 & $\mathrm{~J}$ & 360 \\
\hline Bis(2-chloro-1-methylethyl)ether & 1800 & $\mathrm{UD}$ & 1800 & 230000 & UD & 230000 & 360 & $\mathrm{U}$ & 360 \\
\hline Bis(2-Chloroethoxy)methane & 1800 & UD & 1800 & 230000 & UD & 230000 & 360 & $\mathrm{U}$ & 360 \\
\hline Bis(2-chloroethyl) ether & 1800 & $\mathrm{UD}$ & 1800 & 230000 & UD & 230000 & 360 & $\mathrm{U}$ & 360 \\
\hline Bis(2-ethylhexyl) phthalate & 1800 & $\mathrm{UD}$ & 1800 & 230000 & $\mathrm{UD}$ & 230000 & 28 & JB & 360 \\
\hline Butylbenzylphthalate & 1800 & $\mathrm{UD}$ & 1800 & 230000 & UD & 230000 & 360 & $\mathrm{U}$ & 360 \\
\hline Carbazole & 430 & JD & 1800 & 180000 & JD & 230000 & 24 & $\mathrm{~J}$ & 360 \\
\hline Chrysene & 8300 & $\mathrm{D}$ & 1800 & 630000 & $\mathrm{D}$ & 230000 & 100 & $\mathrm{~J}$ & 360 \\
\hline Di-n-butylphthalate & 1800 & UD & 1800 & 230000 & $\mathrm{UD}$ & 230000 & 38 & $\mathrm{~J}$ & 360 \\
\hline Di-n-octylphthalate & 1800 & UD & 1800 & 230000 & UD & 230000 & 360 & $\mathrm{U}$ & 360 \\
\hline Dibenz[a,h]anthracene & 1700 & JD & 1800 & 120000 & JD & 230000 & 32 & $\mathrm{~J}$ & 360 \\
\hline Dibenzofuran & 110 & JD & 1800 & 86000 & JD & 230000 & 360 & $\mathrm{U}$ & 360 \\
\hline Diethylphthalate & 1800 & $\mathrm{UD}$ & 1800 & 230000 & $\mathrm{UD}$ & 230000 & 360 & $\mathrm{U}$ & 360 \\
\hline Dimethyl phthalate & 1800 & UD & 1800 & 230000 & $\mathrm{UD}$ & 230000 & 360 & $\mathrm{U}$ & 360 \\
\hline Fluoranthene & 12000 & $\mathrm{D}$ & 1800 & 130000 & $\mathrm{D}$ & 230000 & 220 & $\mathrm{~J}$ & 360 \\
\hline \begin{tabular}{|l|} 
Fluorene \\
\end{tabular} & 430 & $\mathrm{JD}$ & 1800 & 160000 & $\mathrm{JD}$ & 230000 & 360 & $\mathrm{U}$ & 360 \\
\hline Hexachlorobenzene & 1800 & $\mathrm{UD}$ & 1800 & 230000 & $\mathrm{UD}$ & 230000 & 360 & $\mathrm{U}$ & 360 \\
\hline Hexachlorobutadiene & 1800 & $\mathrm{UD}$ & 1800 & 230000 & $\mathrm{UD}$ & 230000 & 360 & $\mathrm{U}$ & 360 \\
\hline Hexachlorocyclopentadiene & 1800 & $\mathrm{UD}$ & 1800 & 230000 & $\mathrm{UD}$ & 230000 & 360 & $\mathrm{U}$ & 360 \\
\hline Hexachloroethane & 1800 & $\mathrm{UD}$ & 1800 & 230000 & $\mathrm{UD}$ & 230000 & 360 & $\mathrm{U}$ & 360 \\
\hline Indeno(1,2,3-cd)pyrene & 3200 & $\mathrm{D}$ & 1800 & 260000 & $\mathrm{D}$ & 230000 & 58 & $\mathrm{~J}$ & 360 \\
\hline Isophorone & 1800 & $\mathrm{UD}$ & 1800 & 230000 & $\mathrm{UD}$ & 230000 & 360 & $\mathrm{U}$ & 360 \\
\hline N-Nitroso-di-n-dipropylamine & 1800 & $\mathrm{UD}$ & 1800 & 230000 & $\mathrm{UD}$ & 230000 & 360 & $\mathrm{U}$ & 360 \\
\hline N-Nitrosodiphenylamine & 1800 & $\mathrm{UD}$ & 1800 & 230000 & $\mathrm{UD}$ & 230000 & 360 & $\mathrm{U}$ & 360 \\
\hline Naphthalene & 1800 & $\mathrm{UD}$ & 1800 & 73000 & $\mathrm{JD}$ & 230000 & 360 & $\mathrm{U}$ & 360 \\
\hline Nitrobenzene & 1800 & UD & 1800 & 230000 & UD & 230000 & 360 & $\mathrm{U}$ & 360 \\
\hline Pentachlorophenol & 4500 & UD & 4500 & 580000 & UD & 580000 & 910 & $\mathrm{U}$ & 910 \\
\hline \begin{tabular}{|l|} 
Phenanthrene \\
\end{tabular} & 5600 & $\mathrm{D}$ & 1800 & 120000 & $\mathrm{D}$ & 230000 & 180 & $\mathrm{~J}$ & 360 \\
\hline \begin{tabular}{|l|} 
Phenol \\
\end{tabular} & 1800 & UD & 1800 & 230000 & UD & 230000 & 360 & $\mathrm{U}$ & 360 \\
\hline \begin{tabular}{|l} 
Pyrene \\
\end{tabular} & 13000 & $\mathrm{D}$ & 1800 & 120000 & $\mathrm{D}$ & 230000 & 190 & $\mathrm{~J}$ & 360 \\
\hline
\end{tabular}


Table A-1. 128-F-2 Confirmatory Sample Results. (12 Pages)

\begin{tabular}{|c|c|c|c|c|c|c|c|}
\hline \multirow[t]{2}{*}{ Constituent } & \multicolumn{3}{|c|}{$\begin{array}{c}\text { J02581 } \\
\text { Area } 2 \text { Paint } \\
\text { Sample Date 12/7/04 }\end{array}$} & \multirow[t]{2}{*}{ Constituent } & \multicolumn{3}{|c|}{$\begin{array}{c}\text { J02581 } \\
\text { Area } 2 \text { Paint } \\
\text { Sample Date 12/7/04 }\end{array}$} \\
\hline & $\mu \mathrm{g} / \mathrm{kg}$ & $\mathbf{Q}$ & PQL & & $\mu \mathrm{g} / \mathrm{kg}$ & $\mathbf{Q}$ & PQL \\
\hline \multicolumn{4}{|l|}{ PAH } & \multicolumn{4}{|c|}{ SVOAs } \\
\hline Acenaphthene & 42400 & $\mathrm{D}$ & 5270 & 1,2,4-Trichlorobenzene & 2000 & UD & 2000 \\
\hline Acenaphthylene & 14200 & $\mathrm{D}$ & 527 & 1,2-Dichlorobenzene & 2000 & UD & 2000 \\
\hline Anthracene & 516 & $\mathrm{D}$ & 52.7 & 1,3-Dichlorobenzene & 2000 & UD & 2000 \\
\hline Benzo(a)anthracene & 155 & $\mathrm{D}$ & 52.7 & 1,4-Dichlorobenzene & 2000 & $\mathrm{UD}$ & 2000 \\
\hline Benzo(a)pyrene & 119 & $\mathrm{D}$ & 52.7 & 2,4,5-Trichlorophenol & 5000 & UD & 5000 \\
\hline Benzo(b)fluoranthene & 77.5 & $\mathrm{D}$ & 52.7 & 2,4,6-Trichlorophenol & 2000 & UD & 2000 \\
\hline Benzo(ghi)perylene & 55.9 & $\mathrm{D}$ & 52.7 & 2,4-Dichlorophenol & 2000 & $\mathrm{UD}$ & 2000 \\
\hline Benzo(k)fluoranthene & 50.6 & JD & 52.7 & 2,4-Dimethylphenol & 2000 & $\mathrm{UD}$ & 2000 \\
\hline Chrysene & 163 & $\mathrm{D}$ & 52.7 & 2,4-Dinitrophenol & 5000 & UD & 5000 \\
\hline Dibenz[a,h]anthracene & 52.7 & UD & 52.7 & 2,4-Dinitrotoluene & 2000 & UD & 2000 \\
\hline Fluoranthene & 4240 & $\mathrm{D}$ & 105.4 & 2,6-Dinitrotoluene & 2000 & UD & 2000 \\
\hline \begin{tabular}{|l|} 
Fluorene \\
\end{tabular} & 17800 & $\mathrm{D}$ & 52.7 & 2-Chloronaphthalene & 2000 & $\mathrm{UD}$ & 2000 \\
\hline Indeno(1,2,3-cd)pyrene & 52.7 & UD & 52.7 & 2-Chlorophenol & 2000 & UD & 2000 \\
\hline Naphthalene & 325000 & $\mathrm{D}$ & 5270 & 2-Methylnaphthalene & 5200 & $\mathrm{D}$ & 2000 \\
\hline \begin{tabular}{|l|} 
Phenanthrene \\
\end{tabular} & 38300 & $\mathrm{D}$ & 527 & 2-Methylphenol (cresol, o-) & 2000 & UD & 2000 \\
\hline Pyrene & 365 & $\mathrm{D}$ & 105.4 & 2-Nitroaniline & 5000 & UD & 5000 \\
\hline \multicolumn{4}{|l|}{ PCBs } & 2-Nitrophenol & 2000 & $\mathrm{UD}$ & 2000 \\
\hline Aroclor-1016 & 74 & $\mathrm{U}$ & 74 & 3+4 Methylphenol (cresol, & 2000 & UD & 2000 \\
\hline Aroclor-1221 & 74 & $\mathrm{U}$ & 74 & 3,3'-Dichlorobenzidine & 2000 & UD & 2000 \\
\hline \begin{tabular}{|l|} 
Aroclor-1232 \\
\end{tabular} & 74 & $\mathrm{U}$ & 74 & 3-Nitroaniline & 5000 & $\mathrm{UD}$ & 5000 \\
\hline Aroclor-1242 & 74 & $\mathrm{U}$ & 74 & 4,6-Dinitro-2-methylphenol & 5000 & UD & 5000 \\
\hline \begin{tabular}{|l|} 
Aroclor-1248 \\
\end{tabular} & 74 & $\mathrm{U}$ & 74 & 4-Bromophenylphenyl ether & 2000 & UD & 2000 \\
\hline Aroclor-1254 & 74 & $\mathrm{U}$ & 74 & 4-Chloro-3-methylphenol & 2000 & UD & 2000 \\
\hline \begin{tabular}{|l|} 
Aroclor-1260 \\
\end{tabular} & 74 & $\mathrm{U}$ & 74 & 4-Chloroaniline & 2000 & $\mathrm{UD}$ & 2000 \\
\hline \multicolumn{4}{|c|}{ Pesticides } & 4-Chlorophenylphenyl ether & 2000 & UD & 2000 \\
\hline Aldrin & 18 & UD & 18 & 4-Nitroaniline & 5000 & UD & 5000 \\
\hline Alpha-BHC & 18 & UD & 18 & 4-Nitrophenol & 5000 & UD & 5000 \\
\hline alpha-Chlordane & 18 & $\mathrm{UD}$ & 18 & Acenaphthene & 2000 & $\mathrm{UD}$ & 2000 \\
\hline \begin{tabular}{|l} 
beta-1,2,3,4,5,6- \\
Hexachlorocyclohexane
\end{tabular} & 18 & $\mathrm{UD}$ & 18 & Acenaphthylene & 2000 & UD & 2000 \\
\hline \begin{tabular}{|l} 
Delta-BHC \\
\end{tabular} & 18 & UD & 18 & Anthracene & 2000 & UD & 2000 \\
\hline \begin{tabular}{|l|} 
Dichlorodiphenyldichloroethane \\
\end{tabular} & 37 & $\mathrm{UD}$ & 37 & Benzo(a)anthracene & 180 & $\mathrm{JD}$ & 2000 \\
\hline Dichlorodiphenyldichloroethylene & 37 & $\mathrm{UD}$ & 37 & Benzo(a)pyrene & 140 & $\mathrm{JD}$ & 2000 \\
\hline \begin{tabular}{|l|l} 
Dichlorodiphenyltrichloroethane \\
\end{tabular} & 37 & $\mathrm{UD}$ & 37 & \begin{tabular}{|l} 
Benzo(b)fluoranthene \\
\end{tabular} & 100 & $\mathrm{JD}$ & 2000 \\
\hline Dieldrin & 37 & UD & 37 & Benzo(ghi)perylene & 2000 & UD & 2000 \\
\hline \begin{tabular}{|l|} 
Endosulfan I \\
\end{tabular} & 18 & $\mathrm{UD}$ & 18 & \begin{tabular}{|l} 
Benzo(k)fluoranthene \\
\end{tabular} & 120 & $\mathrm{JD}$ & 2000 \\
\hline Endosulfan II & 37 & $\mathrm{UD}$ & 37 & Bis(2-chloro-1- & 2000 & $\mathrm{UD}$ & 2000 \\
\hline Endosulfan sulfate & 37 & $\mathrm{UD}$ & 37 & Bis(2-Chloroethoxy)methane & 2000 & UD & 2000 \\
\hline Endrin & 37 & UD & 37 & Bis(2-chloroethyl) ether & 2000 & UD & 2000 \\
\hline Endrin aldehyde & 37 & UD & 37 & Bis(2-ethylhexyl) phthalate & 200 & JBD & 2000 \\
\hline Endrin ketone & 37 & UD & 37 & Butylbenzylphthalate & 2000 & UD & 2000 \\
\hline Gamma-BHC (Lindane) & 18 & $\mathrm{UD}$ & 18 & Carbazole & 2000 & UD & 2000 \\
\hline gamma-Chlordane & 18 & UD & 18 & Chrysene & 200 & JD & 2000 \\
\hline Heptachlor & 18 & UD & 18 & Di-n-butylphthalate & 110 & $\mathrm{JD}$ & 2000 \\
\hline Heptachlor epoxide & 18 & UD & 18 & Di-n-octylphthalate & 2000 & UD & 2000 \\
\hline Methoxychlor & 180 & UD & 180 & Dibenz[a,h]anthracene & 2000 & UD & 2000 \\
\hline Toxaphene & 1800 & UD & 1800 & Dibenzofuran & 2000 & UD & 2000 \\
\hline
\end{tabular}


Table A-1. 128-F-2 Confirmatory Sample Results. (12 Pages)

\begin{tabular}{|l|c|c|c|}
\hline \multirow{4}{*}{ Constituent } & \multicolumn{3}{c|}{$\begin{array}{c}\text { J02581 } \\
\text { Area 2 Paint } \\
\text { Sample Date 12/7/04 }\end{array}$} \\
\cline { 2 - 4 } & Hg/kg & Q & PQL \\
\hline \multicolumn{3}{|c|}{ SVOAs (continued) } \\
\hline Diethylphthalate & 2000 & UD & 2000 \\
\hline Dimethyl phthalate & 2000 & UD & 2000 \\
\hline Fluoranthene & 390 & JD & 2000 \\
\hline Fluorene & 2000 & UD & 2000 \\
\hline Hexachlorobenzene & 2000 & UD & 2000 \\
\hline Hexachlorobutadiene & 2000 & UD & 2000 \\
\hline Hexachlorocyclopentadiene & 2000 & UD & 2000 \\
\hline Hexachloroethane & 2000 & UD & 2000 \\
\hline Indeno(1,2,3-cd)pyrene & 2000 & UD & 2000 \\
\hline Isophorone & 2000 & UD & 2000 \\
\hline N-Nitroso-di-n-dipropylamine & 2000 & UD & 2000 \\
\hline N-Nitrosodiphenylamine & 2000 & UD & 2000 \\
\hline Naphthalene & 8500 & D & 2000 \\
\hline Nitrobenzene & 2000 & UD & 2000 \\
\hline Pentachlorophenol & 5000 & UD & 5000 \\
\hline Phenanthrene & 310 & JD & 2000 \\
\hline Phenol & 2000 & UD & 2000 \\
\hline Pyrene & 370 & JD & 2000 \\
\hline
\end{tabular}


Table A-2. 128-F-2 In-Process Riverbank Excavation Berm Data (16 Pages)

\begin{tabular}{|c|c|c|c|c|c|c|c|c|c|c|c|c|c|c|c|c|c|c|c|c|}
\hline \multirow{2}{*}{ Sample Location } & \multirow{2}{*}{$\begin{array}{c}\text { Sample } \\
\text { Number } \\
\end{array}$} & \multirow{2}{*}{$\begin{array}{c}\text { Sample } \\
\text { Date }\end{array}$} & \multicolumn{3}{|c|}{ Americium-241 } & \multicolumn{3}{|c|}{ Cesium-137 } & \multicolumn{3}{|c|}{ Cobalt-60 } & \multicolumn{3}{|c|}{ Europium-152 } & \multicolumn{3}{|c|}{ Europium-154 } & \multicolumn{3}{|c|}{ Europium-155 } \\
\hline & & & $\mathrm{pCi} / \mathrm{g}$ & $\mathbf{Q}$ & MDA & $\mathrm{pCi} / \mathrm{g}$ & $\mathbf{Q}$ & MDA & $\mathrm{pCi} / \mathrm{g}$ & $\mathbf{Q}$ & MDA & $\mathrm{pCi} / \mathrm{g}$ & $\mathbf{Q}$ & MDA & pCi/g & $\mathbf{Q}$ & MDA & $\mathrm{pCi} / \mathrm{g}$ & Q & MDA \\
\hline Riverbank & J13NX7 & $11 / 8 / 06$ & 0.048 & $\mathrm{U}$ & 0.048 & 0.053 & $\mathrm{U}$ & 0.053 & 0.051 & $\mathrm{U}$ & 0.051 & 0.13 & $\mathrm{U}$ & 0.13 & 0.16 & $\mathrm{U}$ & 0.16 & 0.086 & $\mathrm{U}$ & 0.086 \\
\hline Riverbank & J13NX6 & 11/8/06 & 0.28 & $\mathrm{U}$ & 0.28 & 0.081 & $\mathrm{U}$ & 0.081 & 0.091 & $\mathrm{U}$ & 0.091 & 0.21 & $\mathrm{U}$ & 0.21 & 0.30 & $\mathrm{U}$ & 0.30 & 0.21 & $\mathrm{U}$ & 0.21 \\
\hline Riverbank & NX5 & 11/8/06 & 078 & $\mathrm{U}$ & 0.078 & 092 & $\mathrm{U}$ & 0.092 & 0.12 & $\mathrm{U}$ & 0.12 & & $\mathrm{U}$ & 0.15 & 0.38 & $\mathrm{U}$ & 0.38 & 0.089 & $\mathrm{U}$ & 0.089 \\
\hline Riverbank & $\mathrm{LX} 4$ & $/ 1 / 06$ & 073 & \begin{tabular}{l|l}
$\mathrm{U}$ \\
\end{tabular} & 0.073 & 0.187 & & 0.055 & 0.078 & $\mathrm{U}$ & 0.078 & 0.13 & & 0.099 & 0.25 & $\mathrm{U}$ & 0.25 & 14 & $\mathrm{U}$ & .14 \\
\hline Riverbank & $\mathrm{X} 3$ & $1 / 06$ & 10 & \begin{tabular}{l|l}
$\mathrm{U}$ \\
\end{tabular} & 0.10 & 0.27 & $\mathrm{U}$ & 0.27 & 0.16 & $\mathrm{U}$ & 0.16 & 0.2 & \begin{tabular}{l|l}
$\mathrm{U}$ \\
\end{tabular} & (1) & 0.46 & $\mathrm{U}$ & 6 & 13 & $\mathrm{U}$ & 13 \\
\hline Riverbank & $\mathrm{X} 2$ & 06 & 43 & $\mathrm{U}$ & 0.43 & 0.13 & $\mathrm{U}$ & 0. & 0.13 & $=$ & 3 & 0.3 & $\mathrm{U}$ & 0 & 0.36 & $\mathrm{U}$ & 5 & 30 & $\mathrm{U}$ & 30 \\
\hline Riverbank & $\mathrm{X} 1$ & $1 / 06$ & 0.29 & $\mathrm{U}$ & 0.29 & 0.071 & $\mathrm{U}$ & 0.071 & 0.080 & $\mathrm{U}$ & 0.080 & 0.19 & $\mathrm{U}$ & 0.1 & 0.26 & $\mathrm{U}$ & 0.26 & 18 & $\mathrm{U}$ & 0.18 \\
\hline Rive & $\mathrm{X} 0$ & 106 & 072 & $\mathrm{U}$ & 0.072 & 0.069 & $\mathrm{U}$ & 0.069 & 0.077 & $\mathrm{U}$ & 077 & 5 & $\mathrm{U}$ & 0 & 0.15 & $\mathrm{U}$ & 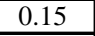 & 11 & $\mathrm{U}$ & 1 \\
\hline Riverbank & LP5 & $26 / 06$ & 0.26 & \begin{tabular}{l|l}
$\mathrm{U}$ \\
\end{tabular} & 0.26 & 0.11 & $\mathrm{U}$ & 0.11 & 0.085 & $\mathrm{U}$ & 0.085 & 0.18 & \begin{tabular}{l|l}
$\mathrm{U}$ \\
\end{tabular} & 0.18 & 0.26 & $\mathrm{U}$ & .26 & .21 & $\mathrm{U}$ & 21 \\
\hline Riverbank & LP4 & $/ 26 / 06$ & 079 & \begin{tabular}{l|l}
$\mathrm{U}$ \\
\end{tabular} & 0.079 & 0.062 & $\mathrm{U}$ & 0.062 & 0.074 & $\mathrm{U}$ & 0.074 & 0.14 & \begin{tabular}{l|l}
$\mathrm{U}$ \\
\end{tabular} & 0.14 & 0.18 & $\mathrm{U}$ & 0.18 & 0.13 & $\mathrm{U}$ & 0.13 \\
\hline Riverbank & LP3 & $10 / 26 / 06$ & 0.47 & \begin{tabular}{l|l}
$\mathrm{U}$ \\
\end{tabular} & 0.47 & 0.12 & $\mathrm{U}$ & 0.12 & 0.13 & $\mathrm{U}$ & 0.13 & 0.32 & \begin{tabular}{l|l}
$\mathrm{U}$ \\
\end{tabular} & 0.32 & 0.46 & $\mathrm{U}$ & 0.46 & 0.33 & $\mathrm{U}$ & 0.33 \\
\hline Riverbank & J13LP2 & $10 / 26 / 06$ & 0.11 & $\mathrm{U}$ & 0.11 & 0.15 & $\mathrm{U}$ & 0.15 & 0.17 & $\mathrm{U}$ & 0.17 & 0.20 & $\mathrm{U}$ & 0.20 & 0.56 & $\mathrm{U}$ & 0.56 & 0.13 & $\mathrm{U}$ & 0.13 \\
\hline Riverbank & J13LP1 & $10 / 26 / 06$ & 0.21 & $\mathrm{U}$ & 0.21 & 0.063 & $\mathrm{U}$ & 0.063 & 0.071 & $\mathrm{U}$ & 0.071 & 0.15 & $\mathrm{U}$ & 0.15 & 0.20 & $\mathrm{U}$ & 0.20 & 0.17 & $\mathrm{U}$ & 0.17 \\
\hline Riverbank & J13LP0 & $10 / 26 / 06$ & 0.085 & $\mathrm{U}$ & 0.085 & 0.060 & $\mathrm{U}$ & 0.060 & 0.070 & $\mathrm{U}$ & 0.070 & 0.15 & $\mathrm{U}$ & 0.15 & 0.19 & $\mathrm{U}$ & 0.19 & 0.18 & $\mathrm{U}$ & 0.18 \\
\hline Riverbank & J13LN9 & $10 / 26 / 06$ & 0.30 & $\mathrm{U}$ & 0.30 & 0.061 & $\mathrm{U}$ & 0.061 & 0.071 & $\mathrm{U}$ & 0.071 & 0.15 & $\mathrm{U}$ & 0.15 & 0.21 & $\mathrm{U}$ & 0.21 & 0.17 & $\mathrm{U}$ & 0.17 \\
\hline Riverbank & J13LN8 & $10 / 26 / 06$ & 0.37 & $\mathrm{U}$ & 0.37 & 0.087 & $\mathrm{U}$ & 0.087 & 0.099 & $\mathrm{U}$ & 0.099 & 0.24 & $\mathrm{U}$ & 0.24 & 0.33 & $\mathrm{U}$ & 0.33 & 0.25 & $\mathrm{U}$ & 0.25 \\
\hline Riverbank & J13LN7 & $10 / 26 / 06$ & 0.086 & $\mathrm{U}$ & 0.086 & 0.10 & $\mathrm{U}$ & 0.10 & 0.13 & $\mathrm{U}$ & 0.13 & 0.15 & $\mathrm{U}$ & 0.15 & 0.36 & $\mathrm{U}$ & 0.36 & 0.096 & $\mathrm{U}$ & 0.096 \\
\hline
\end{tabular}

\begin{tabular}{|c|c|c|c|c|c|c|c|c|c|c|c|c|c|c|c|c|c|c|c|c|}
\hline \multirow{2}{*}{ Sample Location } & \multirow{2}{*}{$\begin{array}{l}\text { Sample } \\
\text { Number } \\
\end{array}$} & \multirow{2}{*}{$\begin{array}{c}\text { Sample } \\
\text { Date }\end{array}$} & \multicolumn{3}{|c|}{ Gross Alpha } & \multicolumn{3}{|c|}{ Gross Beta } & \multicolumn{3}{|c|}{ Potassium-40 } & \multicolumn{3}{|c|}{ Radium-226 } & \multicolumn{3}{|c|}{ Radium-228 } & \multicolumn{3}{|c|}{ Thorium-228 } \\
\hline & & & $\mathrm{pCi} / \mathrm{g}$ & $\mathbf{Q}$ & MDA & $\mathrm{pCi} / \mathrm{g}$ & $\mathbf{Q}$ & MDA & $\mathrm{pCi} / \mathrm{g}$ & $\mathbf{Q}$ & MDA & $\mathrm{pCi} / \mathrm{g}$ & $\mathbf{Q}$ & MDA & $\mathrm{pCi} / \mathrm{g}$ & $\mathbf{Q}$ & MDA & $\mathrm{pCi} / \mathrm{g}$ & $\mathbf{Q}$ & MDA \\
\hline Riverbank & J13NX7 & $11 / 8 / 06$ & 3.93 & $\mathrm{U}$ & 5.9 & 19.0 & & 6.4 & 10.1 & & 0.54 & 0.398 & & 0.092 & 3453 & & 0.29 & 0.501 & & 0.056 \\
\hline Riverbank & J13NX6 & $11 / 8 / 06$ & -0.846 & $\mathrm{U}$ & 4.7 & 13.1 & & 5.5 & 13.1 & & 2.5 & 0.358 & & 0.15 & 0.681 & & 0.40 & 0.480 & & 0.12 \\
\hline Riverbank & J13NX5 & 11/8/06 & 5.82 & & 5.6 & 15.5 & & 9.6 & 11.6 & & 1.2 & 0.334 & & 0.18 & 0.845 & & 0.41 & 0.348 & & 0.069 \\
\hline Riverbank & J13LX4 & 11/1/06 & 4.70 & $\mathrm{U}$ & 5.6 & 20.1 & & 5.4 & 11.8 & & 0.49 & 0.390 & & 0.14 & 0.746 & & 0.33 & 0.589 & & 0.078 \\
\hline Riverbank & J13LX3 & 11/1/06 & 4.93 & & 4.9 & 22.4 & & 6.4 & 11.1 & & 1.9 & 0.423 & & 0.28 & 0.73 & $\mathrm{U}$ & 0.73 & 0.402 & & 0.093 \\
\hline Riverbank & J13LX2 & 11/1/06 & 13.0 & & 4.7 & 22.6 & & 5.5 & 12.1 & & 1.0 & 0.647 & & 0.16 & 0.617 & & 0.42 & 0.879 & & 0.16 \\
\hline Riverbank & J13LX1 & $\begin{array}{l}11 / 1 / 06 \\
\end{array}$ & 4.25 & $\mathrm{U}$ & 4.9 & 14.5 & & 9.5 & 12.0 & & 0.54 & 0.297 & & 0.15 & 0.824 & & 0.27 & 0.623 & & 0.082 \\
\hline Riverbank & J13LX0 & 11/1/06 & 11.2 & & 5.1 & 23.0 & & 5.9 & 11.0 & & 0.68 & 0.299 & & 0.13 & 0.588 & & 0.24 & 0.566 & & 0.071 \\
\hline Riverbank & J13LP5 & $10 / 26 / 06$ & 4.88 & $\mathrm{U}$ & 7.5 & 17.2 & & 5.6 & 10.3 & & 0.76 & 0.426 & & 0.16 & 6.28 & & 0.25 & 0.555 & & 0.12 \\
\hline Riverbank & J13LP4 & $10 / 26 / 06$ & 5.27 & $\mathrm{U}$ & 7.8 & 23.0 & & 5.6 & 12.6 & & 0.66 & 0.582 & & 0.13 & 0.880 & & 0.31 & 0.656 & & 0.076 \\
\hline Riverbank & J13LP3 & $10 / 26 / 06$ & 6.77 & & 6.3 & 20.7 & & 5.7 & 14.7 & & 1.4 & 0.525 & & 0.19 & 0.550 & & 0.45 & 0.671 & & 0.18 \\
\hline Riverbank & J13LP2 & $10 / 26 / 06$ & 10.3 & & 4.6 & 23.9 & & 5.6 & 16.4 & & 2.0 & 0.545 & & 0.23 & 1.12 & & 0.61 & 0.589 & & 0.1 \\
\hline Riverbank & J13LP1 & $10 / 26 / 06$ & 8.27 & & 5.3 & 17.4 & & 5.4 & 10.5 & & 0.68 & 0.456 & & 0.13 & 0.568 & & 0.24 & 0.717 & & 0.11 \\
\hline Riverbank & J13LP0 & $10 / 26 / 06$ & 43.2 & & 4.6 & 33.8 & & 6.3 & 10.5 & & 0.70 & 1.02 & & 0.12 & 1.94 & & 0.27 & 2.13 & & 0.12 \\
\hline Riverbank & J13LN9 & $10 / 26 / 06$ & 17.3 & & 4.5 & 22.4 & & 5.5 & 14.2 & & 0.58 & 0.579 & & 0.11 & 0.977 & & 0.26 & 0.811 & & 0.081 \\
\hline Riverbank & J13LN8 & $10 / 26 / 06$ & 4.88 & $\mathrm{U}$ & 4.9 & 20.3 & & 9.5 & 14.5 & & 0.99 & 0.475 & & 0.14 & 0.338 & & 0.29 & 0.868 & & 0.13 \\
\hline Riverbank & J13LN7 & $10 / 26 / 06$ & 12.2 & & 5.2 & 21.3 & & 5.9 & 16.1 & & 1.3 & 0.454 & & 0.19 & 1.57 & & 0.45 & 0.498 & & 0.079 \\
\hline
\end{tabular}


Table A-2. 128-F-2 In-Process Riverbank Excavation Berm Data (16 Pages)

\begin{tabular}{|c|c|c|c|c|c|c|c|c|c|c|c|}
\hline \multirow{2}{*}{ Sample Location } & \multirow{2}{*}{$\begin{array}{c}\text { Sample } \\
\text { Number }\end{array}$} & \multirow{2}{*}{$\begin{array}{c}\text { Sample } \\
\text { Date }\end{array}$} & \multicolumn{2}{|c|}{ Thorium-232 } & \multicolumn{3}{c|}{ Uranium-235 } & \multicolumn{3}{c|}{ Uranium-238 } \\
\cline { 7 - 11 } & pCi/g & Q & MDA & pCi/g & Q & MDA & pCi/g & Q & MDA \\
\hline Riverbank & J13NX7 & $11 / 8 / 06$ & 0.453 & & 0.29 & 0.17 & U & 0.17 & 5.7 & U & 5.7 \\
\hline Riverbank & J13NX6 & $11 / 8 / 06$ & 0.681 & & 0.40 & 0.31 & $\mathrm{U}$ & 0.31 & 10 & $\mathrm{U}$ & 10 \\
\hline Riverbank & J13NX5 & $11 / 8 / 06$ & 0.845 & & 0.41 & 0.13 & $\mathrm{U}$ & 0.13 & 13 & $\mathrm{U}$ & 13 \\
\hline Riverbank & J13LX4 & $11 / 1 / 06$ & 0.746 & & 0.33 & 0.24 & $\mathrm{U}$ & 0.24 & 9.7 & $\mathrm{U}$ & 9.7 \\
\hline Riverbank & J13LX3 & $11 / 1 / 06$ & 0.73 & $\mathrm{U}$ & 0.73 & 0.19 & $\mathrm{U}$ & 0.19 & 17 & $\mathrm{U}$ & 17 \\
\hline Riverbank & J13LX2 & $11 / 1 / 06$ & 0.617 & & 0.42 & 0.44 & $\mathrm{U}$ & 0.44 & 13 & $\mathrm{U}$ & 13 \\
\hline Riverbank & J13LX1 & $11 / 1 / 06$ & 0.824 & & 0.27 & 0.28 & $\mathrm{U}$ & 0.28 & 9.0 & $\mathrm{U}$ & 9.0 \\
\hline Riverbank & J13LX0 & $11 / 1 / 06$ & 0.588 & & 0.24 & 0.23 & $\mathrm{U}$ & 0.23 & 7.4 & $\mathrm{U}$ & 7.4 \\
\hline Riverbank & J13LP5 & $10 / 26 / 06$ & 0.628 & & 0.25 & 0.30 & $\mathrm{U}$ & 0.30 & 9.5 & $\mathrm{U}$ & 9.5 \\
\hline Riverbank & J13LP4 & $10 / 26 / 06$ & 0.880 & & 0.31 & 0.20 & $\mathrm{U}$ & 0.20 & 8.8 & $\mathrm{U}$ & 8.8 \\
\hline Riverbank & J13LP3 & $10 / 26 / 06$ & 0.550 & & 0.45 & 0.49 & $\mathrm{U}$ & 0.49 & 16 & $\mathrm{U}$ & 16 \\
\hline Riverbank & J13LP2 & $10 / 26 / 06$ & 1.12 & & 0.61 & 0.19 & $\mathrm{U}$ & 0.19 & 18 & $\mathrm{U}$ & 18 \\
\hline Riverbank & J13LP1 & $10 / 26 / 06$ & 0.568 & & 0.24 & 0.24 & $\mathrm{U}$ & 0.24 & 7.7 & $\mathrm{U}$ & 7.4 \\
\hline Riverbank & J13LP0 & $10 / 26 / 06$ & 1.94 & & 0.27 & 0.23 & $\mathrm{U}$ & 0.23 & 7.6 & $\mathrm{U}$ & 7.6 \\
\hline Riverbank & J13LN9 & $10 / 26 / 06$ & 0.977 & & 0.26 & 0.26 & $\mathrm{U}$ & 0.26 & 7.9 & $\mathrm{U}$ & 7.9 \\
\hline Riverbank & J13LN8 & $10 / 26 / 06$ & 0.338 & & 0.29 & 0.37 & $\mathrm{U}$ & 0.37 & 11 & $\mathrm{U}$ & 11 \\
\hline Riverbank & J13LN7 & $10 / 26 / 06$ & 1.57 & & 0.45 & 0.15 & $\mathrm{U}$ & 0.15 & 15 & $\mathrm{U}$ & 15 \\
\hline
\end{tabular}


Table A-2. 128-F-2 In-Process Riverbank Excavation Berm Data (16 Pages)

\begin{tabular}{|c|c|c|c|c|c|c|c|c|c|c|c|c|c|c|c|c|c|c|c|c|c|c|}
\hline \multirow{2}{*}{ Sample Location } & \multirow{2}{*}{$\begin{array}{c}\text { Sample } \\
\text { Number }\end{array}$} & \multirow{2}{*}{$\begin{array}{c}\text { Sample } \\
\text { Date }\end{array}$} & \multicolumn{2}{|c|}{ Aluminum } & \multicolumn{3}{|c|}{ Antimony } & \multicolumn{3}{|c|}{ Arsenic } & \multicolumn{3}{|c|}{ Barium } & \multicolumn{3}{|c|}{ Beryllium } & \multicolumn{3}{|c|}{ Boron } & \multicolumn{3}{|c|}{ Cadmium } \\
\hline & & & $\mathrm{mg} / \mathrm{kg}$ & \begin{tabular}{l|l|}
$\mathbf{Q}$ & $\mathbf{P Q L}$ \\
\end{tabular} & $\mathrm{mg} / \mathrm{kg}$ & $\mathbf{Q}$ & PQL & $\mathrm{mg} / \mathrm{kg}$ & $\mathbf{Q}$ & PQL & mg/kg & $\mathbf{Q}$ & PQL & $\mathrm{mg} / \mathrm{kg}$ & $\mathbf{Q}$ & PQL & mg/kg & $\mathbf{Q}$ & PQL & $\mathrm{mg} / \mathrm{kg}$ & $\mathbf{Q}$ & PQL \\
\hline Riverbank & J13NX7 & $11 / 8 / 06$ & 5230 & 6.7 & 0.79 & $\mathrm{U}$ & 0.79 & 2.7 & & 0.98 & 61.9 & & 0.03 & 0.31 & & 0.03 & 0.67 & & 0.60 & 0.09 & $\mathrm{U}$ & 0.09 \\
\hline Riverbank & J13NX6 & $11 / 8 / 06$ & 4520 & 6.8 & 0.81 & $\mathrm{U}$ & 0.81 & 2.2 & & 1.0 & 54.0 & & 0.03 & 0.27 & & 0.03 & 0.93 & & 0.62 & 0.1 & $\mathrm{U}$ & 0.1 \\
\hline Riverbank & J13NX5 & $11 / 8 / 06$ & 4820 & 7.3 & 0.87 & $\mathrm{U}$ & 0.87 & 3.3 & & 1.1 & 63.0 & & 0.03 & 0.28 & & 0.03 & 0.66 & $\mathrm{U}$ & 0.66 & 0.10 & $\mathrm{U}$ & 0.10 \\
\hline Riverbank & J13LX4 & $11 / 1 / 06$ & 6250 & 7.9 & 0.94 & $\mathrm{U}$ & 0.94 & 3.4 & & 1.2 & 105 & & 0.04 & 0.59 & & 0.04 & 0.91 & & 0.71 & 0.70 & & 0.11 \\
\hline Riverbank & J13LX3 & $11 / 1 / 06$ & 6500 & 7.2 & 0.85 & $\mathrm{U}$ & 0.85 & 3.3 & & 1.1 & 65.6 & & 0.03 & 0.54 & & 0.03 & 0.64 & $\mathrm{U}$ & 0.64 & 0.64 & & 0.10 \\
\hline Riverbank & J13LX2 & $11 / 1 / 06$ & 9600 & 7.9 & 0.93 & $\mathrm{U}$ & 0.93 & 7.4 & & 1.2 & 107 & & 0.04 & 0.61 & & 0.04 & 1.2 & & 0.071 & 2.1 & & 0.11 \\
\hline Riverbank & J13LX1 & $11 / 1 / 06$ & 9150 & 8.3 & 2.4 & & 0.99 & 8.6 & & 1.2 & 121 & & 0.04 & 0.49 & & 0.04 & 1.6 & & 0.75 & 2.0 & & 0.12 \\
\hline Riverbank & J13LX0 & $11 / 1 / 06$ & 6390 & 7.8 & 0.92 & $\mathrm{U}$ & 0.92 & 7.6 & & 1.1 & 108 & & 0.04 & 0.36 & & 0.04 & 1.4 & & 0.70 & 0.29 & & 0.11 \\
\hline Riverbank & J13LP5 & $10 / 26 / 06$ & 5920 & 7.2 & 2.2 & & 1.1 & 14.4 & & 1.2 & 230 & & 0.06 & 0.1 & & 0.03 & 1.9 & & 1.2 & 0.67 & & 0.1 \\
\hline Riverbank & J13LP4 & $10 / 26 / 06$ & 6350 & 7.5 & 1.2 & $\mathrm{U}$ & 1.2 & 17.6 & & 1.2 & 91.8 & & 0.07 & 0.11 & & 0.03 & 1.2 & $\mathrm{U}$ & 1.2 & 0.32 & & 0.1 \\
\hline Riverbank & J13LP3 & $10 / 26 / 06$ & 7990 & 7.8 & 1.2 & $\mathrm{U}$ & 1.2 & 6.0 & & 1.3 & 132 & & 0.07 & 0.16 & & 0.03 & 1.9 & & 1.3 & 0.99 & & 0.10 \\
\hline Riverbank & J13LP2 & $10 / 26 / 06$ & 6230 & 8.1 & 1.3 & $\mathrm{U}$ & 1.3 & 6.9 & & 1.3 & 88.2 & & 0.07 & 0.14 & & 0.04 & 1.3 & $\mathrm{U}$ & 1.3 & 2.2 & & 0.11 \\
\hline Riverbank & J13LP1 & $10 / 26 / 06$ & 13000 & 8.0 & 1.7 & & 1.3 & 9.4 & & 1.3 & 108 & & 0.07 & 0.1 & & 0.04 & 1.3 & $\mathrm{U}$ & 1.3 & 1.5 & & 0.11 \\
\hline Riverbank & J13LP0 & $10 / 26 / 06$ & 11900 & 7.2 & 1.1 & $\mathrm{U}$ & 1.1 & 19.5 & & 1.2 & 321 & & 0.06 & 0.32 & & 0.03 & 1.2 & $\mathrm{U}$ & 1.2 & 0.45 & & 0.09 \\
\hline Riverbank & J13LN9 & $10 / 26 / 06$ & 7270 & 7.2 & 2.0 & & 1.1 & 19.6 & & 1.2 & 105 & & 0.06 & 0.17 & & 0.03 & 1.2 & $\mathrm{U}$ & 1.2 & 1.4 & & 0.1 \\
\hline Riverbank & J13LN8 & $10 / 26 / 06$ & 7860 & 7.4 & 1.2 & $\mathrm{U}$ & 1.2 & 4.4 & & 1.2 & 105 & & 0.06 & 0.22 & & 0.03 & 1.2 & $\mathrm{U}$ & 1.2 & 2.6 & & 0.1 \\
\hline Riverbank & J13LN7 & $10 / 26 / 06$ & 6670 & 6.9 & 1.1 & $\mathrm{U}$ & 1.1 & 10.0 & & 1.1 & 120 & & 0.06 & 0.19 & & 0.03 & 1.1 & $\mathrm{U}$ & 1.1 & 2.1 & & 0.09 \\
\hline
\end{tabular}

\begin{tabular}{|c|c|c|c|c|c|c|c|c|c|c|c|c|c|c|c|c|c|c|c|c|c|c|c|}
\hline \multirow{2}{*}{ Sample Location } & \multirow{2}{*}{$\begin{array}{c}\text { Sample } \\
\text { Number }\end{array}$} & \multirow{2}{*}{$\begin{array}{c}\text { Sample } \\
\text { Date }\end{array}$} & \multicolumn{3}{|c|}{ Calcium } & \multicolumn{3}{|c|}{ Chromium } & \multicolumn{3}{|c|}{ Cobalt } & \multicolumn{3}{|c|}{ Copper } & \multicolumn{3}{|c|}{$\begin{array}{l}\text { Hexavalent } \\
\text { Chromium }\end{array}$} & \multicolumn{3}{|c|}{ Iron } & \multicolumn{3}{|c|}{ Lead } \\
\hline & & & $\mathrm{mg} / \mathrm{kg}$ & $\mathbf{Q}$ & PQL & $\mathrm{mg} / \mathrm{kg}$ & $\mathbf{Q}$ & PQL & $\mathrm{mg} / \mathrm{kg}$ & $\mathbf{Q}$ & PQL & $\mathrm{mg} / \mathrm{kg}$ & $\mathbf{Q}$ & PQL & $\mathrm{mg} / \mathrm{kg}$ & $\mathbf{Q}$ & PQL & $\mathrm{mg} / \mathrm{kg}$ & $\mathbf{Q}$ & \begin{tabular}{|l|} 
PQL \\
\end{tabular} & $\mathrm{mg} / \mathrm{kg}$ & $\mathbf{Q}$ & PQL \\
\hline Riverbank & $\begin{array}{l}\mathrm{J} 13 \mathrm{NX} 7 \\
\end{array}$ & $\begin{array}{l}11 / 8 / 06 \\
\end{array}$ & 2930 & & 3.9 & 8.0 & & \begin{tabular}{|l|}
0.38 \\
\end{tabular} & 6.1 & & 0.16 & 10.9 & & \begin{tabular}{|l|}
0.22 \\
\end{tabular} & 0.26 & & \begin{tabular}{|l|}
0.22 \\
\end{tabular} & 16600 & & 7.7 & 4.4 & & \begin{tabular}{|l|}
0.50 \\
\end{tabular} \\
\hline Riverbank & J13NX6 & $11 / 8 / 06$ & 2680 & & 4.1 & 6.6 & & \begin{tabular}{|l|}
0.39 \\
\end{tabular} & 5.4 & & 0.16 & 9.7 & & \begin{tabular}{|l|}
0.23 \\
\end{tabular} & 0.23 & $\mathrm{U}$ & \begin{tabular}{|l|}
0.23 \\
\end{tabular} & 14000 & & 7.9 & 3.9 & & \begin{tabular}{|l|}
0.52 \\
\end{tabular} \\
\hline Riverbank & J13NX5 & $11 / 8 / 06$ & 2870 & & 4.3 & 8.0 & & \begin{tabular}{|l|}
0.42 \\
\end{tabular} & 6.2 & & 0.17 & 10.9 & & \begin{tabular}{|l|l|}
0.24 \\
\end{tabular} & 0.24 & $\mathrm{U}$ & \begin{tabular}{|l|}
0.24 \\
\end{tabular} & 15400 & & 8.5 & 4.3 & & 0.56 \\
\hline Riverbank & J13LX4 & $11 / 1 / 06$ & 3920 & & 4.7 & 42.2 & & 0.45 & 5.5 & & 0.19 & 40.2 & & 0.26 & 2.6 & $\mathrm{U}$ & 2.6 & 15700 & & 9.2 & 68.9 & & 0.60 \\
\hline Riverbank & J13LX3 & $11 / 1 / 06$ & 3360 & & 4.2 & 23.4 & & 0.41 & 5.7 & & 0.17 & 64.3 & & 0.24 & 0.24 & $\mathrm{U}$ & \begin{tabular}{|l|}
0.24 \\
\end{tabular} & 15900 & & 8.3 & 102 & & 0.54 \\
\hline Riverbank & J13LX2 & $11 / 1 / 06$ & 4010 & & 4.7 & 253 & & 0.45 & 7.6 & & 0.19 & 201 & & 0.26 & 0.26 & $\mathrm{U}$ & \begin{tabular}{|l|}
0.26 \\
\end{tabular} & 24200 & & 9.1 & 293 & & 0.60 \\
\hline Riverbank & J13LX1 & $\begin{array}{l}11 / 1 / 06 \\
\end{array}$ & 4470 & & 4.9 & 104 & & \begin{tabular}{|l|}
0.47 \\
\end{tabular} & 8.5 & & 0.20 & 322 & & \begin{tabular}{|l|}
0.28 \\
\end{tabular} & 0.32 & & \begin{tabular}{|l|}
0.27 \\
\end{tabular} & 26400 & & 9.6 & 404 & & 0.63 \\
\hline Riverbank & J13LX0 & $\begin{array}{l}11 / 1 / 06 \\
\end{array}$ & 9380 & & 4.6 & 25.5 & & \begin{tabular}{|l|}
0.44 \\
\end{tabular} & 7.1 & & 0.18 & 60.0 & & 0.26 & 54.7 & & 12.9 & 22200 & & 9.0 & 35.1 & & \begin{tabular}{|l|}
0.59 \\
\end{tabular} \\
\hline Riverbank & J13LP5 & $10 / 26 / 06$ & 7030 & & 2.7 & 33.5 & & 0.22 & 6.6 & & 0.16 & 578 & & \begin{tabular}{|l|l|}
0.25 \\
\end{tabular} & 0.26 & & \begin{tabular}{|l|}
0.22 \\
\end{tabular} & 30800 & & 7.7 & 1820 & & \begin{tabular}{|l|}
0.38 \\
\end{tabular} \\
\hline Riverbank & J13LP4 & $10 / 26 / 06$ & 4300 & & 2.8 & 22.2 & & 0.23 & 5.9 & & 0.16 & 20.5 & & \begin{tabular}{|l|}
0.26 \\
\end{tabular} & 0.82 & & \begin{tabular}{|l|}
0.23 \\
\end{tabular} & 17800 & & 8.0 & 44.1 & & \begin{tabular}{|l|}
0.39 \\
\end{tabular} \\
\hline Riverbank & J13LP3 & $10 / 26 / 06$ & 6010 & & 3.0 & 273 & & \begin{tabular}{|l|}
0.24 \\
\end{tabular} & 6.1 & & 0.17 & 43.9 & & \begin{tabular}{|l|}
0.27 \\
\end{tabular} & 0.24 & $\mathrm{U}$ & \begin{tabular}{|l|}
0.24 \\
\end{tabular} & 17300 & & 8.3 & 86.4 & & \begin{tabular}{|l|}
0.41 \\
\end{tabular} \\
\hline Riverbank & J13LP2 & $10 / 26 / 06$ & 3370 & & 3.0 & 786 & & 0.25 & 7.4 & & 0.18 & 148 & & \begin{tabular}{|l|}
0.28 \\
\end{tabular} & 4.9 & & \begin{tabular}{|l|}
0.25 \\
\end{tabular} & 20600 & & 8.6 & 83.2 & & \begin{tabular}{|l|l|}
0.42 \\
\end{tabular} \\
\hline Riverbank & J13LP1 & $10 / 26 / 06$ & 3990 & & 3.0 & 328 & & 0.25 & 6.8 & & 0.18 & 160 & & 0.28 & 0.58 & & 0.24 & 25100 & & 8.6 & 140 & & 0.42 \\
\hline Riverbank & J13LP0 & $10 / 26 / 06$ & 3080 & & 2.7 & 148 & & \begin{tabular}{|l|}
0.22 \\
\end{tabular} & 12.3 & & 0.16 & 47.7 & & \begin{tabular}{|l|l|}
0.25 \\
\end{tabular} & 5.3 & & \begin{tabular}{|l|}
0.22 \\
\end{tabular} & 44900 & & 7.7 & 6.4 & & \begin{tabular}{|l|l|}
0.38 \\
\end{tabular} \\
\hline Riverbank & J13LN9 & $10 / 26 / 06$ & 3410 & & 2.7 & 329 & & \begin{tabular}{|l|}
0.22 \\
\end{tabular} & 7.7 & & 0.16 & 75.6 & & \begin{tabular}{|l|}
0.25 \\
\end{tabular} & 5.1 & & \begin{tabular}{|l|}
0.22 \\
\end{tabular} & 24700 & & 7.7 & 210 & & 0.38 \\
\hline Riverbank & J13LN8 & $10 / 26 / 06$ & 3830 & & 2.8 & 434 & & \begin{tabular}{|l|}
0.23 \\
\end{tabular} & 7.9 & & 0.16 & 39.4 & & \begin{tabular}{|l|}
0.26 \\
\end{tabular} & 1.8 & & 0.23 & 19900 & & 7.9 & 83.3 & & \begin{tabular}{|l|}
0.39 \\
\end{tabular} \\
\hline Riverbank & J13LN7 & $10 / 26 / 06$ & 3850 & & 2.6 & 121 & & \begin{tabular}{|l|}
0.21 \\
\end{tabular} & 7.1 & & 0.15 & 45.8 & & \begin{tabular}{|l|l|}
0.24 \\
\end{tabular} & 1.3 & & \begin{tabular}{|l|}
0.21 \\
\end{tabular} & 19200 & & 7.4 & 127 & & \begin{tabular}{|l|}
0.37 \\
\end{tabular} \\
\hline
\end{tabular}


Table A-2. 128-F-2 In-Process Riverbank Excavation Berm Data (16 Pages)

\begin{tabular}{|c|c|c|c|c|c|c|c|c|c|c|c|c|c|c|c|c|c|c|c|c|c|c|c|}
\hline \multirow{2}{*}{ Sample Location } & \multirow{2}{*}{$\begin{array}{c}\text { Sample } \\
\text { Number }\end{array}$} & \multirow{2}{*}{$\begin{array}{c}\text { Sample } \\
\text { Date }\end{array}$} & \multicolumn{3}{|c|}{ Magnesium } & \multicolumn{3}{|c|}{ Manganese } & \multicolumn{3}{|c|}{ Mercury } & \multicolumn{3}{|c|}{ Molybdenum } & \multicolumn{3}{|c|}{ Nickel } & \multicolumn{3}{|c|}{ Potassium } & \multicolumn{3}{|c|}{ Selenium } \\
\hline & & & $\mathrm{mg} / \mathrm{kg}$ & $\mathbf{Q}$ & PQL & $\mathrm{mg} / \mathrm{kg}$ & $\mathbf{Q}$ & PQL & $\mathrm{mg} / \mathrm{kg}$ & $\mathbf{Q}$ & PQL & $\mathrm{mg} / \mathrm{kg}$ & \begin{tabular}{l|l}
$\mathbf{Q}$ & \\
\end{tabular} & PQL & $\mathrm{mg} / \mathrm{kg}$ & $\mathbf{Q}$ & PQL & $\mathrm{mg} / \mathrm{kg}$ & $\mathbf{Q}$ & PQL & $\mathrm{mg} / \mathrm{kg}$ & $\mathbf{Q}$ & PQL \\
\hline Riverbank & J13NX7 & 11/8/06 & 3480 & & 1.5 & 289 & & 0.13 & 0.02 & $\mathrm{U}$ & 0.02 & 0.50 & $\mathrm{U}$ & 0.50 & 10.1 & & 0.69 & 914 & & 6.7 & 1.4 & & 1.4 \\
\hline Riverbank & J13NX6 & $11 / 8 / 06$ & 3030 & & 1.5 & 257 & & 0.13 & 0.02 & $\mathrm{U}$ & 0.02 & 0.52 & \begin{tabular}{l|l} 
\\
\end{tabular} & 0.52 & 9.1 & & 0.71 & 786 & & 6.9 & 1.4 & $\mathrm{U}$ & 1.4 \\
\hline Riverbank & J13NX5 & $11 / 8 / 06$ & 3280 & & 1.6 & 277 & & 0.14 & 0.02 & $\mathrm{U}$ & \begin{tabular}{l|l|}
0.02 \\
\end{tabular} & 0.56 & \begin{tabular}{l|l} 
\\
\end{tabular} & 0.56 & 11.1 & & 0.76 & 827 & & 7.4 & 1.5 & $\mathrm{U}$ & 1.5 \\
\hline Riverbank & J13LX4 & $11 / 1 / 06$ & 3740 & & 1.7 & 234 & & 0.15 & 1.6 & & 0.02 & 0.60 & $\mathrm{U}$ & 0.60 & 15.3 & & 0.82 & 966 & & 8.0 & 1.6 & $\mathrm{U}$ & 1.6 \\
\hline Riverbank & J13LX3 & 11/1/06 & 4270 & & 1.6 & 208 & & 0.14 & 0.12 & & 0.02 & 0.54 & $\mathrm{U}$ & 0.54 & 29.9 & & 0.75 & 968 & & 7.2 & 1.5 & $\mathrm{U}$ & 1.5 \\
\hline Riverbank & J13LX2 & $11 / 1 / 06$ & 4420 & & 1.7 & 638 & & 0.15 & 2.2 & & 0.06 & 0.68 & & 0.60 & 29.8 & & 0.82 & 1050 & & 7.9 & 1.6 & $\mathrm{U}$ & 1.6 \\
\hline Riverbank & J13LX1 & $11 / 1 / 06$ & 4900 & & 1.8 & 436 & & 0.16 & 1.0 & & 0.02 & 1.7 & & 0.63 & 104 & & 0.87 & 1060 & & 8.4 & 1.7 & $\mathrm{U}$ & 1.7 \\
\hline Riverbank & J13LX0 & $11 / 1 / 06$ & 4010 & & 1.7 & 326 & & 0.15 & 0.44 & & 0.02 & 0.59 & \begin{tabular}{l|l} 
\\
\end{tabular} & 0.59 & 23.3 & & 0.81 & 951 & & 7.8 & 1.6 & $\mathrm{U}$ & 1.6 \\
\hline Riverbank & J13LP5 & $10 / 26 / 06$ & 4110 & & 2.3 & 423 & & 0.06 & 0.04 & & 0.01 & 1.2 & & 0.32 & 26.0 & & 0.41 & 921 & & 6.7 & 0.83 & \begin{tabular}{|l|}
$\mathrm{U}$ \\
\end{tabular} & 0.83 \\
\hline Riverbank & J13LP4 & $10 / 26 / 06$ & 3890 & & 2.4 & 280 & & 0.07 & 0.02 & $\mathrm{U}$ & 0.02 & 0.69 & & 0.33 & 14.8 & & 0.43 & 930 & & 7.0 & 0.86 & $\mathrm{U}$ & 0.86 \\
\hline Riverbank & J13LP3 & $10 / 26 / 06$ & 4250 & & 2.5 & 242 & & 0.07 & 0.06 & & \begin{tabular}{l|l|}
0.02 \\
\end{tabular} & 0.61 & & 0.34 & 15.7 & & 0.45 & 971 & & 7.3 & 0.89 & $\mathrm{U}$ & 0.89 \\
\hline Riverbank & J13LP2 & $10 / 26 / 06$ & 3440 & & 2.5 & 185 & & 0.07 & 0.36 & & 0.02 & 0.94 & & 0.35 & 15.9 & & 0.46 & 904 & & 7.5 & 0.92 & $\mathrm{U}$ & 0.92 \\
\hline Riverbank & J13LP1 & $10 / 26 / 06$ & 3800 & & 2.5 & 211 & & 0.07 & 0.25 & & \begin{tabular}{l|l|}
0.02 \\
\end{tabular} & 0.92 & & 0.35 & 19.6 & & 0.46 & 722 & & 7.5 & 1.1 & & 0.91 \\
\hline Riverbank & J13LP0 & $10 / 26 / 06$ & 3380 & & 2.3 & 1410 & & 0.06 & 0.02 & & \begin{tabular}{l|}
0.02 \\
\end{tabular} & 2.7 & & 0.32 & 43.4 & & 0.41 & 647 & & 6.7 & 1.3 & & 0.82 \\
\hline Riverbank & J13LN9 & $10 / 26 / 06$ & 3920 & & 2.3 & 289 & & 0.06 & 0.04 & & 0.02 & 0.87 & & 0.32 & 13.6 & & 0.41 & 843 & & 6.7 & 0.82 & $\mathrm{U}$ & 0.82 \\
\hline Riverbank & J13LN8 & $10 / 26 / 06$ & 4500 & & 2.3 & 235 & & 0.06 & 0.08 & & \begin{tabular}{l|l|}
0.02 \\
\end{tabular} & 0.71 & & 0.32 & 14.8 & & 0.42 & 1230 & & 6.9 & 0.84 & $\mathrm{U}$ & 0.84 \\
\hline Riverbank & J13LN7 & $10 / 26 / 06$ & 4070 & & 2.2 & 392 & & 0.06 & 0.02 & & \begin{tabular}{l|l}
0.01 \\
\end{tabular} & 0.54 & & 0.30 & 13.4 & & 0.40 & 1080 & & 6.5 & 0.79 & $\mathrm{U}$ & 0.79 \\
\hline
\end{tabular}

\begin{tabular}{|c|c|c|c|c|c|c|c|c|c|c|c|c|c|c|c|c|c|c|c|c|}
\hline \multirow{2}{*}{ Sample Location } & \multirow{2}{*}{$\begin{array}{c}\text { Sample } \\
\text { Number }\end{array}$} & \multirow{2}{*}{$\begin{array}{c}\text { Sample } \\
\text { Date }\end{array}$} & \multicolumn{3}{|c|}{ Silicon } & \multicolumn{3}{|c|}{ Silver } & \multicolumn{3}{|c|}{ Sodium } & \multicolumn{3}{|c|}{ Vanadium } & \multicolumn{3}{|c|}{ Zinc } & \multicolumn{3}{|c|}{ TPH } \\
\hline & & & $\mathrm{mg} / \mathrm{kg}$ & $\mathbf{Q}$ & PQL & $\mathrm{mg} / \mathrm{kg}$ & $\mathbf{Q}$ & PQL & $\mathrm{mg} / \mathrm{kg}$ & $\mathbf{Q}$ & PQL & $\mathrm{mg} / \mathrm{kg}$ & $\mathbf{Q}$ & PQL & $\mathrm{mg} / \mathrm{kg}$ & $\mathbf{Q}$ & PQL & $\mathrm{mg} / \mathrm{kg}$ & $\mathbf{Q}$ & PQL \\
\hline Riverbank & J13NX7 & $11 / 8 / 06$ & 699 & & 2.0 & 0.16 & $\mathrm{U}$ & 0.16 & 83.9 & & 0.79 & 39.3 & & 0.19 & 36.1 & & 0.13 & 146 & $\mathrm{U}$ & 146 \\
\hline Riverbank & J13NX6 & $11 / 8 / 06$ & 649 & & 2.1 & 0.16 & $\mathrm{U}$ & 0.16 & 76.7 & & 0.81 & 34.4 & & 0.19 & 31.5 & & 0.13 & 152 & $\mathrm{U}$ & 152 \\
\hline Riverbank & J13NX5 & $11 / 8 / 06$ & 719 & & 2.2 & 0.17 & $\mathrm{U}$ & 0.17 & 78.7 & & 0.87 & 39.2 & & 0.21 & 55.2 & & 0.14 & 161 & $\mathrm{U}$ & 161 \\
\hline Riverbank & J13LX4 & $11 / 1 / 06$ & 714 & & 2.4 & 0.19 & $\mathrm{U}$ & 0.19 & 106 & & 0.94 & 33.7 & & 0.22 & 181 & & 0.15 & 197 & & 175 \\
\hline Riverbank & J13LX3 & $11 / 1 / 06$ & 950 & & 2.2 & 0.17 & $\mathrm{U}$ & 0.17 & 109 & & 0.85 & 32.4 & & 0.20 & 146 & & 0.14 & 159 & $\mathrm{U}$ & 159 \\
\hline Riverbank & J13LX2 & $11 / 1 / 06$ & 643 & & 2.4 & 0.19 & $\mathrm{U}$ & 0.19 & 138 & & 0.93 & 39.5 & & 0.22 & 526 & & 0.15 & 172 & $\mathrm{U}$ & 172 \\
\hline Riverbank & J13LX1 & $11 / 1 / 06$ & 743 & & 2.5 & 0.20 & $\mathrm{U}$ & 0.20 & 148 & & 0.99 & 33.2 & & 0.24 & 399 & & 0.16 & 199 & & 181 \\
\hline Riverbank & J13LX0 & $11 / 1 / 06$ & 924 & & 2.4 & 0.18 & $\mathrm{U}$ & 0.18 & 133 & & 0.92 & 29.5 & & 0.22 & 117 & & 0.15 & 170 & $\mathrm{U}$ & 170 \\
\hline Riverbank & J13LP5 & $10 / 26 / 06$ & 415 & & 1.3 & 3.6 & & 0.35 & 177 & & 0.67 & 41.8 & & 0.16 & 477 & & 0.1 & 147 & $\mathrm{U}$ & 147 \\
\hline Riverbank & J13LP4 & $10 / 26 / 06$ & 504 & & 1.3 & 0.36 & $\mathrm{U}$ & 0.36 & 127 & & 0.69 & 37.3 & & 0.16 & 106 & & 0.1 & 155 & $\mathrm{U}$ & 155 \\
\hline Riverbank & J13LP3 & $10 / 26 / 06$ & 490 & & 1.4 & 0.38 & $\mathrm{U}$ & 0.38 & 224 & & 0.72 & 37.9 & & 0.17 & 259 & & 0.10 & 162 & $\mathrm{U}$ & 162 \\
\hline Riverbank & J13LP2 & $10 / 26 / 06$ & 610 & & 1.4 & 0.39 & $\mathrm{U}$ & 0.39 & 88.1 & & 0.74 & 32.6 & & 0.18 & 523 & & 0.11 & 164 & $\mathrm{U}$ & 164 \\
\hline Riverbank & J13LP1 & $10 / 26 / 06$ & 596 & & 1.4 & 0.39 & $\mathrm{U}$ & 0.39 & 158 & & 0.74 & 60.3 & & 0.18 & 477 & & 0.11 & 163 & $\mathrm{U}$ & 163 \\
\hline Riverbank & J13LP0 & $10 / 26 / 06$ & 603 & & 1.3 & 0.35 & $\mathrm{U}$ & 0.35 & 74.6 & & 0.66 & 118 & & 0.16 & 52.1 & & 0.09 & 146 & $\mathrm{U}$ & 146 \\
\hline Riverbank & J13LN9 & $10 / 26 / 06$ & 632 & & 1.3 & 0.35 & $\mathrm{U}$ & 0.35 & 122 & & 0.67 & 32.9 & & 0.16 & 680 & & 0.1 & 146 & $\mathrm{U}$ & 146 \\
\hline Riverbank & J13LN8 & $10 / 26 / 06$ & 613 & & 1.3 & 0.36 & $\mathrm{U}$ & 0.36 & 100 & & 0.68 & 34.5 & & 0.16 & 479 & & 0.1 & 151 & $\mathrm{U}$ & 151 \\
\hline Riverbank & J13LN7 & $10 / 26 / 06$ & 756 & & 1.2 & 0.33 & $\mathrm{U}$ & 0.33 & 149 & & 0.64 & 29.3 & & 0.15 & 642 & & 0.09 & 139 & $\mathrm{U}$ & 139 \\
\hline
\end{tabular}


Table A-2. 128-F-2 In-Process Riverbank Excavation Berm Data (16 Pages)

\begin{tabular}{|c|c|c|c|c|c|c|c|c|c|c|c|c|c|c|c|}
\hline \multirow[t]{2}{*}{ Constituents } & \multicolumn{3}{|c|}{$\begin{array}{c}\text { J13NX7 } \\
\text { Riverbank } \\
\text { Sample Date 11/8/06 }\end{array}$} & \multicolumn{3}{|c|}{$\begin{array}{c}\text { J13NX6 } \\
\text { Riverbank } \\
\text { Sample Date 11/8/06 }\end{array}$} & \multicolumn{3}{|c|}{$\begin{array}{c}\text { J13NX5 } \\
\text { Riverbank } \\
\text { Sample Date 11/8/06 }\end{array}$} & \multicolumn{3}{|c|}{$\begin{array}{c}\text { J13LX4 } \\
\text { Riverbank } \\
\text { Sample Date 11/1/06 }\end{array}$} & \multicolumn{3}{|c|}{$\begin{array}{c}\text { J13LX3 } \\
\text { Riverbank } \\
\text { Sample Date 11/1/06 }\end{array}$} \\
\hline & $\mu \mathrm{g} / \mathrm{kg}$ & $\mathbf{Q}$ & PQL & $\mu \mathrm{g} / \mathrm{kg}$ & $\mathbf{Q}$ & PQL & $\mu \mathrm{g} / \mathrm{kg}$ & $\mathbf{Q}$ & PQL & $\mu \mathrm{g} / \mathrm{kg}$ & $\mathbf{Q}$ & PQL & $\mu \mathrm{g} / \mathrm{kg}$ & $\mathbf{Q}$ & PQL \\
\hline \multicolumn{16}{|c|}{ Polychlorinated Biphenyls (PCBs) } \\
\hline Aroclor-1016 & 15 & $\mathrm{U}$ & 15 & 15 & $\mathrm{U}$ & 15 & 16 & $\mathrm{U}$ & 16 & 18 & $\mathrm{U}$ & 18 & 16 & $\mathrm{U}$ & 16 \\
\hline Aroclor-1221 & 15 & $\mathrm{U}$ & 15 & 15 & $\mathrm{U}$ & 15 & 16 & $\mathrm{U}$ & 16 & 18 & $\mathrm{U}$ & 18 & 16 & $\mathrm{U}$ & 16 \\
\hline Aroclor-1232 & 15 & $\mathrm{U}$ & 15 & 15 & $\mathrm{U}$ & 15 & 16 & $\mathrm{U}$ & 16 & 18 & $\mathrm{U}$ & 18 & 16 & $\mathrm{U}$ & 16 \\
\hline Aroclor-1242 & 15 & $\mathrm{U}$ & 15 & 15 & $\mathrm{U}$ & 15 & 16 & $\mathrm{U}$ & 16 & 18 & $\mathrm{U}$ & 18 & 16 & $\mathrm{U}$ & 16 \\
\hline Aroclor-1248 & 15 & $\mathrm{U}$ & 15 & 15 & $\mathrm{U}$ & 15 & 16 & $\mathrm{U}$ & 16 & 18 & $\mathrm{U}$ & 18 & 16 & $\mathrm{U}$ & 16 \\
\hline Aroclor-1254 & 15 & $\mathrm{U}$ & 15 & 15 & $\mathrm{U}$ & 15 & 16 & $\mathrm{U}$ & 16 & 18 & $\mathrm{U}$ & 18 & 16 & $\mathrm{U}$ & 16 \\
\hline Aroclor-1260 & 15 & $\mathrm{U}$ & 15 & 15 & $\mathrm{U}$ & 15 & 16 & $\mathrm{U}$ & 16 & 42 & & 18 & 16 & $\bar{U}$ & 16 \\
\hline \multicolumn{16}{|c|}{ Semivolatile Organic Analytes (SVOAs) } \\
\hline 1,2,4-Trichlorobenzene & 370 & $\mathrm{U}$ & 370 & 380 & $\mathrm{U}$ & 380 & 400 & $\mathrm{U}$ & 400 & 8800 & $\mathrm{U}$ & 8800 & 400 & $\mathrm{U}$ & 400 \\
\hline 1,2-Dichlorobenzene & 370 & $\mathrm{U}$ & 370 & 380 & $\mathrm{U}$ & 380 & 400 & $\mathrm{U}$ & 400 & 8800 & $\mathrm{U}$ & 8800 & 400 & $\mathrm{U}$ & 400 \\
\hline 1,3-Dichlorobenzene & 370 & $\mathrm{U}$ & 370 & 380 & $\mathrm{U}$ & 380 & 400 & $\mathrm{U}$ & 400 & 8800 & $\mathrm{U}$ & 8800 & 400 & $\mathrm{U}$ & 400 \\
\hline 1,4-Dichlorobenzene & 370 & $\mathrm{U}$ & 370 & 380 & $\mathrm{U}$ & 380 & 400 & $\mathrm{U}$ & 400 & 8800 & $\mathrm{U}$ & 8800 & 400 & $\mathrm{U}$ & 400 \\
\hline 2,4,5-Trichlorophenol & 920 & $\mathrm{U}$ & 920 & 950 & $\mathrm{U}$ & 950 & 1000 & $\mathrm{U}$ & 1000 & 22000 & $\mathrm{U}$ & 22000 & 1000 & $\mathrm{U}$ & 1000 \\
\hline 2,4,6-Trichlorophenol & 370 & $\mathrm{U}$ & 370 & 380 & $\mathrm{U}$ & 380 & 400 & $\mathrm{U}$ & 400 & 8800 & $\mathrm{U}$ & 8800 & 400 & $\mathrm{U}$ & 400 \\
\hline 2,4-Dichlorophenol & 370 & $\mathrm{U}$ & 370 & 380 & $\mathrm{U}$ & 380 & 400 & $\mathrm{U}$ & 400 & 8800 & $\mathrm{U}$ & 8800 & 400 & $\mathrm{U}$ & 400 \\
\hline 2,4-Dimethylphenol & 370 & $\mathrm{U}$ & 370 & 380 & $\mathrm{U}$ & 380 & 400 & $\mathrm{U}$ & 400 & 8800 & $\mathrm{U}$ & 8800 & 400 & $\mathrm{U}$ & 400 \\
\hline 2,4-Dinitrophenol & 920 & $\mathrm{U}$ & 920 & 950 & $\mathrm{U}$ & 950 & 1000 & $\mathrm{U}$ & 1000 & 22000 & $\mathrm{U}$ & 22000 & 1000 & $\mathrm{U}$ & 1000 \\
\hline 2,4-Dinitrotoluene & 370 & $\mathrm{U}$ & 370 & 380 & $\mathrm{U}$ & 380 & 400 & $\mathrm{U}$ & 400 & 8800 & $\mathrm{U}$ & 8800 & 400 & $\mathrm{U}$ & 400 \\
\hline 2,6-Dinitrotoluene & 370 & $\mathrm{U}$ & 370 & 380 & $\mathrm{U}$ & 380 & 400 & $\mathrm{U}$ & 400 & 8800 & $\mathrm{U}$ & 8800 & 400 & $\mathrm{U}$ & 400 \\
\hline 2-Chloronaphthalene & 370 & $\mathrm{U}$ & 370 & 380 & $\mathrm{U}$ & 380 & 400 & $\mathrm{U}$ & 400 & 8800 & $\mathrm{U}$ & 8800 & 400 & $\mathrm{U}$ & 400 \\
\hline 2-Chlorophenol & 370 & $\mathrm{U}$ & 370 & 380 & $\mathrm{U}$ & 380 & 400 & $\mathrm{U}$ & 400 & 8800 & $\mathrm{U}$ & 8800 & 400 & $\mathrm{U}$ & 400 \\
\hline 2-Methylnaphthalene & 370 & $\mathrm{U}$ & 370 & 380 & $\mathrm{U}$ & 380 & 400 & $\mathrm{U}$ & 400 & 8800 & $\mathrm{U}$ & 8800 & 400 & $\mathrm{U}$ & 400 \\
\hline 2-Methylphenol (cresol, o-) & 370 & $\mathrm{U}$ & 370 & 380 & $\mathrm{U}$ & 380 & 400 & $\mathrm{U}$ & 400 & 8800 & $\mathrm{U}$ & 8800 & 400 & $\mathrm{U}$ & 400 \\
\hline 2-Nitroaniline & 920 & $\mathrm{U}$ & 920 & 950 & $\mathrm{U}$ & 950 & 1000 & $\mathrm{U}$ & 1000 & 22000 & $\mathrm{U}$ & 22000 & 1000 & $\mathrm{U}$ & 1000 \\
\hline 2-Nitrophenol & 370 & $\mathrm{U}$ & 370 & 380 & $\mathrm{U}$ & 380 & 400 & $\mathrm{U}$ & 400 & 8800 & $\mathrm{U}$ & 8800 & 400 & $\mathrm{U}$ & 400 \\
\hline 3+4 Methylphenol (cresol, m+p) & 370 & $\mathrm{U}$ & 370 & 380 & $\mathrm{U}$ & 380 & 400 & $\mathrm{U}$ & 400 & 8800 & $\bar{U}$ & 8800 & 400 & $\bar{U}$ & 400 \\
\hline 3,3'-Dichlorobenzidine & 370 & $\mathrm{U}$ & 370 & 380 & $\mathrm{U}$ & 380 & 400 & $\mathrm{U}$ & 400 & 8800 & $\mathrm{U}$ & 8800 & 400 & $\mathrm{U}$ & 400 \\
\hline 3-Nitroaniline & 920 & $\mathrm{U}$ & 920 & 950 & $\mathrm{U}$ & 950 & 1000 & $\mathrm{U}$ & 1000 & 22000 & $\mathrm{U}$ & 22000 & 1000 & $\mathrm{U}$ & 1000 \\
\hline 4,6-Dinitro-2-methylphenol & 920 & $\mathrm{U}$ & 920 & 950 & $\mathrm{U}$ & 950 & 1000 & $\mathrm{U}$ & 1000 & 22000 & $\mathrm{U}$ & 22000 & 1000 & $\mathrm{U}$ & 1000 \\
\hline 4-Bromophenylphenyl ether & 370 & $\mathrm{U}$ & 370 & 380 & $\mathrm{U}$ & 380 & 400 & $\mathrm{U}$ & 400 & 8800 & $\mathrm{U}$ & 8800 & 400 & $\mathrm{U}$ & 400 \\
\hline 4-Chloro-3-methylphenol & 370 & $\mathrm{U}$ & 370 & 380 & $\mathrm{U}$ & 380 & 400 & $\mathrm{U}$ & 400 & 8800 & $\mathrm{U}$ & 8800 & 400 & $\mathrm{U}$ & 400 \\
\hline 4-Chloroaniline & 370 & $\mathrm{U}$ & 370 & 380 & $\mathrm{U}$ & 380 & 400 & $\mathrm{U}$ & 400 & 8800 & $\mathrm{U}$ & 8800 & 400 & $\mathrm{U}$ & 400 \\
\hline 4-Chlorophenylphenyl ether & 370 & $\mathrm{U}$ & 370 & 380 & $\mathrm{U}$ & 380 & 400 & $\mathrm{U}$ & 400 & 8800 & $\mathrm{U}$ & 8800 & 400 & $\mathrm{U}$ & 400 \\
\hline 4-Nitroaniline & 920 & $\mathrm{U}$ & 920 & 950 & $\mathrm{U}$ & 950 & 1000 & $\mathrm{U}$ & 1000 & 22000 & $\mathrm{U}$ & 22000 & 1000 & $\mathrm{U}$ & 1000 \\
\hline 4-Nitrophenol & 920 & $\mathrm{U}$ & 920 & 950 & $\mathrm{U}$ & 950 & 1000 & $\mathrm{U}$ & 1000 & 22000 & $\mathrm{U}$ & 22000 & 1000 & $\mathrm{U}$ & 1000 \\
\hline Acenaphthene & 370 & $\mathrm{U}$ & 370 & 380 & $\mathrm{U}$ & 380 & 400 & $\mathrm{U}$ & 400 & 8800 & $\mathrm{U}$ & 8800 & 40 & $\mathrm{~J}$ & 400 \\
\hline Acenaphthylene & 370 & $\mathrm{U}$ & 370 & 380 & $\mathrm{U}$ & 380 & 400 & $\mathrm{U}$ & 400 & 8800 & $\mathrm{U}$ & 8800 & 400 & $\mathrm{U}$ & 400 \\
\hline Anthracene & 370 & $\mathrm{U}$ & 370 & 380 & $\mathrm{U}$ & 380 & 400 & $\mathrm{U}$ & 400 & 8800 & $\mathrm{U}$ & 8800 & 62 & $\mathrm{~J}$ & 400 \\
\hline
\end{tabular}


Table A-2. 128-F-2 In-Process Riverbank Excavation Berm Data (16 Pages)

\begin{tabular}{|c|c|c|c|c|c|c|c|c|c|c|c|c|c|c|c|}
\hline \multirow[t]{2}{*}{ Constituents } & \multicolumn{3}{|c|}{$\begin{array}{c}\text { J13NX7 } \\
\text { Riverbank } \\
\text { Sample Date 11/8/06 } \\
\end{array}$} & \multicolumn{3}{|c|}{$\begin{array}{c}\text { J13NX6 } \\
\text { Riverbank } \\
\text { Sample Date 11/8/06 } \\
\end{array}$} & \multicolumn{3}{|c|}{$\begin{array}{c}\text { J13NX5 } \\
\text { Riverbank } \\
\text { Sample Date 11/8/06 } \\
\end{array}$} & \multicolumn{3}{|c|}{$\begin{array}{c}\text { J13LX4 } \\
\text { Riverbank } \\
\text { Sample Date 11/1/06 } \\
\end{array}$} & \multicolumn{3}{|c|}{$\begin{array}{c}\text { J13LX3 } \\
\text { Riverbank } \\
\text { Sample Date 11/1/06 }\end{array}$} \\
\hline & $\mu \mathrm{g} / \mathrm{kg}$ & $\mathbf{Q}$ & PQL & $\mu \mathrm{g} / \mathrm{kg}$ & $\mathbf{Q}$ & PQL & $\mu \mathrm{g} / \mathrm{kg}$ & $\mathbf{Q}$ & PQL & $\mu \mathrm{g} / \mathrm{kg}$ & $\mathbf{Q}$ & PQL & $\mu \mathrm{g} / \mathrm{kg}$ & $\mathbf{Q}$ & PQL \\
\hline \multicolumn{16}{|c|}{ SVOAs (continued) } \\
\hline Benzo(a)anthracene & 370 & $\mathrm{U}$ & 370 & 380 & $\mathrm{U}$ & 380 & 400 & $\mathrm{U}$ & 400 & 8800 & $\mathrm{U}$ & 8800 & 150 & $\mathrm{~J}$ & 400 \\
\hline Benzo(a)pyrene & 370 & $\mathrm{U}$ & 370 & 380 & $\mathrm{U}$ & 380 & 400 & $\mathrm{U}$ & 400 & 8800 & $\mathrm{U}$ & 8800 & 130 & $\mathrm{~J}$ & 400 \\
\hline Benzo(b)fluoranthene & 370 & $\mathrm{U}$ & 370 & 380 & $\mathrm{U}$ & 380 & 400 & $\mathrm{U}$ & 400 & 8800 & $\mathrm{U}$ & 8800 & 99 & $\mathrm{~J}$ & 400 \\
\hline Benzo(ghi)perylene & 370 & $\mathrm{U}$ & 370 & 380 & $\mathrm{U}$ & 380 & 400 & $\mathrm{U}$ & 400 & 8800 & $\mathrm{U}$ & 8800 & 74 & $\mathrm{~J}$ & 400 \\
\hline Benzo(k)fluoranthene & 370 & $\mathrm{U}$ & 370 & 380 & $\mathrm{U}$ & 380 & 400 & $\mathrm{U}$ & 400 & 8800 & $\mathrm{U}$ & 8800 & 100 & $\mathrm{~J}$ & 400 \\
\hline Bis(2-chloro-1-methylethyl)ether & 370 & $\mathrm{U}$ & 370 & 380 & $\mathrm{U}$ & 380 & 400 & $\mathrm{U}$ & 400 & 8800 & $\mathrm{U}$ & 8800 & 400 & $\mathrm{U}$ & 400 \\
\hline Bis(2-Chloroethoxy)methane & 370 & $\mathrm{U}$ & 370 & 380 & $\mathrm{U}$ & 380 & 400 & $\mathrm{U}$ & 400 & 8800 & $\mathrm{U}$ & 8800 & 400 & $\mathrm{U}$ & 400 \\
\hline Bis(2-chloroethyl) ether & 370 & $\mathrm{U}$ & 370 & 380 & $\mathrm{U}$ & 380 & 400 & $\mathrm{U}$ & 400 & 8800 & $\mathrm{U}$ & 8800 & 400 & $\mathrm{U}$ & 400 \\
\hline Bis(2-ethylhexyl) phthalate & 370 & $\mathrm{U}$ & 370 & 380 & $\mathrm{U}$ & 380 & 23 & $\mathrm{~J}$ & 400 & 8800 & $\mathrm{U}$ & 8800 & 78 & $\mathrm{JB}$ & 400 \\
\hline Butylbenzylphthalate & 370 & $\mathrm{U}$ & 370 & 380 & $\mathrm{U}$ & 380 & 400 & $\mathrm{U}$ & 400 & 8800 & $\mathrm{U}$ & 8800 & 400 & $\mathrm{U}$ & 400 \\
\hline Carbazole & 370 & $\mathrm{U}$ & 370 & 380 & $\mathrm{U}$ & 380 & 400 & $\mathrm{U}$ & 400 & 8800 & $\mathrm{U}$ & 8800 & 25 & $\mathrm{~J}$ & 400 \\
\hline Chrysene & 370 & $\mathrm{U}$ & 370 & 380 & $\mathrm{U}$ & 380 & 400 & $\mathrm{U}$ & 400 & 8800 & $\mathrm{U}$ & 8800 & 180 & $\mathrm{~J}$ & 400 \\
\hline Di-n-butylphthalate & 370 & $\mathrm{U}$ & 370 & 380 & $\mathrm{U}$ & 380 & 400 & $\mathrm{U}$ & 400 & 8800 & $\mathrm{U}$ & 8800 & 400 & $\mathrm{U}$ & 400 \\
\hline Di-n-octylphthalate & 370 & $\mathrm{U}$ & 370 & 380 & $\mathrm{U}$ & 380 & 400 & $\mathrm{U}$ & 400 & 8800 & $\mathrm{U}$ & 8800 & 400 & $\mathrm{U}$ & 400 \\
\hline Dibenz[a,h]anthracene & 370 & $\mathrm{U}$ & 370 & 380 & $\mathrm{U}$ & 380 & 400 & $\mathrm{U}$ & 400 & 8800 & $\mathrm{U}$ & 8800 & 400 & $\mathrm{U}$ & 400 \\
\hline \begin{tabular}{|l|} 
Dibenzofuran \\
\end{tabular} & 370 & $\mathrm{U}$ & 370 & 380 & $\mathrm{U}$ & 380 & 400 & $\mathrm{U}$ & 400 & 8800 & $\mathrm{U}$ & 8800 & 400 & $\mathrm{U}$ & 400 \\
\hline Diethylphthalate & 370 & $\mathrm{U}$ & 370 & 380 & $\mathrm{U}$ & 380 & 400 & $\mathrm{U}$ & 400 & 8800 & $\mathrm{U}$ & 8800 & 400 & $\mathrm{U}$ & 400 \\
\hline Dimethyl phthalate & 370 & $\mathrm{U}$ & 370 & 380 & $\mathrm{U}$ & 380 & 400 & $\mathrm{U}$ & 400 & 8800 & $\mathrm{U}$ & 8800 & 400 & $\mathrm{U}$ & 400 \\
\hline \begin{tabular}{|l|} 
Fluoranthene \\
\end{tabular} & 370 & $\mathrm{U}$ & 370 & 380 & $\mathrm{U}$ & 380 & 400 & $\mathrm{U}$ & 400 & 8800 & $\mathrm{U}$ & 8800 & 350 & $\mathrm{~J}$ & 400 \\
\hline Fluorene & 370 & $\mathrm{U}$ & 370 & 380 & $\mathrm{U}$ & 380 & 400 & $\mathrm{U}$ & 400 & 8800 & $\mathrm{U}$ & 8800 & 24 & $\mathrm{~J}$ & 400 \\
\hline Hexachlorobenzene & 370 & $\mathrm{U}$ & 370 & 380 & $\mathrm{U}$ & 380 & 400 & $\mathrm{U}$ & 400 & 8800 & $\mathrm{U}$ & 8800 & 400 & $\mathrm{U}$ & 400 \\
\hline Hexachlorobutadiene & 370 & $\mathrm{U}$ & 370 & 380 & $\mathrm{U}$ & 380 & 400 & $\mathrm{U}$ & 400 & 8800 & $\mathrm{U}$ & 8800 & 400 & $\mathrm{U}$ & 400 \\
\hline Hexachlorocyclopentadiene & 370 & $\mathrm{U}$ & 370 & 380 & $\mathrm{U}$ & 380 & 400 & $\mathrm{U}$ & 400 & 8800 & $\mathrm{U}$ & 8800 & 400 & $\mathrm{U}$ & 400 \\
\hline Hexachloroethane & 370 & $\mathrm{U}$ & 370 & 380 & $\mathrm{U}$ & 380 & 400 & $\mathrm{U}$ & 400 & 8800 & $\mathrm{U}$ & 8800 & 400 & $\mathrm{U}$ & 400 \\
\hline Indeno(1,2,3-cd)pyrene & 370 & $\mathrm{U}$ & 370 & 380 & $\mathrm{U}$ & 380 & 400 & $\mathrm{U}$ & 400 & 8800 & $\mathrm{U}$ & 8800 & 71 & $\mathrm{~J}$ & 400 \\
\hline Isophorone & 370 & $\mathrm{U}$ & 370 & 380 & $\mathrm{U}$ & 380 & 400 & $\mathrm{U}$ & 400 & 8800 & $\mathrm{U}$ & 8800 & 400 & $\mathrm{U}$ & 400 \\
\hline N-Nitroso-di-n-dipropylamine & 370 & $\mathrm{U}$ & 370 & 380 & $\mathrm{U}$ & 380 & 400 & $\mathrm{U}$ & 400 & 8800 & $\mathrm{U}$ & 8800 & 400 & $\mathrm{U}$ & 400 \\
\hline N-Nitrosodiphenylamine & 370 & $\mathrm{U}$ & 370 & 380 & $\mathrm{U}$ & 380 & 400 & $\mathrm{U}$ & 400 & 8800 & $\mathrm{U}$ & 8800 & 400 & $\mathrm{U}$ & 400 \\
\hline Naphthalene & 370 & $\mathrm{U}$ & 370 & 380 & $\mathrm{U}$ & 380 & 400 & $\mathrm{U}$ & 400 & 8800 & $\mathrm{U}$ & 8800 & 400 & $\mathrm{U}$ & 400 \\
\hline Nitrobenzene & 370 & $\mathrm{U}$ & 370 & 380 & $\mathrm{U}$ & 380 & 400 & $\mathrm{U}$ & 400 & 8800 & $\mathrm{U}$ & 8800 & 400 & $\mathrm{U}$ & 400 \\
\hline Pentachlorophenol & 920 & $\mathrm{U}$ & 920 & 950 & $\mathrm{U}$ & 950 & 1000 & $\mathrm{U}$ & 1000 & 22000 & $\mathrm{U}$ & 22000 & 1000 & $\mathrm{U}$ & 1000 \\
\hline \begin{tabular}{|l|} 
Phenanthrene \\
\end{tabular} & 370 & $\mathrm{U}$ & 370 & 380 & $\mathrm{U}$ & 380 & 400 & $\mathrm{U}$ & 400 & 8800 & $\mathrm{U}$ & 8800 & 290 & $\mathrm{~J}$ & 400 \\
\hline Phenol & 370 & $\mathrm{U}$ & 370 & 380 & $\mathrm{U}$ & 380 & 400 & $\mathrm{U}$ & 400 & 8800 & $\mathrm{U}$ & 8800 & 400 & $\mathrm{U}$ & 400 \\
\hline Pyrene & 370 & $\mathrm{U}$ & 370 & 380 & $\mathrm{U}$ & 380 & 400 & $\mathrm{U}$ & 400 & 8800 & $\mathrm{U}$ & 8800 & 350 & $\mathrm{~J}$ & 400 \\
\hline \multicolumn{16}{|c|}{ Pesticides } \\
\hline Alpha-BHC & 1.5 & $\mathrm{U}$ & 1.5 & 1.5 & $\mathrm{U}$ & 1.5 & 1.6 & $\mathrm{U}$ & 1.6 & 1.8 & $\mathrm{U}$ & 1.8 & 1.6 & $\mathrm{U}$ & 1.6 \\
\hline gamma-BHC (Lindane) & 1.5 & $\mathrm{U}$ & 1.5 & 1.5 & $\mathrm{U}$ & 1.5 & 1.6 & $\mathrm{U}$ & 1.6 & 1.8 & $\mathrm{U}$ & 1.8 & 1.6 & $\mathrm{U}$ & 1.6 \\
\hline Beta-BHC & 1.5 & $\mathrm{U}$ & 1.5 & 1.5 & $\mathrm{U}$ & 1.5 & 1.6 & $\mathrm{U}$ & 1.6 & 2.5 & $\mathrm{I}$ & 1.8 & 1.6 & $\mathrm{U}$ & 1.6 \\
\hline
\end{tabular}


Table A-2. 128-F-2 In-Process Riverbank Excavation Berm Data (16 Pages)

\begin{tabular}{|c|c|c|c|c|c|c|c|c|c|c|c|c|c|c|c|}
\hline \multirow[t]{2}{*}{ Constituents } & \multicolumn{3}{|c|}{$\begin{array}{c}\text { J13NX7 } \\
\text { Riverbank } \\
\text { Sample Date 11/8/06 }\end{array}$} & \multicolumn{3}{|c|}{$\begin{array}{c}\text { J13NX6 } \\
\text { Riverbank } \\
\text { Sample Date 11/8/06 }\end{array}$} & \multicolumn{3}{|c|}{$\begin{array}{c}\text { J13NX5 } \\
\text { Riverbank } \\
\text { Sample Date 11/8/06 }\end{array}$} & \multicolumn{3}{|c|}{$\begin{array}{c}\text { J13LX4 } \\
\text { Riverbank } \\
\text { Sample Date 11/1/06 }\end{array}$} & \multicolumn{3}{|c|}{$\begin{array}{c}\text { J13LX3 } \\
\text { Riverbank } \\
\text { Sample Date 11/1/06 } \\
\end{array}$} \\
\hline & $\mu \mathrm{g} / \mathrm{kg}$ & $\mathbf{Q}$ & PQL & $\mu \mathrm{g} / \mathrm{kg}$ & $\mathbf{Q}$ & PQL & $\mu \mathrm{g} / \mathrm{kg}$ & $\mathbf{Q}$ & PQL & $\mu \mathrm{g} / \mathrm{kg}$ & $\mathbf{Q}$ & PQL & $\mu \mathrm{g} / \mathrm{kg}$ & $\mathbf{Q}$ & $\overline{\text { PQL }}$ \\
\hline \multicolumn{16}{|c|}{ Pesticides (continued) } \\
\hline Heptachlor & 1.5 & $\mathrm{U}$ & 1.5 & 1.5 & $\mathrm{U}$ & 1.5 & 1.6 & $\mathrm{U}$ & 1.6 & 1.8 & $\mathrm{U}$ & 1.8 & 1.6 & $\mathrm{U}$ & 1.6 \\
\hline Delta-BHC & 1.5 & $\mathrm{U}$ & 1.5 & 1.5 & $\mathrm{U}$ & 1.5 & 1.6 & $\mathrm{U}$ & 1.6 & 1.8 & $\mathrm{U}$ & 1.8 & 1.6 & $\mathrm{U}$ & 1.6 \\
\hline Aldrin & 1.5 & $\mathrm{U}$ & 1.5 & 1.5 & $\mathrm{U}$ & 1.5 & 1.6 & $\bar{U}$ & 1.6 & 2.3 & & 1.8 & 1.6 & $\bar{U}$ & 1.6 \\
\hline Heptachlor epoxide & 1.5 & $\mathrm{U}$ & 1.5 & 1.5 & $\mathrm{U}$ & 1.5 & 1.6 & $\mathrm{U}$ & 1.6 & 1.8 & $\mathrm{U}$ & 1.8 & 1.6 & $\mathrm{U}$ & 1.6 \\
\hline \begin{tabular}{|l|} 
Endosulfan I \\
\end{tabular} & 1.5 & $\mathrm{U}$ & 1.5 & 1.5 & $\mathrm{U}$ & 1.5 & 1.6 & $\mathrm{U}$ & 1.6 & 1.8 & $\mathrm{U}$ & 1.8 & 1.6 & $\mathrm{U}$ & 1.6 \\
\hline alpha-Chlordane & 1.5 & $\mathrm{U}$ & 1.5 & 1.5 & $\mathrm{U}$ & 1.5 & 1.6 & $\mathrm{U}$ & 1.6 & 1.8 & $\mathrm{U}$ & 1.8 & 1.6 & $\mathrm{U}$ & 1.6 \\
\hline 4,4'-DDE & 1.5 & $\mathrm{U}$ & 1.5 & 1.5 & $\mathrm{U}$ & 1.5 & 1.6 & $\mathrm{U}$ & 1.6 & 4.2 & & 1.8 & 1.6 & $\mathrm{U}$ & 1.6 \\
\hline Deildrin & 1.5 & $\mathrm{U}$ & 1.5 & 1.5 & $\mathrm{U}$ & 1.5 & 1.6 & $\mathrm{U}$ & 1.6 & 1.8 & $\mathrm{U}$ & 1.8 & 1.6 & $\mathrm{U}$ & 1.6 \\
\hline Endrin & 1.5 & $\mathrm{U}$ & 1.5 & 1.5 & $\mathrm{U}$ & 1.5 & 1.6 & $\mathrm{U}$ & 1.6 & 1.8 & $\mathrm{U}$ & 1.8 & 1.6 & $\mathrm{U}$ & 1.6 \\
\hline 4,4'-DDD & 1.5 & $\mathrm{U}$ & 1.5 & 1.5 & $\mathrm{U}$ & 1.5 & 1.6 & $\mathrm{U}$ & 1.6 & 2.5 & & 1.8 & 1.6 & $\mathrm{U}$ & 1.6 \\
\hline Endosulfan II & 1.5 & $\mathrm{U}$ & 1.5 & 1.5 & $\mathrm{U}$ & 1.5 & 1.6 & $\mathrm{U}$ & 1.6 & 1.8 & $\mathrm{U}$ & 1.8 & 1.6 & $\mathrm{U}$ & 1.6 \\
\hline 4,4'-DDT & 1.5 & $\mathrm{U}$ & 1.5 & 1.5 & $\mathrm{U}$ & 1.5 & 1.6 & $\mathrm{U}$ & 1.6 & 1.8 & $\mathrm{U}$ & 1.8 & 1.6 & $\mathrm{U}$ & 1.6 \\
\hline Endrin aldehyde & 2.0 & & 1.5 & 1.5 & $\mathrm{U}$ & 1.5 & 1.6 & $\mathrm{U}$ & 1.6 & 8.9 & & 1.8 & 1.6 & $\mathrm{U}$ & 1.6 \\
\hline Methoxychlor & 2.4 & & 1.5 & 1.5 & $\mathrm{U}$ & 1.5 & 1.6 & $\mathrm{U}$ & 1.6 & 3.8 & I & 1.8 & 1.6 & $\mathrm{U}$ & 1.6 \\
\hline \begin{tabular}{|l|} 
Endrin ketone \\
\end{tabular} & 1.5 & $\mathrm{U}$ & 1.5 & 1.5 & $\mathrm{U}$ & 1.5 & 1.6 & $\mathrm{U}$ & 1.6 & 1.8 & $\mathrm{U}$ & 1.8 & 1.6 & $\mathrm{U}$ & 1.6 \\
\hline Toxaphene & 15 & $\mathrm{U}$ & 15 & 15 & $\mathrm{U}$ & 15 & 16 & $\mathrm{U}$ & 16 & 18 & $\mathrm{U}$ & 18 & 16 & $\mathrm{U}$ & 16 \\
\hline \multicolumn{16}{|c|}{ PAHs (Polycyclic Aromatic Hydrocarbons) } \\
\hline Naphthalene & 36.8 & $\mathrm{U}$ & 36.8 & 38.2 & $\mathrm{U}$ & 38.2 & 40.5 & $\mathrm{U}$ & 40.5 & 44.2 & $\mathrm{U}$ & 44.2 & 40.0 & $\mathrm{U}$ & 40.0 \\
\hline Acenaphthylene & 36.8 & $\mathrm{U}$ & 36.8 & 38.2 & $\mathrm{U}$ & 38.2 & 40.5 & $\mathrm{U}$ & 40.5 & 44.2 & $\mathrm{U}$ & 44.2 & 40.0 & $\mathrm{U}$ & 40.0 \\
\hline Acenaphthene & 36.8 & $\mathrm{U}$ & 36.8 & 38.2 & $\mathrm{U}$ & 38.2 & 25 & $\mathrm{~J}$ & 40.5 & 44.2 & $\mathrm{U}$ & 44.2 & 40.0 & $\mathrm{U}$ & 40.0 \\
\hline Fluorene & 3.68 & $\mathrm{U}$ & 3.68 & 3.82 & $\mathrm{U}$ & 3.82 & 4.05 & $\mathrm{U}$ & 4.05 & 39 & & 4.42 & 18 & & 4.0 \\
\hline Phenanthrene & 2.6 & $\mathrm{~J}$ & 3.68 & 3.8 & $\mathrm{~J}$ & 3.82 & 2.3 & $\mathrm{~J}$ & 4.05 & 49 & & 4.42 & 160 & & 4.0 \\
\hline Anthracene & 3.68 & $\mathrm{U}$ & 3.68 & 3.82 & $\mathrm{U}$ & 3.82 & 2.0 & $\mathrm{~J}$ & 4.05 & 6.4 & & 4.42 & 31 & & 4.0 \\
\hline Fluoranthrene & 2.0 & $\mathrm{~J}$ & 3.68 & 3.82 & $\mathrm{U}$ & 3.82 & 10 & $\mathrm{~J}$ & 4.05 & 120 & & 4.42 & 230 & & 4.0 \\
\hline Indeno (1,2,3-cd)pyrene & 3.68 & $\mathrm{U}$ & 3.68 & 3.82 & $\mathrm{U}$ & 3.82 & 4.05 & $\mathrm{U}$ & 4.05 & 4.42 & $\mathrm{U}$ & 4.42 & 83 & & 4.0 \\
\hline Pyrene & 3.68 & $\mathrm{U}$ & 3.68 & 3.82 & $\mathrm{U}$ & 3.82 & 5.5 & & 4.05 & 38 & & 4.42 & 210 & & 4.0 \\
\hline Benzo(a)anthracene & 3.68 & $\mathrm{U}$ & 3.68 & 3.82 & $\mathrm{U}$ & 3.82 & 1.6 & $\mathrm{~J}$ & 4.05 & 4.42 & $\mathrm{U}$ & 4.42 & 90 & & 4.0 \\
\hline Chrysene & 3.68 & $\mathrm{U}$ & 3.68 & 2.8 & $\mathrm{~J}$ & 3.82 & 2.0 & $\mathrm{~J}$ & 4.05 & 20 & & 4.42 & 90 & & 4.0 \\
\hline Benzo(b)fluoranthrene & 3.68 & $\mathrm{U}$ & 3.68 & 3.82 & $\mathrm{U}$ & 3.82 & 1.1 & $\mathrm{~J}$ & 4.05 & 34 & & 4.42 & 58 & & 4.0 \\
\hline Benzo(k)fluoranthrene & 3.68 & $\mathrm{U}$ & 3.68 & 3.82 & $\mathrm{U}$ & 3.82 & 4.05 & $\mathrm{U}$ & 4.05 & 12 & & 4.42 & 41 & & 4.0 \\
\hline
\end{tabular}


Table A-2. 128-F-2 In-Process Riverbank Excavation Berm Data (16 Pages)

\begin{tabular}{|c|c|c|c|c|c|c|c|c|c|c|c|c|c|c|c|}
\hline \multirow[t]{2}{*}{ Constituents } & \multicolumn{3}{|c|}{$\begin{array}{c}\text { J13LX2 } \\
\text { Riverbank } \\
\text { Sample Date 11/1/06 }\end{array}$} & \multicolumn{3}{|c|}{$\begin{array}{c}\text { J13LX1 } \\
\text { Riverbank } \\
\text { Sample Date 11/1/06 }\end{array}$} & \multicolumn{3}{|c|}{$\begin{array}{c}\text { J13LX0 } \\
\text { Riverbank } \\
\text { Sample Date 11/1/06 }\end{array}$} & \multicolumn{3}{|c|}{$\begin{array}{c}\text { J13LP5 } \\
\text { Riverbank } \\
\text { Sample Date 10/26/06 }\end{array}$} & \multicolumn{3}{|c|}{$\begin{array}{c}\text { J13LP4 } \\
\text { Riverbank } \\
\text { Sample Date 10/26/06 } \\
\end{array}$} \\
\hline & $\mu \mathrm{g} / \mathrm{kg}$ & $\mathbf{Q}$ & PQL & $\mu \mathrm{g} / \mathrm{kg}$ & $\mathbf{Q}$ & PQL & $\mu \mathrm{g} / \mathrm{kg}$ & $\mathbf{Q}$ & PQL & $\mu \mathrm{g} / \mathrm{kg}$ & $\mathbf{Q}$ & PQL & $\mu \mathrm{g} / \mathrm{kg}$ & $\mathbf{Q}$ & PQL \\
\hline \multicolumn{16}{|c|}{ PCBs } \\
\hline \begin{tabular}{|l|} 
Aroclor-1016 \\
\end{tabular} & 17 & $\mathrm{U}$ & 17 & 18 & $\mathrm{U}$ & 18 & 17 & $\mathrm{U}$ & 17 & 15 & $\mathrm{U}$ & 14 & 16 & $\mathrm{U}$ & 16 \\
\hline \begin{tabular}{|l|} 
Aroclor-1221 \\
\end{tabular} & 17 & $\mathrm{U}$ & 17 & 18 & $\mathrm{U}$ & 18 & 17 & $\mathrm{U}$ & 17 & 15 & $\mathrm{U}$ & 14 & 16 & $\mathrm{U}$ & 16 \\
\hline \begin{tabular}{|l|} 
Aroclor-1232 \\
\end{tabular} & 17 & $\mathrm{U}$ & 17 & 18 & $\mathrm{U}$ & 18 & 17 & $\mathrm{U}$ & 17 & 15 & $\mathrm{U}$ & 14 & 16 & $\mathrm{U}$ & 16 \\
\hline \begin{tabular}{|l|} 
Aroclor-1242 \\
\end{tabular} & 17 & $\mathrm{U}$ & 17 & 18 & $\mathrm{U}$ & 18 & 17 & $\mathrm{U}$ & 17 & 15 & $\mathrm{U}$ & 14 & 16 & $\mathrm{U}$ & 16 \\
\hline \begin{tabular}{|l|} 
Aroclor-1248 \\
\end{tabular} & 17 & $\mathrm{U}$ & 17 & 18 & $\mathrm{U}$ & 18 & 17 & $\mathrm{U}$ & 17 & 15 & $\mathrm{U}$ & 14 & 16 & $\mathrm{U}$ & 16 \\
\hline \begin{tabular}{|l|} 
Aroclor-1254 \\
\end{tabular} & 17 & $\mathrm{U}$ & 17 & 18 & $\mathrm{U}$ & 18 & 17 & $\mathrm{U}$ & 17 & 15 & $\mathrm{U}$ & 14 & 16 & $\mathrm{U}$ & 16 \\
\hline \begin{tabular}{|l|} 
Aroclor-1260 \\
\end{tabular} & 17 & $\mathrm{U}$ & 17 & 18 & $\mathrm{U}$ & 18 & 17 & $\mathrm{U}$ & 17 & 15 & $\mathrm{U}$ & 14 & 16 & $\mathrm{U}$ & 16 \\
\hline \multicolumn{16}{|c|}{ SVOAs } \\
\hline 1,2,4-Trichlorobenzene & 8600 & $\mathrm{U}$ & 8600 & 9100 & $\mathrm{U}$ & 9100 & 430 & $\mathrm{U}$ & 430 & 370 & $\mathrm{U}$ & 370 & 390 & $\mathrm{U}$ & 390 \\
\hline 1,2-Dichlorobenzene & 8600 & $\mathrm{U}$ & 8600 & 9100 & $\mathrm{U}$ & 9100 & 430 & $\mathrm{U}$ & 430 & 370 & $\mathrm{U}$ & 370 & 390 & $\mathrm{U}$ & 390 \\
\hline 1,3-Dichlorobenzene & 8600 & $\mathrm{U}$ & 8600 & 9100 & $\mathrm{U}$ & 9100 & 430 & $\mathrm{U}$ & 430 & 370 & $\mathrm{U}$ & 370 & 390 & $\mathrm{U}$ & 390 \\
\hline 1,4-Dichlorobenzene & 8600 & $\mathrm{U}$ & 8600 & 9100 & $\mathrm{U}$ & 9100 & 430 & $\mathrm{U}$ & 430 & 370 & $\mathrm{U}$ & 370 & 390 & $\mathrm{U}$ & 390 \\
\hline 2,4,5-Trichlorophenol & 22000 & $\mathrm{U}$ & 22000 & 23000 & $\mathrm{U}$ & 23000 & 1100 & $\mathrm{U}$ & 1100 & 920 & $\mathrm{U}$ & 920 & 970 & $\mathrm{U}$ & 970 \\
\hline 2,4,6-Trichlorophenol & 8600 & $\mathrm{U}$ & 8600 & 9100 & $\mathrm{U}$ & 9100 & 430 & $\mathrm{U}$ & 430 & 370 & $\mathrm{U}$ & 370 & 390 & $\mathrm{U}$ & 390 \\
\hline 2,4-Dichlorophenol & 8600 & $\mathrm{U}$ & 8600 & 9100 & $\mathrm{U}$ & 9100 & 430 & $\mathrm{U}$ & 430 & 370 & $\mathrm{U}$ & 370 & 390 & $\mathrm{U}$ & 390 \\
\hline 2,4-Dimethylphenol & 8600 & $\mathrm{U}$ & 8600 & 9100 & $\mathrm{U}$ & 9100 & 430 & $\mathrm{U}$ & 430 & 370 & $\mathrm{U}$ & 370 & 390 & $\mathrm{U}$ & 390 \\
\hline 2,4-Dinitrophenol & 22000 & $\mathrm{U}$ & 22000 & 23000 & $\mathrm{U}$ & 23000 & 1100 & $\mathrm{U}$ & 1100 & 920 & $\mathrm{U}$ & 920 & 970 & $\mathrm{U}$ & 970 \\
\hline 2,4-Dinitrotoluene & 8600 & $\mathrm{U}$ & 8600 & 9100 & $\mathrm{U}$ & 9100 & 430 & $\mathrm{U}$ & 430 & 370 & $\mathrm{U}$ & 370 & 390 & $\mathrm{U}$ & 390 \\
\hline 2,6-Dinitrotoluene & 8600 & $\mathrm{U}$ & 8600 & 9100 & $\mathrm{U}$ & 9100 & 430 & $\mathrm{U}$ & 430 & 370 & $\mathrm{U}$ & 370 & 390 & $\mathrm{U}$ & 390 \\
\hline 2-Chloronaphthalene & 8600 & $\mathrm{U}$ & 8600 & 9100 & $\mathrm{U}$ & 9100 & 430 & $\mathrm{U}$ & 430 & 370 & $\mathrm{U}$ & 370 & 390 & $\mathrm{U}$ & 390 \\
\hline 2-Chlorophenol & 8600 & $\mathrm{U}$ & 8600 & 9100 & $\mathrm{U}$ & 9100 & 430 & $\mathrm{U}$ & 430 & 370 & $\mathrm{U}$ & 370 & 390 & $\mathrm{U}$ & 390 \\
\hline 2-Methylnaphthalene & 8600 & $\mathrm{U}$ & 8600 & 9100 & $\mathrm{U}$ & 9100 & 430 & $\mathrm{U}$ & 430 & 370 & $\mathrm{U}$ & 370 & 390 & $\mathrm{U}$ & 390 \\
\hline 2-Methylphenol (cresol, o-) & 8600 & $\mathrm{U}$ & 8600 & 9100 & $\mathrm{U}$ & 9100 & 430 & $\mathrm{U}$ & 430 & 370 & $\mathrm{U}$ & 370 & 390 & $\mathrm{U}$ & 390 \\
\hline 2-Nitroaniline & 22000 & $\mathrm{U}$ & 22000 & 23000 & $\mathrm{U}$ & 23000 & 1100 & $\mathrm{U}$ & 1100 & 920 & $\mathrm{U}$ & 920 & 970 & $\mathrm{U}$ & 970 \\
\hline 2-Nitrophenol & 8600 & $\mathrm{U}$ & 8600 & 9100 & $\mathrm{U}$ & 9100 & 430 & $\mathrm{U}$ & 430 & 370 & $\mathrm{U}$ & 370 & 390 & $\mathrm{U}$ & 390 \\
\hline 3+4 Methylphenol (cresol, $\mathrm{m}+\mathrm{p})$ & 8600 & $\mathrm{U}$ & 8600 & 9100 & $\mathrm{U}$ & 9100 & 430 & $\mathrm{U}$ & 430 & 370 & $\mathrm{U}$ & 370 & 390 & $\mathrm{U}$ & 390 \\
\hline 3,3'-Dichlorobenzidine & 8600 & $\mathrm{U}$ & 8600 & 9100 & $\mathrm{U}$ & 9100 & 430 & $\mathrm{U}$ & 430 & 370 & $\mathrm{U}$ & 370 & 390 & $\mathrm{U}$ & 390 \\
\hline 3-Nitroaniline & 22000 & $\mathrm{U}$ & 22000 & 23000 & $\mathrm{U}$ & 23000 & 1100 & $\mathrm{U}$ & 1100 & 920 & $\mathrm{U}$ & 920 & 970 & $\mathrm{U}$ & 970 \\
\hline 4,6-Dinitro-2-methylphenol & 22000 & $\mathrm{U}$ & 22000 & 23000 & $\mathrm{U}$ & 23000 & 1100 & $\mathrm{U}$ & 1100 & 920 & $\mathrm{U}$ & 920 & 970 & $\mathrm{U}$ & 970 \\
\hline 4-Bromophenylphenyl ether & 8600 & $\mathrm{U}$ & 8600 & 9100 & $\mathrm{U}$ & 9100 & 430 & $\mathrm{U}$ & 430 & 370 & $\mathrm{U}$ & 370 & 390 & $\mathrm{U}$ & 390 \\
\hline 4-Chloro-3-methylphenol & 8600 & $\mathrm{U}$ & 8600 & 9100 & $\mathrm{U}$ & 9100 & 430 & $\mathrm{U}$ & 430 & 370 & $\mathrm{U}$ & 370 & 390 & $\mathrm{U}$ & 390 \\
\hline 4-Chloroaniline & 8600 & $\mathrm{U}$ & 8600 & 9100 & $\mathrm{U}$ & 9100 & 430 & $\mathrm{U}$ & 430 & 370 & $\mathrm{U}$ & 370 & 390 & $\mathrm{U}$ & 390 \\
\hline 4-Chlorophenylphenyl ether & 8600 & $\mathrm{U}$ & 8600 & 9100 & $\mathrm{U}$ & 9100 & 430 & $\mathrm{U}$ & 430 & 370 & $\mathrm{U}$ & 370 & 390 & $\mathrm{U}$ & 390 \\
\hline 4-Nitroaniline & 22000 & $\mathrm{U}$ & 22000 & 23000 & $\mathrm{U}$ & 23000 & 1100 & $\mathrm{U}$ & 1100 & 920 & $\mathrm{U}$ & 920 & 970 & $\mathrm{U}$ & 970 \\
\hline 4-Nitrophenol & 22000 & $\mathrm{U}$ & 22000 & 23000 & $\mathrm{U}$ & 23000 & 1100 & $\mathrm{U}$ & 1100 & 920 & $\mathrm{U}$ & 920 & 970 & $\mathrm{U}$ & 970 \\
\hline Acenaphthene & 8600 & $\mathrm{U}$ & 8600 & 9100 & $\mathrm{U}$ & 9100 & 430 & $\mathrm{U}$ & 430 & 370 & $\mathrm{U}$ & 370 & 390 & $\mathrm{U}$ & 390 \\
\hline Acenaphthylene & 8600 & $\mathrm{U}$ & 8600 & 9100 & $\mathrm{U}$ & 9100 & 430 & $\mathrm{U}$ & 430 & 370 & $\mathrm{U}$ & 370 & 390 & $\mathrm{U}$ & 390 \\
\hline Anthracene & 8600 & $\mathrm{U}$ & 8600 & 9100 & $\mathrm{U}$ & 9100 & 430 & $\mathrm{U}$ & 430 & 370 & $\mathrm{U}$ & 370 & 390 & $\mathrm{U}$ & 390 \\
\hline
\end{tabular}


Table A-2. 128-F-2 In-Process Riverbank Excavation Berm Data (16 Pages)

\begin{tabular}{|c|c|c|c|c|c|c|c|c|c|c|c|c|c|c|c|}
\hline \multirow[t]{2}{*}{ Constituents } & \multicolumn{3}{|c|}{$\begin{array}{c}\text { J13LX2 } \\
\text { Riverbank } \\
\text { Sample Date 11/1/06 }\end{array}$} & \multicolumn{3}{|c|}{$\begin{array}{c}\text { J13LX1 } \\
\text { Riverbank } \\
\text { Sample Date 11/1/06 }\end{array}$} & \multicolumn{3}{|c|}{$\begin{array}{c}\text { J13LX0 } \\
\text { Riverbank } \\
\text { Sample Date 11/1/06 }\end{array}$} & \multicolumn{3}{|c|}{$\begin{array}{c}\text { J13LP5 } \\
\text { Riverbank } \\
\text { Sample Date 10/26/06 }\end{array}$} & \multicolumn{3}{|c|}{$\begin{array}{c}\text { J13LP4 } \\
\text { Riverbank } \\
\text { Sample Date 10/26/06 }\end{array}$} \\
\hline & $\mu \mathrm{g} / \mathrm{kg}$ & $\mathbf{Q}$ & PQL & $\mu \mathrm{g} / \mathrm{kg}$ & $\bar{Q}$ & PQL & $\mu g / \mathbf{k g}$ & $\mathbf{Q}$ & PQL & $\mu \mathrm{g} / \mathrm{kg}$ & $\mathbf{Q}$ & PQL & $\mu \mathrm{g} / \mathrm{kg}$ & $\mathbf{Q}$ & PQL \\
\hline \multicolumn{16}{|c|}{ SVOAs (continued) } \\
\hline Benzo(a)anthracene & 8600 & $\mathrm{U}$ & 8600 & 9100 & $\mathrm{U}$ & 9100 & 430 & $\mathrm{U}$ & 430 & 370 & $\mathrm{U}$ & 370 & 390 & $\mathrm{U}$ & 390 \\
\hline Benzo(a)pyrene & 8600 & $\mathrm{U}$ & 8600 & 9100 & $\bar{U}$ & 9100 & 430 & $\mathrm{U}$ & 430 & 370 & $\mathrm{U}$ & 370 & 390 & $\mathrm{U}$ & 390 \\
\hline Benzo(b)fluoranthene & 8600 & $\mathrm{U}$ & 8600 & 9100 & $\bar{U}$ & 9100 & 430 & $\mathrm{U}$ & 430 & 370 & $\mathrm{U}$ & 370 & 390 & $\mathrm{U}$ & 390 \\
\hline Benzo(ghi)perylene & 8600 & $\mathrm{U}$ & 8600 & 9100 & $\bar{U}$ & 9100 & 430 & $\mathrm{U}$ & 430 & 370 & $\mathrm{U}$ & 370 & 390 & $\mathrm{U}$ & 390 \\
\hline Benzo(k)fluoranthene & 8600 & $\mathrm{U}$ & 8600 & 9100 & $\bar{U}$ & 9100 & 430 & $\mathrm{U}$ & 430 & 370 & $\mathrm{U}$ & 370 & 390 & $\mathrm{U}$ & 390 \\
\hline Bis(2-chloro-1-methylethyl)ether & 8600 & $\mathrm{U}$ & 8600 & 9100 & $\bar{U}$ & 9100 & 430 & $\mathrm{U}$ & 430 & 370 & $\mathrm{U}$ & 370 & 390 & $\mathrm{U}$ & 390 \\
\hline Bis(2-Chloroethoxy)methane & 8600 & $\mathrm{U}$ & 8600 & 9100 & $\overline{\mathrm{U}}$ & 9100 & 430 & $\mathrm{U}$ & 430 & 370 & $\mathrm{U}$ & 370 & 390 & $\mathrm{U}$ & 390 \\
\hline Bis(2-chloroethyl) ether & 8600 & $\mathrm{U}$ & 8600 & 9100 & $\bar{U}$ & 9100 & 430 & $\mathrm{U}$ & 430 & 370 & $\mathrm{U}$ & 370 & 390 & $\mathrm{U}$ & 390 \\
\hline Bis(2-ethylhexyl) phthalate & 8600 & $\mathrm{U}$ & 8600 & 9100 & $\mathrm{U}$ & 9100 & 160 & JB & 430 & 69 & $\mathrm{JB}$ & 370 & 71 & JB & 390 \\
\hline Butylbenzylphthalate & 8600 & $\mathrm{U}$ & 8600 & 9100 & $\bar{U}$ & 9100 & 430 & $\mathrm{U}$ & 430 & 370 & $\mathrm{U}$ & 370 & 390 & $\mathrm{U}$ & 390 \\
\hline Carbazole & 8600 & $\mathrm{U}$ & 8600 & 9100 & $\overline{\mathrm{U}}$ & 9100 & 430 & $\mathrm{U}$ & 430 & 370 & $\mathrm{U}$ & 370 & 390 & $\mathrm{U}$ & 390 \\
\hline Chrysene & 8600 & $\mathrm{U}$ & 8600 & 9100 & $\bar{U}$ & 9100 & 430 & $\mathrm{U}$ & 430 & 29 & $\mathrm{~J}$ & 370 & 390 & $\mathrm{U}$ & 390 \\
\hline Di-n-butylphthalate & 8600 & $\mathrm{U}$ & 8600 & 9100 & $\bar{U}$ & 9100 & 43 & $\mathrm{~J}$ & 430 & 45 & JB & 370 & 46 & JB & 390 \\
\hline Di-n-octylphthalate & 8600 & $\mathrm{U}$ & 8600 & 9100 & $\bar{U}$ & 9100 & 430 & $\mathrm{U}$ & 430 & 370 & $\mathrm{U}$ & 370 & 390 & $\mathrm{U}$ & 390 \\
\hline Dibenz[a,h]anthracene & 8600 & $\mathrm{U}$ & 8600 & 9100 & $\bar{U}$ & 9100 & 430 & $\mathrm{U}$ & 430 & 370 & $\mathrm{U}$ & 370 & 390 & $\mathrm{U}$ & 390 \\
\hline Dibenzofuran & 8600 & $\mathrm{U}$ & 8600 & 9100 & $\bar{U}$ & 9100 & 430 & $\mathrm{U}$ & 430 & 370 & $\mathrm{U}$ & 370 & 390 & $\mathrm{U}$ & 390 \\
\hline Diethylphthalate & 8600 & $\mathrm{U}$ & 8600 & 9100 & $\bar{U}$ & 9100 & 430 & $\mathrm{U}$ & 430 & 370 & $\mathrm{U}$ & 370 & 390 & $\mathrm{U}$ & 390 \\
\hline Dimethyl phthalate & 8600 & $\mathrm{U}$ & 8600 & 9100 & $\bar{U}$ & 9100 & 430 & $\mathrm{U}$ & 430 & 370 & $\mathrm{U}$ & 370 & 390 & $\mathrm{U}$ & 390 \\
\hline Fluoranthene & 8600 & $\mathrm{U}$ & 8600 & 9100 & $\mathrm{U}$ & 9100 & 430 & $\mathrm{U}$ & 430 & 370 & $\mathrm{U}$ & 370 & 390 & $\mathrm{U}$ & 390 \\
\hline Fluorene & 8600 & $\mathrm{U}$ & 8600 & 9100 & $\mathrm{U}$ & 9100 & 430 & $\mathrm{U}$ & 430 & 370 & $\mathrm{U}$ & 370 & 390 & $\mathrm{U}$ & 390 \\
\hline Hexachlorobenzene & 8600 & $\mathrm{U}$ & 8600 & 9100 & $\bar{U}$ & 9100 & 430 & $\mathrm{U}$ & 430 & 370 & $\mathrm{U}$ & 370 & 390 & $\mathrm{U}$ & 390 \\
\hline Hexachlorobutadiene & 8600 & $\mathrm{U}$ & 8600 & 9100 & $\mathrm{U}$ & 9100 & 430 & $\mathrm{U}$ & 430 & 370 & $\mathrm{U}$ & 370 & 390 & $\mathrm{U}$ & 390 \\
\hline Hexachlorocyclopentadiene & 8600 & $\mathrm{U}$ & 8600 & 9100 & $\mathrm{U}$ & 9100 & 430 & $\mathrm{U}$ & 430 & 370 & $\mathrm{U}$ & 370 & 390 & $\mathrm{U}$ & 390 \\
\hline Hexachloroethane & 8600 & $\mathrm{U}$ & 8600 & 9100 & $\mathrm{U}$ & 9100 & 430 & $\mathrm{U}$ & 430 & 370 & $\mathrm{U}$ & 370 & 390 & $\mathrm{U}$ & 390 \\
\hline Indeno(1,2,3-cd)pyrene & 8600 & $\mathrm{U}$ & 8600 & 9100 & $\mathrm{U}$ & 9100 & 430 & $\mathrm{U}$ & 430 & 370 & $\mathrm{U}$ & 370 & 390 & $\mathrm{U}$ & 390 \\
\hline Isophorone & 8600 & $\mathrm{U}$ & 8600 & 9100 & $\mathrm{U}$ & 9100 & 430 & $\mathrm{U}$ & 430 & 370 & $\mathrm{U}$ & 370 & 390 & $\mathrm{U}$ & 390 \\
\hline N-Nitroso-di-n-dipropylamine & 8600 & $\mathrm{U}$ & 8600 & 9100 & $\mathrm{U}$ & 9100 & 430 & $\mathrm{U}$ & 430 & 370 & $\mathrm{U}$ & 370 & 390 & $\mathrm{U}$ & 390 \\
\hline N-Nitrosodiphenylamine & 8600 & $\mathrm{U}$ & 8600 & 9100 & $\mathrm{U}$ & 9100 & 430 & $\mathrm{U}$ & 430 & 370 & $\mathrm{U}$ & 370 & 390 & $\mathrm{U}$ & 390 \\
\hline Naphthalene & 8600 & $\mathrm{U}$ & 8600 & 9100 & $\mathrm{U}$ & 9100 & 430 & $\mathrm{U}$ & 430 & 370 & $\mathrm{U}$ & 370 & 390 & $\mathrm{U}$ & 390 \\
\hline Nitrobenzene & 8600 & $\mathrm{U}$ & 8600 & 9100 & $\mathrm{U}$ & 9100 & 430 & $\mathrm{U}$ & 430 & 370 & $\mathrm{U}$ & 370 & 390 & $\mathrm{U}$ & 390 \\
\hline \begin{tabular}{|l|} 
Pentachlorophenol \\
\end{tabular} & 22000 & $\mathrm{U}$ & 22000 & 23000 & $\mathrm{U}$ & 23000 & 1100 & $\mathrm{U}$ & 1100 & 920 & $\mathrm{U}$ & 920 & 970 & $\mathrm{U}$ & 970 \\
\hline \begin{tabular}{|l} 
Phenanthrene \\
\end{tabular} & 8600 & $\mathrm{U}$ & 8600 & 9100 & $\mathrm{U}$ & 9100 & 430 & $\mathrm{U}$ & 430 & 48 & $\mathrm{~J}$ & 370 & 390 & $\mathrm{U}$ & 390 \\
\hline \begin{tabular}{|l|} 
Phenol \\
\end{tabular} & 8600 & $\mathrm{U}$ & 8600 & 9100 & $\mathrm{U}$ & 9100 & 430 & $\mathrm{U}$ & 430 & 370 & $\mathrm{U}$ & 370 & 390 & $\mathrm{U}$ & 390 \\
\hline Pyrene & 8600 & $\mathrm{U}$ & 8600 & 9100 & $\mathrm{U}$ & 9100 & 29 & $\mathrm{~J}$ & 430 & 370 & $\mathrm{U}$ & 370 & 390 & $\mathrm{U}$ & 390 \\
\hline \multicolumn{16}{|c|}{ Pesticides } \\
\hline Alpha-BHC & 1.7 & $\mathrm{U}$ & 1.7 & 1.8 & $\bar{U}$ & 1.8 & 1.7 & $\mathrm{U}$ & 1.7 & 1.5 & $\mathrm{U}$ & 1.5 & 1.6 & $\mathrm{U}$ & 1.6 \\
\hline gamma-BHC (Lindane) & 1.7 & $\mathrm{U}$ & 1.7 & 1.8 & $\overline{\mathrm{U}}$ & 1.8 & 1.7 & $\mathrm{U}$ & 1.7 & 1.5 & $\mathrm{U}$ & 1.5 & 1.6 & $\mathrm{U}$ & 1.6 \\
\hline Beta-BHC & 1.7 & $\mathrm{U}$ & 1.7 & 2.0 & I & 1.8 & 1.7 & $\mathrm{U}$ & 1.7 & 1.5 & $\mathrm{U}$ & 1.5 & 1.6 & $\mathrm{U}$ & 1.6 \\
\hline
\end{tabular}


Table A-2. 128-F-2 In-Process Riverbank Excavation Berm Data (16 Pages)

\begin{tabular}{|c|c|c|c|c|c|c|c|c|c|c|c|c|c|c|c|}
\hline \multirow[t]{2}{*}{ Constituents } & \multicolumn{3}{|c|}{$\begin{array}{c}\text { J13LX2 } \\
\text { Riverbank } \\
\text { Sample Date 11/1/06 }\end{array}$} & \multicolumn{3}{|c|}{$\begin{array}{c}\text { J13LX1 } \\
\text { Riverbank } \\
\text { Sample Date 11/1/06 } \\
\end{array}$} & \multicolumn{3}{|c|}{$\begin{array}{c}\text { J13LX0 } \\
\text { Riverbank } \\
\text { Sample Date 11/1/06 } \\
\end{array}$} & \multicolumn{3}{|c|}{$\begin{array}{c}\text { J13LP5 } \\
\text { Riverbank } \\
\text { Sample Date 10/26/06 }\end{array}$} & \multicolumn{3}{|c|}{$\begin{array}{c}\text { J13LP4 } \\
\text { Riverbank } \\
\text { Sample Date 10/26/06 }\end{array}$} \\
\hline & $\mu \mathrm{g} / \mathrm{kg}$ & $\mathbf{Q}$ & PQL & $\mu \mathrm{g} / \mathrm{kg}$ & $\mathbf{Q}$ & PQL & $\mu \mathrm{g} / \mathrm{kg}$ & $\mathbf{Q}$ & PQL & $\mu \mathrm{g} / \mathrm{kg}$ & $\mathbf{Q}$ & PQL & $\mu \mathrm{g} / \mathrm{kg}$ & $\mathbf{Q}$ & PQL \\
\hline \multicolumn{16}{|c|}{ Pesticides (continued) } \\
\hline Heptachlor & 1.7 & $\mathrm{U}$ & 1.7 & 1.8 & $\mathrm{U}$ & 1.8 & 1.7 & $\bar{U}$ & 1.7 & 1.5 & $\mathrm{U}$ & 1.5 & 1.6 & $\mathrm{U}$ & 1.6 \\
\hline Delta-BHC & 1.7 & $\mathrm{U}$ & 1.7 & 1.8 & $\mathrm{U}$ & 1.8 & 1.2 & $\mathrm{~J}$ & 1.7 & 1.5 & $\mathrm{U}$ & 1.5 & 1.6 & $\mathrm{U}$ & 1.6 \\
\hline Aldrin & 1.7 & $\mathrm{U}$ & 1.7 & 1.8 & $\mathrm{U}$ & 1.8 & 0.82 & JI & 1.7 & 1.5 & $\mathrm{U}$ & 1.5 & 1.6 & $\mathrm{U}$ & 1.6 \\
\hline Heptachlor epoxide & 1.7 & $\mathrm{U}$ & 1.7 & 1.8 & $\mathrm{U}$ & 1.8 & 1.2 & $\mathrm{~J}$ & 1.7 & 1.5 & $\mathrm{U}$ & 1.5 & 1.6 & $\mathrm{U}$ & 1.6 \\
\hline gamma-Chlordane & 1.7 & $\mathrm{U}$ & 1.7 & 1.8 & $\mathrm{U}$ & 1.8 & 1.7 & $\bar{U}$ & 1.7 & 1.5 & $\mathrm{U}$ & 1.5 & 1.6 & $\mathrm{U}$ & 1.6 \\
\hline Endosulfan I & 1.7 & $\mathrm{U}$ & 1.7 & 1.8 & $\mathrm{U}$ & 1.8 & 0.94 & $\mathrm{~J}$ & 1.7 & 1.5 & $\mathrm{U}$ & 1.5 & 1.6 & $\mathrm{U}$ & 1.6 \\
\hline alpha-Chlordane & 1.7 & $\mathrm{U}$ & 1.7 & 1.8 & $\mathrm{U}$ & 1.8 & 0.52 & JI & 1.7 & 1.5 & $\mathrm{U}$ & 1.5 & 1.6 & $\mathrm{U}$ & 1.6 \\
\hline 4,4'-DDE & 1.7 & $\mathrm{U}$ & 1.7 & 1.8 & $\mathrm{U}$ & 1.8 & 1.7 & $\mathrm{U}$ & 1.7 & 1.5 & $\mathrm{U}$ & 1.5 & 1.6 & $\mathrm{U}$ & 1.6 \\
\hline Deildrin & 1.7 & $\bar{U}$ & 1.7 & 1.8 & $\mathrm{U}$ & 1.8 & 1.7 & $\bar{U}$ & 1.7 & 1.5 & $\mathrm{U}$ & 1.5 & 1.6 & $\bar{U}$ & 1.6 \\
\hline Endrin & 1.7 & $\mathrm{U}$ & 1.7 & 1.8 & $\mathrm{U}$ & 1.8 & 1.7 & $\mathrm{U}$ & 1.7 & 1.5 & $\mathrm{U}$ & 1.5 & 1.6 & $\mathrm{U}$ & 1.6 \\
\hline 4,4'-DDD & 1.7 & $\mathrm{U}$ & 1.7 & 1.8 & $\mathrm{U}$ & 1.8 & 4.8 & & 1.7 & 1.5 & $\mathrm{U}$ & 1.5 & 1.6 & $\mathrm{U}$ & 1.6 \\
\hline Endosulfan II & 1.7 & $\mathrm{U}$ & 1.7 & 1.8 & $\mathrm{U}$ & 1.8 & 1.7 & $\mathrm{U}$ & 1.7 & 1.5 & $\mathrm{U}$ & 1.5 & 1.6 & $\mathrm{U}$ & 1.6 \\
\hline 4,4’-DDT & 1.7 & $\mathrm{U}$ & 1.7 & 1.8 & $\mathrm{U}$ & 1.8 & 18 & I & 1.7 & 1.5 & $\mathrm{U}$ & 1.5 & 1.6 & $\mathrm{U}$ & 1.6 \\
\hline Endrin aldehyde & 1.7 & $\mathrm{U}$ & 1.7 & 1.8 & $\mathrm{U}$ & 1.8 & 24 & I & 1.7 & 1.5 & $\mathrm{U}$ & 1.5 & 1.6 & $\mathrm{U}$ & 1.6 \\
\hline Endosulfan sulfate & 1.7 & $\mathrm{U}$ & 1.7 & 1.8 & $\mathrm{U}$ & 1.8 & 1.7 & $\mathrm{U}$ & 1.7 & 1.5 & $\mathrm{U}$ & 1.5 & 1.6 & $\mathrm{U}$ & 1.6 \\
\hline Methoxychlor & 2.6 & I & 1.7 & 1.8 & $\mathrm{U}$ & 1.8 & 12 & & 1.7 & 1.5 & $\mathrm{U}$ & 1.5 & 1.6 & $\mathrm{U}$ & 1.6 \\
\hline Endrin ketone & 1.7 & $\mathrm{U}$ & 1.7 & 1.8 & $\mathrm{U}$ & 1.8 & 3.3 & $\mathrm{I}$ & 1.7 & 1.5 & $\mathrm{U}$ & 1.5 & 1.6 & $\mathrm{U}$ & 1.6 \\
\hline \begin{tabular}{|l} 
Toxaphene \\
\end{tabular} & 17 & $\mathrm{U}$ & 17 & 18 & $\mathrm{U}$ & 18 & 17 & $\mathrm{U}$ & 17 & 15 & $\mathrm{U}$ & 15 & 16 & $\mathrm{U}$ & 16 \\
\hline \multicolumn{16}{|c|}{ PAHs (Polycyclic Aromatic Hydrocarbons) } \\
\hline Naphthalene & 43.1 & $\mathrm{U}$ & 43.1 & 180 & & 45.6 & 42.9 & $\bar{U}$ & 42.9 & 16 & $\mathrm{~J}$ & 33.7 & 38.8 & $\mathrm{U}$ & 38.8 \\
\hline Acenaphthylene & 43.1 & $\mathrm{U}$ & 43.1 & 37 & $\mathrm{~J}$ & 45.6 & 42.9 & $\mathrm{U}$ & 42.9 & 19 & $\mathrm{~J}$ & 33.7 & 38.8 & $\mathrm{U}$ & 38.8 \\
\hline Acenaphthene & 43.1 & $\mathrm{U}$ & 43.1 & 190 & & 45.6 & 61 & & 42.9 & 33.7 & $\mathrm{U}$ & 33.7 & 38.8 & $\mathrm{U}$ & 38.8 \\
\hline Fluorene & 19 & & 4.31 & 3.9 & $\mathrm{~J}$ & 4.56 & 110 & & 4.29 & 23 & & 3.37 & 3.88 & $\mathrm{U}$ & 3.88 \\
\hline Phenanthrene & 150 & & 4.31 & 81 & & 4.56 & 87 & & 4.29 & 31 & $\mathrm{~B}$ & 3.37 & 7.9 & $\mathrm{~B}$ & 3.88 \\
\hline \begin{tabular}{|l} 
Anthracene \\
\end{tabular} & 30 & & 4.31 & 4.1 & $\mathrm{~J}$ & 4.56 & 4.29 & $\mathrm{U}$ & 4.29 & 1.6 & $\mathrm{~J}$ & 3.37 & 3.88 & $\mathrm{U}$ & $\begin{array}{l}0.80 \\
3.88\end{array}$ \\
\hline Fluoranthrene & 190 & & 4.31 & 4.8 & & 4.56 & 12 & & 4.29 & 47 & & 3.37 & 1.6 & $\mathrm{~J}$ & 3.88 \\
\hline Indeno (1,2,3-cd)pyrene & 30 & & 4.31 & 4.56 & $\mathrm{U}$ & 4.56 & 4.29 & $\mathrm{U}$ & 4.29 & 60 & & 3.37 & 1.8 & $\mathrm{~J}$ & 3.88 \\
\hline Pyrene & 180 & & 4.31 & 20 & & 4.56 & 6.4 & & 4.29 & 23 & & 3.37 & 1.6 & $\mathrm{~J}$ & 3.88 \\
\hline Benzo(a)anthracene & 80 & & 4.31 & 13 & & 4.56 & 10 & & 4.29 & 20 & & 3.37 & 1.7 & $\mathrm{~J}$ & 3.88 \\
\hline Chrysene & 150 & & 4.31 & 4.56 & $\mathrm{U}$ & 4.56 & 4.29 & $\mathrm{U}$ & 4.29 & 35 & & 3.37 & 2.5 & $\mathrm{~J}$ & 3.88 \\
\hline Benzo(b)fluoranthrene & 46 & & 4.31 & 17 & & 4.56 & 19 & & 4.29 & 100 & & 3.37 & 1.9 & $\mathrm{~J}$ & 3.88 \\
\hline Benzo(k)fluoranthrene & 33 & & 4.31 & 4.56 & $\mathrm{U}$ & 4.56 & 4.29 & $\mathrm{U}$ & 4.29 & 24 & & 3.37 & 3.88 & $\mathrm{U}$ & 3.88 \\
\hline Benzo(a)pyrene & 92 & & 4.31 & 6.3 & & 4.56 & 4.29 & $\mathrm{U}$ & 4.29 & 46 & & 3.37 & 3.6 & $\mathrm{~J}$ & 3.88 \\
\hline Dibenzo(a,h)anthracene & 11 & & 4.31 & 4.56 & $\mathrm{U}$ & 4.56 & 16 & & 4.29 & 11 & & 3.37 & 3.88 & $\mathrm{U}$ & 3.88 \\
\hline Benzo(ghi)perylene & 45 & & 4.31 & 20 & & 4.56 & 4.29 & $\overline{\mathrm{U}}$ & 4.29 & 68 & & 3.37 & 2.8 & $\mathrm{~J}$ & 3.88 \\
\hline
\end{tabular}


Table A-2. 128-F-2 In-Process Riverbank Excavation Berm Data (16 Pages)

\begin{tabular}{|c|c|c|c|c|c|c|c|c|c|c|c|c|c|c|c|}
\hline \multirow[t]{2}{*}{ Constituents } & \multicolumn{3}{|c|}{$\begin{array}{c}\text { J13LP3 } \\
\text { Riverbank } \\
\text { Sample Date 10/26/06 }\end{array}$} & \multicolumn{3}{|c|}{$\begin{array}{c}\text { J13LP2 } \\
\text { Riverbank } \\
\text { Sample Date 10/26/06 }\end{array}$} & \multicolumn{3}{|c|}{$\begin{array}{c}\text { J13LP1 } \\
\text { Riverbank } \\
\text { Sample Date 10/26/06 } \\
\end{array}$} & \multicolumn{3}{|c|}{$\begin{array}{c}\text { J13LP0 } \\
\text { Riverbank } \\
\text { Sample Date 10/26/06 }\end{array}$} & \multicolumn{3}{|c|}{$\begin{array}{c}\text { J13LN9 } \\
\text { Riverbank } \\
\text { Sample Date 10/26/06 } \\
\end{array}$} \\
\hline & $\mu \mathrm{g} / \mathrm{kg}$ & $\mathbf{Q}$ & PQL & $\mu \mathrm{g} / \mathrm{kg}$ & $\mathbf{Q}$ & PQL & $\mu \mathrm{g} / \mathrm{kg}$ & $\mathbf{Q}$ & PQL & $\mu \mathrm{g} / \mathrm{kg}$ & $\mathbf{Q}$ & PQL & $\mu \mathrm{g} / \mathrm{kg}$ & $\mathbf{Q}$ & PQL \\
\hline \multicolumn{16}{|c|}{ PCBs } \\
\hline Aroclor-1016 & 16 & $\mathrm{U}$ & 16 & 16 & $\mathrm{U}$ & 16 & 16 & $\mathrm{U}$ & 16 & 15 & $\mathrm{U}$ & 15 & 15 & $\mathrm{U}$ & 15 \\
\hline Aroclor-1221 & 16 & $\mathrm{U}$ & 16 & 16 & $\mathrm{U}$ & 16 & 16 & $\mathrm{U}$ & 16 & 15 & $\mathrm{U}$ & 15 & 15 & $\mathrm{U}$ & 15 \\
\hline Aroclor-1232 & 16 & $\mathrm{U}$ & 16 & 16 & $\mathrm{U}$ & 16 & 16 & $\mathrm{U}$ & 16 & 15 & $\mathrm{U}$ & 15 & 15 & $\mathrm{U}$ & 15 \\
\hline Aroclor-1242 & 16 & $\mathrm{U}$ & 16 & 16 & $\mathrm{U}$ & 16 & 16 & $\mathrm{U}$ & 16 & 15 & $\mathrm{U}$ & 15 & 15 & $\mathrm{U}$ & 15 \\
\hline Aroclor-1248 & 16 & $\mathrm{U}$ & 16 & 16 & $\mathrm{U}$ & 16 & 16 & $\mathrm{U}$ & 16 & 15 & $\mathrm{U}$ & 15 & 15 & $\mathrm{U}$ & 15 \\
\hline Aroclor-1254 & 16 & $\mathrm{U}$ & 16 & 110 & & 16 & 16 & $\mathrm{U}$ & 16 & 15 & $\mathrm{U}$ & 15 & 15 & $\mathrm{U}$ & 15 \\
\hline Aroclor-1260 & 16 & $\mathrm{U}$ & 16 & 16 & $\mathrm{U}$ & 16 & 16 & $\mathrm{U}$ & 16 & 15 & $\mathrm{U}$ & 15 & 15 & $\mathrm{U}$ & 15 \\
\hline \multicolumn{16}{|c|}{ SVOAs } \\
\hline 1,2,4-Trichlorobenzene & 400 & $\mathrm{U}$ & 400 & 410 & $\mathrm{U}$ & 410 & 410 & $\mathrm{U}$ & 410 & 370 & $\mathrm{U}$ & 370 & 370 & $\mathrm{U}$ & 370 \\
\hline 1,2-Dichlorobenzene & 400 & $\mathrm{U}$ & 400 & 410 & $\mathrm{U}$ & 410 & 410 & $\mathrm{U}$ & 410 & 370 & $\mathrm{U}$ & 370 & 370 & $\mathrm{U}$ & 370 \\
\hline 1,3-Dichlorobenzene & 400 & $\mathrm{U}$ & 400 & 410 & $\mathrm{U}$ & 410 & 410 & $\mathrm{U}$ & 410 & 370 & $\mathrm{U}$ & 370 & 370 & $\mathrm{U}$ & 370 \\
\hline 1,4-Dichlorobenzene & 400 & $\mathrm{U}$ & 400 & 410 & $\mathrm{U}$ & 410 & 410 & $\mathrm{U}$ & 410 & 370 & $\mathrm{U}$ & 370 & 370 & $\mathrm{U}$ & 370 \\
\hline 2,4,5-Trichlorophenol & 1000 & $\mathrm{U}$ & 1000 & 1000 & $\mathrm{U}$ & 1000 & 1000 & $\mathrm{U}$ & 1000 & 920 & $\mathrm{U}$ & 920 & 920 & $\mathrm{U}$ & 920 \\
\hline 2,4,6-Trichlorophenol & 400 & $\mathrm{U}$ & 400 & 410 & $\mathrm{U}$ & 410 & 410 & $\mathrm{U}$ & 410 & 370 & $\mathrm{U}$ & 370 & 370 & $\mathrm{U}$ & 370 \\
\hline 2,4-Dichlorophenol & 400 & $\mathrm{U}$ & 400 & 410 & $\mathrm{U}$ & 410 & 410 & $\mathrm{U}$ & 410 & 370 & $\mathrm{U}$ & 370 & 370 & $\mathrm{U}$ & 370 \\
\hline 2,4-Dimethylphenol & 400 & $\mathrm{U}$ & 400 & 410 & $\mathrm{U}$ & 410 & 410 & $\mathrm{U}$ & 410 & 370 & $\mathrm{U}$ & 370 & 370 & $\mathrm{U}$ & 370 \\
\hline 2,4-Dinitrophenol & 1000 & $\mathrm{U}$ & 1000 & 1000 & $\mathrm{U}$ & 1000 & 1000 & $\mathrm{U}$ & 1000 & 920 & $\mathrm{U}$ & 920 & 920 & $\mathrm{U}$ & 920 \\
\hline 2,4-Dinitrotoluene & 400 & $\mathrm{U}$ & 400 & 410 & $\mathrm{U}$ & 410 & 410 & $\mathrm{U}$ & 410 & 370 & $\mathrm{U}$ & 370 & 370 & $\mathrm{U}$ & 370 \\
\hline 2,6-Dinitrotoluene & 400 & $\mathrm{U}$ & 400 & 410 & $\mathrm{U}$ & 410 & 410 & $\mathrm{U}$ & 410 & 370 & $\mathrm{U}$ & 370 & 370 & $\mathrm{U}$ & 370 \\
\hline 2-Chloronaphthalene & 400 & $\mathrm{U}$ & 400 & 410 & $\mathrm{U}$ & 410 & 410 & $\mathrm{U}$ & 410 & 370 & $\mathrm{U}$ & 370 & 370 & $\mathrm{U}$ & 370 \\
\hline 2-Chlorophenol & 400 & $\mathrm{U}$ & 400 & 410 & $\mathrm{U}$ & 410 & 410 & $\mathrm{U}$ & 410 & 370 & $\mathrm{U}$ & 370 & 370 & $\mathrm{U}$ & 370 \\
\hline 2-Methylnaphthalene & 400 & $\mathrm{U}$ & 400 & 410 & $\mathrm{U}$ & 410 & 410 & $\mathrm{U}$ & 410 & 370 & $\mathrm{U}$ & 370 & 370 & $\mathrm{U}$ & 370 \\
\hline 2-Methylphenol (cresol, o-) & 400 & $\mathrm{U}$ & 400 & 410 & $\mathrm{U}$ & 410 & 410 & $\mathrm{U}$ & 410 & 370 & $\mathrm{U}$ & 370 & 370 & $\mathrm{U}$ & 370 \\
\hline 2-Nitroaniline & 1000 & $\mathrm{U}$ & 1000 & 1000 & $\mathrm{U}$ & 1000 & 1000 & $\mathrm{U}$ & 1000 & 920 & $\mathrm{U}$ & 920 & 920 & $\mathrm{U}$ & 920 \\
\hline 2-Nitrophenol & 400 & $\mathrm{U}$ & 400 & 410 & $\mathrm{U}$ & 410 & 410 & $\mathrm{U}$ & 410 & 370 & $\mathrm{U}$ & 370 & 370 & $\mathrm{U}$ & 370 \\
\hline 3+4 Methylphenol (cresol, m+p) & 400 & $\mathrm{U}$ & 400 & 410 & $\mathrm{U}$ & 410 & 410 & $\mathrm{U}$ & 410 & 370 & $\mathrm{U}$ & 370 & 370 & $\mathrm{U}$ & 370 \\
\hline 3,3'-Dichlorobenzidine & 400 & $\mathrm{U}$ & 400 & 410 & $\mathrm{U}$ & 410 & 410 & $\mathrm{U}$ & 410 & 370 & $\mathrm{U}$ & 370 & 370 & $\mathrm{U}$ & 370 \\
\hline 3-Nitroaniline & 1000 & $\mathrm{U}$ & 1000 & 1000 & $\mathrm{U}$ & 1000 & 1000 & $\bar{U}$ & 1000 & 920 & $\mathrm{U}$ & 920 & 920 & $\bar{U}$ & 920 \\
\hline 4,6-Dinitro-2-methylphenol & 1000 & $\mathrm{U}$ & 1000 & 1000 & $\mathrm{U}$ & 1000 & 1000 & $\mathrm{U}$ & 1000 & 920 & $\mathrm{U}$ & 920 & 920 & $\mathrm{U}$ & 920 \\
\hline 4-Bromophenylphenyl ether & 400 & $\mathrm{U}$ & 400 & 410 & $\mathrm{U}$ & 410 & 410 & $\mathrm{U}$ & 410 & 370 & $\mathrm{U}$ & 370 & 370 & $\mathrm{U}$ & 370 \\
\hline 4-Chloro-3-methylphenol & 400 & $\mathrm{U}$ & 400 & 410 & $\mathrm{U}$ & 410 & 410 & $\mathrm{U}$ & 410 & 370 & $\mathrm{U}$ & 370 & 370 & $\mathrm{U}$ & 370 \\
\hline 4-Chloroaniline & 400 & $\mathrm{U}$ & 400 & 410 & $\mathrm{U}$ & 410 & 410 & $\mathrm{U}$ & 410 & 370 & $\mathrm{U}$ & 370 & 370 & $\mathrm{U}$ & 370 \\
\hline 4-Chlorophenylphenyl ether & 400 & $\mathrm{U}$ & 400 & 410 & $\mathrm{U}$ & 410 & 410 & $\mathrm{U}$ & 410 & 370 & $\mathrm{U}$ & 370 & 370 & $\mathrm{U}$ & 370 \\
\hline 4-Nitroaniline & 1000 & $\mathrm{U}$ & 1000 & 1000 & $\mathrm{U}$ & 1000 & 1000 & $\mathrm{U}$ & 1000 & 920 & $\mathrm{U}$ & 920 & 920 & $\mathrm{U}$ & 920 \\
\hline 4-Nitrophenol & 1000 & $\mathrm{U}$ & 1000 & 1000 & $\mathrm{U}$ & 1000 & 1000 & $\mathrm{U}$ & 1000 & 920 & $\mathrm{U}$ & 920 & 920 & $\mathrm{U}$ & 920 \\
\hline Acenaphthene & 400 & $\mathrm{U}$ & 400 & 410 & $\mathrm{U}$ & 410 & 410 & $\mathrm{U}$ & 410 & 370 & $\mathrm{U}$ & 370 & 370 & $\mathrm{U}$ & 370 \\
\hline Acenaphthylene & 400 & $\mathrm{U}$ & 400 & 410 & $\mathrm{U}$ & 410 & 410 & $\mathrm{U}$ & 410 & 370 & $\mathrm{U}$ & 370 & 370 & $\mathrm{U}$ & 370 \\
\hline Anthracene & 400 & $\mathrm{U}$ & 400 & 410 & $\mathrm{U}$ & 410 & 410 & $\mathrm{U}$ & 410 & 370 & $\mathrm{U}$ & 370 & 370 & $\mathrm{U}$ & 370 \\
\hline
\end{tabular}


Table A-2. 128-F-2 In-Process Riverbank Excavation Berm Data (16 Pages)

\begin{tabular}{|c|c|c|c|c|c|c|c|c|c|c|c|c|c|c|c|}
\hline \multirow[t]{2}{*}{ Constituents } & \multicolumn{3}{|c|}{$\begin{array}{c}\text { J13LP3 } \\
\text { Riverbank } \\
\text { Sample Date 10/26/06 }\end{array}$} & \multicolumn{3}{|c|}{$\begin{array}{c}\text { J13LP2 } \\
\text { Riverbank } \\
\text { Sample Date 10/26/06 }\end{array}$} & \multicolumn{3}{|c|}{$\begin{array}{c}\text { J13LP1 } \\
\text { Riverbank } \\
\text { Sample Date 10/26/06 }\end{array}$} & \multicolumn{3}{|c|}{$\begin{array}{c}\text { J13LP0 } \\
\text { Riverbank } \\
\text { Sample Date 10/26/06 }\end{array}$} & \multicolumn{3}{|c|}{$\begin{array}{c}\text { J13LN9 } \\
\text { Riverbank } \\
\text { Sample Date 10/26/06 }\end{array}$} \\
\hline & $\mu \mathrm{g} / \mathrm{kg}$ & $\mathbf{Q}$ & PQL & $\mu \mathrm{g} / \mathrm{kg}$ & $\mathbf{Q}$ & PQL & $\mu \mathrm{g} / \mathrm{kg}$ & $\mathbf{Q}$ & PQL & $\mu \mathrm{g} / \mathrm{kg}$ & $\mathbf{Q}$ & PQL & $\mu \mathrm{g} / \mathrm{kg}$ & $\mathbf{Q}$ & PQL \\
\hline \multicolumn{16}{|c|}{ SVOAs (continued) } \\
\hline Benzo(a)anthracene & 400 & $\mathrm{U}$ & 400 & 410 & $\bar{U}$ & 410 & 410 & $\mathrm{U}$ & 410 & 370 & $\mathrm{U}$ & 370 & 370 & $\mathrm{U}$ & 370 \\
\hline Benzo(a)pyrene & 400 & $\mathrm{U}$ & 400 & 410 & $\bar{U}$ & 410 & 410 & $\mathrm{U}$ & 410 & 370 & $\mathrm{U}$ & 370 & 370 & $\mathrm{U}$ & 370 \\
\hline Benzo(b)fluoranthene & 400 & $\mathrm{U}$ & 400 & 410 & $\overline{\mathrm{U}}$ & 410 & 410 & $\mathrm{U}$ & 410 & 370 & $\mathrm{U}$ & 370 & 370 & $\mathrm{U}$ & 370 \\
\hline Benzo(ghi)perylene & 400 & $\mathrm{U}$ & 400 & 410 & $\overline{\mathrm{U}}$ & 410 & 410 & $\mathrm{U}$ & 410 & 370 & $\mathrm{U}$ & 370 & 370 & $\mathrm{U}$ & 370 \\
\hline Benzo(k)fluoranthene & 400 & $\mathrm{U}$ & 400 & 410 & $\bar{U}$ & 410 & 410 & $\mathrm{U}$ & 410 & 370 & $\mathrm{U}$ & 370 & 370 & $\mathrm{U}$ & 370 \\
\hline Bis(2-chloro-1-methylethyl)ether & 400 & $\mathrm{U}$ & 400 & 410 & $\mathrm{U}$ & 410 & 410 & $\mathrm{U}$ & 410 & 370 & $\mathrm{U}$ & 370 & 370 & $\mathrm{U}$ & 370 \\
\hline Bis(2-Chloroethoxy)methane & 400 & $\mathrm{U}$ & 400 & 410 & $\overline{\mathrm{U}}$ & 410 & 410 & $\mathrm{U}$ & 410 & 370 & $\mathrm{U}$ & 370 & 370 & $\mathrm{U}$ & 370 \\
\hline Bis(2-chloroethyl) ether & 400 & $\mathrm{U}$ & 400 & 410 & $\bar{U}$ & 410 & 410 & $\mathrm{U}$ & 410 & 370 & $\mathrm{U}$ & 370 & 370 & $\mathrm{U}$ & 370 \\
\hline Bis(2-ethylhexyl) phthalate & 84 & JB & 400 & 74 & JB & 410 & 73 & JB & 410 & 110 & JB & 370 & 59 & JB & 370 \\
\hline Butylbenzylphthalate & 400 & $\mathrm{U}$ & 400 & 410 & $\mathrm{U}$ & 410 & 410 & $\mathrm{U}$ & 410 & 370 & $\mathrm{U}$ & 370 & 370 & $\mathrm{U}$ & 370 \\
\hline Carbazole & 400 & $\mathrm{U}$ & 400 & 410 & $\mathrm{U}$ & 410 & 410 & $\mathrm{U}$ & 410 & 370 & $\mathrm{U}$ & 370 & 370 & $\mathrm{U}$ & 370 \\
\hline Chrysene & 400 & $\mathrm{U}$ & 400 & 410 & $\mathrm{U}$ & 410 & 410 & $\mathrm{U}$ & 410 & 370 & $\mathrm{U}$ & 370 & 370 & $\mathrm{U}$ & 370 \\
\hline Di-n-butylphthalate & 50 & JB & 400 & 71 & $\mathrm{JB}$ & 410 & 42 & JB & 410 & 76 & JB & 370 & 40 & JB & 370 \\
\hline Di-n-octylphthalate & 400 & $\mathrm{U}$ & 400 & 410 & $\mathrm{U}$ & 410 & 410 & $\mathrm{U}$ & 410 & 370 & $\mathrm{U}$ & 370 & 370 & $\mathrm{U}$ & 370 \\
\hline Dibenz[a,h]anthracene & 400 & $\mathrm{U}$ & 400 & 410 & $\bar{U}$ & 410 & 410 & $\mathrm{U}$ & 410 & 370 & $\mathrm{U}$ & 370 & 370 & $\mathrm{U}$ & 370 \\
\hline Dibenzofuran & 400 & $\mathrm{U}$ & 400 & 410 & $\mathrm{U}$ & 410 & 410 & $\mathrm{U}$ & 410 & 370 & $\mathrm{U}$ & 370 & 370 & $\mathrm{U}$ & 370 \\
\hline Diethylphthalate & 400 & $\mathrm{U}$ & 400 & 410 & $\mathrm{U}$ & 410 & 410 & $\mathrm{U}$ & 410 & 370 & $\mathrm{U}$ & 370 & 370 & $\mathrm{U}$ & 370 \\
\hline Dimethyl phthalate & 400 & $\mathrm{U}$ & 400 & 410 & $\overline{\mathrm{U}}$ & 410 & 410 & $\mathrm{U}$ & 410 & 370 & $\mathrm{U}$ & 370 & 370 & $\mathrm{U}$ & 370 \\
\hline Fluoranthene & 400 & $\mathrm{U}$ & 400 & 410 & $\overline{\mathrm{U}}$ & 410 & 410 & $\mathrm{U}$ & 410 & 370 & $\mathrm{U}$ & 370 & 370 & $\mathrm{U}$ & 370 \\
\hline Fluorene & 400 & $\mathrm{U}$ & 400 & 410 & $\bar{U}$ & 410 & 410 & $\mathrm{U}$ & 410 & 370 & $\mathrm{U}$ & 370 & 370 & $\mathrm{U}$ & 370 \\
\hline Hexachlorobenzene & 400 & $\mathrm{U}$ & 400 & 410 & $\mathrm{U}$ & 410 & 410 & $\mathrm{U}$ & 410 & 370 & $\mathrm{U}$ & 370 & 370 & $\mathrm{U}$ & 370 \\
\hline Hexachlorobutadiene & 400 & $\mathrm{U}$ & 400 & 410 & $\mathrm{U}$ & 410 & 410 & $\mathrm{U}$ & 410 & 370 & $\mathrm{U}$ & 370 & 370 & $\mathrm{U}$ & 370 \\
\hline Hexachlorocyclopentadiene & 400 & $\mathrm{U}$ & 400 & 410 & $\overline{\mathrm{U}}$ & 410 & 410 & $\mathrm{U}$ & 410 & 370 & $\mathrm{U}$ & 370 & 370 & $\mathrm{U}$ & 370 \\
\hline Hexachloroethane & 400 & $\mathrm{U}$ & 400 & 410 & $\mathrm{U}$ & 410 & 410 & $\mathrm{U}$ & 410 & 370 & $\mathrm{U}$ & 370 & 370 & $\mathrm{U}$ & 370 \\
\hline Indeno(1,2,3-cd)pyrene & 400 & $\mathrm{U}$ & 400 & 410 & $\overline{\mathrm{U}}$ & 410 & 410 & $\mathrm{U}$ & 410 & 370 & $\mathrm{U}$ & 370 & 370 & $\mathrm{U}$ & 370 \\
\hline Isophorone & 400 & $\mathrm{U}$ & 400 & 410 & $\bar{U}$ & 410 & 410 & $\mathrm{U}$ & 410 & 370 & $\mathrm{U}$ & 370 & 370 & $\mathrm{U}$ & 370 \\
\hline N-Nitroso-di-n-dipropylamine & 400 & $\mathrm{U}$ & 400 & 410 & $\mathrm{U}$ & 410 & 410 & $\mathrm{U}$ & 410 & 370 & $\mathrm{U}$ & 370 & 370 & $\mathrm{U}$ & 370 \\
\hline N-Nitrosodiphenylamine & 400 & $\mathrm{U}$ & 400 & 410 & $\bar{U}$ & 410 & 410 & $\mathrm{U}$ & 410 & 370 & $\mathrm{U}$ & 370 & 370 & $\mathrm{U}$ & 370 \\
\hline Naphthalene & 400 & $\mathrm{U}$ & 400 & 410 & $\bar{U}$ & 410 & 410 & $\mathrm{U}$ & 410 & 370 & $\mathrm{U}$ & 370 & 370 & $\mathrm{U}$ & 370 \\
\hline Nitrobenzene & 400 & $\mathrm{U}$ & 400 & 410 & $\bar{U}$ & 410 & 410 & $\mathrm{U}$ & 410 & 370 & $\mathrm{U}$ & 370 & 370 & $\mathrm{U}$ & 370 \\
\hline Pentachlorophenol & 1000 & $\mathrm{U}$ & 1000 & 1000 & $\mathrm{U}$ & 1000 & 1000 & $\mathrm{U}$ & 1000 & 920 & $\mathrm{U}$ & 920 & 920 & $\mathrm{U}$ & 920 \\
\hline Phenanthrene & 22 & $\mathrm{~J}$ & 400 & 410 & $\bar{U}$ & 410 & 410 & $\mathrm{U}$ & 410 & 370 & $\mathrm{U}$ & 370 & 370 & $\mathrm{U}$ & 370 \\
\hline Phenol & 400 & $\mathrm{U}$ & 400 & 410 & $\bar{U}$ & 410 & 410 & $\mathrm{U}$ & 410 & 370 & $\mathrm{U}$ & 370 & 370 & $\mathrm{U}$ & 370 \\
\hline Pyrene & 400 & $\mathrm{U}$ & 400 & 410 & $\bar{U}$ & 410 & 410 & $\mathrm{U}$ & 410 & 370 & $\mathrm{U}$ & 370 & 370 & $\mathrm{U}$ & 370 \\
\hline \multicolumn{16}{|c|}{ Pesticides } \\
\hline Alpha-BHC & 1.6 & $\mathrm{U}$ & 1.6 & 1.6 & $\mathrm{U}$ & 1.6 & 1.6 & $\mathrm{U}$ & 1.6 & 1.5 & $\mathrm{U}$ & 1.5 & 1.5 & $\mathrm{U}$ & 1.5 \\
\hline gamma-BHC (Lindane) & 1.6 & $\mathrm{U}$ & 1.6 & 1.6 & $\mathrm{U}$ & 1.6 & 1.6 & $\mathrm{U}$ & 1.6 & 1.5 & $\mathrm{U}$ & 1.5 & 1.5 & $\mathrm{U}$ & 1.5 \\
\hline Beta-BHC & 1.6 & $\mathrm{U}$ & 1.6 & 1.6 & $\mathrm{U}$ & 1.6 & 1.6 & $\mathrm{U}$ & 1.6 & 1.5 & $\mathrm{U}$ & 1.5 & 1.5 & $\mathrm{U}$ & 1.5 \\
\hline
\end{tabular}


Table A-2. 128-F-2 In-Process Riverbank Excavation Berm Data (16 Pages)

\begin{tabular}{|c|c|c|c|c|c|c|c|c|c|c|c|c|c|c|c|}
\hline \multirow[t]{2}{*}{ Constituents } & \multicolumn{3}{|c|}{$\begin{array}{c}\text { J13LP3 } \\
\text { Riverbank } \\
\text { Sample Date } 10 / 26 / 06\end{array}$} & \multicolumn{3}{|c|}{$\begin{array}{c}\text { J13LP2 } \\
\text { Riverbank } \\
\text { Sample Date 10/26/06 }\end{array}$} & \multicolumn{3}{|c|}{$\begin{array}{c}\text { J13LP1 } \\
\text { Riverbank } \\
\text { Sample Date 10/26/06 }\end{array}$} & \multicolumn{3}{|c|}{$\begin{array}{c}\text { J13LP0 } \\
\text { Riverbank } \\
\text { Sample Date } 10 / 26 / 06 \\
\end{array}$} & \multicolumn{3}{|c|}{$\begin{array}{c}\text { J13LN9 } \\
\text { Riverbank } \\
\text { Sample Date 10/26/06 } \\
\end{array}$} \\
\hline & $\mu \mathrm{g} / \mathrm{kg}$ & $\mathbf{Q}$ & PQL & $\mu \mathrm{g} / \mathrm{kg}$ & $\mathbf{Q}$ & PQL & $\mu \mathrm{g} / \mathrm{kg}$ & $\mathbf{Q}$ & PQL & $\mu \mathrm{g} / \mathrm{kg}$ & $\mathbf{Q}$ & PQL & $\mu \mathrm{g} / \mathrm{kg}$ & $\mathbf{Q}$ & PQL \\
\hline \multicolumn{16}{|c|}{ Pesticides (continued) } \\
\hline Heptachlor & 1.6 & $\mathrm{U}$ & 1.6 & 1.6 & $\mathrm{U}$ & 1.6 & 1.6 & $\mathrm{U}$ & 1.6 & 1.5 & $\mathrm{U}$ & 1.5 & 1.5 & $\mathrm{U}$ & 1.5 \\
\hline Delta-BHC & 1.6 & $\mathrm{U}$ & 1.6 & 1.6 & $\mathrm{U}$ & 1.6 & 1.6 & $\mathrm{U}$ & 1.6 & 1.5 & $\mathrm{U}$ & 1.5 & 1.5 & $\mathrm{U}$ & 1.5 \\
\hline Aldrin & 1.6 & $\mathrm{U}$ & 1.6 & 1.6 & $\mathrm{U}$ & 1.6 & 1.6 & $\mathrm{U}$ & 1.6 & 1.5 & $\bar{U}$ & 1.5 & 1.5 & $\bar{U}$ & 1.5 \\
\hline Heptachlor epoxide & 1.6 & $\mathrm{U}$ & 1.6 & 1.6 & $\mathrm{U}$ & 1.6 & 1.6 & $\mathrm{U}$ & 1.6 & 1.5 & $\mathrm{U}$ & 1.5 & 1.5 & $\mathrm{U}$ & 1.5 \\
\hline \begin{tabular}{|l|} 
gamma-Chlordane \\
\end{tabular} & 1.6 & $\mathrm{U}$ & 1.6 & 3.4 & $\mathrm{I}$ & 1.6 & 1.6 & $\mathrm{U}$ & 1.6 & 1.5 & $\mathrm{U}$ & 1.5 & 1.5 & $\mathrm{U}$ & 1.5 \\
\hline Endosulfan I & 1.6 & $\mathrm{U}$ & 1.6 & 1.6 & $\mathrm{U}$ & 1.6 & 1.6 & $\mathrm{U}$ & 1.6 & 1.5 & $\mathrm{U}$ & 1.5 & 1.5 & $\mathrm{U}$ & 1.5 \\
\hline alpha-Chlordane & 1.6 & $\mathrm{U}$ & 1.6 & 1.6 & $\mathrm{U}$ & 1.6 & 1.6 & $\mathrm{U}$ & 1.6 & 1.5 & $\mathrm{U}$ & 1.5 & 1.5 & $\mathrm{U}$ & 1.5 \\
\hline 4,4'-DDE & 1.6 & $\mathrm{U}$ & 1.6 & 4.9 & & 1.6 & 1.6 & $\mathrm{U}$ & 1.6 & 1.5 & $\mathrm{U}$ & 1.5 & 1.5 & $\mathrm{U}$ & 1.5 \\
\hline Deildrin & 1.6 & $\mathrm{U}$ & 1.6 & 1.6 & $\mathrm{U}$ & 1.6 & 1.6 & $\mathrm{U}$ & 1.6 & 1.5 & $\mathrm{U}$ & 1.5 & 1.5 & $\mathrm{U}$ & 1.5 \\
\hline Endrin & 1.6 & $\mathrm{U}$ & 1.6 & 1.6 & $\mathrm{U}$ & 1.6 & 1.6 & $\mathrm{U}$ & 1.6 & 1.5 & $\mathrm{U}$ & 1.5 & 1.5 & $\mathrm{U}$ & 1.5 \\
\hline 4,4'-DDD & 1.6 & $\mathrm{U}$ & 1.6 & 1.6 & $\mathrm{U}$ & 1.6 & 1.6 & $\mathrm{U}$ & 1.6 & 1.5 & $\mathrm{U}$ & 1.5 & 1.5 & $\mathrm{U}$ & 1.5 \\
\hline Endosulfan II & 1.6 & $\mathrm{U}$ & 1.6 & 1.6 & $\mathrm{U}$ & 1.6 & 1.6 & $\mathrm{U}$ & 1.6 & 1.5 & $\mathrm{U}$ & 1.5 & 1.5 & $\mathrm{U}$ & 1.5 \\
\hline 4,4'-DDT & 1.6 & $\mathrm{U}$ & 1.6 & 1.6 & $\mathrm{U}$ & 1.6 & 1.6 & $\mathrm{U}$ & 1.6 & 1.5 & $\mathrm{U}$ & 1.5 & 1.5 & $\mathrm{U}$ & 1.5 \\
\hline Endrin aldehyde & 1.6 & $\mathrm{U}$ & 1.6 & 1.8 & $\mathrm{I}$ & 1.6 & 1.6 & $\mathrm{U}$ & 1.6 & 1.5 & $\mathrm{U}$ & 1.5 & 1.5 & $\mathrm{U}$ & 1.5 \\
\hline Endosulfan sulfate & 1.6 & $\mathrm{U}$ & 1.6 & 1.6 & $\mathrm{U}$ & 1.6 & 1.6 & $\mathrm{U}$ & 1.6 & 1.5 & $\mathrm{U}$ & 1.5 & 1.5 & $\mathrm{U}$ & 1.5 \\
\hline Methoxychlor & 1.6 & $\mathrm{U}$ & 1.6 & 1.9 & $\mathrm{I}$ & 1.6 & 2.4 & $\mathrm{I}$ & 1.6 & 1.5 & $\mathrm{U}$ & 1.5 & 1.9 & I & 1.5 \\
\hline \begin{tabular}{|l} 
Endrin ketone \\
\end{tabular} & 1.6 & $\mathrm{U}$ & 1.6 & 1.6 & $\mathrm{U}$ & 1.6 & 1.6 & $\mathrm{U}$ & 1.6 & 1.5 & $\mathrm{U}$ & 1.5 & 1.5 & $\mathrm{U}$ & 1.5 \\
\hline Toxaphene & 16 & $\mathrm{U}$ & 16 & 16 & $\mathrm{U}$ & 16 & 16 & $\mathrm{U}$ & 16 & 15 & $\mathrm{U}$ & 15 & 15 & $\mathrm{U}$ & 15 \\
\hline \multicolumn{16}{|c|}{ PAHs (Polycyclic Aromatic Hydrocarbons) } \\
\hline Naphthalene & 43 & & 40.0 & 41.2 & $\mathrm{U}$ & 41.2 & 40.7 & $\mathrm{U}$ & 40.7 & 36.6 & $\mathrm{U}$ & 36.6 & 36.6 & $\mathrm{U}$ & 36.6 \\
\hline Acenaphthylene & 40.0 & $\mathrm{U}$ & 40.0 & 41.2 & $\mathrm{U}$ & 41.2 & 40.7 & $\mathrm{U}$ & 40.7 & 36.6 & $\mathrm{U}$ & 36.6 & 36.6 & $\mathrm{U}$ & 36.6 \\
\hline Acenaphthene & 61 & & 40.0 & 41.2 & $\mathrm{U}$ & 41.2 & 40.7 & $\mathrm{U}$ & 40.7 & 36.6 & $\mathrm{U}$ & 36.6 & 36.6 & $\mathrm{U}$ & 36.6 \\
\hline \begin{tabular}{|l} 
Fluorene \\
\end{tabular} & 4.00 & $\mathrm{U}$ & 4.00 & 4.12 & $\mathrm{U}$ & 4.12 & 4.07 & $\mathrm{U}$ & 4.07 & 3.66 & $\mathrm{U}$ & 3.66 & 3.66 & $\mathrm{U}$ & 3.66 \\
\hline \begin{tabular}{|l|} 
Phenanthrene \\
\end{tabular} & 13 & $\mathrm{~B}$ & 4.00 & 4.7 & $\mathrm{~B}$ & 4.12 & 1.8 & $\mathrm{JB}$ & 4.07 & 3.6 & $\mathrm{JB}$ & 3.66 & 4.2 & $\mathrm{~B}$ & 3.66 \\
\hline Anthracene & 2.5 & $\mathrm{~J}$ & 4.00 & 4.12 & $\mathrm{U}$ & 4.12 & 1.1 & $\mathrm{~J}$ & 4.07 & 3.66 & $\mathrm{U}$ & 3.66 & 3.66 & $\mathrm{U}$ & 3.66 \\
\hline Fluoranthrene & 69 & & 4.00 & 3.3 & $\mathrm{~J}$ & 4.12 & 16 & & 4.07 & 3.66 & $\mathrm{U}$ & 3.66 & 3.1 & $\mathrm{~J}$ & 3.66 \\
\hline Indeno (1,2,3-cd)pyrene & 2.0 & $\mathrm{~J}$ & 4.00 & 2.4 & $\mathrm{~J}$ & 4.12 & 12 & & 4.07 & 2.6 & $\mathrm{~J}$ & 3.66 & 9.9 & & 3.66 \\
\hline Pyrene & 2.9 & $\mathrm{~J}$ & 4.00 & 3.4 & $\mathrm{~J}$ & 4.12 & 4.07 & $\mathrm{U}$ & 4.07 & 3.66 & $\mathrm{U}$ & 3.66 & 1.5 & $\mathrm{~J}$ & 3.66 \\
\hline \begin{tabular}{|l} 
Benzo(a)anthracene \\
\end{tabular} & 4.2 & & 4.00 & 5.9 & & 4.12 & 1.3 & $\mathrm{~J}$ & 4.07 & 3.66 & $\mathrm{U}$ & 3.66 & 5.3 & & 3.66 \\
\hline Chrysene & 10 & & 4.00 & 18 & & 4.12 & 3.6 & $\mathrm{~J}$ & 4.07 & 1.6 & $\mathrm{~J}$ & 3.66 & 14 & & 3.66 \\
\hline Benzo(b)fluoranthrene & 3.5 & $\mathrm{~J}$ & 4.00 & 7.1 & & 4.12 & 1.1 & $\mathrm{~J}$ & 4.07 & 3.66 & $\mathrm{U}$ & 3.66 & 10 & & 3.66 \\
\hline Benzo(k)fluoranthrene & 4.00 & $\mathrm{U}$ & 4.00 & 2.0 & $\mathrm{~J}$ & 4.12 & 1.2 & $\mathrm{~J}$ & 4.07 & 3.66 & $\mathrm{U}$ & 3.66 & 4.0 & & 3.66 \\
\hline Benzo(a)pyrene & 4.00 & $\mathrm{U}$ & 4.00 & 5.2 & & 4.12 & 1.4 & $\mathrm{~J}$ & 4.07 & 3.66 & $\mathrm{U}$ & 3.66 & 10 & & 3.66 \\
\hline Dibenzo(a,h)anthracene & 4.00 & $\mathrm{U}$ & 4.00 & 4.12 & $\mathrm{U}$ & 4.12 & 4.07 & $\mathrm{U}$ & 4.07 & 3.66 & $\mathrm{U}$ & 3.66 & 1.3 & $\mathrm{~J}$ & 3.66 \\
\hline Benzo(ghi)perylene & 1.6 & $\mathrm{~J}$ & 4.00 & 7.1 & & 4.12 & 5.2 & & 4.07 & 3.66 & $\mathrm{U}$ & 3.66 & 8.5 & & 3.66 \\
\hline
\end{tabular}


Table A-2. 128-F-2 In-Process Riverbank Excavation Berm Data (16 Pages)

\begin{tabular}{|c|c|c|c|c|c|c|}
\hline \multirow[t]{2}{*}{ Constituents } & \multicolumn{3}{|c|}{$\begin{array}{c}\text { J13LN8 } \\
\text { Riverbank } \\
\text { Sample Date } 10 / 26 / 06 \\
\end{array}$} & \multicolumn{3}{|c|}{$\begin{array}{c}\text { J13LN7 } \\
\text { Riverbank } \\
\text { Sample Date 10/26/06 } \\
\end{array}$} \\
\hline & $\mu \mathrm{g} / \mathrm{kg}$ & $\mathbf{Q}$ & PQL & $\mu \mathrm{g} / \mathrm{kg}$ & $\mathbf{Q}$ & PQL \\
\hline \multicolumn{7}{|c|}{ PCBs } \\
\hline Aroclor-1016 & 15 & $\mathrm{U}$ & 15 & 14 & $\mathrm{U}$ & 14 \\
\hline Aroclor-1221 & 15 & $\mathrm{U}$ & 15 & 14 & $\mathrm{U}$ & 14 \\
\hline Aroclor-1232 & 15 & $\mathrm{U}$ & 15 & 14 & $\mathrm{U}$ & 14 \\
\hline Aroclor-1242 & 15 & $\mathrm{U}$ & 15 & 14 & $\mathrm{U}$ & 14 \\
\hline Aroclor-1248 & 15 & $\mathrm{U}$ & 15 & 14 & $\mathrm{U}$ & 14 \\
\hline Aroclor-1254 & 15 & $\mathrm{U}$ & 15 & 14 & $\mathrm{U}$ & 14 \\
\hline Aroclor-1260 & 15 & $\mathrm{U}$ & 15 & 14 & $\mathrm{U}$ & 14 \\
\hline \multicolumn{7}{|c|}{ SVOAs } \\
\hline 1,2,4-Trichlorobenzene & 380 & $\mathrm{U}$ & 380 & 350 & $\mathrm{U}$ & 350 \\
\hline 1,2-Dichlorobenzene & 380 & $\mathrm{U}$ & 380 & 350 & $\mathrm{U}$ & 350 \\
\hline 1,3-Dichlorobenzene & 380 & $\mathrm{U}$ & 380 & 350 & $\bar{U}$ & 350 \\
\hline 1,4-Dichlorobenzene & 380 & $\mathrm{U}$ & 380 & 350 & $\mathrm{U}$ & 350 \\
\hline 2,4,5-Trichlorophenol & 940 & $\mathrm{U}$ & 940 & 880 & $\mathrm{U}$ & 880 \\
\hline 2,4,6-Trichlorophenol & 380 & $\mathrm{U}$ & 380 & 350 & $\mathrm{U}$ & 350 \\
\hline 2,4-Dichlorophenol & 380 & $\mathrm{U}$ & 380 & 350 & $\mathrm{U}$ & 350 \\
\hline 2,4-Dimethylphenol & 380 & $\mathrm{U}$ & 380 & 350 & $\mathrm{U}$ & 350 \\
\hline 2,4-Dinitrophenol & 940 & $\mathrm{U}$ & 940 & 880 & $\mathrm{U}$ & 880 \\
\hline 2,4-Dinitrotoluene & 380 & $\mathrm{U}$ & 380 & 350 & $\mathrm{U}$ & 350 \\
\hline 2,6-Dinitrotoluene & 380 & $\mathrm{U}$ & 380 & 350 & $\mathrm{U}$ & 350 \\
\hline 2-Chloronaphthalene & 380 & $\mathrm{U}$ & 380 & 350 & $\mathrm{U}$ & 350 \\
\hline 2-Chlorophenol & 380 & $\mathrm{U}$ & 380 & 350 & $\mathrm{U}$ & 350 \\
\hline 2-Methylnaphthalene & 380 & $\mathrm{U}$ & 380 & 350 & $\mathrm{U}$ & 350 \\
\hline 2-Methylphenol (cresol, o-) & 380 & $\mathrm{U}$ & 380 & 350 & $\mathrm{U}$ & 350 \\
\hline 2-Nitroaniline & 940 & $\mathrm{U}$ & 940 & 880 & $\mathrm{U}$ & 880 \\
\hline 2-Nitrophenol & 380 & $\mathrm{U}$ & 380 & 350 & $\mathrm{U}$ & 350 \\
\hline 3+4 Methylphenol (cresol, $\mathrm{m}+\mathrm{p}$ ) & 380 & $\mathrm{U}$ & 380 & 350 & $\bar{U}$ & 350 \\
\hline 3,3'-Dichlorobenzidine & 380 & $\mathrm{U}$ & 380 & 350 & $\mathrm{U}$ & 350 \\
\hline 3-Nitroaniline & 940 & $\mathrm{U}$ & 940 & 880 & $\mathrm{U}$ & 880 \\
\hline 4,6-Dinitro-2-methylphenol & 940 & $\mathrm{U}$ & 940 & 880 & $\mathrm{U}$ & 880 \\
\hline 4-Bromophenylphenyl ether & 380 & $\mathrm{U}$ & 380 & 350 & $\mathrm{U}$ & 350 \\
\hline 4-Chloro-3-methylphenol & 380 & $\mathrm{U}$ & 380 & 350 & $\mathrm{U}$ & 350 \\
\hline 4-Chloroaniline & 380 & $\mathrm{U}$ & 380 & 350 & $\mathrm{U}$ & 350 \\
\hline 4-Chlorophenylphenyl ether & 380 & $\mathrm{U}$ & 380 & 350 & $\mathrm{U}$ & 350 \\
\hline 4-Nitroaniline & 940 & $\mathrm{U}$ & 940 & 880 & $\mathrm{U}$ & 880 \\
\hline 4-Nitrophenol & 940 & $\mathrm{U}$ & 940 & 880 & $\mathrm{U}$ & 880 \\
\hline Acenaphthene & 380 & $\mathrm{U}$ & 380 & 350 & $\mathrm{U}$ & 350 \\
\hline Acenaphthylene & 380 & $\mathrm{U}$ & 380 & 350 & $\mathrm{U}$ & 350 \\
\hline Anthracene & 380 & $\mathrm{U}$ & 380 & 350 & $\mathrm{U}$ & 350 \\
\hline
\end{tabular}


Table A-2. 128-F-2 In-Process Riverbank Excavation Berm Data (16 Pages)

\begin{tabular}{|c|c|c|c|c|c|c|}
\hline \multirow[t]{2}{*}{ Constituents } & \multicolumn{3}{|c|}{$\begin{array}{c}\text { J13LN8 } \\
\text { Riverbank } \\
\text { Sample Date } \mathbf{1 0 / 2 6 / 0 6}\end{array}$} & \multicolumn{3}{|c|}{$\begin{array}{c}\text { J13LN7 } \\
\text { Riverbank } \\
\text { Sample Date 10/26/06 }\end{array}$} \\
\hline & $\mu \mathrm{g} / \mathrm{kg}$ & $\mathbf{Q}$ & PQL & $\mu \mathrm{g} / \mathrm{kg}$ & $\mathbf{Q}$ & PQL \\
\hline \multicolumn{7}{|c|}{ SVOAs (continued) } \\
\hline Benzo(a)anthracene & 380 & $\mathrm{U}$ & 380 & 350 & $\mathrm{U}$ & 350 \\
\hline Benzo(a)pyrene & 380 & $\mathrm{U}$ & 380 & 350 & $\mathrm{U}$ & 350 \\
\hline Benzo(b)fluoranthene & 380 & $\mathrm{U}$ & 380 & 350 & $\mathrm{U}$ & 350 \\
\hline Benzo(ghi)perylene & 380 & $\mathrm{U}$ & 380 & 350 & $\mathrm{U}$ & 350 \\
\hline Benzo(k)fluoranthene & 380 & $\mathrm{U}$ & 380 & 350 & $\mathrm{U}$ & 350 \\
\hline Bis(2-chloro-1-methylethyl)ether & 380 & $\mathrm{U}$ & 380 & 350 & $\mathrm{U}$ & 350 \\
\hline Bis(2-Chloroethoxy)methane & 380 & $\mathrm{U}$ & 380 & 350 & $\mathrm{U}$ & 350 \\
\hline Bis(2-chloroethyl) ether & 380 & $\mathrm{U}$ & 380 & 350 & $\mathrm{U}$ & 350 \\
\hline Bis(2-ethylhexyl) phthalate & 63 & JB & 380 & 78 & JB & 350 \\
\hline Butylbenzylphthalate & 380 & $\mathrm{U}$ & 380 & 350 & $\mathrm{U}$ & 350 \\
\hline Carbazole & 380 & $\mathrm{U}$ & 380 & 350 & $\mathrm{U}$ & 350 \\
\hline Chrysene & 380 & $\mathrm{U}$ & 380 & 350 & $\mathrm{U}$ & 350 \\
\hline Di-n-butylphthalate & 36 & JB & 380 & 38 & JB & 350 \\
\hline Di-n-octylphthalate & 380 & $\mathrm{U}$ & 380 & 350 & $\mathrm{U}$ & 350 \\
\hline Dibenz[a,h]anthracene & 380 & $\mathrm{U}$ & 380 & 350 & $\mathrm{U}$ & 350 \\
\hline Dibenzofuran & 380 & $\mathrm{U}$ & 380 & 350 & $\mathrm{U}$ & 350 \\
\hline Diethylphthalate & 380 & $\mathrm{U}$ & 380 & 350 & $\mathrm{U}$ & 350 \\
\hline Dimethyl phthalate & 380 & $\mathrm{U}$ & 380 & 350 & $\mathrm{U}$ & 350 \\
\hline Fluoranthene & 380 & $\mathrm{U}$ & 380 & 350 & $\mathrm{U}$ & 350 \\
\hline Fluorene & 380 & $\mathrm{U}$ & 380 & 350 & $\mathrm{U}$ & 350 \\
\hline Hexachlorobenzene & 380 & $\mathrm{U}$ & 380 & 350 & $\mathrm{U}$ & 350 \\
\hline Hexachlorobutadiene & 380 & $\mathrm{U}$ & 380 & 350 & $\mathrm{U}$ & 350 \\
\hline Hexachlorocyclopentadiene & 380 & $\mathrm{U}$ & 380 & 350 & $\mathrm{U}$ & 350 \\
\hline Hexachloroethane & 380 & $\mathrm{U}$ & 380 & 350 & $\mathrm{U}$ & 350 \\
\hline Indeno(1,2,3-cd)pyrene & 380 & $\mathrm{U}$ & 380 & 350 & $\mathrm{U}$ & 350 \\
\hline Isophorone & 380 & $\mathrm{U}$ & 380 & 350 & $\mathrm{U}$ & 350 \\
\hline N-Nitroso-di-n-dipropylamine & 380 & $\mathrm{U}$ & 380 & 350 & $\mathrm{U}$ & 350 \\
\hline N-Nitrosodiphenylamine & 380 & $\mathrm{U}$ & 380 & 350 & $\mathrm{U}$ & 350 \\
\hline Naphthalene & 380 & $\mathrm{U}$ & 380 & 350 & $\mathrm{U}$ & 350 \\
\hline Nitrobenzene & 380 & $\mathrm{U}$ & 380 & 350 & $\mathrm{U}$ & 350 \\
\hline Pentachlorophenol & 940 & $\mathrm{U}$ & 940 & 880 & $\mathrm{U}$ & 880 \\
\hline Phenanthrene & 380 & $\mathrm{U}$ & 380 & 350 & $\mathrm{U}$ & 350 \\
\hline Phenol & 380 & $\mathrm{U}$ & 380 & 350 & $\mathrm{U}$ & 350 \\
\hline Pyrene & 380 & $\mathrm{U}$ & 380 & 350 & $\mathrm{U}$ & 350 \\
\hline \multicolumn{7}{|c|}{ Pesticides } \\
\hline Alpha-BHC & 1.5 & $\mathrm{U}$ & 1.5 & 1.4 & $\mathrm{U}$ & 1.4 \\
\hline gamma-BHC (Lindane) & 1.5 & $\mathrm{U}$ & 1.5 & 1.4 & $\mathrm{U}$ & 1.4 \\
\hline Beta-BHC & 1.5 & $\mathrm{U}$ & 1.5 & 1.4 & $\mathrm{U}$ & 1.4 \\
\hline
\end{tabular}


Table A-2. 128-F-2 In-Process Riverbank Excavation Berm Data (16 Pages)

\begin{tabular}{|c|c|c|c|c|c|c|}
\hline \multirow[t]{2}{*}{ Constituents } & \multicolumn{3}{|c|}{$\begin{array}{c}\text { J13LN8 } \\
\text { Riverbank } \\
\text { Sample Date } 10 / 26 / 06\end{array}$} & \multicolumn{3}{|c|}{$\begin{array}{c}\text { J13LN7 } \\
\text { Riverbank } \\
\text { Sample Date } 10 / 26 / 06\end{array}$} \\
\hline & $\mu \mathrm{\mu g} / \mathrm{kg}$ & $\mathbf{Q}$ & PQL & $\mu \mathrm{g} / \mathrm{kg}$ & $\mathbf{Q}$ & PQL \\
\hline \multicolumn{7}{|c|}{ Pesticides (continued) } \\
\hline Heptachlor & 1.5 & $\overline{\mathrm{U}}$ & 1.5 & 1.4 & $\mathrm{U}$ & 1.4 \\
\hline Delta-BHC & 1.5 & $\overline{\mathrm{U}}$ & 1.5 & 1.4 & $\mathrm{U}$ & 1.4 \\
\hline Aldrin & 1.5 & $\mathrm{U}$ & 1.5 & 1.4 & $\mathrm{U}$ & 1.4 \\
\hline Heptachlor epoxide & 1.5 & $\overline{\mathrm{U}}$ & 1.5 & 1.4 & $\mathrm{U}$ & 1.4 \\
\hline gamma-Chlordane & 1.5 & $\mathrm{U}$ & 1.5 & 1.4 & $\mathrm{U}$ & 1.4 \\
\hline Endosulfan I & 1.5 & $\bar{U}$ & 1.5 & 1.4 & $\mathrm{U}$ & 1.4 \\
\hline alpha-Chlordane & 1.5 & $\mathrm{U}$ & 1.5 & 1.4 & $\mathrm{U}$ & 1.4 \\
\hline $4,4^{\prime}$-DDE & 1.5 & $\overline{\mathrm{U}}$ & 1.5 & 1.4 & $\mathrm{U}$ & 1.4 \\
\hline Deildrin & 1.5 & $\mathrm{U}$ & 1.5 & 1.4 & $\mathrm{U}$ & 1.4 \\
\hline Endrin & 1.5 & $\overline{\mathrm{U}}$ & 1.5 & 1.4 & $\mathrm{U}$ & 1.4 \\
\hline 4,4'-DDD & 1.5 & $\mathrm{U}$ & 1.5 & 1.4 & $\mathrm{U}$ & 1.4 \\
\hline Endosulfan II & 1.5 & $\bar{U}$ & 1.5 & 1.4 & $\mathrm{U}$ & 1.4 \\
\hline 4,4'-DDT & 1.5 & $\mathrm{U}$ & 1.5 & 1.4 & $\mathrm{U}$ & 1.4 \\
\hline Endrin aldehyde & 1.5 & $\mathrm{U}$ & 1.5 & 1.7 & & 1.4 \\
\hline Endosulfan sulfate & 1.5 & $\mathrm{U}$ & 1.5 & 1.4 & $\mathrm{U}$ & 1.4 \\
\hline Methoxychlor & 1.5 & $\mathrm{U}$ & 1.5 & 1.7 & $\mathrm{I}$ & 1.4 \\
\hline Endrin ketone & 1.5 & $\mathrm{U}$ & 1.5 & 1.4 & $\mathrm{U}$ & 1.4 \\
\hline Toxaphene & 15 & $\mathrm{U}$ & 15 & 14 & $\mathrm{U}$ & 14 \\
\hline \multicolumn{7}{|c|}{ PAHs (Polycyclic Aromatic Hydrocarbons) } \\
\hline Naphthalene & 37.8 & $\overline{\mathrm{U}}$ & 37.8 & 35.2 & $\mathrm{U}$ & 35.2 \\
\hline Acenaphthylene & 37.8 & $\mathrm{U}$ & 37.8 & 35.2 & $\mathrm{U}$ & 35.2 \\
\hline Acenaphthene & 37.8 & $\mathrm{U}$ & 37.8 & 35.2 & $\mathrm{U}$ & 35.2 \\
\hline Fluorene & 15 & & 3.78 & 1.3 & $\mathrm{~J}$ & 3.52 \\
\hline Phenanthrene & 7.2 & $\mathrm{~B}$ & 3.78 & 2.6 & $\mathrm{JB}$ & 3.52 \\
\hline Anthracene & 3.78 & $\mathrm{U}$ & 3.78 & 3.52 & $\mathrm{U}$ & 3.52 \\
\hline Fluoranthrene & 2.6 & $\mathrm{~J}$ & 3.78 & 21 & & 3.52 \\
\hline Indeno (1,2,3-cd)pyrene & 8.4 & & 3.78 & 3.52 & $\mathrm{U}$ & 3.52 \\
\hline Pyrene & 0.94 & $\bar{J}$ & 3.78 & 1.1 & $\mathrm{~J}$ & 3.52 \\
\hline Benzo(a)anthracene & 5.3 & & 3.78 & 2.6 & $\mathrm{~J}$ & 3.52 \\
\hline Chrysene & 12 & & 3.78 & 4.7 & & 3.52 \\
\hline Benzo(b)fluoranthrene & 10 & & 3.78 & 4.3 & & 3.52 \\
\hline Benzo(k)fluoranthrene & 3.5 & $\mathrm{~J}$ & 3.78 & 1.4 & $\mathrm{~J}$ & 3.52 \\
\hline Benzo(a)pyrene & 6.3 & & 3.78 & 2.8 & $\mathrm{~J}$ & 3.52 \\
\hline Dibenzo(a,h)anthracene & 1.4 & $\mathrm{~J}$ & 3.78 & 3.52 & $\mathrm{U}$ & 3.52 \\
\hline Benzo(ghi)perylene & 7.3 & & 3.78 & 3.2 & $\mathrm{~J}$ & 3.52 \\
\hline
\end{tabular}


Table A-3. 128-F-2 In-Process Low-Lying Area Sampling Results (2 Pages).

\begin{tabular}{|c|c|c|c|c|c|c|c|c|c|c|c|c|c|c|c|c|c|c|c|c|c|c|c|}
\hline \multirow{2}{*}{$\begin{array}{c}\text { Sample } \\
\text { Location }\end{array}$} & \multirow{2}{*}{$\begin{array}{c}\text { Sample } \\
\text { Number }\end{array}$} & \multirow{2}{*}{$\begin{array}{c}\text { Sample } \\
\text { Date } \\
\end{array}$} & \multicolumn{3}{|c|}{ Aluminum } & \multicolumn{3}{|c|}{ Antimony } & \multicolumn{3}{|c|}{ Arsenic } & \multicolumn{3}{|c|}{ Barium } & \multicolumn{3}{|c|}{ Beryllium } & \multicolumn{3}{|c|}{ Boron } & \multicolumn{3}{|c|}{ Cadmium } \\
\hline & & & $\mathrm{mg} / \mathrm{kg}$ & $\mathbf{Q}$ & PQL & $\mathrm{mg} / \mathrm{kg}$ & \begin{tabular}{|l|}
$\mathbf{Q}$ \\
\end{tabular} & PQL & $\mathrm{mg} / \mathrm{kg}$ & $\mathbf{Q}$ & PQL & $\mathrm{mg} / \mathrm{kg}$ & $\mathbf{Q}$ & PQL & $\mathrm{mg} / \mathrm{kg}$ & $\mathbf{Q}$ & PQL & $\mathrm{mg} / \mathrm{kg}$ & $\mathbf{Q}$ & PQL & $\mathrm{mg} / \mathrm{kg}$ & $\mathbf{Q}$ & $\overline{\text { PQL }}$ \\
\hline $\mathrm{A}^{\prime}-1$ & J13PB1 & $11 / 16 / 06$ & 8060 & & 7.6 & 0.90 & $\mathrm{U}$ & 0.90 & 7.0 & & 1.1 & 134 & & 0.04 & 0.40 & & 0.04 & 0.68 & $\mathrm{U}$ & 0.68 & 0.32 & & 0.11 \\
\hline $\mathrm{A}^{\prime}-2$ & J13PB2 & $11 / 16 / 06$ & 4880 & & 7.8 & 0.92 & $\mathrm{U}$ & 0.92 & 3.7 & & 1.1 & 76.6 & & 0.04 & 0.25 & & 0.04 & 0.70 & $\mathrm{U}$ & 0.70 & 0.11 & $\mathrm{U}$ & 0.11 \\
\hline $\mathrm{A}^{\prime}-3$ & J13PB3 & $11 / 16 / 06$ & 7350 & & 7.7 & 0.91 & $\mathrm{U}$ & 0.91 & 6.3 & & 1.1 & 111 & & 0.04 & 0.38 & & 0.04 & 0.69 & $\mathrm{U}$ & 0.69 & 0.19 & & 0.11 \\
\hline $\mathrm{A}^{\prime}-4$ & J13PB4 & $11 / 16 / 06$ & 11100 & & 7.2 & 0.85 & $\mathrm{U}$ & 0.85 & 9.2 & & 1.1 & 190 & & 0.03 & 0.53 & & 0.03 & 0.65 & $\mathrm{U}$ & 0.65 & 0.10 & $\mathrm{U}$ & 0.10 \\
\hline $\mathrm{A}^{\prime}-5$ & J13PB5 & $11 / 16 / 06$ & 5530 & & 7.6 & 0.90 & $\mathrm{U}$ & 0.90 & 11.2 & & 1.1 & 86.5 & & 0.04 & 0.32 & & 0.04 & 2.3 & & 0.68 & 1.1 & & 0.11 \\
\hline $\mathrm{A}^{\prime}-6$ & J13PB6 & $11 / 16 / 06$ & 9480 & & 8.3 & 1.2 & & 0.98 & 5.0 & & 1.2 & 80.1 & & 0.04 & 0.33 & & 0.04 & 0.75 & $\mathrm{U}$ & 0.75 & 1.5 & & 0.12 \\
\hline $\mathrm{A}^{\prime}-7$ & J13PB7 & $11 / 16 / 06$ & 7990 & & 7.5 & 1.5 & & 0.89 & 9.7 & & 1.1 & 130 & & 0.04 & 0.37 & & 0.04 & 0.68 & $\mathrm{U}$ & 0.68 & 3.3 & & 0.11 \\
\hline $\mathrm{A}^{\prime}-8$ & J13PB8 & $11 / 16 / 06$ & 7780 & & 7.7 & 1.6 & & 0.09 & 19.6 & & 1.1 & 101 & & 0.04 & 0.34 & & 0.04 & 0.69 & $\mathrm{U}$ & 0.69 & 2.8 & & 0.11 \\
\hline $\mathrm{A}^{\prime}-9$ & J13РB9 & $11 / 16 / 06$ & 7200 & & 6.7 & 1.5 & & 0.79 & 4.7 & & 0.98 & 76.3 & & 0.03 & 0.30 & & 0.03 & 0.60 & $\mathrm{U}$ & 0.60 & 2.0 & & 0.1 \\
\hline$A^{\prime}-10$ & J13PC0 & $11 / 16 / 06$ & 6330 & & 6.9 & 0.82 & $\mathrm{U}$ & 0.82 & 6.7 & & 1.0 & 84.4 & & 0.03 & 0.34 & & 0.03 & 0.62 & $\mathrm{U}$ & 0.62 & 1.3 & & 0.1 \\
\hline $\begin{array}{c}\text { Duplicate of } \\
\text { J13PC0 }\end{array}$ & J13PC1 & 11/16/06 & 5990 & & 6.8 & 1.1 & & 0.81 & 6.4 & & 1.0 & 82.6 & & 0.03 & 0.32 & & 0.03 & 0.62 & $\mathrm{U}$ & 0.62 & 1.2 & & 0.1 \\
\hline $\mathrm{A}^{\prime}-11$ & J13J42 & $11 / 16 / 06$ & 7660 & & 7.8 & 1.1 & & 0.93 & 9.4 & & 1.2 & 107 & & 0.04 & 0.33 & & 0.04 & 0.71 & $\mathrm{U}$ & 0.71 & 1.7 & & 0.11 \\
\hline $\mathrm{A}^{\prime}-12$ & $\mathrm{~J} 13 \mathrm{~J} 43$ & 11/16/06 & 5250 & & 7.7 & 0.91 & $\mathrm{U}$ & 0.91 & 4.3 & & 1.1 & 74.7 & & 0.04 & 0.20 & & 0.04 & 0.69 & $\mathrm{U}$ & 0.69 & 0.30 & & 0.11 \\
\hline A-13 & J13PC4 & 11/16/06 & 6810 & & 6.3 & 0.75 & $\mathrm{U}$ & 0.75 & 2.7 & & 0.92 & 128 & & 0.03 & 0.30 & & 0.03 & 0.57 & $\mathrm{U}$ & 0.57 & 0.09 & $\mathrm{U}$ & 0.09 \\
\hline A-14 & J13PC5 & 11/16/06 & 6980 & & 6.2 & 0.74 & $\mathrm{U}$ & 0.74 & 9.6 & & 0.92 & 83.7 & & 0.03 & 0.31 & & 0.03 & 0.56 & $\mathrm{U}$ & 0.56 & 0.09 & $\mathrm{U}$ & 0.09 \\
\hline A-15 & J13PC6 & 11/16/06 & 7030 & & 6.5 & 0.77 & $\mathrm{U}$ & 0.77 & 4.1 & & 0.95 & 82.2 & & 0.03 & 0.29 & & 0.03 & 0.58 & $\mathrm{U}$ & 0.58 & 0.09 & $\mathrm{U}$ & 0.09 \\
\hline A-16 & J13PC7 & 11/16/06 & 7690 & & 6.3 & 0.75 & $\mathrm{U}$ & 0.75 & 3.8 & & 3.8 & 76.4 & & 0.03 & 0.32 & & 0.03 & 0.57 & $\mathrm{U}$ & 0.57 & 0.09 & $\mathrm{U}$ & 0.09 \\
\hline A-11 (in River) & J13PC2 & 11/16/06 & 3800 & & 7.0 & 0.83 & $\mathrm{U}$ & 0.83 & 4.4 & & 1.0 & 70.4 & & 0.03 & 0.25 & & 0.03 & 0.63 & $\mathrm{U}$ & 0.63 & 0.68 & & 0.1 \\
\hline A-12 (in River) & J13PC3 & 11/16/06 & 3540 & & 7.2 & 0.86 & $\mathrm{U}$ & 0.9 & 3.1 & & 1.0 & 55.1 & $\mathrm{C}$ & 0.03 & 0.20 & & 0.03 & 0.65 & $\mathrm{U}$ & 0.65 & 0.35 & & 0.1 \\
\hline
\end{tabular}

\begin{tabular}{|c|c|c|c|c|c|c|c|c|c|c|c|c|c|c|c|c|c|c|c|c|c|c|c|}
\hline \multirow{2}{*}{$\begin{array}{l}\text { Sample } \\
\text { Location }\end{array}$} & \multirow{2}{*}{$\begin{array}{l}\text { Sample } \\
\text { Number }\end{array}$} & \multirow{2}{*}{$\begin{array}{l}\text { Sample } \\
\text { Date }\end{array}$} & \multicolumn{3}{|c|}{ Calcium } & \multicolumn{3}{|c|}{ Chromium } & \multicolumn{3}{|c|}{ Cobalt } & \multicolumn{3}{|c|}{ Copper } & \multicolumn{3}{|c|}{$\begin{array}{l}\text { Hexavalent } \\
\text { Chromium }\end{array}$} & \multicolumn{3}{|c|}{ Iron } & \multicolumn{3}{|c|}{ Lead } \\
\hline & & & $\mathrm{mg} / \mathrm{kg}$ & $\mathbf{Q}$ & PQL & $\mathrm{mg} / \mathrm{kg}$ & \begin{tabular}{l|l}
$\mathbf{Q}$ & \\
\end{tabular} & PQL & $\mathrm{mg} / \mathrm{kg}$ & $\mathbf{Q} \mathbf{Q}$ & PQL & $\mathrm{mg} / \mathrm{kg}$ & $\mathbf{Q}$ & $\mathbf{P Q L}$ & \begin{tabular}{|l|}
$\mathbf{m g} / \mathbf{k g}$ \\
\end{tabular} & $\mathbf{Q}$ & PQL & $\mathrm{mg} / \mathrm{kg}$ & $\mathbf{Q}$ & PQL & $\mathbf{m g} / \mathbf{k g}$ & $\mathbf{Q}$ & PQL \\
\hline $\mathrm{A}^{\prime}-1$ & J13PB1 & $11 / 16 / 06$ & 3090 & & 4.5 & 172 & & 0.43 & 6.6 & & 0.18 & 46.6 & & 0.25 & 10.3 & & 0.25 & 16800 & & 8.7 & 73.5 & & 0.57 \\
\hline $\mathrm{A}^{\prime}-2$ & J13PB2 & $11 / 16 / 06$ & 1660 & & 4.6 & 18.9 & & 0.44 & 3.1 & & 0.18 & 12.9 & & 0.26 & 0.31 & & 0.26 & 10900 & & 9.0 & 2.2 & & 0.59 \\
\hline $\mathrm{A}^{\prime}-3$ & 3PB3 & $11 / 16 / 06$ & 2620 & & 4.5 & 170 & & 0.44 & 7.1 & & 0.18 & 21.9 & & 0.25 & 6.6 & & 0.25 & 16100 & & 8.9 & 12.7 & & 0.58 \\
\hline $\mathrm{A}^{\prime}-4$ & 3PB4 & $\begin{array}{ll}11 / 16 / 06 \\
\end{array}$ & 2430 & & 4.3 & 140 & & 0.41 & 10.2 & & 0.17 & 34.9 & & 0.24 & 2.5 & & 0.24 & 21600 & & 8.3 & 7.2 & & 0.55 \\
\hline $\mathrm{A}^{\prime}-5$ & 3PB5 & $\begin{array}{l}11 / 16 / 06 \\
\end{array}$ & 2330 & & 4.5 & 146 & & 0.43 & 10.3 & & 0.18 & 31.2 & & 0.25 & 1.2 & & 0.25 & 17200 & & 8.8 & 22.5 & & 0.57 \\
\hline $\mathrm{A}^{\prime}-6$ & 3PB6 & $\begin{array}{l}11 / 16 / 06 \\
\end{array}$ & 4810 & & 4.9 & 21.2 & & 0.47 & 7.6 & & 0.20 & 35.9 & & 0.27 & 0.36 & & 0.27 & 20400 & & 9.6 & 80.8 & & 0.63 \\
\hline $\mathrm{A}^{\prime}-7$ & 3PB7 & 11/16/06 & 5750 & & 4.5 & 16.4 & & 0.43 & 8.6 & & 0.18 & 58.7 & & 0.25 & 0.47 & & 0.25 & 22900 & & 8.7 & 199 & & 0.57 \\
\hline $\mathrm{A}^{\prime}-8$ & J13PB8 & 11/16/06 & 3570 & & 4.6 & 327 & & 0.44 & 8.8 & & 0.18 & 74.3 & & 0.26 & 5.0 & & 0.26 & 21600 & & 8.9 & 225 & & 0.59 \\
\hline $\mathrm{A}^{\prime}-9$ & PB9 & $11 / 16 / 06$ & 4370 & & 4.0 & 121 & & 0.38 & 8.0 & & 0.16 & 74.3 & & 0.22 & 2.7 & & 0.22 & 20600 & & 7.8 & 180 & & 0.51 \\
\hline $\mathrm{A}^{\prime}-10$ & $3 \mathrm{PC0}$ & $11 / 16 / 06$ & 3920 & & 4.1 & 225 & & 0.39 & 7.6 & & 0.16 & 40.1 & & 0.23 & 6.0 & & 0.23 & 20000 & & 8.0 & 107 & & 0.52 \\
\hline $\begin{array}{l}\text { Duplicate of } \\
\text { J13PC0 }\end{array}$ & PC1 & $11 / 16 / 06$ & 3980 & & 4.0 & 223 & & 0.39 & 7.5 & & 0.16 & 38.8 & & 0.23 & 5.7 & & 0.22 & 19900 & & 7.9 & 99.3 & & 0.52 \\
\hline $\mathrm{A}^{\prime}-11$ & $3 \mathrm{~J} 42$ & $11 / 16 / 06$ & 3280 & & 4.6 & 380 & & 0.45 & 7.4 & & 0.19 & 45.2 & & 0.26 & 6.3 & & 0.26 & 20000 & & 9.1 & 133 & & 0.59 \\
\hline $\mathrm{A}^{\prime}-12$ & $3 \mathrm{~J} 43$ & $11 / 16 / 06$ & 5090 & & 4.5 & 15.9 & & 0.44 & 5.1 & & 0.18 & 51.0 & & 0.25 & 0.27 & & 0.26 & 13600 & & 8.9 & 49.5 & & 0.58 \\
\hline A-13 & 3PC4 & $11 / 16 / 06$ & 2760 & & 3.7 & 28.2 & & 0.36 & 5.8 & & 0.15 & 25.3 & & 0.21 & 0.92 & & 0.21 & 14900 & & 7.3 & 12.2 & & 0.48 \\
\hline A-14 & J13PC5 & $11 / 16 / 06$ & 2440 & & 3.7 & 18.1 & & 0.35 & 4.6 & & 0.15 & 18.9 & & 0.21 & 0.90 & & 0.21 & 18300 & & 7.2 & 3.5 & & 0.47 \\
\hline A-15 & J13PC6 & $11 / 16 / 06$ & 16000 & & 3.8 & 21.4 & & 0.37 & 6.0 & & 0.15 & 12.5 & & 0.21 & 0.65 & & 0.21 & 16300 & & 7.5 & 6.1 & & 0.49 \\
\hline A-16 & J13PC7 & $11 / 16 / 06$ & 3150 & & 3.7 & 16.5 & & 0.36 & 6.5 & & 0.15 & 12.2 & & 0.21 & 0.29 & & 0.21 & 17100 & & 7.3 & 4.9 & & 0.48 \\
\hline A-11 (in River) & J13PC2 & $11 / 16 / 06$ & 2630 & & 4.1 & 14.0 & & 0.40 & 5.6 & & 0.17 & 12.1 & & 0.23 & 0.37 & & 0.23 & 14000 & & 8.1 & 15.4 & & 0.53 \\
\hline A-12 (in River) & J13PC3 & $11 / 16 / 06$ & 2070 & $\mathrm{C}$ & 4.2 & 16.5 & & 0.41 & 4.2 & & 0.18 & 9.3 & & 0.24 & 1.6 & & 0.24 & 11900 & & 8.3 & 8.1 & & 0.55 \\
\hline
\end{tabular}


Table A-3. 128-F-2 In-Process Low-Lying Area Sampling Results (2 Pages).

\begin{tabular}{|c|c|c|c|c|c|c|c|c|c|c|c|c|c|c|c|c|c|c|c|c|c|c|c|}
\hline \multirow{2}{*}{$\begin{array}{c}\text { Sample } \\
\text { Location }\end{array}$} & \multirow{2}{*}{$\begin{array}{c}\text { Sample } \\
\text { Number }\end{array}$} & \multirow{2}{*}{$\begin{array}{c}\text { Sample } \\
\text { Date }\end{array}$} & \multicolumn{3}{|c|}{ Magnesium } & \multicolumn{3}{|c|}{ Manganese } & \multicolumn{3}{|c|}{ Mercury } & \multicolumn{3}{|c|}{ Molybdenum } & \multicolumn{3}{|c|}{ Nickel } & \multicolumn{3}{|c|}{ Potassium } & \multicolumn{3}{|c|}{ Selenium } \\
\hline & & & $\mathrm{mg} / \mathrm{kg}$ & $\mathbf{Q}$ & PQL & $\mathrm{mg} / \mathrm{kg}$ & \begin{tabular}{|l|l|}
$\mathbf{Q}$ \\
\end{tabular} & PQL & $\mathrm{mg} / \mathrm{kg}$ & $\mathbf{Q}$ & \begin{tabular}{|l|} 
PQL \\
\end{tabular} & $\mathrm{mg} / \mathrm{kg}$ & $\mathbf{Q}$ & PQL & $\mathrm{mg} / \mathrm{kg}$ & $\mathbf{Q}$ & PQL & $\mathrm{mg} / \mathrm{kg}$ & $\mathbf{Q}$ & \begin{tabular}{|l|} 
PQL \\
\end{tabular} & $\mathrm{mg} / \mathrm{kg}$ & $\mathbf{Q}$ & \begin{tabular}{|l|} 
PQL \\
\end{tabular} \\
\hline $\mathrm{A}^{\prime}-1$ & J13PB1 & $\begin{array}{l}11 / 16 / 06 \\
\end{array}$ & 3610 & & 1.6 & 242 & & 0.14 & 0.14 & & \begin{tabular}{|l|}
0.02 \\
\end{tabular} & 0.57 & $\mathrm{U}$ & 0.57 & 24.0 & & 0.79 & 699 & & 7.6 & 1.5 & $\mathrm{U}$ & 1.5 \\
\hline $\mathrm{A}^{\prime}-2$ & J13PB2 & $\begin{array}{l}11 / 16 / 06 \\
\end{array}$ & 2370 & & 1.7 & 128 & & 0.15 & 0.02 & $\mathrm{U}$ & 0.02 & 0.59 & $\mathrm{U}$ & 0.59 & 7.4 & & 0.81 & 435 & & 7.8 & 1.6 & $\mathrm{U}$ & 1.6 \\
\hline $\mathrm{A}^{\prime}-3$ & J13PB3 & $\begin{array}{l}11 / 16 / 06 \\
\end{array}$ & 3940 & & 1.7 & 439 & & 0.15 & 0.02 & $\mathrm{U}$ & 0.02 & 0.58 & $\mathrm{U}$ & 0.58 & 23.6 & & 0.80 & 674 & & 7.7 & 1.6 & $\mathrm{U}$ & 1.6 \\
\hline $\mathrm{A}^{\prime}-4$ & J13PB4 & $\begin{array}{l}11 / 16 / 06 \\
\end{array}$ & 3940 & & 1.6 & 568 & & 0.14 & 0.02 & $\mathrm{U}$ & 0.02 & 0.86 & & 0.55 & 29.3 & & 0.75 & 654 & & 7.2 & 1.5 & $\mathrm{U}$ & 1.5 \\
\hline $\mathrm{A}^{\prime}-5$ & J13PB5 & $\begin{array}{l}11 / 16 / 06 \\
\end{array}$ & 6040 & & 1.7 & 712 & & 0.14 & 0.02 & $\mathrm{U}$ & 0.02 & 0.69 & & 0.57 & 49.0 & & 0.79 & 516 & & 7.6 & 1.5 & $\mathrm{U}$ & 1.5 \\
\hline $\mathrm{A}^{\prime}-6$ & J13PB6 & $\begin{array}{ll}11 / 16 / 06 \\
\end{array}$ & 4390 & & 1.8 & 695 & & 0.16 & 0.02 & $\mathrm{U}$ & 0.02 & 0.63 & $\mathrm{U}$ & 0.63 & 20.0 & & 0.86 & 892 & & 8.3 & 1.7 & $\mathrm{U}$ & 1.7 \\
\hline $\mathrm{A}^{\prime}-7$ & J13PB7 & $\begin{array}{ll}11 / 16 / 06 \\
\end{array}$ & 5470 & & 1.6 & 495 & & 0.14 & 0.16 & & \begin{tabular}{|l|}
0.02 \\
\end{tabular} & 0.69 & & 0.57 & 15.2 & & 0.79 & 1100 & & 7.6 & 1.5 & $\mathrm{U}$ & 1.5 \\
\hline $\mathrm{A}^{\prime}-8$ & J13PB8 & $\begin{array}{ll}11 / 16 / 06 \\
\end{array}$ & 4280 & & 1.7 & 356 & & 0.15 & 0.02 & & \begin{tabular}{|l|}
0.02 \\
\end{tabular} & 0.59 & $\mathrm{U}$ & 0.59 & 15.1 & & 0.80 & 1060 & & 7.8 & 1.6 & $\mathrm{U}$ & 1.6 \\
\hline $\mathrm{A}^{\prime}-9$ & J13PB9 & $\begin{array}{ll}11 / 16 / 06 \\
\end{array}$ & 4890 & & 1.5 & 346 & & 0.13 & 0.04 & & \begin{tabular}{|l|}
0.02 \\
\end{tabular} & 0.51 & $\mathrm{U}$ & 0.51 & 15.2 & & 0.70 & 863 & & 6.7 & 1.4 & $\mathrm{U}$ & 1.4 \\
\hline $\mathrm{A}^{\prime}-10$ & J13PC0 & $\begin{array}{ll}11 / 16 / 06 \\
\end{array}$ & 4060 & & 1.5 & 279 & & 0.13 & 0.02 & & \begin{tabular}{|l|}
0.02 \\
\end{tabular} & 0.52 & $\mathrm{U}$ & 0.52 & 12.2 & & 0.72 & 1150 & & 6.9 & 1.4 & $\mathrm{U}$ & 1.4 \\
\hline $\begin{array}{c}\text { Duplicate of } \\
\text { J13PC0 }\end{array}$ & J13PC1 & $11 / 16 / 06$ & 3940 & & 1.5 & 271 & & 0.13 & 0.02 & $\mathrm{U}$ & 0.02 & 0.52 & $\mathrm{U}$ & 0.52 & 11.5 & & 0.71 & 1130 & & 6.9 & 1.4 & $\mathrm{U}$ & 1.4 \\
\hline $\mathrm{A}^{\prime}-11$ & J13J42 & $11 / 16 / 06$ & 4170 & & 1.7 & 222 & & 0.15 & 0.05 & & \begin{tabular}{|l|}
0.02 \\
\end{tabular} & 0.59 & $\mathrm{U}$ & 0.59 & 13.8 & & 0.82 & 993 & & 7.9 & 1.6 & $\mathrm{U}$ & 1.6 \\
\hline $\mathrm{A}^{\prime}-12$ & J13J43 & $11 / 16 / 06$ & 3720 & & 1.7 & 215 & & 0.15 & 0.03 & & \begin{tabular}{|l|}
0.02 \\
\end{tabular} & 0.58 & $\mathrm{U}$ & 0.58 & 11.7 & & 0.80 & 805 & & 7.7 & 1.6 & $\mathrm{U}$ & 1.6 \\
\hline A-13 & J13PC4 & $11 / 16 / 06$ & 3480 & & 1.4 & 318 & & 0.12 & 0.02 & & \begin{tabular}{|l|}
0.02 \\
\end{tabular} & 0.48 & $\mathrm{U}$ & $\begin{array}{ll}0.48 \\
\end{array}$ & 11.1 & & 0.66 & 734 & & 6.3 & 1.3 & $\mathrm{U}$ & 1.3 \\
\hline A-14 & J13PC5 & $11 / 16 / 06$ & 3030 & & 1.4 & 157 & & 0.12 & 0.02 & $\mathrm{U}$ & \begin{tabular}{|l|}
0.02 \\
\end{tabular} & 0.47 & $\mathrm{U}$ & 0.47 & 11.3 & & 0.65 & 698 & & 6.3 & 1.3 & $\mathrm{U}$ & 1.3 \\
\hline A-15 & $\begin{array}{l}\text { J13PC6 } \\
\end{array}$ & $11 / 16 / 06$ & 4950 & & 1.4 & 264 & & 0.12 & 0.02 & $\mathrm{U}$ & \begin{tabular}{|l|}
0.02 \\
\end{tabular} & 0.49 & $\mathrm{U}$ & 0.49 & 13.3 & & 0.67 & 1660 & & 6.5 & 1.3 & $\mathrm{U}$ & 1.3 \\
\hline A-16 & $\begin{array}{l}\text { J13PC7 } \\
\end{array}$ & $11 / 16 / 06$ & 4670 & & 1.4 & 271 & & 0.12 & 0.02 & $\mathrm{U}$ & 0.02 & 0.48 & $\mathrm{U}$ & 0.48 & 15.5 & & 0.66 & 1480 & & 6.3 & 1.3 & $\mathrm{U}$ & 1.3 \\
\hline A-11 (in River) & J13PC2 & $11 / 16 / 06$ & 2350 & & 1.5 & 578 & & 0.13 & 0.06 & & 0.02 & 0.53 & $\mathrm{U}$ & 0.53 & 9.4 & & 0.73 & 375 & & 7.0 & 1.4 & $\mathrm{U}$ & 1.4 \\
\hline A-12 (in River) & J13PC3 & $11 / 16 / 06$ & 2290 & C & 1.5 & 318 & & 0.13 & 0.02 & $\mathrm{U}$ & 0.02 & 0.55 & $\mathrm{U}$ & 0.5 & 7.4 & & 0.75 & 362 & & 7.2 & 1.5 & $\mathrm{U}$ & 1.4 \\
\hline
\end{tabular}

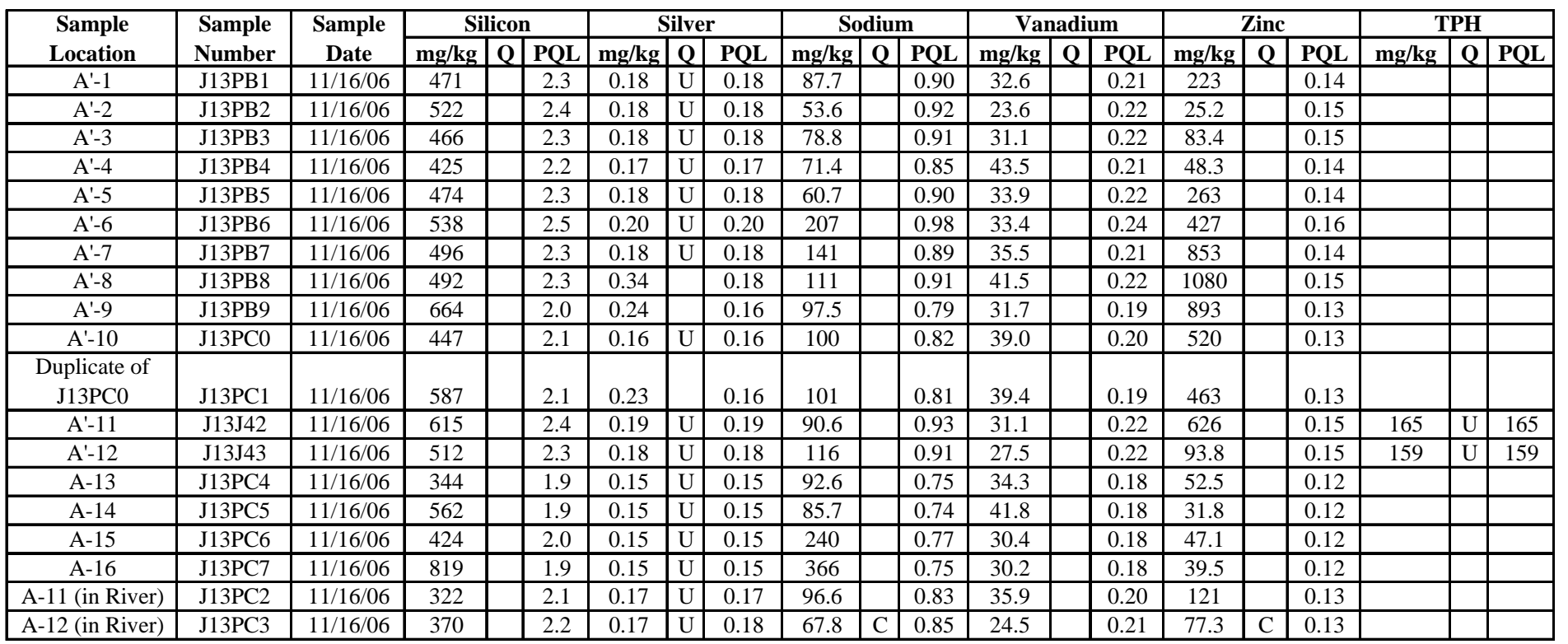


Table A-4. 128-F-2 Area C Verification Sampling Results. (10 pages)

\begin{tabular}{|c|c|c|c|c|c|c|c|c|c|c|c|c|c|c|c|c|c|c|c|c|c|c|c|}
\hline \multirow{2}{*}{$\begin{array}{c}\text { Sample } \\
\text { Location }\end{array}$} & \multirow{2}{*}{$\begin{array}{c}\text { HEIS } \\
\text { Number }\end{array}$} & \multirow{2}{*}{$\begin{array}{c}\text { Sample } \\
\text { Date }\end{array}$} & \multicolumn{3}{|c|}{ Aluminum } & \multicolumn{3}{|c|}{ Antimony } & \multicolumn{3}{|c|}{ Arsenic } & \multicolumn{3}{|c|}{ Barium } & \multicolumn{3}{|c|}{ Beryllium } & \multicolumn{3}{|c|}{ Boron } & \multicolumn{3}{|c|}{ Cadmium } \\
\hline & & & $\mathrm{mg} / \mathrm{kg}$ & $\mathbf{Q}$ & PQL & $\mathrm{mg} / \mathrm{kg}$ & $\mathbf{Q}$ & PQL & $\mathrm{mg} / \mathbf{k g}$ & $\mathbf{Q}$ & PQL & $\mathrm{mg} / \mathrm{kg}$ & \begin{tabular}{l|l}
$\mathbf{Q}$ & \\
\end{tabular} & PQL & $\mathrm{mg} / \mathrm{kg}$ & Q & PQL & $\mathrm{mg} / \mathrm{kg}$ & \begin{tabular}{l|l}
$\mathbf{Q}$ \\
\end{tabular} & PQL & $\mathrm{mg} / \mathbf{k g}$ & $\mathbf{Q}$ & PQL \\
\hline $\mathrm{C}-1$ & J14JC0 & $2 / 10 / 2007$ & 7320 & $\mathrm{C}$ & 8 & 0.95 & $\mathrm{U}$ & 0.95 & 6 & & 1.2 & 112 & \begin{tabular}{l|l}
$\mathrm{C}$ \\
\end{tabular} & 0.04 & 0.37 & & 0.04 & 0.72 & UC & 0.72 & 2.5 & & 0.11 \\
\hline $\mathrm{C}-2$ & J14JC1 & $2 / 10 / 2007$ & 5210 & $\mathrm{C}$ & 6.6 & 0.79 & $\mathrm{U}$ & 0.79 & 2.2 & & 0.98 & 62.3 & \begin{tabular}{l|l}
$\mathrm{C}$ & \\
\end{tabular} & 0.03 & 0.36 & & 0.03 & 0.7 & $\begin{array}{ll}\mathrm{C} \\
\end{array}$ & 0.6 & 0.24 & & 0.09 \\
\hline $\begin{array}{c}\text { Duplicate of } \\
\text { J14JC1 }\end{array}$ & J14JD2 & /2007 & 4070 & C & 6.4 & 0.76 & $\mathrm{U}$ & 0.76 & 3.2 & & 0.94 & 55.3 & C & 0.03 & 0.31 & & 0.03 & 0.58 & UC & 0.58 & 0.26 & & 0.09 \\
\hline $\mathrm{C}-3$ & J14JC2 & $2 / 10 / 2007$ & 12300 & $\mathrm{C}$ & 7 & 1.2 & & 0.82 & 37.1 & & 1 & 786 & \begin{tabular}{l|l}
$\mathrm{C}$ \\
\end{tabular} & 0.03 & 0.8 & & 0.03 & 0.98 & \begin{tabular}{|l|}
$\mathrm{C}$ \\
\end{tabular} & 0.63 & 1.2 & & 0.1 \\
\hline C-3 test pit & J14JF6 & $2 / 10 / 2007$ & 7740 & $\mathrm{C}$ & 6.5 & 0.78 & $\mathrm{U}$ & 0.78 & 6.1 & & 0.96 & 258 & \begin{tabular}{l|l} 
C & \\
\end{tabular} & 0.03 & 0.61 & & 0.03 & 0.59 & UC & 0.59 & 0.35 & & 0.09 \\
\hline C-4 & J14JC3 & $2 / 10 / 2007$ & 7100 & $\mathrm{C}$ & 7 & 3.8 & & 0.83 & 6.2 & & 1 & 96.4 & $\mathrm{C}$ & 0.03 & 0.32 & & 0.03 & 0.63 & UC & 0.63 & 0.56 & & 0.1 \\
\hline $\mathrm{C}-5$ & J14JC4 & $2 / 10 / 2007$ & 9520 & $\mathrm{C}$ & 6.9 & 0.81 & $\mathrm{U}$ & 0.81 & 22 & & 1 & 662 & $\mathrm{C}$ & 0.03 & 0.78 & & 0.03 & 0.73 & \begin{tabular}{|l|}
$\mathrm{C}$ \\
\end{tabular} & 0.62 & 1.6 & & 0.1 \\
\hline C-6 & J14JC5 & $/ 2007$ & 5540 & $\mathrm{C}$ & 8 & 0.96 & $\mathrm{U}$ & 0.96 & 8.3 & & 1.2 & 65.9 & \begin{tabular}{l|l}
$\mathrm{C}$ & \\
\end{tabular} & 0.04 & 0.26 & & 0.04 & 0.91 & $\mathrm{C}$ & 0.73 & 0.82 & & 0.11 \\
\hline C-7 & J14JC6 & $2 / 10 / 2007$ & 6920 & $\mathrm{C}$ & 6.5 & 0.76 & $\mathrm{U}$ & 0.76 & 3.1 & & 0.95 & 185 & $\mathrm{C}$ & 0.03 & 0.29 & & 0.03 & 0.58 & UC & 0.58 & 0.09 & $\mathrm{U}$ & 0.09 \\
\hline $\mathrm{C}-8$ & J14JC7 & $2 / 13 / 2007$ & 9140 & & 7 & 0.83 & $\mathrm{U}$ & 0.83 & 4.3 & & 1 & 120 & $\mathrm{C}$ & 0.03 & 0.53 & & 0.03 & 0.74 & & 0.63 & 2.3 & & 0.1 \\
\hline C-9 & J14JC8 & $2 / 13 / 2007$ & 6900 & & 6.5 & 0.77 & $\mathrm{U}$ & 0.77 & 4.4 & & 0.95 & 120 & \begin{tabular}{l|l}
$\mathrm{C}$ \\
\end{tabular} & 0.03 & 0.42 & & 0.03 & 0.58 & $\mathrm{U}$ & 0.58 & 0.13 & & 0.09 \\
\hline C-10 & J14JC9 & $2 / 13 / 2007$ & 5840 & & 6.8 & 0.81 & $\mathrm{U}$ & 0.81 & 7.3 & & 1 & 63.6 & $\mathrm{C}$ & 0.03 & 0.36 & & 0.03 & 0.62 & $\mathrm{U}$ & 0.62 & 0.96 & & 0.1 \\
\hline C-11 & J14JD0 & $2 / 13 / 2007$ & 4310 & & 6.1 & 0.72 & $\mathrm{U}$ & 0.72 & 2.4 & & 0.9 & 41.3 & $\mathrm{C}$ & 0.03 & 0.34 & & 0.03 & 0.55 & $\mathrm{U}$ & 0.55 & 0.09 & $\mathrm{U}$ & 0.09 \\
\hline $\mathrm{C}-12$ & J14JD1 & $2 / 13 / 2007$ & 5710 & & 6.7 & 0.8 & $\mathrm{U}$ & 0.8 & 2.6 & & 0.99 & 56.5 & $\mathrm{C}$ & 0.03 & 0.49 & & 0.03 & 0.61 & $\mathrm{U}$ & 0.61 & 0.01 & $\mathrm{U}$ & 0.1 \\
\hline Equip blank & J14JD3 & $2 / 13 / 2007$ & 46.4 & $\mathrm{C}$ & 2 & 0.24 & $\mathrm{U}$ & 0.24 & 0.44 & & 0.29 & 1.4 & $\mathrm{C}$ & 0.009 & 0.009 & $\mathrm{U}$ & 0.009 & 0.18 & UC & 0.19 & 0.03 & $\mathrm{U}$ & 0.03 \\
\hline
\end{tabular}

\begin{tabular}{|c|c|c|c|c|c|c|c|c|c|c|c|c|c|c|c|c|c|c|c|c|c|c|c|}
\hline \multirow{2}{*}{$\begin{array}{c}\text { Sample } \\
\text { Location }\end{array}$} & \multirow{2}{*}{$\begin{array}{c}\text { HEIS } \\
\text { Number }\end{array}$} & \multirow{2}{*}{$\begin{array}{c}\text { Sample } \\
\text { Date }\end{array}$} & \multicolumn{3}{|c|}{ Calcium } & \multicolumn{3}{|c|}{ Chromium } & \multicolumn{3}{|c|}{ Cobalt } & \multicolumn{3}{|c|}{ Copper } & \multicolumn{3}{|c|}{$\begin{array}{l}\text { Hexavalent } \\
\text { Chromium }\end{array}$} & \multicolumn{3}{|c|}{ Iron } & \multicolumn{3}{|c|}{ Lead } \\
\hline & & & $\mathrm{mg} / \mathrm{kg}$ & $\bar{Q}$ & PQL & $\mathrm{mg} / \mathrm{kg}$ & $\bar{Q}$ & PQL & \begin{tabular}{l|}
$\mathrm{mg} / \mathbf{k g}$ \\
\end{tabular} & $\mathbf{Q}$ & PQL & $\mathrm{mg} / \mathrm{kg}$ & \begin{tabular}{l|l}
$\mathbf{Q}$ & \\
\end{tabular} & PQL & $\mathrm{mg} / \mathrm{kg}$ & Q & PQL & $\mathrm{mg} / \mathrm{kg}$ & $\bar{Q}$ & PQL & $\mathrm{mg} / \mathrm{kg}$ & $\mathbf{Q}$ & PQL \\
\hline $\mathrm{C}-1$ & $\begin{array}{l}\mathrm{J} 14 \mathrm{JC} 0 \\
\end{array}$ & $2 / 10 / 2007$ & 4230 & $\mathrm{C}$ & 4.8 & 354 & & 0.46 & 8.1 & & 0.19 & 61.5 & & 0.27 & 12.4 & & 0.27 & 20700 & & 9.3 & 160 & & 0.61 \\
\hline $\mathrm{C}-2$ & J14JC1 & $2 / 10 / 2007$ & 4250 & $\mathrm{C}$ & 3.9 & 15 & & 0.38 & 6.6 & & 0.16 & 15.1 & & 0.22 & 0.22 & $\mathrm{U}$ & 0.22 & 17000 & & 7.7 & 10.1 & & 0.5 \\
\hline $\begin{array}{c}\text { Duplicate of } \\
\text { J14JC1 }\end{array}$ & J14JD2 & $2 / 10 / 2007$ & 3710 & C & 3.8 & 15 & & 0.36 & 5.6 & & 0.15 & 14.4 & & 0.21 & 0.35 & & 0.21 & 14300 & & 7.4 & 11.9 & & 0.49 \\
\hline $\mathrm{C}-3$ & J14JC2 & $2 / 10 / 2007$ & 3230 & C & 4.1 & 179 & & 0.4 & 33.9 & & 0.16 & 68.2 & & 0.23 & 4 & & 0.23 & 47000 & & 8 & 8 & & 0.53 \\
\hline C-3 test pit & J14JF6 & $2 / 10 / 2007$ & 2520 & $\mathrm{C}$ & 3.9 & 71.5 & & 0.37 & 9 & & 0.16 & 19.4 & & 0.22 & 0.41 & & 0.22 & 17600 & & 7.6 & 4.7 & & 0.5 \\
\hline C-4 & J14JC3 & $2 / 10 / 2007$ & 3690 & C & 4.1 & 172 & & 0.4 & 6 & & 0.17 & 22.4 & & 0.23 & 6.7 & & 0.23 & 15000 & & 8.1 & 37.6 & & 0.53 \\
\hline $\mathrm{C}-5$ & J14JC4 & $2 / 10 / 2007$ & 2870 & $\mathrm{C}$ & 4.1 & 131 & & 0.39 & 24.8 & & 0.16 & 49.3 & & 0.23 & 2.9 & & 233 & 26800 & & 7.9 & 8.9 & & 0.85 \\
\hline C-6 & J14JC5 & $2 / 10 / 2007$ & 3200 & $\mathrm{C}$ & 4.8 & 209 & & 0.46 & 6.2 & & 0.19 & 43.6 & & 0.27 & 5.7 & & 0.27 & 15800 & & 9.3 & 106 & & 0.61 \\
\hline C-7 & J14JC6 & $2 / 10 / 2007$ & 2200 & C & 3.8 & 22.4 & & 0.37 & 3.6 & & 0.15 & 15.8 & & 0.21 & 1 & & 0.21 & 11500 & & 7.5 & 3.9 & & 0.49 \\
\hline $\mathrm{C}-8$ & J14JC7 & $2 / 13 / 2007$ & 3560 & C & 4.2 & 671 & $\mathrm{C}$ & 0.4 & 6.9 & & 0.17 & 27.6 & & 0.23 & 1.4 & & 0.24 & 18100 & & 8.1 & 42.2 & & 0.53 \\
\hline C-9 & J14JC8 & $2 / 13 / 2007$ & 2670 & C & 3.8 & 55.8 & $\mathrm{C}$ & 0.37 & 5.8 & & 0.15 & 34.6 & & 0.22 & 0.22 & $\mathrm{U}$ & 0.22 & 16800 & & 7.5 & 15.9 & & 0.49 \\
\hline C-10 & J14JC9 & $2 / 13 / 2007$ & 3940 & $\mathrm{C}$ & 4 & 42.2 & $\mathrm{C}$ & 0.39 & 5.6 & & 0.16 & 28.4 & & 0.23 & 4.8 & & 0.23 & 15000 & & 7.9 & 64.4 & & 0.52 \\
\hline C-11 & J14JD0 & $2 / 13 / 2007$ & 5490 & $\mathrm{C}$ & 3.6 & 8.2 & $\mathrm{C}$ & 0.35 & 4.6 & & 0.14 & 10.6 & & 0.2 & 0.21 & $\mathrm{U}$ & 0.21 & 11800 & & 7.1 & 2.5 & & 0.46 \\
\hline C-12 & J14JD1 & $2 / 13 / 2007$ & 2850 & C & 4 & 9.2 & $\mathrm{C}$ & 0.38 & 6.1 & & 0.16 & 12.2 & & 0.22 & 0.29 & & 0.23 & 17600 & & 7.8 & 3.8 & & 0.51 \\
\hline Equip blank & J14JD3 & $2 / 13 / 2007$ & 24.1 & $\mathrm{C}$ & 1.2 & 0.11 & $\mathrm{U}$ & 0.11 & 0.05 & \begin{tabular}{|l|}
$\mathrm{U}$ \\
\end{tabular} & 0.05 & 0.07 & $\mathrm{U}$ & 0.07 & 0.2 & $\mathrm{U}$ & 0.2 & 106 & & 2.3 & 0.26 & & 0.15 \\
\hline
\end{tabular}


Table A-4. 128-F-2 Area C Verification Sampling Results. (10 pages)

\begin{tabular}{|c|c|c|c|c|c|c|c|c|c|c|c|c|c|c|c|c|c|c|c|c|c|c|c|}
\hline \multirow{2}{*}{$\begin{array}{c}\text { Sample } \\
\text { Location }\end{array}$} & \multirow{2}{*}{$\begin{array}{c}\text { HEIS } \\
\text { Number }\end{array}$} & \multirow{2}{*}{$\begin{array}{c}\text { Sample } \\
\text { Date }\end{array}$} & \multicolumn{3}{|c|}{ Magnesium } & \multicolumn{3}{|c|}{ Manganese } & \multicolumn{3}{|c|}{ Mercury } & \multicolumn{3}{|c|}{ Molybdenum } & \multicolumn{3}{|c|}{ Nickel } & \multicolumn{3}{|c|}{ Potassium } & \multicolumn{3}{|c|}{ Selenium } \\
\hline & & & $\mathrm{mg} / \mathrm{kg}$ & $\mathbf{Q}$ & PQL & $\mathrm{mg} / \mathrm{kg}$ & $\mathbf{Q}$ & PQL & $\mathrm{mg} / \mathrm{kg}$ & $\mathbf{Q}$ & PQL & $\mathrm{mg} / \mathrm{kg}$ & $\mathbf{Q}$ & PQL & $\mathrm{mg} / \mathrm{kg}$ & $\mathbf{Q}$ & PQL & $\mathrm{mg} / \mathrm{kg}$ & $\mathbf{Q}$ & PQL & $\mathrm{mg} / \mathrm{kg}$ & $\mathbf{Q}$ & PQL \\
\hline C-1 & J14JC0 & $2 / 10 / 2007$ & 4560 & & 1.8 & 247 & & 0.15 & 0.06 & & 0.02 & 0.61 & U & 0.61 & 12.6 & & 0.84 & 980 & $\mathrm{C}$ & 8.1 & 1.6 & $\mathrm{U}$ & 1.6 \\
\hline $\mathrm{C}-2$ & $14 \mathrm{JC} 1$ & $2 / 10 / 2007$ & 3750 & & 1.4 & 307 & & 0.13 & 0.01 & $\mathrm{U}$ & 0.01 & 0.5 & $\mathrm{U}$ & 0.5 & 8.7 & & 0.69 & 1020 & $\mathrm{C}$ & 6.7 & 1.4 & $\mathrm{U}$ & 1.4 \\
\hline Duplicate of & & & & & & & & & & & & & & & & & & & & & & & \\
\hline J14JC1 & J14JD2 & $2 / 10 / 2007$ & 3090 & & 1.4 & 266 & & 0.12 & 0.02 & $\mathrm{U}$ & 0.02 & 0.49 & $\mathrm{U}$ & 0.49 & 7.9 & & 0.67 & 864 & $\mathrm{C}$ & 6.4 & 1.3 & $\mathrm{U}$ & 1.3 \\
\hline $\mathrm{C}-3$ & $\begin{array}{l}\text { J14JC2 } \\
\end{array}$ & $2 / 10 / 2007$ & 4090 & & 1.5 & 5680 & & 0.13 & 0.02 & & 0.02 & 7.4 & & 0.53 & 92.9 & & 0.72 & 744 & \begin{tabular}{l|l}
$\mathrm{C}$ \\
\end{tabular} & 7 & 1.4 & $\mathrm{U}$ & 1.4 \\
\hline C-3 test pit & $\begin{array}{l}\text { J14JF6 } \\
\end{array}$ & $2 / 10 / 2007$ & 1790 & & 1.4 & 996 & & 0.12 & 0.02 & $\mathrm{U}$ & 0.02 & 0.85 & & 0.5 & 29.4 & & 0.68 & 369 & C & 6.6 & 1.3 & $\mathrm{U}$ & 1.3 \\
\hline C-4 & $\begin{array}{l}\text { J14JC3 } \\
\end{array}$ & $2 / 10 / 2007$ & 4180 & & 1.5 & 391 & & 0.13 & 0.02 & $\mathrm{U}$ & 0.02 & 0.53 & & 0.53 & 15.2 & & 0.73 & 1020 & C & 7 & 1.4 & $\mathrm{U}$ & 1.4 \\
\hline C-5 & J14JC4 & $2 / 10 / 2007$ & 3300 & & 1.5 & 5740 & & 0.13 & 0.02 & & 0.01 & 3.2 & & 0.52 & 74.6 & & 0.72 & 618 & $\mathrm{C}$ & 6.9 & 1.4 & $\mathrm{U}$ & 1.4 \\
\hline C-6 & $14 \mathrm{JC5}$ & $2 / 10 / 2007$ & 3200 & & 1.8 & 201 & & 0.15 & 0.04 & & 0.02 & 0.88 & & 0.61 & 11.8 & & 0.84 & 693 & C & 8.1 & 1.6 & $\mathrm{U}$ & 1.6 \\
\hline C-7 & J14JC6 & $2 / 10 / 2007$ & 2440 & & 1.4 & 193 & & 0.12 & 0.01 & $\mathrm{U}$ & 0.01 & 0.49 & $\mathrm{U}$ & 0.49 & 15.7 & & 0.67 & 416 & C & 6.5 & 1.3 & $\mathrm{U}$ & 1.3 \\
\hline C-8 & $\begin{array}{l}\text { J14JC7 } \\
\end{array}$ & $2 / 13 / 2007$ & 4700 & & 1.5 & 190 & & 0.13 & 0.05 & & 0.02 & 0.53 & $\mathrm{U}$ & 0.53 & 15.4 & & 0.73 & 1180 & $\mathrm{C}$ & 7.1 & 1.4 & $\mathrm{U}$ & 1.4 \\
\hline$C-9$ & IJC8 & $2 / 13 / 2007$ & 3400 & & 1.4 & 416 & & 0.12 & 0.02 & & 0.02 & 0.56 & & 0.49 & 15.6 & & 0.68 & 723 & $\mathrm{C}$ & 6.5 & 1.3 & $\mathrm{U}$ & 1.3 \\
\hline C-10 & $4 \mathrm{JC} 9$ & $2 / 13 / 2007$ & 3690 & & 1.5 & 177 & & 0.13 & 0.02 & & 0.02 & 0.52 & $\mathrm{U}$ & 0.52 & 10.4 & & 0.71 & 858 & $\mathrm{C}$ & 6.9 & 1.4 & $\mathrm{U}$ & 1.4 \\
\hline C-11 & J14JD0 & $2 / 13 / 2007$ & 3300 & & 1.3 & 226 & & 0.12 & 0.01 & $\mathrm{U}$ & 0.01 & 0.46 & $\mathrm{U}$ & 0.46 & 9 & & 0.64 & 690 & C & 6.1 & 1.2 & $\mathrm{U}$ & 1.2 \\
\hline C-12 & J14JD1 & $2 / 13 / 2007$ & 3530 & & 1.5 & 267 & & 0.13 & 0.01 & $\mathrm{U}$ & 0.01 & 0.51 & & 0.51 & 9.8 & & 0.7 & 987 & $\begin{array}{ll}\mathrm{C} \\
\end{array}$ & 6.8 & 1.4 & $\mathrm{U}$ & $\begin{array}{ll}1.4 \\
\end{array}$ \\
\hline Equip blank & J14JD3 & $2 / 13 / 2007$ & 7.2 & & 0.43 & 3.5 & & 0.04 & 0.02 & $\mathrm{U}$ & 0.02 & 0.15 & $\mathrm{U}$ & 0.15 & 0.21 & $\mathrm{U}$ & 0.21 & 18.3 & C & 2 & 0.41 & $\mathrm{U}$ & 0.41 \\
\hline
\end{tabular}

Table A-4. 128-F-2 Area C Verification Sampling Results. (10 pages)

\begin{tabular}{|c|c|c|c|c|c|c|c|c|c|c|c|c|c|c|c|c|c|c|c|c|}
\hline \multirow{2}{*}{$\begin{array}{l}\text { Sample } \\
\text { Location }\end{array}$} & \multirow{2}{*}{$\begin{array}{c}\text { HEIS } \\
\text { Number }\end{array}$} & \multirow{2}{*}{$\begin{array}{l}\text { Sample } \\
\text { Date }\end{array}$} & \multicolumn{3}{|c|}{ Silicon } & \multicolumn{3}{|c|}{ Silver } & \multicolumn{3}{|c|}{ Sodium } & \multicolumn{3}{|c|}{ Vanadium } & \multicolumn{3}{|c|}{ Zinc } & \multicolumn{3}{|c|}{$\begin{array}{c}\text { Total petroleum } \\
\text { hydrocarbons }\end{array}$} \\
\hline & & & $\mathrm{mg} / \mathbf{k g}$ & 0 & $\overline{\text { POL }}$ & $\mathrm{mg} / \mathbf{k g}$ & $\mathbf{0}$ & $\overline{\text { POL }}$ & \begin{tabular}{|l}
$\mathrm{mg} / \mathrm{kg}$ \\
\end{tabular} & 0 & PQL & $\mathrm{mg} / \mathbf{k g}$ & 0 & $\overline{\text { POL }}$ & $\mathrm{mg} / \mathrm{kg}$ & 0 & $\overline{\text { POL }}$ & $\mathbf{m g} / \mathbf{k g}$ & 0 & POL \\
\hline $\mathrm{C}-1$ & J14JC0 & $2 / 10 / 2007$ & 654 & $\mathrm{C}$ & 2.4 & 0.19 & $\mathrm{U}$ & 0.19 & 105 & $\mathrm{C}$ & 0.95 & 39.5 & & 0.23 & 806 & $\mathrm{C}$ & 0.15 & 180 & $\mathrm{U}$ & 180 \\
\hline $\mathrm{C}-2$ & J14JC1 & $2 / 10 / 2007$ & 1000 & $\mathrm{C}$ & 2 & 0.16 & $\mathrm{U}$ & 0.16 & 96.9 & $\mathrm{C}$ & 0.79 & 39 & & 0.19 & 64.9 & $\begin{array}{ll}\mathrm{C} \\
\end{array}$ & 0.13 & 147 & $\mathrm{U}$ & 147 \\
\hline Duplicate of & & & & & & & & & & & & & & & & & & & & \\
\hline J14JC1 & J14JD2 & $2 / 10 / 2007$ & 537 & C & 1.9 & 0.15 & $\mathrm{U}$ & 0.15 & 70.5 & C & 0.76 & 33.8 & & 0.18 & 70.3 & C & 0.12 & 143 & $\mathrm{U}$ & 143 \\
\hline $\mathrm{C}-3$ & J14JC2 & $2 / 10 / 2007$ & 912 & $\mathrm{C}$ & 2.1 & 0.16 & $\overline{\mathrm{U}}$ & 0.16 & 57 & $\mathrm{C}$ & 0.82 & 124 & & 0.2 & 44.3 & $\mathrm{C}$ & 0.13 & 139 & $\mathrm{U}$ & 139 \\
\hline C-3 test pit & J14JF6 & $2 / 10 / 2007$ & 294 & $\mathrm{C}$ & 2 & 0.16 & $\mathrm{U}$ & 0.16 & 82.3 & $\mathrm{C}$ & 0.78 & 41.1 & & 0.19 & 35.5 & $\begin{array}{ll}\mathrm{C} \\
\end{array}$ & 0.12 & & & \\
\hline $\mathrm{C}-4$ & J14JC3 & $2 / 10 / 2007$ & 517 & $\mathrm{C}$ & 2.1 & 0.17 & $\mathrm{U}$ & 0.17 & 106 & $\mathrm{C}$ & 0.83 & 26.6 & & 0.2 & 145 & $\mathrm{C}$ & 0.13 & 155 & $\mathrm{U}$ & 155 \\
\hline C-5 & J14JC4 & $2 / 10 / 2007$ & 482 & $\mathrm{C}$ & 2.1 & 0.16 & $\mathrm{U}$ & 0.16 & 60.7 & $\mathrm{C}$ & 0.81 & 58.2 & & 0.2 & 82.4 & $\mathrm{C}$ & 0.13 & 152 & $\mathrm{U}$ & 152 \\
\hline C-6 & J14JC5 & $2 / 10 / 2007$ & 730 & $\mathrm{C}$ & 2.4 & 0.19 & $\mathrm{U}$ & 0.19 & 123 & $\mathrm{C}$ & 0.96 & 25.7 & & 0.23 & 427 & $\begin{array}{ll}\mathrm{C} \\
\end{array}$ & 0.15 & 180 & $\mathrm{U}$ & 180 \\
\hline $\mathrm{C}-7$ & J14JC6 & $2 / 10 / 2007$ & 396 & $\mathrm{C}$ & 2 & 0.15 & $\mathrm{U}$ & 0.15 & 70.7 & $\mathrm{C}$ & 0.76 & 21.7 & & 0.18 & 27.3 & $\mathrm{C}$ & 0.12 & 143 & $\mathrm{U}$ & 143 \\
\hline $\mathrm{C}-8$ & J14JC7 & $2 / 13 / 2007$ & 653 & $\mathrm{C}$ & 2.1 & 0.17 & $\mathrm{UC}$ & 0.17 & 149 & $\mathrm{C}$ & 2.1 & 32.9 & & 0.2 & 277 & $\mathrm{C}$ & 0.13 & 158 & $\mathrm{U}$ & 158 \\
\hline C-9 & J14JC8 & $2 / 13 / 2007$ & 566 & $\mathrm{C}$ & 2 & 0.15 & UC & 0.15 & 95.2 & $\mathrm{C}$ & 1.9 & 36.5 & & 0.18 & 66.7 & \begin{tabular}{|l|}
$\mathrm{C}$ \\
\end{tabular} & 0.12 & 147 & $\mathrm{U}$ & 147 \\
\hline C-10 & J14JC9 & $2 / 13 / 2007$ & 636 & $\mathrm{C}$ & 2.1 & 0.16 & $\mathrm{UC}$ & 0.16 & 131 & $\mathrm{C}$ & 2 & 31.6 & & 0.19 & 376 & C & 0.13 & 154 & $\mathrm{U}$ & 154 \\
\hline C-11 & 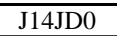 & $2 / 13 / 2007$ & 474 & $\mathrm{C}$ & 1.9 & 0.14 & $\mathrm{UC}$ & 0.14 & 92.6 & $\mathrm{C}$ & 1.8 & 27.3 & & 0.17 & 27.8 & $\mathrm{C}$ & 0.12 & 138 & $\mathrm{U}$ & 138 \\
\hline C-12 & J14JD1 & $2 / 13 / 2007$ & 534 & $\mathrm{C}$ & 2 & 0.16 & $\mathrm{UC}$ & 0.16 & 110 & $\mathrm{C}$ & 2 & 43.4 & & 0.19 & 35.1 & C & 0.13 & 153 & $\mathrm{U}$ & 153 \\
\hline Equip blank & J14JD3 & $2 / 13 / 2007$ & 36.8 & $\mathrm{C}$ & 0.6 & 0.05 & $\mathrm{U}$ & 0.05 & 7.8 & $\mathrm{C}$ & 0.24 & 0.105 & & 0.06 & 0.52 & C & 0.04 & & & \\
\hline
\end{tabular}


Table A-4. 128-F-2 Area C Verification Sampling Results. (10 pages)

\begin{tabular}{|c|c|c|c|c|c|c|c|c|c|c|c|c|}
\hline \multirow[t]{2}{*}{ Constituent } & \multicolumn{3}{|c|}{$\begin{array}{c}\text { J14JC1 } \\
\text { Sample Location C-2 } \\
\text { Sample Date 2/10/07 }\end{array}$} & \multicolumn{3}{|c|}{$\begin{array}{c}\text { J14JD2 } \\
\text { Duplicate of J14JC1 } \\
\text { Sample Date 2/10/07 }\end{array}$} & \multicolumn{3}{|c|}{$\begin{array}{c}\text { J14JC0 } \\
\text { Sample Location C-1 } \\
\text { Sample Date 2/10/07 }\end{array}$} & \multicolumn{3}{|c|}{$\begin{array}{c}\text { J14JC2 } \\
\text { Sample Location C-3 } \\
\text { Sample Date 2/10/07 }\end{array}$} \\
\hline & $\mu g / k g$ & $\mathbf{Q}$ & PQL & $\mu \mathrm{g} / \mathrm{kg}$ & $\mathbf{Q}$ & PQL & $\mu g / k g$ & $\mathbf{Q}$ & PQL & $\mu g / \mathrm{kg}$ & $\mathbf{Q}$ & PQL \\
\hline \multicolumn{13}{|c|}{ Polychlorinated Biphenyls } \\
\hline Aroclor-1016 & 13 & $\mathrm{U}$ & 13 & 13 & $\mathrm{U}$ & 13 & 13 & $\mathrm{U}$ & 13 & 13 & $\mathrm{U}$ & 13 \\
\hline \begin{tabular}{|l|} 
Aroclor-1221 \\
\end{tabular} & 13 & $\mathrm{U}$ & 13 & 13 & $\mathrm{U}$ & 13 & 13 & $\mathrm{U}$ & 13 & 13 & \begin{tabular}{|l|}
$\mathrm{U}$ \\
\end{tabular} & 13 \\
\hline \begin{tabular}{|l|} 
Aroclor-1232 \\
\end{tabular} & 13 & $\mathrm{U}$ & 13 & 13 & $\mathrm{U}$ & 13 & 13 & $\mathrm{U}$ & 13 & 13 & $\mathrm{U}$ & 13 \\
\hline Aroclor-1242 & 13 & $\mathrm{U}$ & 13 & 13 & $\mathrm{U}$ & 13 & 13 & $\mathrm{U}$ & 13 & 13 & $\mathrm{U}$ & 13 \\
\hline Aroclor-1248 & 13 & $\mathrm{U}$ & 13 & 13 & $\mathrm{U}$ & 13 & 13 & $\mathrm{U}$ & 13 & 13 & $\mathrm{U}$ & 13 \\
\hline Aroclor-1254 & 13 & $\mathrm{U}$ & 13 & 13 & $\mathrm{U}$ & 13 & 13 & $\mathrm{U}$ & 13 & 13 & $\mathrm{U}$ & 13 \\
\hline \begin{tabular}{|l|} 
Aroclor-1260 \\
\end{tabular} & 13 & $\mathrm{U}$ & 13 & 13 & $\mathrm{U}$ & 13 & 13 & $\mathrm{U}$ & 13 & 13 & \begin{tabular}{|l|}
$\mathrm{U}$ \\
\end{tabular} & 13 \\
\hline \multicolumn{13}{|c|}{ Pesticides } \\
\hline Aldrin & 0.4 & $\mathrm{~J}$ & 1.8 & & & & 0.72 & $\mathrm{~J}$ & 2.2 & 1.9 & $\mathrm{U}$ & 1.9 \\
\hline Alpha-BHC & 1.8 & $\mathrm{U}$ & 1.8 & & & & 2.2 & $\mathrm{U}$ & 2.2 & 1.9 & $\mathrm{U}$ & 1.9 \\
\hline Alpha-Chlordane & 1.8 & $\mathrm{U}$ & 1.8 & & & & 2.2 & $\mathrm{U}$ & 2.2 & 1.9 & $\mathrm{U}$ & 1.9 \\
\hline Beta-BHC & 1.8 & $\mathrm{U}$ & 1.8 & & & & 2.2 & $\mathrm{U}$ & 2.2 & 1.9 & $\mathrm{U}$ & 1.9 \\
\hline Delta-BHC & 1.8 & $\mathrm{U}$ & 1.8 & & & & 2.2 & $\mathrm{U}$ & 2.2 & 1.9 & \begin{tabular}{|l|}
$\mathrm{U}$ \\
\end{tabular} & 1.9 \\
\hline Dichlorodiphenyldichloroethane & 1.8 & $\mathrm{U}$ & 1.8 & & & & 2.2 & $\mathrm{U}$ & 2.2 & 1.9 & $\mathrm{U}$ & 1.9 \\
\hline \begin{tabular}{|l} 
Dichlorodiphenyldichloroethylene \\
\end{tabular} & 1.8 & $\mathrm{U}$ & 1.8 & & & & 2.2 & $\mathrm{U}$ & 2.2 & 1.9 & $\mathrm{U}$ & 1.9 \\
\hline Dichlorodiphenyltrichloroethane & 1.8 & $\mathrm{U}$ & 1.8 & & & & 2.2 & $\mathrm{U}$ & 2.2 & 1.9 & $\mathrm{U}$ & 1.9 \\
\hline Dieldrin & 1.8 & $\mathrm{U}$ & 1.8 & & & & 2.2 & $\mathrm{U}$ & 2.2 & 1.9 & $\mathrm{U}$ & 1.9 \\
\hline Endosulfan I & 1.8 & $\mathrm{U}$ & 1.8 & & & & 2.2 & $\mathrm{U}$ & 2.2 & 1.9 & $\mathrm{U}$ & 1.9 \\
\hline Endosulfan II & 1.8 & $\mathrm{U}$ & 1.8 & & & & 2.2 & $\mathrm{U}$ & 2.2 & 1.9 & $\mathrm{U}$ & 1.9 \\
\hline Endosulfan sulfate & 1.8 & $\mathrm{U}$ & 1.8 & & & & 2.2 & $\mathrm{U}$ & 2.2 & 1.9 & $\mathrm{U}$ & 1.9 \\
\hline Endrin & 1.8 & $\mathrm{U}$ & 1.8 & & & & 0.49 & $\mathrm{~J}$ & 2.2 & 1.9 & $\mathrm{U}$ & 1.9 \\
\hline Endrin aldehyde & 1.8 & $\mathrm{U}$ & 1.8 & & & & 2.2 & $\mathrm{U}$ & 2.2 & 1.9 & $\mathrm{U}$ & 1.9 \\
\hline Endrin ketone & 1.8 & $\mathrm{U}$ & 1.8 & & & & 2.2 & $\mathrm{U}$ & 2.2 & 1.9 & $\mathrm{U}$ & 1.9 \\
\hline Gamma-BHC (Lindane) & 1.8 & $\mathrm{U}$ & 1.8 & & & & 2.2 & $\mathrm{U}$ & 2.2 & 1.9 & $\mathrm{U}$ & 1.9 \\
\hline gamma-Chlordane & 1.8 & $\mathrm{U}$ & 1.8 & & & & 2.2 & $\mathrm{U}$ & 2.2 & 1.9 & $\mathrm{U}$ & 1.9 \\
\hline Heptachlor & 1.8 & $\mathrm{U}$ & 1.8 & & & & 2.2 & $\mathrm{U}$ & 2.2 & 1.9 & $\mathrm{U}$ & 1.9 \\
\hline Heptachlor epoxide & 1.8 & $\mathrm{U}$ & 1.8 & & & & 2.2 & $\mathrm{U}$ & 2.2 & 1.9 & $\mathrm{U}$ & 1.9 \\
\hline Methoxychlor & 1.8 & $\mathrm{U}$ & 1.8 & & & & 2.2 & $\mathrm{U}$ & 2.2 & 1.9 & $\mathrm{U}$ & 1.9 \\
\hline Toxaphene & 18 & $\mathrm{U}$ & 18 & & & & 22 & $\mathrm{U}$ & 22 & 19 & $\mathrm{U}$ & 19 \\
\hline \multicolumn{13}{|c|}{ Semivolatile Organic Analytes } \\
\hline 1,2,4-Trichlorobenzene & 370 & $\mathrm{U}$ & 370 & & & & 450 & $\mathrm{U}$ & 450 & 390 & $\mathrm{U}$ & 390 \\
\hline 1,2-Dichlorobenzene & 370 & $\mathrm{U}$ & 370 & & & & 450 & $\mathrm{U}$ & 450 & 390 & $\mathrm{U}$ & 390 \\
\hline 1,3-Dichlorobenzene & 370 & $\mathrm{U}$ & 370 & & & & 450 & $\mathrm{U}$ & 450 & 390 & $\mathrm{U}$ & 390 \\
\hline 1,4-Dichlorobenzene & 370 & $\mathrm{U}$ & 370 & & & & 450 & $\mathrm{U}$ & 450 & 390 & $\mathrm{U}$ & 390 \\
\hline 2,4,5-Trichlorophenol & 920 & $\mathrm{U}$ & 920 & & & & 1100 & $\mathrm{U}$ & 1100 & 970 & $\mathrm{U}$ & 970 \\
\hline 2,4,6-Trichlorophenol & 370 & $\mathrm{U}$ & 370 & & & & 450 & $\mathrm{U}$ & 450 & 390 & $\mathrm{U}$ & 390 \\
\hline 2,4-Dichlorophenol & 370 & $\mathrm{U}$ & 370 & & & & 450 & $\mathrm{U}$ & 450 & 390 & $\mathrm{U}$ & 390 \\
\hline 2,4-Dimethylphenol & 370 & $\mathrm{U}$ & 370 & & & & 450 & $\mathrm{U}$ & 450 & 390 & $\mathrm{U}$ & 390 \\
\hline 2,4-Dinitrophenol & 920 & $\mathrm{U}$ & 920 & & & & 1100 & $\mathrm{U}$ & 1100 & 970 & $\mathrm{U}$ & 970 \\
\hline 2,4-Dinitrotoluene & 370 & $\mathrm{U}$ & 370 & & & & 450 & $\mathrm{U}$ & 450 & 390 & $\mathrm{U}$ & 390 \\
\hline 2,6-Dinitrotoluene & 370 & $\mathrm{U}$ & 370 & & & & 450 & $\mathrm{U}$ & 450 & 390 & $\mathrm{U}$ & 390 \\
\hline 2-Chloronaphthalene & 370 & $\mathrm{U}$ & 370 & & & & 450 & $\mathrm{U}$ & 450 & 390 & $\mathrm{U}$ & 390 \\
\hline 2-Chlorophenol & 370 & $\mathrm{U}$ & 370 & & & & 450 & $\mathrm{U}$ & 450 & 390 & $\mathrm{U}$ & 390 \\
\hline 2-Methylnaphthalene & 370 & $\mathrm{U}$ & 370 & & & & 450 & $\mathrm{U}$ & 450 & 390 & $\mathrm{U}$ & 390 \\
\hline 2-Methylphenol (cresol, o-) & 370 & $\mathrm{U}$ & 370 & & & & 450 & $\mathrm{U}$ & 450 & 390 & $\mathrm{U}$ & 390 \\
\hline 2-Nitroaniline & 920 & $\mathrm{U}$ & 920 & & & & 1100 & $\mathrm{U}$ & 1100 & 970 & $\mathrm{U}$ & 970 \\
\hline 2-Nitrophenol & 370 & $\mathrm{U}$ & 370 & & & & 450 & $\mathrm{U}$ & 450 & 390 & $\mathrm{U}$ & 390 \\
\hline
\end{tabular}


Table A-4. 128-F-2 Area C Verification Sampling Results. (10 pages)

\begin{tabular}{|c|c|c|c|c|c|c|c|c|c|c|c|c|}
\hline \multirow[t]{2}{*}{ Constituent } & \multicolumn{3}{|c|}{$\begin{array}{c}\text { J14JC1 } \\
\text { Sample Location C-2 } \\
\text { Sample Date 2/10/07 }\end{array}$} & \multicolumn{3}{|c|}{$\begin{array}{c}\text { J14JD2 } \\
\text { Duplicate of J14JC1 } \\
\text { Sample Date 2/10/07 }\end{array}$} & \multicolumn{3}{|c|}{$\begin{array}{c}\text { J14JC0 } \\
\text { Sample Location C-1 } \\
\text { Sample Date 2/10/07 }\end{array}$} & \multicolumn{3}{|c|}{$\begin{array}{c}\text { J14JC2 } \\
\text { Sample Location C-3 } \\
\text { Sample Date 2/10/07 }\end{array}$} \\
\hline & $\mu \mathrm{g} / \mathrm{kg}$ & $\mathbf{Q}$ & PQL & $\mu \mathrm{g} / \mathrm{kg}$ & $\mathbf{Q}$ & PQL & $\mu \mathrm{g} / \mathrm{kg}$ & $\mathbf{Q}$ & PQL & $\mu \mathrm{g} / \mathrm{kg}$ & $\mathbf{Q}$ & PQL \\
\hline \multicolumn{13}{|c|}{ Semivolatile Organic Analytes (continued) } \\
\hline 3,3'-Dichlorobenzidine & 370 & $\mathrm{U}$ & 370 & & & & 450 & $\mathrm{U}$ & 450 & 390 & $\mathrm{U}$ & 390 \\
\hline 4-Methylphenol (p-cresol) & 370 & $\mathrm{U}$ & 370 & & & & 450 & $\mathrm{U}$ & 450 & 390 & $\mathrm{U}$ & 390 \\
\hline 3-Nitroaniline & 920 & $\mathrm{U}$ & 920 & & & & 1100 & $\mathrm{U}$ & 1100 & 970 & $\mathrm{U}$ & 970 \\
\hline 4,6-Dinitro-2-methylphenol & 920 & $\mathrm{U}$ & 920 & & & & 1100 & $\mathrm{U}$ & 1100 & 970 & $\mathrm{U}$ & 970 \\
\hline 4-Bromophenyl-phenylether & 370 & $\mathrm{U}$ & 370 & & & & 450 & $\mathrm{U}$ & 450 & 390 & $\mathrm{U}$ & 390 \\
\hline 4-Chloro-3-methylphenol & 370 & $\mathrm{U}$ & 370 & & & & 450 & $\mathrm{U}$ & 450 & 390 & $\mathrm{U}$ & 390 \\
\hline 4-Chloroaniline & 370 & $\mathrm{U}$ & 370 & & & & 450 & $\mathrm{U}$ & 450 & 390 & $\mathrm{U}$ & 390 \\
\hline 4-Chlorophenyl-phenylether & 370 & $\mathrm{U}$ & 370 & & & & 450 & $\mathrm{U}$ & 450 & 390 & $\mathrm{U}$ & 390 \\
\hline 4-Nitroaniline & 920 & $\mathrm{U}$ & 920 & & & & 1100 & $\mathrm{U}$ & 1100 & 970 & $\mathrm{U}$ & 970 \\
\hline 4-Nitrophenol & 920 & $\mathrm{U}$ & 920 & & & & 1100 & $\mathrm{U}$ & 1100 & 970 & $\mathrm{U}$ & 970 \\
\hline Acenaphthene & 370 & $\mathrm{U}$ & 370 & & & & 450 & $\mathrm{U}$ & 450 & 390 & $\mathrm{U}$ & 390 \\
\hline Acenaphthylene & 370 & $\mathrm{U}$ & 370 & & & & 450 & $\mathrm{U}$ & 450 & 390 & $\mathrm{U}$ & 390 \\
\hline Anthracene & 370 & $\mathrm{U}$ & 370 & & & & 450 & $\mathrm{U}$ & 450 & 390 & $\mathrm{U}$ & 390 \\
\hline Benzo(a)anthracene & 370 & $\mathrm{U}$ & 370 & & & & 450 & $\mathrm{U}$ & 450 & 390 & $\mathrm{U}$ & 390 \\
\hline Benzo(a)pyrene & 370 & $\mathrm{U}$ & 370 & & & & 450 & $\mathrm{U}$ & 450 & 390 & $\mathrm{U}$ & 390 \\
\hline Benzo(b)fluoranthene & 370 & $\mathrm{U}$ & 370 & & & & 450 & $\mathrm{U}$ & 450 & 390 & $\mathrm{U}$ & 390 \\
\hline Benzo(g,h,i)perylene & 370 & $\mathrm{U}$ & 370 & & & & 450 & $\mathrm{U}$ & 450 & 390 & $\mathrm{U}$ & 390 \\
\hline Benzo(k)fluoranthene & 370 & $\mathrm{U}$ & 370 & & & & 450 & $\mathrm{U}$ & 450 & 390 & $\mathrm{U}$ & 390 \\
\hline Bis(2-chloro-1-methylethyl)ether & 370 & $\mathrm{U}$ & 370 & & & & 450 & $\mathrm{U}$ & 450 & 390 & $\mathrm{U}$ & 390 \\
\hline Bis(2-chloroethoxy)methane & 370 & $\mathrm{U}$ & 370 & & & & 450 & $\mathrm{U}$ & 450 & 390 & $\mathrm{U}$ & 390 \\
\hline Bis(2-chloroethyl) ether & 370 & $\mathrm{U}$ & 370 & & & & 450 & $\mathrm{U}$ & 450 & 390 & $\mathrm{U}$ & 390 \\
\hline Bis(2-ethylhexyl) phthalate & 71 & $\mathrm{JB}$ & 370 & & & & 47 & $\mathrm{JB}$ & 450 & 36 & $\mathrm{JB}$ & 390 \\
\hline Butylbenzylphthalate & 370 & $\mathrm{U}$ & 370 & & & & 450 & $\mathrm{U}$ & 450 & 390 & $\mathrm{U}$ & 390 \\
\hline Carbazole & 370 & $\mathrm{U}$ & 370 & & & & 450 & $\mathrm{U}$ & 450 & 390 & $\mathrm{U}$ & 390 \\
\hline Chrysene & 370 & $\mathrm{U}$ & 370 & & & & 450 & $\mathrm{U}$ & 450 & 390 & $\mathrm{U}$ & 390 \\
\hline Dibenz(a,h)anthracene & 53 & $\mathrm{~J}$ & 370 & & & & 23 & $\mathrm{~J}$ & 450 & 36 & $\mathrm{~J}$ & 390 \\
\hline Dibenzofuran & 370 & $\mathrm{U}$ & 370 & & & & 450 & $\mathrm{U}$ & 450 & 390 & $\mathrm{U}$ & 390 \\
\hline Diethylphthalate & 370 & $\mathrm{U}$ & 370 & & & & 450 & $\mathrm{U}$ & 450 & 390 & $\mathrm{U}$ & 390 \\
\hline Dimethylphthalate & 370 & $\mathrm{U}$ & 370 & & & & 450 & $\mathrm{U}$ & 450 & 390 & $\mathrm{U}$ & 390 \\
\hline Di-n-butylphthalate & 370 & $\mathrm{U}$ & 370 & & & & 450 & $\mathrm{U}$ & 450 & 390 & $\mathrm{U}$ & 390 \\
\hline Di-n-octylphthalate & 370 & $\mathrm{U}$ & 370 & & & & 450 & $\mathrm{U}$ & 450 & 390 & $\mathrm{U}$ & 390 \\
\hline Fluoranthene & 370 & $\mathrm{U}$ & 370 & & & & 450 & $\mathrm{U}$ & 450 & 390 & $\mathrm{U}$ & 390 \\
\hline Fluorene & 370 & $\mathrm{U}$ & 370 & & & & 450 & $\mathrm{U}$ & 450 & 390 & $\mathrm{U}$ & 390 \\
\hline Hexachlorobenzene & 370 & $\mathrm{U}$ & 370 & & & & 450 & $\mathrm{U}$ & 450 & 390 & $\mathrm{U}$ & 390 \\
\hline Hexachlorobutadiene & 370 & $\mathrm{U}$ & 370 & & & & 450 & $\mathrm{U}$ & 450 & 390 & $\mathrm{U}$ & 390 \\
\hline Hexachlorocyclopentadiene & 370 & $\mathrm{U}$ & 370 & & & & 450 & $\mathrm{U}$ & 450 & 390 & $\mathrm{U}$ & 390 \\
\hline Hexachloroethane & 370 & $\mathrm{U}$ & 370 & & & & 450 & $\mathrm{U}$ & 450 & 390 & $\mathrm{U}$ & 390 \\
\hline Indeno(1,2,3-cd)pyrene & 370 & $\mathrm{U}$ & 370 & & & & 450 & $\mathrm{U}$ & 450 & 390 & $\mathrm{U}$ & 390 \\
\hline Isophorone & 370 & $\mathrm{U}$ & 370 & & & & 450 & $\mathrm{U}$ & 450 & 390 & $\mathrm{U}$ & 390 \\
\hline Naphthalene & 370 & $\mathrm{U}$ & 370 & & & & 450 & $\mathrm{U}$ & 450 & 390 & $\mathrm{U}$ & 390 \\
\hline Nitrobenzene & 370 & $\mathrm{U}$ & 370 & & & & 450 & $\mathrm{U}$ & 450 & 390 & $\mathrm{U}$ & 390 \\
\hline N-Nitroso-di-n-dipropylamine & 370 & $\mathrm{U}$ & 370 & & & & 450 & $\mathrm{U}$ & 450 & 390 & $\mathrm{U}$ & 390 \\
\hline N-Nitrosodiphenylamine & 370 & $\mathrm{U}$ & 370 & & & & 450 & $\mathrm{U}$ & 450 & 390 & $\mathrm{U}$ & 390 \\
\hline Pentachlorophenol & 920 & $\mathrm{U}$ & 920 & & & & 1100 & $\mathrm{U}$ & 1100 & 970 & $\mathrm{U}$ & 970 \\
\hline Phenanthrene & 370 & $\mathrm{U}$ & 370 & & & & 450 & $\mathrm{U}$ & 450 & 390 & $\mathrm{U}$ & 390 \\
\hline Phenol & 370 & $\mathrm{U}$ & 370 & & & & 450 & $\mathrm{U}$ & 450 & 390 & $\mathrm{U}$ & 390 \\
\hline Pyrene & 370 & $\mathrm{U}$ & 370 & & & & 450 & $\mathrm{U}$ & 450 & 390 & $\mathrm{U}$ & 390 \\
\hline
\end{tabular}


Table A-4. 128-F-2 Area C Verification Sampling Results. (10 pages)

\begin{tabular}{|c|c|c|c|c|c|c|c|c|c|c|c|c|}
\hline \multirow[t]{2}{*}{ Constituent } & \multicolumn{3}{|c|}{$\begin{array}{c}\text { J14JF6 } \\
\text { Sample Location C-3 } \\
\text { Test Pit } \\
\text { Sample Date } 2 / 10 / 07\end{array}$} & \multicolumn{3}{|c|}{$\begin{array}{c}\text { J14JC3 } \\
\text { Sample Location C-4 } \\
\text { Sample Date 2/10/07 }\end{array}$} & \multicolumn{3}{|c|}{$\begin{array}{c}\text { J14JC4 } \\
\text { Sample Location C-5 } \\
\text { Sample Date 2/10/07 }\end{array}$} & \multicolumn{3}{|c|}{$\begin{array}{c}\text { J14JC5 } \\
\text { Sample Location C-6 } \\
\text { Sample Date 2/10/07 }\end{array}$} \\
\hline & $\mu \mathrm{g} / \mathrm{kg}$ & $\mathbf{Q}$ & PQL & $\mu \mathrm{g} / \mathrm{kg}$ & $\mathbf{Q}$ & PQL & $\mu g / \mathrm{kg}$ & $\mathbf{Q}$ & PQL & $\mu \mathrm{g} / \mathrm{kg}$ & $\mathbf{Q}$ & PQL \\
\hline \multicolumn{13}{|c|}{ Polychlorinated Biphenyls } \\
\hline Aroclor-1016 & & & & 13 & $\mathrm{U}$ & 13 & 13 & $\mathrm{U}$ & 13 & 13 & $\mathrm{U}$ & 13 \\
\hline \begin{tabular}{|l|} 
Aroclor-1221 \\
\end{tabular} & & & & 13 & $\mathrm{U}$ & 13 & 13 & $\mathrm{U}$ & 13 & 13 & $\mathrm{U}$ & 13 \\
\hline Aroclor-1232 & & & & 13 & $\mathrm{U}$ & 13 & 13 & $\mathrm{U}$ & 13 & 13 & $\mathrm{U}$ & 13 \\
\hline \begin{tabular}{|l|} 
Aroclor-1242 \\
\end{tabular} & & & & 13 & $\mathrm{U}$ & 13 & 13 & $\mathrm{U}$ & 13 & 13 & $\mathrm{U}$ & 13 \\
\hline Aroclor-1248 & & & & 13 & $\mathrm{U}$ & 13 & 13 & $\mathrm{U}$ & 13 & 13 & $\mathrm{U}$ & 13 \\
\hline Aroclor-1254 & & & & 13 & $\mathrm{U}$ & 13 & 13 & $\mathrm{U}$ & 13 & 13 & $\mathrm{U}$ & 13 \\
\hline Aroclor-1260 & & & & 13 & $\mathrm{U}$ & 13 & 13 & $\mathrm{U}$ & 13 & 13 & $\mathrm{U}$ & 13 \\
\hline \multicolumn{13}{|c|}{ Pesticides } \\
\hline Aldrin & & & & 1.9 & $\mathrm{U}$ & 1.9 & 0.37 & $\mathrm{~J}$ & 1.9 & 2.3 & $\mathrm{U}$ & 2.3 \\
\hline Alpha-BHC & & & & 1.9 & $\mathrm{U}$ & 1.9 & 1.9 & $\mathrm{U}$ & 1.9 & 2.3 & $\mathrm{U}$ & 2.3 \\
\hline Alpha-Chlordane & & & & 1.9 & $\mathrm{U}$ & 1.9 & 1.9 & $\mathrm{U}$ & 1.9 & 2.3 & $\mathrm{U}$ & 2.3 \\
\hline Beta-BHC & & & & 1.9 & $\mathrm{U}$ & 1.9 & 1.9 & $\mathrm{U}$ & 1.9 & 2.3 & $\mathrm{U}$ & 2.3 \\
\hline Delta-BHC & & & & 1.9 & $\mathrm{U}$ & 1.9 & 1.9 & $\mathrm{U}$ & 1.9 & 2.3 & $\mathrm{U}$ & 2.3 \\
\hline \begin{tabular}{|l} 
Dichlorodiphenyldichloroethane \\
\end{tabular} & & & & 0.53 & $\mathrm{~J}$ & 1.9 & 1.9 & $\mathrm{U}$ & 1.9 & 2.3 & $\mathrm{U}$ & 2.3 \\
\hline \begin{tabular}{|l} 
Dichlorodiphenyldichloroethylene \\
\end{tabular} & & & & 0.33 & $\mathrm{~J}$ & 1.9 & 1.9 & $\mathrm{U}$ & 1.9 & 2.3 & $\mathrm{U}$ & 2.3 \\
\hline Dichlorodiphenyltrichloroethane & & & & 1.9 & $\mathrm{U}$ & 1.9 & 1.9 & $\mathrm{U}$ & 1.9 & 2.3 & $\mathrm{U}$ & 2.3 \\
\hline \begin{tabular}{|l|} 
Dieldrin \\
\end{tabular} & & & & 1.9 & $\mathrm{U}$ & 1.9 & 1.9 & $\mathrm{U}$ & 1.9 & 2.3 & $\mathrm{U}$ & 2.3 \\
\hline Endosulfan I & & & & 0.47 & $\mathrm{~J}$ & 1.9 & 1.9 & $\mathrm{U}$ & 1.9 & 2.3 & $\mathrm{U}$ & 2.3 \\
\hline Endosulfan II & & & & 1.9 & $\mathrm{U}$ & 1.9 & 1.9 & $\mathrm{U}$ & 1.9 & 2.3 & $\mathrm{U}$ & 2.3 \\
\hline Endosulfan sulfate & & & & 1.9 & $\mathrm{U}$ & 1.9 & 1.9 & $\mathrm{U}$ & 1.9 & 2.3 & $\mathrm{U}$ & 2.3 \\
\hline Endrin & & & & 1.9 & $\mathrm{U}$ & 1.9 & 1.9 & $\mathrm{U}$ & 1.9 & 2.3 & $\mathrm{U}$ & 2.3 \\
\hline \begin{tabular}{|l} 
Endrin aldehyde \\
\end{tabular} & & & & 0.63 & $\mathrm{~J}$ & 1.9 & 1.9 & $\mathrm{U}$ & 1.9 & 2.3 & $\mathrm{U}$ & 2.3 \\
\hline Endrin ketone & & & & 1.9 & $\mathrm{U}$ & 1.9 & 1.9 & $\mathrm{U}$ & 1.9 & 2.3 & $\mathrm{U}$ & 2.3 \\
\hline Gamma-BHC (Lindane) & & & & 1.9 & $\mathrm{U}$ & 1.9 & 1.9 & $\mathrm{U}$ & 1.9 & 2.3 & $\mathrm{U}$ & 2.3 \\
\hline gamma-Chlordane & & & & 0.33 & $\mathrm{~J}$ & 1.9 & 1.9 & $\mathrm{U}$ & 1.9 & 2.3 & $\mathrm{U}$ & 2.3 \\
\hline Heptachlor & & & & 1.9 & $\mathrm{U}$ & 1.9 & 1.9 & $\mathrm{U}$ & 1.9 & 2.3 & $\mathrm{U}$ & 2.3 \\
\hline Heptachlor epoxide & & & & 1.9 & $\mathrm{U}$ & 1.9 & 1.9 & $\mathrm{U}$ & 1.9 & 2.3 & $\mathrm{U}$ & 2.3 \\
\hline Methoxychlor & & & & 1.9 & $\mathrm{U}$ & 1.9 & 1.9 & $\mathrm{U}$ & 1.9 & 2.3 & $\mathrm{U}$ & 2.3 \\
\hline Toxaphene & & & & 19 & $\mathrm{U}$ & 19 & 19 & $\mathrm{U}$ & 19 & 23 & $\mathrm{U}$ & 23 \\
\hline \multicolumn{13}{|c|}{ Semivolatile Organic Analytes } \\
\hline 1,2,4-Trichlorobenzene & & & & 390 & $\mathrm{U}$ & 390 & 380 & $\mathrm{U}$ & 380 & 450 & $\mathrm{U}$ & 450 \\
\hline 1,2-Dichlorobenzene & & & & 390 & $\mathrm{U}$ & 390 & 380 & $\mathrm{U}$ & 380 & 450 & $\mathrm{U}$ & 450 \\
\hline 1,3-Dichlorobenzene & & & & 390 & $\mathrm{U}$ & 390 & 380 & $\mathrm{U}$ & 380 & 450 & $\mathrm{U}$ & 450 \\
\hline 1,4-Dichlorobenzene & & & & 390 & $\mathrm{U}$ & 390 & 380 & $\mathrm{U}$ & 380 & 450 & $\mathrm{U}$ & 450 \\
\hline 2,4,5-Trichlorophenol & & & & 980 & $\mathrm{U}$ & 980 & 950 & $\mathrm{U}$ & 950 & 1100 & $\mathrm{U}$ & 1100 \\
\hline 2,4,6-Trichlorophenol & & & & 390 & $\mathrm{U}$ & 390 & 380 & $\mathrm{U}$ & 380 & 450 & $\mathrm{U}$ & 450 \\
\hline 2,4-Dichlorophenol & & & & 390 & $\mathrm{U}$ & 390 & 380 & $\mathrm{U}$ & 380 & 450 & $\mathrm{U}$ & 450 \\
\hline 2,4-Dimethylphenol & & & & 390 & $\mathrm{U}$ & 390 & 380 & $\mathrm{U}$ & 380 & 450 & $\mathrm{U}$ & 450 \\
\hline 2,4-Dinitrophenol & & & & 980 & $\mathrm{U}$ & 980 & 950 & $\mathrm{U}$ & 950 & 1100 & $\mathrm{U}$ & 1100 \\
\hline 2,4-Dinitrotoluene & & & & 390 & $\mathrm{U}$ & 390 & 380 & $\mathrm{U}$ & 380 & 450 & $\mathrm{U}$ & 450 \\
\hline 2,6-Dinitrotoluene & & & & 390 & $\mathrm{U}$ & 390 & 380 & $\mathrm{U}$ & 380 & 450 & $\mathrm{U}$ & 450 \\
\hline 2-Chloronaphthalene & & & & 390 & $\mathrm{U}$ & 390 & 380 & $\mathrm{U}$ & 380 & 450 & $\mathrm{U}$ & 450 \\
\hline 2-Chlorophenol & & & & 390 & $\mathrm{U}$ & 390 & 380 & $\mathrm{U}$ & 380 & 450 & $\mathrm{U}$ & 450 \\
\hline 2-Methylnaphthalene & & & & 390 & $\mathrm{U}$ & 390 & 380 & $\mathrm{U}$ & 380 & 450 & $\mathrm{U}$ & 450 \\
\hline 2-Methylphenol (cresol, o-) & & & & 390 & $\mathrm{U}$ & 390 & 380 & $\mathrm{U}$ & 380 & 450 & $\mathrm{U}$ & 450 \\
\hline 2-Nitroaniline & & & & 980 & $\mathrm{U}$ & 980 & 950 & $\mathrm{U}$ & 950 & 1100 & $\mathrm{U}$ & 1100 \\
\hline 2-Nitrophenol & & & & 390 & $\mathrm{U}$ & 390 & 380 & $\mathrm{U}$ & 380 & 450 & $\mathrm{U}$ & 450 \\
\hline
\end{tabular}


Table A-4. 128-F-2 Area C Verification Sampling Results. (10 pages)

\begin{tabular}{|c|c|c|c|c|c|c|c|c|c|c|c|c|}
\hline \multirow[t]{2}{*}{ Constituent } & \multicolumn{3}{|c|}{$\begin{array}{c}\text { J14JF6 } \\
\text { Sample Location C-3 } \\
\text { Test Pit } \\
\text { Sample Date } 2 / 10 / 07\end{array}$} & \multicolumn{3}{|c|}{$\begin{array}{c}\text { J14JC3 } \\
\text { Sample Location C-4 } \\
\text { Sample Date 2/10/07 }\end{array}$} & \multicolumn{3}{|c|}{$\begin{array}{c}\text { J14JC4 } \\
\text { Sample Location C-5 } \\
\text { Sample Date 2/10/07 }\end{array}$} & \multicolumn{3}{|c|}{$\begin{array}{c}\text { J14JC5 } \\
\text { Sample Location C-6 } \\
\text { Sample Date 2/10/07 }\end{array}$} \\
\hline & $\mu \mathrm{g} / \mathrm{kg}$ & $\mathbf{Q}$ & $\overline{\text { PQL }}$ & $\mu g / k g$ & $\mathbf{Q}$ & PQL & $\mu g / k g$ & $\mathbf{Q}$ & PQL & $\mu g / \mathrm{kg}$ & $\mathbf{Q}$ & PQL \\
\hline \multicolumn{13}{|c|}{ Semivolatile Organic Analytes (continued) } \\
\hline 3,3'-Dichlorobenzidine & & & & 390 & $\mathrm{U}$ & 390 & 380 & $\mathrm{U}$ & 380 & 450 & $\mathrm{U}$ & 450 \\
\hline 4-Methylphenol (p-cresol) & & & & 390 & $\mathrm{U}$ & 390 & 380 & $\mathrm{U}$ & 380 & 450 & $\mathrm{U}$ & 450 \\
\hline 3-Nitroaniline & & & & 980 & $\mathrm{U}$ & 980 & 950 & $\mathrm{U}$ & 950 & 1100 & $\mathrm{U}$ & 1100 \\
\hline \begin{tabular}{|l} 
4,6-Dinitro-2-methylphenol \\
\end{tabular} & & & & 980 & $\mathrm{U}$ & 980 & 950 & $\mathrm{U}$ & 950 & 1100 & $\mathrm{U}$ & 1100 \\
\hline 4-Bromophenyl-phenylether & & & & 390 & $\mathrm{U}$ & 390 & 380 & $\mathrm{U}$ & 380 & 450 & $\mathrm{U}$ & 450 \\
\hline 4-Chloro-3-methylphenol & & & & 390 & $\mathrm{U}$ & 390 & 380 & $\mathrm{U}$ & 380 & 450 & $\mathrm{U}$ & 450 \\
\hline 4-Chloroaniline & & & & 390 & $\mathrm{U}$ & 390 & 380 & $\mathrm{U}$ & 380 & 450 & U & 450 \\
\hline 4-Chlorophenyl-phenylether & & & & 390 & $\mathrm{U}$ & 390 & 380 & $\mathrm{U}$ & 380 & 450 & $\mathrm{U}$ & 450 \\
\hline 4-Nitroaniline & & & & 980 & $\mathrm{U}$ & 980 & 950 & $\mathrm{U}$ & 950 & 1100 & $\mathrm{U}$ & 1100 \\
\hline 4-Nitrophenol & & & & 980 & $\mathrm{U}$ & 980 & 950 & $\mathrm{U}$ & 950 & 1100 & U & 1100 \\
\hline Acenaphthene & & & & 390 & $\mathrm{U}$ & 390 & 380 & $\mathrm{U}$ & 380 & 450 & U & 450 \\
\hline Acenaphthylene & & & & 390 & $\mathrm{U}$ & 390 & 380 & $\mathrm{U}$ & 380 & 450 & U & 450 \\
\hline Anthracene & & & & 390 & $\mathrm{U}$ & 390 & 380 & $\mathrm{U}$ & 380 & 450 & U & 450 \\
\hline Benzo(a)anthracene & & & & 390 & $\mathrm{U}$ & 390 & 380 & $\mathrm{U}$ & 380 & 450 & $\mathrm{U}$ & 450 \\
\hline Benzo(a)pyrene & & & & 390 & $\mathrm{U}$ & 390 & 380 & $\mathrm{U}$ & 380 & 450 & $\mathrm{U}$ & 450 \\
\hline Benzo(b)fluoranthene & & & & 390 & $\mathrm{U}$ & 390 & 380 & $\mathrm{U}$ & 380 & 450 & $\mathrm{U}$ & 450 \\
\hline \begin{tabular}{|l} 
Benzo(g,h,i)perylene \\
\end{tabular} & & & & 390 & $\mathrm{U}$ & 390 & 380 & $\mathrm{U}$ & 380 & 450 & $\mathrm{U}$ & 450 \\
\hline Benzo(k)fluoranthene & & & & 390 & $\mathrm{U}$ & 390 & 380 & $\mathrm{U}$ & 380 & 450 & $\mathrm{U}$ & 450 \\
\hline Bis(2-chloro-1-methylethyl)ether & & & & 390 & $\mathrm{U}$ & 390 & 380 & $\mathrm{U}$ & 380 & 450 & $\mathrm{U}$ & 450 \\
\hline Bis(2-chloroethoxy)methane & & & & 390 & $\mathrm{U}$ & 390 & 380 & $\mathrm{U}$ & 380 & 450 & $\mathrm{U}$ & 450 \\
\hline Bis(2-chloroethyl) ether & & & & 390 & $\mathrm{U}$ & 390 & 380 & $\mathrm{U}$ & 380 & 450 & $\mathrm{U}$ & 450 \\
\hline Bis(2-ethylhexyl) phthalate & & & & 41.457 & $\mathrm{JB}$ & 390 & 37 & $\mathrm{JB}$ & 380 & 320 & JB & 450 \\
\hline Butylbenzylphthalate & & & & 390 & $\mathrm{U}$ & 390 & 380 & $\mathrm{U}$ & 380 & 450 & $\mathrm{U}$ & 450 \\
\hline \begin{tabular}{|l} 
Carbazole \\
\end{tabular} & & & & 390 & $\mathrm{U}$ & 390 & 380 & $\mathrm{U}$ & 380 & 450 & $\mathrm{U}$ & 450 \\
\hline Chrysene & & & & 390 & $\mathrm{U}$ & 390 & 380 & $\mathrm{U}$ & 380 & 450 & $\mathrm{U}$ & 450 \\
\hline \begin{tabular}{|l} 
Dibenz(a,h)anthracene \\
\end{tabular} & & & & 32.565 & $\mathrm{~J}$ & 390 & 380 & $\mathrm{U}$ & 380 & 450 & $\mathrm{U}$ & 450 \\
\hline Dibenzofuran & & & & 390 & $\mathrm{U}$ & 390 & 380 & $\mathrm{U}$ & 380 & 450 & $\mathrm{U}$ & 450 \\
\hline \begin{tabular}{|l} 
Diethylphthalate \\
\end{tabular} & & & & 390 & $\mathrm{U}$ & 390 & 380 & $\mathrm{U}$ & 380 & 450 & $\mathrm{U}$ & 450 \\
\hline Dimethylphthalate & & & & 390 & $\mathrm{U}$ & 390 & 380 & $\mathrm{U}$ & 380 & 450 & $\mathrm{U}$ & 450 \\
\hline Di-n-butylphthalate & & & & 390 & $\mathrm{U}$ & 390 & 380 & $\mathrm{U}$ & 380 & 450 & $\mathrm{U}$ & 450 \\
\hline \begin{tabular}{|l|} 
Di-n-octylphthalate \\
\end{tabular} & & & & 390 & \begin{tabular}{|l|}
$\mathrm{U}$ \\
\end{tabular} & 390 & 380 & $\mathrm{U}$ & 380 & 450 & U & 450 \\
\hline Fluoranthene & & & & 390 & $\mathrm{U}$ & 390 & 380 & $\mathrm{U}$ & 380 & 450 & $\mathrm{U}$ & 450 \\
\hline Fluorene & & & & 390 & $\mathrm{U}$ & 390 & 380 & $\mathrm{U}$ & 380 & 450 & $\mathrm{U}$ & 450 \\
\hline Hexachlorobenzene & & & & 390 & $\mathrm{U}$ & 390 & 380 & $\mathrm{U}$ & 380 & 450 & $\mathrm{U}$ & 450 \\
\hline Hexachlorobutadiene & & & & 390 & $\mathrm{U}$ & 390 & 380 & $\mathrm{U}$ & 380 & 450 & $\mathrm{U}$ & 450 \\
\hline Hexachlorocyclopentadiene & & & & 390 & $\mathrm{U}$ & 390 & 380 & $\mathrm{U}$ & 380 & 450 & $\mathrm{U}$ & 450 \\
\hline Hexachloroethane & & & & 390 & $\mathrm{U}$ & 390 & 380 & $\mathrm{U}$ & 380 & 450 & $\mathrm{U}$ & 450 \\
\hline Indeno(1,2,3-cd)pyrene & & & & 390 & $\mathrm{U}$ & 390 & 380 & $\mathrm{U}$ & 380 & 450 & $\mathrm{U}$ & 450 \\
\hline Isophorone & & & & 390 & $\mathrm{U}$ & 390 & 380 & $\mathrm{U}$ & 380 & 450 & $\mathrm{U}$ & 450 \\
\hline \begin{tabular}{|l} 
Naphthalene \\
\end{tabular} & & & & 390 & \begin{tabular}{|l|}
$\mathrm{U}$ \\
\end{tabular} & 390 & 380 & $\mathrm{U}$ & 380 & 450 & U & 450 \\
\hline Nitrobenzene & & & & 390 & $\mathrm{U}$ & 390 & 380 & $\mathrm{U}$ & 380 & 450 & $\mathrm{U}$ & 450 \\
\hline \begin{tabular}{|l} 
N-Nitroso-di-n-dipropylamine \\
\end{tabular} & & & & 390 & $\mathrm{U}$ & 390 & 380 & $\mathrm{U}$ & 380 & 450 & $\mathrm{U}$ & 450 \\
\hline N-Nitrosodiphenylamine & & & & 390 & $\mathrm{U}$ & 390 & 380 & $\mathrm{U}$ & 380 & 450 & $\mathrm{U}$ & 450 \\
\hline Pentachlorophenol & & & & 980 & $\mathrm{U}$ & 980 & 950 & $\mathrm{U}$ & 950 & 1100 & $\mathrm{U}$ & 1100 \\
\hline Phenanthrene & & & & 390 & $\mathrm{U}$ & 390 & 380 & $\mathrm{U}$ & 380 & 450 & $\mathrm{U}$ & 450 \\
\hline Phenol & & & & 390 & $\mathrm{U}$ & 390 & 380 & $\mathrm{U}$ & 380 & 450 & $\mathrm{U}$ & 450 \\
\hline Pyrene & & & & 390 & $\mathrm{U}$ & 390 & 380 & $\mathrm{U}$ & 380 & 450 & $\mathrm{U}$ & 450 \\
\hline
\end{tabular}


Table A-4. 128-F-2 Area C Verification Sampling Results. (10 pages)

\begin{tabular}{|c|c|c|c|c|c|c|c|c|c|c|c|c|}
\hline \multirow[t]{2}{*}{ Constituent } & \multicolumn{3}{|c|}{$\begin{array}{c}\text { J14JC6 } \\
\text { Sample Location C-7 } \\
\text { Sample Date } 2 / 10 / 07\end{array}$} & \multicolumn{3}{|c|}{$\begin{array}{c}\text { J14JC7 } \\
\text { Sample Location C-8 } \\
\text { Sample Date 2/13/07 }\end{array}$} & \multicolumn{3}{|c|}{$\begin{array}{c}\text { J14JC8 } \\
\text { Sample Location C-9 } \\
\text { Sample Date } 2 / 13 / 07\end{array}$} & \multicolumn{3}{|c|}{$\begin{array}{c}\text { J14JC9 } \\
\text { Sample Location C-10 } \\
\text { Sample Date } 2 / 13 / 07\end{array}$} \\
\hline & $\mu g / \mathbf{k g}$ & $\mathbf{Q}$ & PQL & $\mu g / k g$ & $\mathbf{Q}$ & PQL & $\mu \mathrm{g} / \mathrm{kg}$ & $\mathbf{Q}$ & PQL & $\mu \mathrm{g} / \mathrm{kg}$ & $\mathbf{Q}$ & PQL \\
\hline \multicolumn{13}{|c|}{ Polychlorinated Biphenyls } \\
\hline Aroclor-1016 & 13 & $\mathrm{U}$ & 13 & 16 & $\mathrm{U}$ & 16 & 15 & $\mathrm{U}$ & 15 & 16 & $\mathrm{U}$ & 16 \\
\hline Aroclor-1221 & 13 & $\mathrm{U}$ & 13 & 16 & $\mathrm{U}$ & 16 & 15 & $\mathrm{U}$ & 15 & 16 & $\mathrm{U}$ & 16 \\
\hline Aroclor-1232 & 13 & $\mathrm{U}$ & 13 & 16 & $\mathrm{U}$ & 16 & 15 & $\mathrm{U}$ & 15 & 16 & $\mathrm{U}$ & 16 \\
\hline Aroclor-1242 & 13 & $\mathrm{U}$ & 13 & 16 & $\mathrm{U}$ & 16 & 15 & $\mathrm{U}$ & 15 & 16 & $\mathrm{U}$ & 16 \\
\hline Aroclor-1248 & 13 & $\mathrm{U}$ & 13 & 16 & $\mathrm{U}$ & 16 & 15 & $\mathrm{U}$ & 15 & 16 & $\mathrm{U}$ & 16 \\
\hline Aroclor-1254 & 13 & $\mathrm{U}$ & 13 & 16 & $\mathrm{U}$ & 16 & 15 & $\mathrm{U}$ & 15 & 16 & $\mathrm{U}$ & 16 \\
\hline Aroclor-1260 & 13 & $\mathrm{U}$ & 13 & 16 & $\mathrm{U}$ & 16 & 15 & $\mathrm{U}$ & 15 & 16 & $\mathrm{U}$ & 16 \\
\hline \multicolumn{13}{|c|}{ Pesticides } \\
\hline Aldrin & 0.57 & $\mathrm{~J}$ & 1.8 & 0.44 & $\mathrm{~J}$ & 2.2 & 1.8 & $\mathrm{U}$ & 1.8 & 0.66 & $\mathrm{~J}$ & 1.9 \\
\hline Alpha-BHC & 1.8 & $\mathrm{U}$ & 1.8 & 2.2 & $\mathrm{U}$ & 2.2 & 1.8 & $\mathrm{U}$ & 1.8 & 0.43 & $\mathrm{~J}$ & 1.9 \\
\hline Alpha-Chlordane & 1.8 & $\mathrm{U}$ & 1.8 & 2.2 & $\mathrm{U}$ & 2.2 & 1.8 & $\mathrm{U}$ & 1.8 & 1.9 & $\mathrm{U}$ & 1.9 \\
\hline Beta-BHC & 1.8 & $\mathrm{U}$ & 1.8 & 0.72 & $\mathrm{~J}$ & 2.2 & 0.51 & $\mathrm{~J}$ & 1.8 & 1.9 & $\mathrm{U}$ & 1.9 \\
\hline Delta-BHC & 1.8 & $\mathrm{U}$ & 1.8 & 2.2 & $\mathrm{U}$ & 2.2 & 1.8 & $\mathrm{U}$ & 1.8 & 1.9 & $\mathrm{U}$ & 1.9 \\
\hline Dichlorodiphenyldichloroethane & 1.8 & $\mathrm{U}$ & 1.8 & 2.2 & $\mathrm{U}$ & 2.2 & 1.8 & $\mathrm{U}$ & 1.8 & 1.9 & $\mathrm{U}$ & 1.9 \\
\hline Dichlorodiphenyldichloroethylene & 1.8 & $\mathrm{U}$ & 1.8 & 2.2 & $\mathrm{U}$ & 2.2 & 0.4 & $\mathrm{~J}$ & 1.8 & 1.9 & $\mathrm{U}$ & 1.9 \\
\hline Dichlorodiphenyltrichloroethane & 1.8 & $\mathrm{U}$ & 1.8 & 2.2 & $\mathrm{U}$ & 2.2 & 1.9 & & 1.9 & 1.9 & $\mathrm{U}$ & 1.9 \\
\hline Dieldrin & 1.8 & $\mathrm{U}$ & 1.8 & 2.2 & $\mathrm{U}$ & 2.2 & 1.8 & $\mathrm{U}$ & 1.8 & 1.9 & $\mathrm{U}$ & 1.9 \\
\hline Endosulfan I & 1.8 & $\mathrm{U}$ & 1.8 & 2.2 & $\mathrm{U}$ & 2.2 & 1.8 & $\mathrm{U}$ & 1.8 & 1.9 & $\mathrm{U}$ & 1.9 \\
\hline Endosulfan II & 1.8 & $\mathrm{U}$ & 1.8 & 2.2 & $\mathrm{U}$ & 2.2 & 0.37 & $\mathrm{~J}$ & 1.8 & 1.9 & $\mathrm{U}$ & 1.9 \\
\hline Endosulfan sulfate & 1.8 & $\mathrm{U}$ & 1.8 & 2.2 & $\mathrm{U}$ & 2.2 & 1.8 & $\mathrm{U}$ & 1.8 & 1.9 & $\mathrm{U}$ & 1.9 \\
\hline Endrin & 1.8 & $\mathrm{U}$ & 1.8 & 2.2 & $\mathrm{U}$ & 2.2 & 1.8 & $\mathrm{U}$ & 1.8 & 1.9 & $\mathrm{U}$ & 1.9 \\
\hline Endrin aldehyde & 0.6 & $\mathrm{~J}$ & 1.8 & 2.2 & $\mathrm{U}$ & 2.2 & 1.8 & $\mathrm{U}$ & 1.8 & 1.9 & $\mathrm{U}$ & 1.9 \\
\hline Endrin ketone & 1.8 & $\mathrm{U}$ & 1.8 & 2.2 & $\mathrm{U}$ & 2.2 & 1.8 & $\mathrm{U}$ & 1.8 & 1.9 & $\mathrm{U}$ & 1.9 \\
\hline Gamma-BHC (Lindane) & 1.8 & $\mathrm{U}$ & 1.8 & 2.2 & $\mathrm{U}$ & 2.2 & 1.8 & $\mathrm{U}$ & 1.8 & 1.9 & $\mathrm{U}$ & 1.9 \\
\hline gamma-Chlordane & 1.8 & $\mathrm{U}$ & 1.8 & 2.2 & $\mathrm{U}$ & 2.2 & 1.8 & $\mathrm{U}$ & 1.8 & 1.9 & $\mathrm{U}$ & 1.9 \\
\hline Heptachlor & 1.8 & $\mathrm{U}$ & 1.8 & 2.2 & $\mathrm{U}$ & 2.2 & 1.8 & $\mathrm{U}$ & 1.8 & 1.9 & $\mathrm{U}$ & 1.9 \\
\hline Heptachlor epoxide & 1.8 & $\mathrm{U}$ & 1.8 & 2.2 & $\mathrm{U}$ & 2.2 & 1.8 & $\mathrm{U}$ & 1.8 & 1.9 & $\mathrm{U}$ & 1.9 \\
\hline Methoxychlor & 1.8 & $\mathrm{U}$ & 1.8 & 2.2 & $\mathrm{U}$ & 2.2 & 1.8 & $\mathrm{U}$ & 1.8 & 1.9 & $\mathrm{U}$ & 1.9 \\
\hline Toxaphene & 18 & $\mathrm{U}$ & 18 & 20 & $\mathrm{U}$ & 20 & 18 & $\mathrm{U}$ & 18 & 19 & $\mathrm{U}$ & 19 \\
\hline \multicolumn{13}{|c|}{ Semivolatile Organic Analytes } \\
\hline 1,2,4-Trichlorobenzene & 360 & $\mathrm{U}$ & 360 & 400 & $\mathrm{U}$ & 400 & 370 & $\mathrm{U}$ & 370 & 390 & $\mathrm{U}$ & 390 \\
\hline 1,2-Dichlorobenzene & 360 & $\mathrm{U}$ & 360 & 400 & $\mathrm{U}$ & 400 & 370 & $\mathrm{U}$ & 370 & 390 & $\mathrm{U}$ & 390 \\
\hline 1,3-Dichlorobenzene & 360 & $\mathrm{U}$ & 360 & 400 & $\mathrm{U}$ & 400 & 370 & $\mathrm{U}$ & 370 & 390 & $\mathrm{U}$ & 390 \\
\hline 1,4-Dichlorobenzene & 360 & $\mathrm{U}$ & 360 & 400 & $\mathrm{U}$ & 400 & 370 & $\mathrm{U}$ & 370 & 390 & $\mathrm{U}$ & 390 \\
\hline 2,4,5-Trichlorophenol & 890 & $\mathrm{U}$ & 890 & 1000 & $\mathrm{U}$ & 1000 & 920 & $\mathrm{U}$ & 920 & 970 & $\mathrm{U}$ & 970 \\
\hline 2,4,6-Trichlorophenol & 360 & $\mathrm{U}$ & 360 & 400 & $\mathrm{U}$ & 400 & 370 & $\mathrm{U}$ & 370 & 390 & $\mathrm{U}$ & 390 \\
\hline 2,4-Dichlorophenol & 360 & $\mathrm{U}$ & 360 & 400 & $\mathrm{U}$ & 400 & 370 & $\mathrm{U}$ & 370 & 390 & $\mathrm{U}$ & 390 \\
\hline 2,4-Dimethylphenol & 360 & $\mathrm{U}$ & 360 & 400 & $\mathrm{U}$ & 400 & 370 & $\mathrm{U}$ & 370 & 390 & $\mathrm{U}$ & 390 \\
\hline 2,4-Dinitrophenol & 890 & $\mathrm{U}$ & 890 & 1000 & $\mathrm{U}$ & 1000 & 920 & $\mathrm{U}$ & 920 & 970 & $\mathrm{U}$ & 970 \\
\hline 2,4-Dinitrotoluene & 360 & $\mathrm{U}$ & 360 & 400 & $\mathrm{U}$ & 400 & 370 & $\mathrm{U}$ & 370 & 390 & $\mathrm{U}$ & 390 \\
\hline 2,6-Dinitrotoluene & 360 & $\mathrm{U}$ & 360 & 400 & $\mathrm{U}$ & 400 & 370 & $\mathrm{U}$ & 370 & 390 & $\mathrm{U}$ & 390 \\
\hline 2-Chloronaphthalene & 360 & $\mathrm{U}$ & 360 & 400 & $\mathrm{U}$ & 400 & 370 & $\mathrm{U}$ & 370 & 390 & $\mathrm{U}$ & 390 \\
\hline 2-Chlorophenol & 360 & $\mathrm{U}$ & 360 & 400 & $\mathrm{U}$ & 400 & 370 & $\mathrm{U}$ & 370 & 390 & $\mathrm{U}$ & 390 \\
\hline 2-Methylnaphthalene & 360 & $\mathrm{U}$ & 360 & 400 & $\mathrm{U}$ & 400 & 370 & $\mathrm{U}$ & 370 & 390 & $\mathrm{U}$ & 390 \\
\hline 2-Methylphenol (cresol, o-) & 360 & $\mathrm{U}$ & 360 & 400 & $\mathrm{U}$ & 400 & 370 & $\mathrm{U}$ & 370 & 390 & $\mathrm{U}$ & 390 \\
\hline 2-Nitroaniline & 890 & $\mathrm{U}$ & 890 & 1000 & $\mathrm{U}$ & 1000 & 920 & $\mathrm{U}$ & 920 & 970 & $\mathrm{U}$ & 970 \\
\hline 2-Nitrophenol & 360 & $\mathrm{U}$ & 360 & 400 & $\mathrm{U}$ & 400 & 370 & $\mathrm{U}$ & 370 & 390 & $\mathrm{U}$ & 390 \\
\hline
\end{tabular}


Table A-4. 128-F-2 Area C Verification Sampling Results. (10 pages)

\begin{tabular}{|c|c|c|c|c|c|c|c|c|c|c|c|c|}
\hline \multirow[t]{2}{*}{ Constituent } & \multicolumn{3}{|c|}{$\begin{array}{c}\text { J14JC6 } \\
\text { Sample Location C-7 } \\
\text { Sample Date 2/10/07 }\end{array}$} & \multicolumn{3}{|c|}{$\begin{array}{c}\text { J14JC7 } \\
\text { Sample Location C-8 } \\
\text { Sample Date 2/13/07 }\end{array}$} & \multicolumn{3}{|c|}{$\begin{array}{c}\text { J14JC8 } \\
\text { Sample Location C-9 } \\
\text { Sample Date 2/13/07 }\end{array}$} & \multicolumn{3}{|c|}{$\begin{array}{c}\text { J14JC9 } \\
\text { Sample Location C-10 } \\
\text { Sample Date 2/13/07 }\end{array}$} \\
\hline & $\mu \mathrm{g} / \mathrm{kg}$ & $\mathbf{Q}$ & PQL & $\mu \mathrm{g} / \mathrm{kg}$ & $\mathbf{Q}$ & PQL & $\mu \mathrm{g} / \mathrm{kg}$ & $\mathbf{Q}$ & PQL & $\mu \mathrm{g} / \mathrm{kg}$ & $\mathbf{Q}$ & PQL \\
\hline \multicolumn{13}{|c|}{ Semivolatile Organic Analytes (continued) } \\
\hline 3,3'-Dichlorobenzidine & 360 & $\mathrm{U}$ & 360 & 400 & $\mathrm{U}$ & 400 & 370 & $\mathrm{U}$ & 370 & 390 & $\mathrm{U}$ & 390 \\
\hline 4-Methylphenol (p-cresol) & 360 & $\mathrm{U}$ & 360 & 400 & $\mathrm{U}$ & 400 & 370 & $\mathrm{U}$ & 370 & 390 & $\mathrm{U}$ & 390 \\
\hline 3-Nitroaniline & 890 & $\mathrm{U}$ & 890 & 1000 & $\mathrm{U}$ & 1000 & 920 & $\mathrm{U}$ & 920 & 970 & $\mathrm{U}$ & 970 \\
\hline 4,6-Dinitro-2-methylphenol & 890 & $\mathrm{U}$ & 890 & 1000 & $\mathrm{U}$ & 1000 & 920 & $\mathrm{U}$ & 920 & 970 & $\mathrm{U}$ & 970 \\
\hline 4-Bromophenyl-phenylether & 360 & $\mathrm{U}$ & 360 & 400 & $\mathrm{U}$ & 400 & 370 & $\mathrm{U}$ & 370 & 390 & $\mathrm{U}$ & 390 \\
\hline 4-Chloro-3-methylphenol & 360 & $\mathrm{U}$ & 360 & 400 & $\mathrm{U}$ & 400 & 370 & $\mathrm{U}$ & 370 & 390 & $\mathrm{U}$ & 390 \\
\hline 4-Chloroaniline & 360 & $\mathrm{U}$ & 360 & 400 & $\mathrm{U}$ & 400 & 370 & $\mathrm{U}$ & 370 & 390 & $\mathrm{U}$ & 390 \\
\hline 4-Chlorophenyl-phenylether & 360 & $\mathrm{U}$ & 360 & 400 & $\mathrm{U}$ & 400 & 370 & $\mathrm{U}$ & 370 & 390 & $\mathrm{U}$ & 390 \\
\hline 4-Nitroaniline & 890 & $\mathrm{U}$ & 890 & 1000 & $\mathrm{U}$ & 1000 & 920 & $\mathrm{U}$ & 920 & 970 & $\mathrm{U}$ & 970 \\
\hline 4-Nitrophenol & 890 & $\mathrm{U}$ & 890 & 1000 & $\mathrm{U}$ & 1000 & 920 & $\mathrm{U}$ & 920 & 970 & $\mathrm{U}$ & 970 \\
\hline Acenaphthene & 360 & $\mathrm{U}$ & 360 & 400 & $\mathrm{U}$ & 400 & 370 & $\mathrm{U}$ & 370 & 390 & $\mathrm{U}$ & 390 \\
\hline Acenaphthylene & 360 & $\mathrm{U}$ & 360 & 400 & $\mathrm{U}$ & 400 & 370 & $\mathrm{U}$ & 370 & 390 & $\mathrm{U}$ & 390 \\
\hline Anthracene & 360 & $\mathrm{U}$ & 360 & 400 & $\mathrm{U}$ & 400 & 370 & $\mathrm{U}$ & 370 & 390 & $\mathrm{U}$ & 390 \\
\hline Benzo(a)anthracene & 360 & $\mathrm{U}$ & 360 & 400 & $\mathrm{U}$ & 400 & 370 & $\mathrm{U}$ & 370 & 390 & $\mathrm{U}$ & 390 \\
\hline Benzo(a)pyrene & 360 & $\mathrm{U}$ & 360 & 400 & $\mathrm{U}$ & 400 & 19 & $\mathrm{~J}$ & 370 & 390 & $\mathrm{U}$ & 390 \\
\hline Benzo(b)fluoranthene & 360 & $\mathrm{U}$ & 360 & 400 & $\mathrm{U}$ & 400 & 21 & $\mathrm{~J}$ & 370 & 390 & $\mathrm{U}$ & 390 \\
\hline Benzo(g,h,i)perylene & 360 & $\mathrm{U}$ & 360 & 400 & $\mathrm{U}$ & 400 & 22 & $\mathrm{~J}$ & 370 & 390 & $\mathrm{U}$ & 390 \\
\hline Benzo(k)fluoranthene & 360 & $\mathrm{U}$ & 360 & 400 & $\mathrm{U}$ & 400 & 20 & $\mathrm{~J}$ & 370 & 390 & $\mathrm{U}$ & 390 \\
\hline Bis(2-chloro-1-methylethyl)ether & 360 & $\mathrm{U}$ & 360 & 400 & $\mathrm{U}$ & 400 & 370 & $\mathrm{U}$ & 370 & 390 & $\mathrm{U}$ & 390 \\
\hline Bis(2-chloroethoxy)methane & 360 & $\mathrm{U}$ & 360 & 400 & $\mathrm{U}$ & 400 & 370 & $\mathrm{U}$ & 370 & 390 & $\mathrm{U}$ & 390 \\
\hline Bis(2-chloroethyl) ether & 360 & $\mathrm{U}$ & 360 & 400 & $\mathrm{U}$ & 400 & 370 & $\mathrm{U}$ & 370 & 390 & $\mathrm{U}$ & 390 \\
\hline Bis(2-ethylhexyl) phthalate & 160 & JB & 360 & 53 & JB & 400 & 65 & JB & 370 & 113 & JB & 390 \\
\hline Butylbenzylphthalate & 360 & $\mathrm{U}$ & 360 & 400 & $\mathrm{U}$ & 400 & 370 & $\mathrm{U}$ & 370 & 390 & $\mathrm{U}$ & 390 \\
\hline Carbazole & 360 & $\mathrm{U}$ & 360 & 400 & $\mathrm{U}$ & 400 & 370 & $\mathrm{U}$ & 370 & 390 & $\mathrm{U}$ & 390 \\
\hline Chrysene & 360 & $\mathrm{U}$ & 360 & 400 & $\mathrm{U}$ & 400 & 18 & $\mathrm{~J}$ & 370 & 390 & $\mathrm{U}$ & 390 \\
\hline Dibenz(a,h)anthracene & 360 & $\mathrm{U}$ & 360 & 92 & JB & 400 & 34 & JB & 370 & 21 & JB & 390 \\
\hline Dibenzofuran & 360 & $\mathrm{U}$ & 360 & 400 & $\mathrm{U}$ & 400 & 370 & $\mathrm{U}$ & 370 & 390 & $\mathrm{U}$ & 390 \\
\hline Diethylphthalate & 360 & $\mathrm{U}$ & 360 & 400 & $\mathrm{U}$ & 400 & 370 & $\mathrm{U}$ & 370 & 390 & $\mathrm{U}$ & 390 \\
\hline Dimethylphthalate & 360 & $\mathrm{U}$ & 360 & 400 & $\mathrm{U}$ & 400 & 370 & $\mathrm{U}$ & 370 & 390 & $\mathrm{U}$ & 390 \\
\hline Di-n-butylphthalate & 360 & $\mathrm{U}$ & 360 & 400 & $\mathrm{U}$ & 400 & 370 & $\mathrm{U}$ & 370 & 390 & $\mathrm{U}$ & 390 \\
\hline Di-n-octylphthalate & 360 & $\mathrm{U}$ & 360 & 400 & $\mathrm{U}$ & 400 & 370 & $\mathrm{U}$ & 370 & 390 & $\mathrm{U}$ & 390 \\
\hline Fluoranthene & 360 & $\mathrm{U}$ & 360 & 400 & $\mathrm{U}$ & 400 & 370 & $\mathrm{U}$ & 370 & 390 & $\mathrm{U}$ & 390 \\
\hline Fluorene & 360 & $\mathrm{U}$ & 360 & 400 & $\mathrm{U}$ & 400 & 370 & $\mathrm{U}$ & 370 & 390 & $\mathrm{U}$ & 390 \\
\hline Hexachlorobenzene & 360 & $\mathrm{U}$ & 360 & 400 & $\mathrm{U}$ & 400 & 370 & $\mathrm{U}$ & 370 & 390 & $\mathrm{U}$ & 390 \\
\hline Hexachlorobutadiene & 360 & $\mathrm{U}$ & 360 & 400 & $\mathrm{U}$ & 400 & 370 & $\mathrm{U}$ & 370 & 390 & $\mathrm{U}$ & 390 \\
\hline Hexachlorocyclopentadiene & 360 & $\mathrm{U}$ & 360 & 400 & $\mathrm{U}$ & 400 & 370 & $\mathrm{U}$ & 370 & 390 & $\mathrm{U}$ & 390 \\
\hline Hexachloroethane & 360 & $\mathrm{U}$ & 360 & 400 & $\mathrm{U}$ & 400 & 370 & $\mathrm{U}$ & 370 & 390 & $\mathrm{U}$ & 390 \\
\hline Indeno(1,2,3-cd)pyrene & 360 & $\mathrm{U}$ & 360 & 400 & $\mathrm{U}$ & 400 & 19 & $\mathrm{~J}$ & 370 & 390 & $\mathrm{U}$ & 390 \\
\hline Isophorone & 360 & $\mathrm{U}$ & 360 & 400 & $\mathrm{U}$ & 400 & 370 & $\mathrm{U}$ & 370 & 390 & $\mathrm{U}$ & 390 \\
\hline Naphthalene & 360 & $\mathrm{U}$ & 360 & 400 & $\mathrm{U}$ & 400 & 370 & $\mathrm{U}$ & 370 & 390 & $\mathrm{U}$ & 390 \\
\hline Nitrobenzene & 360 & $\mathrm{U}$ & 360 & 400 & $\mathrm{U}$ & 400 & 370 & $\mathrm{U}$ & 370 & 390 & $\mathrm{U}$ & 390 \\
\hline N-Nitroso-di-n-dipropylamine & 360 & $\mathrm{U}$ & 360 & 400 & $\mathrm{U}$ & 400 & 370 & $\mathrm{U}$ & 370 & 390 & $\mathrm{U}$ & 390 \\
\hline N-Nitrosodiphenylamine & 360 & $\mathrm{U}$ & 360 & 400 & $\mathrm{U}$ & 400 & 370 & $\mathrm{U}$ & 370 & 390 & $\mathrm{U}$ & 390 \\
\hline Pentachlorophenol & 890 & $\mathrm{U}$ & 890 & 1000 & $\mathrm{U}$ & 1000 & 920 & $\mathrm{U}$ & 920 & 970 & $\mathrm{U}$ & 970 \\
\hline Phenanthrene & 360 & $\mathrm{U}$ & 360 & 400 & $\mathrm{U}$ & 400 & 370 & $\mathrm{U}$ & 370 & 390 & $\mathrm{U}$ & 390 \\
\hline Phenol & 360 & $\mathrm{U}$ & 360 & 400 & $\mathrm{U}$ & 400 & 370 & $\mathrm{U}$ & 370 & 390 & $\mathrm{U}$ & 390 \\
\hline Pyrene & 360 & $\mathrm{U}$ & 360 & 400 & $\mathrm{U}$ & 400 & 370 & $\mathrm{U}$ & 370 & 390 & $\mathrm{U}$ & 390 \\
\hline
\end{tabular}


Table A-4. 128-F-2 Area C Verification Sampling Results. (10 pages)

\begin{tabular}{|c|c|c|c|c|c|c|}
\hline \multirow[t]{2}{*}{ Constituent } & \multicolumn{3}{|c|}{$\begin{array}{c}\text { J14JD0 } \\
\text { Sample Location C-11 } \\
\text { Sample Date } 2 / 13 / 07\end{array}$} & \multicolumn{3}{|c|}{$\begin{array}{c}\text { J14JD1 } \\
\text { Sample Location C-12 } \\
\text { Sample Date 2/13/07 }\end{array}$} \\
\hline & $\mu g / \mathrm{kg}$ & $\mathbf{Q}$ & PQL & $\mu \mathrm{g} / \mathrm{kg}$ & $\mathbf{Q}$ & PQL \\
\hline \multicolumn{7}{|c|}{ Polychlorinated Biphenyls } \\
\hline Aroclor-1016 & 14 & $\mathrm{U}$ & 14 & 31 & UD & 31 \\
\hline Aroclor-1221 & 14 & $\mathrm{U}$ & 14 & 31 & UD & 31 \\
\hline Aroclor-1232 & 14 & $\mathrm{U}$ & 14 & 31 & UD & 31 \\
\hline Aroclor-1242 & 14 & $\mathrm{U}$ & 14 & 31 & UD & 31 \\
\hline Aroclor-1248 & 14 & $\mathrm{U}$ & 14 & 31 & UD & 31 \\
\hline Aroclor-1254 & 14 & $\mathrm{U}$ & 14 & 31 & UD & 31 \\
\hline Aroclor-1260 & 14 & $\mathrm{U}$ & 14 & 400 & $\mathrm{D}$ & 31 \\
\hline \multicolumn{7}{|c|}{ Pesticides } \\
\hline Aldrin & 1.7 & $\mathrm{U}$ & 1.7 & 1.9 & $\mathrm{U}$ & 1.9 \\
\hline Alpha-BHC & 1.7 & $\mathrm{U}$ & 1.7 & 1.9 & $\mathrm{U}$ & 1.9 \\
\hline Alpha-Chlordane & 1.7 & $\mathrm{U}$ & 1.7 & 1.9 & $\mathrm{U}$ & 1.9 \\
\hline Beta-BHC & 1.7 & $\mathrm{U}$ & 1.7 & 0.58 & $\mathrm{~J}$ & 1.9 \\
\hline Delta-BHC & 1.7 & $\mathrm{U}$ & 1.7 & 1.9 & $\mathrm{U}$ & 1.9 \\
\hline Dichlorodiphenyldichloroethane & 1.7 & $\mathrm{U}$ & 1.7 & 1.9 & $\mathrm{U}$ & 1.9 \\
\hline Dichlorodiphenyldichloroethylene & 1.7 & $\mathrm{U}$ & 1.7 & 1.9 & $\mathrm{U}$ & 1.9 \\
\hline Dichlorodiphenyltrichloroethane & 1.7 & $\mathrm{U}$ & 1.7 & 8.7 & & 1.9 \\
\hline \begin{tabular}{|l|} 
Dieldrin \\
\end{tabular} & 1.7 & $\mathrm{U}$ & 1.7 & 2.6 & & 1.9 \\
\hline Endosulfan I & 1.7 & $\mathrm{U}$ & 1.7 & 1.9 & $\mathrm{U}$ & 1.9 \\
\hline Endosulfan II & 1.7 & $\mathrm{U}$ & 1.7 & 9.2 & & 1.9 \\
\hline Endosulfan sulfate & 1.7 & $\mathrm{U}$ & 1.7 & 8.9 & & 1.9 \\
\hline Endrin & 1.7 & $\mathrm{U}$ & 1.7 & 0.38 & $\overline{\mathrm{J}}$ & 1.9 \\
\hline Endrin aldehyde & 0.52 & $\mathrm{~J}$ & 1.7 & 8.5 & & 1.9 \\
\hline Endrin ketone & 1.7 & $\mathrm{U}$ & 1.7 & 1.9 & $\mathrm{U}$ & 1.9 \\
\hline Gamma-BHC (Lindane) & 1.7 & $\mathrm{U}$ & 1.7 & 1.9 & $\bar{U}$ & 1.9 \\
\hline gamma-Chlordane & 1.7 & $\mathrm{U}$ & 1.7 & 2.4 & & 1.9 \\
\hline Heptachlor & 1.7 & $\mathrm{U}$ & 1.7 & 1.9 & $\mathrm{U}$ & 1.9 \\
\hline Heptachlor epoxide & 1.7 & $\mathrm{U}$ & 1.7 & 1.9 & $\bar{U}$ & 1.9 \\
\hline Methoxychlor & 1.7 & $\mathrm{U}$ & 1.7 & 8.9 & & 1.9 \\
\hline Toxaphene & 17 & $\mathrm{U}$ & 17 & 19 & $\mathrm{U}$ & 19 \\
\hline \multicolumn{7}{|c|}{ Semivolatile Organic Analytes } \\
\hline 1,2,4-Trichlorobenzene & 340 & $\mathrm{U}$ & 340 & 380 & $\mathrm{U}$ & 380 \\
\hline 1,2-Dichlorobenzene & 340 & $\mathrm{U}$ & 340 & 380 & $\mathrm{U}$ & 380 \\
\hline 1,3-Dichlorobenzene & 340 & $\mathrm{U}$ & 340 & 380 & $\mathrm{U}$ & 380 \\
\hline 1,4-Dichlorobenzene & 340 & $\mathrm{U}$ & 340 & 380 & $\mathrm{U}$ & 380 \\
\hline 2,4,5-Trichlorophenol & 860 & $\mathrm{U}$ & 860 & 960 & $\mathrm{U}$ & 960 \\
\hline 2,4,6-Trichlorophenol & 340 & $\mathrm{U}$ & 340 & 380 & $\mathrm{U}$ & 380 \\
\hline 2,4-Dichlorophenol & 340 & $\mathrm{U}$ & 340 & 380 & $\mathrm{U}$ & 380 \\
\hline 2,4-Dimethylphenol & 340 & $\mathrm{U}$ & 340 & 380 & $\mathrm{U}$ & 380 \\
\hline 2,4-Dinitrophenol & 860 & $\mathrm{U}$ & 860 & 960 & $\mathrm{U}$ & 960 \\
\hline 2,4-Dinitrotoluene & 340 & $\mathrm{U}$ & 340 & 380 & $\mathrm{U}$ & 380 \\
\hline 2,6-Dinitrotoluene & 340 & $\mathrm{U}$ & 340 & 380 & $\mathrm{U}$ & 380 \\
\hline 2-Chloronaphthalene & 340 & $\mathrm{U}$ & 340 & 380 & $\mathrm{U}$ & 380 \\
\hline 2-Chlorophenol & 340 & $\mathrm{U}$ & 340 & 380 & $\mathrm{U}$ & 380 \\
\hline 2-Methylnaphthalene & 340 & $\mathrm{U}$ & 340 & 380 & $\mathrm{U}$ & 380 \\
\hline 2-Methylphenol (cresol, o-) & 340 & $\mathrm{U}$ & 340 & 380 & $\mathrm{U}$ & 380 \\
\hline 2-Nitroaniline & 860 & $\mathrm{U}$ & 860 & 960 & $\bar{U}$ & 960 \\
\hline
\end{tabular}


Table A-4. 128-F-2 Area C Verification Sampling Results. (10 pages)

\begin{tabular}{|c|c|c|c|c|c|c|}
\hline \multirow[t]{2}{*}{ Constituent } & \multicolumn{3}{|c|}{$\begin{array}{c}\text { J14JD0 } \\
\text { Sample Location C-11 } \\
\text { Sample Date 2/13/07 }\end{array}$} & \multicolumn{3}{|c|}{$\begin{array}{c}\text { J14JD1 } \\
\text { Sample Location C-12 } \\
\text { Sample Date 2/13/07 }\end{array}$} \\
\hline & $\mu \mathrm{g} / \mathrm{kg}$ & $\mathbf{Q}$ & PQL & $\mu g / k g$ & $\mathbf{Q}$ & PQL \\
\hline \multicolumn{7}{|c|}{ Semivolatile Organic Analytes (continued) } \\
\hline 3,3'-Dichlorobenzidine & 340 & $\mathrm{U}$ & 340 & 380 & $\mathrm{U}$ & 380 \\
\hline 4-Methylphenol (p-cresol) & 340 & $\mathrm{U}$ & 340 & 380 & $\mathrm{U}$ & 380 \\
\hline 3-Nitroaniline & 860 & $\mathrm{U}$ & 860 & 960 & $\mathrm{U}$ & 960 \\
\hline 4,6-Dinitro-2-methylphenol & 860 & $\mathrm{U}$ & 860 & 960 & $\mathrm{U}$ & 960 \\
\hline 4-Bromophenyl-phenylether & 340 & $\mathrm{U}$ & 340 & 380 & $\mathrm{U}$ & 380 \\
\hline 4-Chloro-3-methylphenol & 340 & $\mathrm{U}$ & 340 & 380 & $\mathrm{U}$ & 380 \\
\hline 4-Chloroaniline & 340 & $\mathrm{U}$ & 340 & 380 & $\mathrm{U}$ & 380 \\
\hline 4-Chlorophenyl-phenylether & 340 & $\mathrm{U}$ & 340 & 380 & $\mathrm{U}$ & 380 \\
\hline 4-Nitroaniline & 860 & $\mathrm{U}$ & 860 & 960 & $\mathrm{U}$ & 960 \\
\hline 4-Nitrophenol & 860 & $\mathrm{U}$ & 860 & 960 & $\mathrm{U}$ & 960 \\
\hline Acenaphthene & 340 & $\mathrm{U}$ & 340 & 380 & $\mathrm{U}$ & 380 \\
\hline \begin{tabular}{|l} 
Acenaphthylene \\
\end{tabular} & 340 & $\mathrm{U}$ & 340 & 380 & $\mathrm{U}$ & 380 \\
\hline Anthracene & 340 & $\mathrm{U}$ & 340 & 380 & $\mathrm{U}$ & 380 \\
\hline Benzo(a)anthracene & 340 & $\mathrm{U}$ & 340 & 380 & $\mathrm{U}$ & 380 \\
\hline Benzo(a)pyrene & 340 & $\mathrm{U}$ & 340 & 380 & $\mathrm{U}$ & 380 \\
\hline Benzo(b)fluoranthene & 340 & $\mathrm{U}$ & 340 & 380 & $\mathrm{U}$ & 380 \\
\hline Benzo(g,h,i)perylene & 340 & $\mathrm{U}$ & 340 & 380 & $\mathrm{U}$ & 380 \\
\hline Benzo(k)fluoranthene & 340 & $\mathrm{U}$ & 340 & 380 & $\mathrm{U}$ & 380 \\
\hline Bis(2-chloro-1-methylethyl)ether & 340 & $\mathrm{U}$ & 340 & 380 & $\mathrm{U}$ & 380 \\
\hline Bis(2-chloroethoxy)methane & 340 & $\mathrm{U}$ & 340 & 380 & $\mathrm{U}$ & 380 \\
\hline Bis(2-chloroethyl) ether & 340 & $\mathrm{U}$ & 340 & 380 & $\mathrm{U}$ & 380 \\
\hline Bis(2-ethylhexyl) phthalate & 76 & JB & 340 & 78 & $\mathrm{JB}$ & 380 \\
\hline Butylbenzylphthalate & 340 & $\mathrm{U}$ & 340 & 380 & $\mathrm{U}$ & 380 \\
\hline Carbazole & 340 & $\mathrm{U}$ & 340 & 380 & $\mathrm{U}$ & 380 \\
\hline \begin{tabular}{|l|} 
Chrysene \\
\end{tabular} & 340 & $\mathrm{U}$ & 340 & 380 & $\mathrm{U}$ & 380 \\
\hline Dibenz(a,h)anthracene & 39 & JB & 340 & 25 & JB & 380 \\
\hline Dibenzofuran & 340 & $\mathrm{U}$ & 340 & 380 & $\mathrm{U}$ & 380 \\
\hline Diethylphthalate & 340 & $\mathrm{U}$ & 340 & 380 & $\mathrm{U}$ & 380 \\
\hline Dimethylphthalate & 340 & $\mathrm{U}$ & 340 & 380 & $\mathrm{U}$ & 380 \\
\hline \begin{tabular}{|l} 
Di-n-butylphthalate \\
\end{tabular} & 340 & $\mathrm{U}$ & 340 & 380 & $\mathrm{U}$ & 380 \\
\hline Di-n-octylphthalate & 340 & $\mathrm{U}$ & 340 & 380 & $\mathrm{U}$ & 380 \\
\hline Fluoranthene & 340 & $\bar{U}$ & 340 & 380 & $\mathrm{U}$ & 380 \\
\hline \begin{tabular}{|l|} 
Fluorene \\
\end{tabular} & 340 & $\mathrm{U}$ & 340 & 380 & $\mathrm{U}$ & 380 \\
\hline Hexachlorobenzene & 340 & $\mathrm{U}$ & 340 & 380 & $\mathrm{U}$ & 380 \\
\hline Hexachlorobutadiene & 340 & $\mathrm{U}$ & 340 & 380 & $\mathrm{U}$ & 380 \\
\hline Hexachlorocyclopentadiene & 340 & $\mathrm{U}$ & 340 & 380 & $\mathrm{U}$ & 380 \\
\hline Hexachloroethane & 340 & $\mathrm{U}$ & 340 & 380 & $\mathrm{U}$ & 380 \\
\hline Indeno(1,2,3-cd)pyrene & 340 & $\mathrm{U}$ & 340 & 380 & $\mathrm{U}$ & 380 \\
\hline Isophorone & 340 & $\mathrm{U}$ & 340 & 380 & $\mathrm{U}$ & 380 \\
\hline Naphthalene & 340 & $\mathrm{U}$ & 340 & 380 & $\mathrm{U}$ & 380 \\
\hline Nitrobenzene & 340 & $\mathrm{U}$ & 340 & 380 & $\mathrm{U}$ & 380 \\
\hline N-Nitroso-di-n-dipropylamine & 340 & $\mathrm{U}$ & 340 & 380 & $\mathrm{U}$ & 380 \\
\hline N-Nitrosodiphenylamine & 340 & $\mathrm{U}$ & 340 & 380 & $\mathrm{U}$ & 380 \\
\hline \begin{tabular}{|l|} 
Pentachlorophenol \\
\end{tabular} & 860 & $\mathrm{U}$ & 860 & 960 & $\mathrm{U}$ & 960 \\
\hline Phenanthrene & 340 & $\mathrm{U}$ & 340 & 380 & $\mathrm{U}$ & 380 \\
\hline Phenol & 340 & $\mathrm{U}$ & 340 & 380 & $\mathrm{U}$ & 380 \\
\hline Pyrene & 340 & $\mathrm{U}$ & 340 & 380 & $\mathrm{U}$ & 380 \\
\hline
\end{tabular}


Table A-5. 128-F-2 Stockpile Verification Sampling Results. (6 pages)

\begin{tabular}{|c|c|c|c|c|c|c|c|c|c|c|c|c|c|c|c|c|c|c|c|c|}
\hline \multirow{2}{*}{$\begin{array}{c}\text { Sample } \\
\text { Location }\end{array}$} & \multirow{2}{*}{$\begin{array}{c}\text { HEIS } \\
\text { Number }\end{array}$} & \multirow{2}{*}{$\begin{array}{c}\text { Sample } \\
\text { Date }\end{array}$} & \multicolumn{3}{|c|}{ Americium-241 } & \multicolumn{3}{|c|}{ Barium-133 } & \multicolumn{3}{|c|}{ Cesium-137 } & \multicolumn{3}{|c|}{ Cobalt-60 } & \multicolumn{3}{|c|}{ Europium-152 } & \multicolumn{3}{|c|}{ Europium-154 } \\
\hline & & & $\mathrm{pCi} / \mathrm{g}$ & $\mathbf{Q}$ & MDA & $\mathrm{pCi} / \mathrm{g}$ & $\mathbf{Q}$ & MDA & $\mathrm{pCi} / \mathrm{g}$ & $\mathbf{Q}$ & MDA & $\mathrm{pCi} / \mathrm{g}$ & $\mathbf{Q}$ & MDA & $\mathrm{pCi} / \mathrm{g}$ & $\mathbf{Q}$ & MDA & $\mathrm{pCi} / \mathrm{g}$ & $\mathbf{Q}$ & MDA \\
\hline North Area & J163R5 & $12 / 11 / 2007$ & 0.064 & $\mathrm{U}$ & 0.064 & 0.076 & $\mathrm{U}$ & 0.076 & 0.071 & $\mathrm{U}$ & 0.071 & 0.068 & $\mathrm{U}$ & 0.068 & 0.165 & $\mathrm{U}$ & 55 & .244 & $\mathrm{U}$ & .244 \\
\hline Centra & & & 0.06 & $\mathrm{U}$ & 0.06 & 0.068 & $\mathrm{JI}$ & 68 & 55 & $\mathrm{U}$ & 0.055 & 0.063 & $\mathrm{U}$ & & 0.147 & $\mathrm{U}$ & 47 & 0.19 & $\mathrm{U}$ & 0.19 \\
\hline South Area & $3 \mathrm{R} 7$ & $11 / 2007$ & 072 & & 0.072 & 09 & 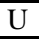 & & 96 & $\mathrm{U}$ & 96 & 38 & & 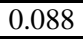 & .247 & $\mathrm{U}$ & 247 & 0.294 & $\mathrm{U}$ & 0.294 \\
\hline $\begin{array}{c}\text { Duplicate of } \\
\text { J163R7 } \\
\end{array}$ & J163R8 & $2 / 11 / 2007$ & 0.073 & $\mathrm{U}$ & 0.073 & 0.074 & $\mathrm{U}$ & 0.074 & 0.091 & $\mathrm{U}$ & 0.091 & 0.067 & $\mathrm{U}$ & 0.067 & 0.191 & $\mathrm{U}$ & 0.191 & 0.232 & U & 0.232 \\
\hline
\end{tabular}

\begin{tabular}{|c|c|c|c|c|c|c|c|c|c|c|c|c|c|c|c|c|c|c|c|c|}
\hline \multirow{2}{*}{$\begin{array}{c}\text { Sample } \\
\text { Location }\end{array}$} & \multirow{2}{*}{$\begin{array}{c}\text { HEIS } \\
\text { Number }\end{array}$} & \multirow{2}{*}{$\begin{array}{c}\text { Sample } \\
\text { Date }\end{array}$} & \multicolumn{3}{|c|}{ Europium-155 } & \multicolumn{3}{|c|}{ Gross alpha } & \multicolumn{3}{|c|}{ Gross beta } & \multicolumn{3}{|c|}{ Potassium-40 } & \multicolumn{3}{|c|}{ Radium-226 } & \multicolumn{3}{|c|}{ Radium-228 } \\
\hline & & & $\mathrm{pCi} / \mathrm{g}$ & $\mathbf{Q}$ & MDA & $\mathrm{pCi} / \mathrm{g}$ & $\mathbf{Q}$ & MDA & $\mathrm{pCi} / \mathrm{g}$ & $\mathbf{Q}$ & MDA & $\mathrm{pCi} / \mathrm{g}$ & $\mathbf{Q}$ & MDA & $\mathrm{pCi} / \mathrm{g}$ & $\mathbf{Q}$ & MDA & $\mathrm{pCi} / \mathrm{g}$ & $\mathbf{Q}$ & MDA \\
\hline North Area & J163R5 & $12 / 11 / 2007$ & 0.139 & $\mathrm{U}$ & 0.139 & 15.2 & & 8.38 & 14 & & 5.63 & 10.4 & & 0.778 & 0.395 & & 0.1 & 0.686 & & 0.243 \\
\hline Central Area & J163R6 & $12 / 11 / 2007$ & 0.136 & $\mathrm{U}$ & 0.136 & 9.87 & & 9.54 & 16.2 & & 5.33 & 10.8 & & 0.597 & 0.329 & & 0.118 & 0.58 & & 0.195 \\
\hline South Area & J163R7 & $12 / 11 / 2007$ & 0.184 & $\mathrm{U}$ & 0.184 & 11.6 & & 6.75 & 17.6 & & 5.95 & 11.1 & & 0.945 & 0.394 & & 0.186 & 0.627 & & 0.337 \\
\hline $\begin{array}{c}\text { Duplicate of } \\
\text { J163R7 }\end{array}$ & J163R8 & $12 / 11 / 2007$ & 0.158 & $\mathrm{U}$ & 0.158 & 17.8 & & 7.86 & 15.2 & & 5.31 & 11.5 & & 0.677 & 0.446 & & 0.129 & 0.822 & & 0.315 \\
\hline
\end{tabular}

\begin{tabular}{|c|c|c|c|c|c|c|c|c|c|c|c|c|c|c|c|c|c|}
\hline \multirow{2}{*}{$\begin{array}{c}\text { Sample } \\
\text { Location }\end{array}$} & \multirow{2}{*}{$\begin{array}{c}\text { HEIS } \\
\text { Number }\end{array}$} & \multirow{2}{*}{$\begin{array}{c}\text { Sample } \\
\text { Date }\end{array}$} & \multicolumn{3}{|c|}{ Silver 108-metastable } & \multicolumn{3}{|c|}{ Thorium-228 } & \multicolumn{3}{|c|}{ Thorium-232 } & \multicolumn{3}{|c|}{ Uranium-235 GEA } & \multicolumn{3}{|c|}{ Uranium-238 GEA } \\
\hline & & & $\mathrm{pCi} / \mathrm{g}$ & $\mathbf{Q}$ & MDA & $\mathrm{pCi} / \mathrm{g}$ & $\mathbf{Q}$ & MDA & pCi/g & $\mathbf{Q}$ & MDA & pCi/g & $\mathbf{Q}$ & MDA & pCi/g & $\mathbf{Q}$ & MDA \\
\hline North Area & J163R5 & \begin{tabular}{|l|}
$12 / 11 / 2007$ \\
\end{tabular} & 0.054 & $\mathrm{U}$ & 0.054 & 0.502 & & 0.081 & 0.686 & & 0.243 & 0.258 & $\mathrm{U}$ & 0.258 & 7.48 & $\mathbf{U}$ & 7.48 \\
\hline Central Area & J163R6 & \begin{tabular}{|l|}
$12 / 11 / 2007$ \\
\end{tabular} & 0.046 & $\mathrm{U}$ & 0.046 & 0.455 & & 0.071 & 0.58 & & 0.195 & 0.221 & $\mathrm{U}$ & 0.221 & 7.08 & $\mathrm{U}$ & 7.08 \\
\hline South Area & J163R7 & \begin{tabular}{|l|}
$12 / 11 / 2007$ \\
\end{tabular} & 0.066 & $\mathrm{U}$ & 0.066 & 0.558 & & 0.101 & 0.627 & & 0.337 & 0.294 & $\mathrm{U}$ & 0.294 & 11.2 & $\mathrm{U}$ & 11.2 \\
\hline $\begin{array}{c}\text { Duplicate of } \\
\text { J163R7 }\end{array}$ & J163R8 & $12 / 11 / 2007$ & 0.053 & $\mathrm{U}$ & 0.053 & 0.649 & & 0.077 & 0.822 & & 0.315 & 0.253 & $\mathrm{U}$ & 0.253 & 8.25 & $\mathrm{U}$ & 8.25 \\
\hline
\end{tabular}


Table A-5. 128-F-2 Stockpile Verification Sampling Results. (6 pages)

\begin{tabular}{|c|c|c|c|c|c|c|c|c|c|c|c|c|c|c|c|c|c|c|c|c|c|c|c|}
\hline \multirow{2}{*}{$\begin{array}{c}\text { Sample } \\
\text { Location }\end{array}$} & \multirow{2}{*}{$\begin{array}{c}\text { HEIS } \\
\text { Number }\end{array}$} & \multirow{2}{*}{$\begin{array}{c}\text { Sample } \\
\text { Date }\end{array}$} & \multicolumn{3}{|c|}{ Aluminum } & \multicolumn{3}{|c|}{ Antimony } & \multicolumn{3}{|c|}{ Arsenic } & \multicolumn{3}{|c|}{ Barium } & \multicolumn{3}{|c|}{ Beryllium } & \multicolumn{3}{|c|}{ Boron } & \multicolumn{3}{|c|}{ Cadmium } \\
\hline & & & $\mathrm{mg} / \mathrm{kg}$ & $\mathbf{Q}$ & PQL & $\mathrm{mg} / \mathrm{kg}$ & $\mathbf{Q}$ & PQL & $\mathrm{mg} / \mathrm{kg}$ & $\mathbf{Q}$ & PQL & $\mathrm{mg} / \mathrm{kg}$ & $\mathbf{Q}$ & PQL & $\mathrm{mg} / \mathrm{kg}$ & $\mathbf{Q}$ & PQL & $\mathrm{mg} / \mathrm{kg}$ & $\mathbf{Q}$ & PQL & $\mathrm{mg} / \mathrm{kg}$ & $\mathbf{Q}$ & PQL \\
\hline North Area & J163R5 & $12 / 11 / 2007$ & 4600 & & 3.5 & 1.2 & $\bar{C}$ & 0.26 & 2.4 & $\overline{\mathrm{C}}$ & 0.44 & 61.1 & & 0.09 & 0.04 & $\overline{\mathrm{U}}$ & 0.04 & 0.44 & $\mathrm{U}$ & 0.44 & 0.04 & $\mathrm{U}$ & 0.04 \\
\hline Central Area & J163R6 & $12 / 11 / 2007$ & 4970 & & 3.9 & 0.73 & $\mathrm{C}$ & 0.3 & 5.2 & $\mathrm{C}$ & 0.49 & 64.8 & & 0.1 & 0.05 & $\mathrm{U}$ & 0.05 & 0.49 & $\mathrm{U}$ & 0.49 & 0.05 & $\mathrm{U}$ & 0.05 \\
\hline South Area & J163R7 & $12 / 11 / 2007$ & 5470 & & 4 & 1.5 & $\mathrm{C}$ & 0.3 & 2.3 & $\mathrm{C}$ & 0.5 & 68.1 & & 0.1 & 0.05 & $\mathrm{U}$ & 0.05 & 0.5 & $\mathrm{U}$ & 0.5 & 0.05 & $\mathrm{U}$ & 0.05 \\
\hline Duplicate of & & & & & & & & & & & & & & & & & & & & & & & \\
\hline J163R7 & J163R8 & $12 / 11 / 2007$ & 5980 & & 4.2 & 0.46 & C & 0.32 & 2.3 & C & 0.53 & 68.2 & & 0.11 & 0.05 & $\mathrm{U}$ & 0.05 & 0.53 & $\mathrm{U}$ & 0.53 & 0.05 & $\mathrm{U}$ & 0.05 \\
\hline Equip blank & J163R9 & $12 / 11 / 2007$ & 41.5 & & 3.7 & 1 & $\mathrm{C}$ & 0.28 & 0.81 & $\mathrm{C}$ & 0.46 & 1.2 & & 0.09 & 0.05 & $\mathrm{U}$ & 0.05 & 0.46 & $\mathrm{U}$ & 0.46 & 0.05 & $\mathrm{U}$ & 0.05 \\
\hline
\end{tabular}

\begin{tabular}{|c|c|c|c|c|c|c|c|c|c|c|c|c|c|c|c|c|c|c|c|c|c|c|c|}
\hline \multirow{2}{*}{$\begin{array}{c}\text { Sample } \\
\text { Location }\end{array}$} & \multirow{2}{*}{$\begin{array}{c}\text { HEIS } \\
\text { Number }\end{array}$} & \multirow{2}{*}{$\begin{array}{c}\text { Sample } \\
\text { Date }\end{array}$} & \multicolumn{3}{|c|}{ Calcium } & \multicolumn{3}{|c|}{ Chromium } & \multicolumn{3}{|c|}{ Cobalt } & \multicolumn{3}{|c|}{ Copper } & \multicolumn{3}{|c|}{$\begin{array}{l}\text { Hexavalent } \\
\text { Chromium }\end{array}$} & \multicolumn{3}{|c|}{ Iron } & \multicolumn{3}{|c|}{ Lead } \\
\hline & & & $\mathrm{mg} / \mathrm{kg}$ & $\bar{Q}$ & PQL & $\mathrm{mg} / \mathbf{k g}$ & $\mathbf{Q}$ & PQL & $\mathrm{mg} / \mathrm{kg}$ & $\mathbf{Q}$ & PQL & $\mathrm{mg} / \mathrm{kg}$ & $\mathbf{Q}$ & PQL & $\mathrm{mg} / \mathrm{kg}$ & $\mathbf{Q}$ & PQL & $\mathbf{m g} / \mathbf{k g}$ & $\mathbf{Q}$ & PQL & $\mathrm{mg} / \mathrm{kg}$ & \begin{tabular}{|l|}
$\mathbf{Q}$ \\
\end{tabular} & PQL \\
\hline North Area & J163R5 & $12 / 11 / 2007$ & 4540 & $\overline{\mathrm{C}}$ & 3.5 & 15.7 & & 0.17 & 5.8 & & 0.17 & 14.8 & & 0.17 & 0.22 & $\mathrm{U}$ & 0.22 & 17000 & & 3.9 & 11.9 & \begin{tabular}{|l|}
$\mathrm{C}$ \\
\end{tabular} & 0.26 \\
\hline Central Area & J163R6 & $12 / 11 / 2007$ & 4470 & $\mathrm{C}$ & 3.9 & 32.2 & & 0.2 & 6.1 & & 0.2 & 23.9 & & 0.2 & 0.73 & & 0.22 & 18100 & & 4.4 & 24.8 & \begin{tabular}{|l|}
$\mathrm{C}$ \\
\end{tabular} & 0.3 \\
\hline South Area & J163R7 & $12 / 11 / 2007$ & 3610 & $\mathrm{C}$ & 4 & 12.2 & & 0.2 & 5.7 & & 0.2 & 13.4 & & 0.2 & 0.24 & & 0.22 & 16400 & & 4.5 & 12.8 & \begin{tabular}{|l|}
$\mathrm{C}$ \\
\end{tabular} & 0.3 \\
\hline $\begin{array}{c}\text { Duplicate of } \\
\end{array}$ & J163R8 & $12 / 11 / 2007$ & 3840 & $C$ & 42 & 113 & & 0.21 & 6.3 & & 021 & 135 & & 0.21 & 0.22 & $\mathrm{U}$ & 0.22 & 17400 & & 47 & 87 & C & 0.32 \\
\hline Equip blank & J163R9 & $12 / 11 / 2007$ & 18.2 & $\mathrm{C}$ & 3.7 & 0.19 & $\mathrm{U}$ & 0.19 & 0.19 & $\mathrm{U}$ & 0.19 & 0.25 & & 0.19 & & & & 111 & & 4.2 & 0.2 & \begin{tabular}{|c|}
$\mathrm{C}$ \\
\end{tabular} & 0.28 \\
\hline
\end{tabular}

\begin{tabular}{|c|c|c|c|c|c|c|c|c|c|c|c|c|c|c|c|c|c|c|c|c|c|c|c|}
\hline \multirow{2}{*}{$\begin{array}{c}\text { Sample } \\
\text { Location }\end{array}$} & \multirow{2}{*}{$\begin{array}{c}\text { HEIS } \\
\text { Number }\end{array}$} & \multirow{2}{*}{$\begin{array}{c}\text { Sample } \\
\text { Date }\end{array}$} & \multicolumn{3}{|c|}{ Magnesium } & \multicolumn{3}{|c|}{ Manganese } & \multicolumn{3}{|c|}{ Mercury } & \multicolumn{3}{|c|}{ Molybdenum } & \multicolumn{3}{|c|}{ Nickel } & \multicolumn{3}{|c|}{ Potassium } & \multicolumn{3}{|c|}{ Selenium } \\
\hline & & & $\mathrm{mg} / \mathrm{kg}$ & $\mathbf{Q}$ & PQL & $\mathrm{mg} / \mathrm{kg}$ & $\mathbf{Q}$ & PQL & $\mathbf{m g} / \mathbf{k g}$ & $\mathbf{Q}$ & PQL & $\mathrm{mg} / \mathrm{kg}$ & \begin{tabular}{|l|}
$\mathbf{Q}$ \\
\end{tabular} & PQL & $\mathrm{mg} / \mathrm{kg}$ & $\mathbf{Q}$ & PQL & $\mathrm{mg} / \mathrm{kg}$ & $\mathbf{Q}$ & PQL & $\mathrm{mg} / \mathrm{kg}$ & \begin{tabular}{l|l}
$\mathbf{Q}$ \\
\end{tabular} & PQL \\
\hline North Area & J163R5 & $12 / 11 / 2007$ & 3760 & & 3.2 & 247 & & 0.03 & 0.05 & & 0.01 & 0.26 & $\mathrm{U}$ & 0.26 & 11.7 & & 0.17 & 846 & & 43.1 & 0.52 & $\mathrm{U}$ & 0.53 \\
\hline Central Area & J163R6 & $12 / 11 / 2007$ & 3900 & & 3.9 & 277 & & 0.04 & 0.02 & & 0.01 & 1.3 & \begin{tabular}{|l|}
$\mathrm{C}$ \\
\end{tabular} & 0.3 & 10.5 & & 0.2 & 806 & & 48.6 & 0.59 & $\mathrm{U}$ & 0.59 \\
\hline South Area & $\begin{array}{l}\text { J163R7 } \\
\end{array}$ & $12 / 11 / 2007$ & 3460 & & 3.7 & 263 & & 0.04 & 0.04 & & 0.009 & 1 & \begin{tabular}{|l|}
$\mathrm{C}$ \\
\end{tabular} & 0.3 & 9.6 & & 0.2 & 1160 & & 49.4 & 0.95 & $\mathrm{C}$ & 0.6 \\
\hline $\begin{array}{c}\text { Duplicate of } \\
\text { J163R7 }\end{array}$ & J163R8 & $12 / 11 / 2007$ & 3800 & & 3.9 & 276 & & 0.04 & 0.03 & & 0.01 & 1.2 & C & 0.32 & 9.8 & & 0.21 & 1200 & & 51.8 & 0.63 & $\mathrm{U}$ & 0.63 \\
\hline Equip blank & J163R9 & $12 / 11 / 2007$ & 7.2 & & 3.4 & 3.8 & & 0.04 & 0.008 & $\mathrm{U}$ & 0.008 & 0.6 & \begin{tabular}{|l|}
$\mathrm{C}$ \\
\end{tabular} & 0.28 & 0.19 & $\mathrm{U}$ & 0.19 & 62 & & 45.6 & 0.56 & $\mathrm{U}$ & 0.56 \\
\hline
\end{tabular}

\begin{tabular}{|c|c|c|c|c|c|c|c|c|c|c|c|c|c|c|c|c|c|c|c|c|}
\hline \multirow{2}{*}{$\begin{array}{l}\text { Sample } \\
\text { Location }\end{array}$} & \multirow{2}{*}{$\begin{array}{c}\text { HEIS } \\
\text { Number }\end{array}$} & \multirow{2}{*}{$\begin{array}{l}\text { Sample } \\
\text { Date }\end{array}$} & \multicolumn{3}{|c|}{ Silicon } & \multicolumn{3}{|c|}{ Silver } & \multicolumn{3}{|c|}{ Sodium } & \multicolumn{3}{|c|}{ Vanadium } & \multicolumn{3}{|c|}{ Zinc } & \multicolumn{3}{|c|}{$\begin{array}{c}\text { Total petroleum } \\
\text { hydrocarbons }\end{array}$} \\
\hline & & & $\mathrm{mg} / \mathrm{kg}$ & $\mathbf{Q}$ & PQL & $\mathrm{mg} / \mathrm{kg}$ & $\mathbf{Q}$ & PQL & $\mathrm{ng} / \mathrm{kg}$ & $\mathbf{Q}$ & PQL & $\mathrm{mg} / \mathrm{kg}$ & \begin{tabular}{l|l}
$\mathbf{Q}$ \\
\end{tabular} & PQL & $\mathrm{mg} / \mathrm{kg}$ & $\mathbf{Q}$ & PQL & $\mathrm{mg} / \mathrm{kg}$ & $\mathbf{Q}$ & PQL \\
\hline North Area & J163R5 & $12 / 11 / 2007$ & 2110 & & 3.5 & 0.09 & $\mathrm{U}$ & 0.09 & 221 & $\mathrm{C}$ & 1.7 & 42.7 & & 0.12 & 54 & $\mathrm{C}$ & 0.52 & 143 & $\mathrm{U}$ & 143 \\
\hline Central Area & & & 2240 & & 3.9 & 1 & $\mathrm{U}$ & 01 & 191 & $\mathrm{C}$ & 2 & 42 & & & 124 & $\mathrm{C}$ & 0.59 & 144 & $\mathrm{U}$ & 144 \\
\hline Sout & 7 & $1 / 2007$ & 270 & & 4 & 0.1 & $\mathrm{U}$ & 0.1 & 172 & $\mathrm{C}$ & 2 & 37.6 & & 0.14 & 72.7 & $\mathrm{C}$ & 0.6 & 142 & $\mathrm{U}$ & 142 \\
\hline Duplicate of & & & & & & & & & & & & & & & & & & & & \\
\hline J163R7 & J163R8 & $12 / 11 / 2007$ & 3100 & & 4.2 & 0.11 & $\mathrm{U}$ & 0.11 & 198 & C & 2.1 & 40.9 & & 0.15 & 49 & C & 0.63 & 141 & $\mathrm{U}$ & 141 \\
\hline Equip blank & J163R9 & $12 / 11 / 2007$ & 61.4 & & 3.7 & 0.09 & $\mathrm{U}$ & 0.09 & 11.8 & $\mathrm{C}$ & 1.9 & 0.13 & $\mathrm{U}$ & 0.13 & 0.91 & $\mathrm{C}$ & 0.56 & & & \\
\hline
\end{tabular}


Table A-5. 128-F-2 Stockpile Verification Sampling Results. (6 pages)

\begin{tabular}{|c|c|c|c|c|c|c|c|c|c|c|c|c|}
\hline \multirow[t]{2}{*}{ Constituent } & \multicolumn{3}{|c|}{$\begin{array}{l}\text { J163R5, North Area, } \\
\text { Sample Date 12/11/07 }\end{array}$} & \multicolumn{3}{|c|}{$\begin{array}{c}\text { J163R6, Central Area, } \\
\text { Sample Date 12/11/07 }\end{array}$} & \multicolumn{3}{|c|}{$\begin{array}{l}\text { J163R7, South Area, } \\
\text { Sample Date 12/11/07 }\end{array}$} & \multicolumn{3}{|c|}{$\begin{array}{l}\text { J163R8, South Area, } \\
\text { Sample Date 12/11/07 }\end{array}$} \\
\hline & $\mu g / \mathbf{k g}$ & \begin{tabular}{l|l}
$\mathbf{Q}$ \\
\end{tabular} & PQL & $\mu \mathrm{g} / \mathrm{kg}$ & \begin{tabular}{|l|}
$\mathbf{Q}$ \\
\end{tabular} & PQL & $\mu \mathrm{g} / \mathrm{kg}$ & \begin{tabular}{|l|}
$\mathbf{Q}$ \\
\end{tabular} & PQL & $\mu g / \mathrm{kg}$ & \begin{tabular}{|l|}
$\mathbf{Q}$ \\
\end{tabular} & PQL \\
\hline \multicolumn{13}{|c|}{ Polychlorinated Biphenyls } \\
\hline Aroclor-1016 & 14 & $\mathrm{U}$ & 14 & 14 & $\mathrm{U}$ & 14 & 14 & $\mathrm{U}$ & 14 & 14 & $\mathrm{U}$ & 14 \\
\hline \begin{tabular}{|l|} 
Aroclor-1221 \\
\end{tabular} & 14 & $\mathrm{U}$ & 14 & 14 & $\mathrm{U}$ & 14 & 14 & \begin{tabular}{|l|}
$\mathrm{U}$ \\
\end{tabular} & 14 & 14 & $\mathrm{U}$ & 14 \\
\hline Aroclor-1232 & 14 & $\mathrm{U}$ & 14 & 14 & $\mathrm{U}$ & 14 & 14 & $\mathrm{U}$ & 14 & 14 & $\mathrm{U}$ & 14 \\
\hline Aroclor-1242 & 14 & $\mathrm{U}$ & 14 & 14 & $\mathrm{U}$ & 14 & 14 & \begin{tabular}{|l|}
$\mathrm{U}$ \\
\end{tabular} & 14 & 14 & $\mathrm{U}$ & 14 \\
\hline \begin{tabular}{|l|} 
Aroclor-1248 \\
\end{tabular} & 14 & $\mathrm{U}$ & 14 & 14 & $\mathrm{U}$ & 14 & 14 & \begin{tabular}{|l|}
$\mathrm{U}$ \\
\end{tabular} & 14 & 14 & $\mathrm{U}$ & 14 \\
\hline \begin{tabular}{|l|} 
Aroclor-1254 \\
\end{tabular} & 16 & & 14 & 15 & & 14 & 48 & & 14 & 23 & & 14 \\
\hline Aroclor-1260 & 14 & $\mathrm{U}$ & 14 & 14 & $\mathrm{U}$ & 14 & 14 & \begin{tabular}{|l|l}
$\mathrm{U}$ \\
\end{tabular} & 14 & 14 & $\mathrm{U}$ & 14 \\
\hline \multicolumn{13}{|c|}{ Pesticides } \\
\hline Aldrin & 1.4 & UD & 1.4 & 1.4 & UD & 1.4 & 1.4 & UD & 1.4 & 1.4 & UD & 1.4 \\
\hline Alpha-BHC & 1.4 & UD & 1.4 & 1.4 & UD & 1.4 & 1.4 & UD & 1.4 & 1.4 & UD & 1.4 \\
\hline Alpha-Chlordane & 1.4 & UD & 1.4 & 1.4 & UD & 1.4 & 1.4 & UD & 1.4 & 1.4 & UD & 1.4 \\
\hline \begin{tabular}{|l|} 
Beta-BHC \\
\end{tabular} & 1.4 & UD & 1.4 & 1.4 & UD & 1.4 & 1.4 & UD & 1.4 & 1.4 & UD & 1.4 \\
\hline Delta-BHC & 1.4 & UD & 1.4 & 1.4 & UD & 1.4 & 1.4 & UD & 1.4 & 1.4 & UD & 1.4 \\
\hline Dichlorodiphenyldichloroethane & 1.4 & UD & 1.4 & 1.4 & UD & 1.4 & 1.4 & UD & 1.4 & 1.4 & UD & 1.4 \\
\hline \begin{tabular}{|l|} 
Dichlorodiphenyldichloroethylene \\
\end{tabular} & 2.2 & JD & 1.4 & 1.4 & UD & 1.4 & 1.4 & UD & 1.4 & 1.4 & UD & 1.4 \\
\hline Dichlorodiphenyltrichloroethane & 6.6 & JD & 1.4 & 1.4 & UD & 1.4 & 1.4 & UD & 1.4 & 1.4 & UD & 1.4 \\
\hline Dieldrin & 1.4 & UD & 1.4 & 1.4 & UD & 1.4 & 1.4 & UD & 1.4 & 1.4 & UD & 1.4 \\
\hline Endosulfan I & 1.4 & UD & 1.4 & 1.4 & UD & 1.4 & 1.4 & UD & 1.4 & 1.4 & UD & 1.4 \\
\hline Endosulfan II & 1.4 & UD & 1.4 & 1.4 & UD & 1.4 & 1.4 & UD & 1.4 & 1.4 & UD & 1.4 \\
\hline Endosulfan sulfate & 1.4 & UD & 1.4 & 1.4 & UD & 1.4 & 1.4 & UD & 1.4 & 1.4 & UD & 1.4 \\
\hline Endrin & 1.4 & UD & 1.4 & 1.4 & UD & 1.4 & 1.4 & UD & 1.4 & 1.4 & UD & 1.4 \\
\hline Endrin aldehyde & 1.4 & UD & 1.4 & 1.4 & UD & 1.4 & 1.4 & UD & 1.4 & 1.4 & UD & 1.4 \\
\hline Endrin ketone & 1.4 & UD & 1.4 & 1.4 & UD & 1.4 & 1.4 & UD & 1.4 & 1.4 & UD & 1.4 \\
\hline Gamma-BHC (Lindane) & 1.4 & UD & 1.4 & 1.4 & UD & 1.4 & 1.4 & UD & 1.4 & 1.4 & UD & 1.4 \\
\hline gamma-Chlordane & 1.4 & UD & 1.4 & 1.4 & UD & 1.4 & 1.4 & UD & 1.4 & 1.4 & UD & 1.4 \\
\hline Heptachlor & 1.4 & UD & 1.4 & 1.4 & UD & 1.4 & 1.4 & UD & 1.4 & 1.4 & UD & 1.4 \\
\hline Heptachlor epoxide & 1.4 & UD & 1.4 & 1.4 & UD & 1.4 & 1.4 & UD & 1.4 & 1.4 & UD & 1.4 \\
\hline Methoxychlor & 1.4 & UD & 1.4 & 1.4 & UD & 1.4 & 1.4 & UD & 1.4 & 1.4 & UD & 1.4 \\
\hline Toxaphene & 14 & UD & 14 & 14 & UD & 14 & 14 & UD & 14 & 14 & UD & 14 \\
\hline \multicolumn{13}{|c|}{ Semivolatile Organic Analytes } \\
\hline 1,2,4-Trichlorobenzene & 360 & $\mathrm{U}$ & 360 & 360 & $\mathrm{U}$ & 360 & 360 & $\mathrm{U}$ & 360 & 360 & $\mathrm{U}$ & 360 \\
\hline 1,2-Dichlorobenzene & 360 & $\mathrm{U}$ & 360 & 360 & $\mathrm{U}$ & 360 & 360 & $\mathrm{U}$ & 360 & 360 & $\mathrm{U}$ & 360 \\
\hline \begin{tabular}{|l}
$1,3-D i c h l o r o b e n z e n e$ \\
\end{tabular} & 360 & $\mathrm{U}$ & 360 & 360 & \begin{tabular}{|l|}
$\mathrm{U}$ \\
\end{tabular} & 360 & 360 & $\mathrm{U}$ & 360 & 360 & $\mathrm{U}$ & 360 \\
\hline 1,4-Dichlorobenzene & 360 & $\mathrm{U}$ & 360 & 360 & $\mathrm{U}$ & 360 & 360 & \begin{tabular}{|l|}
$\mathrm{U}$ \\
\end{tabular} & 360 & 360 & $\mathrm{U}$ & 360 \\
\hline 2,4,5-Trichlorophenol & 900 & $\mathrm{U}$ & 900 & 900 & \begin{tabular}{|l|}
$\mathrm{U}$ \\
\end{tabular} & 900 & 900 & \begin{tabular}{|l|l}
$\mathrm{U}$ \\
\end{tabular} & 900 & 890 & $\mathrm{U}$ & 890 \\
\hline 2,4,6-Trichlorophenol & 360 & $\mathrm{U}$ & 360 & 360 & $\mathrm{U}$ & 360 & 360 & \begin{tabular}{|l|}
$\mathrm{U}$ \\
\end{tabular} & 360 & 360 & $\mathrm{U}$ & 360 \\
\hline 2,4-Dichlorophenol & 360 & $\mathrm{U}$ & 360 & 360 & $\mathrm{U}$ & 360 & 360 & $\mathrm{U}$ & 360 & 360 & $\mathrm{U}$ & 360 \\
\hline 2,4-Dimethylphenol & 360 & $\mathrm{U}$ & 360 & 360 & \begin{tabular}{|l|}
$\mathrm{U}$ \\
\end{tabular} & 360 & 360 & \begin{tabular}{|l|}
$\mathrm{U}$ \\
\end{tabular} & 360 & 360 & $\mathrm{U}$ & 360 \\
\hline 2,4-Dinitrophenol & 360 & $\mathrm{U}$ & 360 & 360 & \begin{tabular}{|l|}
$\mathrm{U}$ \\
\end{tabular} & 360 & 360 & $\mathrm{U}$ & 360 & 360 & $\mathrm{U}$ & 360 \\
\hline 2,4-Dinitrotoluene & 360 & $\mathrm{U}$ & 360 & 360 & $\mathrm{U}$ & 360 & 360 & \begin{tabular}{|l|l}
$\mathrm{U}$ \\
\end{tabular} & 360 & 360 & $\mathrm{U}$ & 360 \\
\hline 2,6-Dinitrotoluene & 360 & $\mathrm{U}$ & 360 & 360 & $\mathrm{U}$ & 360 & 360 & $\mathrm{U}$ & 360 & 360 & $\mathrm{U}$ & 360 \\
\hline 2-Chloronaphthalene & 360 & $\mathrm{U}$ & 360 & 360 & $\mathrm{U}$ & 360 & 360 & $\mathrm{U}$ & 360 & 360 & $\mathrm{U}$ & 360 \\
\hline 2-Chlorophenol & 360 & $\mathrm{U}$ & 360 & 360 & $\mathrm{U}$ & 360 & 360 & $\mathrm{U}$ & 360 & 360 & $\mathrm{U}$ & 360 \\
\hline 2-Methylnaphthalene & 360 & $\mathrm{U}$ & 360 & 360 & \begin{tabular}{|l|}
$\mathrm{U}$ \\
\end{tabular} & 360 & 360 & $\mathrm{U}$ & 360 & 360 & $\mathrm{U}$ & 360 \\
\hline \begin{tabular}{|l} 
2-Methylphenol (cresol, o-) \\
\end{tabular} & 360 & $\mathrm{U}$ & 360 & 360 & \begin{tabular}{|l|}
$\mathrm{U}$ \\
\end{tabular} & 360 & 360 & $\mathrm{U}$ & 360 & 360 & $\mathrm{U}$ & 360 \\
\hline 2-Nitroaniline & 900 & $\mathrm{U}$ & 900 & 900 & \begin{tabular}{|l|}
$\mathrm{U}$ \\
\end{tabular} & 900 & 900 & $\mathrm{U}$ & 900 & 890 & $\mathrm{U}$ & 890 \\
\hline 2-Nitrophenol & 360 & $\mathrm{U}$ & 360 & 360 & \begin{tabular}{|l|}
$\mathrm{U}$ \\
\end{tabular} & 360 & 360 & $\mathrm{U}$ & 360 & 360 & $\mathrm{U}$ & 360 \\
\hline
\end{tabular}


Table A-5. 128-F-2 Stockpile Verification Sampling Results. (6 pages)

\begin{tabular}{|c|c|c|c|c|c|c|c|c|c|c|c|c|}
\hline \multirow[t]{2}{*}{ Constituent } & \multicolumn{3}{|c|}{$\begin{array}{l}\text { J163R5, North Area, } \\
\text { Sample Date 12/11/07 }\end{array}$} & \multicolumn{3}{|c|}{$\begin{array}{c}\text { J163R6, Central Area, } \\
\text { Sample Date 12/11/07 }\end{array}$} & \multicolumn{3}{|c|}{$\begin{array}{l}\text { J163R7, South Area, } \\
\text { Sample Date 12/11/07 }\end{array}$} & \multicolumn{3}{|c|}{$\begin{array}{l}\text { J163R8, South Area, } \\
\text { Sample Date 12/11/07 }\end{array}$} \\
\hline & $\mu \mathrm{g} / \mathrm{kg}$ & $\mathbf{Q}$ & PQL & $\mu \mathrm{gg} / \mathrm{kg}$ & $\mathbf{Q}$ & PQL & $\mu \mathrm{g} / \mathrm{kg}$ & $\mathbf{Q}$ & PQL & $\mu \mathrm{g} / \mathrm{kg}$ & $\mathbf{Q}$ & PQL \\
\hline \multicolumn{13}{|c|}{ Semivolatile Organic Analytes (continued) } \\
\hline 3,3'-Dichlorobenzidine & 360 & $\mathrm{U}$ & 360 & 360 & $\mathrm{U}$ & 360 & 360 & $\mathrm{U}$ & 360 & 360 & $\mathrm{U}$ & 360 \\
\hline 4-Methylphenol (p-cresol) & 360 & $\mathrm{U}$ & 360 & 360 & $\mathrm{U}$ & 360 & 360 & $\mathrm{U}$ & 360 & 360 & $\mathrm{U}$ & 360 \\
\hline 3-Nitroaniline & 900 & $\mathrm{U}$ & 900 & 900 & $\mathrm{U}$ & 900 & 900 & $\mathrm{U}$ & 900 & 890 & $\mathrm{U}$ & 890 \\
\hline 4,6-Dinitro-2-methylphenol & 900 & $\mathrm{U}$ & 900 & 900 & $\mathrm{U}$ & 900 & 900 & $\mathrm{U}$ & 900 & 890 & $\mathrm{U}$ & 890 \\
\hline 4-Bromophenyl-phenylether & 360 & $\mathrm{U}$ & 360 & 360 & $\mathrm{U}$ & 360 & 360 & $\mathrm{U}$ & 360 & 360 & $\mathrm{U}$ & 360 \\
\hline 4-Chloro-3-methylphenol & 360 & $\mathrm{U}$ & 360 & 360 & $\mathrm{U}$ & 360 & 360 & $\mathrm{U}$ & 360 & 360 & $\mathrm{U}$ & 360 \\
\hline 4-Chloroaniline & 360 & $\mathrm{U}$ & 360 & 360 & $\mathrm{U}$ & 360 & 360 & $\mathrm{U}$ & 360 & 360 & $\mathrm{U}$ & 360 \\
\hline 4-Chlorophenyl-phenylether & 360 & $\mathrm{U}$ & 360 & 360 & $\mathrm{U}$ & 360 & 360 & $\mathrm{U}$ & 360 & 360 & $\mathrm{U}$ & 360 \\
\hline 4-Nitroaniline & 900 & $\mathrm{U}$ & 900 & 900 & $\mathrm{U}$ & 900 & 900 & $\mathrm{U}$ & 900 & 890 & $\mathrm{U}$ & 890 \\
\hline 4-Nitrophenol & 900 & $\mathrm{U}$ & 900 & 900 & $\mathrm{U}$ & 900 & 900 & $\mathrm{U}$ & 900 & 890 & $\mathrm{U}$ & 890 \\
\hline Acenaphthene & 27 & $\mathrm{~J}$ & 360 & 360 & $\mathrm{U}$ & 360 & 360 & $\mathrm{U}$ & 360 & 360 & $\mathrm{U}$ & 360 \\
\hline Acenaphthylene & 360 & $\mathrm{U}$ & 360 & 360 & $\mathrm{U}$ & 360 & 360 & $\mathrm{U}$ & 360 & 360 & $\mathrm{U}$ & 360 \\
\hline Anthracene & 60 & $\mathrm{~J}$ & 360 & 360 & $\mathrm{U}$ & 360 & 360 & $\mathrm{U}$ & 360 & 360 & $\mathrm{U}$ & 360 \\
\hline Benzo(a)anthracene & 320 & $\mathrm{~J}$ & 360 & 20 & $\mathrm{~J}$ & 360 & 21 & $\mathrm{~J}$ & 360 & 360 & $\mathrm{U}$ & 360 \\
\hline Benzo(a)pyrene & 280 & $\mathrm{~J}$ & 360 & 23 & $\mathrm{~J}$ & 360 & 20 & $\mathrm{~J}$ & 360 & 360 & $\mathrm{U}$ & 360 \\
\hline Benzo(b)fluoranthene & 210 & $\mathrm{~J}$ & 360 & 19 & $\mathrm{~J}$ & 360 & 20 & $\mathrm{~J}$ & 360 & 360 & $\mathrm{U}$ & 360 \\
\hline Benzo(g,h,i)perylene & 160 & $\mathrm{~J}$ & 360 & 18 & $\mathrm{~J}$ & 360 & 19 & $\mathrm{~J}$ & 360 & 360 & $\mathrm{U}$ & 360 \\
\hline Benzo(k)fluoranthene & 230 & $\mathrm{~J}$ & 360 & 360 & $\mathrm{U}$ & 360 & 26 & $\mathrm{~J}$ & 360 & 360 & $\mathrm{U}$ & 360 \\
\hline Bis(2-chloro-1-methylethyl)ether & 360 & $\mathrm{U}$ & 360 & 360 & $\mathrm{U}$ & 360 & 360 & $\mathrm{U}$ & 360 & 360 & $\mathrm{U}$ & 360 \\
\hline Bis(2-chloroethoxy)methane & 360 & $\mathrm{U}$ & 360 & 360 & $\mathrm{U}$ & 360 & 360 & $\mathrm{U}$ & 360 & 360 & $\mathrm{U}$ & 360 \\
\hline Bis(2-chloroethyl) ether & 360 & $\mathrm{U}$ & 360 & 360 & $\mathrm{U}$ & 360 & 360 & $\mathrm{U}$ & 360 & 360 & $\mathrm{U}$ & 360 \\
\hline Bis(2-ethylhexyl) phthalate & 27 & $\mathrm{~J}$ & 360 & 37 & $\mathrm{~J}$ & 360 & 110 & $\mathrm{~J}$ & 360 & 27 & $\mathrm{~J}$ & 360 \\
\hline Butylbenzylphthalate & 360 & $\mathrm{U}$ & 360 & 360 & $\mathrm{U}$ & 360 & 360 & $\mathrm{U}$ & 360 & 360 & $\mathrm{U}$ & 360 \\
\hline Carbazole & 30 & $\mathrm{~J}$ & 360 & 360 & $\mathrm{U}$ & 360 & 360 & $\mathrm{U}$ & 360 & 360 & $\mathrm{U}$ & 360 \\
\hline Chrysene & 370 & & 360 & 26 & $\mathrm{~J}$ & 360 & 37 & $\mathrm{~J}$ & 360 & 360 & $\mathrm{U}$ & 360 \\
\hline Dibenz(a,h)anthracene & 360 & $\mathrm{U}$ & 360 & 360 & $\mathrm{U}$ & 360 & 71 & $\mathrm{~J}$ & 360 & 360 & $\mathrm{U}$ & 360 \\
\hline Dibenzofuran & 360 & $\mathrm{U}$ & 360 & 360 & $\mathrm{U}$ & 360 & 360 & $\mathrm{U}$ & 360 & 360 & $\mathrm{U}$ & 360 \\
\hline Diethylphthalate & 77 & $\mathrm{~J}$ & 360 & 360 & $\mathrm{U}$ & 360 & 360 & $\mathrm{U}$ & 360 & 360 & $\mathrm{U}$ & 360 \\
\hline Dimethylphthalate & 360 & $\mathrm{U}$ & 360 & 360 & $\mathrm{U}$ & 360 & 360 & $\mathrm{U}$ & 360 & 360 & $\mathrm{U}$ & 360 \\
\hline Di-n-butylphthalate & 360 & $\mathrm{U}$ & 360 & 360 & $\mathrm{U}$ & 360 & 360 & $\mathrm{U}$ & 360 & 360 & $\mathrm{U}$ & 360 \\
\hline Di-n-octylphthalate & 360 & $\mathrm{U}$ & 360 & 360 & $\mathrm{U}$ & 360 & 360 & $\mathrm{U}$ & 360 & 360 & $\mathrm{U}$ & 360 \\
\hline Fluoranthene & 670 & & 360 & 39 & $\mathrm{~J}$ & 360 & 19 & $\mathrm{~J}$ & 360 & 360 & $\mathrm{U}$ & 360 \\
\hline Fluorene & 19 & $\mathrm{~J}$ & 360 & 360 & $\mathrm{U}$ & 360 & 360 & $\mathrm{U}$ & 360 & 360 & $\mathrm{U}$ & 360 \\
\hline Hexachlorobenzene & 360 & $\mathrm{U}$ & 360 & 360 & $\mathrm{U}$ & 360 & 360 & $\mathrm{U}$ & 360 & 360 & $\mathrm{U}$ & 360 \\
\hline Hexachlorobutadiene & 360 & $\mathrm{U}$ & 360 & 360 & $\mathrm{U}$ & 360 & 360 & $\mathrm{U}$ & 360 & 360 & $\mathrm{U}$ & 360 \\
\hline Hexachlorocyclopentadiene & 360 & $\mathrm{U}$ & 360 & 360 & $\mathrm{U}$ & 360 & 360 & $\mathrm{U}$ & 360 & 360 & $\mathrm{U}$ & 360 \\
\hline Hexachloroethane & 360 & $\mathrm{U}$ & 360 & 360 & $\mathrm{U}$ & 360 & 360 & $\mathrm{U}$ & 360 & 360 & $\mathrm{U}$ & 360 \\
\hline Indeno(1,2,3-cd)pyrene & 150 & $\mathrm{~J}$ & 360 & 360 & $\mathrm{U}$ & 360 & 360 & $\mathrm{U}$ & 360 & 360 & $\mathrm{U}$ & 360 \\
\hline Isophorone & 360 & $\mathrm{U}$ & 360 & 360 & $\mathrm{U}$ & 360 & 360 & $\mathrm{U}$ & 360 & 360 & $\mathrm{U}$ & 360 \\
\hline Naphthalene & 360 & $\mathrm{U}$ & 360 & 360 & $\mathrm{U}$ & 360 & 360 & $\mathrm{U}$ & 360 & 360 & $\mathrm{U}$ & 360 \\
\hline Nitrobenzene & 360 & $\mathrm{U}$ & 360 & 360 & $\mathrm{U}$ & 360 & 360 & $\mathrm{U}$ & 360 & 360 & $\mathrm{U}$ & 360 \\
\hline N-Nitroso-di-n-dipropylamine & 360 & $\mathrm{U}$ & 360 & 360 & $\mathrm{U}$ & 360 & 360 & $\mathrm{U}$ & 360 & 360 & $\mathrm{U}$ & 360 \\
\hline N-Nitrosodiphenylamine & 360 & $\mathrm{U}$ & 360 & 360 & $\mathrm{U}$ & 360 & 360 & $\mathrm{U}$ & 360 & 360 & $\mathrm{U}$ & 360 \\
\hline Pentachlorophenol & 900 & $\mathrm{U}$ & 900 & 900 & $\mathrm{U}$ & 900 & 900 & $\mathrm{U}$ & 900 & 890 & $\mathrm{U}$ & 890 \\
\hline Phenanthrene & 310 & $\mathrm{~J}$ & 360 & 22 & $\mathrm{~J}$ & 360 & 360 & $\mathrm{U}$ & 360 & 360 & $\mathrm{U}$ & 360 \\
\hline Phenol & 360 & $\mathrm{U}$ & 360 & 360 & $\mathrm{U}$ & 360 & 360 & $\mathrm{U}$ & 360 & 360 & $\mathrm{U}$ & 360 \\
\hline Pyrene & 790 & & 360 & 46 & $\mathrm{~J}$ & 360 & 73 & $\mathrm{~J}$ & 360 & 360 & $\mathrm{U}$ & 360 \\
\hline
\end{tabular}


Table A-5. 128-F-2 Stockpile Verification Sampling Results. (6 pages)

\begin{tabular}{|c|c|c|c|}
\hline \multirow[t]{2}{*}{ Constituent } & \multicolumn{3}{|c|}{$\begin{array}{l}\text { J163R9, Equip Blank, } \\
\text { Sample Date 12/11/07 }\end{array}$} \\
\hline & $\mu \mathrm{g} / \mathrm{kg}$ & $\mathbf{Q}$ & PQL \\
\hline \multicolumn{4}{|l|}{ Aroclor-1016 } \\
\hline \multicolumn{4}{|l|}{ Aroclor-1221 } \\
\hline \multicolumn{4}{|l|}{ Aroclor-1232 } \\
\hline \multicolumn{4}{|l|}{ Aroclor-1242 } \\
\hline \multicolumn{4}{|l|}{ Aroclor-1248 } \\
\hline \multicolumn{4}{|l|}{ Aroclor-1254 } \\
\hline Aroclor-1260 & & & \\
\hline
\end{tabular}

\begin{tabular}{|l|l|l|l|}
\hline Aldrin & & & \\
\hline Alpha-BHC & & & \\
\hline Alpha-Chlordane & & & \\
\hline Beta-BHC & & & \\
\hline Delta-BHC & & & \\
\hline Dichlorodiphenyldichloroethane & & & \\
\hline Dichlorodiphenyldichloroethylene & & & \\
\hline Dichlorodiphenyltrichloroethane & & & \\
\hline Dieldrin & & & \\
\hline Endosulfan I & & & \\
\hline Endosulfan II & & & \\
\hline Endosulfan sulfate & & & \\
\hline Endrin & & & \\
\hline Endrin aldehyde & & & \\
\hline Endrin ketone & & & \\
\hline Gamma-BHC (Lindane) & & & \\
\hline gamma-Chlordane & & & \\
\hline Heptachlor & & & \\
\hline Heptachlor epoxide & & & \\
\hline Methoxychlor & & & \\
\hline Toxaphene & & & \\
\hline
\end{tabular}

\section{Semivolatile Organic Analytes}

\begin{tabular}{|l|c|c|c|}
\hline 1,2,4-Trichlorobenzene & 330 & $\mathrm{U}$ & 330 \\
\hline 1,2-Dichlorobenzene & 330 & $\mathrm{U}$ & 330 \\
\hline 1,3-Dichlorobenzene & 330 & $\mathrm{U}$ & 330 \\
\hline 1,4-Dichlorobenzene & 330 & $\mathrm{U}$ & 330 \\
\hline 2,4,5-Trichlorophenol & 830 & $\mathrm{U}$ & 830 \\
\hline 2,4,6-Trichlorophenol & 330 & $\mathrm{U}$ & 330 \\
\hline 2,4-Dichlorophenol & 330 & $\mathrm{U}$ & 330 \\
\hline 2,4-Dimethylphenol & 330 & $\mathrm{U}$ & 330 \\
\hline 2,4-Dinitrophenol & 830 & $\mathrm{U}$ & 830 \\
\hline 2,4-Dinitrotoluene & 330 & $\mathrm{U}$ & 330 \\
\hline 2,6-Dinitrotoluene & 330 & $\mathrm{U}$ & 330 \\
\hline 2-Chloronaphthalene & 330 & $\mathrm{U}$ & 330 \\
\hline 2-Chlorophenol & 330 & $\mathrm{U}$ & 330 \\
\hline 2-Methylnaphthalene & 330 & $\mathrm{U}$ & 330 \\
\hline 2-Methylphenol (cresol, o-) & 330 & $\mathrm{U}$ & 330 \\
\hline 2-Nitroaniline & 830 & $\mathrm{U}$ & 830 \\
\hline 2-Nitrophenol & 330 & $\mathrm{U}$ & 330 \\
\hline
\end{tabular}


Table A-5. 128-F-2 Stockpile Verification Sampling Results. (6 pages)

\begin{tabular}{|c|c|c|c|}
\hline \multirow[t]{2}{*}{ Constituent } & \multicolumn{3}{|c|}{$\begin{array}{l}\text { J163R9, Equip Blank, } \\
\text { Sample Date 12/11/07 }\end{array}$} \\
\hline & $\mu \mathrm{g} / \mathrm{kg}$ & $\mathbf{Q}$ & PQL \\
\hline \multicolumn{4}{|c|}{ Semivolatile Organic Analytes (continued) } \\
\hline 3,3'-Dichlorobenzidine & 330 & $\mathrm{U}$ & 330 \\
\hline 4-Methylphenol (p-cresol) & 330 & $\mathrm{U}$ & 330 \\
\hline 3-Nitroaniline & 830 & $\mathrm{U}$ & 830 \\
\hline 4,6-Dinitro-2-methylphenol & 830 & $\mathrm{U}$ & 830 \\
\hline 4-Bromophenyl-phenylether & 330 & $\mathrm{U}$ & 330 \\
\hline 4-Chloro-3-methylphenol & 330 & $\mathrm{U}$ & 330 \\
\hline 4-Chloroaniline & 330 & $\mathrm{U}$ & 330 \\
\hline 4-Chlorophenyl-phenylether & 330 & $\mathrm{U}$ & 330 \\
\hline 4-Nitroaniline & 830 & $\mathrm{U}$ & 830 \\
\hline 4-Nitrophenol & 830 & $\mathrm{U}$ & 830 \\
\hline Acenaphthene & 330 & $\mathrm{U}$ & 330 \\
\hline Acenaphthylene & 330 & $\mathrm{U}$ & 330 \\
\hline Anthracene & 330 & $\mathrm{U}$ & 330 \\
\hline Benzo(a)anthracene & 330 & $\mathrm{U}$ & 330 \\
\hline Benzo(a)pyrene & 330 & $\mathrm{U}$ & 330 \\
\hline Benzo(b)fluoranthene & 330 & $\mathrm{U}$ & 330 \\
\hline Benzo(g,h,i)perylene & 330 & $\mathrm{U}$ & 330 \\
\hline Benzo(k)fluoranthene & 330 & $\mathrm{U}$ & 330 \\
\hline Bis(2-chloro-1-methylethyl)ether & 330 & $\mathrm{U}$ & 330 \\
\hline Bis(2-chloroethoxy)methane & 330 & $\mathrm{U}$ & 330 \\
\hline Bis(2-chloroethyl) ether & 330 & $\mathrm{U}$ & 330 \\
\hline Bis(2-ethylhexyl) phthalate & 50 & $\mathrm{~J}$ & 330 \\
\hline Butylbenzylphthalate & 330 & $\mathrm{U}$ & 330 \\
\hline Carbazole & 330 & $\mathrm{U}$ & 330 \\
\hline Chrysene & 330 & $\mathrm{U}$ & 330 \\
\hline Dibenz(a,h)anthracene & 21 & $\mathrm{~J}$ & 330 \\
\hline Dibenzofuran & 330 & $\mathrm{U}$ & 330 \\
\hline Diethylphthalate & 330 & $\mathrm{U}$ & 330 \\
\hline Dimethylphthalate & 330 & $\mathrm{U}$ & 330 \\
\hline Di-n-butylphthalate & 330 & $\mathrm{U}$ & 330 \\
\hline Di-n-octylphthalate & 330 & $\mathrm{U}$ & 330 \\
\hline Fluoranthene & 330 & $\mathrm{U}$ & 330 \\
\hline Fluorene & 330 & $\mathrm{U}$ & 330 \\
\hline Hexachlorobenzene & 330 & $\mathrm{U}$ & 330 \\
\hline Hexachlorobutadiene & 330 & $\mathrm{U}$ & 330 \\
\hline Hexachlorocyclopentadiene & 330 & $\mathrm{U}$ & 330 \\
\hline Hexachloroethane & 330 & $\mathrm{U}$ & 330 \\
\hline Indeno(1,2,3-cd)pyrene & 330 & $\mathrm{U}$ & 330 \\
\hline Isophorone & 330 & $\mathrm{U}$ & 330 \\
\hline Naphthalene & 330 & $\mathrm{U}$ & 330 \\
\hline Nitrobenzene & 330 & $\mathrm{U}$ & 330 \\
\hline N-Nitroso-di-n-dipropylamine & 330 & $\mathrm{U}$ & 330 \\
\hline N-Nitrosodiphenylamine & 330 & $\mathrm{U}$ & 330 \\
\hline Pentachlorophenol & 830 & $\mathrm{U}$ & 830 \\
\hline Phenanthrene & 330 & $\mathrm{U}$ & 330 \\
\hline Phenol & 330 & $\mathrm{U}$ & 330 \\
\hline Pyrene & 330 & $\mathrm{U}$ & 330 \\
\hline
\end{tabular}


Table A-6. 128-F-2 Asbestos Verification Data Results.

\begin{tabular}{|c|c|c|c|}
\hline Sample Area & $\begin{array}{c}\text { HEIS } \\
\text { Number }\end{array}$ & $\begin{array}{c}\text { Sample } \\
\text { Date }\end{array}$ & Asbestos Result \\
\hline A-1 & $\mathrm{J} 14 \mathrm{~KB} 8$ & $02 / 28 / 07$ & None detected. \\
\hline$A-2$ & J14KB9 & $02 / 28 / 07$ & None detected. \\
\hline$A-3$ & J14KC0 & $02 / 28 / 07$ & Trace. \\
\hline$A-4$ & J14KC1 & $02 / 28 / 07$ & None detected. \\
\hline$A-5$ & J14KC2 & $02 / 28 / 07$ & Trace. \\
\hline A-6 & J14KC3 & $03 / 01 / 07$ & Trace. \\
\hline$A-7$ & $\mathrm{~J} 14 \mathrm{KC} 4$ & $03 / 01 / 07$ & Trace. \\
\hline A-8 & J14KC5 & 03/01/07 & Trace. \\
\hline$A-9$ & J14KC6 & $03 / 01 / 07$ & Trace. \\
\hline$A-10$ & J14KC7 & $03 / 05 / 07$ & Trace. \\
\hline A-11 & J14KC8 & $03 / 05 / 07$ & Trace. \\
\hline $\begin{array}{l}\text { Duplicate of } \\
\text { J14KC8 }\end{array}$ & J14KC9 & 03/05/07 & None detected. \\
\hline A-12 & J14KD0 & $03 / 05 / 07$ & None detected. \\
\hline B-1 & J14L15 & $03 / 06 / 07$ & None detected. \\
\hline B-2 & J14L16 & $03 / 06 / 07$ & None detected. \\
\hline B-3 & J14L17 & $03 / 06 / 07$ & None detected. \\
\hline B-4 & J14L18 & $03 / 06 / 07$ & Trace. \\
\hline B-5 & J14L19 & $03 / 06 / 07$ & None detected. \\
\hline $\begin{array}{l}\text { Duplicate of } \\
\text { J14L19 }\end{array}$ & J14L20 & 03/06/07 & None detected. \\
\hline B-6 & J14L21 & 03/06/07 & Trace. \\
\hline B-7 & J14L22 & $03 / 06 / 07$ & Trace. \\
\hline B-8 & J14L23 & 03/06/07 & Trace. \\
\hline B-9 & J14L24 & $03 / 06 / 07$ & None detected. \\
\hline B-10 & J14L25 & $03 / 06 / 07$ & Trace. \\
\hline $\mathrm{C}-1$ & J14J97 & $02 / 10 / 07$ & None detected. \\
\hline $\mathrm{C}-2$ & J14J98 & $02 / 10 / 07$ & Trace. \\
\hline $\begin{array}{l}\text { Duplicate of } \\
\text { J14J98 }\end{array}$ & J14JB9 & 02/10/07 & None detected. \\
\hline $\mathrm{C}-3$ & J14J99 & 02/10/07 & Trace. \\
\hline $\mathrm{C}-4$ & J14JB0 & $02 / 10 / 07$ & None detected. \\
\hline $\mathrm{C}-5$ & J14JB1 & $02 / 10 / 07$ & None detected. \\
\hline $\mathrm{C}-6$ & J14JB2 & $02 / 10 / 07$ & None detected. \\
\hline $\mathrm{C}-7$ & J14JB3 & $02 / 10 / 07$ & None detected. \\
\hline $\mathrm{C}-8$ & J14JB4 & $02 / 13 / 07$ & None detected. \\
\hline $\mathrm{C}-9$ & J14JB5 & $02 / 13 / 07$ & None detected. \\
\hline $\mathrm{C}-10$ & J14JB6 & 02/13/07 & None detected. \\
\hline C-11 & J14JB7 & $02 / 13 / 07$ & None detected. \\
\hline C-12 & J14JB8 & $02 / 13 / 07$ & None detected. \\
\hline D-north & J163R2 & $12 / 11 / 07$ & None detected. \\
\hline D-central & J163R3 & $12 / 11 / 07$ & None detected. \\
\hline D-south & J163R4 & $12 / 11 / 07$ & None detected. \\
\hline $\begin{array}{l}\text { Duplicate of } \\
\text { J163R4 }\end{array}$ & J163T0 & $12 / 11 / 07$ & None detected. \\
\hline $\mathrm{E}$ & J163T1 & $12 / 11 / 07$ & None detected. \\
\hline
\end{tabular}


Table A-7. Sample location A-7 prior to additional remediation.

\begin{tabular}{|c|c|c|c|c|c|c|c|c|c|c|c|c|c|c|c|c|c|c|c|c|}
\hline \multirow{2}{*}{$\begin{array}{c}\text { Sample } \\
\text { Location }\end{array}$} & \multirow{2}{*}{$\begin{array}{c}\text { HEIS } \\
\text { Number }\end{array}$} & \multirow{2}{*}{$\begin{array}{c}\text { Sample } \\
\text { Date }\end{array}$} & \multicolumn{3}{|c|}{ Americium-241 } & \multicolumn{3}{|c|}{ Cesium-137 } & \multicolumn{3}{|c|}{ Cobalt-60 } & \multicolumn{3}{|c|}{ Europium-152 } & \multicolumn{3}{|c|}{ Europium-154 } & \multicolumn{3}{|c|}{ Europium-155 } \\
\hline & & & $\mathrm{pCi} / \mathrm{g}$ & 0 & MDA & $\mathrm{i} / \mathrm{g}$ & $\mathbf{Q}$ & MDA & $\mathrm{Ci} / \mathrm{g}$ & $\mathbf{Q}$ & MDA & $\mathrm{pCi} / \mathrm{g}$ & $\mathbf{Q}$ & MDA & $\mathrm{pCi} / \mathrm{g}$ & $\mathbf{Q}$ & MDA & $\mathrm{pCi} / \mathrm{g}$ & $\mathbf{Q}$ & MDA \\
\hline A7 & J14KB1 & $3 / 1 / 2007$ & 0.3 & $U$ & 0.3 & 0.091 & $\mathrm{U}$ & 0.091 & 0.094 & U & 0.094 & 0.23 & $\mathrm{U}$ & 0.23 & 0.31 & $\mathrm{U}$ & 0.31 & 0.21 & $\mathrm{U}$ & 0.21 \\
\hline
\end{tabular}

\begin{tabular}{|c|c|c|c|c|c|c|c|c|c|c|c|c|c|c|c|c|c|c|c|c|}
\hline \multirow{2}{*}{\begin{tabular}{|c|} 
Sample \\
Location
\end{tabular}} & \multirow{2}{*}{$\begin{array}{c}\text { HEIS } \\
\text { Number }\end{array}$} & \multirow{2}{*}{$\begin{array}{c}\text { Sample } \\
\text { Date }\end{array}$} & \multicolumn{3}{|c|}{ Gross alpha } & \multicolumn{3}{|c|}{ Gross beta } & \multicolumn{3}{|c|}{ Potassium-40 } & \multicolumn{3}{|c|}{ Radium-226 } & \multicolumn{3}{|c|}{ Radium-228 } & \multicolumn{3}{|c|}{ Silver 108-metastable } \\
\hline & & & $\mathrm{pCi} / \mathrm{g}$ & $\mathbf{Q}$ & MDA & $\mathrm{pCi} / \mathrm{g}$ & $\mathbf{Q}$ & MDA & $\mathrm{pCi} / \mathrm{g}$ & $\mathbf{Q}$ & MDA & $\mathrm{pCi} / \mathrm{g}$ & $\mathbf{Q}$ & MDA & $\mathrm{pCi} / \mathrm{g}$ & $\mathbf{Q}$ & MDA & $\mathrm{pCi} / \mathrm{g}$ & $\mathbf{Q}$ & MDA \\
\hline A7 & J14KB1 & $3 / 1 / 2007$ & 10.2 & & 5.7 & 22 & & 8.9 & 13.5 & & 0.79 & 0.427 & & 0.16 & 0.665 & & 0.38 & 0.063 & $\mathrm{U}$ & 0.063 \\
\hline
\end{tabular}

\begin{tabular}{|c|c|c|c|c|c|c|c|c|c|c|c|c|c|c|}
\hline \multirow{2}{*}{$\begin{array}{c}\text { Sample } \\
\text { Location }\end{array}$} & \multirow{2}{*}{$\begin{array}{c}\text { HEIS } \\
\text { Number }\end{array}$} & \multirow{2}{*}{$\begin{array}{c}\text { Sample } \\
\text { Date }\end{array}$} & \multicolumn{3}{|c|}{ Thorium-228 GEA } & \multicolumn{3}{|c|}{ Thorium-232 GEA } & \multicolumn{3}{|c|}{ Uranium-235 GEA } & \multicolumn{3}{|c|}{ Uranium-238 GEA } \\
\hline & & & & $\mathbf{Q}$ & 28 & & 8 & & & 8 & & & $\mathbf{Q}$ & MDA \\
\hline 87 & J14KB1 & $3 / 1 / 2007$ & 0.783 & & 0.1 & 0.665 & & 0.38 & 0.3 & $\mathrm{U}$ & 0.3 & 1. & $\mathrm{U}$ & 1. \\
\hline
\end{tabular}


Table A-7. Sample location A-7 prior to additional remediation.

\begin{tabular}{|c|c|c|c|c|c|c|c|c|c|c|c|c|c|c|c|c|c|c|c|c|c|c|c|}
\hline \multirow{2}{*}{$\begin{array}{c}\text { Sample } \\
\text { Location }\end{array}$} & \multirow{2}{*}{$\begin{array}{c}\text { HEIS } \\
\text { Number }\end{array}$} & \multirow{2}{*}{$\begin{array}{c}\text { Sample } \\
\text { Date }\end{array}$} & \multicolumn{3}{|c|}{ Aluminum } & \multicolumn{3}{|c|}{ Antimony } & \multicolumn{3}{|c|}{ Arsenic } & \multicolumn{3}{|c|}{ Barium } & \multicolumn{3}{|c|}{ Beryllium } & \multicolumn{3}{|c|}{ Boron } & \multicolumn{3}{|c|}{ Cadmium } \\
\hline & & & $\mathrm{mg} / \mathrm{kg}$ & $\mathbf{Q}$ & PQL & $\mathrm{g} / \mathbf{k g}$ & $\mathbf{Q}$ & PQL & $\mathrm{mg} / \mathbf{k g}$ & $\mathbf{Q}$ & PQL & $\mathrm{mg} / \mathrm{kg}$ & $\mathbf{Q}$ & PQL & $\mathrm{mg} / \mathrm{kg}$ & $\mathbf{Q}$ & PQL & $\mathrm{mg} / \mathrm{kg}$ & $\mathbf{Q}$ & PQL & $\mathrm{mg} / \mathrm{kg}$ & $\mathbf{Q}$ & PQL \\
\hline A7 & J14KB1 & $3 / 1 / 2007$ & 6180 & & 4.3 & 0.94 & U & 0.94 & 2.6 & & 1.2 & 78.8 & & 0.06 & 0.25 & & 0.03 & 1.4 & & 1.1 & 2.5 & $\begin{array}{ll}C \\
\end{array}$ & 0.09 \\
\hline \multirow{2}{*}{$\begin{array}{c}\text { Sample } \\
\text { Location }\end{array}$} & \multirow{2}{*}{$\begin{array}{c}\text { HEIS } \\
\text { Number }\end{array}$} & \multirow{2}{*}{$\begin{array}{c}\text { Sample } \\
\text { Date }\end{array}$} & \multicolumn{3}{|c|}{ Calcium } & \multicolumn{3}{|c|}{ Chromium } & \multicolumn{3}{|c|}{ Cobalt } & \multicolumn{3}{|c|}{ Copper } & \multicolumn{3}{|c|}{$\begin{array}{l}\text { Hexavalent } \\
\text { Chromium }\end{array}$} & \multicolumn{3}{|c|}{ Iron } & \multicolumn{3}{|c|}{ Lead } \\
\hline & & & $\mathrm{mg} / \mathrm{kg}$ & $\bar{Q}$ & PQL & $\mathrm{mg} / \mathrm{kg}$ & $\mathbf{Q}$ & PQL & $\mathrm{mg} / \mathrm{kg}$ & $\mathbf{Q}$ & PQL & $\mathrm{mg} / \mathrm{kg}$ & $\mathbf{Q}$ & PQL & $\mathrm{mg} / \mathrm{kg}$ & $\mathbf{Q}$ & PQL & $\mathrm{mg} / \mathrm{kg}$ & $\mathbf{Q}$ & PQL & $\mathrm{mg} / \mathrm{kg}$ & $\mathbf{Q}$ & PQL \\
\hline A7 & J14KB1 & $3 / 1 / 2007$ & 3890 & & 2.2 & 46.9 & & 0.23 & 5.7 & & 0.26 & 19.6 & & 0.35 & 0.42 & & 0.22 & 15600 & & 8.7 & 25.4 & & 0.82 \\
\hline
\end{tabular}

\begin{tabular}{|c|c|c|c|c|c|c|c|c|c|c|c|c|c|c|c|c|c|c|c|c|c|c|c|}
\hline \multirow{2}{*}{$\begin{array}{c}\text { Sample } \\
\text { Location }\end{array}$} & \multirow{2}{*}{$\begin{array}{c}\text { HEIS } \\
\text { Number }\end{array}$} & \multirow{2}{*}{$\begin{array}{c}\text { Sample } \\
\text { Date }\end{array}$} & \multicolumn{3}{|c|}{ Magnesium } & \multicolumn{3}{|c|}{ Manganese } & \multicolumn{3}{|c|}{ Mercury } & \multicolumn{3}{|c|}{ Molybdenum } & \multicolumn{3}{|c|}{ Nickel } & \multicolumn{3}{|c|}{ Potassium } & \multicolumn{3}{|c|}{ Selenium } \\
\hline & & & $\mathrm{mg} / \mathrm{kg}$ & $\mathbf{Q}$ & PQL & $\mathrm{mg} / \mathrm{kg}$ & $\mathbf{Q}$ & PQL & $\mathbf{m g} / \mathbf{k g}$ & $\mathbf{Q}$ & PQL & $\mathrm{mg} / \mathrm{kg}$ & Q & PQL & $\mathrm{mg} / \mathrm{kg}$ & $\mathbf{Q}$ & PQL & $\mathrm{mg} / \mathbf{k g}$ & $\mathbf{Q}$ & PQL & $\mathrm{mg} / \mathrm{kg}$ & \begin{tabular}{|l|}
$\mathbf{Q}$ \\
\end{tabular} & PQL \\
\hline A7 & J14KB1 & $3 / 1 / 2007$ & 4080 & & 2.1 & 272 & $\mathrm{C}$ & 0.06 & 0.87 & & 0.02 & 0.38 & U & 0.38 & 12.7 & & 0.56 & 1010 & & 14.3 & 1.1 & \begin{tabular}{|l|}
$U$ \\
\end{tabular} & 1.1 \\
\hline
\end{tabular}

\begin{tabular}{|c|c|c|c|c|c|c|c|c|c|c|c|c|c|c|c|c|c|c|c|c|}
\hline \multirow{2}{*}{$\begin{array}{c}\text { Sample } \\
\text { Location }\end{array}$} & \multirow{2}{*}{$\begin{array}{l}\text { HEIS } \\
\text { Number }\end{array}$} & \multirow{2}{*}{$\begin{array}{l}\text { Sample } \\
\text { Date }\end{array}$} & \multicolumn{3}{|c|}{ Silicon } & \multicolumn{3}{|c|}{ Silver } & \multicolumn{3}{|c|}{ Sodium } & \multicolumn{3}{|c|}{ Vanadium } & \multicolumn{3}{|c|}{ Zinc } & \multicolumn{3}{|c|}{$\begin{array}{l}\text { Total petroleum } \\
\text { hydrocarbons }\end{array}$} \\
\hline & & & $\mathrm{mg} / \mathrm{kg}$ & $\mathbf{Q}$ & PQL & $\mathrm{mg} / \mathrm{kg}$ & $\mathbf{Q}$ & PQL & $\mathrm{mg} / \mathrm{kg}$ & \begin{tabular}{l|}
$\mathbf{Q}$ \\
\end{tabular} & PQL & $\mathrm{mg} / \mathrm{kg}$ & $\mathbf{Q}$ & PQL & $\mathrm{mg} / \mathrm{kg}$ & $\mathbf{Q}$ & PQL & $\mathrm{mg} / \mathrm{kg}$ & $\mathbf{Q}$ & PQL \\
\hline A7 & J14KB1 & $3 / 1 / 2007$ & 460 & C & 1.2 & 0.26 & $\mathrm{U}$ & 0.26 & 113 & C & 1.3 & 31.9 & & 0.29 & 88.3 & C & 0.09 & 141 & $\mathrm{U}$ & 141 \\
\hline
\end{tabular}


Table A-7. Sample location A-7 prior to additional remediation.

\begin{tabular}{|c|c|c|c|c|c|c|c|}
\hline \multirow[t]{2}{*}{ Constituent } & \multicolumn{3}{|c|}{$\begin{array}{c}\text { J14KB1, Sample } \\
\text { Location A7, Sample } \\
\text { Date 3/1/07 }\end{array}$} & \multirow[t]{2}{*}{ Constituent } & \multicolumn{3}{|c|}{$\begin{array}{c}\text { J14KB1, Sample Location } \\
\text { A7, Sample Date 3/1/07 }\end{array}$} \\
\hline & $\mu \mathrm{g} / \mathrm{kg}$ & $\mathbf{Q}$ & PQL & & $\mu \mathrm{g} / \mathrm{kg}$ & $\mathbf{Q}$ & PQL \\
\hline \multicolumn{4}{|c|}{ Polychlorinated Biphenyls } & \multicolumn{4}{|c|}{ Semivolatile Organic Analytes (continued) } \\
\hline Aroclor-1016 & 14 & $\mathrm{U}$ & 14 & 3,3'-Dichlorobenzidine & 350 & $\mathrm{U}$ & 350 \\
\hline \begin{tabular}{|l|} 
Aroclor-1221 \\
\end{tabular} & 14 & $\mathrm{U}$ & 14 & 4-Methylphenol (p-cresol) & 350 & $\mathrm{U}$ & 350 \\
\hline \begin{tabular}{|l|} 
Aroclor-1232 \\
\end{tabular} & 14 & $\mathrm{U}$ & 14 & 3-Nitroaniline & 890 & $\mathrm{U}$ & 890 \\
\hline \begin{tabular}{|l|} 
Aroclor-1242 \\
\end{tabular} & 14 & $\mathrm{U}$ & 14 & 4,6-Dinitro-2-methylphenol & 890 & $\mathrm{U}$ & 890 \\
\hline Aroclor-1248 & 14 & $\mathrm{U}$ & 14 & 4-Bromophenyl-phenylether & 350 & $\mathrm{U}$ & 350 \\
\hline \begin{tabular}{|l|} 
Aroclor-1254 \\
\end{tabular} & 14 & $\mathrm{U}$ & 14 & 4-Chloro-3-methylphenol & 350 & $\mathrm{U}$ & 350 \\
\hline Aroclor-1260 & 14 & $\mathrm{U}$ & 14 & 4-Chloroaniline & 350 & $\mathrm{U}$ & 350 \\
\hline \multicolumn{4}{|c|}{ Pesticides } & 4-Chlorophenyl-phenylether & 350 & $\mathrm{U}$ & 350 \\
\hline Aldrin & 1.4 & $\mathrm{U}$ & 1.4 & 4-Nitroaniline & 890 & $\mathrm{U}$ & 890 \\
\hline Alpha-BHC & 1.4 & $\mathrm{U}$ & 1.4 & \begin{tabular}{|l|} 
4-Nitrophenol \\
\end{tabular} & 890 & $\mathrm{U}$ & 890 \\
\hline Alpha-Chlordane & 1.4 & $\mathrm{U}$ & 1.4 & Acenaphthene & 350 & $\mathrm{U}$ & 350 \\
\hline Beta-BHC & 2.1 & $\mathrm{D}$ & 1.4 & \begin{tabular}{|l|} 
Acenaphthylene \\
\end{tabular} & 350 & $\mathrm{U}$ & 350 \\
\hline Delta-BHC & 1.4 & $\mathrm{U}$ & 1.4 & Anthracene & 18 & $\mathrm{~J}$ & 350 \\
\hline \begin{tabular}{|l|} 
Dichlorodiphenyldichloroethane \\
\end{tabular} & 1.4 & $\mathrm{U}$ & 1.4 & Benzo(a)anthracene & 47 & $\mathrm{~J}$ & 350 \\
\hline \begin{tabular}{|l|} 
Dichlorodiphenyldichloroethylene \\
\end{tabular} & 1.4 & $\mathrm{U}$ & 1.4 & Benzo(a)pyrene & 38 & $\mathrm{~J}$ & 350 \\
\hline Dichlorodiphenyltrichloroethane & 1.4 & $\mathrm{U}$ & 1.4 & Benzo(b)fluoranthene & 30 & $\mathrm{~J}$ & 350 \\
\hline Dieldrin & 1.4 & $\mathrm{U}$ & 1.4 & Benzo(g,h,i)perylene & 19 & $\mathrm{~J}$ & 350 \\
\hline \begin{tabular}{|l|} 
Endosulfan I \\
\end{tabular} & 1.4 & $\mathrm{U}$ & 1.4 & Benzo(k)fluoranthene & 44 & $\mathrm{~J}$ & 350 \\
\hline \begin{tabular}{|l|} 
Endosulfan II \\
\end{tabular} & 1.4 & $\mathrm{U}$ & 1.4 & Bis(2-chloro-1-methylethyl)ether & 350 & $\mathrm{U}$ & 350 \\
\hline Endosulfan sulfate & 1.4 & $\mathrm{U}$ & 1.4 & Bis(2-chloroethoxy)methane & 350 & $\mathrm{U}$ & 350 \\
\hline Endrin & 1.4 & $\mathrm{U}$ & 1.4 & Bis(2-chloroethyl) ether & 350 & $\mathrm{U}$ & 350 \\
\hline \begin{tabular}{|l} 
Endrin aldehyde \\
\end{tabular} & 1.4 & $\mathrm{U}$ & 1.4 & \begin{tabular}{|l} 
Bis(2-ethylhexyl) phthalate \\
\end{tabular} & 50 & $\mathrm{JB}$ & 350 \\
\hline \begin{tabular}{|l|} 
Endrin ketone \\
\end{tabular} & 1.4 & $\mathrm{U}$ & 1.4 & Butylbenzylphthalate & 350 & $\mathrm{U}$ & 350 \\
\hline Gamma-BHC (Lindane) & 1.4 & $\mathrm{U}$ & 1.4 & Carbazole & 350 & $\mathrm{U}$ & 350 \\
\hline gamma-Chlordane & 1.4 & $\mathrm{U}$ & 1.4 & \begin{tabular}{|l|} 
Chrysene \\
\end{tabular} & 58 & $\mathrm{~J}$ & 350 \\
\hline \begin{tabular}{|l|} 
Heptachlor \\
\end{tabular} & 1.4 & $\mathrm{U}$ & 1.4 & Dibenz(a,h)anthracene & 49 & $\mathrm{~J}$ & 350 \\
\hline Heptachlor epoxide & 1.4 & $\mathrm{U}$ & 1.4 & \begin{tabular}{|l|} 
Dibenzofuran \\
\end{tabular} & 350 & $\mathrm{U}$ & 350 \\
\hline Methoxychlor & 1.4 & $\mathrm{U}$ & 1.4 & \begin{tabular}{|l} 
Diethylphthalate \\
\end{tabular} & 350 & $\mathrm{U}$ & 350 \\
\hline \begin{tabular}{|l} 
Toxaphene \\
\end{tabular} & 14 & $\mathrm{U}$ & 14 & \begin{tabular}{|l|} 
Dimethylphthalate \\
\end{tabular} & 350 & $\mathrm{U}$ & 350 \\
\hline \multicolumn{4}{|c|}{ Semivolatile Organic Analytes } & Di-n-butylphthalate & 350 & $\mathrm{U}$ & 350 \\
\hline 1,2,4-Trichlorobenzene & 350 & $\mathrm{U}$ & 350 & \begin{tabular}{|l|} 
Di-n-octylphthalate \\
\end{tabular} & 350 & $\mathrm{U}$ & 350 \\
\hline 1,2-Dichlorobenzene & 350 & $\mathrm{U}$ & 350 & \begin{tabular}{|l} 
Fluoranthene \\
\end{tabular} & 110 & $\mathrm{~J}$ & 350 \\
\hline 1,3-Dichlorobenzene & 350 & $\mathrm{U}$ & 350 & Fluorene & 350 & $\mathrm{U}$ & 350 \\
\hline 1,4-Dichlorobenzene & 350 & $\mathrm{U}$ & 350 & Hexachlorobenzene & 350 & $\mathrm{U}$ & 350 \\
\hline 2,4,5-Trichlorophenol & 890 & $\mathrm{U}$ & 890 & Hexachlorobutadiene & 350 & $\mathrm{U}$ & 350 \\
\hline 2,4,6-Trichlorophenol & 350 & $\mathrm{U}$ & 350 & Hexachlorocyclopentadiene & 350 & $\mathrm{U}$ & 350 \\
\hline 2,4-Dichlorophenol & 350 & $\mathrm{U}$ & 350 & Hexachloroethane & 350 & $\mathrm{U}$ & 350 \\
\hline 2,4-Dimethylphenol & 350 & $\mathrm{U}$ & 350 & Indeno(1,2,3-cd)pyrene & 21 & $\mathrm{~J}$ & 350 \\
\hline 2,4-Dinitrophenol & 890 & $\mathrm{U}$ & 890 & Isophorone & 350 & $\mathrm{U}$ & 350 \\
\hline 2,4-Dinitrotoluene & 350 & $\mathrm{U}$ & 350 & \begin{tabular}{|l|} 
Naphthalene \\
\end{tabular} & 350 & $\mathrm{U}$ & 350 \\
\hline 2,6-Dinitrotoluene & 350 & $\mathrm{U}$ & 350 & \begin{tabular}{|l|} 
Nitrobenzene \\
\end{tabular} & 350 & $\mathrm{U}$ & 350 \\
\hline 2-Chloronaphthalene & 350 & $\mathrm{U}$ & 350 & \begin{tabular}{|l} 
N-Nitroso-di-n-dipropylamine \\
\end{tabular} & 350 & $\mathrm{U}$ & 350 \\
\hline 2-Chlorophenol & 350 & $\mathrm{U}$ & 350 & N-Nitrosodiphenylamine & 350 & $\mathrm{U}$ & 350 \\
\hline 2-Methylnaphthalene & 350 & $\mathrm{U}$ & 350 & \begin{tabular}{|l|} 
Pentachlorophenol \\
\end{tabular} & 890 & $\mathrm{U}$ & 890 \\
\hline 2-Methylphenol (cresol, o-) & 350 & $\mathrm{U}$ & 350 & \begin{tabular}{|l|} 
Phenanthrene \\
\end{tabular} & 69 & $\mathrm{~J}$ & 350 \\
\hline 2-Nitroaniline & 890 & $\mathrm{U}$ & 890 & Phenol & 350 & $\mathrm{U}$ & 350 \\
\hline 2-Nitrophenol & 350 & $\mathrm{U}$ & 350 & Pyrene & 120 & $\mathrm{~J}$ & 350 \\
\hline
\end{tabular}


APPENDIX B

\section{PHOTOGRAPHS}


Confirmatory Sampling Excavation at the West End of Trench 1. The top arrow shows top soil, the middle arrow is debris, and bottom arrow is concrete debris on east end of trench.

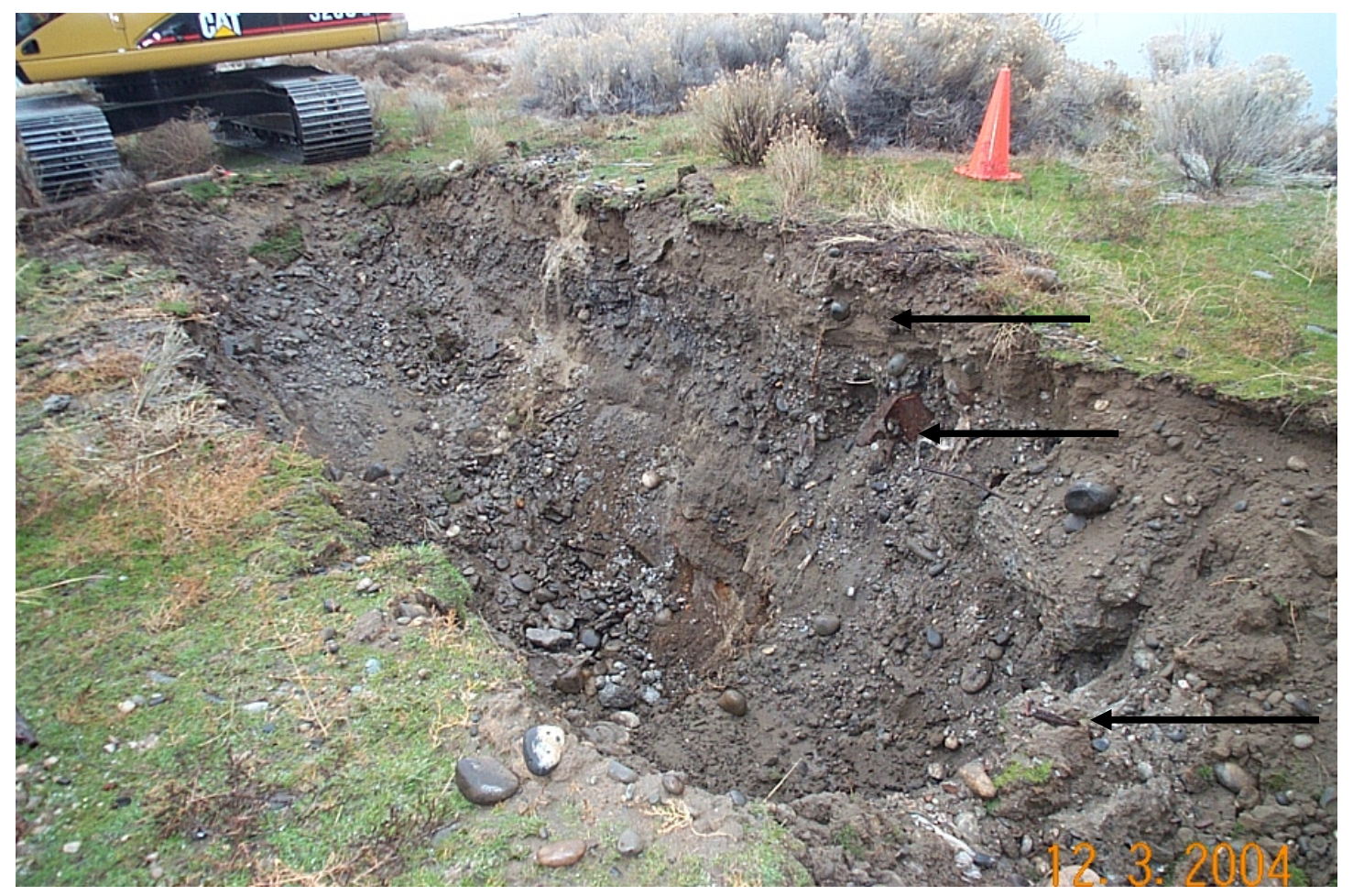

Confirmatory Sample J024R1 Collected from Purple- and Gold-Colored Soil in Trench 1.

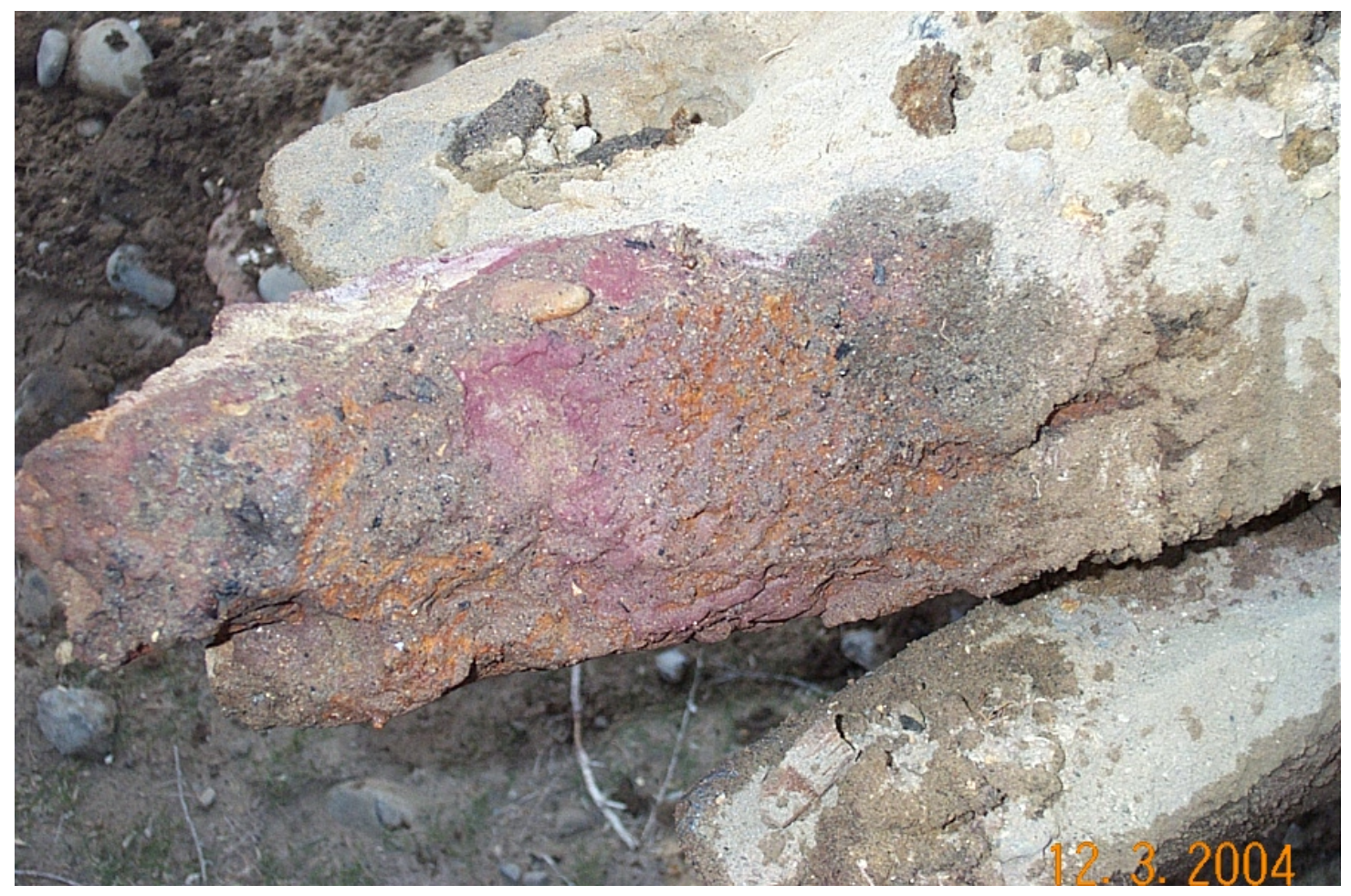


Confirmatory Sampling Excavation in Trench 1. Arrows point to concrete debris.

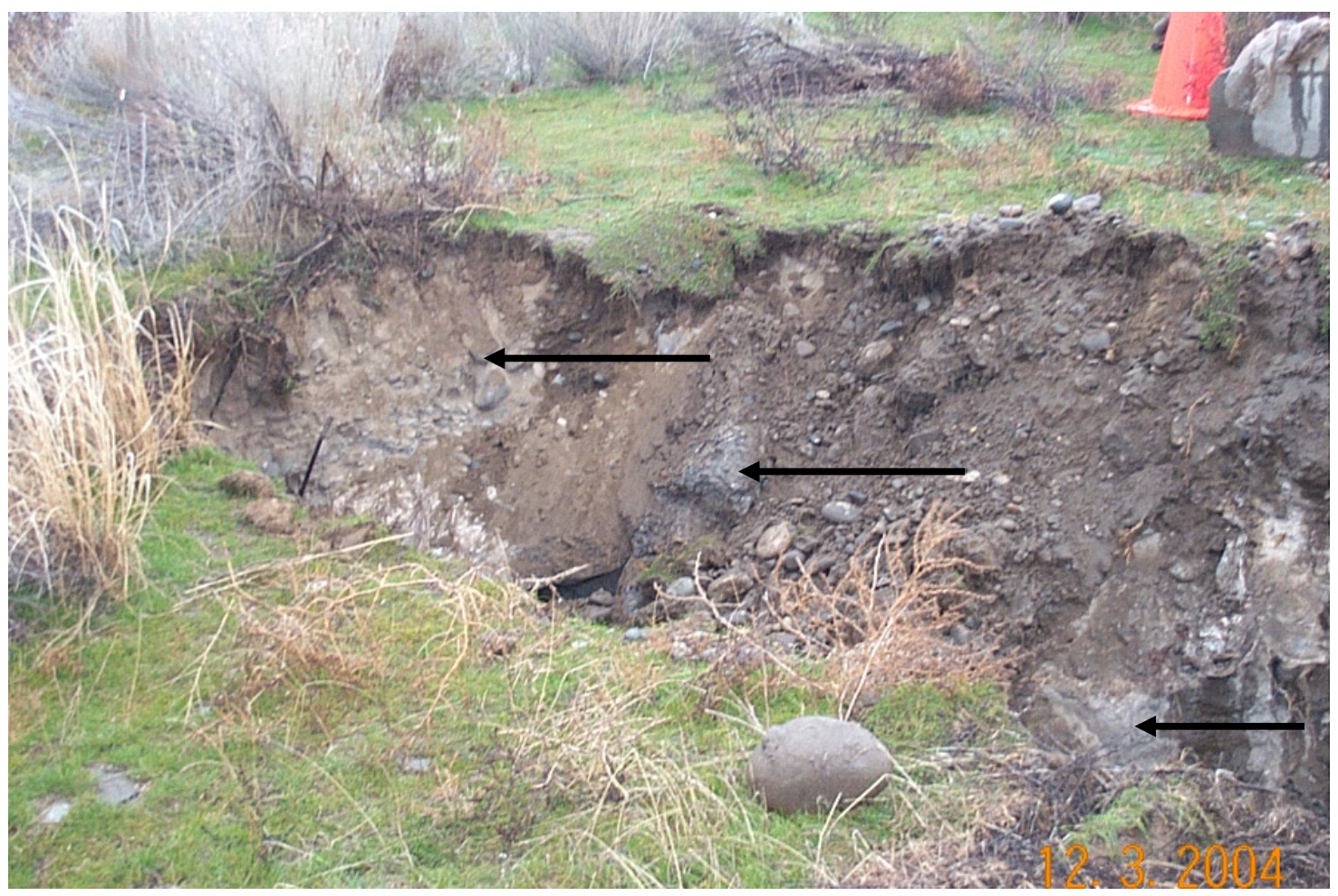

\section{Confirmatory Sampling Excavation in Trench 2 Showing Two Ash Layers.} Sample J024R7 was collected at the upper arrow and J024R8 was collected at the lower arrow.

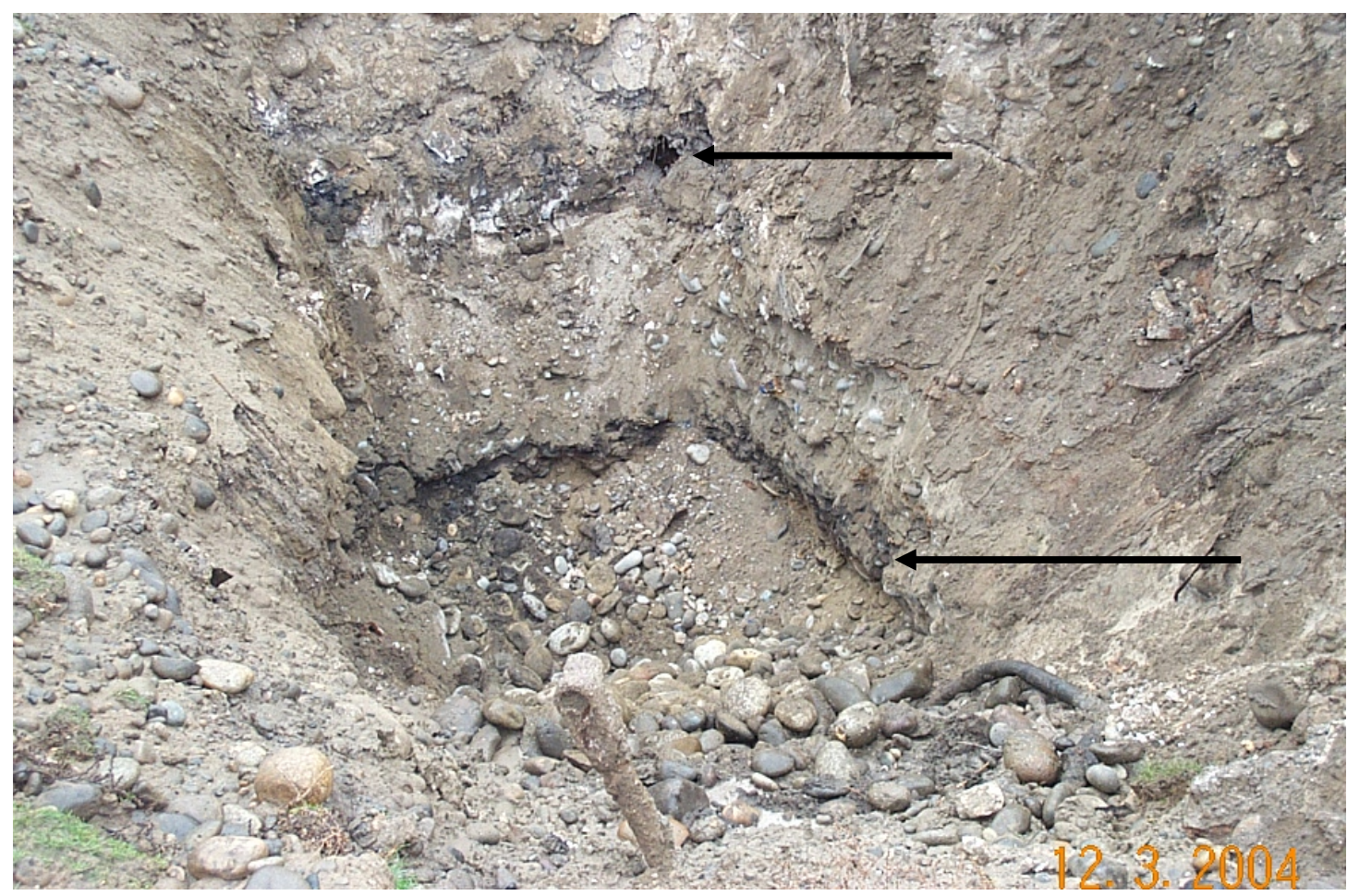




\section{Ballast Found in Trench 3 During Confirmatory Sampling.}

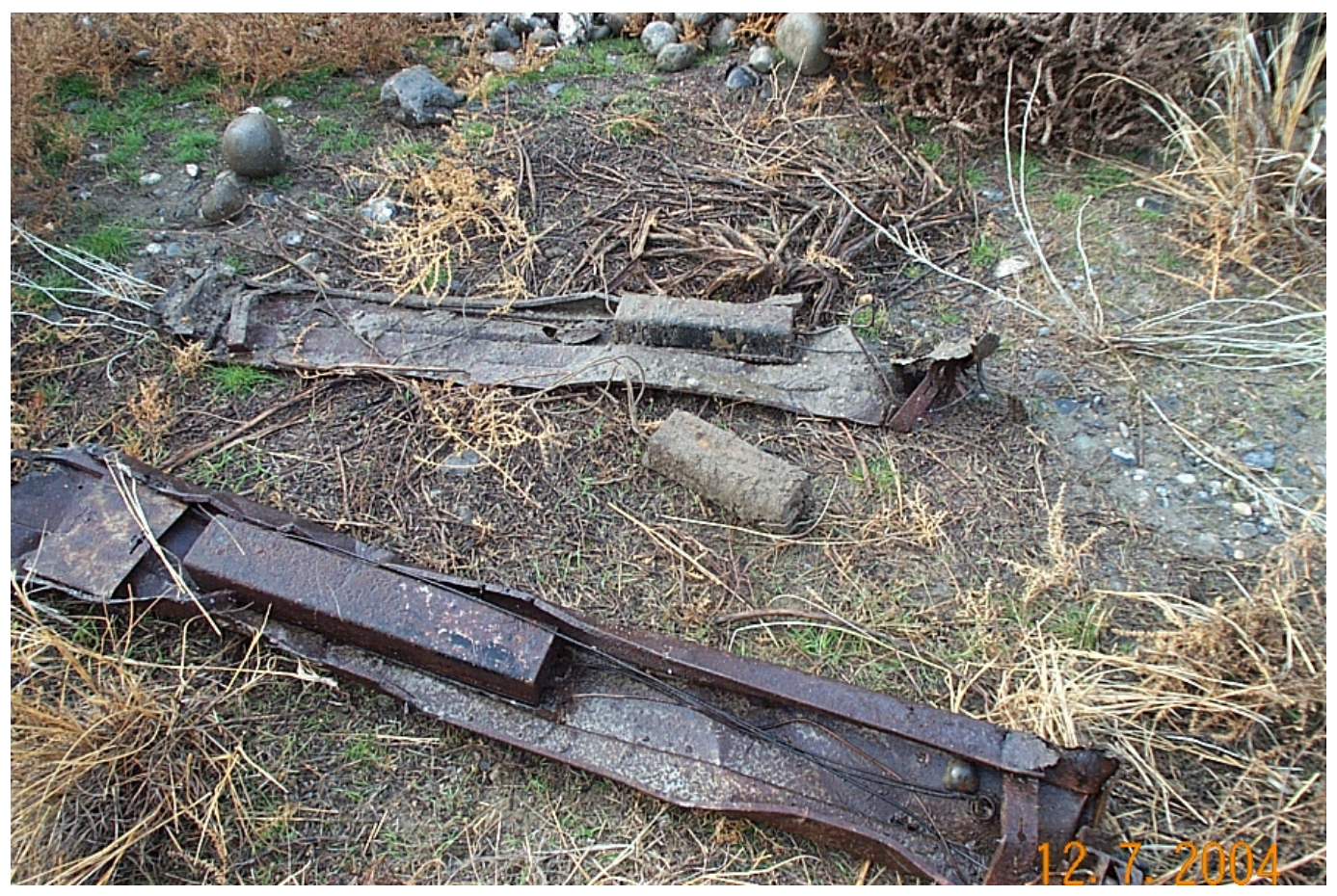

Photograph of Debris in Excavated Sidewall Prior to Phase II Excavation.

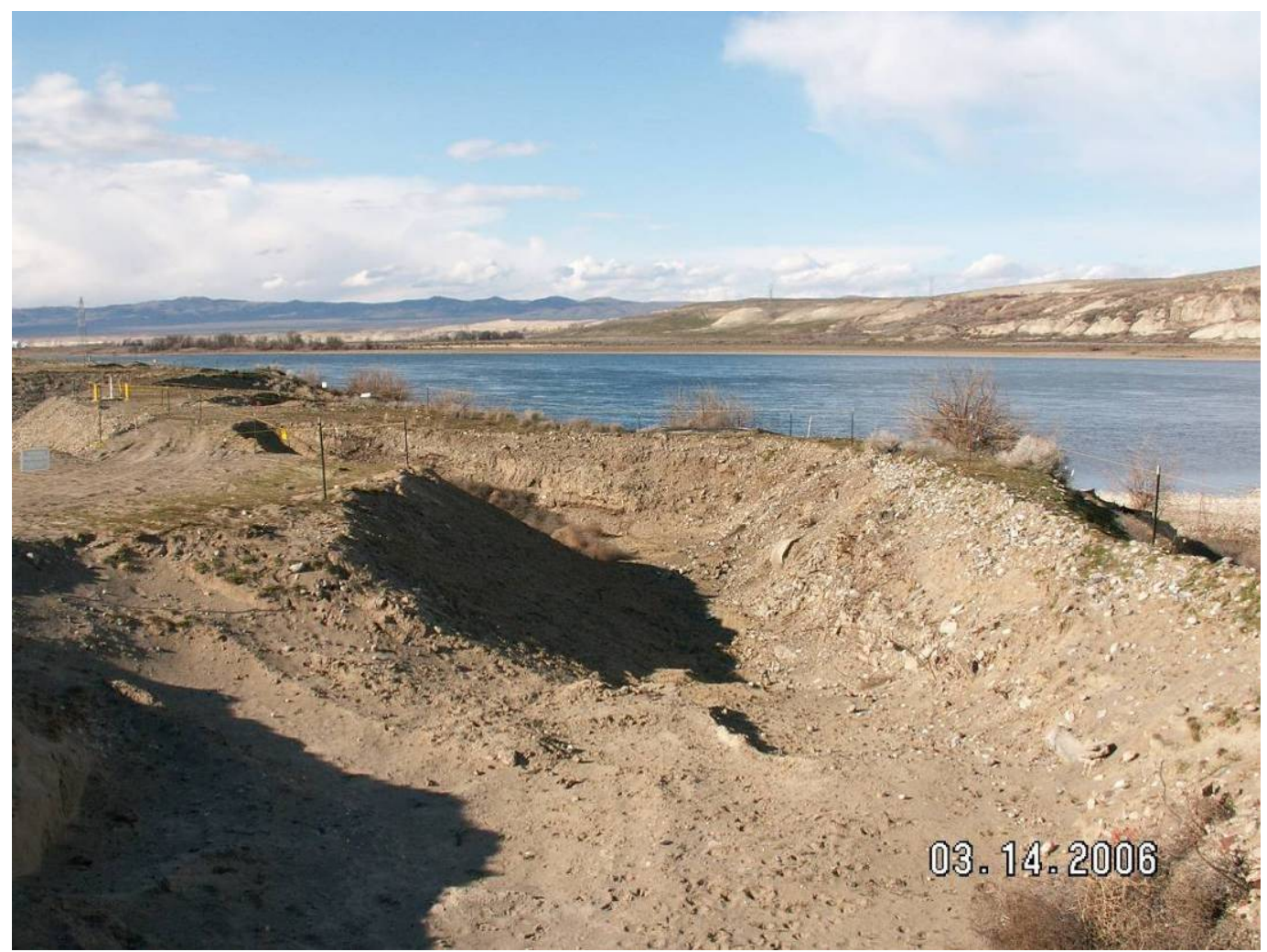


Photograph of Debris in Excavated Sidewall Prior to Phase II Excavation.

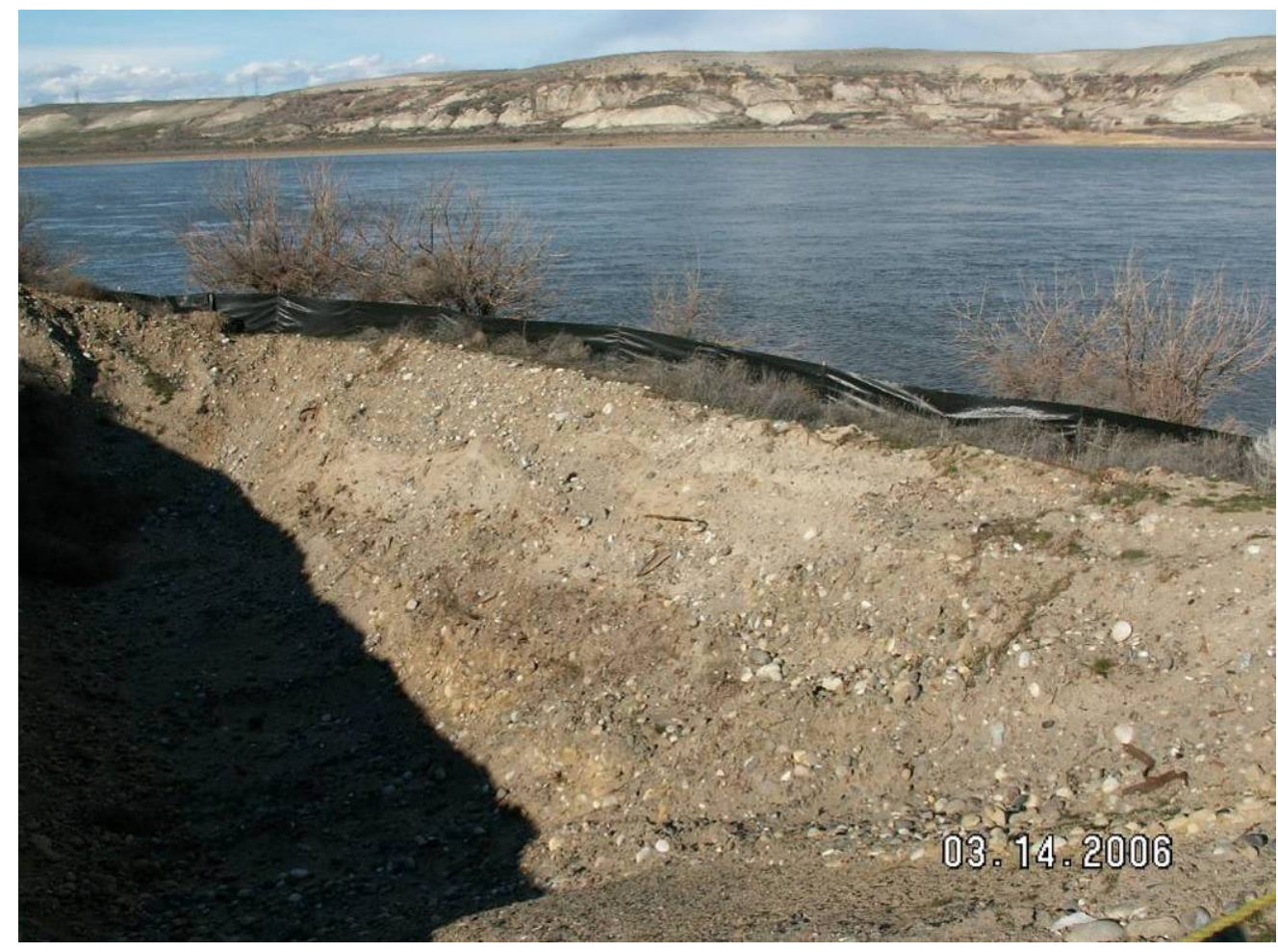

Photograph of Debris Sidewall Prior to Phase II Excavation. Left arrow points to concrete debris in sidewall, right arrow points to big tree that is present in additional photographs.

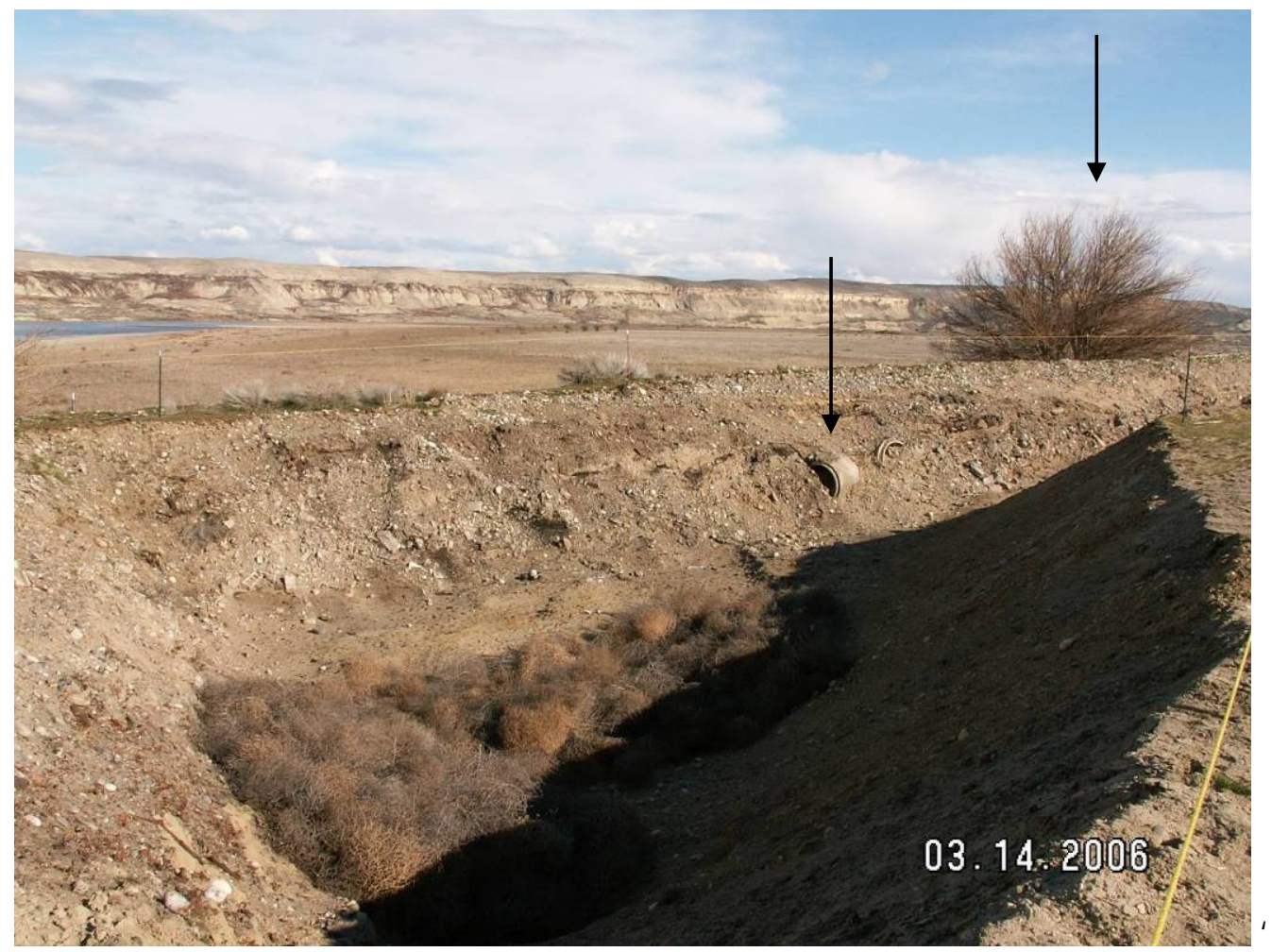


The 128-F-2 Site Shoreline at High Water Prior to Phase II Excavation. Arrow points to big tree.

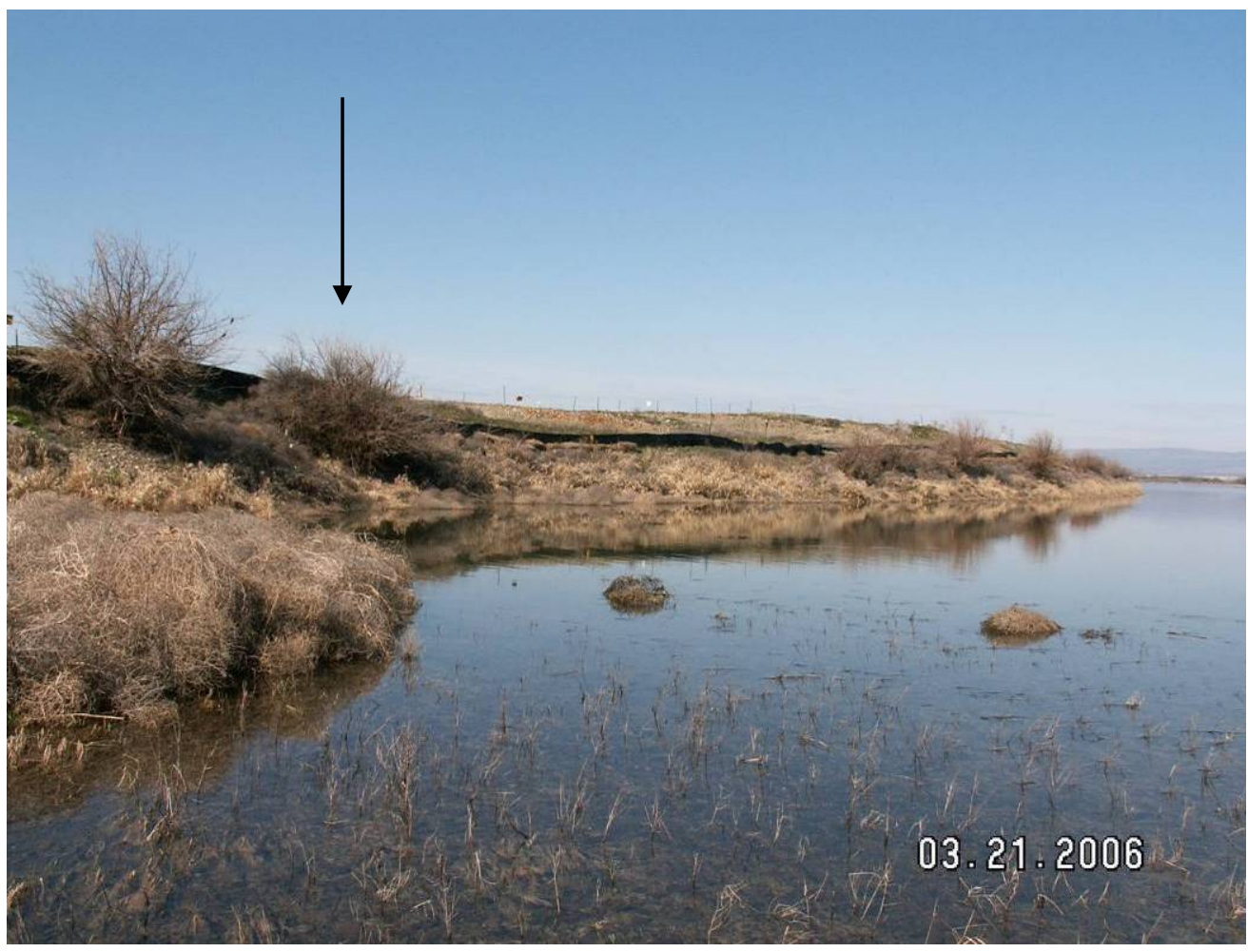

Riverbank Prior to Phase II Removal. Arrow points to big tree.

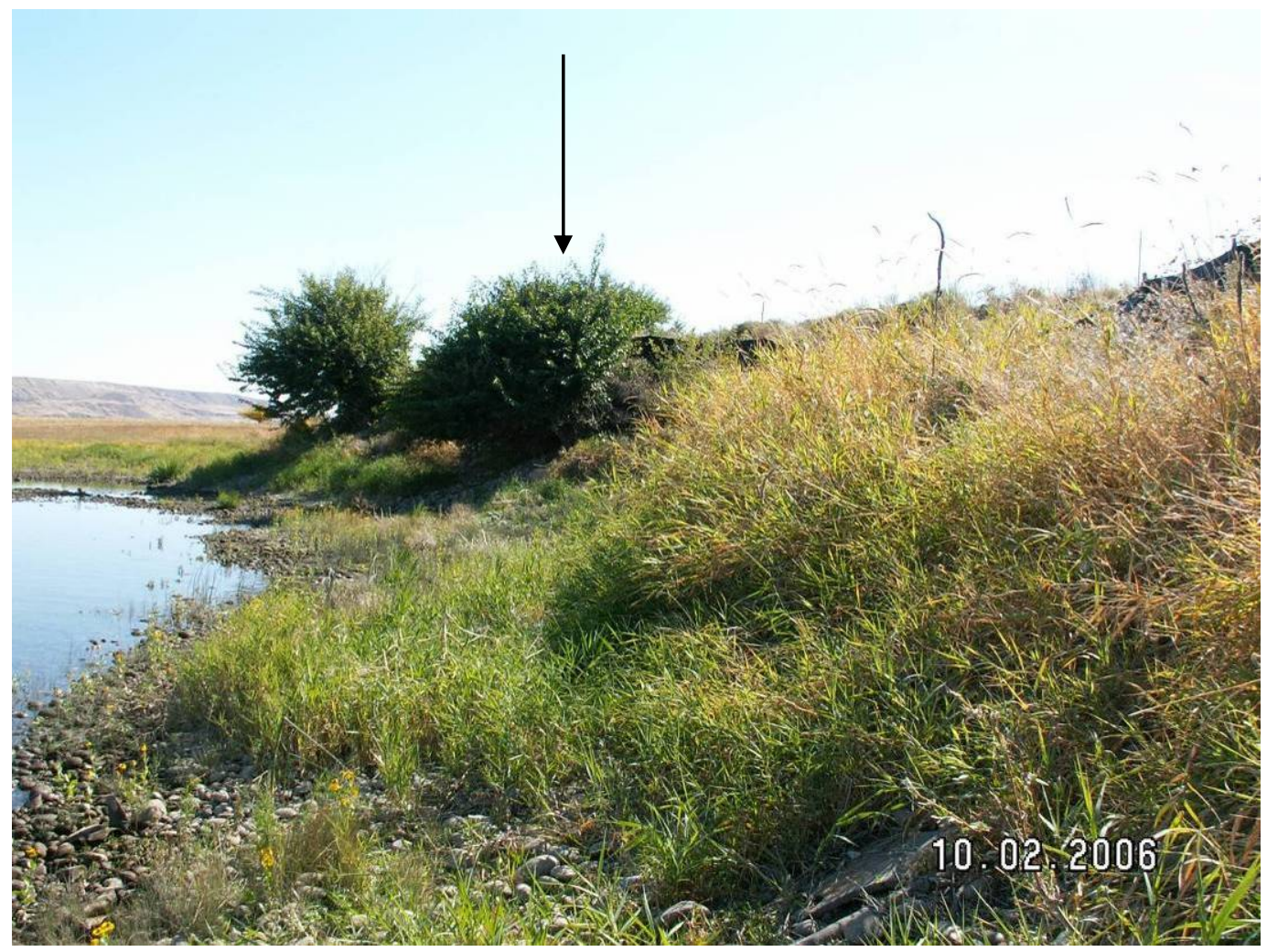


Upper Bank Prior to Phase II Excavation. Big tree is on right.

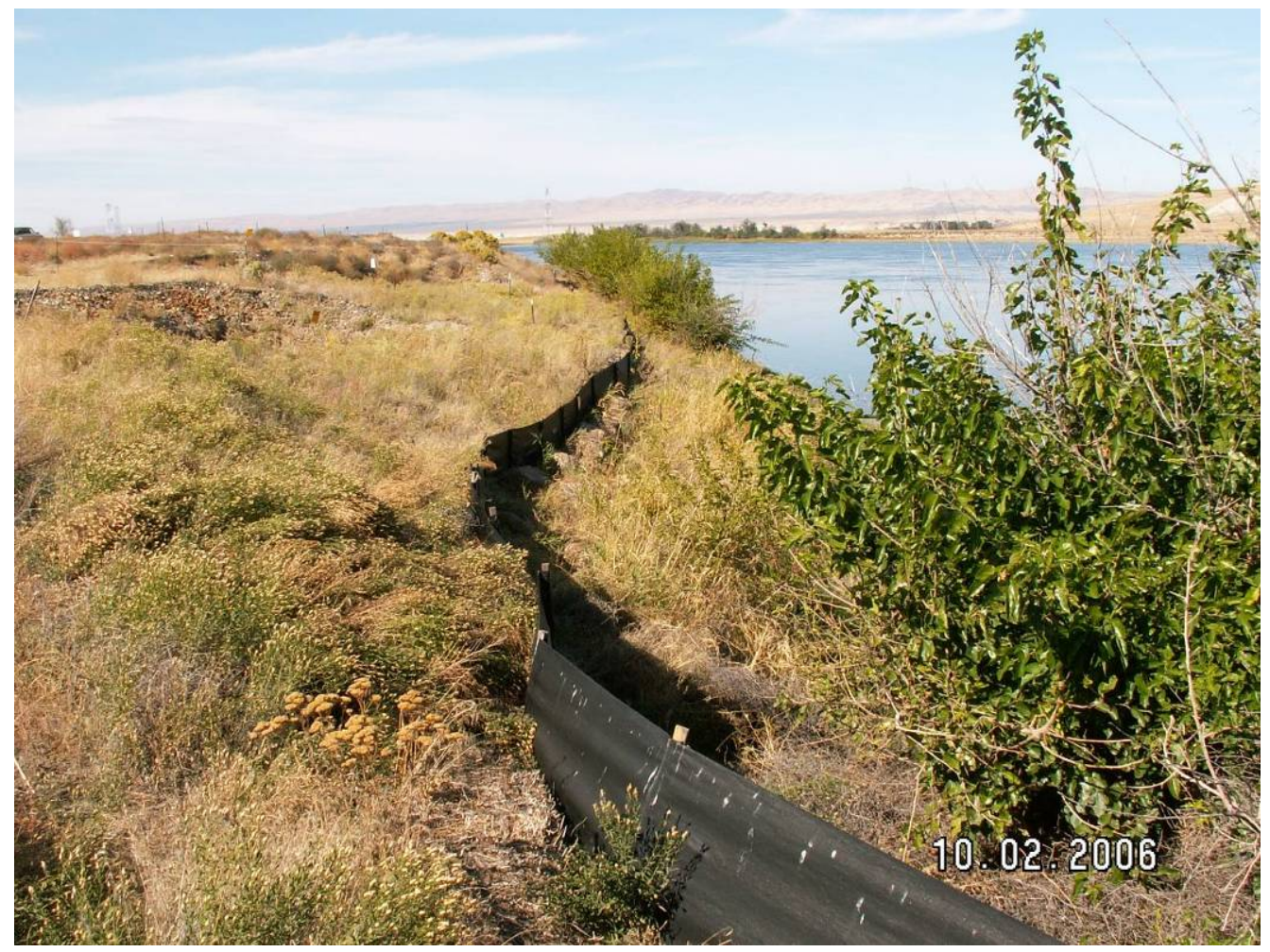

Silt Fence and Hay Bale Removal Prior to Phase II Remediation. Arrow points to big tree.

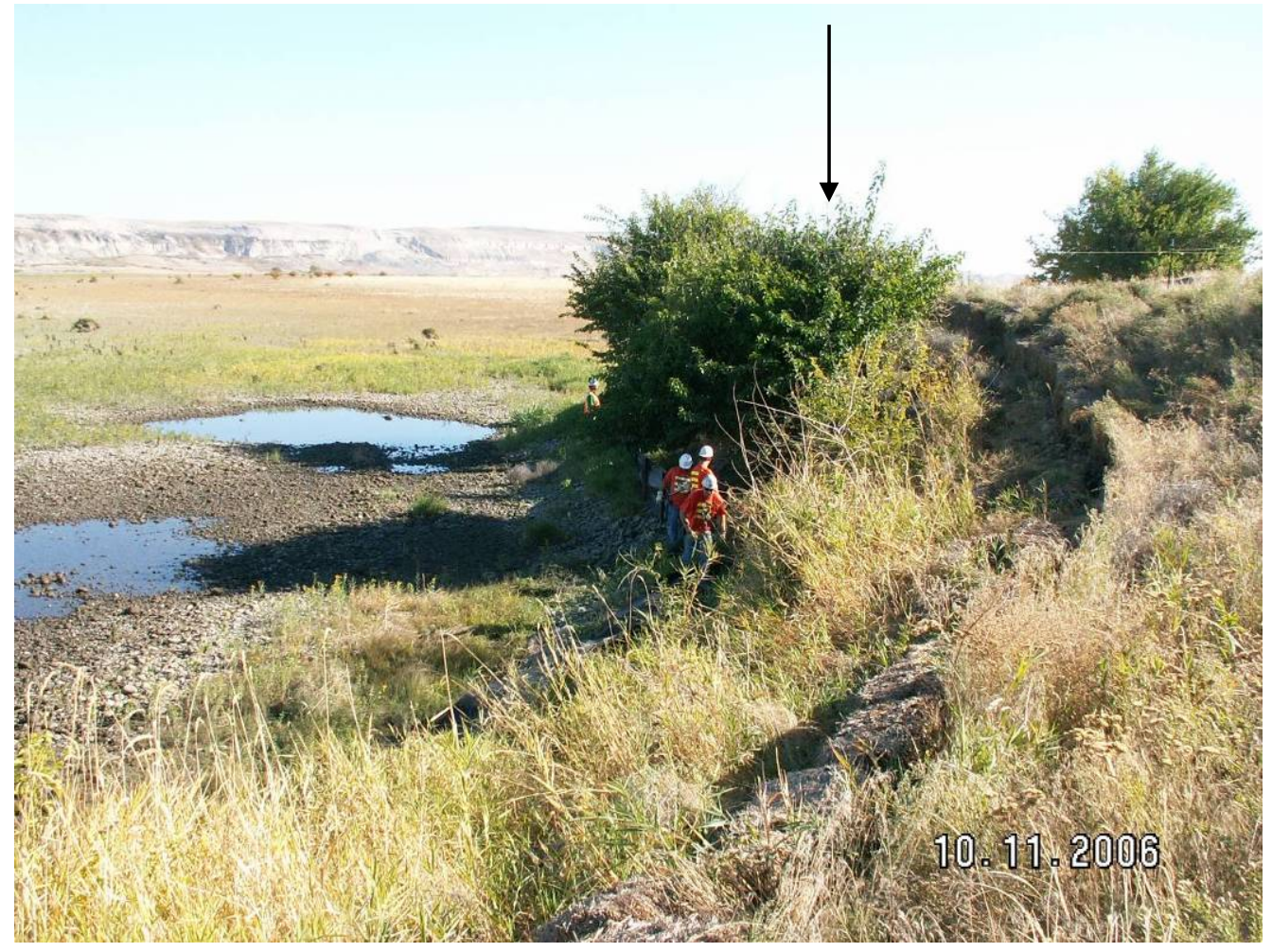




\section{Phase II Excavation at Low Water. Arrow points to soil berm with hay bales on top.}

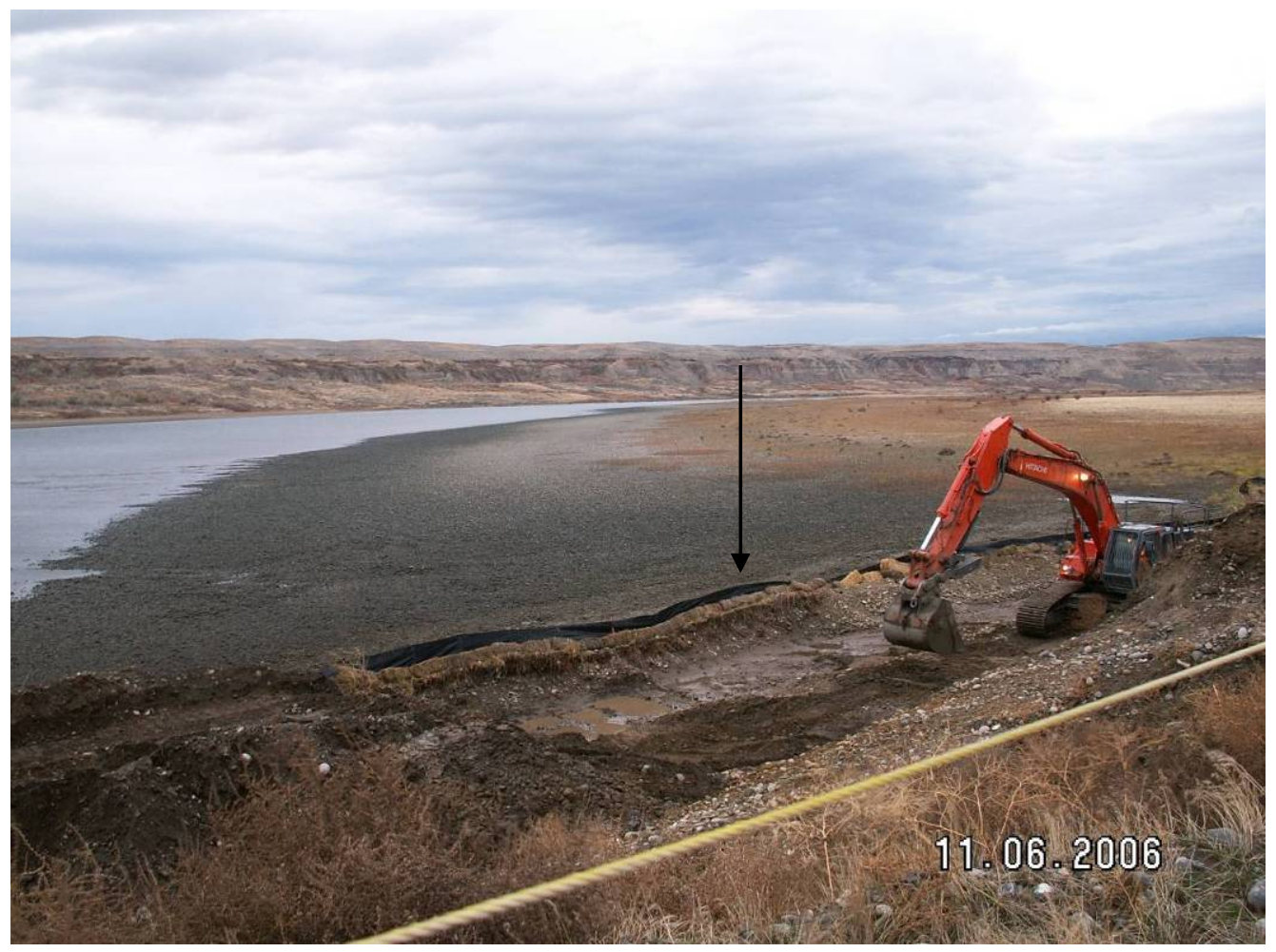

Area C Just Prior to Excavation Below the Ordinary High Water Mark (OHWM). Left arrow points to soil berm, right arrow points to big tree.

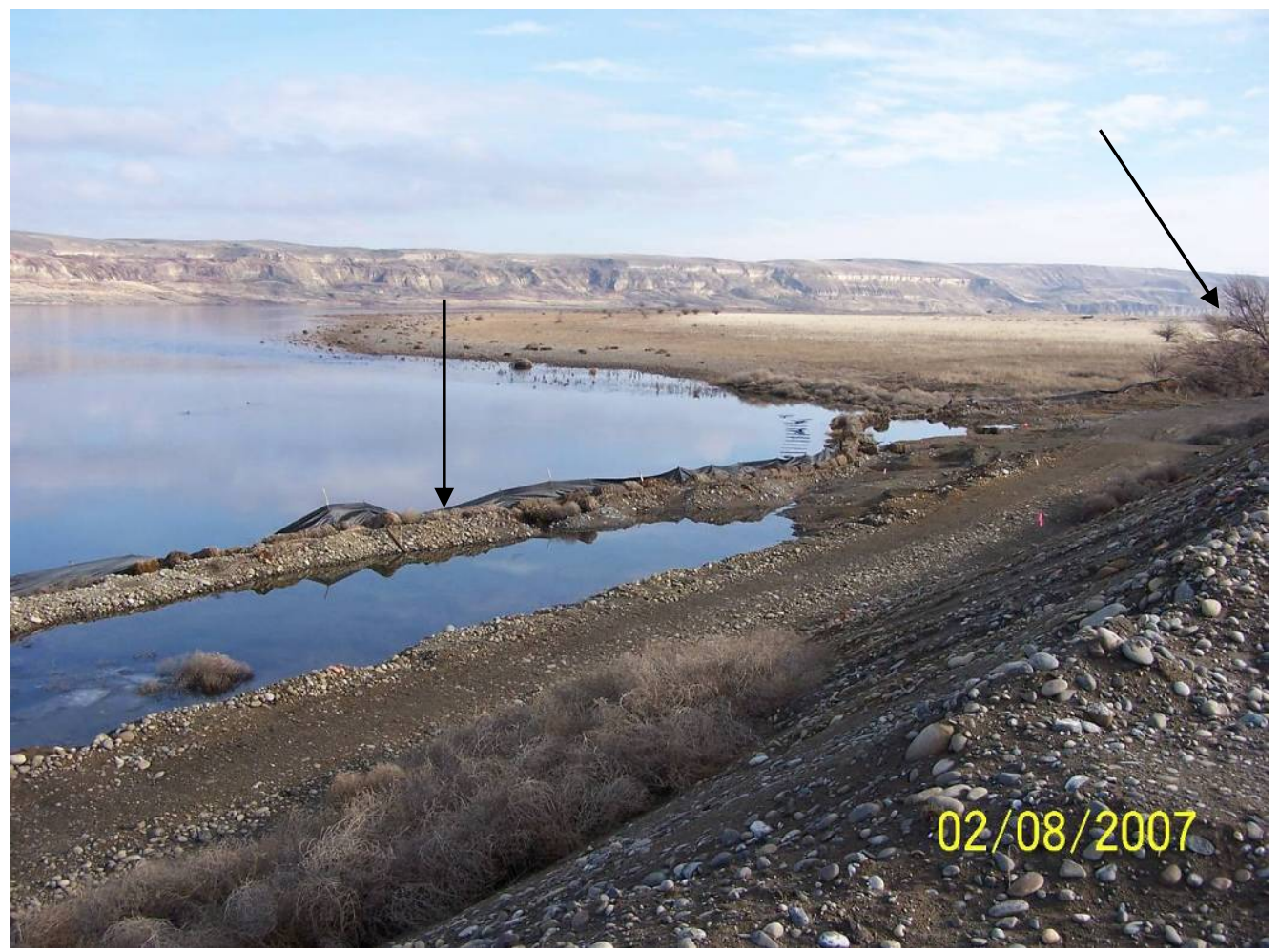


Area C Excavation Below the Ordinary High Water Mark. Arrow points to big tree.

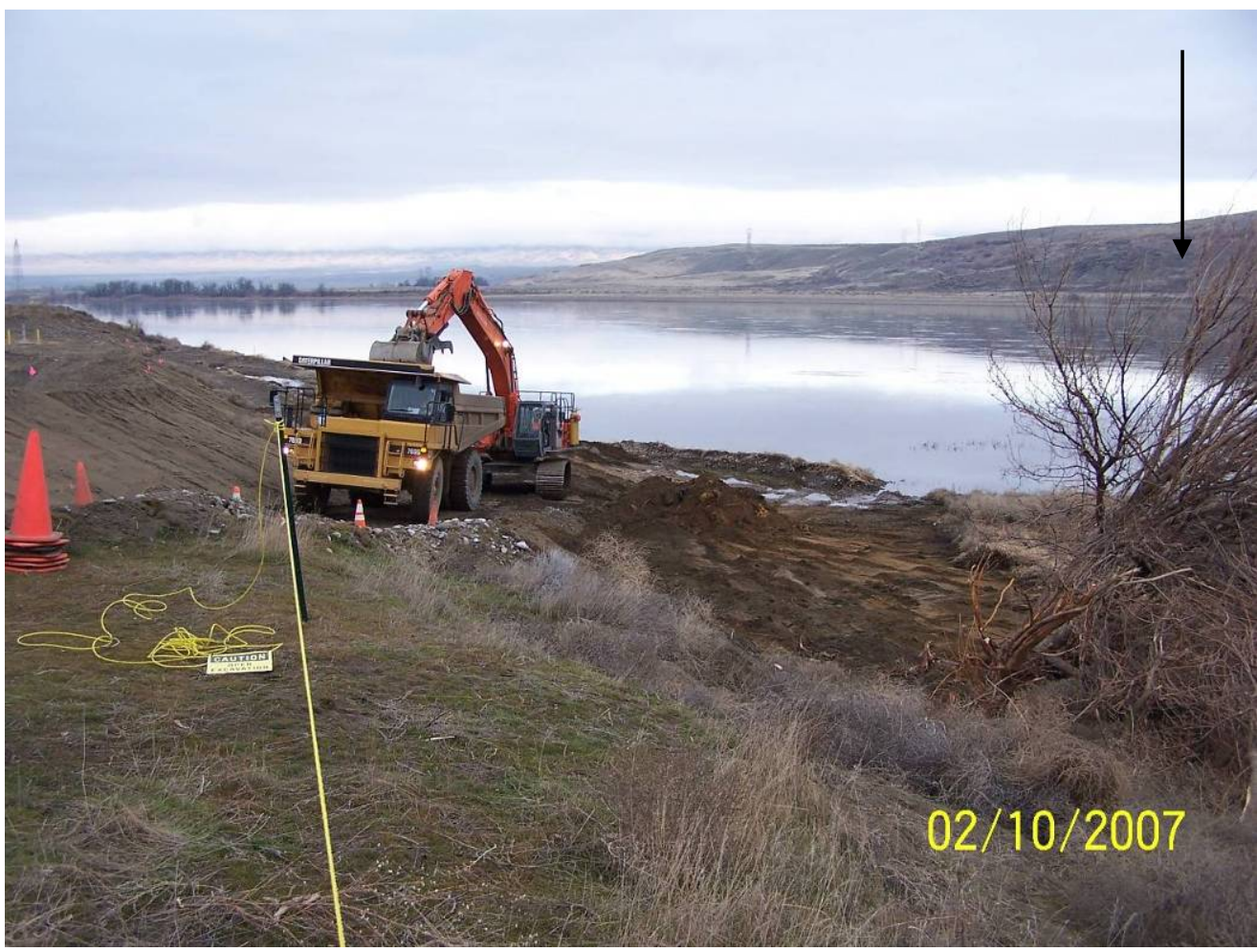

Area C Excavation Below the Ordinary High Water Mark.

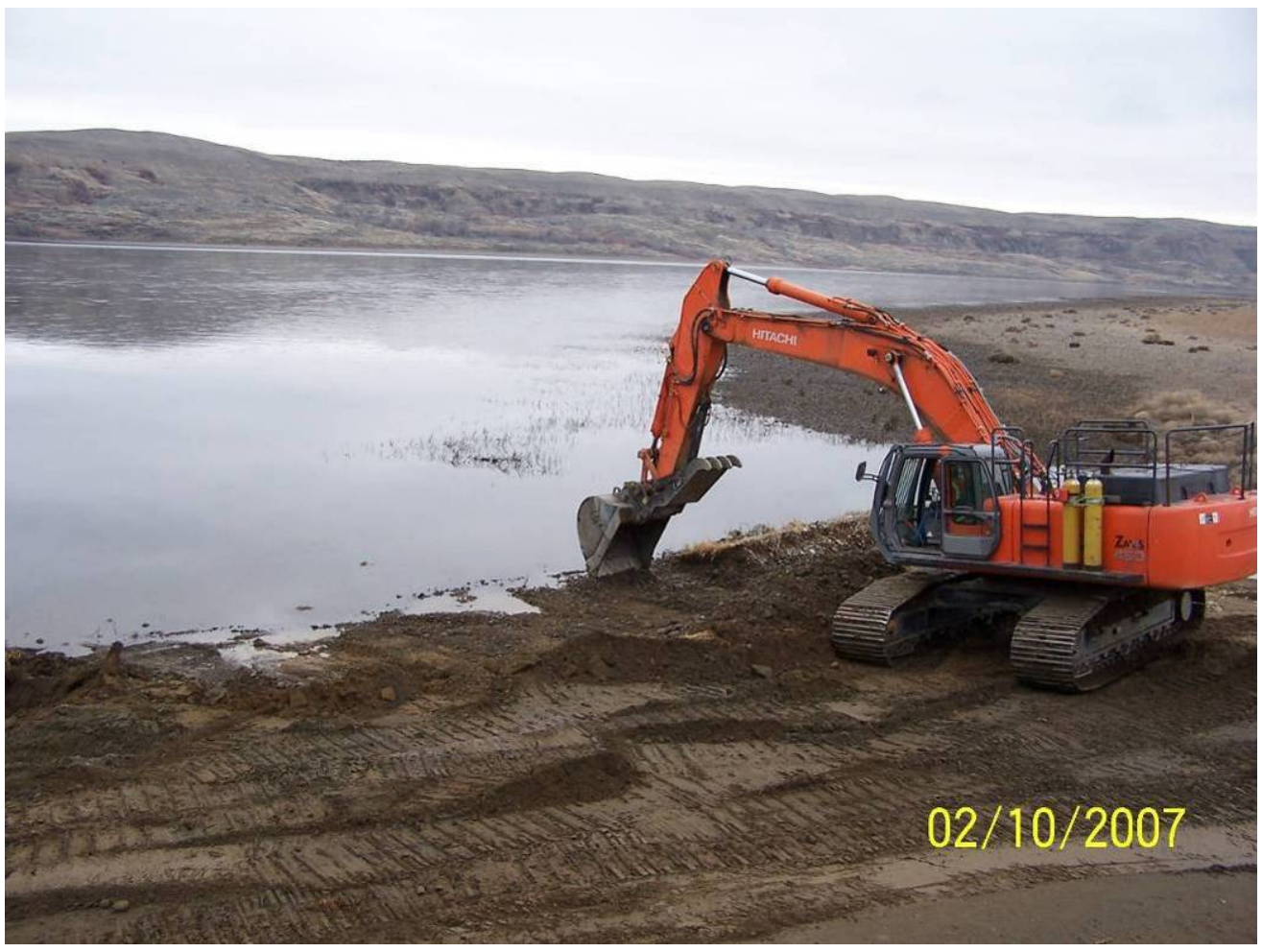




\section{Area C Excavation Below the Ordinary High Water Mark.}

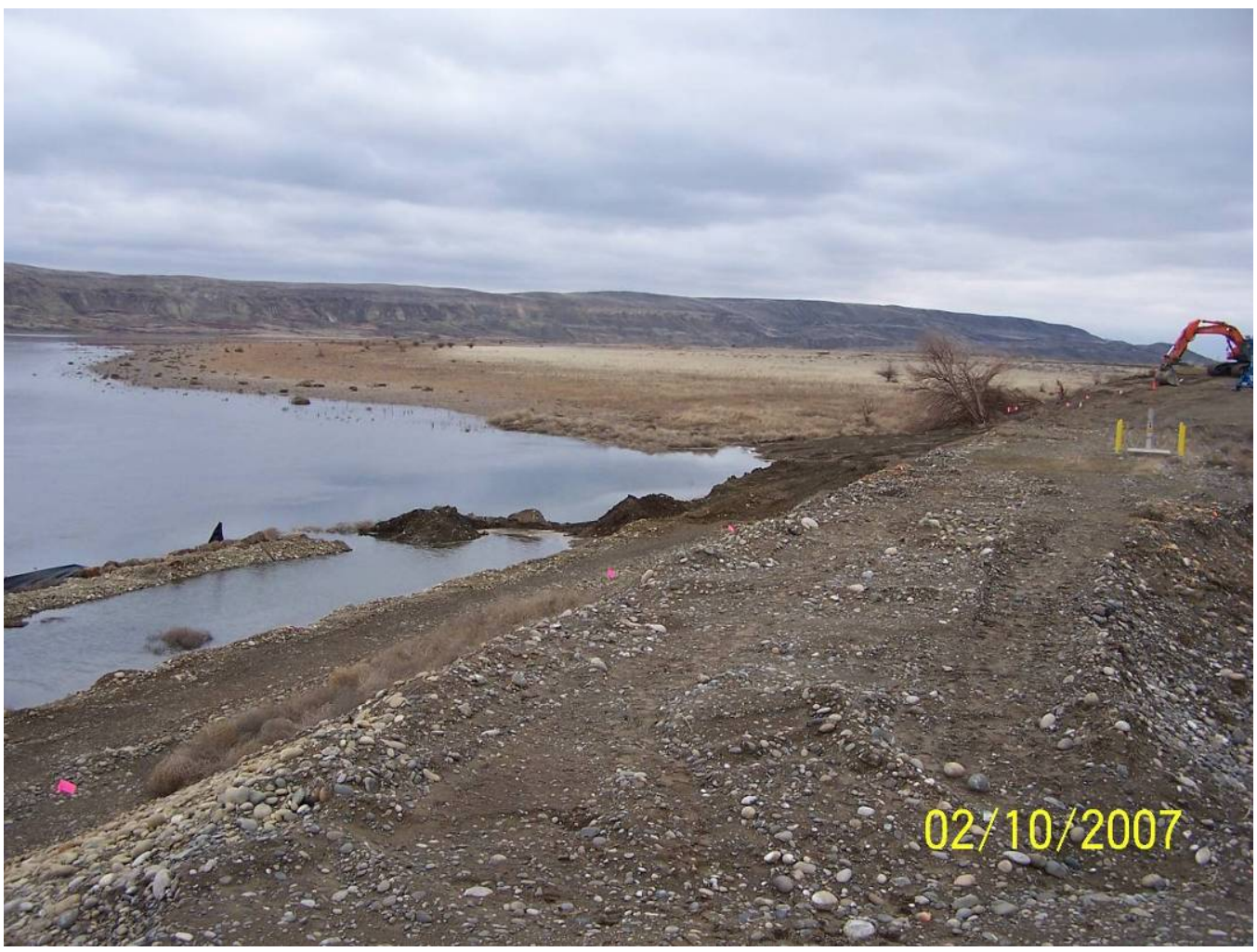

Area C Excavation Below the Ordinary High Water Mark.

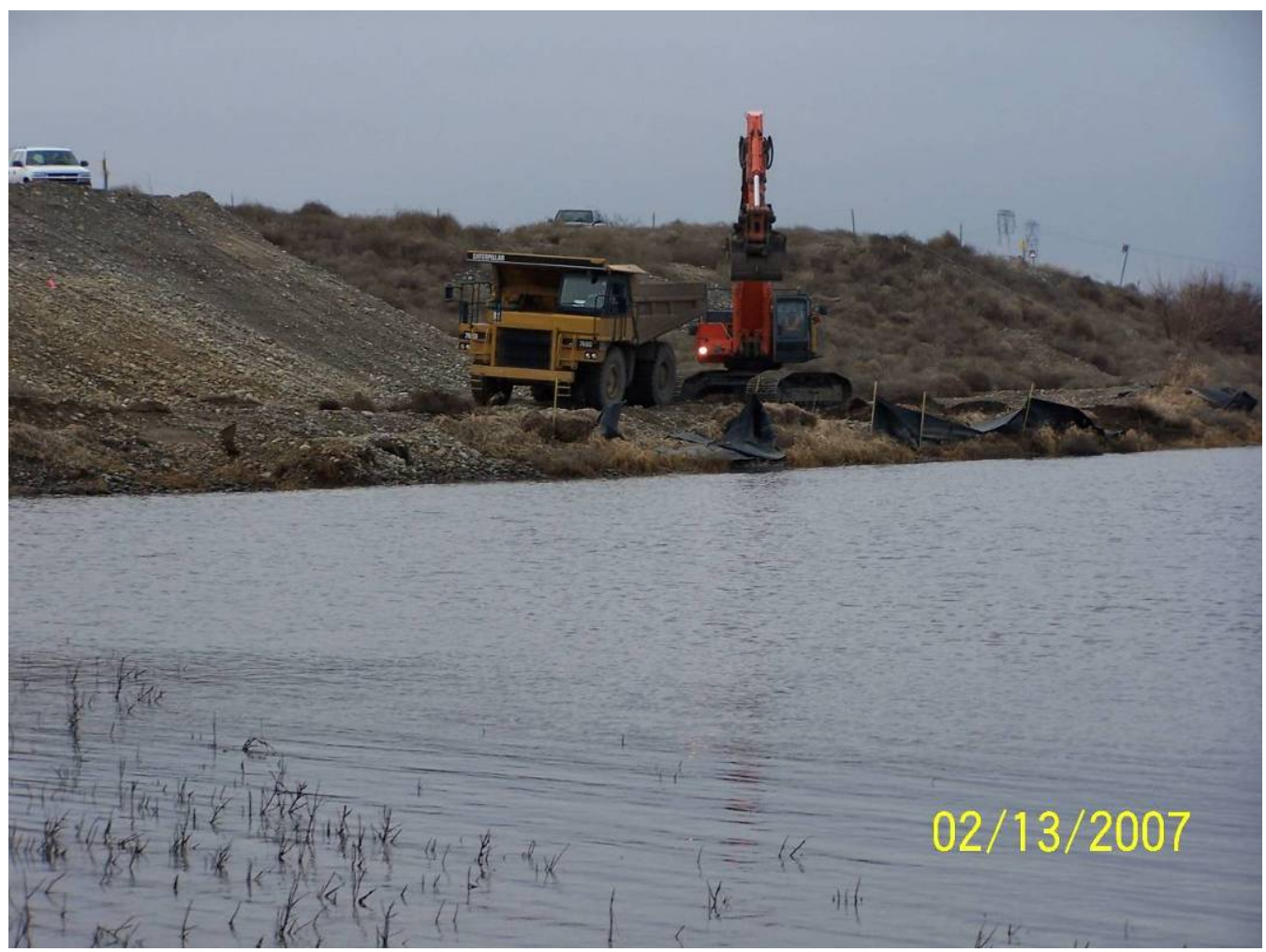




\section{Area C After Excavation Below the OHWM and Subsequent Gravel Stabilization. Arrow points to big tree.}

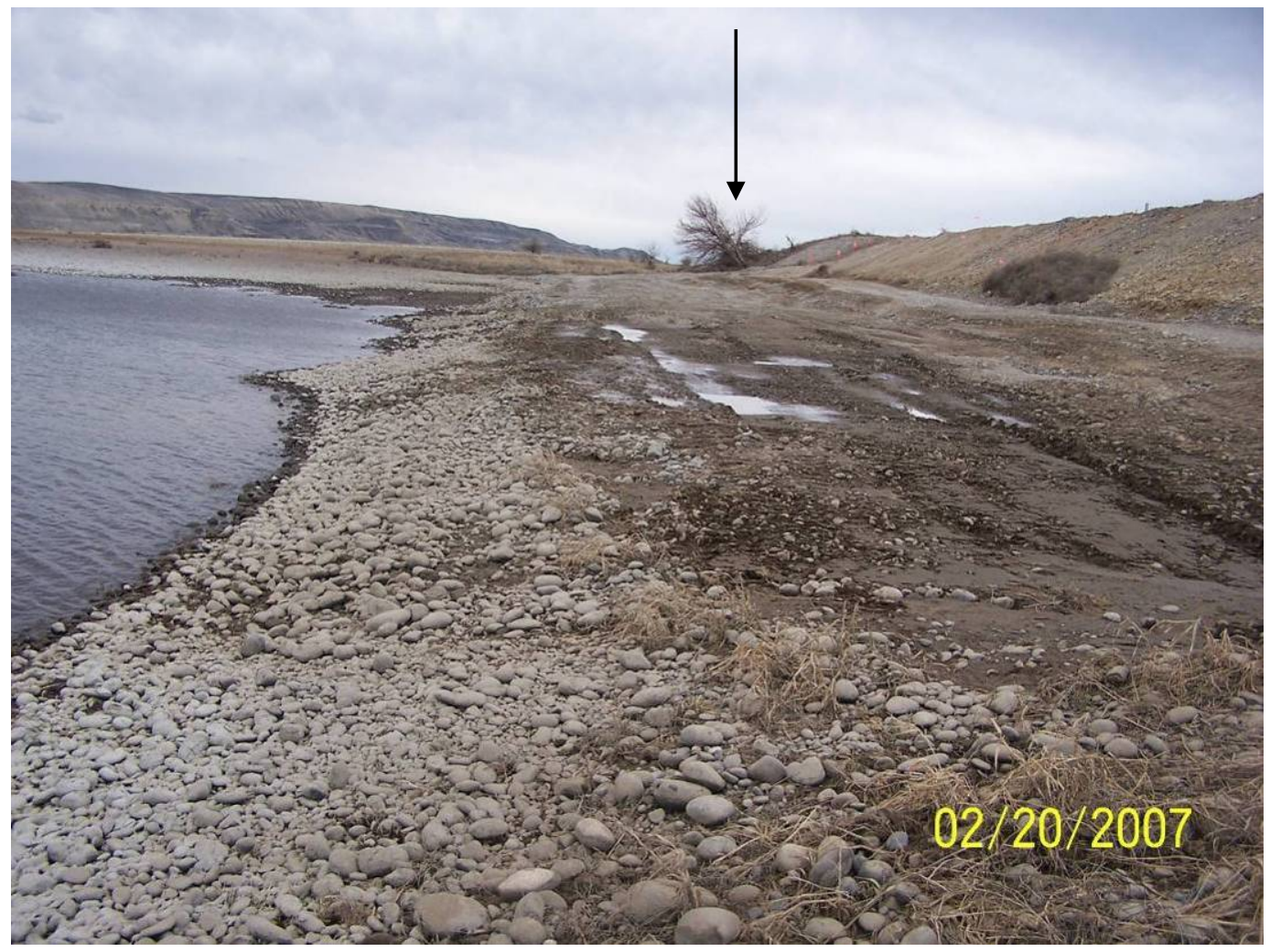




\section{APPENDIX C}

\section{SAMPLE DESIGN FOR HEXAVALENT CHROMIUM SAMPLES IN SAMPLE AREA B}




\section{SUMMARY}

This report summarizes the sampling design used, and associated statistical assumptions, as well as general guidelines for conducting post-sampling data analysis. Sampling plan components presented here include how many sampling locations to choose and where within the sampling area to collect those samples. The type of medium to sample (e.g., soil, groundwater) and how to analyze the samples (in situ, fixed laboratory, etc.) are addressed in other sections of the sampling plan.

Table C-1 summarizes the sampling design developed. Figure C-1 shows sampling locations in the field, and Table C-2 lists sampling location coordinates.

Table C-1. Summary of Sampling Design.

\begin{tabular}{|c|c|}
\hline Primary objective of design & Compare a site mean or median to a fixed threshold \\
\hline Type of sampling design & Nonparametric \\
\hline Sample placement (location) in the field & Systematic with a random start location \\
\hline Working (null) hypothesis & The median(mean) value at the site exceeds the threshold \\
\hline $\begin{array}{l}\text { Formula for calculating number of sampling } \\
\text { locations }\end{array}$ & Sign test - MARSSIM version \\
\hline Calculated total number of samples & 10 \\
\hline Number of samples on map ${ }^{\mathrm{a}}$ & 10 \\
\hline Number of selected sample areas ${ }^{b}$ & 1 \\
\hline 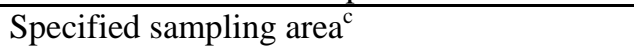 & $3008.90 \mathrm{~m}^{2}\left(32387.5 \mathrm{ft}^{2}\right)$ \\
\hline Size of grid / Area of grid cell ${ }^{\mathrm{d}}$ & $18.6 \mathrm{~m} / 300.9 \mathrm{~m}^{2}\left(61.1538 \mathrm{feet} / 3238.75 \mathrm{ft}^{2}\right)$ \\
\hline Grid pattern & Triangular \\
\hline \multicolumn{2}{|c|}{$\begin{array}{l}\text { This number may differ from the calculated number because of (1) grid edge effects, (2) adding judgment samples, or (3) } \\
\text { selecting or unselecting sample areas. } \\
\text { The number of selected sample areas is the number of colored areas on the map of the site. These sample areas contain the } \\
\text { locations where samples are collected. } \\
\text { The sampling area is the total surface area of the selected colored sample areas on the map of the site. } \\
\text { Size of grid/area of grid cell gives the linear and square dimensions of the grid used to systematically place samples. } \\
\text { Including measurement analyses and fixed overhead costs. See the Cost of Sampling section for an explanation of the costs } \\
\text { presented here. }\end{array}$} \\
\hline
\end{tabular}


Figure C-1. Sample Locations.

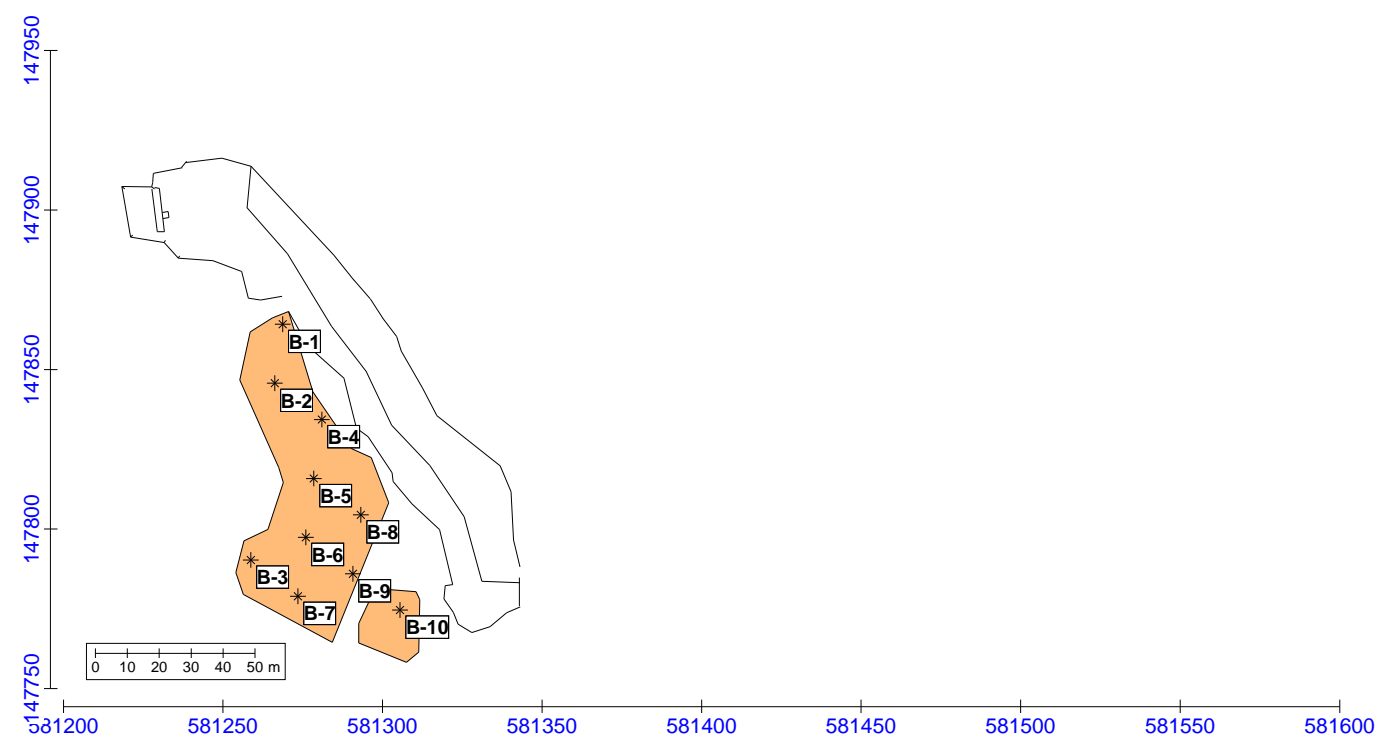

Table C-2. Area B Sampling Location Coordinates.

\begin{tabular}{|c|c|c|c|c|c|}
\hline X Coord & Y Coord & Label & Value & Type & Historical \\
\hline 581268.7291 & 147864.2044 & B-1 & & Systematic & \\
\hline 581266.2329 & 147845.7326 & B-2 & & Systematic & \\
\hline 581258.7443 & 147790.3172 & B-3 & & Systematic & \\
\hline 581280.9818 & 147834.3349 & B-4 & & Systematic & \\
\hline 581278.4856 & 147815.8631 & B-5 & & Systematic & \\
\hline 581275.9894 & 147797.3913 & B-6 & & Systematic & \\
\hline 581273.4933 & 147778.9196 & B-7 & & Systematic & \\
\hline 581293.2346 & 147804.4655 & B-8 & & Systematic & \\
\hline 581290.7384 & 147785.9937 & B-9 & & Systematic & \\
\hline 581305.4873 & 147774.5960 & B-10 & & Systematic & \\
\hline
\end{tabular}

\section{Primary Sampling Objective}

The primary purpose of sampling at this site is to compare a site median or mean value with a fixed threshold. The working hypothesis (or "null” hypothesis) is that the median (mean) value at the site is equal to or exceeds the threshold. The alternative hypothesis is that the median(mean) value is less than the threshold. Visual Sample $\operatorname{Plan}^{1}$ (VSP) calculates the number of samples required to reject the null hypothesis in favor of the alternative one, given a selected sampling approach and inputs to the associated equation.

\footnotetext{
${ }^{1}$ Visual Sample Plan is a site map-based user-interface program that may be downloaded at http://dqo.pnl.gov.
} 


\section{Selected Sampling Approach}

A nonparametric systematic sampling approach with a random start was used to determine the number of samples and to specify sampling locations. A nonparametric formula was chosen because the conceptual model and historical information (e.g., historical data from this site or a very similar site) indicate that typical parametric assumptions may not be true.

Both parametric and nonparametric equations rely on assumptions about the population. Typically, however, nonparametric equations require fewer assumptions and allow for more uncertainty about the statistical distribution of values at the site. The trade-off is that if the parametric assumptions are valid, the required number of samples is usually less than if a nonparametric equation was used.

Locating the sample points over a systematic grid with a random start ensures spatial coverage of the site. Statistical analyses of systematically collected data are valid if a random start to the grid is used. One disadvantage of systematically collected samples is that spatial variability or patterns may not be discovered if the grid spacing is large relative to the spatial patterns.

\section{Number of Total Samples: Calculation Equation and Inputs}

The equation used to calculate the number of samples is based on a Sign test (see Gilbert et al. 2001). For this site, the null hypothesis is rejected in favor of the alternative one if the median(mean) is sufficiently smaller than the threshold. The number of samples to collect is calculated so that if the inputs to the equation are true, the calculated number of samples will cause the null hypothesis to be rejected.

The formula used to calculate the number of samples is:

$$
n=\frac{\left(Z_{1-\alpha}+Z_{1-\beta}\right)^{2}}{4(\operatorname{Sign} P-0.5)^{2}}
$$

where

$$
\operatorname{Sign} P=\Phi\left(\frac{\Delta}{s_{\text {total }}}\right)
$$

$\Phi(\mathrm{z})$ is the cumulative standard normal distribution on $(-\infty, \mathrm{z})$ (see Gilbert et al. 2001 for details),

$n \quad$ is the number of samples,

$S_{\text {total }}$ is the estimated standard deviation of the measured values including analytical error,

$\Delta \quad$ is the width of the gray region, 
$\alpha \quad$ is the acceptable probability of incorrectly concluding the site median (mean) is less than the threshold,

$\beta \quad$ is the acceptable probability of incorrectly concluding the site median (mean) exceeds the threshold,

$Z_{1-\alpha} \quad$ is the value of the standard normal distribution such that the proportion of the distribution less than $Z_{1-\alpha}$ is $1-\alpha$,

$Z_{1-\beta} \quad$ is the value of the standard normal distribution such that the proportion of the distribution less than $Z_{1-\beta}$ is $1-\beta$.

Note: The Multi-Agency Radiation Survey and Site Investigation Manual (MARSSIM) (EPA et al. 2000) suggests that the number of samples should be increased by at least $20 \%$ to account for missing or unusable data and uncertainty in the calculated value of n. VSP allows a user-supplied percent overage as discussed in MARSSIM (EPA et al. 2000, p. 5-33).

The values of these inputs that result in the calculated number of sampling locations are summarized in Table C-3.

Table C-3. VSP User Inputs.

\begin{tabular}{|c|c|c|c|c|c|c|c|}
\hline \multirow{2}{*}{ Analyte } & \multirow{2}{*}{$\mathbf{n}^{\mathbf{a}}$} & \multicolumn{7}{|c|}{ Parameter } \\
\cline { 3 - 8 } & & $\boldsymbol{S}$ & $\Delta$ & $\alpha$ & $\beta$ & $\boldsymbol{Z}_{1-\alpha}{ }^{\mathrm{b}}$ & $\boldsymbol{Z}_{1-\beta}{ }^{\mathrm{c}}$ \\
\hline & 10 & 0.25 & 0.4 & 0.05 & 0.2 & 1.64485 & 0.841621 \\
\hline
\end{tabular}

a The final number of samples has been increased by the MARSSIM Overage of $20 \%$.

b This value is automatically calculated by VSP based upon the user defined value of $\alpha$.

c This value is automatically calculated by VSP based upon the user defined value of $\beta$.

Figure C-2 is a performance goal diagram, described in the U.S. Environmental Protection Agency's (EPA's) QA/G-4 guidance (EPA 2000b). It shows the probability of concluding the sample area is dirty on the vertical axis versus a range of possible true median (mean) values for the site on the horizontal axis. This graph contains all of the inputs to the number of samples equation and pictorially represents the calculation.

The red vertical line is shown at the threshold (action limit) on the horizontal axis. The width of the gray shaded area is equal to $\Delta$; the upper horizontal dashed blue line is positioned at $1-\alpha$ on the vertical axis; the lower horizontal dashed blue line is positioned at $\beta$ on the vertical axis. The vertical green line is positioned at one standard deviation below the threshold. The shape of the red curve corresponds to the estimates of variability. The calculated number of samples results in the curve that passes through the lower bound of $\Delta$ at $\beta$ and the upper bound of $\Delta$ at 1- $\alpha$. If any of the inputs change, the number of samples that result in the correct curve changes.

\section{Statistical Assumptions}

The assumptions associated with the formulas for computing the number of samples are:

1. The computed sign test statistic is normally distributed. 
2. The variance estimate, $S^{2}$, is reasonable and representative of the population being sampled.

3. The population values are not spatially or temporally correlated.

4. The sampling locations will be selected probabilistically.

The first three assumptions will be assessed in a post data collection analysis. The last assumption is valid because the gridded sample locations were selected based on a random start.

Figure C-2. Performance Goal Diagram.

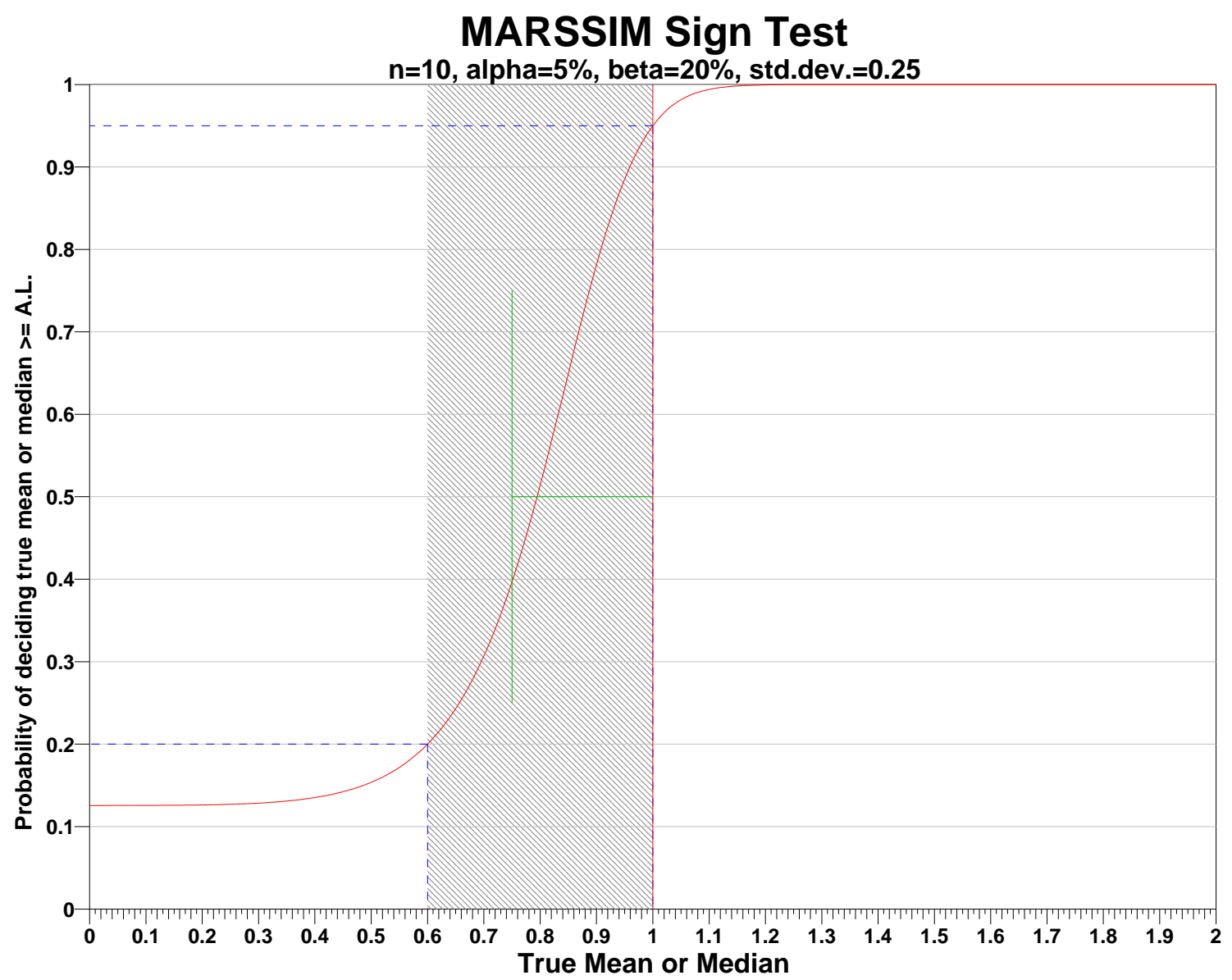

\section{Sensitivity Analysis}

The sensitivity of the calculation of number of samples was explored by varying the standard deviation, lower bound of gray region (\% of action level), beta (\%), probability of mistakenly concluding that $\mu>$ action level and alpha (\%), probability of mistakenly concluding that $\mu<$ action level and examining the resulting changes in the number of samples. Table C-4 shows the results of this analysis. 
Table C-4. Sensitivity Analysis. Number of Samples.

\begin{tabular}{|c|c|c|c|c|c|c|c|}
\hline \multirow{2}{*}{\multicolumn{2}{|c|}{$A L=1$}} & \multicolumn{2}{|c|}{$\alpha=5$} & \multicolumn{2}{|c|}{$\alpha=10$} & \multicolumn{2}{|c|}{$\alpha=15$} \\
\hline & & $s=0.5$ & $s=0.25$ & $s=0.5$ & $s=0.25$ & $s=0.5$ & $s=0.25$ \\
\hline \multirow{3}{*}{ LBGR=90 } & $\beta=15$ & 345 & 90 & 257 & 68 & 206 & 54 \\
\hline & $\beta=20$ & 297 & 77 & 216 & 57 & 170 & 45 \\
\hline & $\beta=25$ & 258 & 68 & 184 & 48 & 141 & 38 \\
\hline \multirow{3}{*}{ LBGR $=80$} & $\beta=15$ & 90 & 27 & 68 & 21 & 54 & 16 \\
\hline & $\beta=20$ & 77 & 23 & 57 & 17 & 45 & 14 \\
\hline & $\beta=25$ & 68 & 21 & 48 & 15 & 38 & 11 \\
\hline \multirow{3}{*}{ LBGR=70 } & $\beta=15$ & 44 & 16 & 33 & 12 & 27 & 10 \\
\hline & $\beta=20$ & 38 & 14 & 28 & 10 & 22 & 8 \\
\hline & $\beta=25$ & 33 & 12 & 23 & 9 & 18 & 6 \\
\hline
\end{tabular}

$\mathrm{s}=$ Standard Deviation

LBGR= Lower Bound of Gray Region (\% of Action Level)

$\beta=$ Beta (\%), Probability of mistakenly concluding that $\mu>$ action level

$\alpha=$ Alpha (\%), Probability of mistakenly concluding that $\mu<$ action level

$\mathrm{AL}=$ Action Level (Threshold)

\section{Recommended Data Analysis Activities}

Post data collection activities generally follow those outlined in EPA's Guidance for Data Quality Assessment (EPA 2000a). The data analysts will become familiar with the context of the problem and goals for data collection and assessment. The data will be verified and validated before being subjected to statistical or other analyses. Graphical and analytical tools will be used to verify to the extent possible the assumptions of any statistical analyses that are performed as well as to achieve a general understanding of the data. The data will be assessed to determine whether they are adequate in both quality and quantity to support the primary objective of sampling.

Because the primary objective for sampling for this site is to compare the site median (mean) value with a threshold value, the data will be assessed in this context. Assuming the data are adequate, at least one statistical test will be done to perform a comparison between the data and the threshold of interest. Results of the exploratory and quantitative assessments of the data will be reported, along with conclusions that may be supported by them.

\section{REFERENCES}

EPA, 2000a, Guidance for Data Quality Assessment: Practical Methods for Data Analysis, EPA/600/R-96/084, U.S. Environmental Protection Agency, Washington, D.C.

EPA, 2000b, Guidance for Data Quality Objectives Process, EPA QA/G-4, EPA/600/R-96/055, U.S. Environmental Protection Agency, Washington, D.C.

EPA, DOD, DOE, and NRC, 2000, Multi-Agency Radiation Survey and Site Investigation Manual (MARSSIM), NUREG-1575, Rev. 1, EPA 402-R-97-016, Rev. 1, DOE/EH-0624, 
Rev.1, U.S. Department of Energy, and U.S. Nuclear Regulatory Commission, Washington, D.C.

Gilbert R.O., J.R. Davidson, J.E. Wilson, and B.A. Pulsipher, 2001, Visual Sample Plan (VSP) Models and Code Verification, PNNL-13450, Pacific Northwest National Laboratory, Richland, Washington. 
APPENDIX D

CALCULATIONS 


\section{APPENDIX D CALCULATION BRIEFS}

The calculations in this appendix are kept in the active Washington Closure Hanford project files and are available upon request. When the project is completed, the files will be stored in a U.S. Department of Energy, Richland Operations Office, repository. These calculations have been prepared in accordance with ENG-1, Engineering Services, ENG-1-4.5, "Project Calculation,” Washington Closure Hanford, Richland, Washington. The following calculations are provided in this appendix:

128-F-2, Area A Cleanup Verification 95\% UCL Calculation, 0100F-CA-V0359, Rev. 0, Washington Closure Hanford, Richland, Washington.

128-F-2, Upland (Area B) Cleanup Verification 95\% UCL, 0100F-CA-V0333, Rev. 0, Washington Closure Hanford, Richland, Washington.

128-F-2, Area A Waste Site Cleanup Verification Hazard Quotient and Carcinogenic Risk Calculation, 0100F-CA-V0360, Rev. 0, Washington Closure Hanford, Richland, Washington.

128-F-2, Upland (Area B) Waste Site Cleanup Verification Hazard Quotient and Carcinogenic Risk Calculation, 0100F-CA-V0334, Rev. 1, Washington Closure Hanford, Richland, Washington.

128-F-2, Staging Pile Waste Site Cleanup Verification Hazard Quotient and Carcinogenic Risk Calculation, 0100F-CA-V0361, Rev. 0, Washington Closure Hanford, Richland, Washington.

\section{DISCLAIMER FOR CALCULATIONS}

The calculations provided in this appendix have been generated to document compliance with established cleanup levels. These calculations should be used in conjunction with other relevant documents in the administrative record. 


\section{CALCULATION COVER SHEET}

Project Title: 100-F Field Remediation

Area: $100-\mathrm{F}$

Discipline: Environmental

${ }^{*}$ Calculation No: 0100F-CA-V0359

Subject: 128-F-2, Area A Cleanup Verification 95\% UCL Calculation

Computer Program: Excel

Program No: Excel 2003

The attached calculations have been generated to document compliance with established cleanup levels. These calculations should be used in conjunction with other relevant documents in the administrative record.

Committed Calculation $\bar{x} \quad$ Preliminary $\Gamma \quad$ Superseded $\Gamma \quad$ Voided $\Gamma$

\begin{tabular}{|c|c|c|c|c|c|c|}
\hline Rev. & Sheet Numbers & Originator & Checker & Reviewer & Approval & Date \\
\hline 0 & $\begin{array}{c}\text { Cover }=1 \\
\text { Sheets }=9 \\
\text { Attm. } 1=18\end{array}$ & h.s.s. s & D. $y^{2}$ & NA & J. D. Fancher & \\
\hline & & & & & 7 & \\
\hline & & & & & & \\
\hline & & & & & & \\
\hline & & & & & & \\
\hline & & & & & & \\
\hline & & & & & & \\
\hline
\end{tabular}

SUMMARY OF REVISION

\begin{tabular}{|l|l|}
\hline & \multicolumn{1}{|c|}{$\mid \mathbf{}$} \\
\hline & \\
\hline & \\
\hline & \\
\hline
\end{tabular}




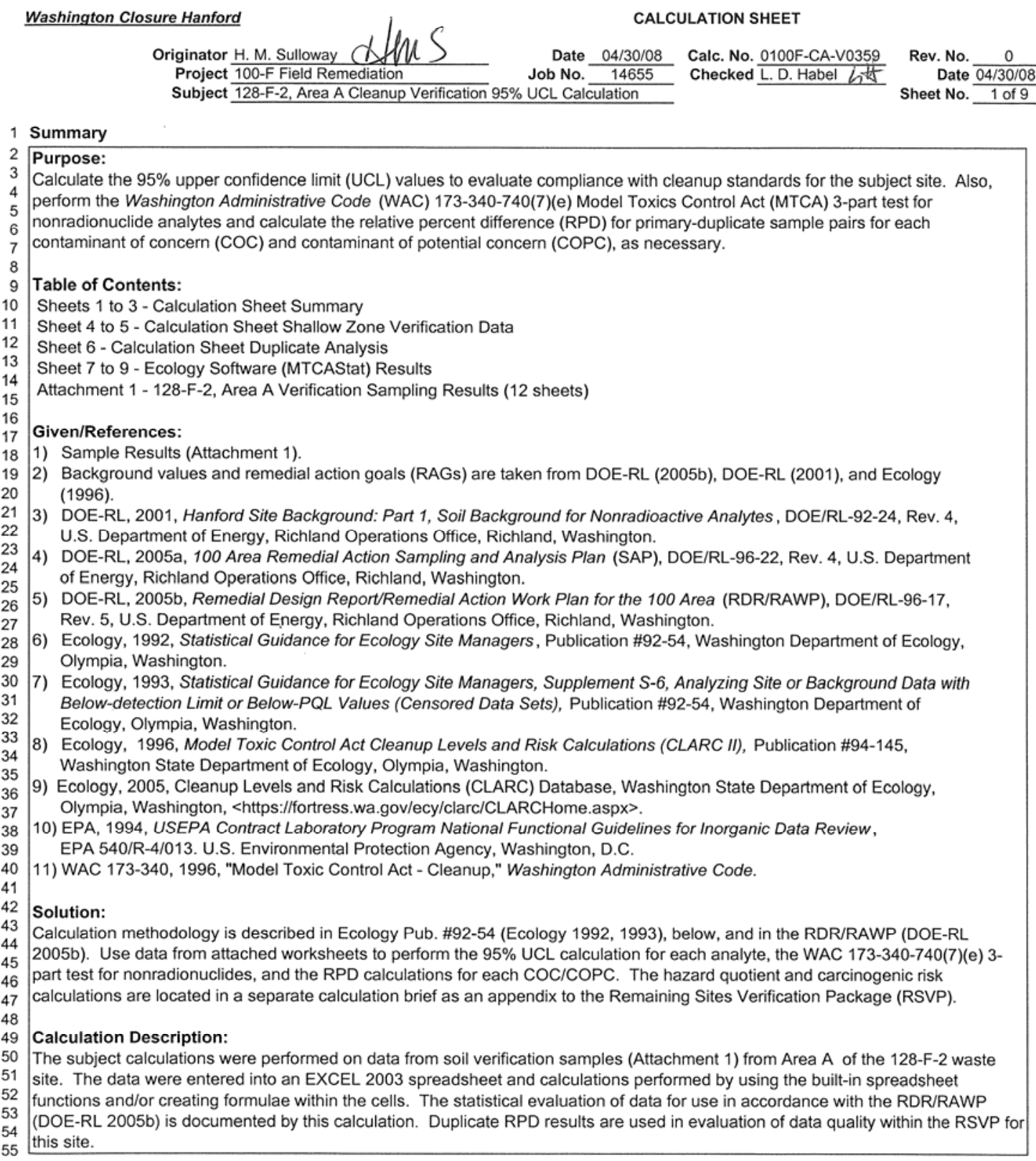


Washington Closure Hanford

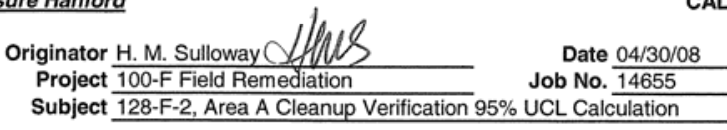

CALCULATION SHEET

\author{
Calc. No. 0100F-CA-V0359 \\ Checked L. D. Habel itt
}
Rev. No. Sheet No. $\frac{0408}{2 \text { of } 9}$

\section{Summary (continued)}

2 Methodology:

3 For nonradioactive analytes with $\leq 50 \%$ of the data below detection limits and all detected radionuclide analytes, the statistical value calculated to evaluate the effectiveness of cleanup is the $95 \%$ UCL. For nonradioactive analytes with $>50 \%$ of the data below detection 5 limits, as determined by direct inspection of the sample results (Attachment 1), the maximum detected value for the data set is used 6 instead of the $95 \%$ UCL, and no further calculations are performed for those data sets. For convenience, these maximum detected values are included in the summary tables that follow. The $95 \%$ UCL was not calculated for data sets with no reported detections. Calculated cleanup levels are not available in Ecology (2005) under WAC 173-340-740(3) for aluminum, calcium, iron, magnesium, potassium, silicon, and sodium; therefore, these constituents are not considered site COCs/COPCs and are also not included in these calculations.

All nonradionuclide data reported as being undetected are set to $1 / 2$ the detection limit value for calculation of the statistics (Ecology 1993). In cases where the laboratory does not report a value below the minimal detectable activity (MDA), half of the MDA is used in the calculation. For the statistical evaluation of duplicate sample pairs, the samples are averaged before being included in the data set, after adjustments for censored data as described above.

For nonradionuclides, the WAC 173-340 statistical guidance suggests that a test for distributional form be performed on the data and the $95 \%$ UCL calculated on the appropriate distribution using Ecology software. For nonradionuclide small data sets

$(n<10)$, the calculations are performed assuming nonparametric distribution, so no tests for distribution are performed. For nonradionuclide data sets of ten or greater, as for the subject site, distributional testing is done using Ecology's MTCAStat software 3 (Ecology 1993). Due to differences in addressing censored data between the RDR/RAWP (DOE-RL 2005b) and MTCAStat coding and due to a limitation in the MTCAStat coding (no direct capability to address variable quantitation limits within a data set), substitutions for censored data are performed before software input and the resulting data set treated as uncensored.

The WAC 173-340-740(7)(e) 3-part test is performed for nonradionuclide analytes only and determines if: 1) the $95 \%$ UCL exceeds the most stringent cleanup limit for each COPC/COC,

2) greater than $10 \%$ of the raw data exceed the most stringent cleanup limit for each $\mathrm{COPC} / \mathrm{COC}$

3) the maximum value of the raw data set exceeds two times the most stringent cleanup limit for each COPC/COC.

The RPD is calculated when both the primary value and the duplicate value for a given analyte are above detection limits and are greater than 5 times the target detection limit (TDL). The TDL is a laboratory detection limit pre-determined for each analytical method and is listed in Table II-1 of the SAP (DOE-RL 2005a). Where direct evaluation of the attached sample data showed that a given analyte was not detected in the primary and/or duplicate sample, further evaluation of the RPD value was not performed. The RPD calculations use the following formula:

For quality assurance/quality control (QAVQ) split and duplicate RPD calculations, a value less than $30 \%$ indicates the data compare favorably. For regulatory splits, a threshold of $35 \%$ is used (EPA 1994). If the RPD is greater than $30 \%$ (or $35 \%$ for regulatory split data), further investigation regarding the usability of the data is performed. No split samples were collected for cleanup verification of 78

$\mathrm{RPD}=[|\mathrm{M}-\mathrm{S}| /((\mathrm{M}+\mathrm{S}) / 2)]^{*} 100$

where, $\quad M=$ Main Sample Value $\quad S=$ Split (or duplicate) Sample Value

the subject site. Additional discussion as necessary is provided in the data quality assessment section of the applicable RSVP. 


\section{Washington Closure Hanford} Originator $\frac{\text { H. M. Sullowayd d W S S }}{100-\text { F Field Remediation }}$ Subject 128-F-2, Area A Cleanup Verification $95 \%$ UCL Calculation

\section{CALCULATION SHEET}

Date $04 / 30 / 08$

14655

ummary (continued)

3 The results presented in the tables that follow include the summary of the results of the $95 \%$ UCL calculations for the shallow zone 4 excavation, the WAC 173-340-740(7)(e) 3-part test evaluation, and the RPD calculations, and are for use in risk analysis and the RSVP 4 excavation,
for this site.

\begin{tabular}{|c|c|c|c|c|}
\hline 7 & \multicolumn{4}{|c|}{ Results Summary - Shallow Zone Excavation } \\
\hline 9 & Analyte & $\begin{array}{l}\text { 95\% UCL } \\
\text { Result }^{\mathrm{a}}\end{array}$ & $\begin{array}{c}\text { Maximum } \\
\text { Value }^{\mathrm{a}}\end{array}$ & Units \\
\hline 10 & Arsenic & 4.4 & & $\mathrm{mg} / \mathrm{kg}$ \\
\hline 11 & Barium & 84.9 & & $\mathrm{mg} / \mathrm{kg}$ \\
\hline 12 & Beryllium & 0.26 & & $\mathrm{mg} / \mathrm{kg}$ \\
\hline 13 & Boron & 1.8 & & $\mathrm{mg} / \mathrm{kg}$ \\
\hline 14 & Chromium & 23.5 & & $\mathrm{mg} / \mathrm{kg}$ \\
\hline 15 & Cobalt & 6.0 & & $\mathrm{mg} / \mathrm{kg}$ \\
\hline 16 & Copper & 23.4 & & $\mathrm{mg} / \mathrm{kg}$ \\
\hline 17 & Hexavalent chromium & 0.53 & & $\mathrm{mg} / \mathrm{kg}$ \\
\hline 18 & Lead & 9.0 & & $\mathrm{mg} / \mathrm{kg}$ \\
\hline 19 & Manganese & 304 & & $\mathrm{mg} / \mathrm{kg}$ \\
\hline 20 & Nickel & 16.7 & & $\mathrm{mg} / \mathrm{kg}$ \\
\hline 21 & Vanadium & 34.2 & & $\mathrm{mg} / \mathrm{kg}$ \\
\hline 22 & Zinc & 47.2 & & $\mathrm{mg} / \mathrm{kg}$ \\
\hline 23 & Bis (2-ethylhexyl) phthalate & 0.085 & & $\mathrm{mg} / \mathrm{kg}$ \\
\hline 24 & Cesium-137 & & 0.218 & $\mathrm{pCi} / \mathrm{g}$ \\
\hline 25 & Antimony & & 0.92 & $\mathrm{mg} / \mathrm{kg}$ \\
\hline 26 & Cadmium & & 0.22 & $\mathrm{mg} / \mathrm{kg}$ \\
\hline 27 & Mercury & & 0.040 & $\mathrm{mg} / \mathrm{kg}$ \\
\hline 28 & Aldrin & & 0.0022 & $\mathrm{mg} / \mathrm{kg}$ \\
\hline 29 & Beta-BHC & & 0.0047 & $\mathrm{mg} / \mathrm{kg}$ \\
\hline 30 & 4,4'-DDE & & 0.0050 & $\mathrm{mg} / \mathrm{kg}$ \\
\hline 31 & 4,4'-DDT & & 0.0055 & $\mathrm{mg} / \mathrm{kg}$ \\
\hline 32 & Endrin aldehyde & & 0.0051 & $\mathrm{mg} / \mathrm{kg}$ \\
\hline 33 & Heptachlor & & 0.0033 & $\mathrm{mg} / \mathrm{kg}$ \\
\hline 34 & Dibenz $(a, h)$ anthracene & & 0.048 & $\mathrm{mg} / \mathrm{kg}$ \\
\hline $\begin{array}{l}35 \\
36\end{array}$ & \multicolumn{4}{|c|}{ WAC 173-340-740(7)(e) Evaluation: } \\
\hline 37 & \multicolumn{4}{|c|}{ WAC 173-340 3-Part Test for most stringent RAG: } \\
\hline 38 & $95 \%$ UCL > Cleanup Limit? & YES & & \\
\hline 39 & $>10 \%$ above Cleanup Limit? & YES & & \\
\hline 40 & Any sample $>2 x$ Cleanup Limit? & NO & & \\
\hline
\end{tabular}

44 as described in the methodology section.

45
46
47
48
49
50
51
52
53
54
55

Calc. No. 0100F-CA-V0359 Checked L. D. Habel int
Rev. No.
Date 04/30/08 Sheet No. 3 of 9


Washington Closure Hanford

Orginator H.M. Sulonay ofth

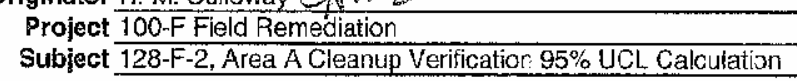

CALCULATION SHEET

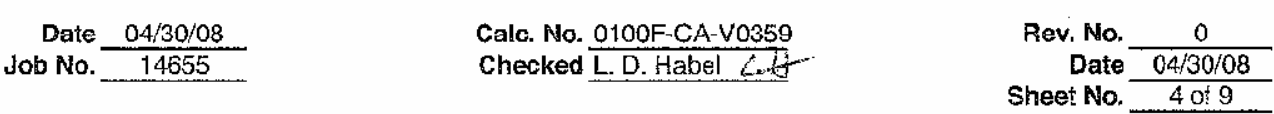

128-F-2 Area A Excavation Shallow Zone Statistical Calculations

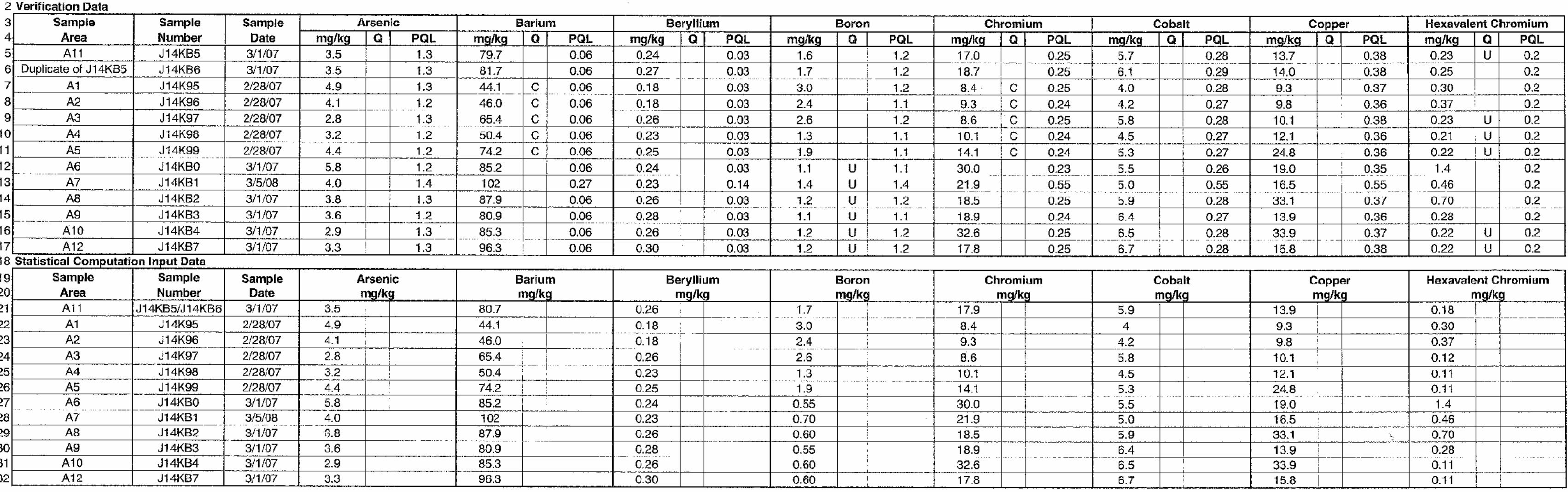

33 Statistical Computations

96.3
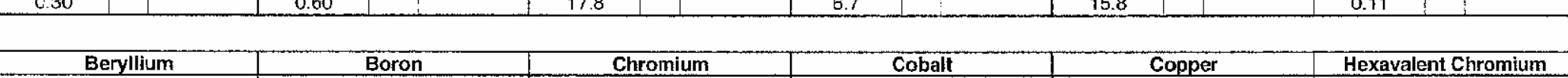

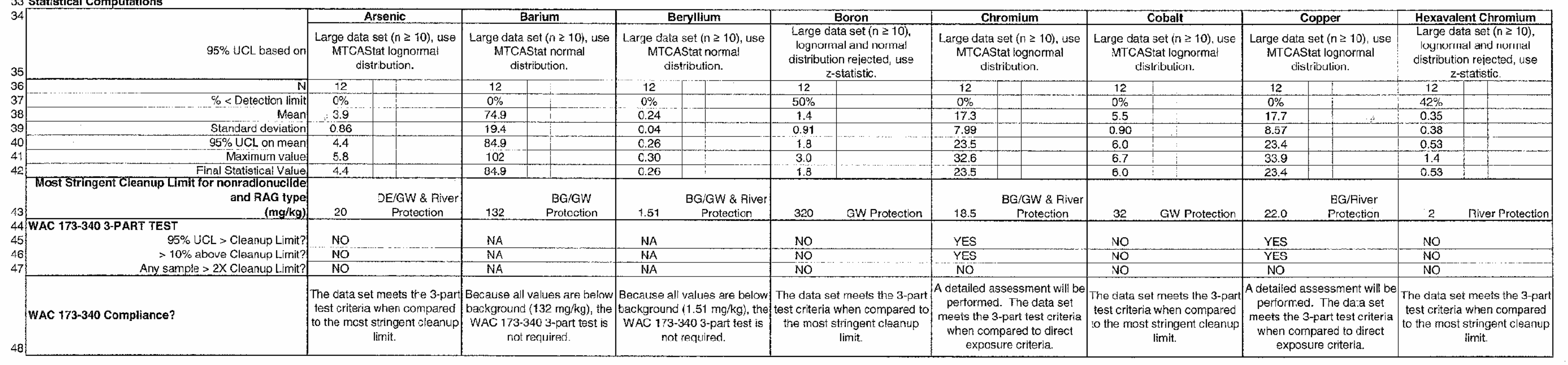




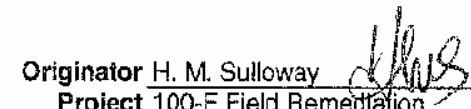

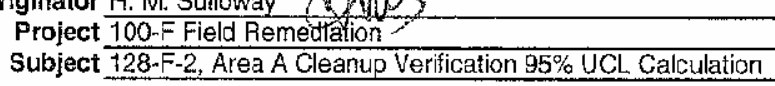

CALCULATION SHEET

Date $04 / 30 / 08$
Job No. 14655
Calc. No. 0100F-CA-V0359
Checked L.D. Habel

Rev. No. $\frac{0}{\text { Date }}$
Sheet No. $\frac{04 / 30 / 08}{5 \text { of } 9}$

\begin{tabular}{|c|c|c|c|c|c|c|c|c|c|c|c|c|c|c|c|c|c|c|}
\hline \multirow{2}{*}{$\begin{array}{l}2 \text { 2 Verification Data } \\
3 \\
4 \\
4\end{array}$} & \multirow{2}{*}{$\begin{array}{l}\text { Sample } \\
\text { Number }\end{array}$} & \multirow{3}{*}{$\begin{array}{l}\text { Sample } \\
\text { Date } \\
\text { Jifopon }\end{array}$} & & \multirow{2}{*}{\multicolumn{2}{|c|}{ Manganese }} & \multirow{2}{*}{\multicolumn{3}{|c|}{ Nickel }} & \multirow{2}{*}{\multicolumn{3}{|c|}{ Vanadium }} & & \multirow{2}{*}{\multicolumn{3}{|c|}{ Bis(2-ethylhexyl) phithalate }} \\
\hline & & & & & & & & & & & & & & & & & & \\
\hline & & & $\frac{m g h g}{7.6}$ & $\frac{P Q L}{0.9}$ & $\frac{m g 9 k 9}{267}$ & \begin{tabular}{l|l} 
& $P L L$ \\
$C$ & 0.06 \\
\end{tabular} & $\frac{\mathrm{mglkg}}{13.9}$ & & $\frac{P Q 6}{0.6}$ & $\frac{\mathrm{m} / \mathrm{kg}}{26.8}$ & & $\frac{P L L}{0.31}$ & $\frac{m g / k g}{49}$ & & $\begin{array}{l}P Q L \\
0.09 \\
\end{array}$ & $\frac{\mathrm{mg} / \mathrm{kg}}{0.085}$ & $\mid \frac{\mathrm{Q}}{\mathrm{JB}}$ & $\begin{array}{l}\frac{P L}{0.38} \\
0.03\end{array}$ \\
\hline Dupplicate of J14KB5 & J14KB6 & $\begin{array}{l}3 / 1 / 2007 \\
2 / 28007\end{array}$ & 7.5 & 0.89 & 276 & 0.06 & 14.8 & & 0.6 & 34.1 & & 0.32 & 50 & 4 & 0.1 & 0.059 & $\mathrm{JB}$ & 0.38 \\
\hline $\begin{array}{l}A_{1} \\
A^{2}\end{array}$ & J14K95 & 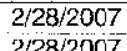 & 3.9 & 0.87 & $\begin{array}{r}200 \\
-195\end{array}$ & 0.06 & $\frac{9.7}{212}$ & & 0.59 & 19 & & 0.31 & 27.3 & $\mathrm{C}$ & 0.09 & 0.024 & JB & 0.36 \\
\hline$\frac{A 2}{A 3}$ & $\begin{array}{l}\frac{J 14 k 96}{J 14 k 9 \%} \\
J 15 \%\end{array}$ & $\begin{array}{l}2 / 28 / 2007 \\
2 / 282007\end{array}$ & $\frac{4.1}{3.8}$ & $\frac{0.8}{0.88}$ & $\frac{195}{274}$ & 0.06 & $\frac{21.2}{92}$ & & $\begin{array}{c}0.57 \\
-06\end{array}$ & $\frac{21.8}{363}$ & & 0.3 & 30.1 & $c$ & $\begin{array}{l}0.09 \\
0.99\end{array}$ & $\begin{array}{l}0.35 \\
0.32\end{array}$ & $u$ & 0.35 \\
\hline$\frac{A 3}{A 4}$ & $\begin{array}{l}J 14 k 9 ? \\
J 14 K 98\end{array}$ & $\begin{array}{l}2 / 288 / 2007 \\
2 / 28 / 2007\end{array}$ & $\begin{array}{l}3.8 \\
3.0 \\
3.0\end{array}$ & $\frac{0.88}{0.83}$ & $\frac{274}{189}$ & $\begin{array}{r}0.06 \\
-006\end{array}$ & $\frac{9.2}{9.5}$ & & 0.6 & $\frac{36.3}{235}$ & & $\frac{0.32}{0.3}$ & $\frac{34.7}{294}$ & $\frac{\mathrm{C}}{\mathrm{C}}$ & $\begin{array}{l}0.09 \\
0.09\end{array}$ & $\frac{0.022}{0.029}$ & $\begin{array}{l}J B i \\
J B\end{array}$ & 0.36 \\
\hline A5 & J14k99 & $2 / 28 / 2007$ & 9.1 & 0.85 & 234 & 0.06 & $\begin{array}{l}9.5 \\
13.6\end{array}$ & & 0.56 & $\frac{23.5}{30.7}$ & & 0.3 & $\frac{29.4}{49.5}$ & $\frac{c}{c}$ & 0.09 & $\begin{array}{l}0.029 \\
0.023 \\
\end{array}$ & JB & \\
\hline A6 & J14KBO & $3 / / 2007$ & 5.6 & 0.81 & 212 & 0.06 & 13.5 & & 0.55 & 39.5 & & 0.29 & 40.6 & C & 0.09 & 0.035 & $\mathrm{JB}$ & 0.35 \\
\hline$A 7$ & J160F5 & $3 / / 2 / 2008$ & 2.8 & 0.82 & 276 & 0.11 & 9.8 & & 0.55 & 36.2 & & 0.38 & 34.8 & 0 & 1.6 & 0.026 & $\mathrm{JB}$ & 0.35 \\
\hline$\frac{A B}{A 9}$ & $\begin{array}{l}\mathrm{J} 14 \mathrm{~KB} 2 \\
114 \mathrm{~KB} 3\end{array}$ & $\frac{3 / 1 / 2007}{3 / 1 / 1007}$ & $\frac{6.7}{58}$ & 0.86 & $\frac{406}{0.06}$ & 0.06 & 16.3 & & $\begin{array}{l}0.58 \\
0.57\end{array}$ & 28.2 & & 0.31 & 40.4 & $\mathrm{C}$ & 0.09 & 0.033 & JB & 0.36 \\
\hline$\frac{A 9}{A 10}$ & $\begin{array}{l}J 14 K B 3 \\
14 K B 4\end{array}$ & 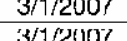 & $\begin{array}{l}5.8 \\
175 \\
7\end{array}$ & $\frac{0.85}{0.85}$ & $\frac{32}{324}$ & \begin{tabular}{l|l}
$C$ & 0.06 \\
\end{tabular} & $\begin{array}{ll}17.4 \\
1.44\end{array}$ & & $\frac{0.57}{0.50}$ & 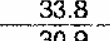 & & $\frac{0.3}{034}$ & 43.6 & $\frac{C}{C}$ & 0.09 & 0.097 & JB & 0.36 \\
\hline \multirow{2}{*}{\multicolumn{19}{|c|}{$\begin{array}{l}7 \mathrm{~A} \\
8 \text { Statistical Computation Inpui Datat }\end{array}$}} \\
\hline & & & & & & & & & & & & & & & & & & \\
\hline $\begin{array}{l}\text { Sample } \\
\text { Area }\end{array}$ & $\begin{array}{l}\text { Sample } \\
\text { Number }\end{array}$ & $\begin{array}{c}\text { Sample } \\
\text { Date }\end{array}$ & \multicolumn{2}{|c|}{$\begin{array}{l}\text { Lead } \\
\mathrm{mg} / \mathrm{kg}\end{array}$} & \multicolumn{2}{|c|}{$\begin{array}{l}\text { Manganese } \\
\mathrm{mg} / \mathrm{kg}\end{array}$} & \multicolumn{3}{|c|}{$\begin{array}{l}\text { Nickel } \\
\text { mg/kg }\end{array}$} & \multicolumn{3}{|c|}{$\begin{array}{c}\text { Vanadium } \\
\text { mg/kg }\end{array}$} & \multicolumn{3}{|c|}{$\begin{array}{c}\text { Zinc } \\
\text { ma/ka }\end{array}$} & \multirow{2}{*}{\multicolumn{3}{|c|}{$\begin{array}{c}\begin{array}{c}\text { Bis(2-ethylhexyl) phthalate } \\
\text { mglkg }\end{array} \\
\end{array}$}} \\
\hline & J14KB5/J14KB6 & $3 / 1 / 07$ & 7.6 & & 272 & & 14.4 & & & 30.5 & & & 49.5 & & & & & \\
\hline$A 1$ & $\mathrm{~J} 14 \mathrm{~K} 95$ & $2 / 28$ & 3.9 & & 200 & & 97 & & & 19.0 & & & 27.3 & & & 0.024 & & \\
\hline 23 & & & 4.1 & & 195 & & 21.2 & & & 21.8 & & & & & & 0.18 & & \\
\hline A3 & 5111 & $2 / 28$ & 3.8 & & 274 & & 9.2 & & & 36.3 & & & 34.7 & & & 0.022 & & \\
\hline $\mathrm{A}_{4}$ & & & 3. & & 189 & & 9.5 & & & 23.5 & & & 29.4 & & & 29 & & \\
\hline $\mathrm{A}^{\mathrm{A}} \mathrm{6}$ & $\frac{J 14 K 99}{514 K B 0}$ & $\frac{2 / 28: 107}{3107}$ & 9. & & 234 & & & & & 30.7 & & & 6 & & & & & \\
\hline $\begin{array}{ll}A 6 \\
A 7\end{array}$ & $\frac{114 K}{116 D}$ & 34107 & 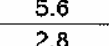 & & $\frac{212}{216}$ & & 13.5 & & & 39.5 & & & 40.6 & & & & & \\
\hline$\frac{A 7}{A B}$ & & & $\frac{2.2}{6.7}$ & & 406 & & $\frac{9.6}{16}$ & & & 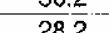 & & & 404 & & & 0 & & \\
\hline & & & & & 年 & & & & & & & & 40.4 & & & 0.007 & & \\
\hline A10 & J14KBA & $3 / 1 / 107$ & 17.5 & & 344 & & 1164 & & & $\frac{5.6}{309}$ & & & 621 & & & .0.057 & & \\
\hline & J14KB3 & 3/1/107 & 7.0 & & 284 & & 18 & & & 31.2 & & & $\frac{279}{179}$ & & & 0.055 & & \\
\hline
\end{tabular}

\section{Statistical Computations}

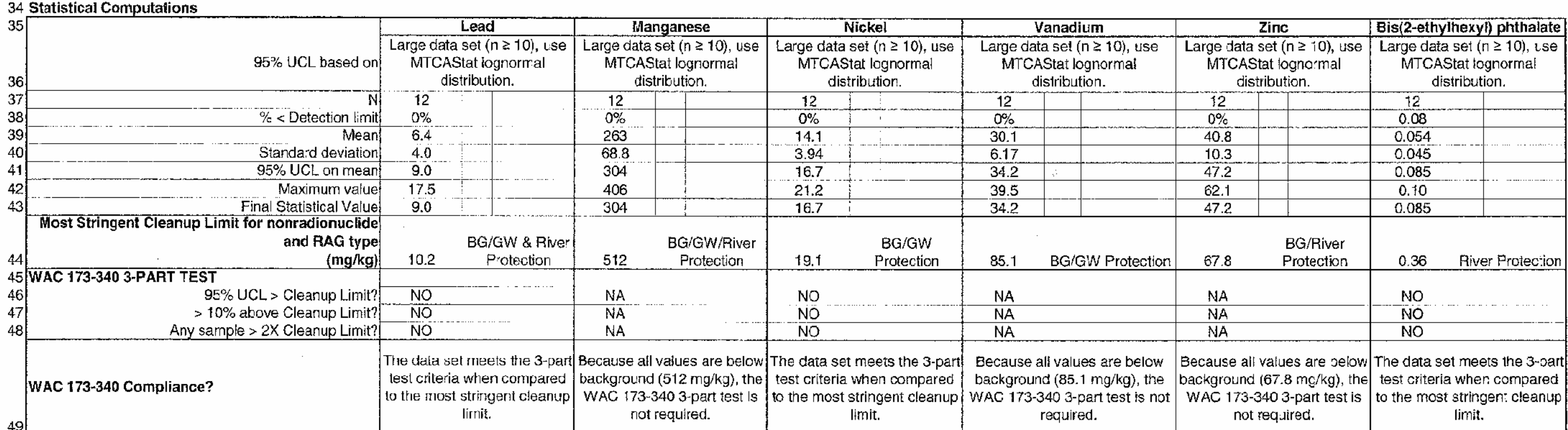


Washington Closure Haniord

Priginator H.M. Sulloway NGWS

Subject 128 -F-2 Area A Cleanup Verification $95 \%$ UCL Calcultion
CALCULATION SHEET

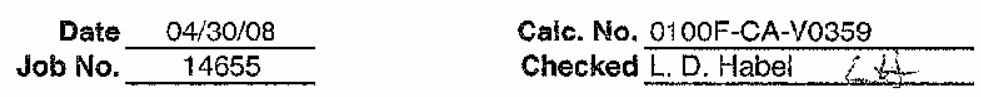

Rev. No. $\frac{0}{04 / 3008}$

Sheet No $\frac{0.60}{6 \text { of } 9}$

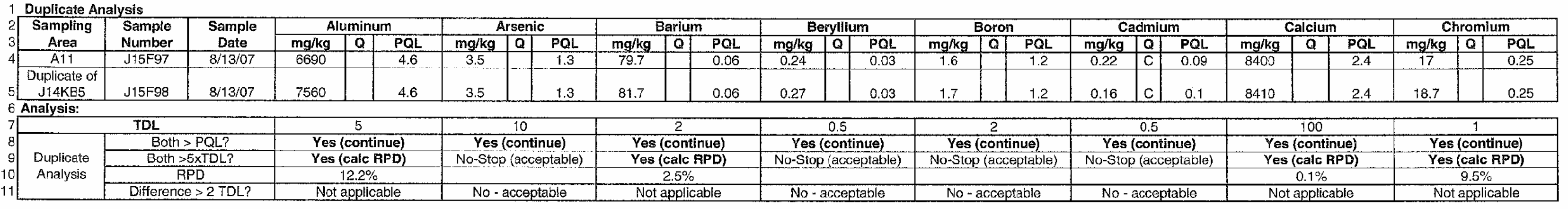

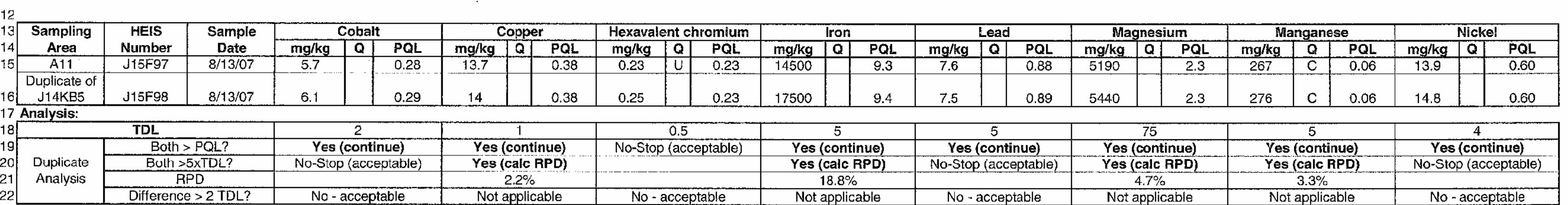

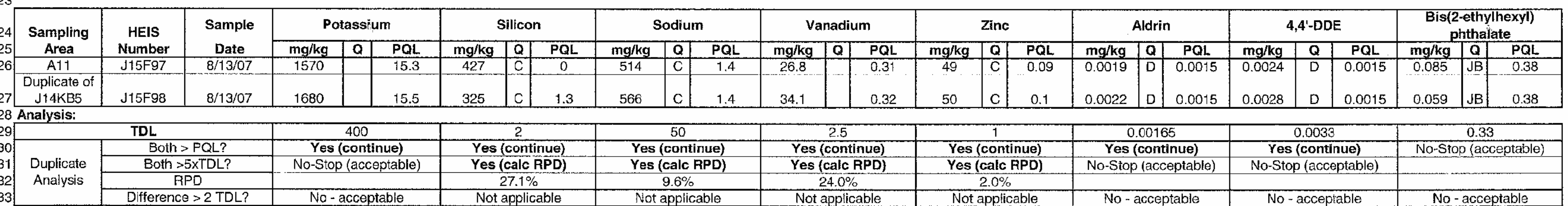

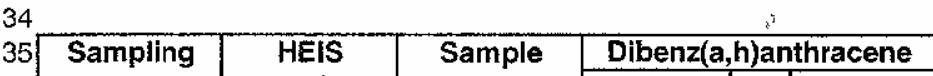

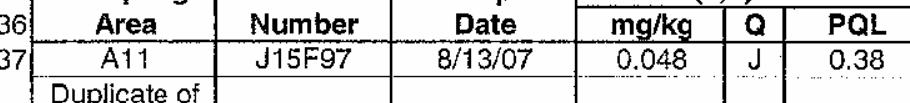

\begin{tabular}{|l|l|l|l|l|l|}
${ }_{38} \begin{array}{c}\text { Duplicate of } \\
\mathrm{J} 14 \mathrm{KBS} 5\end{array}$ & $\mathrm{~J} 15 \mathrm{FF} 98$ & $8 / 13 / 07$ & 0.027 & $\mathrm{~J}$ & 0.38 \\
\hline
\end{tabular}

39 Analysis:

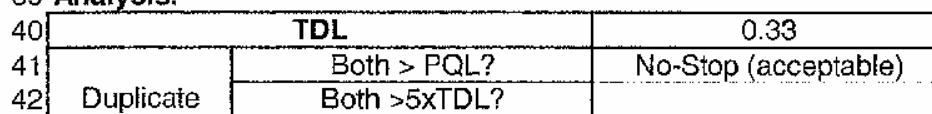

42 Duplicate

43 Anail

\begin{tabular}{|c|c|}
\hline Both $>5 \times X D L ?$ & \\
\hline RPD & \\
\hline Difference $>2$ 2TDL? & No - acceptable \\
\hline
\end{tabular} 


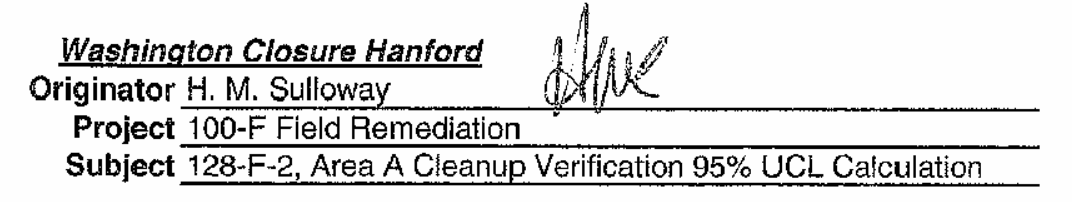

CALCULATION SHEET
Date $04 / 30 / 08$
Calc. No. 0100F-CA-V0359
Rev. No. $\frac{0}{04 / 30108}$

Date $\frac{04 / 30 / 08}{7 \text { of } 9}$

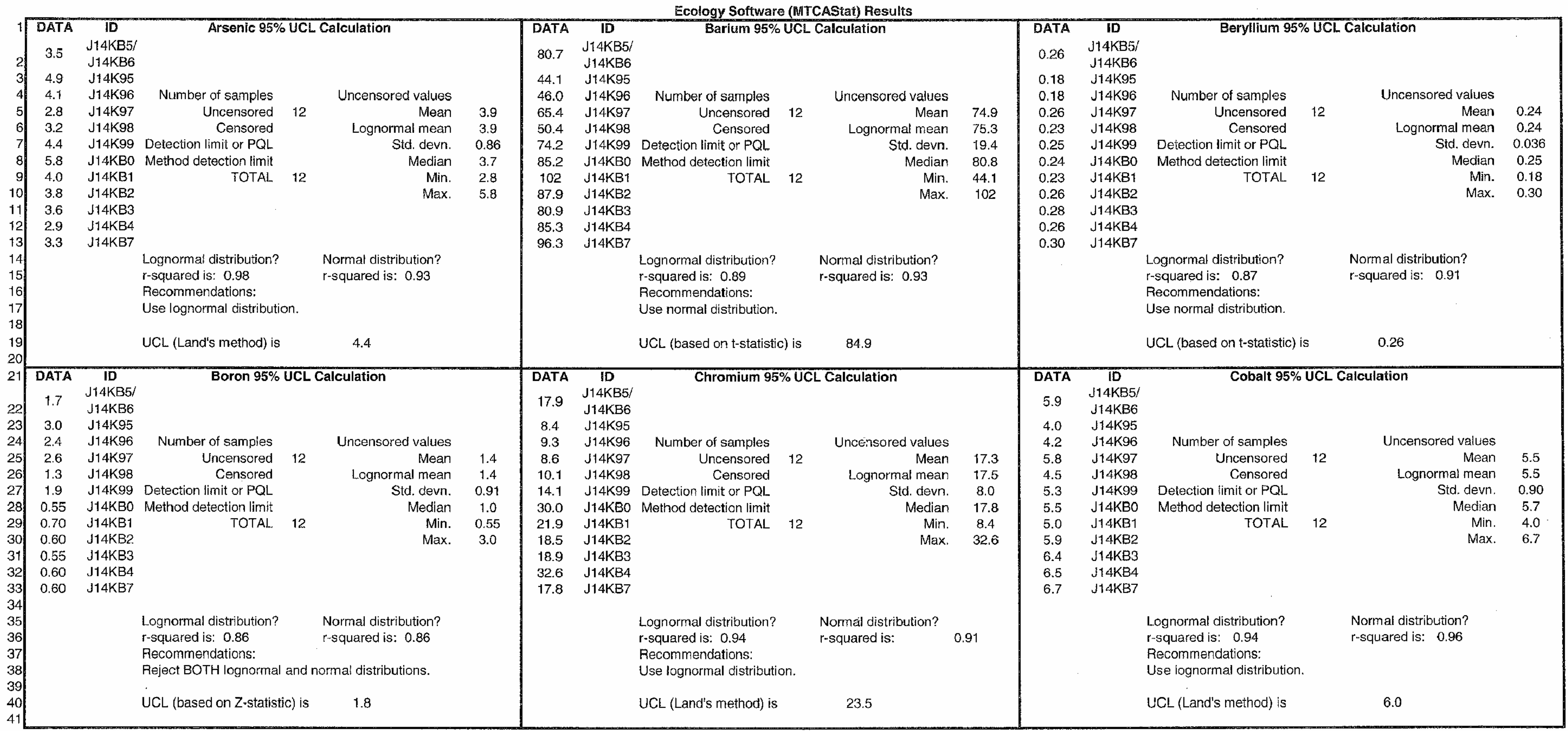


Washinaton Closure Hanford Originator $\frac{\text { H. M. Sulloway }}{100-\text { F Field Remediation }}$ Subject 128-F-2, Area A Cleanup Verification 95\% UCL Cacen

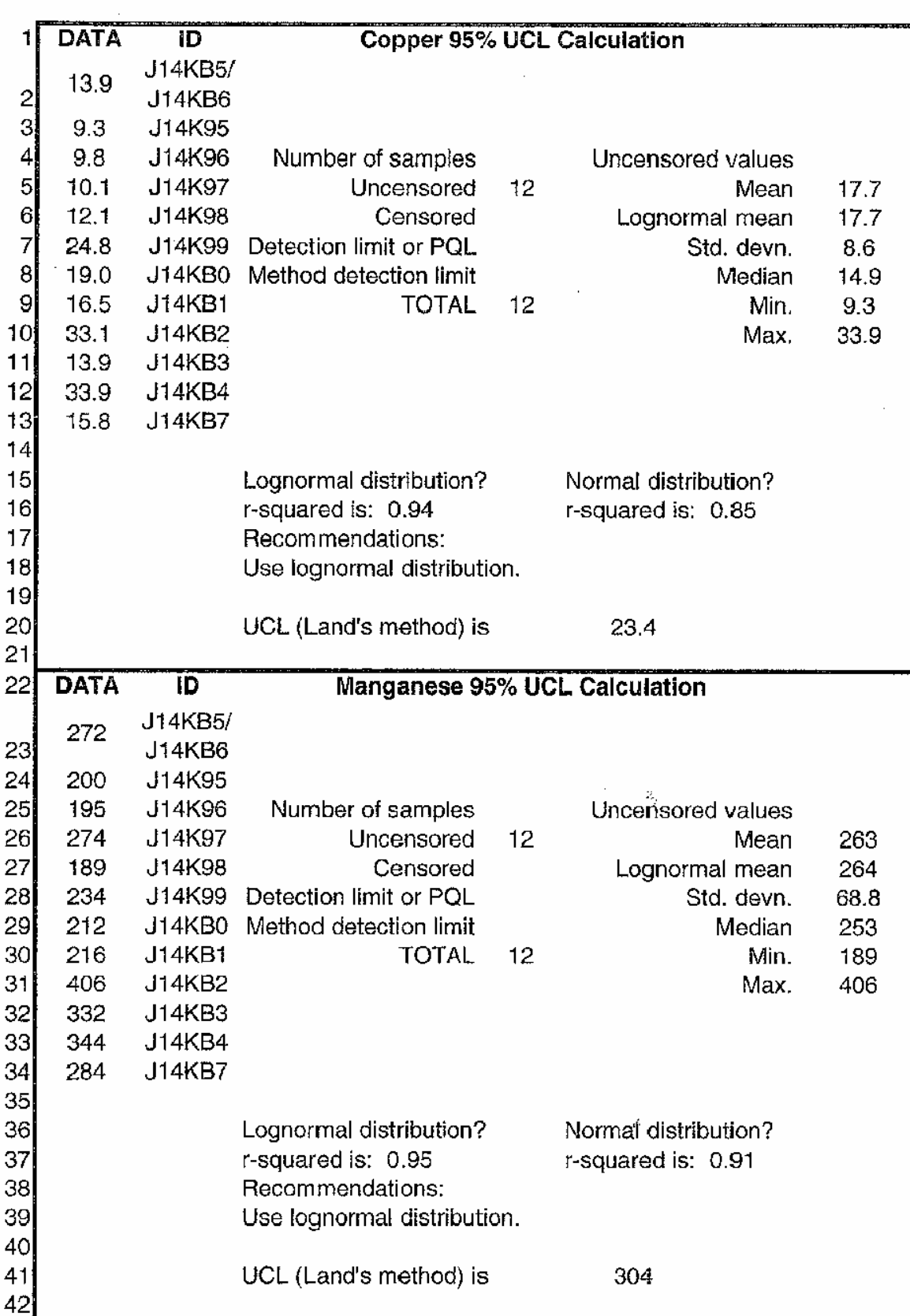

DATA ID

Ecology Soffware (MTCASiat) Results
DATA ID Hexavalent chromium $95 \%$ UCL Calculatio

$0.30 \quad \mathrm{~J} 14 \mathrm{~K} 95$

$\begin{array}{llll}0.37 & \mathrm{~J} 14 \mathrm{~K} 96 & \text { Number of sampies } & \text { Uncensored values }\end{array}$

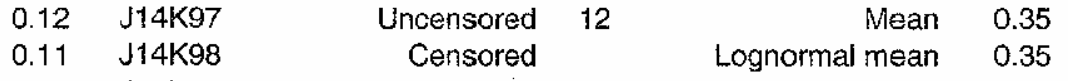

$\begin{array}{lll}0.11 & \mathrm{~J} 14 \mathrm{~K} 99 & \text { Detection limit or } \mathrm{PQL} \\ 1.4 & \mathrm{~J} 14 \mathrm{KBO} & \text { Method detection }\end{array}$

0.46 J14KB1 TOTAL 12

$0.70 \quad \mathrm{~J} 14 \mathrm{~KB} 2$

$\begin{array}{ll}0.70 & \mathrm{~J} 14 \mathrm{~KB} 2 \\ 0.28 & \mathrm{~J} 14 \mathrm{~KB} 3 \\ 0.11 & \mathrm{~J} 14 \mathrm{KBB}\end{array}$

0.11 J14KB4

$0.11 \quad$ J14KB

CALCULATION SHEET

\begin{tabular}{|c|c|c|}
\hline $\begin{array}{rr}\text { Date } & 04 / 30 / 08 \\
\text { Job N No. } & 14655 \\
\end{array}$ & $\begin{array}{l}\text { Calc. No. 0100F-CA-V0359 } \\
\text { Checked L.D. Habel ct }\end{array}$ & $\begin{aligned} & \text { Rev. No. } 0 \\
& \text { Date } 04 / 30 / 08 \\
& 0\end{aligned}$ \\
\hline
\end{tabular}

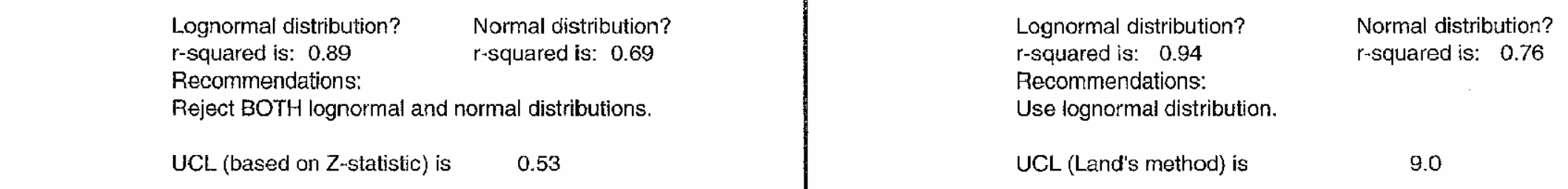

\begin{tabular}{ccc|ccc}
\hline DATA & ID & Nickel 95\% UCL Calculation & DATA & ID & Vanadium $95 \%$ UCL Calculation \\
14.4 & $\mathrm{~J} 14 \mathrm{~KB} 5 /$ & & 30.5 & $\mathrm{~J} 14 \mathrm{~KB} 5 /$ & \\
$\mathrm{J} 14 \mathrm{KBB} 6$ & &
\end{tabular}

9.7 J14K95

9.2 J14K97 Uncensored 12 Mean 14.1

9.5 J14K98 Censored

$\begin{array}{rr}\text { Lognormal mean } & 14.1 \\ \text { Stdern. } & 3.9 \\ \text { Medn } & 14.0\end{array}$

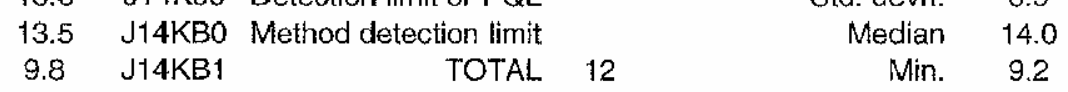

$\begin{array}{ll}16.3 & \mathrm{~J} 14 \mathrm{~KB} 2 \\ 17.4 & \mathrm{~J} 14 \mathrm{KBB}\end{array}$

$\begin{array}{ll}17.4 & \mathrm{~J} 14 \mathrm{~KB} 3 \\ 16.4 & \mathrm{~J} 14 \mathrm{~KB} 4 \\ 18.0 & \mathrm{~J} 14 \mathrm{~KB}\end{array}$

$\begin{array}{ll}16.4 & \mathrm{~J} 14 \mathrm{~KB} 4 \\ 18.0 & \mathrm{~J} 14 \mathrm{~KB} 7\end{array}$

$\begin{array}{ll}21.8 & \mathrm{~J} 14 \mathrm{~K} 96 \\ 36.3 & \mathrm{~J} 14 \mathrm{~K} 97\end{array}$

$\begin{array}{ll}36.3 & \mathrm{~J} 14 \mathrm{~K} 97 \\ 23.5 & \mathrm{~J} 14 \mathrm{~K} 98 \\ 30.7 & \mathrm{~J} 14 \mathrm{~K} 9 \mathrm{~g}\end{array}$

Number of samples

Uncensored t2

Detection limit or $\mathrm{PQL}$

$\begin{array}{lrl} & \text { MEO } \\ \text { KBO } & \text { Method detection limit } \\ \text { TOTAL } & 12\end{array}$

$\mathrm{J} 14 \mathrm{~KB} 2$

J14KB3

$\begin{array}{ll}33.8 & \mathrm{~J} 4 \mathrm{~KB} 3 \\ 30.9 & \mathrm{~J} 14 \mathrm{~KB} 4 \\ 314.2 & \end{array}$

$\begin{array}{ll}30.9 & \mathrm{~J} 14 \mathrm{~KB} 4 \\ 31.2 & \mathrm{~J} 14 \mathrm{~KB} 7\end{array}$

$\begin{array}{ll}\text { Lognormal distribution? } & \text { Normal distribution? } \\ \text { r-squared is: } 0.92 & \text { r-squared is: } 0.94 \\ \text { Recommendations: } & \\ \text { Use lognormal distribution. } & \\ \text { UCL (Land's method) is } & 16.7\end{array}$

UCL (Land's method) is $\quad 16.7$
Lognormal distribution?
r-squared is: 0.93 Recommendations: Use lognormal distribution.

UCL (Land's method) is
Uncensored values Mean
Lognormal mean

Median
Max. heet No. $\frac{04 / 30 / 08}{8 \text { of } 9}$

\begin{tabular}{|cc|} 
& \\
& \\
censored values & \\
Mean & 6.4 \\
Lognormal mean & 6.4 \\
Std. devn. & 4.0 \\
Median & 5.7 \\
Min. & 2.8 \\
Max. & 17.5 \\
& \\
\hline
\end{tabular}

Normal distritiution? r-squared is: 0.96

34.2 


\begin{tabular}{|c|c|}
\hline $04 / 30 / 08$ & \\
\hline & \\
\hline
\end{tabular}

Ecology Sofiware (hTTCAStat) Results

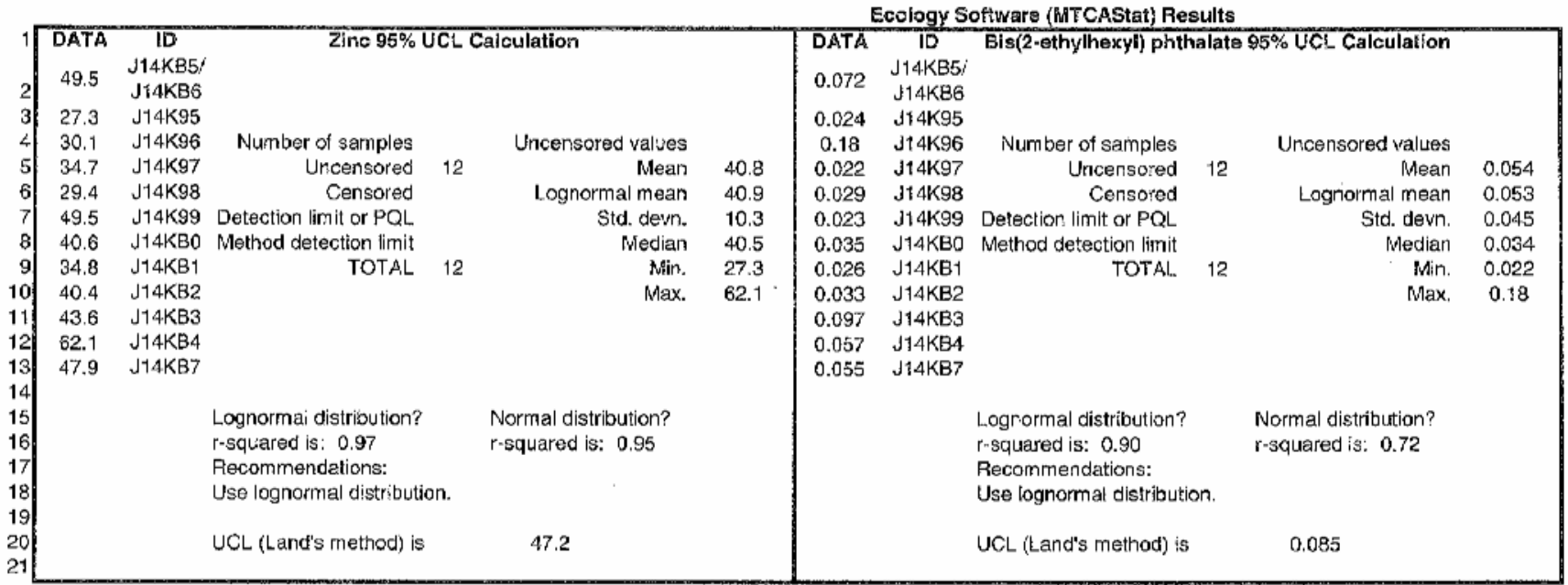


Attachment 1. 128-F-2 Area A Verification Sampling Results.

\begin{tabular}{|c|c|c|c|c|c|c|c|c|c|c|c|c|c|c|c|c|c|c|c|c|c|c|c|}
\hline \multirow{2}{*}{$\begin{array}{c}\begin{array}{c}\text { Sample } \\
\text { Location }\end{array} \\
\text { AlI } \\
\end{array}$} & \multirow{2}{*}{$\begin{array}{c}\text { HEIS } \\
\text { Number } \\
\text { J14KB5 } 5\end{array}$} & \multirow{2}{*}{$\begin{array}{c}\text { Sample } \\
\text { Date } \\
3 / 1 / 2007\end{array}$} & \multicolumn{3}{|c|}{ Americium-2 } & \multicolumn{3}{|c|}{ Cesium-137 } & \multicolumn{3}{|c|}{ Cobalt-60 } & \multicolumn{3}{|c|}{ Europium-152 } & \multirow{2}{*}{\multicolumn{3}{|c|}{ Europium-154 }} & \multirow{2}{*}{\multicolumn{3}{|c|}{ Europium-155 }} & \multirow{2}{*}{\multicolumn{3}{|c|}{ Gross alpha }} \\
\hline & & & $\frac{\mathrm{pCi} / \mathrm{g}}{030}$ & $\mathrm{Q}$ & MDA & $\mathrm{pCi} / \mathrm{g}$ & & MDA & $\mathrm{pCi} / \mathrm{g}$ & & MDA & $\mathrm{pCi} / \mathrm{g}$ & & MDA & & & MDA & & & & $\mathrm{pCi} / \mathrm{g}$ & & MDA \\
\hline Duplicate of & & & & & 0.30 & & U & 0 & 0.11 & & 0.11 & 024 & & 0.24 & & $\mathrm{U}$ & 0.31 & 0.23 & $\mathrm{u}$ & 0.23 & 3.06 & & \\
\hline & KRG & & 0.30 & u & $0.3 \mathrm{c}$ & 0.095 & u & 0.095 & 0.11 & U & 011 & & & & & $\mathrm{U}$ & & & & & & & \\
\hline & & & 0.34 & $\mathrm{U}$ & 0 & 0.10 & & & & & & & & 0. & & \begin{tabular}{|l|l}
$\mathrm{u}$ \\
\end{tabular} & 0.3 & 0.27 & $\frac{\mathrm{U}}{\mathrm{U}}$ & 0.27 & 5.13 & $\frac{U}{U}$ & $\frac{5.2}{5.3}$ \\
\hline A & & & 0.12 & U & & & & & & & & & & 0. & 0.4 & $\mathrm{U}$ & 0.4 & 0.2 & u & 0 & 9.91 & & 4.9 \\
\hline 1 & & & & $\mathrm{I}$ & & & & & 0.0 & & 0. & & & 0. & 0.2 & $\mathrm{U}$ & & 0.1 & & & . & & \\
\hline A & & & & & & 0. & 1 & & 0. & $\sqrt{1}$ & & & & 0 & 0. & $=$ & & 0.1 & & & 2 & & $\frac{4.0}{4.2}$ \\
\hline & & & 0 & & & 0.2 & & & 0.098 & & & & & 0 & & & & 0. & & & 9.74 & & $\frac{4.2}{47}$ \\
\hline A & $\mathrm{J}$ & & & & & 0.1 & & & 0.1 & & & & & & & & & 0. & & & 9 & $\bar{I}$ & $\frac{4.1}{66}$ \\
\hline$A$ & & & & & & 0.0 & & & & & & & & & 0. & & & 0.1 & & & $\frac{.9}{6.32}$ & & $\frac{0.0}{733}$ \\
\hline A & & & 0.2 & & & & & & & & & & & 0. & & & & 0 & & & & & 40 \\
\hline A & & & & & & & & & & & & & & 0 & & & & & & & & & 9 \\
\hline & $\mathrm{J1} 14 \mathrm{~K}$ & $3 / 1 /$ & 0.32 & $\mathrm{U}$ & & & & & 089 & & 0.089 & 0.2 & & 0.2 & 0.26 & & 0.26 & 0.2 & & 0.2 & 0.3 & & $\frac{9}{9}$ \\
\hline $\mathrm{A} 12$ & J14 & $3 / 1 / 2007$ & & & 0.32 & & & 76 & & & 00 & & & & & & & & & & & & \\
\hline
\end{tabular}

\begin{tabular}{|c|c|c|c|c|c|c|c|c|c|c|c|c|c|c|c|c|c|c|c|c|c|c|c|}
\hline \multirow{2}{*}{$\begin{array}{c}\text { Sample } \\
\text { Location } \\
\end{array}$} & \multirow{2}{*}{$\begin{array}{c}\text { HEIS } \\
\text { Number }\end{array}$} & \multirow{2}{*}{$\begin{array}{c}\text { Sample } \\
\text { Date }\end{array}$} & \multicolumn{3}{|c|}{ Gross beta } & \multicolumn{3}{|c|}{ Potassium-40 } & \multicolumn{3}{|c|}{ Radium-226 } & \multicolumn{3}{|c|}{ Radium-228 } & \multicolumn{3}{|c|}{ Silver 108-metastable } & \multicolumn{3}{|c|}{ Thorium-228 GEA } & \multicolumn{3}{|c|}{ Thorium-232 GEA } \\
\hline & & & $\mathrm{pCi} / \mathrm{g}$ & $Q$ & MDA & $\mathrm{pCi} / \mathrm{g}$ & Q & MDA & $\mathrm{pCi} / \mathrm{g}$ & Q & MDA & $\mathrm{pCi} / \mathrm{g}$ & Q & MDA & $\mathrm{pCi} / \mathrm{g}$ & Q & MDA & $\mathrm{pCi} / \mathrm{g}$ & Q & MDA & $\mathrm{pCi} / \mathrm{g}$ & $\mathrm{Q}$ & MDA \\
\hline$A 11$ & J14KB5 & \begin{tabular}{|l|}
$3 / 1 / 2007$ \\
\end{tabular} & 28.6 & & 9.3 & 6.4 & & 0.88 & 0.426 & & 0.18 & 0.784 & & 0.41 & 0.069 & $\mathrm{U}$ & 0.069 & 0.636 & & 0.17 & 0.784 & & 0.41 \\
\hline $\begin{array}{c}\text { Duplicate of } \\
\text { J14KB5 }\end{array}$ & J14KB6 & $3 / 1 / 2007$ & 20.5 & & 9.2 & 6.4 & & 0.88 & 0.426 & & 0.18 & 0.784 & & 0.41 & 0.069 & $\mathrm{U}$ & 0.069 & 0.714 & & 0.15 & 1.03 & & 0.38 \\
\hline $\mathrm{Al}$ & $\mathrm{J14} \mathrm{K} 95$ & $2 / 28 / 2007$ & 20.7 & & 9.3 & 15.4 & & 0.82 & 0.212 & & 0.18 & 0.976 & & 0.5 & 0.072 & $\mathrm{U}$ & 0.072 & 0.804 & & 0.13 & 0.976 & & 0.5 \\
\hline $\mathrm{A} 2$ & $\mathrm{~J} 14 \mathrm{~K} 96$ & $2 / 28 / 2007$ & 20.2 & & 5.4 & 12.5 & & 1.6 & 0.443 & & 0.29 & 0.709 & & 0.42 & 0.092 & $\mathrm{U}$ & 0.092 & 1.04 & & 0.17 & 0.709 & & 0.42 \\
\hline $\mathrm{A} 3$ & $\mathrm{~J} 14 \mathrm{~K} 97$ & $2 / 28 / 2007$ & 17 & & 6.3 & 10.2 & & 0.68 & 0.369 & & 0.13 & 0.661 & & 0.35 & 0.049 & $\mathrm{U}$ & 0.049 & 0.457 & & 0.094 & 0.661 & & 0.35 \\
\hline $\mathrm{A} 4$ & J14K98 & $2 / 28 / 2007$ & 18.4 & & 5.4 & 15 & & 1.3 & 0.356 & & 0.25 & 0.88 & $\mathrm{U}$ & 0.88 & 0.087 & $\mathrm{U}$ & 0.087 & 0.536 & & 0.18 & 0.88 & $\mathrm{U}$ & 0.88 \\
\hline A5 & J14K99 & $2 / 28 / 2007$ & 26.6 & & 7.6 & 13.1 & & 0.93 & 0.549 & & 0.15 & 0.963 & & 0.36 & 0.061 & $\mathrm{U}$ & 0.061 & 0.828 & & 0.15 & 0.963 & & 0.36 \\
\hline $\mathrm{A} 6$ & J14KBO & \begin{tabular}{|l|}
$3 / 1 / 2007$ \\
\end{tabular} & 20.9 & & 5.5 & 14 & & 1.1 & 0.288 & & 0.16 & 0.98 & $\mathrm{U}$ & 0.98 & 0.074 & $\mathrm{U}$ & 0.074 & 0.523 & & 0.14 & 0.98 & $\mathrm{U}$ & 0.98 \\
\hline $\mathrm{A} 7$ & J16DF5 & \begin{tabular}{|l|}
$3 / 5 / 2008$ \\
\end{tabular} & 1.98 & & 5.47 & 14 & & 0.86 & 0.358 & & 0.139 & 0.562 & & 0.307 & 0.055 & $\mathrm{U}$ & 0.055 & 0.64 & & 0.128 & 0.562 & & 0.307 \\
\hline A8 & $\mathrm{J} 14 \mathrm{~KB} 2$ & $3 / 1 / 2007$ & 20.9 & & 5.3 & 21.5 & & 0.59 & 0.944 & & 0.1 & 1.5 & & 0.21 & 0.037 & $\mathrm{U}$ & 0.037 & 1.71 & & 0.092 & 1.5 & & 0.21 \\
\hline $\mathrm{A} 9$ & J14KB3 & \begin{tabular}{|l|}
$3 / 1 / 2007$ \\
\end{tabular} & 28.6 & & 9.3 & 34.2 & & 0.68 & 1.49 & & 0.13 & 2.36 & & 0.33 & 0.058 & $\mathrm{U}$ & 0.058 & 2.66 & & 0.13 & 2.36 & & 0.33 \\
\hline $\mathrm{A} 10$ & $\mathrm{~J} 14 \mathrm{~KB} 4$ & $3 / 1 / 2007$ & 21.4 & & 5.9 & 12.8 & & 0.86 & 0.607 & & 0.15 & 0.839 & & 0.43 & 0.065 & $\mathrm{U}$ & 0.065 & 0.963 & & 0.17 & 0.839 & & 0.43 \\
\hline $\mathrm{A} 12$ & $114 \mathrm{~KB} 7$ & $3 / 1 / 2007$ & 25.3 & & 6.3 & 13.7 & & 0.63 & 0.623 & & 0.15 & 1.05 & & 0.37 & 0.057 & $\mathrm{U}$ & 0.057 & 0.947 & & 0.15 & 1.05 & & 0.37 \\
\hline
\end{tabular}

\begin{tabular}{|c|c|c|c|c|c|c|c|c|}
\hline \multirow{2}{*}{$\begin{array}{c}\text { Sample } \\
\text { Location }\end{array}$} & \multirow{2}{*}{$\begin{array}{c}\text { HEIS } \\
\text { Number } \\
\end{array}$} & \multirow{2}{*}{$\begin{array}{c}\text { Sample } \\
\text { Date }\end{array}$} & \multicolumn{3}{|c|}{ Uranium-235 GEA } & \multicolumn{3}{|c|}{ Uranium-238 GEA } \\
\hline & & & $\mathrm{pCi} / \mathrm{g}$ & $Q$ & MDA & $\mathrm{pCi} / \mathrm{g}$ & $Q$ & MDA \\
\hline All & $\mathrm{J} 14 \mathrm{~KB} 5$ & $3 / 1 / 2007$ & 0.33 & $\mathrm{U}$ & 0.33 & 13 & $\mathrm{U}$ & 13 \\
\hline $\begin{array}{c}\text { Duplicate of } \\
\text { J14KB55 }\end{array}$ & $\mathrm{J} 14 \mathrm{~KB} 6$ & $3 / 1 / 2007$ & 0.36 & $\mathrm{U}$ & 0.36 & 12 & $\mathrm{U}$ & 12 \\
\hline $\mathrm{Al}$ & $\mathrm{J} 14 \mathrm{~K} 95$ & $2 / 28 / 2007$ & 0.39 & $\mathrm{U}$ & 0.39 & 13 & $\mathrm{U}$ & 13 \\
\hline $\mathrm{A} 2$ & J14K96 & $2 / 28 / 2007$ & 0.38 & $\mathrm{U}$ & 0.38 & 16 & $\mathrm{U}$ & 16 \\
\hline $\mathrm{A} 3$ & J14K97 & $2 / 28 / 2007$ & 0.27 & $\mathrm{U}$ & 0.27 & 8.4 & $\mathrm{U}$ & 8.4 \\
\hline $\mathrm{A} 4$ & J14K98 & $2 / 28 / 2007$ & 0.34 & $\mathrm{U}$ & 0.34 & 14 & $\mathrm{U}$ & 14 \\
\hline A5 & J14K99 & $2 / 28 / 2007$ & 0.32 & $\mathrm{U}$ & 0.32 & 11 & $\mathrm{U}$ & 11 \\
\hline $\mathrm{A} 6$ & $\mathrm{J1} 4 \mathrm{~KB} 0$ & $3 / 1 / 2007$ & 0.39 & $\mathrm{U}$ & 0.39 & 14 & $\mathrm{U}$ & 14 \\
\hline A7 & $\mathrm{J} 16 \mathrm{DF} 5$ & $3 / 5 / 2008$ & 0.254 & $\mathrm{U}$ & 0.254 & 10.1 & $\mathrm{U}$ & 10.1 \\
\hline A8 & J14KB2 & $3 / 1 / 2007$ & 0.2 & $\mathrm{U}$ & 0.2 & 6.2 & $\mathrm{U}$ & 6.2 \\
\hline $\mathrm{A} 9$ & $\mathrm{~J} 14 \mathrm{~KB} 3$ & $3 / 1 / 2007$ & 0.33 & $\mathrm{U}$ & 0.33 & 9.2 & $U$ & 9.2 \\
\hline $\mathrm{Al0}$ & $\mathrm{J} 14 \mathrm{~KB} 4$ & $3 / 1 / 2007$ & 0.34 & $\mathrm{U}$ & 0.34 & 10 & $\mathrm{U}$ & 10 \\
\hline $\mathrm{A} 12$ & J14KB7 & $3 / 1 / 2007$ & 0.31 & $\mathrm{U}$ & 0.31 & 11 & $\mathrm{U}$ & 11 \\
\hline
\end{tabular}

Note: Data qualified with B, C, D and/or $\mathrm{J}$, are considered acceptable values.

$\mathrm{GEA}=$ gamma energy analysis $\quad \mathrm{PQL}=$ practical quantitation limit

$\mathrm{MDA}=$ minimum detectable activity

$\mathrm{U}=$ undetected

Attachment

Originator $\frac{1}{\text { H.M. Sulloway }}$ Sheet No. $\frac{1 \text { of } 11}{04 / 30 / 08}$

Checked L. D. Habel Date

Calc. No. $\frac{\text { Dlo0F-CA-V0359 Rev. No. }}{0}$ 
Attachment 1. 128-F-2, Area A Verification Sampling Results.

\begin{tabular}{|c|c|c|c|c|c|c|c|c|c|c|c|c|c|c|c|c|c|c|c|c|c|c|c|}
\hline \multirow{2}{*}{$\begin{array}{c}\text { Sample } \\
\text { Location } \\
\end{array}$} & \multirow{2}{*}{$\begin{array}{c}\text { HEIS } \\
\text { Number }\end{array}$} & \multirow{2}{*}{$\begin{array}{c}\text { Sample } \\
\text { Date }\end{array}$} & \multicolumn{3}{|c|}{ Aluminum } & \multicolumn{3}{|c|}{ Antimony } & \multicolumn{3}{|c|}{ Arsenic } & \multicolumn{3}{|c|}{ Barium } & \multicolumn{3}{|c|}{ Beryllium } & \multicolumn{3}{|c|}{ Boron } & \multicolumn{3}{|c|}{ Cadmium } \\
\hline & & & $\mathrm{mg} / \mathrm{kg}$ & Q & $\mathrm{PQL}$ & $\mathrm{mg} / \mathrm{kg}$ & $Q$ & $\mathrm{PQL}$ & $\mathrm{mg} / \mathrm{kg}$ & Q & PQL & $\mathrm{mg} / \mathrm{kg}$ & $Q$ & PQL & $\mathrm{mg} / \mathrm{kg}$ & Q & PQL & $\mathrm{mg} / \mathrm{kg}$ & Q & PQL & $\mathrm{mg} / \mathrm{kg}$ & Q & PQL \\
\hline All & J14KB5 & $3 / 1 / 2007$ & 6690 & & 4.6 & 1 & $\mathrm{U}$ & 1.0 & 3.5 & & 1.3 & 79.7 & & 0.06 & 0.24 & & 0.03 & 1.6 & & 1.2 & 0.22 & $\mathrm{C}$ & 0.09 \\
\hline $\begin{array}{c}\text { Duplicate of } \\
\text { J14KB5 }\end{array}$ & J14KB6 & 907 & 7560 & & 4.6 & 1 & $\mathrm{U}$ & 1.0 & 3.5 & & 1.3 & 81.7 & & 0.06 & 0.27 & & 0.03 & 1.7 & & 1.2 & 0.16 & C & 0.10 \\
\hline $\mathrm{Al}$ & J14K95 & 2/28/2007 & 4490 & & 4.5 & 0.99 & $\mathrm{U}$ & 1.0 & 4.9 & & 1.3 & 44.1 & C & 0.06 & 0.18 & & 0.03 & 3 & & 1.2 & 0.09 & $\mathrm{U}$ & 0.09 \\
\hline $\mathrm{A} 2$ & J14K96 & 2/28/2007 & 4880 & & 4.4 & 0.95 & \begin{tabular}{|l|}
$\mathrm{U}$ \\
\end{tabular} & 1.0 & 4.1 & & 1.2 & 46 & C & 0.06 & 0.18 & & 0.03 & 2.4 & & 1.1 & 0.09 & $\mathrm{U}$ & 0.09 \\
\hline $\mathrm{A} 3$ & J14K97 & $2 / 28 / 2007$ & 4390 & & 4.6 & 1 & $\mathrm{U}$ & 1.0 & 2.8 & & 1.3 & 65.4 & C & 0.06 & 0.26 & & 0.03 & 2.6 & & 1.2 & 0.09 & $\mathrm{U}$ & 0.09 \\
\hline $\mathrm{A} 4$ & J14K98 & $2 / 28 / 2007$ & 5130 & & 4.3 & 0.95 & $\mathrm{U}$ & 1.0 & 3.2 & & 1.2 & 50.4 & C & 0.06 & 0.23 & & 0.03 & 1.3 & & 1.1 & 0.09 & $\mathrm{U}$ & 0.09 \\
\hline A5 & J14K99 & 2/28/2007 & 6220 & & 4.4 & 0.97 & $\mathrm{U}$ & 1.0 & 4.4 & & 1.2 & 74.2 & C & 0.06 & 0.25 & & 0.03 & 1.9 & & 1.1 & 0.09 & $\mathrm{U}$ & 0.09 \\
\hline A6 & $.114 \mathrm{KBO}$ & $3 / 1 / 2007$ & 7860 & & 4.2 & 0.92 & & 0.9 & 5.8 & & 1.2 & 85.2 & & 0.06 & 0.24 & & 0.03 & 1.1 & $\mathrm{U}$ & 1.1 & 0.09 & $\mathrm{UC}$ & 0.09 \\
\hline A7 & J16DF5 & $3 / 5 / 2008$ & 7670 & & 3.0 & 0.82 & $\mathrm{U}$ & 0.8 & 4.0 & & 1.4 & 102 & & 0.27 & 0.23 & & 0.14 & 1.4 & $\mathrm{U}$ & 1.4 & 0.14 & $\mathrm{U}$ & 0.14 \\
\hline A8 & $\mathrm{J} 14 \mathrm{~KB} 2$ & $3 / 1 / 2007$ & 7100 & & 4.5 & 0.98 & $\mathrm{U}$ & 1.0 & 3.8 & & 1.3 & 87.9 & & 0.06 & 0.26 & & 0.03 & 1.2 & $\mathrm{U}$ & 1.2 & 0.09 & $\mathrm{UC}$ & 0.09 \\
\hline $\mathrm{A} 9$ & J14KB3 & $3 / 1 / 2007$ & 8360 & & 4.4 & 0.97 & $\mathrm{U}$ & 1.0 & 3.6 & & 1.2 & 80.9 & & 0.06 & 0.28 & & 0.03 & 1.1 & $\mathrm{u}$ & 1.1 & 0.09 & $\mathrm{UC}$ & 0.09 \\
\hline $\mathrm{A} 10$ & $\mathrm{~J} 14 \mathrm{~KB} 4$ & $3 / 1 / 2007$ & 7340 & & 4.5 & 0.1 & $\mathrm{U}$ & 1.0 & 2.9 & & 1.3 & 85.3 & & 0.06 & 0.26 & & 0.03 & 1.2 & $\mathrm{U}$ & 1.2 & 0.13 & C & 0.09 \\
\hline $\mathrm{A} 12$ & $\mathrm{~J} 14 \mathrm{~KB} 7$ & $3 / 1 / 2007$ & 8250 & & 4.6 & 1 & $\mathrm{U}$ & 1.0 & 3.3 & & 1.3 & 96.3 & & 0.06 & 0.3 & & 0.03 & 1.2 & \begin{tabular}{|l|}
$\mathrm{U}$ \\
\end{tabular} & 1.2 & 0.20 & C & 0.09 \\
\hline equip blank & J15K94 & $2 / 28 / 2007$ & 43.4 & & 1.4 & 0.3 & $\mathrm{U}$ & 0.3 & 0.38 & $\mathrm{U}$ & 0.38 & 1.2 & & 0.02 & 0.009 & & 0.009 & 0.35 & U & 0.35 & 0.03 & UC, & 0.03 \\
\hline
\end{tabular}

\begin{tabular}{|c|c|c|c|c|c|c|c|c|c|c|c|c|c|c|c|c|c|c|c|c|c|c|c|}
\hline \multirow{2}{*}{$\begin{array}{c}\text { Sample } \\
\text { Location }\end{array}$} & \multirow{2}{*}{$\begin{array}{c}\text { HEIS } \\
\text { Number }\end{array}$} & \multirow{2}{*}{$\begin{array}{c}\text { Sample } \\
\text { Date }\end{array}$} & \multicolumn{3}{|c|}{ Calcium } & \multicolumn{3}{|c|}{ Chromium } & \multicolumn{3}{|c|}{ Cobalt } & \multicolumn{3}{|c|}{ Copper } & \multicolumn{3}{|c|}{$\begin{array}{l}\text { Hexavalent } \\
\text { Chromium }\end{array}$} & \multicolumn{3}{|c|}{ Iron } & \multicolumn{3}{|c|}{ Lead } \\
\hline & & & $\mathrm{mg} / \mathrm{kg}$ & $Q$ & $\mathrm{PQL}$ & $\mathrm{mg} / \mathrm{kg}$ & $Q$ & PQL & \begin{tabular}{|l|}
$\mathrm{mg} / \mathrm{kg}$ \\
\end{tabular} & $Q$ & PQL & $\mathrm{mg} / \mathrm{kg}$ & $Q$ & PQL & $\mathrm{mg} / \mathrm{kg}$ & $\mathrm{Q}$ & PQL & $\mathrm{mg} / \mathrm{kg}$ & $Q$ & PQL & $\mathrm{mg} / \mathrm{kg}$ & $Q$ & PQ1 \\
\hline All & J14KB5 & $3 / 1 / 2007$ & 8400 & & 2.4 & 17 & & 0.25 & 5.7 & & 0.28 & 13.7 & & 0.38 & 0.23 & $\mathrm{U}$ & 0.23 & 14500 & & 9.3 & 7.6 & & 0.88 \\
\hline Dup of & & & & & & & & & & & & & & & & & & & & & & & \\
\hline $314 \mathrm{~KB} 5$ & $\mathrm{~J} 14 \mathrm{~KB} 6$ & $3 / 1 / 2007$ & 8410 & & 2.4 & 18.7 & & 0.25 & 6.1 & & 0.29 & 14 & & 0.38 & 0.25 & & 0.23 & 17500 & & 9.4 & 7.5 & & 0.89 \\
\hline $\mathrm{A} 2$ & J14K96 & $2 / 28 / 2007$ & 7340 & $\mathrm{C}$ & 2.2 & 9.3 & $\mathrm{C}$ & 0.24 & 4.2 & & 0.27 & 9.8 & & 0.36 & 0.37 & & 0.21 & 11100 & C & 8.8 & 4.1 & & 0.83 \\
\hline $\mathrm{A} 3$ & J14K97 & $2 / 28 / 2007$ & 3880 & $\mathrm{C}$ & 2.4 & 8.6 & $\mathrm{C}$ & 0.25 & 5.8 & & 0.28 & 10.1 & & 0.38 & 0.23 & $\mathrm{U}$ & 0.23 & 14900 & $\mathrm{C}$ & 9.4 & 3.8 & & 0.88 \\
\hline $\mathrm{A} 4$ & $\mathrm{~J} 14 \mathrm{~K} 98$ & $2 / 28 / 2007$ & 2590 & $\mathrm{C}$ & 2.2 & 10.1 & $\mathrm{C}$ & 0.24 & 4.5 & & 0.27 & 12.1 & & 0.36 & 0.21 & $\mathrm{U}$ & 0.21 & 11700 & $\mathrm{C}$ & 8.8 & 3 & & 0.83 \\
\hline A5 & J14K99 & $2 / 28 / 2007$ & 5000 & $\mathrm{C}$ & 2.3 & 14.1 & c & 0.24 & 5.3 & & 0.27 & 24.8 & & 0.36 & 0.22 & $\mathrm{U}$ & 0.22 & 15600 & $\mathrm{C}$ & 9.0 & 9.1 & & 0.85 \\
\hline $\mathrm{A} 6$ & $\mathrm{~J} 14 \mathrm{~KB} 0$ & $3 / 1 / 2007$ & 2920 & & 2.2 & 30 & & 0.23 & 5.5 & & 0.26 & 19 & & 0.35 & 1.4 & & 0.21 & 17700 & & 8.5 & 5.6 & & 0.81 \\
\hline AS & J14KB2 & $3 / 1 / 2007$ & 3380 & & 2.3 & 18.5 & & 0.25 & 5.9 & & 0.28 & 33.1 & & 0.37 & 0.70 & & 0.22 & 15400 & & 9.1 & 6.7 & & 0.86 \\
\hline $\mathrm{A9}$ & J14KB3 & $3 / 1 / 2007$ & 4060 & & 2.3 & 18.9 & & 0.24 & 6.4 & & 0.27 & 13.9 & & 0.36 & 0.28 & & 0.22 & 18200 & & 9.0 & 5.8 & & 0.85 \\
\hline $\mathrm{A} 10$ & J14KB4 & $3 / 1 / 2007$ & 6940 & & 2.3 & 32.6 & & 0.25 & 6.5 & & 0.28 & 33.9 & & 0.37 & 0.22 & $\mathrm{U}$ & 0.22 & 16800 & & 9.2 & 17.5 & & 0.87 \\
\hline $\mathrm{A} 12$ & J14KB7 & $3 / 1 / 2007$ & 11100 & & 2.4 & 17.8 & & 0.25 & 6.7 & & 0.28 & 15.8 & & 0.38 & 0.22 & $\mathrm{U}$ & 0.22 & 17700 & & 9.3 & 7 & & 0.88 \\
\hline equip blank & $\mathrm{J} 15 \mathrm{~K} 94$ & $2 / 28 / 2007$ & 32.1 & & 0.7 & 0.17 & & 0.07 & 0.08 & $\mathrm{U}$ & 0.08 & 0.25 & & 011 & 0.21 & & 0.21 & 111 & & 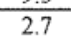 & 26 & U & 0 \\
\hline
\end{tabular}

Attachment

Originator

Checked

Calc. No.

\begin{tabular}{|c|c|c|}
\hline \multirow{3}{*}{$\frac{1}{\frac{1}{\text { H. M. Sulloway }}}$} & \multirow{4}{*}{$\begin{array}{l}\text { Sheet No. } \\
\text { Date } \\
\text { Date } \\
\text { Dev. No. }\end{array}$} & 2 of 11 \\
\hline & & $04 / 30 / 08$ \\
\hline & & $04 / 30 / 08$ \\
\hline & & 0 \\
\hline
\end{tabular}


Attachment 1. 128-F-2, Area A Verification Sampling Results.

\begin{tabular}{|c|c|c|c|c|c|c|c|c|c|c|c|c|c|c|c|c|c|c|c|c|c|c|c|}
\hline \multirow{2}{*}{$\begin{array}{c}\text { Sample } \\
\text { Location }\end{array}$} & \multirow{2}{*}{$\begin{array}{c}\text { HEIS } \\
\text { Number }\end{array}$} & \multirow{2}{*}{$\begin{array}{c}\text { Sample } \\
\text { Date }\end{array}$} & \multicolumn{3}{|c|}{ Magnesium } & \multicolumn{3}{|c|}{ Manganese } & \multicolumn{3}{|c|}{ Mercury } & \multicolumn{3}{|c|}{ Molybdenum } & \multicolumn{3}{|c|}{ Nickel } & \multicolumn{3}{|c|}{ Potassium } & \multicolumn{3}{|c|}{ Selenium } \\
\hline & & & $\mathrm{mg} / \mathrm{kg}$ & $Q$ & $\mathrm{PQL}$ & $\mathrm{mg} / \mathrm{kg}$ & $Q$ & $\mathrm{PQL}$ & $\mathrm{mg} / \mathrm{kg}$ & 0 & $\mathrm{PQL}$ & $\mathrm{mg} / \mathrm{kg}$ & Q & $\mathrm{PQL}$ & $\mathrm{mg} / \mathrm{kg}$ & Q & $\mathrm{PQL}$ & $\mathrm{mg} / \mathrm{kg}$ & Q & PQL & $\mathrm{mg} / \mathrm{kg}$ & Q & $\mathrm{PQL}$ \\
\hline All & J14KB5 & $3 / 1 / 2007$ & 5190 & & 2.3 & 267 & $\mathrm{C}$ & 0.06 & 0.02 & $\mathrm{U}$ & 0.02 & 0.41 & U & 0.41 & 13.9 & & 0.60 & 1570 & & 15.3 & 1.2 & \begin{tabular}{|l|l}
$U$ \\
\end{tabular} & 1.2 \\
\hline $\begin{array}{l}\text { Dup of } \\
.14 \mathrm{~KB} 5\end{array}$ & 4KB6 & $/ 2007$ & 5440 & & 2.3 & 276 & C & 0.06 & 0.02 & $\mathrm{U}$ & 0.02 & 0.4 & $\mathrm{U}$ & 0 & 148 & & 0.60 & 1680 & & 15.5 & 12 & U & 12 \\
\hline $\mathrm{A} 1$ & J14K95 & $2 / 28 / 2007$ & 3750 & & 2.3 & 200 & $\mathrm{C}$ & 0.06 & 0.02 & $\mathrm{U}$ & 0.02 & 0.4 & $\mathrm{U}$ & 0.4 & 9.7 & & 0.59 & 1130 & & 15.1 & 1.1 & \begin{tabular}{|l|}
$\mathrm{U}$ \\
\end{tabular} & 1.1 \\
\hline $\mathrm{A} 2$ & $\mathrm{J14K96}$ & $2 / 28 / 2007$ & 3760 & & 2.2 & 195 & $\mathrm{C}$ & 0.06 & 0.02 & $\mathrm{U}$ & 0.02 & 0.39 & $\mathrm{u}$ & 0.39 & 21.2 & & 0.57 & 1060 & & 14.6 & 1.1 & \begin{tabular}{|l|}
$U$ \\
\end{tabular} & 1.1 \\
\hline $\mathrm{A} 3$ & J14K97 & $2 / 28 / 2007$ & 3360 & & 2.3 & 274 & C & 0.06 & 0.02 & $\mathrm{U}$ & 0.02 & 0.41 & $\mathrm{U}$ & 0.41 & 9.2 & & 0.60 & 1020 & & 15.4 & 1.2 & $\mathrm{U}$ & 1.2 \\
\hline A4 & $\mathrm{J} 14 \mathrm{~K} 98$ & $2 / 28 / 2007$ & 3190 & & 2.2 & 189 & C & 0.06 & 0.03 & & 0.02 & 0.38 & $\mathrm{U}$ & 0.38 & 9.5 & & 0.56 & 724 & & 14.4 & 1.1 & $\mathrm{U}$ & 1.1 \\
\hline $\mathrm{A} 5$ & J14K99 & $2 / 28 / 2007$ & 4160 & & 2.2 & 234 & C & 0.06 & 0.02 & & 0.02 & 0.39 & $\mathrm{U}$ & 0.39 & 13.6 & & 0.57 & 1180 & & 14.8 & 1.1 & $\mathrm{U}$ & 1.1 \\
\hline $\mathrm{A} 6$ & J14KB0 & $3 / 1 / 2007$ & 4340 & & 2.1 & 212 & $\mathrm{C}$ & 0.06 & 0.04 & & 0.02 & 0.38 & $\mathrm{u}$ & 0.38 & 13.5 & & 0.55 & 1060 & & 14.1 & 1.1 & \begin{tabular}{|l|}
$\mathrm{U}$ \\
\end{tabular} & 1.1 \\
\hline A7 & J16DF5 & $3 / 1 / 2007$ & 3890 & & 6.8 & 216 & $\mathrm{C}$ & 0.11 & 0.009 & $\mathrm{U}$ & 0.009 & 0.82 & $\mathrm{U}$ & 0.82 & 9.8 & & 0.55 & 828 & & 10.9 & 1.6 & $\mathrm{U}$ & 1.6 \\
\hline A8 & J14KB2 & $3 / 1 / 2007$ & 4170 & & 2.2 & 406 & $\mathrm{C}$ & 0.06 & 0.02 & $\mathrm{U}$ & 0.02 & 0.4 & $\mathrm{u}$ & 0.4 & 16.3 & & 0.58 & 969 & & 14.2 & 1.1 & $\mathrm{U}$ & 1.1 \\
\hline $\mathrm{A} 9$ & J14KB3 & $3 / 1 / 2007$ & 5260 & & 2.2 & 332 & $\mathrm{C}$ & 0.06 & 0.02 & $\mathrm{U}$ & 0.02 & 0.39 & $\mathrm{U}$ & 0.39 & 17.4 & & 0.57 & 1290 & & 14.8 & 1.1 & $\mathrm{U}$ & 1.1 \\
\hline $\mathrm{A} 10$ & J14KB4 & $3 / 1 / 2007$ & 5340 & & 2.3 & 344 & C & 0.06 & 0.02 & $\mathrm{U}$ & 0.02 & 0.4 & $\mathrm{U}$ & 0.4 & 16.4 & & 0.59 & 1350 & & 15.2 & 1.2 & $\mathrm{U}$ & 1.2 \\
\hline $\mathrm{A} 12$ & J14KB7 & $3 / 1 / 2007$ & 6210 & & 2.3 & 284 & $\mathrm{C}$ & 0.06 & 0.02 & & 0.02 & 0.41 & $\mathrm{U}$ & 0.41 & 18 & & 0.60 & 1310 & & 15.3 & 1.2 & $\mathrm{U}$ & 1.2 \\
\hline & & $2 / 28 / 20$ & & & 0.68 & 32 & 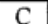 & 27 & 5 & U & & & & & & & & & & & 92 & & \\
\hline
\end{tabular}

\begin{tabular}{|c|c|c|c|c|c|c|c|c|c|c|c|c|c|c|c|c|c|c|c|c|}
\hline \multirow{2}{*}{$\begin{array}{l}\text { Sample } \\
\text { Location }\end{array}$} & \multirow{2}{*}{$\begin{array}{l}\text { HEIS } \\
\text { Number }\end{array}$} & \multirow{2}{*}{$\begin{array}{l}\text { Sample } \\
\text { Date }\end{array}$} & \multicolumn{3}{|c|}{ Silicon } & \multicolumn{3}{|c|}{ Silver } & \multicolumn{3}{|c|}{ Sodium } & \multicolumn{3}{|c|}{ Vanadium } & \multicolumn{3}{|c|}{ Zinc } & \multicolumn{3}{|c|}{$\begin{array}{c}\text { Total petroleum } \\
\text { hydrocarbons }\end{array}$} \\
\hline & & & $\mathrm{mg} / \mathrm{kg}$ & $Q$ & PQL & $\mathrm{mg} / \mathrm{kg}$ & $Q$ & PQL & $\mathrm{mg} / \mathrm{kg}$ & $Q$ & PQL & $\mathrm{mg} / \mathrm{kg}$ & Q & PQL & $\mathrm{mg} / \mathrm{kg}$ & $Q$ & $\mathrm{PQL}$ & $\mathrm{mg} / \mathrm{kg}$ & $Q$ & PQL \\
\hline Al1 & J14KB5 & $3 / 1 / 2007$ & 427 & $\mathrm{C}$ & 0 & 0.28 & $\mathrm{U}$ & 0.28 & 514 & C & 1.4 & 26.8 & & 0.31 & 49 & $\mathrm{C}$ & 0.09 & 150 & $\mathrm{U}$ & 150 \\
\hline $\begin{array}{l}\text { Dup of } \\
\text { J14KB5 }\end{array}$ & J14KB6 & $3 / 1 / 2007$ & 325 & C & 1.3 & 0.29 & $\mathrm{U}$ & 0.29 & 566 & C & 1.4 & 34.1 & & 0.32 & 50 & C & 0.1 & 152 & $\mathrm{u}$ & 152 \\
\hline $\mathrm{Al}$ & J14K95 & $2 / 28 / 2007$ & 565 & & 1.3 & 0.28 & $\mathrm{U}$ & 0.28 & 83.7 & $\mathrm{C}$ & 1.4 & 19 & & 0.31 & 27.3 & $\mathrm{C}$ & 0.09 & 145 & $\mathrm{U}$ & 145 \\
\hline $\mathrm{A} 2$ & J14K96 & $2 / 28 / 2007$ & 603 & & 1.3 & 0.27 & $\mathrm{U}$ & 0.27 & 109 & C & 1.3 & 21.8 & & 0.30 & 30.1 & $\mathrm{C}$ & 0.09 & 142 & $\mathrm{U}$ & 142 \\
\hline $\mathrm{A} 3$ & J14K97 & $2 / 28 / 2007$ & 460 & & 1.3 & 0.28 & $\mathrm{U}$ & 0.28 & 78.5 & $\mathrm{C}$ & 1.4 & 36.3 & & 0.32 & 34.7 & $\mathrm{C}$ & 0.09 & 152 & $\mathrm{U}$ & 152 \\
\hline $\mathrm{A} 4$ & J14K98 & $2 / 28 / 2007$ & 502 & & 1.2 & 0.27 & $\mathrm{U}$ & 0.27 & 73.9 & $\mathrm{C}$ & 1.3 & 23.5 & & 0.30 & 29.4 & $\mathrm{C}$ & 0.09 & 142 & $\mathrm{u}$ & 142 \\
\hline $\mathrm{A5}$ & J14K99 & $2 / 28 / 2007$ & 614 & & 1.3 & 0.27 & $\mathrm{U}$ & 0.27 & 159 & C & 1.3 & 30.7 & & 0.30 & 49.5 & $\mathrm{C}$ & 0.09 & 143 & $\mathrm{U}$ & 143 \\
\hline $\mathrm{A} 6$ & $\mathrm{~J} 14 \mathrm{~KB} 0$ & $3 / 1 / 2007$ & 383 & $C$ & 1.2 & 0.26 & $\mathrm{U}$ & 0.26 & 97.5 & $\mathrm{C}$ & 1.3 & 39.5 & & 0.29 & 40.6 & C & 0.09 & 140 & $\mathrm{U}$ & 140 \\
\hline A7 & J16DF5 & $3 / 1 / 2007$ & 391 & & 10.9 & 0.27 & $\mathrm{U}$ & 0.27 & 97.7 & & 5.5 & 36.2 & & 0.38 & 34.8 & & 1.6 & 140 & $\mathrm{U}$ & 140 \\
\hline$A 8$ & J14KB2 & $3 / 1 / 2007$ & 447 & $\mathrm{C}$ & 1.3 & 0.28 & U & 0.28 & 113 & $\mathrm{C}$ & 1.3 & 28.2 & & 0.31 & 40.4 & $\mathrm{C}$ & 0.09 & 145 & $\mathrm{U}$ & 145 \\
\hline A9 & J14KB3 & $3 / 1 / 2007$ & 424 & C & 1.3 & 0.27 & $\mathrm{U}$ & 0.27 & 274 & C & 1.3 & 33.8 & & 0.30 & 43.6 & $\mathrm{C}$ & 0.09 & 144 & $\mathrm{U}$ & 144 \\
\hline Al0 & J14KB4 & $3 / 1 / 2007$ & 364 & C & 1.3 & 0.28 & $\mathrm{U}$ & 0.28 & 317 & $\mathrm{C}$ & 1.4 & 30.9 & & 0.31 & 62.1 & $\mathrm{C}$ & 0.09 & 150 & $\mathrm{U}$ & 150 \\
\hline $\mathrm{A12}$ & J14KB7 & $3 / 1 / 2007$ & 468 & $\mathrm{C}$ & 1.2 & 0.28 & $\mathrm{U}$ & 0.28 & 279 & $\mathrm{C}$ & 1.4 & 31.2 & & 0.31 & 47.9 & C & 0.09 & 150 & $\mathrm{U}$ & 150 \\
\hline equip blank & J15K94 & $2 / 28 / 2007$ & 33.7 & $\mathrm{C}$ & 0.39 & 0.08 & $\mathrm{U}$ & 0.08 & 7.4 & $\mathrm{C}$ & 0.41 & 0.09 & & 0.09 & 0.65 & C & 0.03 & & & \\
\hline
\end{tabular}

\begin{tabular}{|c|c|c|c|}
\hline Attachment & 1 & _Sheet No. & 3 of 11 \\
\hline Originator & H.M. Sulloway & Date & $04 / 30 / 08$ \\
\hline Checked & L. D. Habel & Date & $04 / 30 / 08$ \\
\hline Calc. No. & $0100 \mathrm{~F}-\mathrm{CA}-\mathrm{V} 0359$ & Rev. No. & 0 \\
\hline
\end{tabular}


Attachment 1. 128-F-2, Area A Verification Sampling Results.

\begin{tabular}{|c|c|c|c|c|c|c|c|c|c|c|c|c|}
\hline \multirow[t]{2}{*}{ Constituent } & \multicolumn{3}{|c|}{$\begin{array}{c}\text { J14K95 } \\
\text { Sample Location A1; } \\
\text { Sample Date } 2 / 28 / 07\end{array}$} & \multicolumn{3}{|c|}{$\begin{array}{c}\text { J14K96 } \\
\text { Sample Location A2; } \\
\text { Sample Date } 2 / 28 / 07\end{array}$} & \multicolumn{3}{|c|}{$\begin{array}{c}\text { J14K97 } \\
\text { Sample Location A3; } \\
\text { Sample Date 2/28/07 }\end{array}$} & \multicolumn{3}{|c|}{$\begin{array}{c}\text { J14K98 } \\
\text { Sample Location A4; } \\
\text { Sample Date 2/28/07 }\end{array}$} \\
\hline & $\mu \mathrm{g} / \mathrm{kg}$ & Q & PQL & $\mu \mathrm{g} / \mathrm{kg}$ & Q & PQL & $\mu \mathrm{g} / \mathrm{kg}$ & $Q$ & PQL & $\mu \mathrm{g} / \mathrm{kg}$ & Q & PQL \\
\hline \multicolumn{13}{|c|}{ Polychlorinated Biphenyls } \\
\hline Aroclor-1016 & 15 & $\mathrm{U}$ & 15 & 14 & $\mathrm{U}$ & 14 & 15 & $\mathrm{U}$ & 15 & 14 & $\bar{U}$ & 14 \\
\hline Aroclor -1221 & 15 & U & 15 & 14 & $\mathrm{U}$ & 14 & 15 & $\mathrm{U}$ & 15 & 14 & $\mathrm{U}$ & 14 \\
\hline Aroclor-1232 & 15 & $\mathrm{U}$ & 15 & 14 & $\mathrm{U}$ & 14 & 15 & $U$ & 15 & 14 & $\mathrm{U}$ & 14 \\
\hline Aroclor- 1242 & 15 & $U$ & 15 & 14 & $\mathrm{U}$ & 14 & 15 & $U$ & 15 & 14 & $\mathrm{U}$ & 14 \\
\hline Aroclor-1248 & 15 & $U$ & 15 & 14 & $\mathrm{U}$ & 14 & 15 & $\mathrm{U}$ & 15 & 14 & $\mathrm{U}$ & 14 \\
\hline Aroclor-1254 & 15 & $\mathrm{U}$ & 15 & 14 & $\mathrm{U}$ & 14 & 15 & U & 15 & 14 & $\bar{U}$ & 14 \\
\hline Aroclor- 1260 & 15 & $\mathrm{U}$ & 15 & 14 & $\mathrm{U}$ & 14 & 15 & $\mathrm{U}$ & 15 & 14 & $\mathrm{U}$ & 14 \\
\hline \multicolumn{13}{|c|}{ Pesticides } \\
\hline Aldrin & 1.5 & UD & 1.5 & 1.4 & UD & 1.4 & 1.5 & UD & 1.5 & 1.4 & UD & 1.4 \\
\hline \begin{tabular}{|l|} 
Alpha-BHC \\
\end{tabular} & 1.5 & UD & 1.5 & 1.4 & UD & 1.4 & 1.5 & UD & 1.5 & 1.4 & UD & 1.4 \\
\hline Alpha-Chlordane & 1.5 & UD & 1.5 & 1.4 & UD & 1.4 & 1.5 & UD & 1.5 & 1.4 & UD & 1.4 \\
\hline Beta-BHC & 4.7 & D & 1.5 & 1.4 & UD & 1.4 & 1.5 & UD & 1.5 & 1.4 & UD & 1.4 \\
\hline Delta-BHC & 1.5 & UD & 1.5 & 1.4 & UD & 1.4 & 1.5 & UD & 1.5 & 1.4 & UD & 1.4 \\
\hline Dichlorodiphenyldichloroethane & 1.5 & UD & 1.5 & 1.4 & UD & 1.4 & 1.5 & UD & 1.5 & 1.4 & UD & 1.4 \\
\hline \begin{tabular}{|l|} 
Dichlorodiphenyldichloroethylene \\
\end{tabular} & 1.5 & UD & 1.5 & 1.4 & UD & 1.4 & 1.5 & UD & 1.5 & 1.4 & UD & 1.4 \\
\hline \begin{tabular}{|l|} 
Dichlorodiphenyltrichloroethane \\
\end{tabular} & 1.5 & UD & 1.5 & 1.4 & UD & 1.4 & 1.5 & UD & 1.5 & 1.4 & UD & 1.4 \\
\hline \begin{tabular}{|l|} 
Dieldrin \\
\end{tabular} & 1.5 & UD & 1.5 & 1.4 & UD & 1.4 & 1.5 & UD & 1.5 & 1.4 & UD & 1.4 \\
\hline Endosulfan I & 1.5 & UD & 1.5 & 1.4 & UD & 1.4 & 1.5 & UD & 1.5 & 1.4 & UD & 1.4 \\
\hline Endosulfan II & 1.5 & UD & 1.5 & 1.4 & UD & 1.4 & 1.5 & UD & 1.5 & 1.4 & UD & 1.4 \\
\hline Endosulfan sulfate & 1.5 & UD & 1.5 & 1.4 & UD & 1.4 & 1.5 & UD & 1.5 & 1.4 & UD & 1.4 \\
\hline \begin{tabular}{|l} 
Endrin \\
\end{tabular} & 1.5 & UD & 1.5 & 1.4 & UD & 1.4 & 1.5 & UD & 1.5 & 1.4 & UD & 1.4 \\
\hline Endrin aldehyde & 1.5 & UD & 1.5 & 1.4 & UD & 1.4 & 1.5 & UD & 1.5 & 1.4 & UD & 1.4 \\
\hline \begin{tabular}{|l} 
Endrin ketone \\
\end{tabular} & 1.5 & UD & 1.5 & 1.4 & UD & 1.4 & 1.5 & UD & 1.5 & 1.4 & UD & 1.4 \\
\hline Gamma-BHC (Lindane) & 1.5 & UD & 1.5 & 1.4 & UD & 1.4 & 1.5 & UD & 1.5 & 1.4 & UD & 1.4 \\
\hline gamma-Chlordane & 1.5 & UD & 1.5 & 1.4 & UD & 1.4 & 1.5 & UD & 1.5 & 1.4 & UD & 1.4 \\
\hline Heptachlor & 1.5 & UD & 1.5 & 1.4 & UD & 1.4 & 1.5 & UD & 1.5 & 1.4 & UD & 1.4 \\
\hline Heptachlor epoxide & 1.5 & UD & 1.5 & 1.4 & UD & 1.4 & 1.5 & UD & 1.5 & 1.4 & UD & 1.4 \\
\hline \begin{tabular}{|l} 
Methoxychlor \\
\end{tabular} & 1.5 & UD & 1.5 & 1.4 & UD & 1.4 & 1.5 & UD & 1.5 & 1.4 & UD & 1.4 \\
\hline \begin{tabular}{|l} 
Toxaphene \\
\end{tabular} & 15 & UD & 15 & 14 & UD & 14 & 15 & UD & 15 & 14 & UD & 14 \\
\hline \multicolumn{13}{|c|}{ Semivolatile Organic Analytes } \\
\hline 1,2,4-Trichlorobenzene & 360 & $\mathrm{U}$ & 360 & 350 & $\mathrm{U}$ & 350 & 380 & $\mathrm{U}$ & 380 & 360 & $\mathrm{U}$ & 360 \\
\hline 1,2-Dichlorobenzene & 360 & $\mathrm{U}$ & 360 & 350 & $\mathrm{U}$ & 350 & 380 & $\mathrm{U}$ & 380 & 360 & U & 360 \\
\hline 1,3-Dichlorobenzene & 360 & $\mathrm{U}$ & 360 & 350 & $\mathrm{U}$ & 350 & 380 & $\mathrm{U}$ & 380 & 360 & $\mathrm{U}$ & 360 \\
\hline 1,4-Dichlorobenzene & 360 & $\mathrm{U}$ & 360 & 350 & $\mathrm{U}$ & 350 & 380 & $\mathrm{U}$ & 380 & 360 & $\mathrm{U}$ & 360 \\
\hline 2,4,5-Trichlorophenol & 910 & $\mathrm{U}$ & 910 & 890 & $\mathrm{U}$ & 890 & 950 & $\mathrm{U}$ & 950 & 890 & $\mathrm{U}$ & 890 \\
\hline 2,4,6-Trichlorophenol & 360 & $\mathrm{U}$ & 360 & 350 & $\mathrm{U}$ & 350 & 380 & $\mathrm{U}$ & 380 & 360 & U & 360 \\
\hline 2,4-Dichlorophenol & 360 & $U$ & 360 & 350 & U & 350 & 380 & $\mathrm{U}$ & 380 & 360 & $\mathrm{U}$ & 360 \\
\hline 2,4-Dimethylphenol & 360 & $U$ & 360 & 350 & $\mathrm{U}$ & 350 & 380 & $U$ & 380 & 360 & U & 360 \\
\hline 2,4-Dinitrophenol & 910 & $\mathrm{U}$ & 910 & 890 & U & 890 & 950 & $U$ & 950 & 890 & $\mathrm{U}$ & 890 \\
\hline 2,4-Dinitrotoluene & 360 & $\mathrm{U}$ & 360 & 350 & U & 350 & 380 & $U$ & 380 & 360 & $\mathrm{U}$ & 360 \\
\hline 2,6-Dinitrotoluene & 360 & $\mathrm{U}$ & 360 & 350 & $\mathrm{U}$ & 350 & 380 & U & 380 & 360 & $\mathrm{U}$ & 360 \\
\hline 2-Chloronaphthalene & 360 & $\mathrm{U}$ & 360 & 350 & $U$ & 350 & 380 & $\mathrm{U}$ & 380 & 360 & $U$ & 360 \\
\hline 2-Chlorophenol & 360 & $\mathrm{U}$ & 360 & 350 & U & 350 & 380 & $U$ & 380 & 360 & $\mathrm{U}$ & 360 \\
\hline 2-Methylnaphthalene & 360 & $\mathrm{U}$ & 360 & 350 & $U$ & 350 & 380 & $\mathrm{U}$ & 380 & 360 & $\mathrm{U}$ & 360 \\
\hline 2-Methylphenol (cresol, o-) & 360 & $\mathrm{U}$ & 360 & 350 & $\mathrm{U}$ & 350 & 380 & $\mathrm{U}$ & 380 & 360 & U & 360 \\
\hline 2-Nitroaniline & 910 & $\mathrm{U}$ & 910 & 890 & $\mathrm{U}$ & 890 & 950 & $\mathrm{U}$ & 950 & 890 & $\mathrm{U}$ & 890 \\
\hline 2-Nitrophenol & 360 & $\mathrm{U}$ & 360 & 350 & $U$ & 350 & 380 & $U$ & 380 & 360 & $\mathrm{U}$ & 360 \\
\hline \multicolumn{7}{|c|}{ Attachment } & \multicolumn{3}{|l|}{1} & \multirow{4}{*}{$\begin{array}{l}\text { Sheet No. } \\
\text { Date } \\
\text { Date } \\
\text { Rev. No. }\end{array}$} & \multicolumn{2}{|c|}{4 of 11} \\
\hline & & & & & \multicolumn{2}{|c|}{ Originator } & \multicolumn{3}{|c|}{ H. M. Sulloway } & & \multicolumn{2}{|c|}{$\frac{40111}{04 / 30 / 08}$} \\
\hline & & & & & \multicolumn{2}{|c|}{ Checked } & L. D. Habe & & & & & $/ 30 / 08$ \\
\hline & & & & & Calc. & & $0100 \mathrm{~F}-\mathrm{CA}$ & 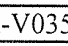 & & & & 0 \\
\hline
\end{tabular}


Attachment 1. 128-F-2 Verification Sampling Results.

\begin{tabular}{|c|c|c|c|c|c|c|c|c|c|c|c|c|}
\hline \multirow[t]{2}{*}{ Constituent } & \multicolumn{3}{|c|}{$\begin{array}{c}\text { J14K95 } \\
\text { Sample Location A1; } \\
\text { Sample Date 2/28/07 }\end{array}$} & \multicolumn{3}{|c|}{$\begin{array}{c}\text { J14K96 } \\
\text { Sample Location A2; } \\
\text { Sample Date 2/28/07 }\end{array}$} & \multicolumn{3}{|c|}{$\begin{array}{c}\text { J14K97 } \\
\text { Sample Location A3; } \\
\text { Sample Date 2/28/07 }\end{array}$} & \multicolumn{3}{|c|}{$\begin{array}{c}\text { J14K98 } \\
\text { Sample Location A4 } \\
\text { Sample Date 2/28/07 }\end{array}$} \\
\hline & $\mu \mathrm{g} / \mathrm{kg}$ & $Q$ & PQL & $\mu \mathrm{g} / \mathrm{kg}$ & $Q$ & PQL & $\mu \mathrm{g} / \mathrm{kg}$ & $Q$ & PQL & $\mu \mathrm{g} / \mathrm{kg}$ & Q & PQL \\
\hline \multicolumn{13}{|c|}{ Semivolatile Organic Analytes (continued) } \\
\hline 3,3'-Dichlorobenzidine & 360 & U & 360 & 350 & U & 350 & 380 & $\mathrm{U}$ & 380 & 360 & $\mathrm{U}$ & 360 \\
\hline 4-Methylphenol (p-cresol) & 360 & $\mathrm{U}$ & 360 & 350 & $\mathrm{U}$ & 350 & 380 & $\mathrm{U}$ & 380 & 360 & $\mathrm{U}$ & 360 \\
\hline 3-Nitroaniline & 910 & $\mathrm{U}$ & 910 & 890 & $\mathrm{U}$ & 890 & 950 & $\mathrm{U}$ & 950 & 890 & $U$ & 890 \\
\hline 4,6-Dinitro-2-methylphenol & 910 & $\mathrm{U}$ & 910 & 890 & $\mathrm{U}$ & 890 & 950 & $\mathrm{U}$ & 950 & 890 & $\mathrm{U}$ & 890 \\
\hline 4-Bromophenyl-phenylether & 360 & $U$ & 360 & 350 & $\mathrm{U}$ & 350 & 380 & $\mathrm{U}$ & 380 & 360 & $\mathrm{U}$ & 360 \\
\hline 4-Chloro-3-methylphenol & 360 & $U$ & 360 & 350 & $\mathrm{U}$ & 350 & 380 & $\mathrm{U}$ & 380 & 360 & $\mathrm{U}$ & 360 \\
\hline \begin{tabular}{|l|} 
4-Chloroaniline \\
\end{tabular} & 360 & $U$ & 360 & 350 & $\mathrm{U}$ & 350 & 380 & $\mathrm{U}$ & 380 & 360 & $U$ & 360 \\
\hline 4-Chlorophenyl-phenylether & 360 & $\mathrm{U}$ & 360 & 350 & U & 350 & 380 & $U$ & 380 & 360 & $\mathrm{U}$ & 360 \\
\hline 4-Nitroaniline & 910 & $U$ & 910 & 890 & $\mathrm{U}$ & 890 & 950 & $\mathrm{U}$ & 950 & 890 & $\mathrm{U}$ & 890 \\
\hline 4-Nitrophenol & 910 & $\mathrm{U}$ & 910 & 890 & $\mathrm{U}$ & 890 & 950 & U & 950 & 890 & $\mathrm{U}$ & 890 \\
\hline Acenaphthene & 360 & $\mathrm{U}$ & 360 & 350 & $\mathrm{U}$ & 350 & 380 & $\mathrm{U}$ & 380 & 360 & $\mathrm{U}$ & 360 \\
\hline \begin{tabular}{|l|} 
Acenaphthylene \\
\end{tabular} & 360 & $\mathrm{U}$ & 360 & 350 & $\mathrm{U}$ & 350 & 380 & $\mathrm{U}$ & 380 & 360 & $\mathrm{U}$ & 360 \\
\hline Anthracene & 360 & $\mathrm{U}$ & 360 & 350 & $\mathrm{U}$ & 350 & 380 & U & 380 & 360 & $\mathrm{U}$ & 360 \\
\hline Benzo(a)anthracene & 360 & $U$ & 360 & 350 & $\mathrm{U}$ & 350 & 380 & U & 380 & 360 & $\mathrm{U}$ & 360 \\
\hline Benzo(a)pyrene & 360 & $\mathrm{U}$ & 360 & 350 & $\mathrm{U}$ & 350 & 380 & U & 380 & 360 & $\mathrm{U}$ & 360 \\
\hline Benzo(b)fluoranthene & 360 & $\mathrm{U}$ & 360 & 350 & $\mathrm{U}$ & 350 & 380 & $\mathrm{U}$ & 380 & 360 & $\mathrm{U}$ & 360 \\
\hline Benzo(g,h,i)perylene & 360 & $U$ & 360 & 350 & $\mathrm{U}$ & 350 & 380 & $\mathrm{U}$ & 380 & 360 & $\mathrm{U}$ & 360 \\
\hline Benzo(k)fluoranthene & 360 & $\mathrm{U}$ & 360 & 350 & $U$ & 350 & 380 & $U$ & 380 & 360 & $\mathrm{U}$ & 360 \\
\hline Bis(2-chloro-1-methylethyl)ether & 360 & $\mathrm{U}$ & 360 & 350 & $U$ & 350 & 380 & $\mathrm{U}$ & 380 & 360 & $\mathrm{U}$ & 360 \\
\hline Bis(2-chloroethoxy)methane & 360 & $U$ & 360 & 350 & $\mathrm{U}$ & 350 & 380 & $\mathrm{U}$ & 380 & 360 & $\mathrm{U}$ & 360 \\
\hline \begin{tabular}{|l|} 
Bis(2-chloroethyl) ether \\
\end{tabular} & 360 & $\mathrm{U}$ & 360 & 350 & $\mathrm{U}$ & 350 & 380 & $\mathrm{U}$ & 380 & 360 & $\mathrm{U}$ & 360 \\
\hline Bis(2-ethylhexyl) phthalate & 24 & $\mathrm{JB}$ & 360 & 350 & $U$ & 350 & 22 & $\mathrm{JB}$ & 380 & 29 & $\mathrm{JB}$ & 360 \\
\hline Butylbenzylphthalate & 360 & $\mathrm{U}$ & 360 & 350 & $\mathrm{U}$ & 350 & 380 & $\mathrm{U}$ & 380 & 360 & $\mathrm{U}$ & 360 \\
\hline Carbazole & 360 & $\mathrm{U}$ & 360 & 350 & $\mathrm{U}$ & 350 & 380 & $\mathrm{U}$ & 380 & 360 & $\mathrm{U}$ & 360 \\
\hline Chrysene & 360 & U & 360 & 350 & $U$ & 350 & 380 & $\mathrm{U}$ & 380 & 360 & $\mathrm{U}$ & 360 \\
\hline Dibenz(a,h)anthracene & 360 & $\mathrm{U}$ & 360 & 350 & $U$ & 350 & 380 & $\mathrm{U}$ & 380 & 360 & $\mathrm{U}$ & 360 \\
\hline \begin{tabular}{|l|} 
Dibenzofuran \\
\end{tabular} & 360 & $\mathrm{U}$ & 360 & 350 & $\mathrm{U}$ & 350 & 380 & U & 380 & 360 & $\mathrm{U}$ & 360 \\
\hline Diethylphthalate & 360 & $U$ & 360 & 350 & U & 350 & 380 & $\mathrm{U}$ & 380 & 360 & $\mathrm{U}$ & 360 \\
\hline Dimethylphthalate & 360 & $\mathrm{U}$ & 360 & 350 & $\mathrm{U}$ & 350 & 380 & $\mathrm{U}$ & 380 & 360 & $\mathrm{U}$ & 360 \\
\hline \begin{tabular}{|l|} 
Di-n-butylphthalate \\
\end{tabular} & 360 & $\mathrm{U}$ & 360 & 350 & $U$ & 350 & 380 & $\mathrm{U}$ & 380 & 360 & $\mathrm{U}$ & 360 \\
\hline \begin{tabular}{|l|l} 
Di-n-octylphthalate \\
\end{tabular} & 360 & $\mathrm{U}$ & 360 & 350 & $U$ & 350 & 380 & $\mathrm{U}$ & 380 & 360 & $\mathrm{U}$ & 360 \\
\hline \begin{tabular}{|l|} 
Fluoranthene \\
\end{tabular} & 360 & $\mathrm{U}$ & 360 & 350 & $\mathrm{U}$ & 350 & 380 & U & 380 & 360 & $\mathrm{U}$ & 360 \\
\hline \begin{tabular}{|l|} 
Fluorene \\
\end{tabular} & 360 & $\mathrm{U}$ & 360 & 350 & $U$ & 350 & 380 & $\mathrm{U}$ & 380 & 360 & $\mathrm{U}$ & 360 \\
\hline Hexachlorobenzene & 360 & $U$ & 360 & 350 & $\mathrm{U}$ & 350 & 380 & $\mathrm{U}$ & 380 & 360 & $\mathrm{U}$ & 360 \\
\hline Hexachlorobutadiene & 360 & $\mathrm{U}$ & 360 & 350 & $\mathrm{U}$ & 350 & 380 & $\mathrm{U}$ & 380 & 360 & $\mathrm{U}$ & 360 \\
\hline Hexachlorocyclopentadiene & 360 & $\mathrm{U}$ & 360 & 350 & $U$ & 350 & 380 & $\mathrm{U}$ & 380 & 360 & $\mathrm{U}$ & 360 \\
\hline \begin{tabular}{|l|} 
Hexachloroethane \\
\end{tabular} & 360 & $\mathrm{U}$ & 360 & 350 & $\mathrm{U}$ & 350 & 380 & $\mathrm{U}$ & 380 & 360 & $\mathrm{U}$ & 360 \\
\hline Indeno( $1,2,3$-cd)pyrene & 360 & $\mathrm{U}$ & 360 & 350 & $\mathrm{U}$ & 350 & 380 & $\mathrm{U}$ & 380 & 360 & $\mathrm{U}$ & 360 \\
\hline \begin{tabular}{|l|} 
Isophorone \\
\end{tabular} & 360 & $\mathrm{U}$ & 360 & 350 & $U$ & 350 & 380 & $\mathrm{U}$ & 380 & 360 & $\mathrm{U}$ & 360 \\
\hline Naphthalene & 360 & $U$ & 360 & 350 & $U$ & 350 & 380 & $\mathrm{U}$ & 380 & 360 & U & 360 \\
\hline \begin{tabular}{|l|} 
Nitrobenzene \\
\end{tabular} & 360 & $U$ & 360 & 350 & $U$ & 350 & 380 & $\mathrm{U}$ & 380 & 360 & $\mathrm{U}$ & 360 \\
\hline \begin{tabular}{|l} 
N-Nitroso-di-n-dipropylamine \\
\end{tabular} & 360 & $U$ & 360 & 350 & $\mathrm{U}$ & 350 & 380 & U & 380 & 360 & $\mathrm{U}$ & 360 \\
\hline \begin{tabular}{|l|} 
N-Nitrosodiphenylamine \\
\end{tabular} & 360 & $U$ & 360 & 350 & $\mathrm{U}$ & 350 & 380 & $\mathrm{U}$ & 380 & 360 & $\mathrm{U}$ & 360 \\
\hline Pentachlorophenol & 910 & $\mathrm{U}$ & 910 & 890 & $\mathrm{U}$ & 890 & 950 & $\mathrm{U}$ & 950 & 890 & $\mathrm{U}$ & 890 \\
\hline \begin{tabular}{|l|} 
Phenanthrene \\
\end{tabular} & 360 & $U$ & 360 & 350 & $\mathrm{U}$ & 350 & 380 & $U$ & 380 & 360 & $U$ & 360 \\
\hline \begin{tabular}{|l|} 
Phenol \\
\end{tabular} & 360 & $U$ & 360 & 350 & $\mathrm{U}$ & 350 & 380 & $U$ & 380 & 360 & $U$ & 360 \\
\hline Pyrene & 360 & $U$ & 360 & 350 & $U$ & 350 & 380 & $\mathrm{U}$ & 380 & 360 & $U$ & 360 \\
\hline & & & & & Atta & ament & 1 & & & Sheet No & & 5 of 11 \\
\hline & & & & & Orig & ator & H. M. Sul & oway & & Date & & $4 / 30 / 08$ \\
\hline & & & & & Che & & L. D. Hab & & & Date & & $4 / 30 / 08$ \\
\hline & & & & & Calc & & $0100 \mathrm{~F}-\mathrm{C} A$ & $\mathrm{a-V0}$ & & Rev. No. & & 0 \\
\hline
\end{tabular}


Attachment 1. 128-F-2, Area A Verification Sampling Results.

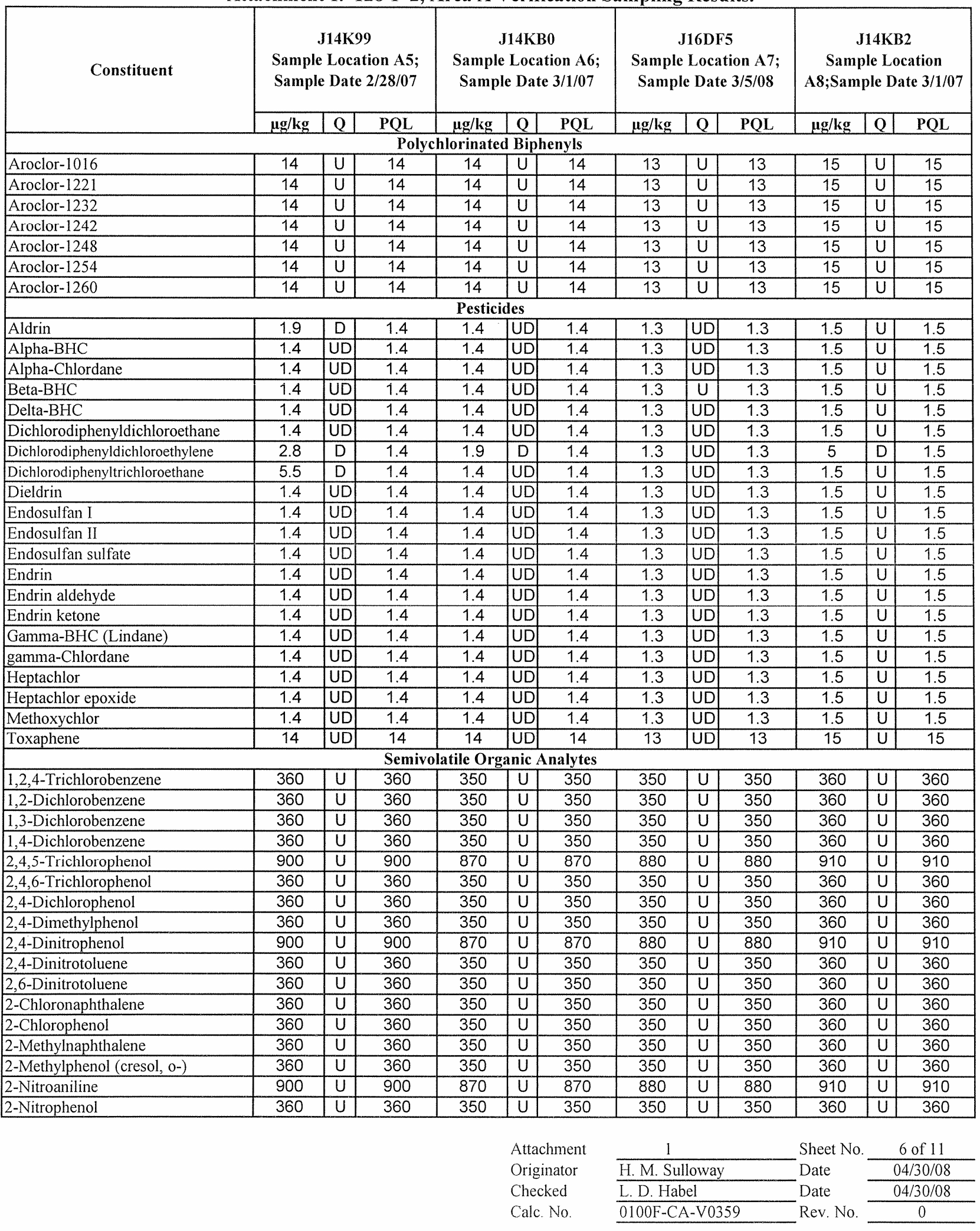


Attachment 1. 128-F-2, Area A Verification Sampling Results.

\begin{tabular}{|c|c|c|c|c|c|c|c|c|c|c|c|c|}
\hline \multirow[t]{2}{*}{ Constituent } & \multicolumn{3}{|c|}{$\begin{array}{c}\text { J14K99 } \\
\text { Sample Location A5; } \\
\text { Sample Date } 2 / 28 / 07\end{array}$} & \multicolumn{3}{|c|}{$\begin{array}{c}\text { J14KB0 } \\
\text { Sample Location A6; } \\
\text { Sample Date } 3 / 1 / 07\end{array}$} & \multicolumn{3}{|c|}{$\begin{array}{c}\text { J16DF5 } \\
\text { Sample Location A7; } \\
\text { Sample Date 3/5/08 }\end{array}$} & \multicolumn{3}{|c|}{$\begin{array}{c}\text { J14KB2 } \\
\text { Sample Location } \\
\text { A8;Sample Date 3/1/07 }\end{array}$} \\
\hline & $\mu \mathrm{g} / \mathrm{kg}$ & $\mathbf{Q}$ & PQL & $\mu \mathrm{g} / \mathrm{kg}$ & \begin{tabular}{|l|l|}
$Q$ & \\
\end{tabular} & PQL & $\mu \mathrm{g} / \mathrm{kg}$ & $Q$ & PQL & \begin{tabular}{l|l}
$\mu \mathrm{g} / \mathrm{kg}$ & \\
\end{tabular} & $Q$ & PQL \\
\hline \multicolumn{13}{|c|}{ Semivolatile Organic Analytes (continued) } \\
\hline 3,3'-Dichlorobenzidine & 360 & $\mathrm{U}$ & 360 & 350 & U & 350 & 350 & $\mathrm{U}$ & 350 & 360 & $\mathrm{U}$ & 360 \\
\hline 4-Methylphenol (p-cresol) & 360 & U & 360 & 350 & $\mathrm{U}$ & 350 & 350 & $\mathrm{U}$ & 350 & 360 & $U$ & 360 \\
\hline 3-Nitroaniline & 900 & U & 900 & 870 & $\mathrm{U}$ & 870 & 880 & $U$ & 880 & 910 & $U$ & 910 \\
\hline \begin{tabular}{|l|} 
4,6-Dinitro-2-methylphenol \\
\end{tabular} & 900 & $U$ & 900 & 870 & $\mathrm{U}$ & 870 & 880 & $U$ & 880 & 910 & $\mathrm{U}$ & 910 \\
\hline 4-Bromophenyl-phenylether & 360 & U & 360 & 350 & $\mathrm{U}$ & 350 & 350 & $\mathrm{U}$ & 350 & 360 & $U$ & 360 \\
\hline \begin{tabular}{|l|} 
4-Chloro-3-methylphenol \\
\end{tabular} & 360 & $\mathrm{U}$ & 360 & 350 & $\mathrm{U}$ & 350 & 350 & $\mathrm{U}$ & 350 & 360 & $U$ & 360 \\
\hline 4-Chloroaniline & 360 & $\mathrm{U}$ & 360 & 350 & $\mathrm{U}$ & 350 & 350 & U & 350 & 360 & $\mathrm{U}$ & 360 \\
\hline 4-Chlorophenyl-phenylether & 360 & $\mathrm{U}$ & 360 & 350 & $\mathrm{U}$ & 350 & 350 & $\bar{U}$ & 350 & 360 & $u$ & 360 \\
\hline \begin{tabular}{|l} 
4-Nitroaniline \\
\end{tabular} & 900 & $\mathrm{U}$ & 900 & 870 & $U$ & 870 & 880 & $U$ & 880 & 910 & $U$ & 910 \\
\hline \begin{tabular}{|l|} 
4-Nitrophenol \\
\end{tabular} & 900 & U & 900 & 870 & $\mathrm{U}$ & 870 & 880 & $\mathrm{U}$ & 880 & 910 & $\mathrm{U}$ & 910 \\
\hline \begin{tabular}{|l} 
Acenaphthene \\
\end{tabular} & 360 & U & 360 & 350 & $\mathrm{U}$ & 350 & 350 & $U$ & 350 & 360 & $\mathrm{U}$ & 360 \\
\hline \begin{tabular}{|l} 
Acenaphthylene \\
\end{tabular} & 360 & U & 360 & 350 & $\mathrm{U}$ & 350 & 350 & $\mathrm{U}$ & 350 & 360 & $U$ & 360 \\
\hline \begin{tabular}{|l} 
Anthracene \\
\end{tabular} & 360 & U & 360 & 350 & $\mathrm{U}$ & 350 & 350 & $U$ & 350 & 360 & $\mathrm{U}$ & 360 \\
\hline Benzo(a)anthracene & 360 & $\mathrm{U}$ & 360 & 350 & $\mathrm{U}$ & 350 & 350 & U & 350 & 360 & $\mathrm{U}$ & 360 \\
\hline Benzo(a)pyrene & 360 & $\mathrm{U}$ & 360 & 350 & U & 350 & 350 & $U$ & 350 & 360 & $U$ & 360 \\
\hline Benzo(b)fluoranthene & 360 & $\mathrm{U}$ & 360 & 350 & U & 350 & 350 & $\mathrm{U}$ & 350 & 360 & $U$ & 360 \\
\hline Benzo(g,h,i)perylene & 360 & $\mathrm{U}$ & 360 & 350 & $U$ & 350 & 350 & $\mathrm{U}$ & 350 & 360 & U & 360 \\
\hline Benzo(k)fluoranthene & 360 & $U$ & 360 & 350 & $\mathrm{U}$ & 350 & 350 & $\mathrm{U}$ & 350 & 360 & $\mathrm{U}$ & 360 \\
\hline Bis(2-chloro-1-methylethyl)ether & 360 & $U$ & 360 & 350 & $U$ & 350 & 350 & $\mathrm{U}$ & 350 & 360 & $\mathrm{U}$ & 360 \\
\hline Bis(2-chloroethoxy)methane & 360 & $\mathrm{U}$ & 360 & 350 & U & 350 & 350 & $U$ & 350 & 360 & $U$ & 360 \\
\hline \begin{tabular}{|l} 
Bis(2-chloroethyl) ether \\
\end{tabular} & 360 & U & 360 & 350 & $\mathrm{U}$ & 350 & 350 & U & 350 & 360 & $U$ & 360 \\
\hline \begin{tabular}{|l} 
Bis(2-ethylhexyl) phthalate \\
\end{tabular} & 23 & $\mathrm{JB}$ & 360 & 35 & $\mathrm{JB}$ & 350 & 26 & $\mathrm{JB}$ & 350 & 33 & $\mathrm{JB}$ & 360 \\
\hline Butylbenzylphthalate & 360 & $U$ & 360 & 350 & $U$ & 350 & 350 & $\mathrm{U}$ & 350 & 360 & U & 360 \\
\hline Carbazole & 360 & $\mathrm{U}$ & 360 & 350 & $U$ & 350 & 350 & $U$ & 350 & 360 & $U$ & 360 \\
\hline \begin{tabular}{|l|} 
Chrysene \\
\end{tabular} & 360 & $U$ & 360 & 350 & $u$ & 350 & 350 & $\mathrm{U}$ & 350 & 360 & $\mathrm{U}$ & 360 \\
\hline Dibenz(a,h)anthracene & 360 & $U$ & 360 & 32 & $\mathrm{~J}$ & 350 & 350 & U & 350 & 360 & $\mathrm{U}$ & 360 \\
\hline \begin{tabular}{|l|} 
Dibenzofuran \\
\end{tabular} & 360 & $U$ & 360 & 350 & $\mathrm{U}$ & 350 & 350 & $\mathrm{U}$ & 350 & 360 & $U$ & 360 \\
\hline Diethylphthalate & 360 & U & 360 & 350 & $U$ & 350 & 350 & $\mathrm{U}$ & 350 & 360 & $U$ & 360 \\
\hline Dimethylphthalate & 360 & $\mathrm{U}$ & 360 & 350 & $\bar{U}$ & 350 & 350 & $\mathrm{U}$ & 350 & 360 & U & 360 \\
\hline Di-n-butylphthalate & 360 & U & 360 & 350 & U & 350 & 350 & $\mathrm{U}$ & 350 & 360 & U & 360 \\
\hline \begin{tabular}{|l|} 
Di-n-octylphthalate \\
\end{tabular} & 360 & $\mathrm{U}$ & 360 & 350 & U & 350 & 350 & $\mathrm{U}$ & 350 & 360 & $\mathrm{U}$ & 360 \\
\hline \begin{tabular}{|l} 
Fluoranthene \\
\end{tabular} & 360 & $\mathrm{U}$ & 360 & 350 & $U$ & 350 & 350 & $U$ & 350 & 360 & U & 360 \\
\hline \begin{tabular}{|l|} 
Fluorene \\
\end{tabular} & 360 & U & 360 & 350 & U & 350 & 350 & $\mathrm{U}$ & 350 & 360 & $\mathrm{U}$ & 360 \\
\hline Hexachlorobenzene & 360 & U & 360 & 350 & $\mathrm{U}$ & 350 & 350 & $\mathrm{U}$ & 350 & 360 & $\mathrm{U}$ & 360 \\
\hline Hexachlorobutadiene & 360 & $U$ & 360 & 350 & $\mathrm{U}$ & 350 & 350 & $\mathrm{U}$ & 350 & 360 & U & 360 \\
\hline Hexachlorocyclopentadiene & 360 & U & 360 & 350 & $U$ & 350 & 350 & $U$ & 350 & 360 & $\mathrm{U}$ & 360 \\
\hline \begin{tabular}{|l} 
Hexachloroethane \\
\end{tabular} & 360 & U & 360 & 350 & $\mathrm{U}$ & 350 & 350 & $U$ & 350 & 360 & $\mathrm{U}$ & 360 \\
\hline Indeno(1,2,3-cd)pyrene & 360 & $\mathrm{U}$ & 360 & 350 & $U$ & 350 & 350 & $\mathrm{U}$ & 350 & 360 & $\bar{U}$ & 360 \\
\hline Isophorone & 360 & $\mathrm{U}$ & 360 & 350 & U & 350 & 350 & $U$ & 350 & 360 & $\mathrm{U}$ & 360 \\
\hline Naphthalene & 360 & $\mathrm{U}$ & 360 & 350 & U & 350 & 350 & $\mathrm{U}$ & 350 & 360 & $U$ & 360 \\
\hline \begin{tabular}{|l} 
Nitrobenzene \\
\end{tabular} & 360 & $\mathrm{U}$ & 360 & 350 & $U$ & 350 & 350 & $U$ & 350 & 360 & $\mathrm{U}$ & 360 \\
\hline N-Nitroso-di-n-dipropylamine & 360 & $U$ & 360 & 350 & $\mathrm{U}$ & 350 & 350 & $U$ & 350 & 360 & $\mathrm{U}$ & 360 \\
\hline N-Nitrosodiphenylamine & 360 & $U$ & 360 & 350 & $\bar{U}$ & 350 & 350 & $U$ & 350 & 360 & $U$ & 360 \\
\hline Pentachlorophenol & 900 & $\mathrm{U}$ & 900 & 870 & $U$ & 870 & 880 & $U$ & 880 & 910 & $U$ & 910 \\
\hline \begin{tabular}{|l|} 
Phenanthrene \\
\end{tabular} & 360 & $U$ & 360 & 350 & $U$ & 350 & 350 & $\mathrm{U}$ & 350 & 360 & $U$ & 360 \\
\hline Phenol & 360 & $\mathrm{U}$ & 360 & 350 & $U$ & 350 & 350 & $\mathrm{U}$ & 350 & 360 & $U$ & 360 \\
\hline Pyrene & 360 & $U$ & 360 & 350 & $U$ & 350 & 350 & $U$ & 350 & 360 & $\mathrm{U}$ & 360 \\
\hline & & & & & Attac & ament & 1 & & & Sheet No. & & $f 11$ \\
\hline & & & & & Origi & lator & H. M. Sull & oway & & & & $4 / 30 / 08$ \\
\hline & & & & & Chec & & L. D. Habe & & & Date & & $4 / 30 / 08$ \\
\hline & & & & & Calc. & & $0100 \mathrm{~F}-\mathrm{CA}$ & $\mathrm{VO}$ & & Rev. No. & & 0 \\
\hline
\end{tabular}


Attachment 1. 128-F-2, Area A Verification Sampling Results.




Attachment 1. 128-F-2, Area A Verification Sampling Results.

\begin{tabular}{|c|c|c|c|c|c|c|c|c|c|c|c|c|}
\hline \multirow[t]{2}{*}{ Constituent } & \multicolumn{3}{|c|}{$\begin{array}{c}\text { J14KB3 } \\
\text { Sample Location A9; } \\
\text { Sample Date 3/1/07 }\end{array}$} & \multicolumn{3}{|c|}{$\begin{array}{c}\text { J14KB4 } \\
\text { Sample Location A10; } \\
\text { Sample Date 3/5/07 }\end{array}$} & \multicolumn{3}{|c|}{$\begin{array}{c}\text { J14KB5 } \\
\text { Sample Location A11; } \\
\text { Sample Date 3/5/07 }\end{array}$} & \multicolumn{3}{|c|}{$\begin{array}{c}\text { J14KB6 } \\
\text { Dup of J14KB5 } \\
\text { Sample Date } 3 / 5 / 07\end{array}$} \\
\hline & $\mu \mathrm{g} / \mathrm{kg}$ & $Q$ & PQL & $\mu \mathrm{g} / \mathrm{kg}$ & $\mathbf{Q}$ & PQL & $\mu \mathrm{g} / \mathrm{kg}$ & $Q$ & PQL & $\mu \mathrm{g} / \mathrm{kg}$ & $Q$ & PQL \\
\hline \multicolumn{13}{|c|}{ Semivolatile Organic Analytes (continued) } \\
\hline 3,3'-Dichlorobenzidine & 360 & $\mathrm{U}$ & 360 & 370 & U & 370 & 380 & $\mathrm{U}$ & 380 & 380 & $U$ & 380 \\
\hline 4-Methylphenol (p-cresol) & 360 & $\mathrm{U}$ & 360 & 370 & $U$ & 370 & 380 & $\mathrm{U}$ & 380 & 380 & $\mathrm{U}$ & 380 \\
\hline 3-Nitroaniline & 910 & $\mathrm{U}$ & 910 & 940 & $\mathrm{U}$ & 940 & 940 & $\mathrm{U}$ & 940 & 950 & $\mathrm{U}$ & 950 \\
\hline 4,6-Dinitro-2-methylphenol & 910 & $\mathrm{U}$ & 910 & 940 & $\mathrm{U}$ & 940 & 940 & $\mathrm{U}$ & 940 & 950 & $\mathrm{U}$ & 950 \\
\hline 4-Bromophenyl-phenylether & 360 & $\mathrm{U}$ & 360 & 370 & $\mathrm{U}$ & 370 & 380 & $\mathrm{U}$ & 380 & 380 & $U$ & 380 \\
\hline 4-Chloro-3-methylphenol & 360 & $U$ & 360 & 370 & $\mathrm{U}$ & 370 & 380 & $\mathrm{U}$ & 380 & 380 & $\mathrm{U}$ & 380 \\
\hline 4-Chloroaniline & 360 & $\mathrm{U}$ & 360 & 370 & $\mathrm{U}$ & 370 & 380 & $\mathrm{U}$ & 380 & 380 & U & 380 \\
\hline 4-Chlorophenyl-phenylether & 360 & $\mathrm{U}$ & 360 & 370 & $\mathrm{U}$ & 370 & 380 & $\mathrm{U}$ & 380 & 380 & $\begin{array}{ll}U \\
\end{array}$ & 380 \\
\hline 4-Nitroaniline & 910 & $\mathrm{U}$ & 910 & 940 & $\mathrm{U}$ & 940 & 940 & $\mathrm{U}$ & 940 & 950 & $U$ & 950 \\
\hline 4-Nitrophenol & 910 & $\mathrm{U}$ & 910 & 940 & $\mathrm{U}$ & 940 & 940 & $\mathrm{U}$ & 940 & 950 & $\mathrm{U}$ & 950 \\
\hline Acenaphthene & 360 & $\mathrm{U}$ & 360 & 370 & $\mathrm{U}$ & 370 & 380 & $\mathrm{U}$ & 380 & 380 & $\mathrm{U}$ & 380 \\
\hline \begin{tabular}{|l|} 
Acenaphthylene \\
\end{tabular} & 360 & $\mathrm{U}$ & 360 & 370 & U & 370 & 380 & $\mathrm{U}$ & 380 & 380 & U & 380 \\
\hline Anthracene & 360 & $U$ & 360 & 370 & U & 370 & 380 & $\mathrm{U}$ & 380 & 380 & $U$ & 380 \\
\hline Benzo(a)anthracene & 360 & $\mathrm{U}$ & 360 & 370 & U & 370 & 380 & $\mathrm{U}$ & 380 & 380 & $\mathrm{U}$ & 380 \\
\hline Benzo(a)pyrene & 360 & $U$ & 360 & 370 & $\mathrm{U}$ & 370 & 380 & $\mathrm{U}$ & 380 & 380 & U & 380 \\
\hline Benzo(b)fluoranthene & 360 & $\mathrm{U}$ & 360 & 370 & $\mathrm{U}$ & 370 & 380 & $\mathrm{U}$ & 380 & 380 & $\mathrm{U}$ & 380 \\
\hline Benzo(g,h,i)perylene & 360 & U & 360 & 370 & U & 370 & 380 & $\mathrm{U}$ & 380 & 380 & $\mathrm{U}$ & 380 \\
\hline Benzo(k)fluoranthene & 360 & U & 360 & 370 & $\mathrm{U}$ & 370 & 380 & $\mathrm{U}$ & 380 & 380 & $\mathrm{U}$ & 380 \\
\hline Bis(2-chloro-1-methylethyl)ether & 360 & $U$ & 360 & 370 & $\mathrm{U}$ & 370 & 380 & $\mathrm{U}$ & 380 & 380 & U & 380 \\
\hline Bis(2-chloroethoxy)methane & 360 & $\mathrm{U}$ & 360 & 370 & $U$ & 370 & 380 & $U$ & 380 & 380 & $\mathrm{U}$ & 380 \\
\hline Bis(2-chloroethyl) ether & 360 & $\mathrm{U}$ & 360 & 370 & $\mathrm{U}$ & 370 & 380 & $\mathrm{U}$ & 380 & 380 & $\mathrm{U}$ & 380 \\
\hline Bis(2-ethylhexyl) phthalate & 97 & $\mathrm{JB}$ & 360 & 57 & $\mathrm{JB}$ & 370 & 85 & $\mathrm{JB}$ & 380 & 59 & $\mathrm{JB}$ & 380 \\
\hline Butylbenzylphthalate & 360 & $\mathrm{U}$ & 360 & 370 & $U$ & 370 & 380 & $\mathrm{U}$ & 380 & 380 & U & 380 \\
\hline Carbazole & 360 & $\mathrm{U}$ & 360 & 370 & $\mathrm{U}$ & 370 & 380 & $U$ & 380 & 380 & U & 380 \\
\hline Chrysene & 360 & $\mathrm{U}$ & 360 & 370 & $U$ & 370 & 380 & $U$ & 380 & 380 & $\mathrm{U}$ & 380 \\
\hline \begin{tabular}{|l} 
Dibenz $(\mathrm{a}, \mathrm{h})$ anthracene \\
\end{tabular} & 19 & $\mathrm{~J}$ & 360 & 31 & $\mathrm{~J}$ & 370 & 48 & $\mathrm{~J}$ & 380 & 27 & $\mathrm{~J}$ & 380 \\
\hline \begin{tabular}{|l|} 
Dibenzofuran \\
\end{tabular} & 360 & $\mathrm{U}$ & 360 & 370 & $\mathrm{U}$ & 370 & 380 & $\mathrm{U}$ & 380 & 380 & U & 380 \\
\hline \begin{tabular}{|l|} 
Diethylphthalate \\
\end{tabular} & 360 & $\mathrm{U}$ & 360 & 370 & $U$ & 370 & 380 & $\mathrm{U}$ & 380 & 380 & U & 380 \\
\hline Dimethylphthalate & 360 & $U$ & 360 & 370 & $\mathrm{U}$ & 370 & 380 & $\mathrm{U}$ & 380 & 380 & $\mathrm{U}$ & 380 \\
\hline Di-n-butylphthalate & 360 & U & 360 & 370 & $\mathrm{U}$ & 370 & 380 & $\mathrm{U}$ & 380 & 380 & $\mathrm{U}$ & 380 \\
\hline Di-n-octylphthalate & 360 & U & 360 & 370 & $\mathrm{U}$ & 370 & 380 & $\mathrm{U}$ & 380 & 380 & $\mathrm{U}$ & 380 \\
\hline \begin{tabular}{|l|} 
Fluoranthene \\
\end{tabular} & 360 & $\mathrm{U}$ & 360 & 370 & $\mathrm{U}$ & 370 & 380 & $\mathrm{U}$ & 380 & 380 & $\mathrm{U}$ & 380 \\
\hline Fluorene & 360 & $\mathrm{U}$ & 360 & 370 & $\mathrm{U}$ & 370 & 380 & $U$ & 380 & 380 & U & 380 \\
\hline Hexachlorobenzene & 360 & $\mathrm{U}$ & 360 & 370 & $\mathrm{U}$ & 370 & 380 & $\mathrm{U}$ & 380 & 380 & $\mathrm{U}$ & 380 \\
\hline Hexachlorobutadiene & 360 & $\mathrm{U}$ & 360 & 370 & $\mathrm{U}$ & 370 & 380 & $\mathrm{U}$ & 380 & 380 & U & 380 \\
\hline Hexachlorocyclopentadiene & 360 & $U$ & 360 & 370 & $\mathrm{U}$ & 370 & 380 & $\mathrm{U}$ & 380 & 380 & $\mathrm{U}$ & 380 \\
\hline Hexachloroethane & 360 & $\mathrm{U}$ & 360 & 370 & $\mathrm{U}$ & 370 & 380 & $U$ & 380 & 380 & $\mathrm{U}$ & 380 \\
\hline Indeno(1,2,3-cd)pyrene & 360 & $U$ & 360 & 370 & $\mathrm{U}$ & 370 & 380 & $\mathrm{U}$ & 380 & 380 & 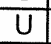 & 380 \\
\hline Isophorone & 360 & $\mathrm{U}$ & 360 & 370 & $\mathrm{U}$ & 370 & 380 & $\mathrm{U}$ & 380 & 380 & $\mathrm{U}$ & 380 \\
\hline Naphthalene & 360 & $\mathrm{U}$ & 360 & 370 & $\mathrm{U}$ & 370 & 380 & $\mathrm{U}$ & 380 & 380 & U & 380 \\
\hline \begin{tabular}{|l} 
Nitrobenzene \\
\end{tabular} & 360 & $\mathrm{U}$ & 360 & 370 & $\mathrm{U}$ & 370 & 380 & $\mathrm{U}$ & 380 & 380 & $\mathrm{U}$ & 380 \\
\hline N-Nitroso-di-n-dipropylamine & 360 & $\mathrm{U}$ & 360 & 370 & $\mathrm{U}$ & 370 & 380 & $\mathrm{U}$ & 380 & 380 & $\mathrm{U}$ & 380 \\
\hline N-Nitrosodiphenylamine & 360 & $\mathrm{U}$ & 360 & 370 & $\mathrm{U}$ & 370 & 380 & $\mathrm{U}$ & 380 & 380 & $U$ & 380 \\
\hline Pentachlorophenol & 910 & $\mathrm{U}$ & 910 & 940 & $\mathrm{U}$ & 940 & 940 & $\mathrm{U}$ & 940 & 950 & U & 950 \\
\hline \begin{tabular}{|l|} 
Phenanthrene \\
\end{tabular} & 360 & $\mathrm{U}$ & 360 & 370 & $\mathrm{U}$ & 370 & 380 & $\mathrm{U}$ & 380 & 380 & $\mathrm{U}$ & 380 \\
\hline Phenol & 360 & $U$ & 360 & 370 & $U$ & 370 & 380 & $\mathrm{U}$ & 380 & 380 & $\mathrm{U}$ & 380 \\
\hline Pyrene & 360 & $\mathrm{U}$ & 360 & 370 & $U$ & 370 & 380 & $\mathrm{U}$ & 380 & 380 & $\mathrm{U}$ & 380 \\
\hline & & & & & Attac & hment & 1 & & & Sheet No. & & of 11 \\
\hline & & & & & Orig & nator & H. M. Sull & way & & Date & & $4 / 30 / 08$ \\
\hline & & & & & Chec & & L. D. Hab & & & & & $4 / 30 / 08$ \\
\hline & & & & & Calc & & $0100 \mathrm{~F}-\mathrm{CA}$ & -V03. & & Rev. No. & & 0 \\
\hline
\end{tabular}


Attachment 1. 100-F-26:8 Verification Sampling Results.

\begin{tabular}{|c|c|c|c|}
\hline \multirow[t]{2}{*}{ Constituent } & \multicolumn{3}{|c|}{$\begin{array}{c}\text { J14KB7 } \\
\text { Sample Location A12; } \\
\text { Sample Date 3/5/07 }\end{array}$} \\
\hline & $\mu \mathrm{g} / \mathrm{kg}$ & $\mathbf{Q}$ & PQL \\
\hline \multicolumn{4}{|c|}{ Polychlorinated Biphenyls } \\
\hline Aroclor-1016 & 15 & $\mathrm{U}$ & 15 \\
\hline Aroclor-1221 & 15 & $\mathrm{U}$ & 15 \\
\hline Aroclor-1232 & 15 & $\mathrm{U}$ & 15 \\
\hline Aroclor-1242 & 15 & $\mathrm{U}$ & 15 \\
\hline Aroclor-1248 & 15 & $\mathrm{U}$ & 15 \\
\hline Aroclor-1254 & 15 & $\mathrm{U}$ & 15 \\
\hline Aroclor-1260 & 15 & $\mathrm{U}$ & 15 \\
\hline \multicolumn{4}{|c|}{ Pesticides } \\
\hline \begin{tabular}{|l|l|} 
Aldrin \\
\end{tabular} & 1.5 & $\mathrm{D}$ & 1.5 \\
\hline Alpha-BHC & 1.5 & UD & 1.5 \\
\hline Alpha-Chlordane & 1.5 & UD & 1.5 \\
\hline Beta-BHC & 1.5 & UD & 1.5 \\
\hline Delta-BHC & 1.5 & UD & 1.5 \\
\hline Dichlorodiphenyldichloroethane & 1.5 & UD & 1.5 \\
\hline \begin{tabular}{|l|} 
Dichlorodiphenyldichloroethylene \\
\end{tabular} & 1.5 & UD & 1.5 \\
\hline \begin{tabular}{|l|} 
Dichlorodiphenyltrichloroethane \\
\end{tabular} & 1.5 & UD & 1.5 \\
\hline \begin{tabular}{|l} 
Dieldrin \\
\end{tabular} & 1.5 & UD & 1.5 \\
\hline Endosulfan I & 1.5 & UD & 1.5 \\
\hline Endosulfan II & 1.5 & UD & 1.5 \\
\hline \begin{tabular}{|l|} 
Endosulfan sulfate \\
\end{tabular} & 1.5 & UD & 1.5 \\
\hline \begin{tabular}{|l|} 
Endrin \\
\end{tabular} & 1.5 & UD & 1.5 \\
\hline Endrin aldehyde & 1.5 & UD & 1.5 \\
\hline Endrin ketone & 1.5 & UD & 1.5 \\
\hline Gamma-BHC (Lindane) & 1.5 & UD & 1.5 \\
\hline gamma-Chlordane & 1.5 & UD & 1.5 \\
\hline \begin{tabular}{|l} 
Heptachlor \\
\end{tabular} & 1.5 & UD & 1.5 \\
\hline Heptachlor epoxide & 1.5 & UD & 1.5 \\
\hline \begin{tabular}{|l} 
Methoxychlor \\
\end{tabular} & 1.5 & UD & 1.5 \\
\hline \begin{tabular}{|l|} 
Toxaphene \\
\end{tabular} & 15 & UD & 15 \\
\hline \multicolumn{4}{|c|}{ Semivolatile Organic Analytes } \\
\hline 1,2,4-Trichlorobenzene & 370 & $\mathrm{U}$ & 370 \\
\hline 1,2-Dichlorobenzene & 370 & $\mathrm{U}$ & 370 \\
\hline 1,3-Dichlorobenzene & 370 & $\mathrm{U}$ & 370 \\
\hline 1,4-Dichlorobenzene & 370 & $\mathrm{U}$ & 370 \\
\hline 2,4,5-Trichlorophenol & 930 & U & 930 \\
\hline 2,4,6-Trichlorophenol & 370 & $\mathrm{U}$ & 370 \\
\hline 2,4-Dichlorophenol & 370 & $\mathrm{U}$ & 370 \\
\hline 2,4-Dimethylphenol & 370 & $\mathrm{U}$ & 370 \\
\hline 2,4-Dinitrophenol & 930 & $U$ & 930 \\
\hline 2,4-Dinitrotoluene & 370 & $\mathrm{U}$ & 370 \\
\hline 2,6-Dinitrotoluene & 370 & $\mathrm{U}$ & 370 \\
\hline 2-Chloronaphthalene & 370 & $\mathrm{U}$ & 370 \\
\hline \begin{tabular}{|l|} 
2-Chlorophenol \\
\end{tabular} & 370 & $\mathrm{U}$ & 370 \\
\hline 2-Methylnaphthalene & 370 & $\mathrm{U}$ & 370 \\
\hline 2-Methylphenol (cresol, o-) & 370 & $\mathrm{U}$ & 370 \\
\hline 2-Nitroaniline & 930 & $\mathrm{U}$ & 930 \\
\hline 2-Nitrophenol & 370 & $\mathrm{U}$ & 370 \\
\hline
\end{tabular}

\begin{tabular}{|c|c|c|c|}
\hline Attachment & 1 & Sheet No & 10 of 11 \\
\hline Originator & H. M. Sulloway & Date & $04 / 30 / 08$ \\
\hline Checked & L. D. Habel & Date & $04 / 30 / 08$ \\
\hline Calc. No. & $0100 \mathrm{~F}-\mathrm{CA}-\mathrm{V} 0359$ & Rev. No. & 0 \\
\hline
\end{tabular}


Attachment 1. 100-F-26:8 Verification Sampling Results.

\begin{tabular}{|c|c|c|c|}
\hline \multirow[t]{2}{*}{ Constituent } & \multicolumn{3}{|c|}{$\begin{array}{c}\text { J14KB7 } \\
\text { Sample Location A12; } \\
\text { Sample Date 3/5/07 }\end{array}$} \\
\hline & $\mu \mathrm{g} / \mathrm{kg}$ & Q & PQL \\
\hline \multicolumn{4}{|c|}{ Semivolatile Organic Analytes (continued) } \\
\hline 3,3'-Dichlorobenzidine & 370 & $\mathrm{U}$ & 370 \\
\hline 4-Methylphenol (p-cresol) & 370 & $\mathrm{U}$ & 370 \\
\hline 3-Nitroaniline & 930 & $\mathrm{U}$ & 930 \\
\hline 4,6-Dinitro-2-methylphenol & 930 & $\mathrm{U}$ & 930 \\
\hline 4-Bromophenyl-phenylether & 370 & $\mathrm{U}$ & 370 \\
\hline 4-Chloro-3-methylphenol & 370 & $\mathrm{U}$ & 370 \\
\hline \begin{tabular}{|l|} 
4-Chloroaniline \\
\end{tabular} & 370 & $\mathrm{U}$ & 370 \\
\hline 4-Chlorophenyl-phenylether & 370 & $U$ & 370 \\
\hline 4-Nitroaniline & 930 & U & 930 \\
\hline 4-Nitrophenol & 930 & $\mathrm{U}$ & 930 \\
\hline Acenaphthene & 370 & $\mathrm{U}$ & 370 \\
\hline Acenaphthylene & 370 & $\mathrm{U}$ & 370 \\
\hline Anthracene & 370 & $\mathrm{U}$ & 370 \\
\hline Benzo(a)anthracene & 370 & $\mathrm{U}$ & 370 \\
\hline Benzo(a)pyrene & 370 & $U$ & 370 \\
\hline Benzo(b)fluoranthene & 370 & $\mathrm{U}$ & 370 \\
\hline Benzo(g,h,i)perylene & 370 & $\mathrm{U}$ & 370 \\
\hline Benzo(k)fluoranthene & 370 & $\mathrm{U}$ & 370 \\
\hline Bis(2-chloro-1-methylethyl)ether & 370 & $\mathrm{U}$ & 370 \\
\hline Bis(2-chloroethoxy)methane & 370 & $U$ & 370 \\
\hline Bis(2-chloroethyl) ether & 370 & $\mathrm{U}$ & 370 \\
\hline Bis(2-ethylhexyl) phthalate & 55 & $\mathrm{JB}$ & 370 \\
\hline Butylbenzylphthalate & 370 & $\mathrm{U}$ & 370 \\
\hline Carbazole & 370 & $U$ & 370 \\
\hline Chrysene & 370 & $U$ & 370 \\
\hline Dibenz $(\mathrm{a}, \mathrm{h})$ anthracene & 27 & $\mathrm{~J}$ & 370 \\
\hline Dibenzofuran & 370 & $\mathrm{U}$ & 370 \\
\hline Diethylphthalate & 370 & $\mathrm{U}$ & 370 \\
\hline Dimethylphthalate & 370 & U & 370 \\
\hline Di-n-butylphthalate & 370 & $\mathrm{U}$ & 370 \\
\hline Di-n-octylphthalate & 370 & U & 370 \\
\hline Fluoranthene & 370 & U & 370 \\
\hline Fluorene & 370 & $\mathrm{U}$ & 370 \\
\hline Hexachlorobenzene & 370 & $\mathrm{U}$ & 370 \\
\hline Hexachlorobutadiene & 370 & $\mathrm{U}$ & 370 \\
\hline Hexachlorocyclopentadiene & 370 & $\mathrm{U}$ & 370 \\
\hline Hexachloroethane & 370 & $\mathrm{U}$ & 370 \\
\hline Indeno(1,2,3-cd)pyrene & 370 & $U$ & 370 \\
\hline Isophorone & 370 & $\mathrm{U}$ & 370 \\
\hline Naphthalene & 370 & U & 370 \\
\hline Nitrobenzene & 370 & $\mathrm{U}$ & 370 \\
\hline N-Nitroso-di-n-dipropylamine & 370 & $\mathrm{U}$ & 370 \\
\hline N-Nitrosodiphenylamine & 370 & $U$ & 370 \\
\hline Pentachlorophenol & 930 & $\mathrm{U}$ & 930 \\
\hline Phenanthrene & 370 & $\mathrm{U}$ & 370 \\
\hline Phenol & 370 & U & 370 \\
\hline Pyrene & 370 & U & 370 \\
\hline
\end{tabular}

\begin{tabular}{|c|c|c|c|}
\hline Attachment & 1 & Sheet No. & 11 of 11 \\
\hline Originator & H. M. Sulloway & Date & $04 / 30 / 08$ \\
\hline Checked & L. D. Habel & Date & $04 / 30 / 08$ \\
\hline Calc. No. & $0100 \mathrm{~F}-\mathrm{CA}-\mathrm{V} 0359$ & Rev. No. & 0 \\
\hline
\end{tabular}




\section{CALCULATION COVER SHEET}

Project Title: 100-F Field Remediation

Job No. 14655

Area: $100-\mathrm{F}$

Discipline: Environmental

*Calculation No: 0100F-CA-V0333

Subject: 128-F-2, Upland (Area B) Cleanup Verification 95\% UCL

Computer Program: Excel

Program No: Excel 2003

The attached calculations have been generated to document compliance with established cleanup levels. These calculations should be used in conjunction with other relevant documents in the administrative record.

Committed Calculation $\bar{x} \quad$ Preliminary $\Gamma \quad$ Superseded $\Gamma \quad$ Voided $\Gamma$

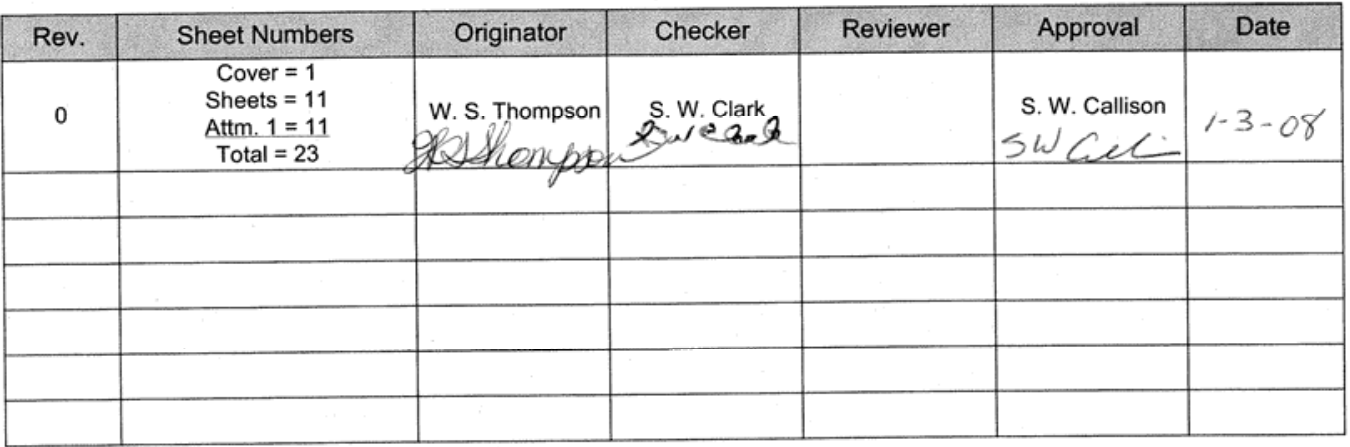

SUMMARY OF REVISION

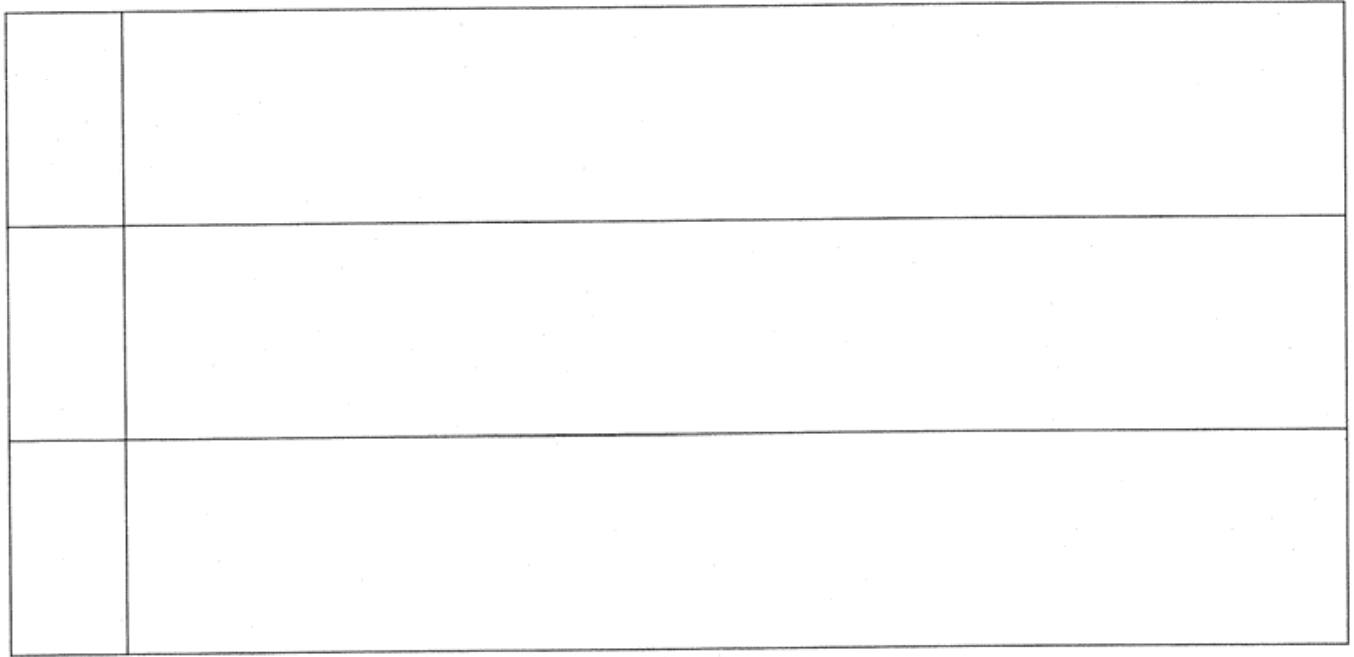

WCH-DE-018 (05/08/2007)

*Obtain Calc. No. from Document Control and Form from Intranet 


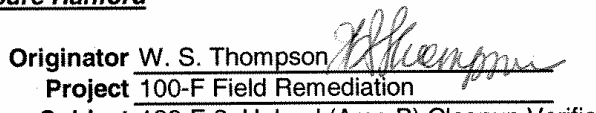

Calculate the $95 \%$ upper confidence limit (UCL) values to evaluate compliance with cleanup standards for the upland portion (Area $B$ ) 4 of the 128-F-2 waste site. Also, perform the Washington Administrative Code (WAC) 173-340-740(7)(e) Model Toxics Control Act (MTCA) 3-part test for nonradionuclide analytes and calculate the relative percent difference (RPD) for primary-duplicate sample pairs for each contaminant of concern (COC) and contaminant of potential concern (COPC), as necessary.

\section{Table of Contents:}

Sheets 1 to 3 - Calculation Sheet Summary

Sheets 4 to 6 - Calculation Sheet Shallow Zone Verification Data

Sheet 7 - Calculation Sheet Duplicate Analysis

Sheets 8 to 11 - Ecology Software (MTCAStat) Results

Attachment 1 - 128-F-2 Upland (Area B) Verification Sampling Results (11 sheets)

\section{Given/References:}

1) Sample Results (Attachment 1).

2) Background values and remedial action goals (RAGs) are taken from DOE-RL (2005b), DOE-RL (2001), and Ecology (1996).

3) DOE-RL, 2001, Hanford Site Background: Part 1, Soil Background for Nonradioactive Analytes, DOE/RL-92-24, Rev. 4, U.S. Department of Energy, Richland Operations Office, Richland, Washington.

4) DOE-RL, 2005a, 100 Area Remedial Action Sampling and Analysis Plan (SAP), DOE/RL-96-22, Rev. 4, U.S. Department of Energy, Richland Operations Office, Richland, Washington.

5) DOE-RL, 2005b, Remedial Design Report/Remedial Action Work Plan for the 100 Area (RDR/RAWP), DOE/RL-96-17, Rev. 5, U.S. Department of Energy, Richland Operations Office, Richland, Washington.

6) Ecology, 1992, Statistical Guidance for Ecology Site Managers, Publication \#92-54, Washington Department of Ecology, Olympia, Washington.

7) Ecology, 1993, Statistical Guidance for Ecology Site Managers, Supplement S-6, Analyzing Site or Background Data with Below-detection Limit or Below-PQL Values (Censored Data Sets), Publication \#92-54, Washington Department of Ecology, Olympia, Washington.

8) Ecology, 1996, Model Toxic Control Act Cleanup Levels and Risk Calculations (CLARC II), Publication \#94-145, Washington State Department of Ecology, Olympia, Washington.

9) Ecology, 2005, Cleanup Levels and Risk Calculations (CLARC) Database, Washington State Department of Ecology, Olympia, Washington, <https://fortress.wa.gov/ecy/clarc/CLARCHome.aspx>.

10) EPA, 1994, USEPA Contract Laboratory Program National Functional Guidelines for Inorganic Data Review, EPA 540/R-4/013. U.S. Environmental Protection Agency, Washington, D.C.

11) WAC 173-340, 1996, "Model Toxic Control Act - Cleanup," Washington Administrative Code.

Solution:

Calculation methodology is described in Ecology Pub. \#92-54 (Ecology 1992, 1993), below, and in the RDR/RAWP (DOE-RL 2005b). Use data from attached worksheets to perform the 95\% UCL calculation for each analyte, the WAC 173-340-740(7)(e) 3-part test for nonradionuclides, and the RPD calculations for each COC/COPC. The hazard quotient and carcinogenic risk calculations are located 6 in a separate calculation brief as an appendix to the Remaining Sites Verification Package (RSVP).

\section{Calculation Description:}

The subject calculations were performed on data from soil verification samples (Attachment 1) from the 128-F-2 Upland (Area B) waste site. The data were entered into an EXCEL 2003 spreadsheet and calculations performed by using the built-in spreadsheet 


\section{CALCULATION SHEET}

\begin{tabular}{|c|c|c|c|}
\hline 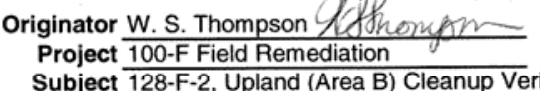 & $\begin{array}{l}\text { Date } \frac{12 / 20 / 07}{14655} \\
\text { Job No. }\end{array}$ & $\begin{array}{l}\text { Calc. No. } 0100-F-C A-V 0333 \\
\text { Checked S. W. Clark 2ule }\end{array}$ & $\begin{array}{l}\text { Rev. No. } \frac{0}{\text { Date } \frac{1 / 30 / 07}{2 \text { of } 11}} \\
\text { Sheet No. }\end{array}$ \\
\hline
\end{tabular}

1 Summary (continued)

2 Methodology:

3 For nonradioactive analytes with $\leq 50 \%$ of the data below detection limits and all detected radionuclide analytes, the statistical value calculated to evaluate the effectiveness of cleanup is the $95 \%$ UCL. For nonradioactive analytes with $>50 \%$ of the data below detection limits, as determined by direct inspection of the sample results (Attachment 1), the maximum detected value for the data set is used instead of the $95 \%$ UCL, and no further calculations are performed for those data sets. For convenience, these maximum detected values are included in the summary tables that follow. The $95 \%$ UCL was not calculated for data sets with no reported detections. Calculated cleanup levels are not available in Ecology (2005) under WAC 173-340-740(3) for aluminum, calcium, iron, magnesium, potassium, silicon, and sodium; therefore, these constituents are not considered site COCs/COPCs and are also not included in these calculations. The $95 \%$ UCL values were also not calculated for radium-226, radium-228, thorium-228, thorium-232, and potassium-40, as these isotopes are not related to the operational history of the site and thus not considered COCs/COPCs.

All nonradionuclide data reported as being undetected are set to $1 / 2$ the detection limit value for calculation of the statistics (Ecology 1993). For radionuclide data, calculation of the statistics was done on the reported value. In cases where the laboratory does not 6 report a value below the minimal detectable activity (MDA), half of the MDA is used in the calculation. For the statistical evaluation of duplicate sample pairs, the samples are averaged before being included in the data set, after adjustments for censored data as described above.

For nonradionuclides, the WAC 173-340 statistical guidance suggests that a test for distributional form be performed on the data and the $95 \%$ UCL calculated on the appropriate distribution using Ecology software. For nonradionuclide small data sets $(n<10)$ and all radionuclide data sets, the calculations are performed assuming nonparametric distribution, so no tests for distribution are performed. For nonradionuclide data sets of ten or greater, as for the subject site, distributional testing is done using Ecology's MTCAStat software (Ecology 1993). Due to differences in addressing censored data between the RDR/RAWP (DOE-RL 2005b) and MTCAStat coding and due to a limitation in the MTCAStat coding (no direct capability to address variable quantitation limits within a data set), substitutions for censored data are performed before software input and the resulting data set treated as uncensored.

The WAC 173-340-740(7)(e) 3-part test is performed for nonradionuclide analytes only and determines if:

1) the $95 \%$ UCL exceeds the most stringent cleanup limit for each COPC/COC,

2) greater than $10 \%$ of the raw data exceed the most stringent cleanup limit for each $\mathrm{COPC} / \mathrm{COC}$,

3) the maximum value of the raw data set exceeds two times the most stringent cleanup limit for each COPC/COC.

The RPD is calculated when both the primary value and the duplicate value for a given analyte are above detection limits and are greater than 5 times the target detection limit (TDL). The TDL is a laboratory detection limit pre-determined for each analytical method and is listed in Table II-1 of the SAP (DOE-RL 2005a). Where direct evaluation of the attached sample data showed that a given analyte was not detected in the primary and/or duplicate sample, further evaluation of the RPD value was not performed. The RPD calculations use the following formula:

$$
\mathrm{RPD}=[|\mathrm{M}-\mathrm{S}| /((\mathrm{M}+\mathrm{S}) / 2)]^{*} 100
$$

where, $\quad M=$ Main Sample Value $\quad S=$ Split (or duplicate) Sample Value

For quality assurance/quality control (QA/QC) split and duplicate RPD calculations, a value less than $30 \%$ indicates the data compare favorably. For regulatory splits, a threshold of $35 \%$ is used (EPA 1994). If the RPD is greater than $30 \%$ (or $35 \%$ for regulatory split data), further investigation regarding the usability of the data is performed. No split samples were collected for cleanup verification of the subject site. Additional discussion is provided in the data quality assessment section of the applicable RSVP, as necessary. 
Originator W. S. Thompson YHAhe Mupic Date 12/20/07 Calc. No. 0100F-CA-V0033 Project 100 -F Field Remediation Job No. 14655 Subject 128-F-2, Upland (Area B) Cleanup Verification 95\% UCL Calculation hecked
Rev. No. $\frac{0}{\text { Date }} \frac{12 / 20 / 07}{3 \text { of } 14}$

1 Summary (continued)

2 Results:

3 The results presented in the tables that follow include the summary of the results of the $95 \%$ UCL calculations for the shallow zone excavation, the WAC 173 340-740(7)(e) 3-part test evaluation, and the RPD calculations, and are for use in risk analysis and the RSVP for this site.

\begin{tabular}{|c|c|c|c|c|c|c|}
\hline \multirow[b]{2}{*}{8} & \multicolumn{4}{|c|}{ Results Summary - Shallow Zone Excavation } & \multicolumn{2}{|c|}{$\begin{array}{l}\text { Relative Percent Difference Results }{ }^{\mathrm{b}} \text { - } \\
\text { QANOC Analysis }\end{array}$} \\
\hline & Analyte & $\begin{array}{c}95 \% \text { UCL } \\
\text { Result }^{\mathrm{a}} \\
\end{array}$ & \begin{tabular}{|c|}
$\begin{array}{c}\text { Maximum } \\
\text { Value }^{\mathrm{a}}\end{array}$ \\
\end{tabular} & Units & Analyte & $\begin{array}{l}\text { Duplicate } \\
\text { Analysis }^{c}\end{array}$ \\
\hline 9 & Arsenic & 3.5 & & $\mathrm{mg} / \mathrm{kg}$ & Aluminum & $0.7 \%$ \\
\hline 10 & Barium & 72.9 & & $\mathrm{mg} / \mathrm{kg}$ & Barium & $3.7 \%$ \\
\hline 11 & Beryllium & 0.39 & & $\mathrm{mg} / \mathrm{kg}$ & Beryllium & $2.5 \%$ \\
\hline 12 & Boron & 2.1 & & $\mathrm{mg} / \mathrm{kg}$ & Calcium & $2.3 \%$ \\
\hline 13 & Chromium, total & 26.7 & & $\mathrm{mg} / \mathrm{kg}$ & Total chromium & $0.8 \%$ \\
\hline 14 & Cobalt & 6.4 & & $\mathrm{mg} / \mathrm{kg}$ & Copper & $1.6 \%$ \\
\hline 15 & Copper & 39.6 & & $\mathrm{mg} / \mathrm{kg}$ & Iron & $0 \%$ \\
\hline 16 & Hexavalent Chromium & 0.80 & & $\mathrm{mg} / \mathrm{kg}$ & Magnesium & $1.4 \%$ \\
\hline 17 & Lead & 10.8 & & $\mathrm{mg} / \mathrm{kg}$ & Manganese & $2.5 \%$ \\
\hline 18 & Manganese & 275 & & $\mathrm{mg} / \mathrm{kg}$ & Silicon & $65.2 \%$ \\
\hline 19 & Nickel & 13.0 & & $\mathrm{mg} / \mathrm{kg}$ & Zinc & $0.6 \%$ \\
\hline 20 & Zinc & 38.7 & & $\mathrm{mg} / \mathrm{kg}$ & Total petroleum hydrocarbons & $9.9 \%$ \\
\hline & Total petroleum hydrocarbons & 48.7 & & $\mathrm{mg} / \mathrm{kg}$ & ${ }^{\mathrm{b}}$ Relative percent difference ev & uation was not \\
\hline 2 & 4,4'-DDE & 0.0064 & & $\mathrm{mg} / \mathrm{kg}$ & required for analytes not inclu & ed in this table \\
\hline & & 0.080 & & $\mathrm{mg} / \mathrm{kg}$ & ${ }^{C}$ These values are discussed ir & the RSVP. \\
\hline
\end{tabular}

Abbreviations/Acronyms:

The following abbreviations and/or acronyms are used in this calculation:

$\mathrm{B}=$ blank contamination (organics)

$\mathrm{BG}=$ background

$\mathrm{C}=$ blank contamination (inorganics)

$\mathrm{COC}=$ contaminant of concern

COPC = contaminant of potential concern

$\mathrm{DE}=$ direct exposure

$\mathrm{GW}=$ groundwater

$\mathrm{J}=$ estimate

MDA = minimal detectable activity

MTCA $=$ Model Toxics Control Act

$\mathrm{PQL}=$ practical quantitation limit

$Q=$ qualifier

$\mathrm{QA} / \mathrm{OC}=$ quality assurance/quality control

RAG = remedial action goal

$\mathrm{RDL}=$ required detection limit

RDR/RAWP = remedial design report/remedial action work plan

RESRAD = RESidual RADioactivity (dose model)

$\mathrm{RPD}=$ relative percent difference

RSVP = remaining sites verification package

SAP $=$ sampling and analysis plan

$\mathrm{TDL}=$ target detection limit

$\mathrm{U}=$ undetected

$\mathrm{UCL}=$ upper confidence limit

$X=$ tentatively identified compound quantified relative to a response factor generated from a daily calibration standard

WAC $=$ Washington Administrative Code 


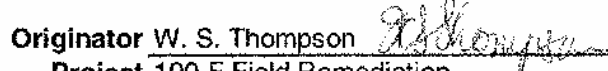

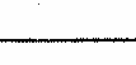
Subject 128-F-2, Upland (Area B) Cleanup Verficication 95\% UCL Calculation

1 100-F-26:8 Excavation Shallow Zone Statistical Calculation

\begin{tabular}{|c|c|c|c|c|c|c|c|c|c|c|c|c|c|c|c|c|c|c|c|c|c|}
\hline \multirow{2}{*}{\begin{tabular}{|c|} 
Sample \\
Area
\end{tabular}} & \multirow{2}{*}{$\begin{array}{l}\text { Sample } \\
\text { Number }\end{array}$} & \multirow{2}{*}{$\begin{array}{c}\text { Sample } \\
\text { Date }\end{array}$} & \multirow{2}{*}{\multicolumn{2}{|c|}{$\begin{array}{c}\text { Arsenic } \\
\mathrm{mol} / \mathrm{kg}\end{array}$}} & \multirow{2}{*}{\multicolumn{3}{|c|}{ Barium }} & \multirow{2}{*}{\multicolumn{2}{|c|}{ Beryllium }} & \multicolumn{3}{|c|}{ Boron } & \multicolumn{3}{|c|}{ Chromium } & \multicolumn{3}{|c|}{ Cobalt } & \multicolumn{3}{|c|}{ Copper } \\
\hline & & & & $\overline{P O L}$ & $\mathrm{mg} / \mathrm{kg}$ & & & & & $\mathrm{mg} / \mathrm{kg}$ & 0 a! & POL & $\mathrm{mg} / \mathrm{kg}$ & & $\mathrm{PQL}$ & $\mathrm{mg} / \mathrm{kg}$ & & $\mathrm{PQL}$ & $\mathrm{mg} / \mathrm{kg}$ & & $P Q L$ \\
\hline & & & 1.7 & 12 & 55.5 & C & 0.06 & 0.44 & 0.03 & 2.6 & & 1.1 & 8.7 & $\mathrm{C}$ & 0.23 & 6.9 & & 0.26 & 12.9 & & 0.35 \\
\hline B.2 & J14L27 & $3 / 6 / 07$ & 4.0 & 1.2 & 65.3 & c & 0.06 & 0.37 & 0.03 & 1.6 & & 1.1 & 11.1 & $c$ & 0.23 & 6.1 & & 0.26 & 12.7 & & 0.35 \\
\hline B-3 & J14L28 & $3 / 6 / 17$ & 4.1 & 1.3 & 76.5 & c & 0.06 & 0.37 & 0.03 & 1.4 & & 1.2 & 15.1 & $\mathrm{c}$ & 0.25 & 6.4 & & 0.29 & 13.9 & & 0.38 \\
\hline B-4 & J14L29 & $3 / 6 / 17$ & 1.9 & 1.2 & 66.1 & c & 0.06 & 0.35 & 0.03 & 1.6 & & 1.1 & 31.3 & c & 0.25 & 5.3 & & 2.6 & 16.6 & & 0.34 \\
\hline B-5 & J14L30 & $3 / 607$ & 3.3 & 12 & 64.4 & C & 0.06 & 0.39 & 0.03 & 2.7 & & 1.1 & 12.9 & o & 0.24 & 6.7 & & 0.27 & 12.5 & & 0.35 \\
\hline Duplicate of $J 14430$ & J14L31 & 36,67 & 4.1 & 1.2 & 66.8 & C & 0.06 & 0.40 & 0.03 & 2.4 & & 1.2 & 12.8 & c & 0.24 & 6.4 & & 0.27 & 12.7 & & 0.37 \\
\hline B-6 & J14L32 & 36107 & 3.2 & 12 & 66.2 & $\mathrm{c}$ & 0.06 & 0.35 & 0.03 & 20 & & 1.1 & 17.7 & c & 0.23 & 6.2 & & 0.26 & 16.1 & & 0.34 \\
\hline$B-7$ & $J 14 L 33$ & 36167 & 1.8 & 1.2 & 49.4 & c & 0.06 & 0.31 & 0.03 & 1.1 & $u$ & 1.1 & 16.9 & $\mathrm{c}$ & 0.23 & 4.9 & & 0.26 & 14.1 & & 0.34 \\
\hline B-8 & J14L 34 & $3 / 6 / 07$ & 2.0 & 1.2 & 68.6 & $\mathrm{c}$ & 0.06 & 0.31 & 0.03 & 1.6 & & 1.1 & 42.4 & $c$ & 0.23 & 5.3 & & 0.26 & 15.4 & & 0.34 \\
\hline B-9 & J14L35 & $3 / 6 / 07$ & 3.9 & 1.2 & 71.8 & c & 0.06 & 0.36 & 0.03 & 1. & & 1.1 & 72.0 & $\mathrm{C}$ & 0.24 & 6.5 & & 0.27 & 14.5 & & 0.36 \\
\hline$\frac{B-9}{B-10}$ & J14L36 & $3 / 6 / 67$ & $\begin{array}{l}3.9 \\
3.8\end{array}$ & 1.2 & 81.6 & C & 0.00 & 0.32 & 0.03 & $\begin{array}{l}1.6 \\
2.0\end{array}$ & & $\frac{1.1}{1.1}$ & 19.6 & 0 & 0.23 & 5.3 & & 0.26 & 110 & & 0.35 \\
\hline
\end{tabular}

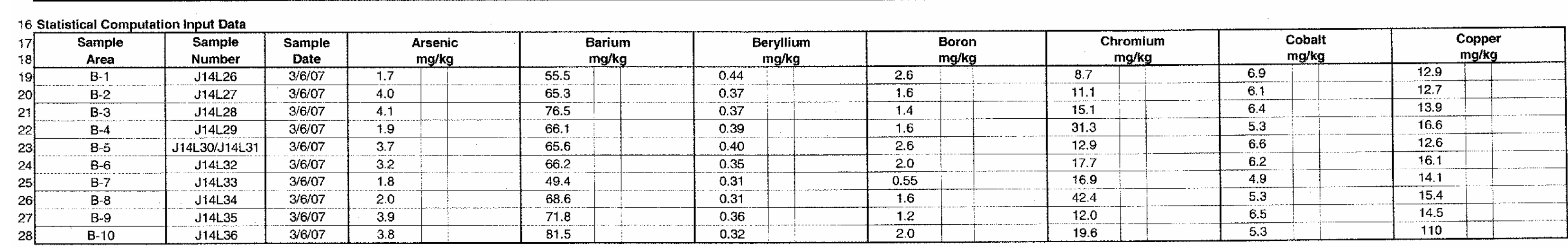

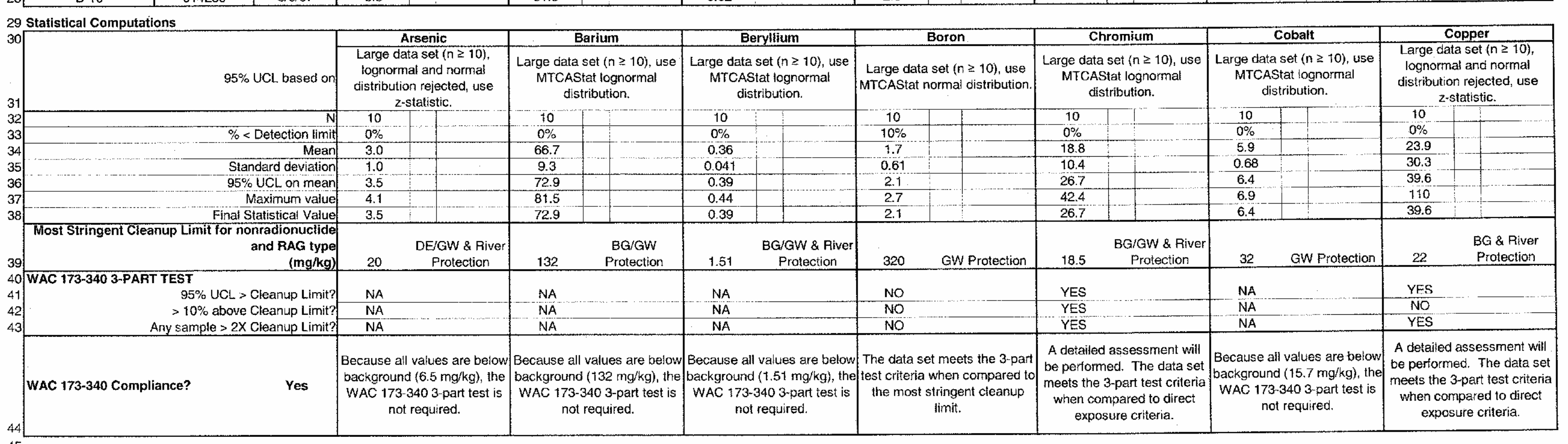


100-F-26:8 Excavation Shallow Zone Statistical Calculation

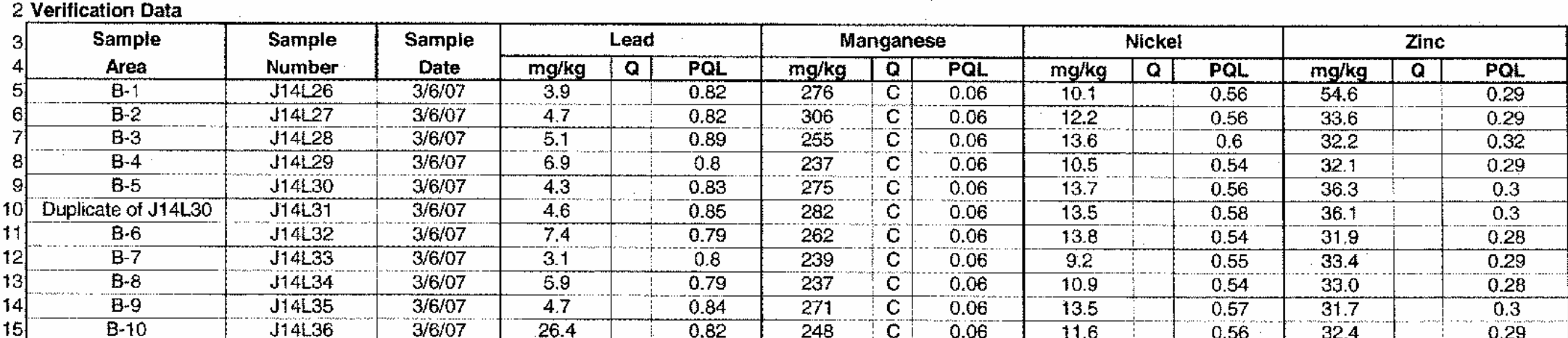

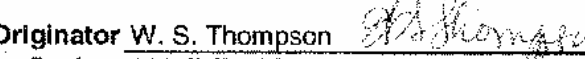

Project $\frac{100-F \text { Field Remediation }}{120}$.

CALCULATION SHEET

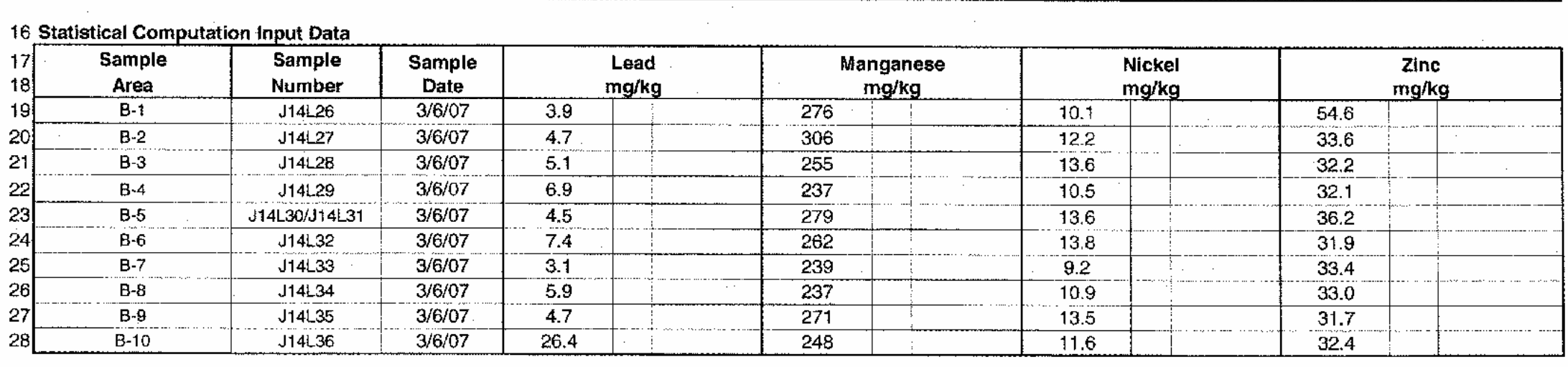

29 Statistical Computation

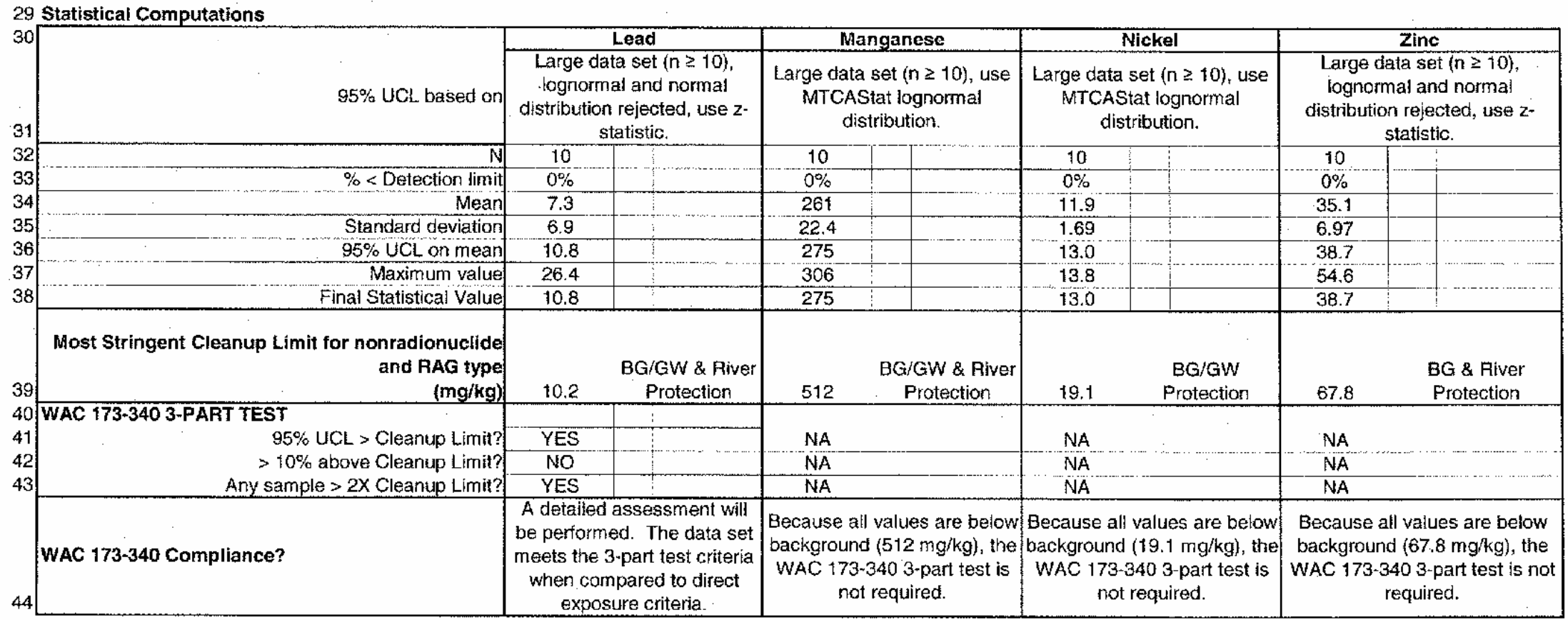

Date
Job No.

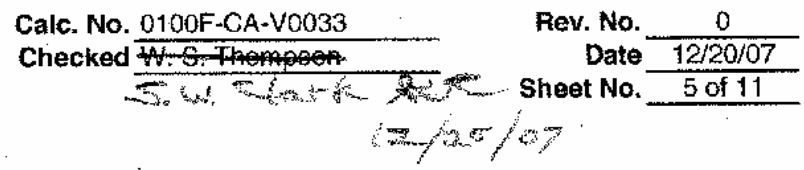
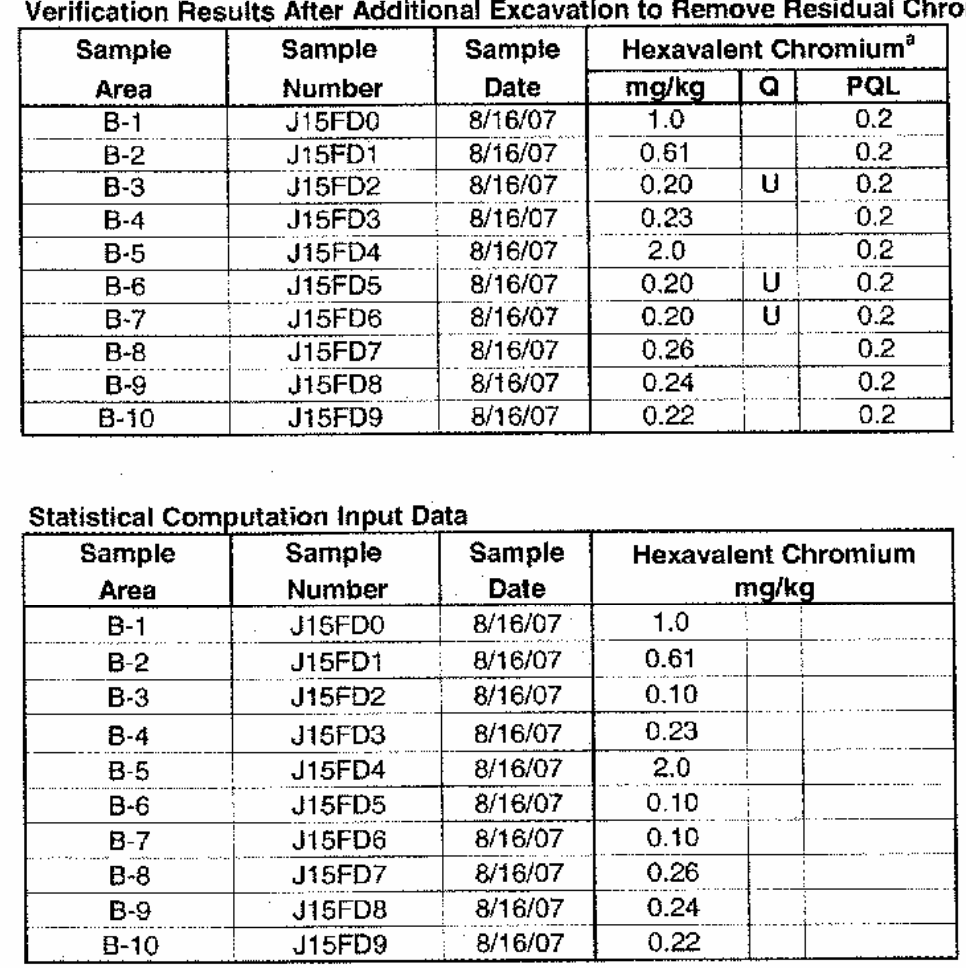

\begin{tabular}{|l|l}
\hline-10 & B 115 \\
\hline
\end{tabular}

\begin{tabular}{|c|c|c|}
\hline $95 \%$ UCL based on & $\begin{array}{l}\text { Hexava } \\
\text { Lagred } \\
\text { lignort } \\
\text { distribut } \\
2\end{array}$ & $\begin{array}{l}\text { ent Chromium } \\
\text { ta set ( } \mathrm{n} \geq 10 \text { ), } \\
\text { al and normal } \\
\text { nn rejected, use } \\
\text { statistic. }\end{array}$ \\
\hline$\%<$ Detection limit & $\frac{10}{30 \%}$ & \\
\hline & 0.49 & \\
\hline $\begin{array}{l}\text { Standard deviation } \\
95 \% \text { UCL on man }\end{array}$ & & \\
\hline $\begin{array}{l}\text { Maximum value } \\
\text { Mand }\end{array}$ & 然 & \\
\hline Final Statistical value & 0.80 & \\
\hline $\begin{array}{r}\text { Most Stringent Cleanup Limit for } \\
\text { nonradionuclide and RAG type } \\
\text { (mg/kg) }\end{array}$ & 2 & River Protection \\
\hline $\begin{array}{l}\text { WAC 173-340 3-PAAT TEST } \\
95 \% \text { UCL > Cleanup Limit? }\end{array}$ & NO & \\
\hline$>10 \%$ above Cleanup Limit? & NO & \\
\hline Any sample $>2 X$ Gieanup Limit? & NO & \\
\hline WAC $173-340$ Compliance? & $\begin{array}{l}\text { The date } \\
\text { part te } \\
\text { compe } \\
\text { stringe }\end{array}$ & $\begin{array}{l}\text { set meets the 3- } \\
\text { t criteria when } \\
\text { ed to the most } \\
\text { t cleanup limit. }\end{array}$ \\
\hline
\end{tabular}


100-F-26:8 Excavation Shallow Zone Statistical Calculations

\begin{tabular}{|c|c|c|c|c|c|c|c|c|c|c|c|c|c|c|c|c|c|c|c|c|c|c|c|}
\hline \multirow{2}{*}{$\begin{array}{c}\text { Sample } \\
\text { Area } \\
\end{array}$} & \multirow{2}{*}{$\begin{array}{l}\text { Sample } \\
\text { Number }\end{array}$} & \multirow{2}{*}{$\begin{array}{c}\text { Sample } \\
\text { Date } \\
\end{array}$} & \multicolumn{3}{|c|}{ Total Petroleum Hycrocarbons } & \multicolumn{3}{|c|}{ Dichtorodiphenyldichloroethylene } & \multicolumn{3}{|c|}{ Bis(2-ethylhexyl) phthalate } & \multicolumn{3}{|c|}{ Dibenz(a,h)anthracene } & \multicolumn{3}{|c|}{ Fluoranthene } & \multicolumn{3}{|c|}{ Phenanthrene } & \multicolumn{3}{|c|}{ Pyrene } \\
\hline & & & $\mathrm{mg} / \mathrm{kg}$ & a & $\mathrm{PQL}$ & $\mu \mathrm{g} / \mathrm{kg}$ & $\mid \mathrm{O}_{1}$ & & $\mu \mathrm{g} / \mathrm{kg}$ & 10 & PQL & $\mu g \mathrm{~kg}$ & La & & pgikg & & POE & $\mu \mathrm{g} / \mathrm{kg}$ & O & POL & $\mu g \mathrm{~kg}$ & & $\mathrm{POL}$ \\
\hline$\frac{8-1}{8-2}$ & & $\frac{3 / 6 / 17}{3 / 6 / 07}$ & 358 & $\frac{G}{c}$ & 0.09 & $\frac{1.3}{8.3}$ & $\frac{\text { UD }}{D}$ & $\frac{1.3}{13}$ & $\frac{55}{36}$ & $\frac{J B}{d B}$ & $\begin{array}{l}350 \\
350 \\
350\end{array}$ & $\frac{23}{23}$ & $\frac{1}{4}$ & 350 & $\begin{array}{ll}350 \\
50\end{array}$ & u & $\frac{350}{350}$ & $\begin{array}{l}350 \\
350 \\
\end{array}$ & $\frac{U}{u}$ & $-\frac{350}{350}$ & $\begin{array}{l}350 \\
350\end{array}$ & $\left|\begin{array}{l}u \\
u\end{array}\right|$ & $\frac{350}{350}$ \\
\hline$\frac{B-2}{B-3}$ & $\frac{13427}{314228}$ & $3 / 6 / 67$ & $\begin{array}{l}35.8 \\
36.8\end{array}$ & $\frac{\mathrm{C}}{\mathrm{C}}$ & $\frac{0.09}{0.1}$ & $\frac{8.3}{1.3}$ & DD & $\frac{1.3}{1.3}$ & $\begin{array}{r}36 \\
34\end{array}$ & $\frac{\sqrt{B}}{\sqrt{B}}$ & $\frac{350}{380}$ & $\frac{23}{380}$ & $\frac{u}{u}$ & $\begin{array}{l}350 \\
380 \\
-10\end{array}$ & $\frac{350}{380}$ & $\frac{0}{0}$ & 380 & $\begin{array}{l}-350 \\
-380\end{array}$ & $\mathrm{U}$ & 380 & 380 & ut & 380 \\
\hline $\mathrm{B}-$ & & & & $c$ & & 1.3 & & 1.3 & & $\sqrt{\mathrm{NB}}$ & & 340 & & & & & & & & & & & 340 \\
\hline B-5 & $\sqrt{114 L 30}$ & & 42.4 & c & $\frac{0.00}{0.09}$ & 1.3 & Wo & $\frac{1.5}{1.3}$ & 250 & $\frac{5}{3 B}$ & $3 t$ & $\frac{340}{23}$ & $\frac{1}{0}$ & 340 & $\frac{40}{360}$ & tu & -360 & 360 & 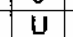 & 360 & 360 & $u$ & 360 \\
\hline Duplicate of $\mathrm{J} 14 \mathrm{~L} 30^{\circ}$ & J14L31 & $\frac{w}{3 i}$ & 38.4 & $\mathrm{c}$ & 0.09 & 1.3 & 范 & 1.3 & $\frac{000}{42}$ & 药 & 370 & 39.924 & $\mathrm{~J}$ & 370 & 370 & u & 370 & 370 & u & 370 & 370 & $u$ & 370 \\
\hline$B-6$ & J1 & & 48.4 & $\mathrm{C}$ & 0.09 & 9.4 & & 1.3 & 39 & $\mathrm{JE}$ & 340 & 340 & $u$ & 340 & 490 & & 340 & 310 & 1 & 340 & 2000 & & 340 \\
\hline & & & & $c$ & 0.09 & 1 & UD & 1.3 & 59 & Jf & 3 & 350 & & 350 & 130 & fus & & 76 & 3 & 350 & 150 & $\mathrm{~J}$ & 350 \\
\hline $\mathrm{B}-8$ & J14L34 & $3 / 6 / 6$ & 43.6 & $c$ & 0.09 & 13 & D & 1.3 & 31 & JE & 340 & 24 & 3 & 340 & 160 & ]$_{3}$ & $\begin{array}{l}160 \\
\end{array}$ & 88. & 0 & 340 & 160 & 1 & 340 \\
\hline$B-9$ & J14L.35 & $\begin{array}{l}3 / 6 / 07 \\
36167\end{array}$ & $\begin{array}{l}38.3 \\
65.9\end{array}$ & 0. & 0.09 & 1.6 & JO & $\begin{array}{l}1.6 \\
1.13\end{array}$ & 110 & JB & $\begin{array}{l}360 \\
350\end{array}$ & 35 & 3 & $\frac{360}{350}$ & $\frac{21}{700}$ & 13 & & $\frac{360}{50}$ & $\mathrm{u}$ & $\begin{array}{r}360 \\
350\end{array}$ & 24 & $J$ & $\begin{array}{l}360 \\
360 \\
250\end{array}$ \\
\hline
\end{tabular}


\begin{tabular}{|c|c|c|c|c|c|}
\hline \multirow{2}{*}{\multicolumn{2}{|c|}{$\begin{array}{c}\text { Fluoranthene } \\
\text { Large data set }(n \geq 10), \\
\text { lognormal and norma: } \\
\text { distributition rejected, use } z- \\
\text { statistic. }\end{array}$}} & \multicolumn{2}{|c|}{$\begin{array}{l}\text { Phenanthrene } \\
\text { Large data sost in } 210) \text {. } \\
\text { lognomal and normal } \\
\text { distribution rejected use } \\
\text { statistic. }\end{array}$} & \multicolumn{2}{|c|}{ 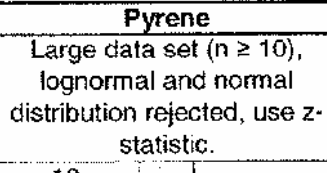 } \\
\hline & & 10 & & $\frac{10}{1 \sin ^{\circ}}$ & \\
\hline $\begin{array}{l}40 \% \\
235\end{array}$ & & $\begin{array}{l}50 \% \\
-591 . \\
91\end{array}$ & & 249 & \\
\hline 232.3 & & 140 & & 254 & \\
\hline$\frac{356}{790}$ & & 520 & : & $\frac{381}{840}$ & \\
\hline 356 & + & 264 & & 381 & \\
\hline 18,000 & Fiver Protection & 240,000 & GW Protection & 48,000 & GW Frotection \\
\hline No & & No & & No & \\
\hline $\begin{array}{l}\text { NO } \\
\text { NO }\end{array}$ & & $\begin{array}{l}\text { No } \\
\text { No } \\
\text { No. }\end{array}$ & & $\frac{\text { NO }}{\text { NO }}$ & \\
\hline $\begin{array}{l}\text { The data } \\
\text { est critir } \\
\text { se most } 5\end{array}$ & $\begin{array}{l}\text { meets the 3-part } \\
\text { then compared to } \\
\text { gent ciearnup limit. }\end{array}$ & $\begin{array}{l}\text { The data st } \\
\text { elst criteria } \\
\text { the most }\end{array}$ & $\begin{array}{l}\text { meets the 3-part } \\
\text { then compared to } \\
\text { ringent cleanup } \\
\text { firmit. }\end{array}$ & $\begin{array}{l}\text { The data } \\
\text { part te: } \\
\text { compa } \\
\text { stringe }\end{array}$ & $\begin{array}{l}\text { et meets the 3- } \\
\text { criteria when } \\
\text { d to the most } \\
\text { cieanup limit. }\end{array}$ \\
\hline
\end{tabular}


Washington Closure Hanford

Originator $W$. S. Thompson ${ }_{\text {Project }} 100$-F Field Remediation Subject $128-\mathrm{F}-2$, Upland (Area B) Cleanup Verification $95 \%$ UCL Calculation

\section{CALCULATION SHEE}

$\begin{aligned} \text { Date } & 12 / 20 / 07 \\ \text { Job No. } & 14655\end{aligned}$
Calc. No. 0100-F-CA-V0333

Rev. No. $\frac{0}{\text { Date }}$

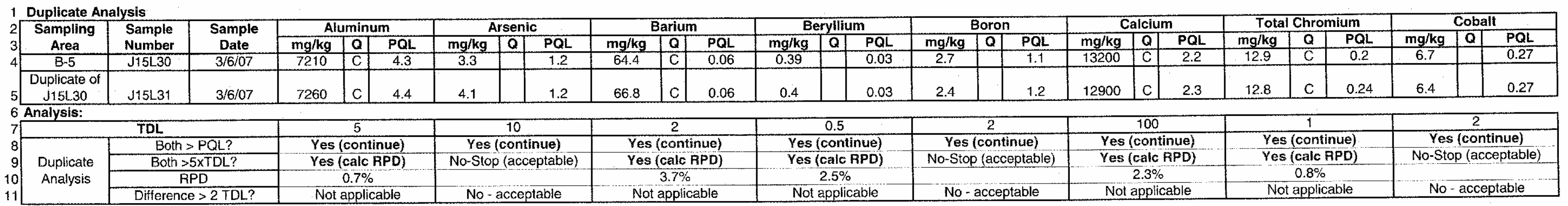

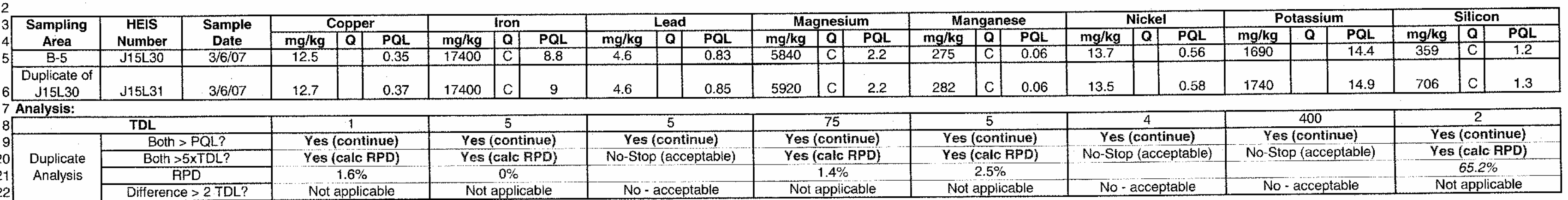

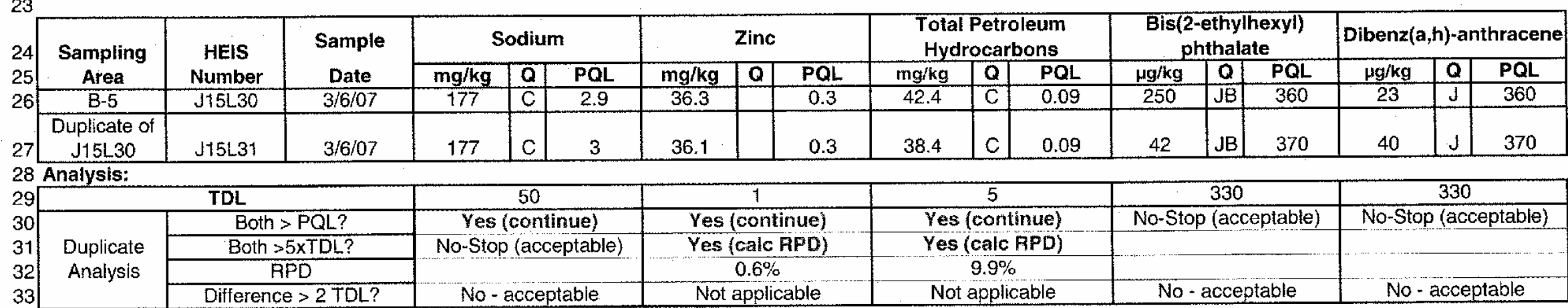


CALCULATION SHEET

Washington Closure Hanford

Originator $\frac{\text { W.S. Thompson }}{100-F \text { Field Remediation }}$

Subject $128-F-2$, Upland (Area B) Cleanup Verification $95 \%$ UCL Calculati

\begin{tabular}{|c|c|c|c|c|c|}
\hline$\overline{\text { DATA }}$ & I & Arsenic $95 \%$ & UCL & Iculation & \\
\hline $\begin{array}{l}1.7 \\
4 \\
4,1\end{array}$ & $\begin{array}{l}J 1426 \\
J 14 L 27\end{array}$ & & & & \\
\hline $\begin{array}{l}4.1 \\
1.9\end{array}$ & $\begin{array}{l}J 14428 \\
\mathbf{J 1 4 L 2 9}\end{array}$ & $\begin{array}{l}\text { Number of samples } \\
\text { Untersored }\end{array}$ & 10 & $\begin{array}{l}\text { Uncensored values } \\
\text { Mean }\end{array}$ & 3.0 \\
\hline $\begin{array}{l}3.7 \\
3.2\end{array}$ & $J 14430 t$ & & & Lognormal mean & \\
\hline $\begin{array}{l}3.2 \\
1.8\end{array}$ & $\begin{array}{l}J 14431 \\
\mathbb{J} 14 L 32\end{array}$ & Detection limit or PQL & & Std. devn. & 1.03 \\
\hline $\begin{array}{c}1.8 \\
2\end{array}$ & J14L.33 & Method detection limit & & Median & $\begin{array}{l}3.5 \\
17\end{array}$ \\
\hline 3.9 & $\sqrt{14 L 34}$ & & 10 & $\begin{array}{l}\text { Main. } \\
\text { Max. }\end{array}$ & 4.1 \\
\hline 3.8 & J14L36 & & & & \\
\hline
\end{tabular}

\begin{tabular}{l|ll}
11 & 3.8 & $\mathrm{~J} 14 \mathrm{~L} 36$ \\
12 & &
\end{tabular}

Lognormal distribution? Normal distribution?

-squared is: 0.836

Recommendations:
Reject BOTH lognormal and normal distributions.

UCL (based on Z-statistic) is 3.5

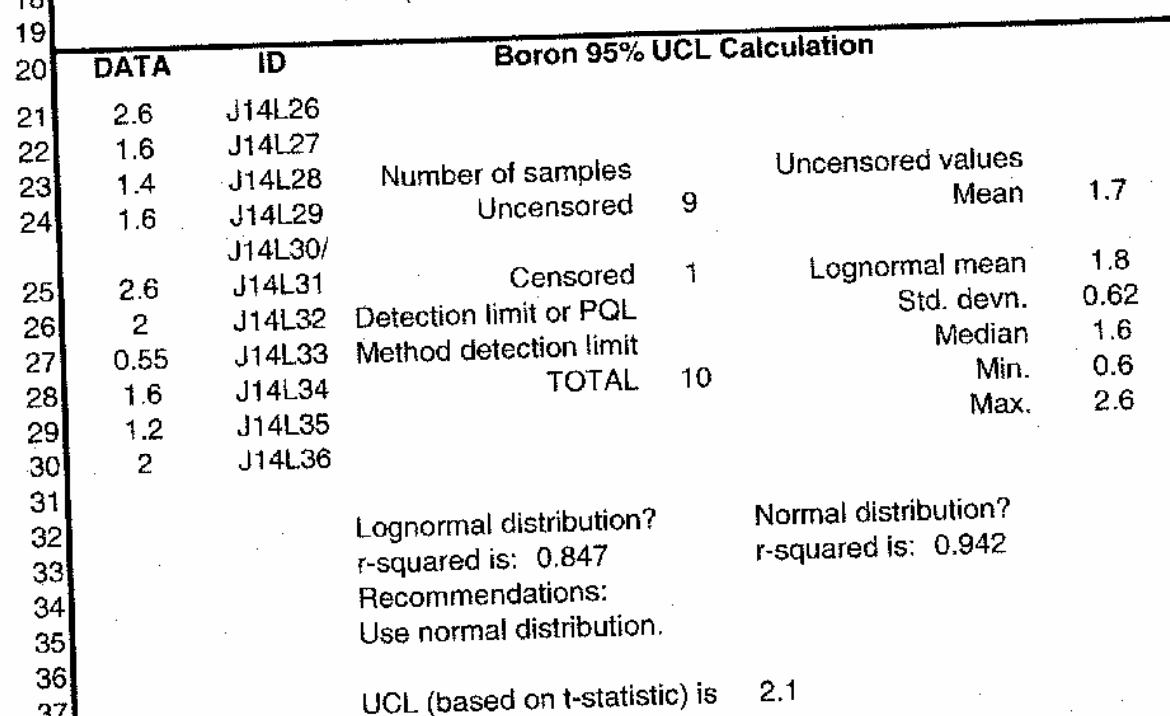

Date $\quad \frac{12 / 20 / 07}{14655}$

Calc. No. 0100-F-CA-V0333

Rev. No. $\frac{0}{\text { Date }}$
Danga

(a) Results

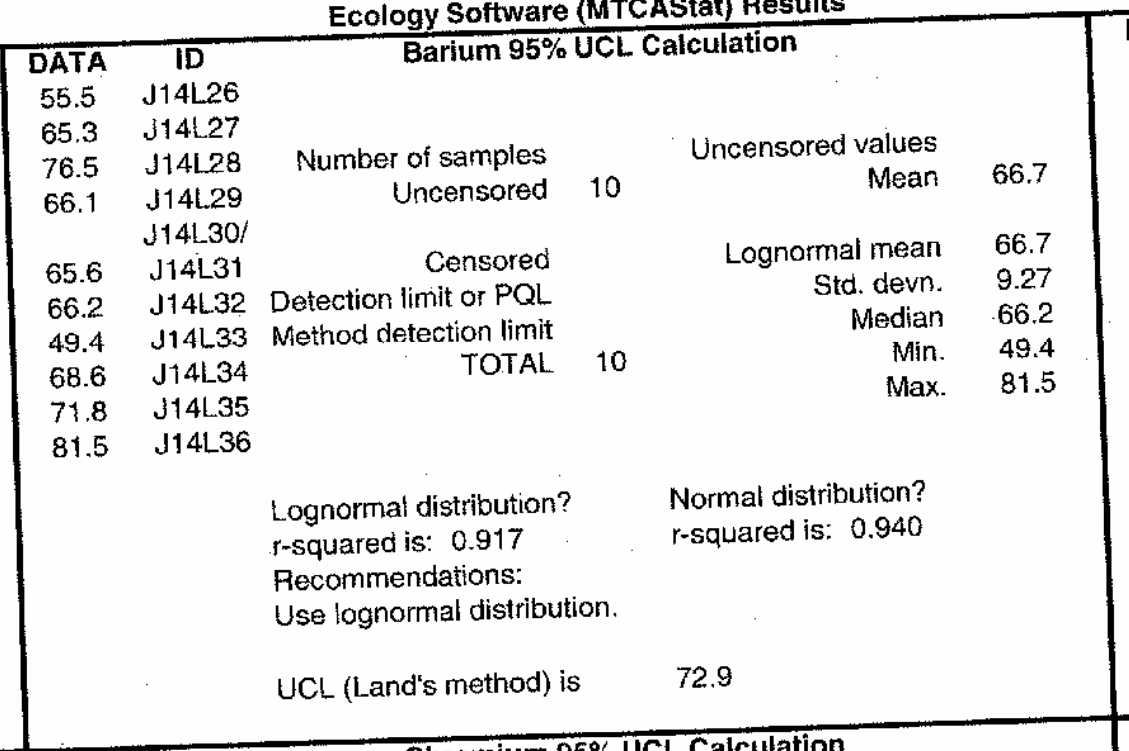

\begin{tabular}{|c|c|c|c|}
\hline DATA & $\mathrm{ID}$ & Beryllium 95 & OUCLC \\
\hline 0.44 & $\begin{array}{ll}J 14 L 26 \\
1141127\end{array}$ & & \\
\hline $\begin{array}{l}0.37 \\
0.37\end{array}$ & J14L28 & Number of samples & \\
\hline 0.39 & $\begin{array}{l}J 14 L 29 \\
J 14 L 30\end{array}$ & & 10 \\
\hline $\begin{array}{c}0.4 \\
0.35\end{array}$ & $J 14 L .3 t$ & $\begin{array}{l}\text { Censored } \\
\text { Dotaction limit or PQL }\end{array}$ & \\
\hline $\begin{array}{l}0.35 \\
0.31\end{array}$ & $\begin{array}{l}J 14 L 32 \\
\mathbb{J 1 4 L 3 3}\end{array}$ & Method detection limit & \\
\hline 0.31 & $J 14 L 34$ & TOTAL & 10 \\
\hline 0.36 & J14L 35 & & \\
\hline
\end{tabular}

Sheet No. $\frac{8 \text { of 11 }}{12}$

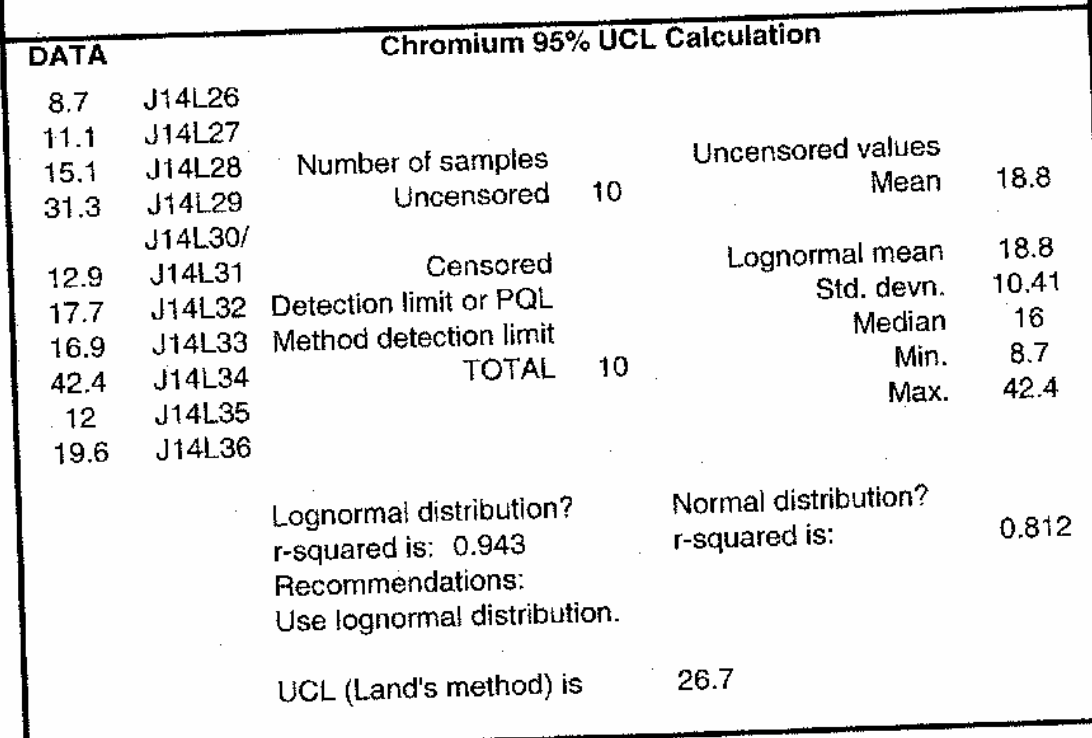

Lognormal distribution?
r-squared is: 0.957
Recormmendations:
Use lognoormal distribution.

UCL (Land's method) is $\quad 0.39$

\begin{tabular}{|c|c|c|c|c|c|}
\hline DATA & ID & Cobalt 95\% & $\mathrm{CLL}$ & tion & \\
\hline 6.9 & $\begin{array}{l}J 14 L 26 \\
J 14227\end{array}$ & & & & \\
\hline $\begin{array}{l}6.1 \\
6.4\end{array}$ & $\begin{array}{ll} \\
J 14 L 28\end{array}$ & Number of samples & & Uncensored values & 6.0 \\
\hline 5.3 & $J 14\llcorner 29$ & Uncensored & 10 & & \\
\hline 6.6 & $\mathrm{~J} 14\llcorner 31$ & Censored & & Lognormal mean & 6.0 \\
\hline 6.2 & $\mathrm{~J} 14 \mathrm{~L} 32$ & Detection limit or $\mathrm{PQL}$ & & $\begin{array}{l}\text { Stad. cern. } \\
\text { Median }\end{array}$ & $\begin{array}{l}0.65 \\
6.2\end{array}$ \\
\hline 4.9 & J14L.33 & Method detection limit & & & 4.9 \\
\hline 5.3 & $J 14\llcorner 34$ & TOTAL & 10 & Max. & 6.9 \\
\hline 6.5 & J14L35 & & & & \\
\hline
\end{tabular}

$\begin{array}{ll}6.5 & \mathrm{~J} 14 \mathrm{~L} 35 \\ 5.3 & \mathrm{~J} 14 \mathrm{~L} 36\end{array}$

Lognormal distribution? Normal distribution?

Recommendations:

Use lognormal distribution.

UCL (Land's method) is $\quad 6.4$

Uncensored values
Mean $\quad 0.36$

Lognormal mean 0.36
Std denn.
0.04

$\begin{array}{cc}\text { Std. devn. } & 0.04 \\ \text { Median } & 0.37 \\ \text { Max } & 0.34\end{array}$

$\begin{array}{cc}\text { Min. } & 0.31 \\ \text { Max. } & 0.44\end{array}$

Normal distribution?
-squared is: 0.953

6.4 


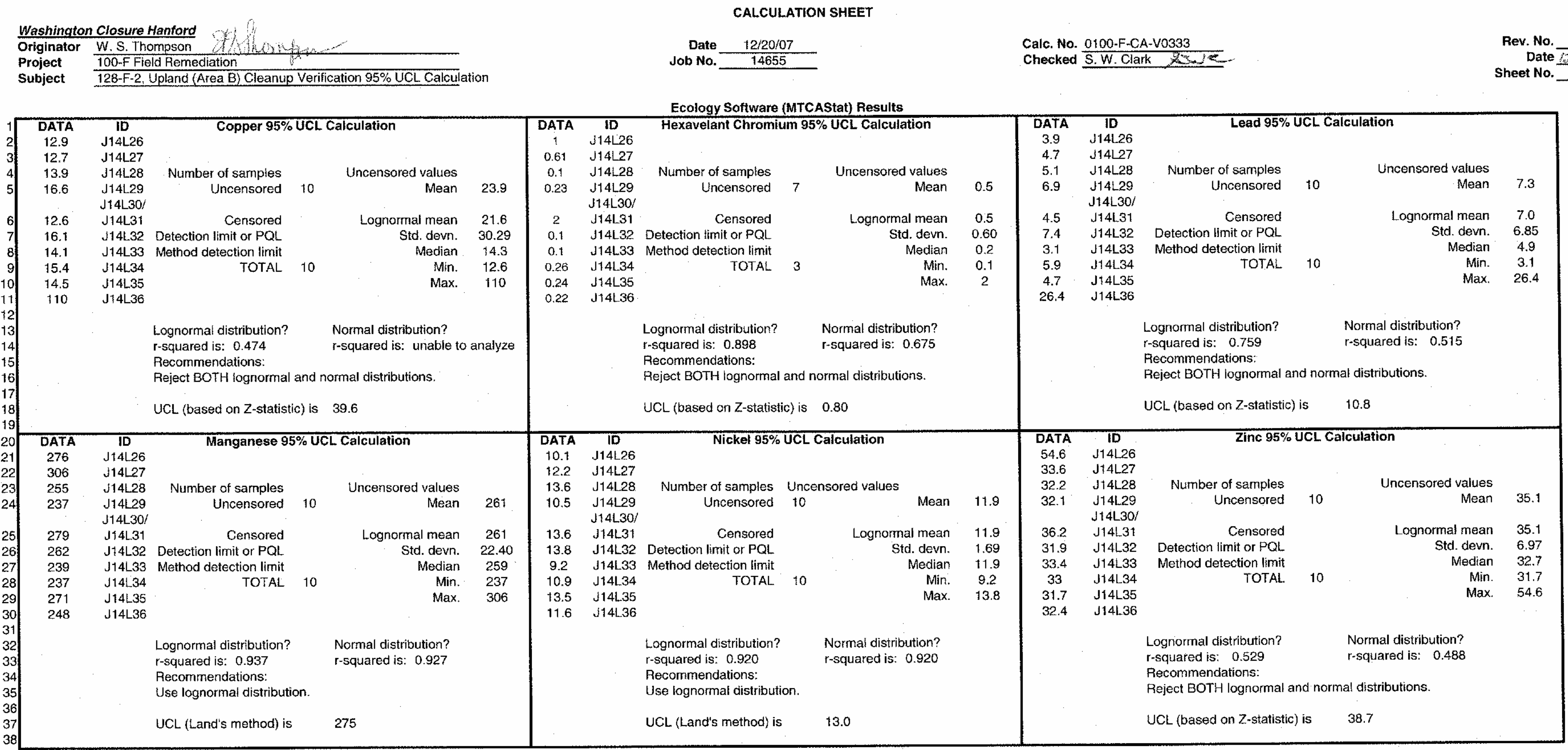


CALCULATION SHEET

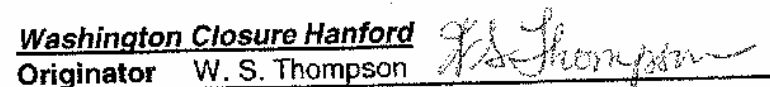

Date $\frac{12 / 20 / 07}{1465}$

Calc. No.0100-F-CA-V0333
Checked S. W. Clark 30 owe

Rev. No.
Date
Sheet No. $\frac{0}{\frac{\tan 10}{100 f 11}}$

$\begin{array}{ll}\begin{array}{l}\text { Project } \\ \text { Subject }\end{array} & \frac{100-F \text { Field Remediation }}{128-F-2, \text { Upland (Area B) Cleanup Verification } 9.5 \% \text { UCL Calculation }}\end{array}$

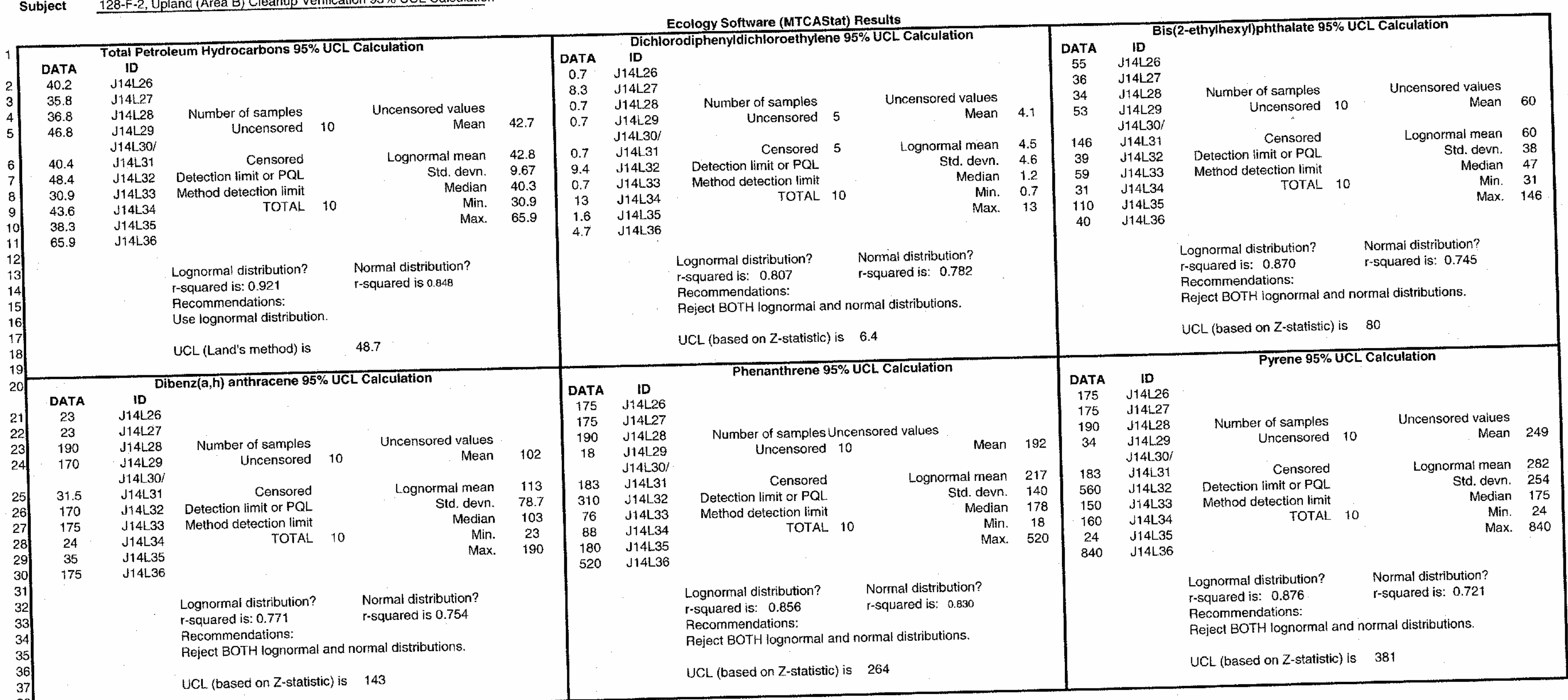




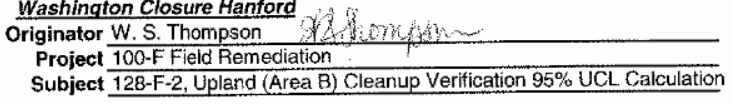

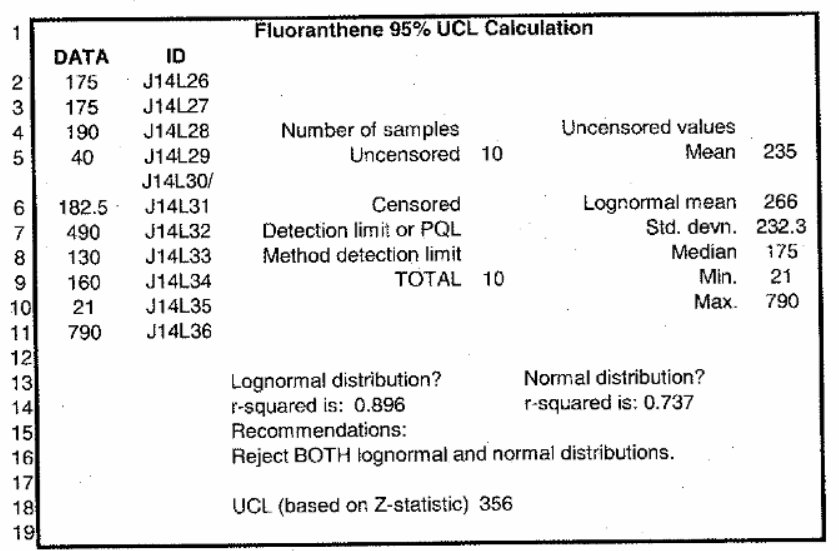

Calc. No. 0100-F-CA-V0333

Date 12/20/07

Ecology Software (MTCAStat) Results

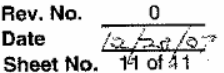


Attachment 1. 128-F-2 Upland (Area B) Verification Sampling Results.

\begin{tabular}{|c|c|c|c|c|c|c|c|c|c|c|c|c|c|c|c|c|c|c|c|c|c|c|c|}
\hline \multirow{2}{*}{$\begin{array}{c}\text { Sample } \\
\text { Location }\end{array}$} & \multirow{2}{*}{$\begin{array}{c}\text { HEIS } \\
\text { Number }\end{array}$} & \multirow{2}{*}{$\begin{array}{c}\text { Semple } \\
\text { Date }\end{array}$} & \multicolumn{3}{|c|}{ Americium-241 GEA } & \multicolumn{3}{|c|}{ Cesium-137 } & \multicolumn{3}{|c|}{ Cobalt-60 } & \multicolumn{3}{|c|}{ Europium-152 } & \multicolumn{3}{|c|}{ Europium-154 } & \multicolumn{3}{|c|}{ Europium-155 } & \multicolumn{3}{|c|}{ Gross alpha } \\
\hline & & & $\mathrm{pCi} / \mathrm{g}$ & $a$ & MDA & $\mathrm{pC} / \mathrm{g}$ & $a$ & MDA & $\mathrm{pCl} / \mathrm{g}$ & $a$ & MDA & $\mathrm{pCV} / \mathrm{g}$ & a & MDA & $\mathrm{pCl} / \mathrm{g}$ & a & MDA & $\mathrm{pCl} / \mathrm{g}$ & a & MDA & $\mathrm{pCl} / \mathrm{g}$ & $\mathbf{Q}$ & MDA \\
\hline $8-1$ & J14L26 & $3 / 6 / 07$ & 0.096 & $\bar{U}$ & 0.096 & 0.1 & 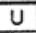 & 0.1 & 0.11 & $\mathrm{U}$ & 0.11 & 0.26 & 0 & 0.26 & 0.34 & $u$ & 0.34 & 0.16 & U & 0.16 & 5.6 & & 5.2 \\
\hline B-2 & J14L27 & $3 / 6 / 07$ & 0.092 & $\bar{U}$ & 0.092 & 0.056 & $\bar{U}$ & 0.056 & 0.071 & $\mathrm{U}$ & 0.071 & 0.14 & U & 0.14 & 0.2 & $u$ & 0.2 & 0.13 & $\mathrm{U}$ & 0.13 & 4.67 & $u$ & 5.2 \\
\hline$B-3$ & J14L28 & $3 / 6 / 07$ & 0.35 & U & 0.35 & 0.11 & $\bar{U}$ & 0.11 & 0.12 & U & 0.12 & 0.25 & 0 & 0.25 & 0.39 & $u$ & 0.39 & 0.27 & $\mathrm{U}$ & 0.27 & 6.72 & & 4.3 \\
\hline B-4 & J14L29 & $3 / 6 / 07$ & 0.24 & $U$ & 0.24 & 0.078 & $U$ & 0.078 & 0.095 & $\mathrm{U}$ & 0.095 & 0.19 & U & 0.19 & 0.26 & $u$ & 0.26 & 0.18 & $U$ & 0.18 & 7.76 & & 4.1 \\
\hline B.5 & J14L30 & $3 / 6 / 07$ & 0.11 & $\bar{U}$ & 0.11 & 0.1 & $U$ & 0.1 & 0.11 & $u$ & 0.11 & 0.28 & 0 & 0.28 & 0.33 & $u$ & 0.33 & 0.24 & 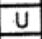 & 0.24 & 5.89 & $u$ & 6 \\
\hline $\begin{array}{l}\text { Duplicate of } \\
\mathrm{J} 14 \mathrm{~L} 30\end{array}$ & & & 0.32 & u & 0.32 & 0.091 & $u$ & 0.091 & 0.11 & $\mathrm{u}$ & 0.11 & 0.24 & ul & 0.24 & 35 & $u$ & 0.35 & 0.25 & $u$ & 0.25 & 2.39 & $u$ & 7.1 \\
\hline$\frac{\pi 14-50}{B-6}$ & $\frac{J 14 L 31}{J 14 L 32}$ & 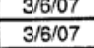 & 0.27 & $\bar{u}$ & 0.27 & 0.097 & $\mathrm{u}$ & 0.097 & 0.1 & U & 0.1 & 0.22 & 0 & 0.22 & 0.29 & U & 0.29 & 0.23 & U & 0.23 & 8.6 & & 6.8 \\
\hline B-7 & J14L33 & $3 / 6 / 07$ & 0.092 & $\mathrm{U}$ & 0.092 & 0.093 & $u$ & 0.093 & 0.1 & $\mathrm{U}$ & 0.1 & 0.23 & $\mathrm{U}$ & 0.23 & 0.26 & $U$ & 0.26 & 0.2 & $U$ & 0.2 & 7.66 & & 5.9 \\
\hline B-8 & J14L34 & $3 / 6 / 07$ & 0.097 & $U$ & 0.097 & 0.12 & $U$ & 0.12 & 0.11 & $u$ & 0.11 & 0.29 & U & 0.29 & 0.39 & U & 0.39 & 0.17 & $U$ & 0.17 & 6.7 & $U$ & 7.2 \\
\hline B-9 & J14L35 & $3 / 6 / 07$ & 0.085 & $U$ & 0.085 & 0.054 & $U$ & 0.054 & 0.071 & $U$ & 0.071 & 0.14 & U & 0.14 & 0.17 & \begin{tabular}{|l}
$U$ \\
\end{tabular} & 0.17 & 0.12 & $u$ & 0.12 & 1.62 & $U$ & 5.7 \\
\hline B-10 & J14L36 & $3 / 6 / 07$ & 0.3 & 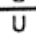 & 0.3 & 0.088 & $u$ & 0.088 & 0.099 & $u$ & 0.099 & 0.22 & U & 0.22 & 0.35 & $U$ & 0.35 & 0.21 & $U$ & 0.21 & 11.7 & & 4.9 \\
\hline
\end{tabular}

\begin{tabular}{|c|c|c|c|c|c|c|c|c|c|c|c|c|c|c|c|c|c|c|c|c|c|c|c|}
\hline \multirow{2}{*}{$\begin{array}{l}\text { Sample } \\
\text { Location }\end{array}$} & \multirow{2}{*}{$\begin{array}{c}\text { HEIS } \\
\text { Number }\end{array}$} & \multirow{2}{*}{$\begin{array}{c}\text { Sample } \\
\text { Date }\end{array}$} & \multicolumn{3}{|c|}{ Gross beta } & \multicolumn{3}{|c|}{ Potassium-40 } & \multicolumn{3}{|c|}{ Radlum-226 } & \multicolumn{3}{|c|}{ Radium-228 } & \multicolumn{3}{|c|}{ Silver 108-metastable } & \multicolumn{3}{|c|}{ Thorium-228 } & \multicolumn{3}{|c|}{ Thorium-232 } \\
\hline & & & $\mathrm{pCl} / \mathrm{g}$ & $a$ & MDA & $\mathrm{pCi} / \mathrm{g}$ & a & MDA & $\mathrm{pCi} / \mathrm{g}$ & Q & MDA & $\mathrm{pCl} / \mathrm{g}$ & a & MDA & $\mathrm{pCi} / \mathrm{g}$ & $a$ & MDA & $\mathrm{pCi} / \mathrm{g}$ & $a$ & MDA & $\mathrm{pCl} / \mathrm{g}$ & \begin{tabular}{l|l} 
a \\
\end{tabular} & MDA \\
\hline B-1 & J14L26 & $3 / 6 / 07$ & 16.9 & & 5.4 & 11.6 & & 1.3 & 0.312 & & 0.17 & 0.84 & 0 & 0.84 & 0.075 & U & 0.075 & 0.629 & & 0.17 & 0.84 & $u$ & 0.84 \\
\hline B.2 & J14L27 & $3 / 6 / 07$ & 18.7 & & 6.4 & 13.3 & & 0.58 & 0.511 & & 0.12 & 0.964 & & 0.25 & 0.044 & U & 0.044 & 0.715 & & 0.071 & 0.964 & & 0.25 \\
\hline B-3 & J14L28 & $3 / 6 / 07$ & 20.8 & & 5.5 & 12.7 & & 1.2 & 0.589 & & 0.18 & 1.28 & & 0.41 & 0.074 & $U$ & 0.074 & 0.735 & & 0.17 & 1.28 & & 0.41 \\
\hline B-4 & J14L29 & $3 / 6 / 07$ & 19.2 & & 7.6 & 10.7 & & 0.65 & 0.162 & & 0.16 & 0.81 & U & 0.81 & 0.057 & $U$ & 0.057 & 0.438 & & 0.13 & 0.81 & $u$ & 0.81 \\
\hline B-5 & J14L30 & $3 / 6 / 07$ & 23.2 & & 6 & 14.1 & & 1.2 & 0.571 & & 0.19 & 1.25 & & 0.47 & 0.078 & $u$ & 0.078 & 0.799 & & 0.21 & 1.25 & & 0.47 \\
\hline Duplicate of & & & & & 5.6 & & & 1 & & & & & & & & $u$ & 1 & & & & 124 & & \\
\hline$\frac{\mathrm{J} 14 \mathrm{~L} 30}{\mathrm{~B}-6}$ & $\frac{\mathrm{J} 14 \mathrm{~L} 31}{\mathrm{~J} 14 \mathrm{~L} 32}$ & $3 / 6 / 07$ & $\frac{19.8}{20}$ & & $\frac{5.6}{5.5}$ & 13.5 & & $\frac{1}{1.1}$ & $\begin{array}{l}0.455 \\
0.363\end{array}$ & & 0.15 & 1.24 & & 0.4 & $\frac{0.071}{0.066}$ & 0 & \begin{tabular}{l|l|}
0.071 \\
0.066
\end{tabular} & $\begin{array}{l}0.813 \\
0.639\end{array}$ & & $\frac{0.14}{0.15}$ & 0.935 & & $\begin{array}{l}0.4 \\
0.38\end{array}$ \\
\hline$\frac{B-6}{B-7}$ & $\begin{array}{l}\text { J14L32 } \\
\text { J14L.33 }\end{array}$ & $\begin{array}{l}3 / 6 / 07 \\
\end{array}$ & $\frac{20}{18.6}$ & & $\frac{5.5}{8.9}$ & 15.1 & & 0.57 & \begin{tabular}{|l|}
0.363 \\
0.355
\end{tabular} & & 0.15 & 0.935 & & 0.38 & $\begin{array}{l}0.066 \\
0.063\end{array}$ & 0 & \begin{tabular}{|l|}
0.006 \\
0.063
\end{tabular} & $\begin{array}{l}0.639 \\
0.381\end{array}$ & & $\frac{0.15}{0.12}$ & 0.477 & & $\frac{0.38}{0.35}$ \\
\hline$\frac{B-7}{B-8}$ & 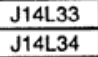 & $\begin{array}{l}3 / 6 / 07 \\
3 / 6 / 07\end{array}$ & $\frac{18.6}{21.7}$ & & 8.7 & 13 & & 1.5 & \begin{tabular}{|l|}
0.355 \\
0.429
\end{tabular} & & $\frac{0.15}{0.18}$ & $\begin{array}{l}0.477 \\
0.626\end{array}$ & & $\frac{0.35}{0.36}$ & 0.081 & U & 0.081 & 0.065 & & 0.17 & 0.626 & & 0.36 \\
\hline B-9 & $\mathrm{J} 14 \mathrm{~L}-35$ & $3 / 6 / 07$ & 18.7 & & 5.4 & 13.8 & & 0.55 & 0.523 & & 0.11 & 0.648 & & 0.26 & 0.039 & u & 0.039 & 0.72 & & 0.066 & 0.648 & & 0.26 \\
\hline B-10 & J14L36 & $3 / 6 / 07$ & 19.2 & & 5.4 & 12.4 & & 0.89 & 0.36 & $U$ & 0.36 & 0.79 & 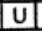 & 0.79 & 0.063 & $\begin{array}{ll} \\
\end{array}$ & 0.063 & 0.513 & & 0.12 & 0.79 & U & 0.79 \\
\hline
\end{tabular}

\begin{tabular}{|c|c|c|c|c|c|c|c|c|}
\hline \multirow{2}{*}{$\begin{array}{c}\text { Sample } \\
\text { Location }\end{array}$} & \multirow{2}{*}{$\begin{array}{c}\text { HEIS } \\
\text { Number }\end{array}$} & \multirow{2}{*}{$\begin{array}{c}\text { Sample } \\
\text { Date }\end{array}$} & \multicolumn{3}{|c|}{ Uranium-235 GEA } & \multicolumn{3}{|c|}{ Uranium-238 GEA } \\
\hline & & & $\mathrm{pCi} / \mathrm{g}$ & $a$ & MDA & $\mathrm{pCl} / \mathrm{g}$ & $Q$ & MDA \\
\hline $\mathrm{B}-1$ & J14L26 & $3 / 6 / 07$ & 0.27 & $u$ & 0.27 & 13 & $u$ & 13 \\
\hline B-2 & $\mathrm{J} 14 \mathrm{~L} 27$ & $3 / 6 / 07$ & 0.22 & $u$ & 0.22 & 7.9 & U & 7.9 \\
\hline $8-3$ & $J 14 L 28$ & $3 / 6 / 07$ & 0.4 & $U$ & 0.4 & 13 & U & 13 \\
\hline B-4 & $\mathrm{J} 14 \mathrm{~L} 29$ & $3 / 6 / 07$ & 0.27 & $U$ & 0.27 & 10 & $U$ & 10 \\
\hline B.5 & J14L30 & 3/6/07 & 0.4 & $\mathrm{U}$ & 0.4 & 13 & $u$ & 13 \\
\hline $\begin{array}{l}\text { Duplicate of } \\
\text { J14L30 }\end{array}$ & J14L31 & $3 / 6 / 07$ & 0.38 & $\mathrm{U}$ & 0.38 & 12 & $u$ & 12 \\
\hline B. 6 & J14L32 & $3 / 6 / 07$ & 0.3 & U & 0.3 & 11 & $U$ & 11 \\
\hline B-7 & J14L33 & $3 / 6 / 07$ & 0.32 & $u$ & 0.32 & 11 & $u$ & 11 \\
\hline B.8 & J14L 34 & $3 / 6 / 07$ & 0.29 & U & 0.29 & 14 & $\mathrm{U}$ & 14 \\
\hline B-9 & & $3 / 6 / 07$ & 0.21 & U & 0.21 & 7.2 & $U$ & 7.2 \\
\hline$\frac{D-10}{B-10}$ & J14L36 & $3 / 6 / 07$ & 0.32 & U & 0.32 & 11 & U & 11 \\
\hline
\end{tabular}

Note: Date qualified with B, C, D and/or J, are consldered acceptable values.

MDA = minimum detectable activity

$\begin{array}{ll}\mathrm{B}=\text { blank contamination (organic) } & \mathrm{PQL}=\text { practical quantitation limit } \\ \mathrm{C}=\text { blank contamination }\end{array}$

$D=$ diluted

GEA = gamma energy analysis

$\mathrm{Q}=$ qualifier

HEIS = Hanford Environmental Information System

$\mathrm{I}=$ interterence

$\mathrm{J}=$ estimate

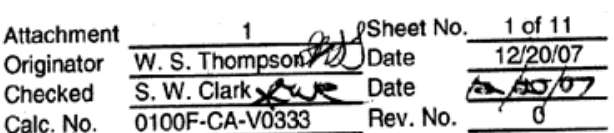

ర్ర 
Attachment 1. 128-F-2 Upland (Area B) Verification Sampling Results.

\begin{tabular}{|c|c|c|c|c|c|c|c|c|c|c|c|c|c|c|c|c|c|c|c|c|c|c|c|}
\hline \multirow{2}{*}{$\begin{array}{c}\text { Sample } \\
\text { Location }\end{array}$} & \multirow{2}{*}{$\begin{array}{c}\text { HEIS } \\
\text { Number }\end{array}$} & \multirow{2}{*}{$\begin{array}{c}\text { Sample } \\
\text { Date }\end{array}$} & \multicolumn{3}{|c|}{ Aluminum } & \multicolumn{3}{|c|}{ Antimony } & \multicolumn{3}{|c|}{ Arsenic } & \multicolumn{3}{|c|}{ Barium } & \multicolumn{3}{|c|}{ Beryllium } & \multicolumn{3}{|c|}{ Boron } & \multicolumn{3}{|c|}{ Cadmium } \\
\hline & & & $\mathrm{mg} / \mathrm{kg}$ & $Q$ & PQL & $\mathrm{mg} / \mathrm{kg}$ & Q & PQL & $\mathrm{mg} / \mathrm{kg}$ & 0 & PQL & $\mathrm{mg} / \mathrm{kg}$ & Q & PQL & $\mathrm{mg} / \mathrm{kg}$ & Q & $\mathrm{PQL}$ & $\mathrm{mg} / \mathrm{kg}$ & Q & PQL & $\mathrm{mg} / \mathrm{kg}$ & Q & $\mathrm{PQL}$ \\
\hline $\begin{array}{l}\text { Equipment } \\
\text { Blank }\end{array}$ & & $3 / 6 / 07$ & & & & & & & & $u$ & 038 & 14 & c & 0.02 & 0.02 & & 0.009 & 0.35 & $\mathrm{u}$ & 0.35 & 0.03 & $U$ & 0.03 \\
\hline$\frac{\text { Blank }}{\text { B-1 }}$ & $\begin{array}{l}\text { J14JT6 } \\
\text { J14L26 }\end{array}$ & $\begin{array}{l}3 / 6 / 07 \\
3 / 6 / 07\end{array}$ & $\frac{54.3}{5140}$ & $\frac{c}{c}$ & $\frac{1.3}{4.3}$ & $\begin{array}{l}0.29 \\
0.94\end{array}$ & $\frac{U}{U}$ & $\frac{0.29}{0.94}$ & $\frac{0.38}{1.7}$ & 0 & $\frac{0.38}{1.2}$ & 5.4 & \begin{tabular}{|l|} 
\\
$c$ \\
\end{tabular} & 0.06 & 0.44 & & 0.03 & 2.6 & & 1.1 & 0.088 & $U$ & 0.09 \\
\hline B-2 & J14L27 & $3 / 6 / 07$ & 6050 & $\mathrm{C}$ & 4.2 & 0.94 & $U$ & 0.94 & 4 & & 1.2 & 65.3 & \begin{tabular}{|l|}
$\mathrm{C}$ \\
\end{tabular} & 0.06 & 0.37 & & 0.03 & 1.6 & & 1.1 & 0.09 & $U$ & 0.09 \\
\hline B-3 & J14L28 & $3 / 6 / 07$ & 7170 & C & 4.6 & 1 & $U$ & 1 & 4.1 & & 1.3 & 76.5 & C & 0.06 & 0.37 & & 0.03 & 1.4 & & 1.2 & 0.01 & $U$ & 0.1 \\
\hline B-4 & J14L29 & $3 / 6 / 07$ & 5770 & C & 4.2 & 0.91 & $\mathrm{U}$ & 0.91 & 1.9 & & 1.2 & 66.1 & c & 0.06 & 0.35 & & 0.03 & 1.6 & & 1.1 & 0.09 & $\mathrm{U}$ & 0.09 \\
\hline B-5 & $\mathrm{J} 14 \mathrm{~L} 30$ & $3 / 6 / 07$ & 7210 & $c$ & 4.3 & 0.95 & $u$ & 0.95 & 3.3 & & 1.2 & 64.4 & c & 0.06 & 0.39 & & 0.03 & 2.7 & & 1.1 & 0.09 & $\mathrm{U}$ & 0.09 \\
\hline Duplicate of & & & & $c$ & & & & & & & & & & & & & 0 & 4 & & 12 & 0.09 & $\mathrm{u}$ & 0.09 \\
\hline$\frac{\mathrm{J} 14 \mathrm{~L} 30}{\mathrm{~B}-6}$ & $\frac{J 14 L 31}{J 14 L 32}$ & $\frac{3 / 6 / 07}{3 / 6 / 07}$ & $\frac{7260}{7290}$ & $\frac{\mathrm{C}}{\mathrm{C}}$ & 4.4 & 0.97 & $u$ & 0.97 & $\frac{4.1}{32}$ & & 1.2 & 66.8 & $C$ & $\frac{0.06}{0.06}$ & $\frac{0.4}{0.35}$ & & $\frac{0.03}{0.03}$ & $\frac{2.4}{2}$ & & 1.1 & 0.09 & U & 0.09 \\
\hline $\begin{array}{l}\text { B-6 } \\
\text { B-7 }\end{array}$ & $\frac{J 14 L 32}{J 14 L 33}$ & $\frac{3 / 6 / 07}{3 / 6 / 07}$ & 5400 & $\frac{C}{c}$ & $\frac{4.1}{4.2}$ & 0.91 & $U$ & 0.91 & $\frac{3.2}{1.8}$ & & $\frac{1.2}{1.2}$ & 66.2 & \begin{tabular}{|l|}
$\mathrm{C}$ \\
$\mathrm{C}$
\end{tabular} & $\frac{0.06}{0.06}$ & 0.31 & & 0.03 & 1.1 & $\mathrm{U}$ & 1.1 & 0.09 & $U$ & 0.09 \\
\hline $\begin{array}{l}B-7 \\
B-8\end{array}$ & J14L34 & $3 / 6 / 07$ & 6070 & $\mathrm{c}$ & $\begin{array}{l}4.2 \\
4.1\end{array}$ & $\frac{0.92}{0.91}$ & $\frac{U}{U}$ & $\frac{0.92}{0.91}$ & $\frac{1.8}{2}$ & & $\frac{1.2}{1.2}$ & $\frac{49.4}{68.6}$ & \begin{tabular}{|l|}
$\mathrm{C}$ \\
$\mathrm{c}$ \\
\end{tabular} & 0.06 & 0.31 & & 0.03 & 1.6 & & 1.1 & 0.09 & $\mathrm{U}$ & 0.09 \\
\hline B-9 & J14L35 & $3 / 6 / 07$ & 7130 & $\mathrm{c}$ & 4.4 & 0.96 & $\mathrm{u}$ & $\frac{0.91}{0.96}$ & 3.9 & & 1.2 & 71.8 & C & 0.06 & 0.36 & & 0.03 & 1.2 & & 1.1 & 0.09 & U & 0.09 \\
\hline 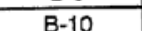 & J14L36 & $3 / 6 / 07$ & 6110 & $\mathrm{C}$ & 4.3 & 0.94 & $U$ & 0.94 & 3.8 & & 1.2 & 81.5 & $\mathrm{c}$ & 0.06 & 0.32 & & 0.03 & 2 & & 1.1 & 0.09 & U & 0.09 \\
\hline
\end{tabular}

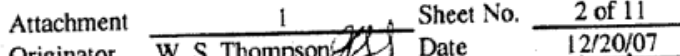

Checked S.W.Clark eque Date 12/20/37

Caic. No. $\frac{\text { OlO0F-CA-V0333 Rev. No. }}{6}$ 
Attachment 1. 128-F-2 Upland (Area B) Verification Sampling Results.

\begin{tabular}{|c|c|c|c|c|c|c|c|c|c|c|c|c|c|c|c|c|c|c|c|c|c|c|}
\hline \multirow{2}{*}{$\begin{array}{c}\text { Sample } \\
\text { Location }\end{array}$} & \multirow{2}{*}{$\begin{array}{c}\text { HEIS } \\
\text { Number }\end{array}$} & \multirow{2}{*}{$\begin{array}{l}\text { Sample } \\
\text { Date }\end{array}$} & \multicolumn{3}{|c|}{ Calcium } & \multicolumn{3}{|c|}{ Chromium } & \multicolumn{3}{|c|}{ Cobalt } & \multicolumn{2}{|c|}{ Copper } & \multicolumn{3}{|c|}{$\begin{array}{l}\text { Hexavalent } \\
\text { Chromium }\end{array}$} & \multicolumn{3}{|c|}{ Iron } & \multicolumn{3}{|c|}{ Lead } \\
\hline & & & $\mathrm{mg} / \mathrm{kg}$ & Q & PQL & $\mathrm{mg} / \mathrm{kg}$ & Q & $\mathrm{PQL}$ & $\mathrm{mg} / \mathrm{kg}$ & Q & $\mathbf{P Q L}$ & $\mathrm{mg} / \mathrm{kg}$ & \begin{tabular}{l|l|}
$Q$ & PQL \\
\end{tabular} & $\mathrm{mg} / \mathrm{kg}$ & Q & PQL & $\mathrm{mg} / \mathrm{kg}$ & Q & PQL & $\mathrm{mg} / \mathrm{kg}$ & Q & PQL \\
\hline Equipment & & & 25.8 & c & 0.69 & 0.15 & & & & & 0 & 0.11 & 0.1 & 0.2 & $u$ & 0.2 & 126 & $c$ & 2.7 & 0.42 & & 0.26 \\
\hline$\frac{B-1}{B-2}$ & \begin{tabular}{|l|} 
J14L26 \\
J14L27 \\
\end{tabular} & $\begin{array}{l}3 / 6 / 07 \\
3 / 6 / 07 \\
\end{array}$ & 6780 & $\mathrm{C}$ & 2.2 & 11.1 & $c$ & 0.23 & 6.1 & & 0.26 & 12.7 & 0.35 & 0.21 & u & 0.21 & 15900 & c & 8.7 & 4.7 & & 0.82 \\
\hline B-3 & J14L28 & $3 / 6 / 07$ & 16600 & $\mathrm{C}$ & 2.4 & 15.1 & C & 0.25 & 6.4 & & 0.29 & 13.9 & 0.38 & 0.23 & 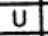 & 0.23 & 16000 & $\mathrm{C}$ & 9.4 & 5.1 & & 0.89 \\
\hline B-4 & J14L29 & $3 / 6 / 07$ & 3540 & $\mathrm{C}$ & 2.1 & 31.3 & $\mathrm{C}$ & 0.25 & 5.3 & & 2.6 & 16.6 & 0.34 & 2.9 & & 0.21 & 15100 & C & 8.5 & 6.9 & & 0.8 \\
\hline B-5 & $\mathrm{J} 14 \mathrm{~L} 30$ & $3 / 6 / 07$ & 13200 & $\mathrm{C}$ & 2.2 & 12.9 & $\mathrm{C}$ & 0.24 & 6.7 & & 0.27 & 12.5 & 0.35 & 0.22 & $u$ & 0.22 & 17400 & C & 8.8 & 4.3 & & 0.83 \\
\hline $\begin{array}{l}\text { Duplicate of } \\
J 14 \mathrm{~L} 30\end{array}$ & & & & & & & & & & & & & & & & & & & & & & \\
\hline$\frac{J 14 L 30}{B \cdot 6}$ & $\begin{array}{l}\mathrm{J} 14 \mathrm{~L} 31 \\
\mathrm{~J} 14 \mathrm{~L} 32\end{array}$ & $\begin{array}{l}3 / 6 / 07 \\
3 / 6 / 07\end{array}$ & $\frac{12900}{8000}$ & C & 2.3 & 12.8 & C & 0.24 & 6.4 & & 0.27 & 12.7 & 0.37 & 0.33 & & 0.22 & 17400 & C & 9 & 4.6 & & 0.85 \\
\hline B-6 & $\frac{\mathrm{J} 14 \mathrm{~L} 32}{\mathrm{~J} 14 \mathrm{~L} 33}$ & $\frac{3 / 6 / 07}{3 / 6 / 07}$ & $\frac{8000}{2760}$ & C & 2.1 & 17.7 & $\mathrm{C}$ & 0.23 & 6.2 & & 0.26 & 16.1 & 0.34 & 0.36 & & 0.21 & 16500 & C & 8.4 & 7.4 & & 0.79 \\
\hline B-7 & $\begin{array}{l}\text { J14L33 } \\
\text { J14L34 }\end{array}$ & $\frac{3 / 6 / 07}{3 / 6 / 07}$ & $\begin{array}{l}2760 \\
3190\end{array}$ & C & 2.2 & 16.9 & $\mathrm{C}$ & 0.23 & 4.9 & & 0.26 & 14.1 & 0.34 & 0.26 & & 0.21 & 13800 & C & 8.5 & 3.1 & & 0.8 \\
\hline B-8 & J14L34 & $\frac{3 / 6 / 07}{3 / 6 / 07}$ & $\begin{array}{l}3190 \\
15400\end{array}$ & C & 2.1 & 42.4 & $\mathrm{C}$ & 0.23 & 5.3 & & 0.26 & 15.4 & 0.34 & 2.7 & & 0.21 & 15500 & c & 8.4 & 5.9 & & 0.79 \\
\hline B-9 & J14L35 & $\begin{array}{l}3 / 6 / 07 \\
3 / 6 / 07\end{array}$ & $\frac{15400}{3920}$ & $\mathrm{C}$ & 2.3 & 12 & C & 0.24 & 6.5 & & 0.27 & 14.5 & 0.36 & 0.29 & & 0.22 & 16800 & C & 8.9 & 4.7 & & 0.84 \\
\hline B-10 & J14L36 & $3 / 6 / 07$ & 3920 & C & 2.2 & 19.6 & C & 0.23 & 5.3 & & 0.26 & 110 & 0.35 & 0.36 & & 0.21 & 15500 & C & 8.7 & 26.4 & & 0.82 \\
\hline B-1 & J15FDO & $8 / 16 / 07$ & & & & & & & & & & & & 1 & & 0.2 & & & & & & \\
\hline $\mathrm{B}-2$ & J15FD1 & $8 / 16 / 07$ & & & & & & & & & & & & 0.61 & & 0.2 & & & & & & \\
\hline B-3 & J15FD2 & $8 / 16 / 07$ & & & & & & & & & & & & 0.2 & $U$ & 0.2 & & & & & & \\
\hline B-4 & J15FD3 & $8 / 16 / 07$ & & & & & & & & & & & & 0.23 & & 0.2 & & & & & & \\
\hline B-5 & J15FD4 & $8 / 16 / 07$ & & & & & & & & & & & & 2 & & 0.2 & & & & & & \\
\hline B-6 & J15FD5 & $8 / 16 / 07$ & & & & & & & & & & & & 0.2 & $U$ & 0.2 & & & & & & \\
\hline B.7 & J15FD6 & $8 / 16 / 07$ & & & & & & & & & & & & 0.2 & $u$ & 0.2 & & & & & & \\
\hline B-8 & J15FD7 & $8 / 16 / 07$ & & & & & & & & & & & & 0.26 & & 0.2 & & & & & & \\
\hline B-9 & J15FD8 & $8 / 16 / 07$ & & & & & & & & & & & & 0.24 & & 0.2 & & & & & & \\
\hline $8-10$ & J15FD9 & $8 / 16 / 07$ & & & & & & & & & & & & 0.22 & & 0.2 & & & & & & \\
\hline
\end{tabular}

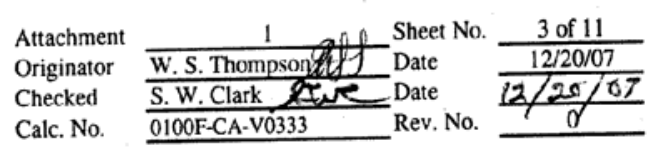


Attachment 1.128-F-2 Upland (Area B) Verification Sampling Results.

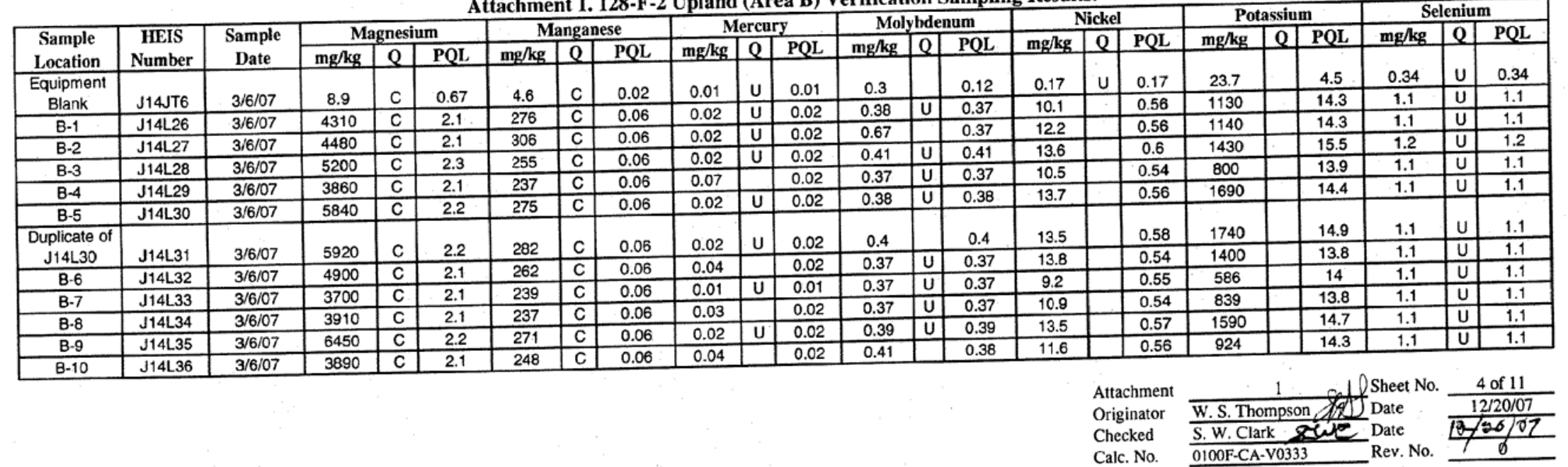


Attachment 1. 128-F-2 Upland (Area B) Verification Sampling Results.

\begin{tabular}{|c|c|c|c|c|c|c|c|c|c|c|c|c|c|c|c|c|c|c|c|c|}
\hline \multirow{2}{*}{$\begin{array}{c}\text { Sample } \\
\text { Location }\end{array}$} & \multirow{2}{*}{$\begin{array}{c}\text { HEIS } \\
\text { Number }\end{array}$} & \multirow{2}{*}{$\begin{array}{c}\text { Sample } \\
\text { Date }\end{array}$} & \multicolumn{3}{|c|}{ Silicon } & \multicolumn{3}{|c|}{ Silver } & \multicolumn{3}{|c|}{ Sodium } & \multicolumn{3}{|c|}{ Vanadium } & \multicolumn{3}{|c|}{ Zinc } & \multicolumn{3}{|c|}{$\begin{array}{l}\text { Total petroleum } \\
\text { hydrocarbons }\end{array}$} \\
\hline & & & $\mathrm{mg} / \mathrm{kg}$ & $Q$ & PQL & $\mathrm{mg} / \mathrm{kg}$ & Q & $\mathrm{PQL}$ & $\mathrm{mg} / \mathrm{kg}$ & 0 & PQL & $\mathrm{mg} / \mathrm{kg}$ & Q & PQL & $\mathrm{mg} / \mathrm{kg}$ & Q & PQL & $\mathrm{mg} / \mathrm{kg}$ & Q & PQL \\
\hline $\begin{array}{l}\text { Equipment } \\
\text { Blank }\end{array}$ & J14JT6 & $3 / 6 / 07$ & 54.1 & C & 0.39 & 0.08 & $\mathrm{u}$ & 0.08 & 8.4 & c & 2.7 & & & & 0.14 & & 0.09 & 1 & C & 0.3 \\
\hline B-1 & $\mathrm{J} 14 \mathrm{~L} 26$ & $3 / 6 / 07$ & 440 & $c$ & 1.2 & 0.26 & $\mathrm{U}$ & 0.26 & 143 & C & 2.9 & 142 & 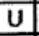 & 142 & 54.6 & & 0.29 & 40.2 & $C$ & 0.09 \\
\hline B-2 & $\mathrm{J} 14 \mathrm{~L} 27$ & $3 / 6 / 07$ & 690 & $\mathrm{C}$ & 1.2 & 0.26 & $U$ & 0.26 & 256 & $\mathrm{C}$ & 2.9 & 140 & U & 140 & 33.6 & & 0.29 & 35.8 & $c$ & 0.09 \\
\hline B-3 & $\mathrm{J} 14 \mathrm{~L} 28$ & $3 / 6 / 07$ & 535 & C & 1.3 & 0.29 & $u$ & 0.29 & 166 & C & 3.2 & 152 & U & 152 & 32.2 & & 0.32 & 36.8 & C & 0.1 \\
\hline B-4 & J14L29 & $3 / 6 / 07$ & 392 & C & 1.2 & 0.26 & $u$ & 0.26 & 99.9 & C & 2.8 & 137 & U & 137 & 32.1 & & 0.29 & 46.8 & C & 0.09 \\
\hline B. 5 & $\mathrm{~J} 14 \mathrm{~L} 30$ & $3 / 6 / 07$ & 359 & $\mathrm{C}$ & 1.2 & 0.27 & 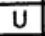 & 0.27 & 177 & $\mathrm{C}$ & 2.9 & 143 & 0 & 143 & 36.3 & & 0.3 & 42.4 & C & 0.09 \\
\hline Duplicate of & & & & & & & & & & & & & ol & 146 & 361 & & 0.3 & 38.4 & $c$ & 0.09 \\
\hline J14L_30 & J14L31 & $3 / 6 / 07$ & 706 & $\mathrm{C}$ & 1.3 & 0.27 & $u$ & 0.27 & 177 & $\mathrm{C}$ & 3 & $\frac{146}{107}$ & $\frac{0}{u}$ & $\frac{146}{137}$ & $\frac{30.1}{31.9}$ & & 0.28 & 48.4 & C & 0.09 \\
\hline B.6 & J14L32 & $3 / 6 / 07$ & 539 & C & 1.2 & 0.26 & $U$ & 0.26 & 213 & C & 2.8 & $\frac{137}{138}$ & $\frac{0}{u}$ & 138 & 33.4 & & 0.29 & 30.9 & C & 0.09 \\
\hline B.7 & J14L33 & $3 / 6 / 07$ & 501 & $c$ & 1.2 & 0.26 & $u$ & 0.26 & 97.5 & C & 2.9 & $\frac{138}{137}$ & $\frac{0}{v}$ & 130 & 33 & & 0.28 & 43.6 & C & 0.09 \\
\hline$B-8$ & J14L34 & $3 / 6 / 07$ & 565 & C & 1.2 & 0.26 & $u$ & 0.26 & 187 & $\mathrm{C}$ & $\frac{2.8}{3}$ & & u & 144 & 31.7 & & 0.3 & 38.3 & $\bar{c}$ & 0.09 \\
\hline B-9 & J14L35 & $3 / 6 / 07$ & 533 & C & 1.3 & 0.27 & $U$ & 0.27 & $\frac{593}{132}$ & $\frac{\mathrm{C}}{\mathrm{c}}$ & $\frac{3}{2.9}$ & $\frac{744}{140}$ & $\mathrm{U}$ & 140 & 32.4 & & 0.29 & 65.9 & $\mathrm{C}$ & 0.09 \\
\hline$B-10$ & $\mathrm{~J} 14 \mathrm{~L} 36$ & $3 / 6 / 07$ & 510 & C & 1.2 & 0.26 & U & 0.26 & 132 & & & & & & & & & & & \\
\hline
\end{tabular}

Attachment

Sriginator W. Thompson Sheet No. $\frac{5 \text { of } 11}{12 / 20 / 07}$

Checked S.W.Clark sewe Date

Calc. No.

Rev. No. 


\begin{tabular}{|c|c|c|c|c|c|c|c|c|c|c|c|c|}
\hline \multirow[t]{2}{*}{ Constituent } & \multicolumn{3}{|c|}{$\begin{array}{c}\text { J14L26 } \\
\text { Sample Location B-1 } \\
\text { Sample Date } 5 / 13 / 07\end{array}$} & \multicolumn{3}{|c|}{$\begin{array}{c}\text { J14L27 } \\
\text { Sample Location B-2 } \\
\text { Sample Date 5/13/07 }\end{array}$} & \multicolumn{3}{|c|}{$\begin{array}{c}\text { J14L28 } \\
\text { Sample Location B-3 } \\
\text { Sample Date 5/13/07 }\end{array}$} & \multicolumn{3}{|c|}{$\begin{array}{c}\text { J14L29 } \\
\text { Sample Location B-4 } \\
\text { Sample Date 5/13/07 }\end{array}$} \\
\hline & $\mu \mathrm{g} / \mathrm{kg}$ & \begin{tabular}{l|l}
$\mathbf{Q}$ & \\
\end{tabular} & $\mathrm{PQL}$ & $\mu \mathrm{g} / \mathrm{kg}$ & $\mathbf{Q}$ & PQL & $\mu \mathrm{g} / \mathrm{kg}$ & Q & PQL & $\mu g / \mathbf{k g}$ & \begin{tabular}{l|l}
$\mathbf{a}$ & \\
\end{tabular} & PQL \\
\hline \multicolumn{13}{|c|}{ Polychlorinated Biphenyls } \\
\hline Aroclor-1016 & 14 & $U$ & 14 & 14 & U & 14 & 15 & U & 15 & 14 & U & 14 \\
\hline Aroclor-1221 & 14 & U & 14 & 14 & $u$ & 14 & 15 & U & 15 & 14 & $\mathrm{U}$ & 14 \\
\hline Aroclor-1232 & 14 & U & 14 & 14 & $\mathrm{U}$ & 14 & 15 & \begin{tabular}{|l|}
$U$ \\
\end{tabular} & 15 & 14 & \begin{tabular}{|l|}
$U$ \\
\end{tabular} & 14 \\
\hline Aroclor-1242 & 14 & $U$ & 14 & 14 & $\mathrm{U}$ & 14 & 15 & U & 15 & 14 & U & 14 \\
\hline \begin{tabular}{|l|} 
Aroclor- 1248 \\
\end{tabular} & 14 & $U$ & 14 & 14 & $\mathrm{U}$ & 14 & 15 & $\mathrm{U}$ & 15 & 14 & $\begin{array}{ll}U \\
\end{array}$ & 14 \\
\hline Aroclor- 1254 & 14 & $\mathrm{U}$ & 14 & 14 & U & 14 & 15 & $\mathrm{U}$ & 15 & 11 & $\mathrm{~J}$ & 14 \\
\hline Arocior- 1260 & 14 & U & 14 & 14 & \begin{tabular}{|l|}
$U$ \\
\end{tabular} & 14 & 15 & U & 15 & 14 & \begin{tabular}{|l|}
$\mathrm{U}$ \\
\end{tabular} & 14 \\
\hline \multicolumn{13}{|c|}{ Pesticides } \\
\hline Aldrin & 1.3 & UD & 1.3 & 1.3 & UD & 1.3 & 1.3 & \begin{tabular}{|l|} 
UD \\
\end{tabular} & 1.3 & 1.3 & UD & 1.3 \\
\hline Alpha-BHC & 1.3 & UD & 1.3 & 1.3 & UD & 1.3 & 1.3 & UD & 1.3 & 1.3 & UD & 1.3 \\
\hline Alpha-Chlordane & 1.3 & UD & 1.3 & 1.3 & UD & 1.3 & 1.3 & UD & 1.3 & 1.3 & UD & 1.3 \\
\hline Beta-BHC & 1.3 & UD & 1.3 & 1.3 & UD & 1.3 & 1.3 & UD & 1.3 & 1.3 & UD & 1.3 \\
\hline Delta-BHC & 1.3 & \begin{tabular}{|l|l|l|l} 
UD \\
\end{tabular} & 1.3 & 1.3 & UD & 1.3 & 1.3 & UD & 1.3 & 1.3 & UD & 1.3 \\
\hline \begin{tabular}{|l|} 
Dichlorodiphenyldichloroethane \\
\end{tabular} & 1.3 & UD & 1.3 & 1.3 & UD & 1.3 & 1.3 & UD & 1.3 & 1.3 & UD & 1.3 \\
\hline \begin{tabular}{|l|} 
Dichlorodiphenyldichloroethylene \\
\end{tabular} & 1.3 & UD & 1.3 & 8.3 & \begin{tabular}{|l|l|}
$D$ \\
\end{tabular} & 1.3 & 1.3 & UD & 1.3 & 1.3 & UD & 1.3 \\
\hline \begin{tabular}{|l|} 
Dichlorodiphenyltrichloroethane \\
\end{tabular} & 1.3 & UD & 1.3 & 45 & \begin{tabular}{|l|l|}
$D$ \\
\end{tabular} & 1.3 & 1.3 & UD & 1.3 & 1.3 & UD & 1.3 \\
\hline \begin{tabular}{|l|} 
Dieldrin \\
\end{tabular} & 1.3 & UD & 1.3 & 1.3 & UD & 1.3 & 1.3 & UD & 1.3 & 1.3 & UD & 1.3 \\
\hline Endosulfan I & 1.3 & \begin{tabular}{|l|l|l|l|l} 
UD \\
\end{tabular} & 1.3 & 4 & $\mathrm{JD}$ & 1.3 & 1.3 & UD & 1.3 & 1.3 & UD & 1.3 \\
\hline Endosulfan II & 1.3 & UD & 1.3 & 1.3 & UD & 1.3 & 1.3 & UD & 1.3 & 1.3 & UD & 1.3 \\
\hline Endosulfan sulfate & 1.3 & UD & 1.3 & 1.3 & UD & 1.3 & 1.3 & UD & 1.3 & 1.3 & UD & 1.3 \\
\hline \begin{tabular}{|l} 
Endrin \\
\end{tabular} & 1.3 & UD & 1.3 & 20 & D & 1.3 & 1.3 & UD & 1.3 & 1.3 & UD & 1.3 \\
\hline \begin{tabular}{|l} 
Endrin aldehyde \\
\end{tabular} & 1.3 & UD & 1.3 & 1.3 & UD & 1.3 & 1.3 & UD & 1.3 & 1.3 & UD & 1.3 \\
\hline \begin{tabular}{|l} 
Endrin ketone \\
\end{tabular} & 1.3 & UD & 1.3 & 1.3 & UD & 1.3 & 1.3 & UD & 1.3 & 1.3 & UD & 1.3 \\
\hline Gamma-BHC (Lindane) & 1.3 & UD & 1.3 & 1.3 & UD & 1.3 & 1.3 & UD & 1.3 & 1.3 & UD & 1.3 \\
\hline gamma-Chlordane & 1.3 & UD & 1.3 & 1.3 & UD & 1.3 & 1.3 & UD & 1.3 & 1.3 & UD & 1.3 \\
\hline Heptachlor & 1.3 & UD & 1.3 & 1.3 & UD & 1.3 & 1.3 & UD & 1.3 & 1.3 & UD & 1.3 \\
\hline Heptachlor epoxide & 1.3 & UD & 1.3 & 1.3 & UD & 1.3 & 1.3 & UD & 1.3 & 1.3 & UD & 1.3 \\
\hline Methoxychlor & 1.3 & UD & 1.3 & 1.3 & UD & 1.3 & 1.3 & UD & 1.3 & 1.3 & UD & 1.3 \\
\hline Toxaphene & 13 & UD & 13 & 13 & UD & 13 & 13 & UD & 13 & 13 & UD & 13 \\
\hline \multicolumn{13}{|c|}{ Semivolatile Organic Analytes } \\
\hline 1,2,4-Trichlorobenzene & 350 & $\mathrm{U}$ & 350 & 350 & $\mathrm{U}$ & 350 & 380 & $U$ & 380 & 340 & $\mathrm{U}$ & 340 \\
\hline 1,2-Dichlorobenzene & 350 & U & 350 & 350 & U & 350 & 380 & U & 380 & 340 & U & 340 \\
\hline \begin{tabular}{|l|}
1,3 -Dichlorobenzene \\
\end{tabular} & 350 & U & 350 & 350 & $U$ & 350 & 380 & $U$ & 380 & 340 & U & 340 \\
\hline 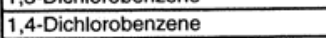 & 350 & U & 350 & 350 & $\mathrm{U}$ & 350 & 380 & $\mathrm{U}$ & 380 & 340 & \begin{tabular}{|l|}
$\mathrm{U}$ \\
\end{tabular} & 340 \\
\hline \begin{tabular}{|l|}
$2,4,5-T r i c h l o r o p h e n o l$ \\
\end{tabular} & 880 & U & 880 & 880 & U & 880 & 950 & \begin{tabular}{|l|}
$\mathrm{U}$ \\
\end{tabular} & 950 & 860 & $\mathrm{U}$ & 860 \\
\hline 2,4,6-Trichlorophenol & 350 & \begin{tabular}{|l|}
$U$ \\
\end{tabular} & 350 & 350 & $\mathrm{U}$ & 350 & 380 & \begin{tabular}{|l|}
$U$ \\
\end{tabular} & 380 & 340 & $\mathrm{U}$ & 340 \\
\hline \begin{tabular}{|l|}
$2,4-$-Dichlorophenol \\
\end{tabular} & 350 & U & 350 & 350 & $\mathrm{U}$ & 350 & 380 & U & 380 & 340 & \begin{tabular}{|l|}
$\mathrm{U}$ \\
\end{tabular} & 340 \\
\hline 2,4-Dimethylphenol & 350 & $\mathrm{U}$ & 350 & 350 & U & 350 & 380 & U & 380 & 340 & U & 340 \\
\hline 2,4-Dinitrophenol & 880 & U & 880 & 880 & U & 880 & 950 & $U$ & 950 & 860 & \begin{tabular}{|l|}
$U$ \\
\end{tabular} & 860 \\
\hline 2,4-Dinitrotoluene & 350 & $\mathrm{U}$ & 350 & 350 & U & 350 & 380 & $\mathrm{U}$ & 380 & 340 & $\mathrm{U}$ & 340 \\
\hline 2,6-Dinitrotoluene & 350 & U & 350 & 350 & $\mathrm{U}$ & 350 & 380 & $U$ & 380 & 340 & U & 340 \\
\hline 2-Chloronaphthalene & 350 & U & 350 & 350 & $\mathrm{U}$ & 350 & 380 & $U$ & 380 & 340 & $\mathrm{U}$ & 340 \\
\hline \begin{tabular}{|l|} 
2-Chlorophenol \\
\end{tabular} & 350 & U & 350 & 350 & $\mathrm{U}$ & 350 & 380 & $U$ & 380 & 340 & U & 340 \\
\hline 2-Methylnaphthalene & 350 & $\mathrm{U}$ & 350 & 350 & $\mathrm{u}$ & 350 & 380 & U & 380 & 340 & \begin{tabular}{|l|}
$U$ \\
\end{tabular} & 340 \\
\hline \begin{tabular}{|l|}
$2-$ Methyiphenol (cresol, o-) \\
\end{tabular} & 350 & U & 350 & 350 & $u$ & 350 & 380 & U & 380 & 340 & \begin{tabular}{l|l}
$U$ \\
\end{tabular} & 340 \\
\hline \begin{tabular}{|l|} 
2-Nitroaniline \\
\end{tabular} & 880 & $\mathrm{U}$ & 880 & 880 & $U$ & 880 & 950 & U & 950 & 860 & U & 860 \\
\hline \multirow[t]{5}{*}{ 2-Nitrophenol } & 350 & U & 350 & 350 & $U$ & 350 & 380 & $U$ & 380 & 340 & \begin{tabular}{|l|}
$U$ \\
\end{tabular} & 340 \\
\hline & & & & & \multirow{4}{*}{\multicolumn{2}{|c|}{$\begin{array}{l}\text { Attachment } \\
\text { Originator } \\
\text { Checked } \\
\text { Calc. No. }\end{array}$}} & \multirow{2}{*}{\multicolumn{3}{|c|}{$\frac{1}{\text { S. Th }}$}} & \multirow{4}{*}{$\begin{array}{l}\text { Sheet No. } \\
\text { Date } \\
\text { Date } \\
\text { Rev. No. }\end{array}$} & \multicolumn{2}{|r|}{6 of 11} \\
\hline & & & & & & & & & & & & $12 / 20 / 07$ \\
\hline & & & & & & & S.W.C & & & & & $100 / 0$ \\
\hline & & & & & & & $0100 \mathrm{~F}-\mathrm{C}$ & $\mathrm{A}-\mathrm{V} 03$ & & & & 01 \\
\hline
\end{tabular}




\begin{tabular}{|c|c|c|c|c|c|c|c|c|c|c|c|c|}
\hline \multirow[t]{2}{*}{ Constituent } & \multicolumn{3}{|c|}{\begin{tabular}{c|} 
J14L30 \\
Sample Location B-5 \\
Sample Date 5/13/07
\end{tabular}} & \multicolumn{3}{|c|}{$\begin{array}{c}\text { J14L31 } \\
\text { Duplicate of J14L30 } \\
\text { Sample Date 8/13/07 }\end{array}$} & \multicolumn{3}{|c|}{$\begin{array}{c}\text { J14L32 } \\
\text { Sample Location B-6 } \\
\text { Sample Date 5/13/07 }\end{array}$} & \multicolumn{3}{|c|}{$\begin{array}{c}\text { J14L33 } \\
\text { Sample Location B-7 } \\
\text { Sample Date 5/13/07 }\end{array}$} \\
\hline & $\mu \mathrm{g} / \mathrm{kg}$ & Q & PQL & $\mu \mathrm{g} / \mathrm{kg}$ & $\mathbf{Q}$ & PQL & $\mu \mathrm{g} / \mathrm{kg}$ & $\mathbf{Q}$ & PQL & $\mu g / \mathrm{kg}$ & $\mathbf{Q}$ & PQL \\
\hline \multicolumn{13}{|c|}{ Polychlorinated Biphenyls } \\
\hline \begin{tabular}{|l|} 
Aroclor-1016 \\
\end{tabular} & 14 & $U$ & 14 & 15 & $\mathrm{U}$ & 15 & 14 & $\mathrm{U}$ & 14 & 14 & $\mathrm{U}$ & 14 \\
\hline Aroclor-1221 & 14 & $U$ & 14 & 15 & $U$ & 15 & 14 & $\mathrm{U}$ & 14 & 14 & $U$ & 14 \\
\hline Aroclor-1232 & 14 & $\mathrm{U}$ & 14 & 15 & $\mathrm{U}$ & 15 & 14 & $\mathrm{U}$ & 14 & 14 & $U$ & 14 \\
\hline Aroclor-1242 & 14 & $U$ & 14 & 15 & $\mathrm{U}$ & 15 & 14 & $\mathrm{U}$ & 14 & 14 & $U$ & 14 \\
\hline Aroclor-1248 & 14 & $\mathrm{U}$ & 14 & 15 & $\mathrm{U}$ & 15 & 14 & $U$ & 14 & 14 & $U$ & 14 \\
\hline Aroclor-1254 & 14 & $\mathrm{U}$ & 14 & 15 & $U$ & 15 & 20 & & 14 & 14 & $U$ & 14 \\
\hline Aroclor- 1260 & 14 & $\mathrm{U}$ & 14 & 15 & $\mathrm{U}$ & 15 & 14 & $\mathrm{U}$ & 14 & 14 & $U$ & 14 \\
\hline \multicolumn{13}{|c|}{ Pesticides } \\
\hline Aldrin & 1.7 & \begin{tabular}{|l|l|}
$\mathrm{D}$ & \\
\end{tabular} & 1.7 & 1.3 & UD & 1.3 & 1.3 & UD & 1.3 & 1.3 & UD & 1.3 \\
\hline Alpha-BHC & 1.3 & UD & 1.3 & 1.3 & UD & 1.3 & 1.3 & UD & 1.3 & 1.3 & UD & 1.3 \\
\hline Alpha-Chlordane & 1.3 & UD & 1.3 & 1.3 & UD & 1.3 & 5.5 & $\mathrm{JD}$ & 1.3 & 1.3 & UD & 1.3 \\
\hline Beta-BHC & 1.3 & UD & 1.3 & 1.3 & UD & 1.3 & 14 & $\mathrm{D}$ & 1.3 & 4.3 & JD & 4.3 \\
\hline Delta-BHC & 1.3 & UD & 1.3 & 1.3 & UD & 1.3 & 1.3 & UD & 1.3 & 1.3 & UD & 1.3 \\
\hline \begin{tabular}{|l|} 
Dichlorodiphenyldichloroethane \\
\end{tabular} & 1.3 & UD & 1.3 & 1.3 & UD & 1.3 & 3 & $\mathrm{JD}$ & 1.3 & 1.3 & UD & 1.3 \\
\hline Dichlorodiphenyldichloroethylene & 1.3 & UD & 1.3 & 1.3 & UD & 1.3 & 9.4 & $\mathrm{D}$ & 1.3 & 1.3 & UD & 1.3 \\
\hline \begin{tabular}{|l|} 
Dichlorodiphenyltrichloroethane \\
\end{tabular} & 1.3 & UD & 1.3 & 1.3 & UD & 1.3 & 33 & $\mathrm{D}$ & 1.3 & 1.3 & UD & 1.3 \\
\hline Dieldrin & 1.3 & UD & 1.3 & 1.3 & UD & 1.3 & 1.3 & UD & 1.3 & 1.3 & UD & 1.3 \\
\hline Endosulfan I & 1.3 & UD & 1.3 & 1.3 & UD & 1.3 & 2.3 & $\mathrm{JD}$ & 1.3 & 1.3 & UD & 1.3 \\
\hline Endosulfan II & 1.3 & UD & 1.3 & 1.3 & UD & 1.3 & 1.3 & UD & 1.3 & 1.3 & UD & 1.3 \\
\hline Endosulfan sulfate & 1.3 & UD & 1.3 & 1.3 & UD & 1.3 & 1.3 & UD & 1.3 & 1.3 & UD & 1.3 \\
\hline \begin{tabular}{|l} 
Endrin \\
\end{tabular} & 1.3 & UD & 1.3 & 1.3 & UD & 1.3 & 7.7 & $\mathrm{D}$ & 1.3 & 1.3 & UD & 1.3 \\
\hline \begin{tabular}{|l} 
Endrin aldehyde \\
\end{tabular} & 1.3 & UD & 1.3 & 1.3 & UD & 1.3 & 1.3 & UD & 1.3 & 1.3 & UD & 1.3 \\
\hline \begin{tabular}{|l} 
Endrin ketone \\
\end{tabular} & 1.3 & UD & 1.3 & 1.3 & UD & 1.3 & 1.4 & $\mathrm{JD}$ & 1.3 & 1.3 & UD & 1.3 \\
\hline Gamma-BHC (Lindane) & 1.3 & UD & 1.3 & 1.3 & UD & 1.3 & 1.3 & UD & 1.3 & 1.3 & UD & 1.3 \\
\hline gamma-Chlordane & 1.3 & UD & 1.3 & 1.3 & UD & 1.3 & 4.6 & $\mathrm{JD}$ & 1.3 & 1.3 & UD & 1.3 \\
\hline Heptachlor & 1.3 & UD & 1.3 & 1.3 & UD & 1.3 & 1.3 & UD & 1.3 & 1.3 & UD & 1.3 \\
\hline Heptachlor epoxide & 1.3 & \begin{tabular}{|l|l|} 
UD \\
\end{tabular} & 1.3 & 1.3 & UD & 1.3 & 1.3 & UD & 1.3 & 1.3 & UD & 1.3 \\
\hline \begin{tabular}{|l} 
Methoxychlor \\
\end{tabular} & 1.3 & UD & 1.3 & 1.3 & UD & 1.3 & 1.3 & UD & 1.3 & 1.3 & UD & 1.3 \\
\hline Toxaphene & 13 & UD & 13 & 13 & UD & 13 & 13 & UD & 13 & 13 & UD & 13 \\
\hline \multicolumn{13}{|c|}{ Semivolatile Organic Analytes } \\
\hline 1,2,4-Trichlorobenzene & 360 & U & 360 & 370 & $U$ & 370 & 340 & $U$ & 340 & 350 & $U$ & 350 \\
\hline 1,2-Dichlorobenzene & 360 & \begin{tabular}{|l|}
$U$ \\
\end{tabular} & 360 & 370 & $\mathrm{U}$ & 370 & 340 & $U$ & 340 & 350 & $U$ & 350 \\
\hline 1,3-Dichlorobenzene & 360 & $\mathrm{U}$ & 360 & 370 & $U$ & 370 & 340 & $\mathrm{U}$ & 340 & 350 & $U$ & 350 \\
\hline \begin{tabular}{|l} 
1,4-Dichlorobenzene \\
\end{tabular} & 360 & $\mathrm{U}$ & 360 & 370 & $U$ & 370 & 340 & $U$ & 340 & 350 & $\mathrm{U}$ & 350 \\
\hline 2,4,5-Trichlorophenol & 900 & $\mathrm{U}$ & 900 & 910 & $U$ & 910 & 860 & $U$ & 860 & 860 & $U$ & 860 \\
\hline 2,4,6-Trichlorophenol & 360 & $\mathrm{U}$ & 360 & 370 & $\mathrm{U}$ & 370 & 340 & $U$ & 340 & 350 & $U$ & 350 \\
\hline 2,4-Dichlorophenol & 360 & $U$ & 360 & 370 & $\mathrm{U}$ & 370 & 340 & $\mathrm{U}$ & 340 & 350 & $U$ & 350 \\
\hline 2,4-Dimethylphenol & 360 & $\mathrm{U}$ & 360 & 370 & $\mathrm{U}$ & 370 & 340 & $\mathrm{U}$ & 340 & 350 & $U$ & 350 \\
\hline 2,4-Dinitrophenol & 900 & $\mathrm{U}$ & 900 & 910 & $U$ & 910 & 860 & $\mathrm{U}$ & 860 & 860 & $U$ & 860 \\
\hline 2,4-Dinitrotoluene & 360 & $\mathrm{U}$ & 360 & 370 & $U$ & 370 & 340 & $U$ & 340 & 350 & $U$ & 350 \\
\hline 2,6-Dinitrotoluene & 360 & U & 360 & 370 & $U$ & 370 & 340 & $\mathrm{U}$ & 340 & 350 & $U$ & 350 \\
\hline \begin{tabular}{|l} 
2-Chloronaphthalene \\
\end{tabular} & 360 & $\mathrm{U}$ & 360 & 370 & $U$ & 370 & 340 & $\mathrm{U}$ & 340 & 350 & $U$ & 350 \\
\hline \begin{tabular}{|l|} 
2-Chlorophenol \\
\end{tabular} & 360 & U & 360 & 370 & $U$ & 370 & 340 & $U$ & 340 & 350 & $\mathrm{U}$ & 350 \\
\hline 2-Methylnaphthalene & 360 & \begin{tabular}{|l|}
$\mathrm{U}$ \\
\end{tabular} & 360 & 370 & $\mathrm{U}$ & 370 & 340 & $U$ & 340 & 350 & $\mathrm{U}$ & 350 \\
\hline 2-Methylphenol (cresol, o-) & 360 & \begin{tabular}{|l|l}
$u$ \\
\end{tabular} & 360 & 370 & $U$ & 370 & 340 & $U$ & 340 & 350 & $U$ & 350 \\
\hline 2-Nitroaniline & 900 & $\mathrm{U}$ & 900 & 910 & $U$ & 910 & 860 & $\mathrm{U}$ & 860 & 860 & $\mathrm{U}$ & 860 \\
\hline \multirow[t]{5}{*}{ 2-Nitrophenol } & 360 & U & 360 & 370 & $\mathrm{U}$ & 370 & 340 & $U$ & 340 & 350 & $\mathrm{U}$ & 350 \\
\hline & & & & \multicolumn{3}{|c|}{ Attachment } & & \multirow{2}{*}{$\begin{array}{l}\text { Sheet No. } \\
\text { Date }\end{array}$} & \multicolumn{2}{|r|}{8 of 11} \\
\hline & & & & & \multicolumn{2}{|c|}{ Originator } & \multicolumn{3}{|c|}{ W.S. Thompson (t) } & & & $2 / 20 / 07$ \\
\hline & & & & & Che & & S. W. Cla & & 2Tue & & & $100 / 0$ \\
\hline & & & & & Calc & No. & $0100 \mathrm{~F}-\mathrm{C}$ & A-V033 & & Rev. No. & & 0 \\
\hline
\end{tabular}




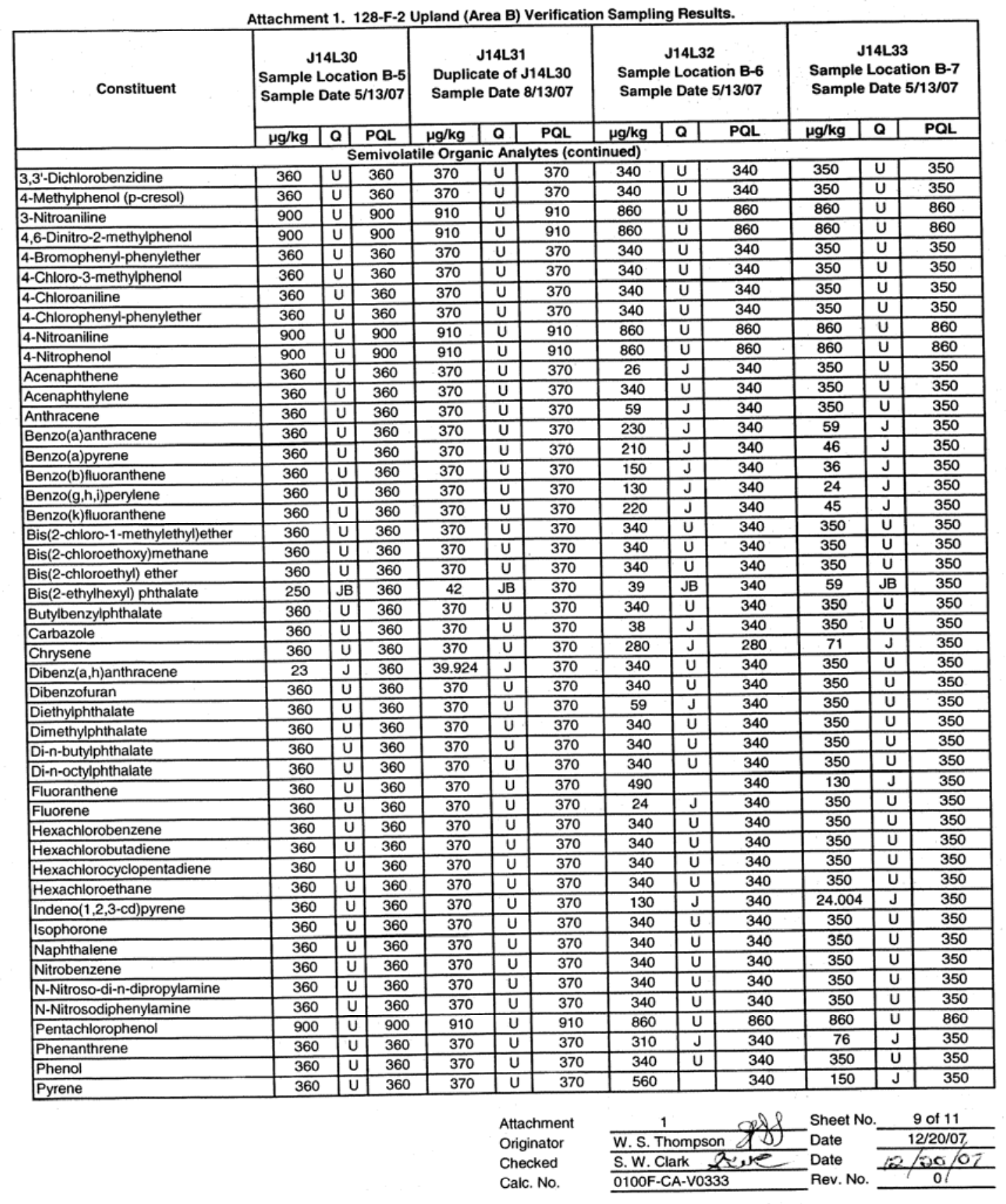




\begin{tabular}{|c|c|c|c|c|c|c|c|c|c|}
\hline \multirow[t]{2}{*}{ Constituent } & \multicolumn{3}{|c|}{$\begin{array}{c}\text { J14L34 } \\
\text { Sample Location B-8 } \\
\text { Sample Date 5/13/07 }\end{array}$} & \multicolumn{3}{|c|}{$\begin{array}{c}\text { J14L35 } \\
\text { Sample Location B-9 } \\
\text { Sample Date 5/13/07 }\end{array}$} & \multicolumn{3}{|c|}{$\begin{array}{c}\text { J14L-36 } \\
\text { Sample Location B-10 } \\
\text { Sample Date 5/13/07 }\end{array}$} \\
\hline & $\mu \mathrm{g} / \mathrm{kg}$ & $\mathbf{Q}$ & PQL & $\mu \mathrm{g} / \mathrm{kg}$ & Q & $\mathrm{PQL}$ & $\mu \mathrm{g} / \mathrm{kg}$ & $\mathbf{a}$ & PQL \\
\hline \multicolumn{10}{|c|}{ Polychlorinated Biphenyls } \\
\hline Aroclor-1016 & 14 & U & 14 & 14 & $U$ & 14 & 14 & $\mathrm{U}$ & 14 \\
\hline Aroclor-1221 & 14 & $\mathrm{U}$ & 14 & 14 & $\mathrm{U}$ & 14 & 14 & $\mathrm{U}$ & 14 \\
\hline Aroclor-1232 & 14 & $\mathrm{U}$ & 14 & 14 & $U$ & 14 & 14 & $\mathrm{U}$ & 14 \\
\hline \begin{tabular}{|l|} 
Aroclor- 1242 \\
\end{tabular} & 14 & \begin{tabular}{|l|}
$\mathrm{U}$ \\
\end{tabular} & 14 & 14 & $U$ & 14 & 14 & $\mathrm{U}$ & 14 \\
\hline Aroclor-1248 & 14 & $\mathrm{U}$ & 14 & 14 & $\mathrm{U}$ & 14 & 14 & $\mathrm{U}$ & 14 \\
\hline Aroclor-1254 & 14 & $U$ & 14 & 14 & $\mathrm{U}$ & 14 & 44 & & 14 \\
\hline Aroclor-1260 & 14 & U & 14 & 14 & $U$ & 14 & 14 & $U$ & 14 \\
\hline \multicolumn{10}{|c|}{ Pesticides } \\
\hline \begin{tabular}{|l} 
Aldrin \\
\end{tabular} & 1.5 & JD & 1.3 & 1.3 & UD & 1.3 & 1.3 & UD & 1.3 \\
\hline Alpha-BHC & 1.3 & UD & 1.3 & 1.3 & UD & 1.3 & 1.3 & UD & 1.3 \\
\hline \begin{tabular}{|l|} 
Alpha-Chlordane \\
\end{tabular} & 1.3 & UD & 1.3 & 1.3 & UD & 1.3 & 3.4 & $\mathrm{JD}$ & 1.3 \\
\hline Beta-BHC & 3.2 & JD & 1.3 & 1.3 & UD & 1.3 & 27 & $\mathrm{D}$ & 1.3 \\
\hline Delta-BHC & 1.3 & UD & 1.3 & 1.3 & UD & 1.3 & 1.3 & UD & 1.3 \\
\hline \begin{tabular}{|l|} 
Dichlorodiphenyldichloroethane \\
\end{tabular} & 1.3 & UD & 1.3 & 1.3 & UD & 1.3 & 1.3 & UD & 1.3 \\
\hline \begin{tabular}{|l|} 
Dichlorodiphenyldichloroethylene \\
\end{tabular} & 13 & \begin{tabular}{|l|}
$\mathrm{D}$ \\
\end{tabular} & 1.3 & 1.6 & $\mathrm{JD}$ & 1.6 & 4.7 & JD & 1.3 \\
\hline \begin{tabular}{|l|} 
Dichlorodiphenyltrichloroethane \\
\end{tabular} & 1.3 & UD & 1.3 & 1.3 & UD & 1.3 & 1.3 & UD & 1.3 \\
\hline \begin{tabular}{|l|l|} 
Dieldrin \\
\end{tabular} & 1.3 & UD & 1.3 & 1.3 & UD & 1.3 & 1.3 & UD & 1.3 \\
\hline Endosulfan I & 1.3 & UD & 1.3 & 1.3 & UD & 1.3 & 3.5 & $\mathrm{JD}$ & 1.3 \\
\hline Endosulfan II & 1.3 & UD & 1.3 & 1.3 & UD & 1.3 & 1.3 & UD & 1.3 \\
\hline Endosulfan sulfate & 1.3 & UD & 1.3 & 1.3 & UD & 1.3 & 2.1 & JD & 1.3 \\
\hline \begin{tabular}{|l} 
Endrin \\
\end{tabular} & 1.3 & UD & 1.3 & 1.3 & UD & 1.3 & 22 & $\mathrm{D}$ & 1.3 \\
\hline \begin{tabular}{|l} 
Endrin aldehyde \\
\end{tabular} & 1.3 & UD & 1.3 & 1.3 & UD & 1.3 & 1.3 & UD & 1.3 \\
\hline Endrin ketone & 1.3 & UD & 1.3 & 1.3 & UD & 1.3 & 4.1 & JD & 1.3 \\
\hline Gamma-BHC (Lindane) & 1.3 & UD & 1.3 & 1.3 & UD & 1.3 & 1.3 & UD & 1.3 \\
\hline \begin{tabular}{|l|} 
gamma-Chlordane \\
\end{tabular} & 1.3 & UD & 1.3 & 1.3 & UD & 1.3 & 1.3 & UD & 1.3 \\
\hline \begin{tabular}{|l} 
Heptachlor \\
\end{tabular} & 1.3 & UD & 1.3 & 1.3 & UD & 1.3 & 1.3 & UD & 1.3 \\
\hline Heptachlor epoxide & 1.3 & UD & 1.3 & 1.3 & UD & 1.3 & 1.3 & UD & 1.3 \\
\hline \begin{tabular}{|l} 
Methoxychlor \\
\end{tabular} & 1.3 & UD & 1.3 & 1.3 & UD & 1.3 & 1.3 & UD & 1.3 \\
\hline Toxaphene & 13 & UD & 13 & 13 & UD & 13 & 13 & UD & 13 \\
\hline \multicolumn{10}{|c|}{ Semivolatile Organic Analytes } \\
\hline 1,2,4-Trichlorobenzene & 340 & $\mathrm{U}$ & 340 & 360 & $\mathrm{U}$ & 360 & 350 & $U$ & 350 \\
\hline 1,2-Dichlorobenzene & 340 & $\mathrm{U}$ & 340 & 360 & \begin{tabular}{|l|l}
$U$ \\
\end{tabular} & 360 & 350 & $U$ & 350 \\
\hline 1,3-Dichlorobenzene & 340 & $\mathrm{U}$ & 340 & 360 & $\mathrm{U}$ & 360 & 350 & $U$ & 350 \\
\hline 1,4-Dichlorobenzene & 340 & $\mathrm{U}$ & 340 & 360 & $\mathrm{U}$ & 360 & 350 & $U$ & 350 \\
\hline 2,4,5-Trichlorophenol & 860 & $\mathrm{U}$ & 860 & 900 & $\mathrm{U}$ & 900 & 880 & $U$ & 880 \\
\hline 2,4,6-Trichlorophenol & 340 & $\mathrm{U}$ & 340 & 360 & $\mathrm{U}$ & 360 & 350 & $\mathrm{U}$ & 350 \\
\hline 2,4-Dichlorophenol & 340 & $U$ & 340 & 360 & $\mathrm{U}$ & 360 & 350 & $U$ & 350 \\
\hline 2,4-Dimethylphenol & 340 & $\mathrm{U}$ & 340 & 360 & $\mathrm{u}$ & 360 & 350 & $\mathrm{U}$ & 350 \\
\hline 2,4-Dinitrophenol & 860 & U & 860 & 900 & $\mathrm{U}$ & 900 & 880 & $\mathrm{U}$ & 880 \\
\hline 2,4-Dinitrotoluene & 340 & $u$ & 340 & 360 & $\mathrm{U}$ & 360 & 350 & $U$ & 350 \\
\hline 2,6-Dinitrotoluene & 340 & U & 340 & 360 & $\mathrm{U}$ & 360 & 350 & $\mathrm{U}$ & 350 \\
\hline 2-Chloronaphthalene & 340 & $\mathrm{U}$ & 340 & 360 & $\mathrm{U}$ & 360 & 350 & $\mathrm{U}$ & 350 \\
\hline \begin{tabular}{|l|} 
2-Chlorophenol \\
\end{tabular} & 340 & \begin{tabular}{|l|}
$U$ \\
\end{tabular} & 340 & 360 & U & 360 & 350 & $\mathrm{U}$ & 350 \\
\hline 2-Methylnaphthalene & 340 & $\mathrm{U}$ & 340 & 360 & U & 360 & 350 & $U$ & 350 \\
\hline 2-Methylphenol (cresol, o-) & 340 & $\mathrm{U}$ & 340 & 360 & U & 360 & 350 & $\mathrm{U}$ & 350 \\
\hline \begin{tabular}{|l|} 
2-Nitroaniline \\
\end{tabular} & 860 & $\mathrm{U}$ & 860 & 900 & $\mathrm{U}$ & 900 & 880 & $\mathrm{U}$ & 880 \\
\hline 2-Nitrophenol & 340 & $\mathrm{U}$ & 340 & 360 & $\mathrm{u}$ & 360 & 350 & $\mathrm{U}$ & 350 \\
\hline \multicolumn{4}{|c|}{ Attachment } & \multicolumn{3}{|c|}{1} & \multirow{4}{*}{$\begin{array}{l}\text { Sheet No. } \\
\text { Date } \\
\text { Date } \\
\text { Rev. No. }\end{array}$} & \multicolumn{2}{|c|}{10 of 11} \\
\hline & & \multicolumn{2}{|c|}{ Originator } & \multicolumn{3}{|c|}{ W.S. Thompson } & & \multicolumn{2}{|c|}{$12 / 20 / 07$} \\
\hline & & \multirow{2}{*}{\multicolumn{2}{|c|}{$\begin{array}{l}\text { Checked } \\
\text { Calc. No. }\end{array}$}} & \multirow{2}{*}{\multicolumn{3}{|c|}{$\begin{array}{l}\text { S. W. Clark } 2 \text { we } \\
0100 \text { F-CA-V0333 }\end{array}$}} & & & $20 / 07$ \\
\hline & & & & & & & & & 0 \\
\hline
\end{tabular}




\begin{tabular}{|c|c|c|c|c|c|c|c|c|c|}
\hline \multirow[t]{2}{*}{ Constituent } & \multicolumn{3}{|c|}{$\begin{array}{c}\text { J14L34 } \\
\text { Sample Location B-8 } \\
\text { Sample Date 5/13/07 }\end{array}$} & \multicolumn{3}{|c|}{$\begin{array}{c}\text { J14L35 } \\
\text { Sample Location B-9 } \\
\text { Sample Date 5/13/07 }\end{array}$} & \multicolumn{3}{|c|}{$\begin{array}{c}\text { J14l36 } \\
\text { Sample Location B-10 } \\
\text { Sample Date 5/13/07 }\end{array}$} \\
\hline & $\mu g / \mathrm{kg}$ & $\mathbf{Q}$ & PQL & $\mu g / \mathrm{kg}$ & $\mathbf{Q}$ & PQL & $\mu g / \mathbf{k g}$ & Q & PQL \\
\hline \multicolumn{10}{|c|}{ Semivolatile Organic Analytes (continued) } \\
\hline 3,3'-Dichlorobenzidine & 340 & $\mathrm{U}$ & 340 & 360 & $\mathrm{U}$ & 360 & 350 & $U$ & 350 \\
\hline 4-Methylphenol (p-cresol) & 340 & $\mathrm{U}$ & 340 & 360 & $\mathrm{U}$ & 360 & 350 & $\mathrm{U}$ & 350 \\
\hline 3-Nitroaniline & 860 & $\mathrm{U}$ & 860 & 900 & $\mathrm{U}$ & 900 & 880 & $\mathrm{U}$ & 880 \\
\hline 4,6-Dinitro-2-methylphenol & 860 & $\mathrm{U}$ & 860 & 900 & $\mathrm{U}$ & 900 & 880 & $U$ & 880 \\
\hline 4-Bromophenyl-phenylether & 340 & $U$ & 340 & 360 & $u$ & 360 & 350 & $U$ & 350 \\
\hline 4-Chloro-3-methylphenol & 340 & $U$ & 340 & 360 & $U$ & 360 & 350 & $\mathrm{U}$ & 350 \\
\hline 4-Chloroaniline & 340 & $U$ & 340 & 360 & $\mathrm{U}$ & 360 & 350 & $\mathrm{U}$ & 350 \\
\hline 4-Chlorophenyl-phenylether & 340 & $\mathrm{U}$ & 340 & 360 & $U$ & 360 & 350 & $U$ & 350 \\
\hline 4-Nitroaniline & 860 & $\mathrm{U}$ & 860 & 900 & $U$ & 900 & 880 & $U$ & 880 \\
\hline 4-Nitrophenol & 860 & $\mathrm{U}$ & 860 & 900 & $\mathrm{U}$ & 900 & 880 & $\mathrm{U}$ & 880 \\
\hline Acenaphthene & 340 & $\mathrm{U}$ & 340 & 360 & $\mathrm{U}$ & 360 & 63 & $\mathrm{~J}$ & 350 \\
\hline \begin{tabular}{|l} 
Acenaphthylene \\
\end{tabular} & 340 & $U$ & 340 & 360 & $U$ & 360 & 350 & $U$ & 350 \\
\hline Anthracene & 340 & $\mathrm{U}$ & 340 & 360 & $\mathrm{U}$ & 360 & 120 & $\mathrm{~J}$ & 350 \\
\hline Benzo(a)anthracene & 65 & $\mathrm{~J}$ & 340 & 360 & $\mathrm{U}$ & 360 & 350 & & 350 \\
\hline Benzo(a)pyrene & 55 & $\mathrm{~J}$ & 340 & 360 & $U$ & 360 & 290 & $\mathrm{~J}$ & 350 \\
\hline \begin{tabular}{|l|} 
Benzo(b)fluoranthene \\
\end{tabular} & 49 & $\mathrm{~J}$ & 340 & 360 & $\mathrm{U}$ & 360 & 230 & $\mathrm{~J}$ & 350 \\
\hline Benzo(g,h,i)perylene & 28 & $\mathrm{~J}$ & 340 & 360 & $u$ & 360 & 190 & $\mathrm{~J}$ & 350 \\
\hline \begin{tabular}{|l} 
Benzo(k)fluoranthene \\
\end{tabular} & 54 & $\mathrm{~J}$ & 340 & 360 & $U$ & 360 & 300 & $\mathrm{~J}$ & 350 \\
\hline \begin{tabular}{|l} 
Bis(2-chloro-1-methylethyl)ether \\
\end{tabular} & 340 & $\mathrm{U}$ & 340 & 360 & $\mathrm{U}$ & 360 & 350 & $\mathrm{U}$ & 350 \\
\hline Bis(2-chloroethoxy)methane & 340 & U & 340 & 360 & $U$ & 360 & 350 & $\mathrm{U}$ & 350 \\
\hline Bis(2-chloroethyl) ether & 340 & \begin{tabular}{|l|l|}
$U$ \\
\end{tabular} & 340 & 360 & $\mathrm{U}$ & 360 & 350 & $U$ & 350 \\
\hline Bis(2-ethylhexyl) phthalate & 31 & $\mathrm{JB}$ & 340 & 110 & $\mathrm{JB}$ & 360 & 40 & $\mathrm{JB}$ & 350 \\
\hline \begin{tabular}{|l|} 
Butylbenzylphthalate \\
\end{tabular} & 340 & $\mathrm{U}$ & 340 & 360 & $\mathrm{U}$ & 360 & 350 & $U$ & 350 \\
\hline Carbazole & 340 & $\mathrm{U}$ & 340 & 360 & $U$ & 360 & 62 & $\mathrm{~J}$ & 350 \\
\hline Chrysene & 80 & $\mathrm{~J}$ & 340 & 360 & $U$ & 360 & 390 & & 350 \\
\hline Dibenz $(a, h)$ anthracene & 24 & $\mathrm{~J}$ & 340 & 35 & $\mathrm{~J}$ & 360 & 350 & $\mathrm{U}$ & 350 \\
\hline Dibenzofuran & 340 & $\mathrm{U}$ & 340 & 360 & $\mathrm{U}$ & 360 & 350 & $U$ & 350 \\
\hline \begin{tabular}{|l|} 
Diethyiphthalate \\
\end{tabular} & 340 & $U$ & 340 & 360 & $\mathrm{U}$ & 360 & 99 & $\mathrm{~J}$ & 350 \\
\hline \begin{tabular}{|l} 
Dimethylphthalate \\
\end{tabular} & 340 & U & 340 & 360 & $\mathrm{U}$ & 360 & 21 & $J$ & 350 \\
\hline \begin{tabular}{|l} 
Di-n-butylphthalate \\
\end{tabular} & 340 & $\mathrm{U}$ & 340 & 360 & $\mathrm{U}$ & 360 & 350 & $\mathrm{U}$ & 350 \\
\hline \begin{tabular}{|l} 
Di-n-octyiphthalate \\
\end{tabular} & 340 & $U$ & 340 & 360 & $\mathrm{U}$ & 360 & 350 & $\mathrm{U}$ & 350 \\
\hline \begin{tabular}{|l} 
Fluoranthene \\
\end{tabular} & 160 & $\mathrm{~J}$ & 160 & 21 & $\mathrm{~J}$ & 360 & 790 & & 350 \\
\hline \begin{tabular}{|l} 
Fluorene \\
\end{tabular} & 340 & $\mathrm{U}$ & 340 & 360 & U & 360 & 46 & $\mathrm{~J}$ & 350 \\
\hline Hexachlorobenzene & 340 & $\mathrm{U}$ & 340 & 360 & $U$ & 360 & 350 & $U$ & 350 \\
\hline Hexachlorobutadiene & 340 & U & 340 & 360 & $\mathrm{U}$ & 360 & 350 & U & 350 \\
\hline Hexachlorocyclopentadiene & 340 & $\mathrm{U}$ & 340 & 360 & $\mathrm{U}$ & 360 & 350 & $\mathrm{U}$ & 350 \\
\hline Hexachloroethane & 340 & $\mathrm{U}$ & 340 & 360 & $\mathrm{U}$ & 360 & 350 & $U$ & 350 \\
\hline \begin{tabular}{|l|} 
Indeno(1,2,3-cd)pyrene \\
\end{tabular} & 27 & $\mathrm{~J}$ & 340 & 360 & $U$ & 360 & 180 & $\mathrm{~J}$ & 350 \\
\hline Isophorone & 340 & $\mathrm{U}$ & 340 & 360 & $\mathrm{U}$ & 360 & 350 & $U$ & 350 \\
\hline \begin{tabular}{|l} 
Naphthalene \\
\end{tabular} & 340 & $\mathrm{U}$ & 340 & 360 & $\mathrm{U}$ & 360 & 350 & $U$ & 350 \\
\hline Nitrobenzene & 340 & U & 340 & 360 & $\mathrm{U}$ & 360 & 350 & $\mathrm{U}$ & 350 \\
\hline N-Nitroso-di-n-dipropylamine & 340 & $\mathrm{U}$ & 340 & 360 & $\mathrm{U}$ & 360 & 350 & $\mathrm{U}$ & 350 \\
\hline N-Nitrosodiphenylamine & 340 & $\mathrm{U}$ & 340 & 360 & $\mathrm{U}$ & 360 & 350 & $\mathrm{U}$ & 350 \\
\hline Pentachlorophenol & 860 & U & 860 & 900 & $\mathrm{U}$ & 900 & 880 & $U$ & 880 \\
\hline Phenanthrene & 88 & $\mathrm{~J}$ & 340 & 360 & $\mathrm{U}$ & 360 & 520 & & 350 \\
\hline Phenol & 340 & U & 340 & 360 & U & 360 & 350 & $\mathrm{U}$ & 350 \\
\hline Pyrene & 160 & $\mathrm{~J}$ & 340 & 24 & \begin{tabular}{|l|}
$J$ \\
\end{tabular} & 360 & 840 & & 350 \\
\hline & & Atta & nment & 1 & & & Sheet I & & 1 of 11 \\
\hline & & & nator & W.S. Tr & omp: & $n(0)$ & Date & & $2 / 20 / 07$ \\
\hline & & Che & ked & S.W.CI & & ave & Date & & $200 / 07$ \\
\hline & & Cal & No. & $0100 \mathrm{~F}-\mathrm{C}$ & $\mathrm{A}-\mathrm{V} 03$ & & Rev. No. & & 0 \\
\hline
\end{tabular}




\section{CALCULATION COVER SHEET}

Project Title: 100-F Area Field Remediation

Job No. 14655

Area: $100-\mathrm{F}$

Discipline: Environmental

*Calculation No: 0100F-CA-V0360

Subject: 128-F-2, Area A Waste Site Cleanup Verification Hazard Quotient and Carcinogenic Risk Calculation

Computer Program: Excel

Program No: Excel 2003

The attached calculations have been generated to document compliance with established cleanup levels. These calculations should be used in conjunction with other relevant documents in the administrative record.

Committed Calculation $\bar{x}$

Preliminary $\Gamma$

Superseded

Voided $\Gamma$

\begin{tabular}{|c|c|c|c|c|c|c|}
\hline Rev. & Sheet Numbers & Originator & Checker & Reviewer & Approval & Date \\
\hline 0 & $\begin{array}{c}\text { Cover }=1 \\
\text { Summary }=3\end{array}$ & H.M Sullg & & $N A$ & J.P. Fancher & $5 / 8 / 88$ \\
\hline & & & & & & \\
\hline & & & & & & \\
\hline & & & & & & \\
\hline & & & & & & \\
\hline & & & & & & \\
\hline
\end{tabular}

SUMMARY OF REVISION

\begin{tabular}{|l|l|}
\hline & \\
& \\
\hline & \\
\hline & \\
\hline & \\
\hline
\end{tabular}

WCH-DE-018 (05/08/2007)

*Obtain Calc. No. from Document Control and Form from Intranet 
Washington Closure Hanford, Ind

CALCULATION SHEET

\begin{tabular}{|c|c|c|c|c|c|c|c|}
\hline Originator: & H M Sulloway & Date: & $04 / 30 / 08$ & Calc No. & $0100 \mathrm{~F}-\mathrm{CA}-\mathrm{V} 0360$ & Rey . & 0 \\
\hline Project: & 100-F Area Field Remediation & Job No: & 14655 & Checked: & L. D. Habel LH & Date: & $04 / 30 / 08$ \\
\hline Subject: & $\begin{array}{l}\text { 128-F-2, Area A Waste Site C } \\
\text { Calculation }\end{array}$ & 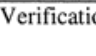 & & & & Sheet I & 1013 \\
\hline
\end{tabular}

\section{PURPOSE:}

Provide documentation to support the calculation of the hazard quotient (HQ) and carcinogenic (excess cancer) risk for area A of the 128-F-2 waste site. In accordance with the remedial action goals (RAGs) in the remedial design report/remedial action work plan (RDR/RAWP) (DOE-RL 2005), the following criteria must be met:

1) An HQ of $<1.0$ for all individual noncarcinogens

2) A cumulative $\mathrm{HQ}$ of $<1.0$ for noncarcinogens

3) An excess cancer risk of $<1 \times 10^{-6}$ for individual carcinogens

4) A cumulative excess cancer risk of $<1 \times 10^{-5}$ for carcinogens.

\section{GIVEN/REFERENCES:}

1) DOE-RL, 2005, Remedial Design Report/Remedial Action Work Plan for the 100 Areas, DOE/RL-96-17, Rev. 5, U.S. Department of Energy, Richland Operations Office, Richland, Washington.

2) WAC 173-340, "Model Toxics Control Act - Cleanup," Washington Administrative Code, 1996.

3) WCH, 2008, Remaining Sites Verification Package for the 128-F-2, 100-F Burning Pit, Attachment to Waste Site Reclassification Form 2008-031, Washington Closure Hanford, Inc., Richland, Washington.

\section{SOLUTION:}

1) Generate an $\mathrm{HQ}$ for each noncarcinogenic constituent detected above background or required detection limit/practical quantitation limit and compare it to the individual HQ of $<1.0$ (DOE-RL 2005).

2) Sum the HQs and compare this value to the cumulative HQ of $<1.0$.

3) Generate an excess cancer risk value for each carcinogenic constituent detected above background or required detection limit/practical quantitation limit and compare it to the excess cancer risk of $<1 \times 10^{-6}$ (DOE-RL 2005).

4) Sum the excess cancer risk value(s) and compare it to the cumulative cancer risk of $<1 \times 10^{-5}$. 
Washington Closure Hanford, Inc. CALCULATION SHEET

\begin{tabular}{|c|c|c|c|c|c|c|c|}
\hline Originator: & H. M. Sullowaycthus & Date: & $04 / 30 / 08$ & Calc. No.: & $0100 \mathrm{~F}-\mathrm{CA}-\mathrm{V} 0360$ & Rev.: & 0 \\
\hline Project: & 100-F Area Field Remediation & Job No: & 14655 & Checked: & L. D. Habel it & Date: & $04 / 30 / 08$ \\
\hline Subject: & \multicolumn{5}{|c|}{$\begin{array}{l}\text { 128-F-2, Area A Waste Site Cleanup Verification Hazard Quotient and Carcinogenic Risk } \\
\text { Calculation }\end{array}$} & \multicolumn{2}{|c|}{ Sheet No. 2 of 3} \\
\hline
\end{tabular}

\section{METHODOLOGY:}

Hazard quotient and carcinogenic risk calculations for area A of the 128-F-2 waste site was conservatively calculated using the higher of the statistically calculated or maximum results for each analyte from the area of verification sampling (WCH 2008). Boron and hexavalent chromium require $\mathrm{HQ}$ and risk calculations because these analytes were detected and a Washington State or Hanford Site background value is not available. Total chromium, copper, and multiple organic contaminants of concern (COCs) (as listed in Table 1) are included because they were detected by laboratory analysis and cannot be attributed to natural occurrence. All other site nonradionuclide COCs were not detected or were quantified below background levels. An example of the HQ and risk calculations is presented below:

1) For example, the maximum value for boron is $1.8 \mathrm{mg} / \mathrm{kg}$, divided by the noncarcinogenic RAG value of $16,000 \mathrm{mg} / \mathrm{kg}$ (boron is identified as a noncarcinogen in WAC 173-340-740[3]), is $1.1 \times 10^{-4}$. Comparing this value, and all other individual values, to the requirement of $<1.0$, this criteria is met.

2) After the HQ calculation is completed for the appropriate analytes, the cumulative HQ can be obtained by summing the individual values. The sum of the HQ values is $1.2 \times 10^{-2}$. Comparing this value to the requirement of $<1.0$, this criteria is met.

3) To calculate the excess cancer risk, the maximum value is divided by the carcinogenic RAG value, then multiplied by $1 \times 10^{-6}$. For example, the maximum value for hexavalent chromium is $0.53 \mathrm{mg} / \mathrm{kg}$, divided by $2.1 \mathrm{mg} / \mathrm{kg}$, and multiplied as indicated, is $2.5 \times 10^{-7}$. Comparing this value and all other individual values to the requirement of $<1 \times 10^{-6}$, this criteria is met.

4) After these calculations are completed for the carcinogenic analytes, the cumulative excess cancer risk can be obtained by summing the individual values. The sum of the excess cancer risk values is $6.7 \times 10^{-7}$. Comparing this value to the requirement of $<1 \times 10^{-5}$, this criterion is met.

\section{RESULTS:}

1) List individual noncarcinogens and corresponding HQs $>1.0$ : None

2) List the cumulative noncarcinogenic $\mathrm{HQ}>1.0$ : None

3) List individual carcinogens and corresponding excess cancer risk $>1 \times 10^{-6}$ : None

4) List the cumulative excess cancer risk for carcinogens $>1 \times 10^{-5}$ : None.

Table 1 shows the results of the calculations. 
Washington Closure Hanford, Inc.

CALCULATION SHEET

\begin{tabular}{|c|c|c|c|c|c|c|c|}
\hline Originator: & H. M. Sulloway Qthe & Date: & $04 / 30 / 08$ & Calc. No.: & 0100F-CA-V0360 & Rev.: & 0 \\
\hline Project: & 100-F Area Field Remediation & Job No: & 14655 & Checked: & L. D. Habel hit & Date: & $04 / 30 / 08$ \\
\hline Subject: & $\begin{array}{l}\text { 128-F-2, Area A V } \\
\text { Calculation }\end{array}$ & & & & & & \\
\hline
\end{tabular}

Table 1. Hazard Quotient and Excess Cancer Risk Results for Area A of the 128-F-2 Waste Site.

\begin{tabular}{|c|c|c|c|c|c|}
\hline Contaminants of Concern ${ }^{a}$ & $\begin{array}{c}\text { Maximum Value } \\
(\mathrm{mg} / \mathrm{kg})\end{array}$ & $\begin{array}{c}\text { Noncarcinogen } \\
\text { RAG }^{\mathbf{b}} \\
(\mathrm{mg} / \mathrm{kg})\end{array}$ & $\begin{array}{c}\text { Hazard } \\
\text { Quotient }\end{array}$ & $\begin{array}{c}\text { Carcinogen } \\
\text { RAG }^{\mathbf{b}} \\
(\mathrm{mg} / \mathrm{kg})\end{array}$ & $\begin{array}{l}\text { Carcinogen } \\
\text { Risk }\end{array}$ \\
\hline \multicolumn{6}{|l|}{ Metals } \\
\hline Boron & 1.8 & 16,000 & 1.1E-04 & -- & 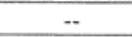 \\
\hline Chromium, total & 23.5 & 80,000 & $2.9 \mathrm{E}-04$ & & \\
\hline Chromium, hexavalent $^{\mathrm{c}}$ & 0.53 & 240 & $2.2 \mathrm{E}-03$ & 2.1 & $2.5 \mathrm{E}-07$ \\
\hline Copper & 23.4 & 2,960 & 7.9E-03 & -- & -- \\
\hline \multicolumn{6}{|l|}{ Semivolatiles } \\
\hline Bis(2-ethylhexyl) phthalate & 0.085 & 1,600 & 5.3E-05 & 71.4 & $1.2 \mathrm{E}-09$ \\
\hline Dibenzo(a,h)anthracene & 0.048 & -- & -- & $0.33^{\mathrm{d}}$ & $1.5 \mathrm{E}-07$ \\
\hline \multicolumn{6}{|l|}{ Pesticides } \\
\hline Aldrin & 0.0022 & 2.40 & $9.2 \mathrm{E}-04$ & 0.0588 & 3.7E-08 \\
\hline BHC, beta (Hexachlorocyclohexane) & 0.0047 & -- & $\cdots$ & 0.556 & 8.5E-09 \\
\hline DDE, 4,4'- & 0.0050 & -- & -- & 2.94 & 1.7E-09 \\
\hline DDT, 4,4'- & 0.0055 & 40 & $1.4 \mathrm{E}-04$ & 2.94 & $1.9 \mathrm{E}-09$ \\
\hline Endrin ketone and Endrin aldehyde & 0.0051 & 24 & 2.1E-04 & -- & $-\cdot$ \\
\hline Heptachlor & 0.0033 & 40 & 8.3E-05 & 0.222 & $1.5 \mathrm{E}-08$ \\
\hline \multicolumn{6}{|l|}{ Totals } \\
\hline \multicolumn{3}{|l|}{ Cumulative Hazard Quotient: } & 1.2E-02 & & \\
\hline \multicolumn{5}{|l|}{ Cumulative Excess Cancer Risk: } & 6.7E-07 \\
\hline
\end{tabular}

Notes:

= From WCH (2008.).

${ }^{b}=$ Value obtained from the RDR/RAWP (DOE-RL 2005) or Washington Administrative Code (WAC) 173-340-740(3), Method B, 1996, unless otherwise noted.

${ }^{\mathrm{c}}=$ Value for the carcinogen RAG calculated based on the inhalation exposure pathway WAC 173-340-750(3), 1996.

${ }^{\mathrm{d}}=$ Carcinogen risk calculated using the cleanup level instead of the required detection limit, per WAC 173-340-740(3), Method B, 1996.

-- = not applicable

RAG = remedial action goal

\section{CONCLUSION:}

This calculation demonstrates that area $\mathrm{A}$ of the 128-F-2 waste site meets the requirements for the hazard quotients and carcinogenic (excess cancer) risk as identified in the RDR/RAWP (DOE-RL 2005). 


\section{CALCULATION COVER SHEET}

Project Title: 100-F Field Remediation

Area: $100-\mathrm{F}$

Discipline: Environmental

*Calculation No: 0100F-CA-V0334

Computer Program: Excel

The attached calculations have brogram No: Excel 2003

should be used in conjunction with other compliance with established cleanup levels. These calculations

Committed Calculation $\bar{x}$

Preliminary

Superseded

Voided $\Gamma$

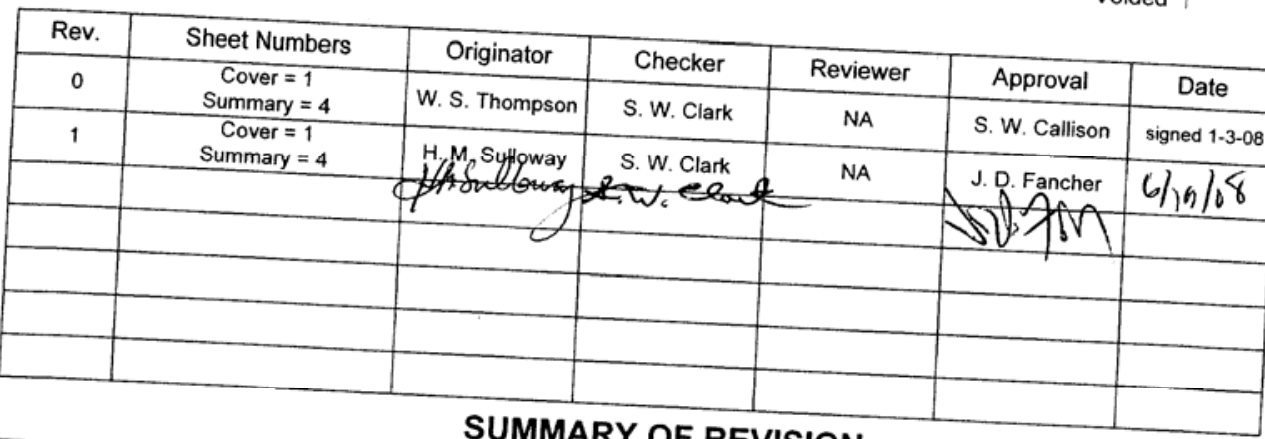

SUMMARY OF REVISION

\begin{tabular}{|l|l|}
\hline 1 & $\begin{array}{l}\text { Pages } 2 \text { and } 3 \text { replaced in entirety. Calculation was revised to provide consistent application of lead } \\
\text { results amongst the hazard quotient calculations for the 128-F-2 waste site. The lead value is reported in } \\
\text { the calculation but is not included in the calculation, as described in the revised methodology section. The } \\
\text { second purpose for the revision to document the use of the RDL as carcinogen RAG for benzo(a)pyrene } \\
\text { and dibenz(a,h)anthracene by adding a footnote. }\end{array}$
\end{tabular}


Washington Closure Hanford, Inc, CALCULATION SHEET

\begin{tabular}{|c|c|c|c|c|c|c|c|}
\hline Originator: & W. S. Thompson TA tho mupa & Date: & $12 / 20 / 07$ & Calc. No.: & 0100F-CA-V0334 & Rev.: & 0 \\
\hline Project: & 100-F Area Field Remediation & Job No: & 14655 & Checked: & S. W. Clark \&wa & Date: & N/50/07 \\
\hline Subject: & \multicolumn{5}{|c|}{$\begin{array}{l}\text { 128-F-2 Upland (Area B) Waste Site Cleanup Verification Hazard Quotient and Carcinogenic Risk } \\
\text { Calculation }\end{array}$} & \multicolumn{2}{|c|}{ Sheet No. 1 of $z_{4}$} \\
\hline
\end{tabular}

\section{PURPOSE:}

Provide documentation to support the calculation of the hazard quotient (HQ) and carcinogenic (excess cancer) risk for the 128-F-2 Upland (Area B) waste site. In accordance with the remedial action goals (RAGs) in the remedial design report/remedial action work plan (RDR/RAWP) (DOE-RL 2005), the following criteria must be met:

1) An $\mathrm{HQ}$ of $<1.0$ for all individual noncarcinogens

2) A cumulative HQ of $<1.0$ for noncarcinogens

3) An excess cancer risk of $<1 \times 10^{-6}$ for individual carcinogens

4) A cumulative excess cancer risk of $<1 \times 10^{-5}$ for carcinogens.

\section{GIVEN/REFERENCES:}

1) DOE-RL, 2005, Remedial Design Report/Remedial Action Work Plan for the 100 Areas, DOE/RL-96-17, Rev. 5, U.S. Department of Energy, Richland Operations Office, Richland, Washington.

2) EPA, 1994, Guidance Manual for the Integrated Exposure Uptake Biokinetic Model for Lead in Children, EPA/540/R-93/081, Publication No. 9285.7-15-1, U.S. Environmental Protection Agency, Washington, D.C.

3) WAC 173-340, "Model Toxics Control Act - Cleanup," Washington Administrative Code, 1996.

4) WCH, 2007a, 128-F-2, Upland (Area B) Cleanup Verification 95\% UCL Calculation, Calc. No. 0100F-CA-V0333, Washington Closure Hanford, Inc., Richland, Washington.

5) WCH, 2007b, Work Instruction for Verification Sampling of the 128-F-2 Waste Site, 100-F Burning Pit, Work Instruction No. 0100F-WI-G0037, Washington Closure Hanford, Inc., Richland, Washington.

\section{SOLUTION:}

1) Generate an HQ for each noncarcinogenic constituent detected above background or required detection limit/practical quantitation limit and compare it to the individual HQ of $<1.0$ (DOE-RL 2005).

2) Sum the HQs and compare this value to the cumulative HQ of $<1.0$.

3) Generate an excess cancer risk value for each carcinogenic constituent detected above background or required detection limit/practical quantitation limit and compare it to the excess cancer risk of $<1 \times 10^{-6}$ (DOE-RL 2005).

4) Sum the excess cancer risk value(s) and compare it to the cumulative cancer risk of $<1 \times 10^{-5}$. 
Washington Closure Hanford, Inc.

Originator: H. M. Sulloway Ific. CALCULATIQN SHEET

\begin{tabular}{c|l|r|r|r|l} 
Project: & $100-\mathrm{F}$ Area Field Remediation & Date: & $6 / 18 / 08$ & Calc. No.: & 0100F-CA-V0334 \\
Subject: & $128-\mathrm{F}-2$ Upland (Area B) Waste Site Cleanup & 14655 & Checked: & S. W. Clark D
\end{tabular}

Calculation

\section{METHODOLOGY:} (WCH 2007b). This hazard quotient and carcinogenic risk calculation is only for the upland portion
(Area B) of the waste site and uses the statistically calculated results for the contaminants of concern available. Lead, antes were detected and a Washington State or Hanford Site bask calculations listed in Table 1) are inclumium, copper, and multiple organic contaminants of attributed to natural occurred because they were detected by laboratory analysis and (COCs) (as quotient because toxic effence. Lead does not have a reference dose for analysis and cannot be daily intake. As toxic effects of lead are correlated with blood-lead for calculation of a hazard daily intake. As a result, the maximum lead concentration is reported but rather than exposure levels or quotient calculation. Total petroleum hydrocarbon (TPH) data are not included in the in the hazard nonradionuclide $\mathrm{CO}$ includes a broad of constituents rather than an individual concluded in the calculations since the HQ and risk calculation wer detected or were quantified below background. All other site the HQ and risk calculations is presented below:

1) $\mathrm{For}$ of $16,000 \mathrm{mg} / \mathrm{kg}$ (boron is identified as a noncarcinogen in WAded by the noncarcinogenic RAG value Comparing this value to the requirement of $<1.0$, this criteria is met.

2) After the HQ calculation is completed for the appropriate analytes, the obtained by summing the individual values. $5.0 \times 10^{-2}$. Comparing this value to the requirement of $<1.0$, this values (shown in Table 1) is

3) To calculate the excess cancer risk then multiplied by $1 \times 10^{-6}$. For example, the statistical value for by the carcinogenic RAG value, divided by $2.1 \mathrm{mg} / \mathrm{kg}$, and multiplied as indicated, is $8.6 \times 10^{-7}$. Comparing this value is $1.8 \mathrm{mg} / \mathrm{kg}$,
individual values to the requirement individual values to the requirement of $<1 \times 10^{-6}$, this criteria is met.

4) After these calculations are completed for the carcingenic analtes, the risk can be obtained by summing the individual values. $9.6 \times 10^{-7}$. Comparing this value to the requirement of $<1 \times 10^{-5}$ this excess cancer risk values is

\section{RESULTS:}

1) List individual noncarcinogens and corresponding $\mathrm{HQs}>1.0$ : None

2) List the cumulative noncarcinogenic $\mathrm{HQ}>1.0$ : None

4) List individual carcinogens and corresponding excess

4) List the cumulative excess cancer risk for carcinogens $>1 \times 10^{-5}: 1 \times 10^{-6}$ : None

Table I shows the results of the calculations. 
$\boldsymbol{s}$.

Washington Closure Hanford/Inc

CALCULATI $\varnothing N$ SHEET

\begin{tabular}{l|l|l|l|l|l|l|l} 
Originator: & H. M. Sulloway flal & Date: & $6 / 18 / 88$ & Calc. No.: & 0100F-CA-V0334 & Rev.:
\end{tabular}

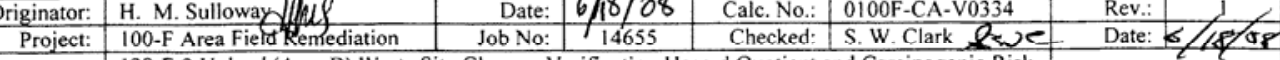

Subject: $128-\mathrm{F}-2$ Upland

Table 1. Hazard Quotient and Excess Cancer Risk Results for the Upland Portion (Area B) of the 128-F-2 Waste Site.

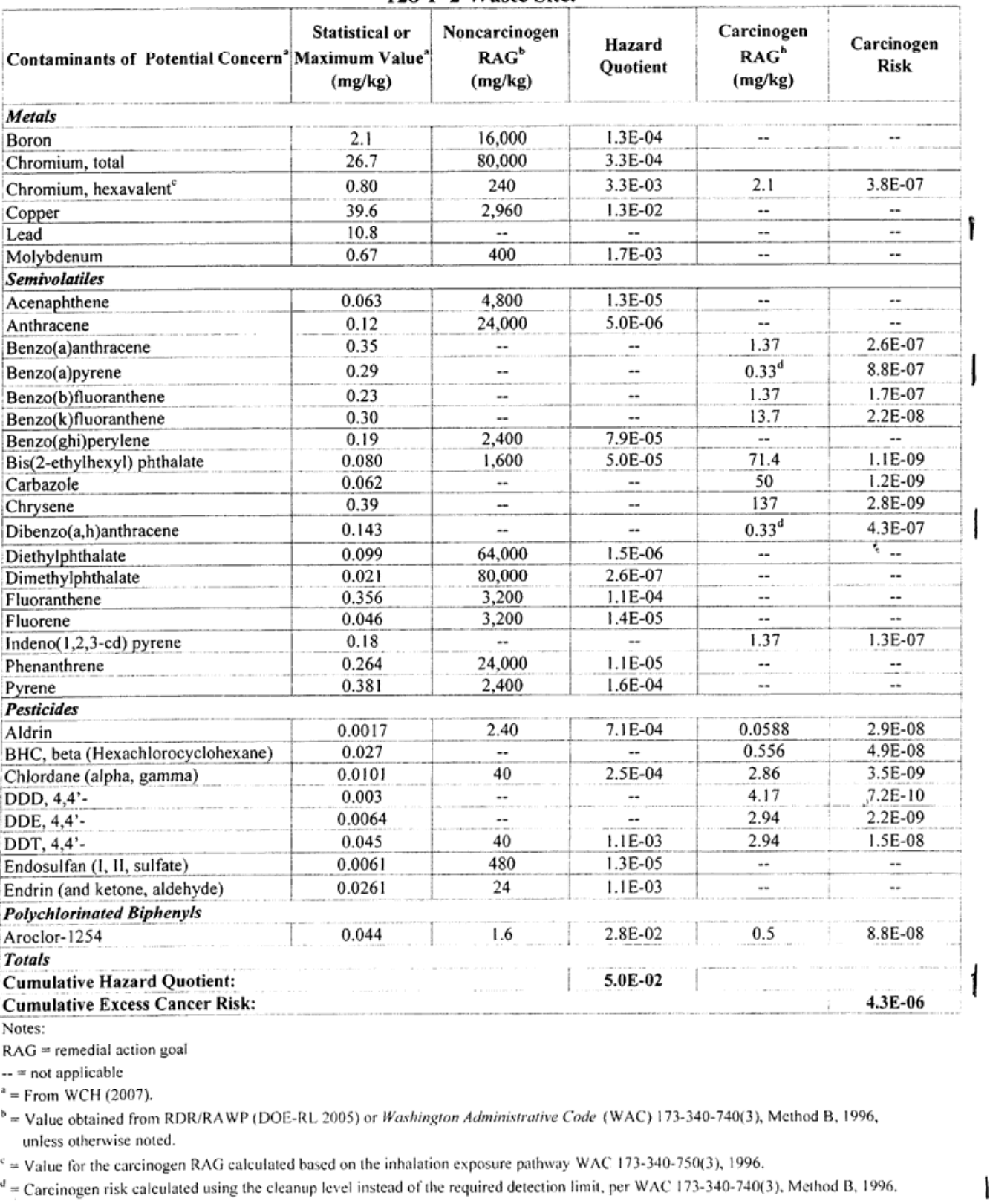


Washington Closure Hanford, Inc CALCULATION SHEET

\begin{tabular}{|c|c|c|c|c|c|c|c|}
\hline Originator: & W.S. Thompson $f(x)$ lowefty & Date: & $12 / 20 / 07$ & Calc. No.: & 0100F-CA-V0334 & Rev.: & 0 \\
\hline Project: & 100-F Area Field Remediation & Job No: & 14655 & Checked: & S. W. Clark 2use & Date: & $1250 / 0$ \\
\hline Subject: & $\begin{array}{l}\text { 128-F-2 Upland (Area B) Waste } \mathrm{Si} \\
\text { Calculation }\end{array}$ & e Cleanup V & rification $\mathrm{H}$ & ard Quotient & nd Carcinogenic Risk & She & 4 \\
\hline
\end{tabular}

\section{CONCLUSION:}

This calculation demonstrates that the upland portion (Area B) of the 128-F-2 waste site meets the requirements for the hazard quotients and carcinogenic (excess cancer) risk as identified in the RDR/RAWP (DOE-RL 2005). 


\section{CALCULATION COVER SHEET}

Area: 100-F

Discipline: Environmental

*Calculation No: 0100F-CA-V0361

Subject: 128-F-2, Staging Pile Waste Site Cleanup Verification Hazard Quotient and Carcinogenic Risk Calculation

Computer Program: Excel

Program No: Excel 2003

The attached calculations have been generated to document compliance with established cleanup levels. These calculations should be used in conjunction with other relevant documents in the administrative record.

Committed Calculation $\times$

Preliminary $\Gamma$

Superseded

Voided

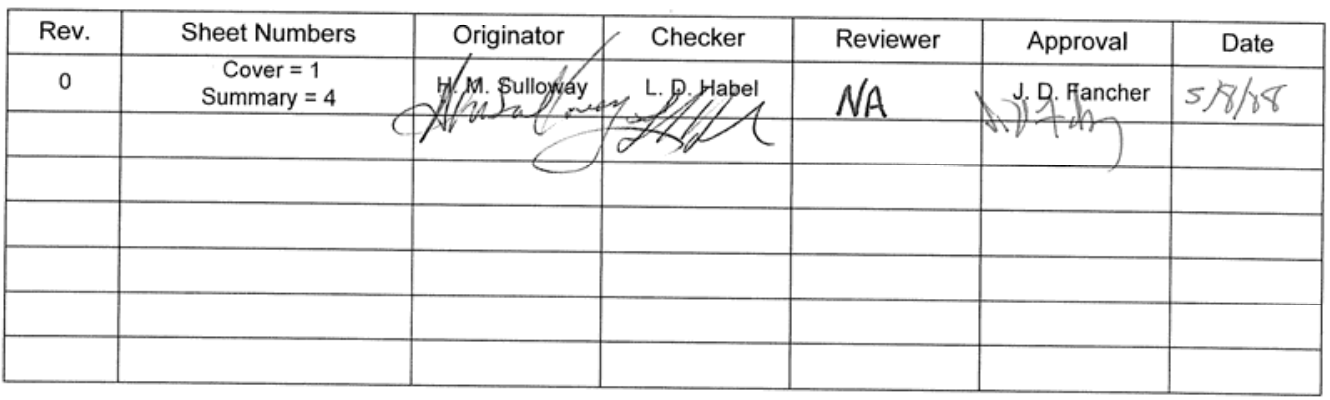

SUMMARY OF REVISION

\begin{tabular}{|l|l|}
\hline & \multicolumn{1}{|c|}{$\mid$} \\
\hline & \\
\hline & \\
\hline & \\
\hline
\end{tabular}


Washington Closure Hanford, Inc. Originator: H. M. Sulloway CfP CALCULATION SHEET

\begin{tabular}{|c|c|c|c|c|c|c|c|}
\hline or: & H. M. Sulloway & Date: & $05 / 01 / 08$ & Calc. No.: & 0100F-CA-V0361 & Rev.: & 0 \\
\hline Project: & 100-F Area FieldRermedjation & Job No: & 14655 & Checked: & L. D. Habel Cमे & Date: & $05 / 01 / 08$ \\
\hline Subj & 128-F-2, Staging & & & & & & \\
\hline
\end{tabular}

\section{METHODOLOGY:}

The HQ and carcinogenic risk calculations were determined for the staging pile footprint of the 128-F-2 waste site. The calculations used the maximum value for each analyte from the results of the 4 composite samples. Of the nonradionuclide contaminants of potential concern (COPC) chromium, copper, lead, selenium, and zinc required the $\mathrm{HQ}$ and risk calculations because they were quantified above background. Additionally, molybdenum required the HQ and risk calculations because this COPC was detected and a Washington State or Hanford Site background value is not available. Hexavalent chromium, Aroclor-1254, 4,4'-DDE, 4,4'-DDT, and multiple semivolatile organic analytes are included because they were detected by laboratory analysis and cannot be attributed to natural occurrence. All other site COPCs were not detected or were quantified below background levels. Lead does not have a reference dose for calculation of a hazard quotient because toxic effects of lead are correlated with blood-lead levels rather than exposure levels or daily intake. As a result, the maximum lead concentration is reported but not included in the hazard quotient calculation. An example of the $\mathrm{HQ}$ and risk calculations is presented below:

1) For example, the maximum value for molybdenum is $1.3 \mathrm{mg} / \mathrm{kg}$, divided by the noncarcinogenic RAG value of $400 \mathrm{mg} / \mathrm{kg}$ (molybdenum is identified as a noncarcinogen in WAC 173-340-740[3]), is $3.3 \times 10^{-3}$. Comparing this value, and all other individual values, to the requirement of $<1.0$, this criteria is met.

2) After the HQ calculation is completed for the appropriate analytes, the cumulative HQ can be obtained by summing the individual values. The sum of the HQ values is $5.3 \times 10^{-2}$. Comparing this value to the requirement of $<1.0$, this criteria is met.

3) To calculate the excess cancer risk, the maximum value is divided by the carcinogenic RAG value, then multiplied by $1 \times 10^{-6}$. For example, the maximum value for hexavalent chromium is $0.73 \mathrm{mg} / \mathrm{kg}$, divided by $2.1 \mathrm{mg} / \mathrm{kg}$, and multiplied as indicated, is $3.5 \times 10^{-7}$. Comparing this value and all other individual values to the requirement of $<1 \times 10^{-6}$, this criteria is met.

4) After these calculations are completed for the carcinogenic analytes, the cumulative excess cancer risk can be obtained by summing the individual values. The sum of the excess cancer risk values is $3.6 \times 10^{-6}$. Comparing this value to the requirement of $<1 \times 10^{-5}$, this criterion is met.

\section{RESULTS:}

1) List individual noncarcinogens and corresponding HQs $>1.0$ : None

2) List the cumulative noncarcinogenic $\mathrm{HQ}>1.0$ : None

3) List individual carcinogens and corresponding excess cancer risk $>1 \times 10^{-6}$ : None

4) List the cumulative excess cancer risk for carcinogens $>1 \times 10^{-5}$ : None.

Table 1 shows the results of the calculations. 
Washington Closure Hanford, Inc.

\begin{tabular}{|c|c|c|c|c|c|c|c|}
\hline Ori & $\mathrm{H}$ & & & & 803 & & \\
\hline Project: & 100-F Area Field Remediation & Job No: & 14655 & Checked: & L. D. Habel $\angle$ \& & $\mathrm{D}$ & $05 / 01 / 08$ \\
\hline Subj & \multicolumn{5}{|c|}{$\begin{array}{l}\text { 128-F-2, Staging Pile Waste Site Cleanup Verification Hazard Quotient and Carcinogenic Risk } \\
\text { Calculation }\end{array}$} & \multicolumn{2}{|c|}{ Sheet No. 3 of 4} \\
\hline
\end{tabular}

Table 1. Hazard Quotient and Excess Cancer Risk Results for the Staging Pile Footprint of the 128-F-2Waste Site.

\begin{tabular}{|c|c|c|c|c|c|}
\hline Contaminants of Concern ${ }^{\mathrm{a}}$ & $\begin{array}{c}\text { Maximum Value } \\
(\mathrm{mg} / \mathrm{kg})\end{array}$ & $\begin{array}{c}\text { Noncarcinogen } \\
\text { RAG }^{\text {b }} \\
(\mathrm{mg} / \mathrm{kg})\end{array}$ & $\begin{array}{l}\text { Hazard } \\
\text { Quotient }\end{array}$ & $\begin{array}{c}\text { Carcinogen } \\
\text { RAG }^{\mathrm{b}} \\
(\mathrm{mg} / \mathrm{kg})\end{array}$ & $\begin{array}{l}\text { Carcinogen } \\
\text { Risk }\end{array}$ \\
\hline \multicolumn{6}{|l|}{ Metals } \\
\hline Chromium, total & 32.2 & 80,000 & $4.0 \mathrm{E}-04$ & & \\
\hline Chromium, hexavalent $^{c}$ & 0.73 & 240 & $3.0 \mathrm{E}-03$ & 2.1 & $3.5 \mathrm{E}-07$ \\
\hline Copper & 23.9 & 2,960 & $8.1 \mathrm{E}-03$ & -- & - \\
\hline Lead & 25 & - & -- & - & - \\
\hline Molybdenum & 1.3 & 400 & 3.3E-03 & - & - \\
\hline Selenium & 0.95 & 400 & $2.4 \mathrm{E}-03$ & - & -- \\
\hline Zinc & 124 & 24,000 & $5.2 \mathrm{E}-03$ & - & - \\
\hline \multicolumn{6}{|l|}{ Semivolatiles } \\
\hline Acenaphthene & 0.027 & 4,800 & $5.6 \mathrm{E}-06$ & - & -- \\
\hline Anthracene & 0.06 & 24,000 & $2.5 \mathrm{E}-06$ & - & -- \\
\hline Benzo(a)anthracene & 0.32 & -- & -- & $1.37^{\mathrm{d}}$ & $2.3 \mathrm{E}-07$ \\
\hline Benzo(a)pyrene & 0.28 & - & - & 0.137 & $2.0 \mathrm{E}-06$ \\
\hline Benzo(b)fluoranthene & 0.21 & -- & - & $1.37^{\mathrm{d}}$ & $1.5 \mathrm{E}-07$ \\
\hline Benzo(k)fluoranthene & 0.230 & -- & -- & $13.7^{\mathrm{d}}$ & $1.7 \mathrm{E}-08$ \\
\hline Benzo(ghi)perylene & 0.16 & 2,400 & $6.7 \mathrm{E}-05$ & -- & -- \\
\hline Bis(2-ethylhexyl) phthalate & 0.11 & 1,600 & $6.9 \mathrm{E}-05$ & 71.4 & $1.5 \mathrm{E}-09$ \\
\hline Carbazole & 0.03 & - & -- & 50 & $6.0 \mathrm{E}-10$ \\
\hline Chrysene & 0.37 & - & -- & $137^{\mathrm{d}}$ & 2.7E-09 \\
\hline Dibenzo(a,h)anthracene & 0.077 & - & - & $0.137^{\mathrm{d}}$ & $5.6 \mathrm{E}-07$ \\
\hline Fluoranthene & 0.67 & 3,200 & $2.1 \mathrm{E}-04$ & -- & -. \\
\hline Fluorene & 0.019 & 3,200 & $5.9 \mathrm{E}-06$ & - & - \\
\hline Indeno(1,2,3-cd) pyrene & 0.15 & - & -- & 1.37 & $1.1 \mathrm{E}-07$ \\
\hline Phenanthrene & 0.31 & 24,000 & $1.3 \mathrm{E}-05$ & - & - \\
\hline Pyrene & 0.79 & 2,400 & $3.3 \mathrm{E}-04$ & - & - \\
\hline \multicolumn{6}{|l|}{ Pesticides } \\
\hline DDE, 4,4'- & 0.0022 & -- & -- & 2.94 & $7.5 \mathrm{E}-10$ \\
\hline DDT, $4,4^{\prime}-$ & 0.0066 & 40 & $1.7 \mathrm{E}-04$ & 2.94 & $2.2 \mathrm{E}-09$ \\
\hline \multicolumn{6}{|l|}{ Polychlorinated Biphenyls } \\
\hline Aroclor-1254 & 0.048 & 1.6 & $3.0 \mathrm{E}-02$ & 0.5 & $9.6 \mathrm{E}-08$ \\
\hline \multicolumn{6}{|l|}{ Totals } \\
\hline \multirow{2}{*}{\multicolumn{5}{|c|}{$\begin{array}{l}\text { Cumulative Hazard Quotient: } \\
\text { Cumulative Excess Cancer Risk: }\end{array}$}} & \\
\hline & & & & & $3.6 \mathrm{E}-06$ \\
\hline \multicolumn{6}{|c|}{$\begin{array}{l}\mathrm{b}=\text { Value obtained from the RDR/RAWP (DOE-RL 2005) or Washington Administrative Code (WAC) 173-340-740(3), Method B, 1996, } \\
\text { unless otherwise noted. }\end{array}$} \\
\hline \multicolumn{6}{|c|}{$\begin{aligned} \mathrm{c}= & \text { Value for the carcinogen RAG calculated based on the inhalation exposure pathway WAC 173-340-750(3), } 1996 . \\
\mathrm{d}= & \text { Calculated with toxicity values updated through May 1, 2008, using the appropriate formulas from WAC 173-340-740 (Ecology 1996). } \\
& \text { Updated toxicity values are available from the EPA Integrated Risk Information System (IRIS) at < http://www.epa.gov/iris }> \\
& \text { or from the Risk Assessment Information System (RAIS) database of the Oak Ridge National Laboratory (ORNL) on the Internet at } \\
& <\text { http://risk.lsd.ornl.gov >. Calculated parameters have been checked against the CLARC Database (Ecology 2005) available on the } \\
& \text { internet at < http://www.ecy.wa.gov>. }\end{aligned}$} \\
\hline
\end{tabular}


Washington Closure Hanford, Inc. C CALCULATION SHEET

\begin{tabular}{|r|l|r|c|r|r|r|r|r|}
\hline Originator: & H. M. Sulloway & Date: & $05 / 01 / 08$ & Calc. No.: & $0100 \mathrm{~F}-\mathrm{CA}-\mathrm{V} 0361$ & Rev.: & 0 \\
\hline Project: & $100-\mathrm{F}$ Area Field Remediation & Job No: & 14655 & Checked: & L. D. Habel & L- & Date: & $05 / 01 / 08$ \\
\hline Subject: & $\begin{array}{l}128-\mathrm{F}-2, \text { Staging Pile Waste Site Cleanup Verification Hazard Quotient and Carcinogenic Risk } \\
\text { Calculation }\end{array}$ & \multirow{2}{*}{ Sheet No. 4 of 4} \\
\hline
\end{tabular}

1

2 CONCLUSION:

4 This calculation demonstrates that staging pile footprint of the 128-F-2 waste site meets the

5 requirements for the hazard quotients and carcinogenic (excess cancer) risk as identified in the

6 RDR/RAWP (DOE-RL 2005). 


\section{APPENDIX E}

\section{VERIFICATION SAMPLING DATA QUALITY ASSESSMENT}




\section{VERIFICATION SAMPLING DATA QUALITY ASSESSMENT}

A data quality assessment (DQA) was performed to compare the verification sampling approach and resulting analytical data with the sampling and data requirements specified in the site-specific sample design (WHC 2007d). This DQA was performed in accordance with sitespecific data quality objectives found in the 100 Area Remedial Action Sampling and Analysis Plan (SAP) (DOE-RL 2005).

A review of the sample design (WCH 2007d), the field logbooks (WCH 2007a, 2007b, 2007c and 2008) and applicable analytical data packages has been performed as part of this DQA. All samples were collected per the sample design. This DQA is limited to the 128-F-2 areas A, B, $\mathrm{D}$, and $\mathrm{E}$ verification sampling data. As discussed in this remaining sites verification package, area $\mathrm{C}$ is to be made into a separate waste site.

To ensure quality data, the SAP data assurance requirements and the data validation procedures for chemical analysis (BHI 2000) are used as appropriate. This review involves evaluation of the data to determine if they are of the right type, quality, and quantity to support the intended use (i.e., closeout decisions). The DQA completes the data life cycle (i.e., planning, implementation, and assessment) that was initiated by the data quality objectives process (EPA 2000).

The closeout sampling approach for the 128-F-2 waste site included a sample design with multiple subunit areas. Verification sample data collected at the 128-F-2 waste site were provided by the laboratories in multiple sample delivery groups (SDGs). The verification SDGs pertaining to areas A, B, D, and E are reviewed in this DQA. SDG K0724 was submitted for third-party validation. No major deficiencies were found in the in the analytical data set and supporting documentation. Minor deficiencies are discussed below.

\section{SDG K0724}

SDG K0724 was submitted for formal third-party validation. No major deficiencies were found in SDG K1066. Minor deficiencies are as follows:

In the gross alpha analysis, one of the laboratory control sample (LCS) recoveries (63\%) is below the acceptance criteria (70\%-130\%). Third-party validation qualified the gross alpha data in the associated samples (J14KB0, J14KB1, J14KB2, J14KB3) as estimated with "J" flags. Estimated data are useable for decision-making purposes.

In the chlorinated pesticide analysis, the analyte toxaphene was not included in the matrix spike (MS), matrix spike duplicate (MSD), or LCS samples. Toxaphene is actually a mixture of compounds rather than a discrete analyte. While the overall concentration of toxaphene can be calculated using several unobstructed peaks in the chromatography, the inclusion of toxaphene in the spiking mixture would be problematic for the other pesticide analytes. The analytical laboratory typically quantifies toxaphene but does not include toxaphene in quality assurance (QA)/quality control (QC) samples. Third-party validation qualified the toxaphene data as estimates with "J" flags. Estimated data are useable for decision-making purposes. 
In the chlorinated pesticide analysis, the MS recovery (28\%) for delta-BHC is below the acceptance criteria (70\%-130\%). Third-party validation qualified the delta-BHC data as estimates with "J" flags. Estimated data are useable for decision-making purposes.

In the chlorinated pesticide analysis, the surrogate recovery (120\%) for tetrachloro-m-xylene is above the acceptance criteria in sample J14KB5. Third-party validation qualified the aldrin, 4,4'DDE, and 4,4-DDT results for sample J14KB5 as estimates with "J" flags. Estimated data are useable for decision-making purposes.

In the chlorinated pesticide analysis, the relative percent difference (RPD) calculated (83\%) for delta-BHC is above the acceptance criteria (less than 30\%). High RPDs in environmental samples are attributed to natural heterogeneities in the sample matrix. Third-party validation qualified all of the delta-BHC results in SDG K0724 as estimates with "J" flags. Estimated data are useable for decision-making purposes.

In the semi-volatile organic compound (SVOC) analysis, the common laboratory contaminant bis(2-ethylhexyl)phthalate was detected in the MB. The associated sample results are all less than 10 times the MB concentration. Third-party validation has raised all of the bis(2-ethylhexyl)phthalate results in SDG K0724 to the contract required detection limit (CRQL) and qualified them as undetected with "U" flags. The data are useable for decision-making purposes.

In the SVOC analysis, the LCS recoveries for hexachlorocyclopentadiene (45\%) and 2,4-dinitrophenol (37\%) are below the acceptance criteria. Third-party validation qualified all of the data for these two analytes in SDG K0724 as estimates with "J" flags. Estimated data are useable for decision-making purposes.

In the SVOC analysis, the RPD calculated for the MS and MSD recoveries for phenol (31.7\%), 4-chloroanaline (49.3\%), 2,4,5-trichlorophenol (58.2\%), 3-nitroanaline (36.8\%), 4-nitrophenol (33\%), 4-nitroanaline (33.5\%), pentachlorophenol (56.9\%), and 3,3,-dichlorobenzidine (51.6\%) are above the acceptance criteria (less than 30\%). High RPDs in environmental samples are attributed to natural heterogeneities in the sample matrix. Third-party validation qualified all of the results for these analytes in SDG K0724 as estimates with "J" flags. Estimated data are useable for decision-making purposes.

In the hexavalent chromium analysis, hexavalent chromium was detected in the equipment blank (EB) sample (J14K94). The concentration in the EB $(0.21 \mathrm{mg} / \mathrm{kg})$ was detected just above the practical quantitation limit (PQL) $(0.20 \mathrm{mg} / \mathrm{kg})$. Third-party validation noted this deficiency but did not qualify any data as a result. This result is probably a laboratory error, but has little effect on the associated field data. The data are useable for decision-making purposes.

In the petroleum hydrocarbon analysis, all of the field samples (140 to $152 \mathrm{mg} / \mathrm{kg} \mathrm{U}$ ) were reported nondetected by the laboratory at a concentration above the required quantitation limit (RQL). The reported detection limits for petroleum hydrocarbon are all below the RAG values (200 mg/kg). Third-party validation did not qualify any data as a result of this deficiency. The data are useable for decision-making purposes. 
In the inductively coupled plasma (ICP) metals analysis, the sodium and zinc concentrations nondetected in sample J14K94 (EB) at a concentration less than 10 times the concentrations detected in the MB. Third-party validation qualified the sodium and zinc concentrations in sample J14K94 as estimates with "UJ" (estimated nondetect) flags. The data are useable for decision-making purposes.

In the ICP metals analysis, the MS recoveries for silicon (155\%), antimony (44\%), and copper (32.8\%) are outside the range of the acceptance criteria (70\% to 130\%). Third party validation qualified all of the data in SDG K0724 for these three analytes as estimates with "J" flags. Estimated data are useable for decision-making purposes.

In the ICP metals analysis, the LCS recovery for silicon (12\%) is outside the range of the acceptance criteria (70\% to $130 \%$ ). Third-party validation qualified all of the data in SDG K0724 for silicon as estimates with "J" flags. Estimated data are useable for decision-making purposes.

In the ICP metals analysis, the RPDs calculated in the laboratory duplicate for lead (112.6\%) and copper (43\%) are above the acceptance criteria (less than 30\%). Elevated RPDs in environmental samples are attributed to natural heterogeneities in the sample matrix. Third-party validation qualified all of the data in SDG K0724 for lead and copper as estimates with "J" flags. Estimated data are useable for decision-making purposes.

\section{SDG K0726}

This SDG comprises 22 field samples (J14L15 through J14L36) collected for verification purposes. These samples were analyzed for alpha-, beta-, and gamma-emitting radionuclides. Eleven of those (J14L26 through J14L36) were also analyzed for SVOCs, hexavalent chromium, ICP metals, mercury, chlorinated pesticides, polychlorinated biphenyls (PCBs), and total petroleum hydrocarbons (TPH). No major deficiencies were found in SDG K0726. Minor deficiencies are as follows:

In the SVOC analysis, recoveries of 2,4-dimethylphenol (49\%) and 1,2,4-trichlorobenzene (56\%) in the MSD are below the acceptance criteria. Sample data for these two analytes in may be considered estimated. Estimated data are useable for decision-making purposes.

In the SVOC analysis, a laboratory error during the preparation of the MS resulted in no recoveries. There were no errors in the preparation of the MSD and LCS samples. These samples confirm quantitation of the analytical system. There is no impact on the field sample data. The data are useable for decision-making purposes.

In the SVOC analysis, the common laboratory contaminant bis(2-ethylhexyl)phthalate was detected in the MB at a concentration below the CRQL. There is no impact on the field sample data. The data are useable for decision-making purposes.

In the ICP metals analysis, barium, calcium, and zinc were detected in the MB below the CRQL. Only sample J14JT6, the EB, had concentrations of these analytes that were not more than 
20-fold greater than the MB results. Analytical results for barium, calcium, and zinc in sample J14JT6 may be considered estimated. There is no impact on the other field data. The data are useable for decision-making purposes.

In the ICP metals analysis, the LCS recovery for silicon was below the acceptance criteria at $53.4 \%$. Analytical results for silicon in SDG K0726 may be considered estimated. The data are useable for decision-making purposes.

In the ICP metals analysis, the MS recoveries for six ICP metals (aluminum, calcium, iron, manganese, antimony, silicon) are out of acceptance criteria. With the exception of antimony, the spiking concentrations are insignificant compared to the native concentrations in the sample from which the MS was prepared. To confirm quantitation, post-digestion spikes (PDSs) and serial dilutions were prepared for all six analytes with acceptable results. In the case of antimony the initial concentration in the native sample is not insignificant compared to the spiking concentration. Sample results reported for antimony in SDG K0726 may be considered estimated. Estimated data are useable for decision-making purposes.

\section{SDG K0717}

This SDG comprises five field samples (J14K95-J14K99) collected for verification purposes. These samples were analyzed for alpha-, beta-, and gamma-emitting radionuclides; hexavalent chromium; ICP metals; mercury; SVOCs; chlorinated pesticides; PCBs; and TPH. A sixth sample, the EB (J14K95), was also prepared and analyzed for ICP metals and mercury. No major deficiencies were found in SDG K1066. Minor deficiencies are as follows:

In the SVOC analysis, one of the surrogates (2,4,6 tribromophenol) in sample J14K98 is below the acceptance criteria at $18 \%$ recovery. However, the data meet the secondary criteria that allow no more than surrogate to be out of range but not below $10 \%$. The data are useable for decision-making purposes.

In the SVOC analysis, the MS recovery for 1,2,4-trichlorobenzene was just below the acceptance criteria (60\%) at 59\%. The MSD and the LCS were both above the lower limit at $68 \%$ and $65 \%$, respectively. The data are useable for decision-making purposes.

In the SVOC analysis, the common laboratory contaminant bis (2-ethylhexyl)phthalate was detected in the MB at a concentration below the CRQL. There is no impact the field sample data. The data are useable for decision-making purposes.

In the PCB analysis, the surrogates tetrachloro-m-xylene and decachlorobiphenyl were above the acceptance criteria (130\%) at $144 \%$ and $140 \%$, respectively. This suggests a possible high bias the field sample data. High-biased data are acceptable for decision-making purposes.

In the ICP metals analysis, the samples were all diluted three-fold due to the nature of the sample matrix being analyzed. This dilution does not impact the final sample data. The data are useable for decision-making purposes. 
In the ICP metals analysis, sodium was detected in the MB at a concentration above the PQL. The field sample concentrations were more than 20 times the MB concentration with the exception of samples J14K97 and J14K98. Sodium concentrations reported for samples J14K97 and J14K98 may be considered estimated. Estimated data are useable for decision-making purposes.

In the ICP metals analysis, the LCS recovery for silicon was below the acceptance criteria at 31.7\%. The MS and MSD met the acceptance criteria. Silicon concentrations reported in SDG K0717 may be considered estimated. Estimated data are useable for decision-making purposes.

In the ICP metals analysis, the MS recoveries for four ICP metals (aluminum, iron, manganese, antimony) are out of acceptance criteria. With the exception of antimony, the spiking concentrations are insignificant compared to the native concentrations in the sample from which the MS was prepared. Therefore, the deficiency in the MS result is a reflection of the analytical variability of the native concentrations rather than a measure of the recoveries from the sample. To confirm quantitation, PDSs and serial dilutions were prepared for all four analytes with acceptable results. In the case of antimony the initial concentration in the native sample is not insignificant compared to the spiking concentration. Sample results reported for antimony in SDG K0717 may be considered estimated. Estimated data are useable for decision-making purposes.

In the chlorinated pesticide analysis, the analyte toxaphene was not included in the MS, MSD, or LCS samples. Toxaphene is actually a mixture of compounds rather than a discrete analyte. While the overall concentration of toxaphene can be calculated using several unobstructed peaks in the chromatography, the inclusion of toxaphene in the spiking mixture would be problematic for the other pesticide analytes. The laboratory typically quantifies toxaphene but does not include toxaphene in QA/QC samples. The toxaphene data are therefore considered estimated but useable for decision-making purposes.

\section{SDG K0919}

This SDG comprises 10 field samples (J15FD0 through J15FD9) collected for verification purposes. These samples were analyzed for hexavalent chromium. No major or minor deficiencies were found in SDG K0919. The data are useable for decision-making purposes.

\section{SDG K1054}

This SDG comprises four field samples (J163R5 through J163R9) collected for verification purposes. These samples were analyzed for alpha-, beta-, and gamma-emitting radionuclides; hexavalent chromium; ICP metals; mercury; chlorinated pesticides; PCBs; and TPH. Five additional samples (J163R5 through J163R9) were also collected and analyzed for SVOCs and ICP metals. No major deficiencies were found in SDG K1054. Minor deficiencies are as follows:

In the SVOC analysis, 7 of 54 surrogates were out of the laboratory criteria. However, all of the samples meet the secondary criteria that allow no more than one surrogate outlier per fraction 
(acid and base neutral) and no recoveries less than 10\%. The data are useable for decisionmaking purposes.

In the ICP metals analysis, three metals (antimony, sodium, zinc) were detected in the MB at concentrations above the PQLs. All of the reported field sample concentrations are more than 20 times the associated MB concentrations. There is no impact the field sample data. The data are useable for decision-making purposes.

In the ICP metals analysis, the MS recoveries for eight ICP metals (aluminum, boron, iron, magnesium, manganese, antimony, silicon, vanadium) are out of acceptance criteria. With the exception of antimony, the spiking concentrations are insignificant compared to the native concentrations in the sample from which the MS was prepared. Therefore, the deficiency in the MS result is a reflection of the analytical variability of the native concentrations rather than a measure of the recoveries from the sample. To confirm quantitation, PDSs and serial dilutions were prepared for all four analytes with acceptable results. In the case of antimony the initial concentration in the native sample is not insignificant compared to the spiking concentration. Sample results reported for antimony in SDG K1054 may be considered estimated. Estimated data are useable for decision-making purposes.

In the ICP metals analysis, the RPDs calculated for four metals (arsenic at 31.6\%, copper at $28.4 \%$, mercury at $54.1 \%$, nickel at $23.9 \%$ ) are out of the acceptance criteria. Elevated RPDs in environmental soil samples are generally attributed to heterogeneities in the sample matrix and not to deficiencies in the laboratory procedures. The data are useable for decision-making purposes.

In the chlorinated pesticide analysis, the analyte toxaphene was not included in the MS, MSD, or LCS samples. As discussed above, the toxaphene data are considered estimated but useable for decision-making purposes.

In the PCB analysis, 1 of 16 surrogate recoveries (tetrachloro-m-xylene at 166\%) is above the acceptance criteria. This result suggests a possible high bias in the data. High-biased data are acceptable for decision-making purposes.

\section{SDG K1138}

This SDG comprises one field sample (J16DF5) collected for verification purposes. This sample was analyzed for alpha-, beta-, and gamma-emitting radionuclides; SVOCs; hexavalent chromium; ICP metals; mercury; chlorinated pesticides; PCBs; and TPH. No major deficiencies were found in SDG K1138. Minor deficiencies are as follows:

In the SVOC analysis, the MS recovery for 1,2,4-trichlorobenzene (59\%) is below the acceptance criteria. The MSD and LCS recoveries are within criteria. The 1,2,4trichlorobenzene result for SDG K1138 may be considered estimated. Estimated data are useable for decision-making purposes. The data are useable for decision-making purposes. 
In the SVOC analysis, the LCS recoveries for isophorone (59\%), 2-methylnaphthalene(56\%), and 4,6-dinitro-2-methylphenol (31\%) are below the acceptance criteria. The MS and MSD recoveries are within criteria. The sample results for these analytes reported in SDG K1138 may be considered estimated. Estimated data are useable for decision-making purposes.

In the SVOC analysis, the LCS recovery for 4-chloro-3-methylphenol (2\%) is significantly below the acceptance criteria. Because the LCS recovery is below $10 \%$, rejection of the data has been considered. Data are not required to be rejected based on the results of a spiked sample only. In this case quantitation is confirmed by the MS and MSD recoveries, which are acceptable. The 4-chloro-3-methylphenol results may be considered estimated. Estimated data are useable for decision-making purposes.

In the ICP metals analysis, sodium was detected in the MB above the PQL concentration. However, the concentration of sodium in the MB is less than $1 / 20^{\text {th }}$ of the concentration in the associated sample. There is no impact on the field data. The data are useable for decisionmaking purposes.

In the ICP metals analysis, the LCS recovery for silicon (35.8\%) is below the acceptance criteria. The sample results for silicon in SDG K1138 may be considered estimated. Estimated data are useable for decision-making purposes.

In the ICP metals analysis, the MS recoveries for five ICP metals (aluminum, iron, manganese, antimony, silicon) are out of acceptance criteria. With the exception of antimony, the spiking concentrations are insignificant compared to the native concentrations in the sample from which the MS was prepared. To confirm quantitation, PDSs and serial dilutions were prepared for all five analytes with acceptable results. In the case of antimony the initial concentration in the native sample is not insignificant compared to the spiking concentration. Sample results reported for antimony in SDG K1138 may be considered estimated. Estimated data are useable for decision-making purposes.

In the hexavalent chromium analysis, the MS (3.3\%) is significantly below the acceptance criteria. Because the MS recovery is below $10 \%$, rejection of the data has been considered. Data are not required to be rejected based on the results of a spiked sample only. In this case quantitation is confirmed by the LCS result. Additional confirmation of the quantitation is found by comparing the only field sample (J16DF5), associated with this MS result, to the duplicate of that sample and to a previous sample (J14KB1, SDG K0724) collected at the same coordinates but at a shallower depth. The sample and duplicate results were $0.46 \mathrm{mg} / \mathrm{kg}$ and $0.59 \mathrm{mg} / \mathrm{kg}$, respectively. The previous sample result is consistent with the current samples at $0.42 \mathrm{mg} / \mathrm{kg}$. There are no QA/QC issues associated with previous sample in SDG K0724. All three results are below the remedial action goal (RAG) values. The hexavalent chromium data in SDG K1138 may be considered estimated. Estimated data are useable for decision-making purposes. 


\section{ASBESTOS SDGs}

Four SDGs contained asbestos data only. There were no deficiencies in any of the asbestos data. The SDGs and associated sample numbers are presented in Table E-1.

Table E-1. Asbestos Samples by Sample Delivery Group.

\begin{tabular}{|l|c|l|}
\hline \multicolumn{1}{|c|}{ SDG } & Number of Field Samples & \multicolumn{1}{|c|}{ Sample Numbers } \\
\hline SDG 07-A-1247 & 8 & J14KC3 to J14KC9, J14KD0 \\
\hline SDG 07-A-1185 & 5 & J14KB8, J14KB9, J14KC0 to J14KC2 \\
\hline SDG 07I-2281-01 & 5 & J163R2 to J163R4, J163T0, J163T1 \\
\hline SDG 07-A-1269 & 11 & J14L15 to J14L25 \\
\hline
\end{tabular}

SDG = sample delivery group

The data are useable for decision-making purposes.

\section{FIELD QUALITY ASSURANCE/QUALITY CONTROL}

RPD evaluations of main sample(s) versus the laboratory duplicate(s) are routinely performed and reported by the laboratory. Any deficiencies in those calculations are reported by SDG in the previous sections.

Field QA/QC measures are used to assess potential sources of error and cross-contamination of samples that could bias results. Field QA/QC samples, listed in the field logbooks (WCH 2007a, 2007b, 2007c, 2008), are summarized in Table E-2. The main and QA/QC sample results are presented in Appendix D.

Table E-2. Field Quality Assurance/Quality Control Samples. (2 Pages)

\begin{tabular}{|c|c|c|}
\hline Main Sample & Duplicate Sample & Equipment Blank \\
\hline J14KC8 & J14KC9 & J15K94 \\
\hline J14L19 & J14L20 & \\
\hline J14KB5 & J14KB6 & \\
\hline J14L30 & J14L31 & J14JT6 \\
\hline J163R7 & J164R8 & J163R9 \\
\hline J163R4 & J163T0 & \\
\hline
\end{tabular}


Field duplicate samples are collected to provide a relative measure of the degree of local heterogeneity in the sampling medium, unlike laboratory duplicates that are used to evaluate precision in the analytical process. The field duplicates are evaluated by computing the RPD of the duplicate samples for each contaminant of concern. The 95\% upper confidence limit (UCL) calculation brief in Appendix D provides details on duplicate pair evaluation and RPD calculation. Only analytes with values above five times the detection limits for both the main and duplicate samples are compared. None of the RPDs calculated exceeded the acceptance criteria of $30 \%$.

RPDs for the remaining analytes are not calculated because an evaluation of the data shows the analytes are not detected in both the main and duplicate sample at more than five times the target detection limit. RPDs of analytes detected at low concentrations (less than five times the detection limit) are not considered to be indicative of the analytical system performance. The data are useable for decision making purposes.

A secondary check of the data variability is used when one or both of the samples being evaluated (main and duplicate) is less than 5 times the target detection limit (TDL), including undetected analytes. In these cases, a control limit of \pm 2 times the TDL is used (Appendix D) to indicate that a visual check of the data is required by the reviewer. The difference was less than 2 times the TDL (for all analytes with one or both of the samples less than 5 times the TDL), and did not required the visual check. However, a visual inspection of all of the data is also performed. No additional major or minor deficiencies are noted. The data are useable for decision-making purposes.

\section{SUMMARY}

Limited, random, or sample matrix-specific influenced batch QC issues such as those discussed above, are a potential for any analysis. The number and types seen in these data sets are within expectations for the matrix types and analyses performed. The DQA review of the 128-F-2 verification sampling data found that the analytical results are accurate within the standard errors associated with the analytical methods, sampling, and sample handling. The DQA review for 128-F-2 waste site concludes that the reviewed data are of the right type, quality, and quantity to support the intended use. Detection limits, precision, accuracy, and sampling data group completeness were assessed to determine if any analytical results should be rejected as a result of QA and QC deficiencies. The analytical data were found acceptable for decision-making purposes. The verification sample analytical data are stored in the Environmental Restoration (ENRE) project-specific database prior to being submitted for inclusion in the Hanford Environmental Information System (HEIS) database. The verification sample analytical data are also summarized in attachment 1 of the appropriate 95\% UCL in Appendix D. 


\section{REFERENCES}

BHI, 2000, Data Validation Procedure for Chemical Analysis, BHI-01435, Rev. 0, Bechtel Hanford, Inc., Richland, Washington.

DOE-RL, 2005, 100 Area Remedial Action Sampling and Analysis Plan, DOE/RL-96-22, Rev. 4, U.S. Department of Energy, Richland Operations Office, Richland, Washington.

EPA, 2000, Guidance for Data Quality Assessment, EPA QA/G-9, QA00 Update, U.S. Environmental Protection Agency, Office of Environmental Information, Washington, D.C.

WCH, 2007a, 100 F Area Remedial Sampling, Logbook EFL-1174-2, pp. 62-71, Washington Closure Hanford, Richland, Washington.

WCH, 2007b, 100 F Area Remedial Sampling, Logbook EFL-1174-3, pp. 57-59, Washington Closure Hanford, Richland, Washington.

WCH, 2007c, 100 F Area Remedial Sampling, Logbook EFL-1174-4, pp. 34-35, Washington Closure Hanford, Richland, Washington.

WCH, 2007d, Work Instruction for Verification Sampling of the 128-F-2 Waste Site, Work Instruction No. 0100F-WI-G0037, Rev 0, Washington Closure Hanford, Richland, Washington.

WCH, 2008, 100-F Remedial Sampling, Logbook ELF-1174-4, pp. 83-85, Washington Closure Hanford, Richland, Washington. 